\title{
DEVELOPMENT AND TESTING OF AN INTEGRATED SIGNAL VALIDATION SYSTEM FOR NUCLEAR POWER PLANTS
}

\author{
Final Report - Volume 2
}

DESCRIPTION OF SIGNAL VALIDATION MODULES

B. R. Upadhyaya and T. W. Kerlin

(Principal Investigators)

$$
\begin{gathered}
\text { K. E. Holbert } \\
\text { O. Glockler } \\
\text { V. M. Morgenstern } \\
\text { Z. Frei } \\
\text { J. Olvera }
\end{gathered}
$$

Department of Nuclear Engineering

The University of Tennessee

Knoxville, Tennessee 37996-2300

Subcontractor

Combustion Engineering, Inc.

P. J. Gaudio, Jr.

September 1989

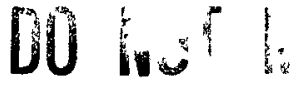
$-21:$ :

Prepared for

THE U.S. DEPARTMENT OF ENERGY

CONTRACT NO. DE-AC02-86NE37959 


\section{DEVELOPMENT AND TESTING OF AN INTEGRATED SIGNAL VALIDATION SYSTEM FOR NUCLEAR POWER PLANTS}

Final Report - Volume 2

DESCRIPTION OF SIGNAL VALIDATION MODULES

B. R. Upadhyaya and T. W. Kerlin

(Principal Investigators)

K. E. Holbert

O. Glockler

V. M. Morgenstern

Z. Frei

J. Olvera

Department of Nuclear Engineering

The University of Tennessee

Knoxville, Tennessee 37996-2300

Subcontractor

Combustion Engineering, Inc.

P. J. Gaudio, Jr.

September 1989

Prepared for

THE U.S. DEPARTMENT OF ENERGY 


\section{DISCLAIMER}

This report was prepared as an account of work sponsored by an agency of the United States Government. Neither the United States Government nor any agency Thereof, nor any of their employees, makes any warranty, express or implied, or assumes any legal liability or responsibility for the accuracy, completeness, or usefulness of any information, apparatus, product, or process disclosed, or represents that its use would not infringe privately owned rights. Reference herein to any specific commercial product, process, or service by trade name, trademark, manufacturer, or otherwise does not necessarily constitute or imply its endorsement, recommendation, or favoring by the United States Government or any agency thereof. The views and opinions of authors expressed herein do not necessarily state or reflect those of the United States Government or any agency thereof. 


\section{DISCLAIMER}

Portions of this document may be illegible in electronic image products. Images are produced from the best available original document. 


\section{SUMMARY}

This report is volume 2 of a three-volume final report on the U.S. Department of Energy sponsored research and development project on Signal Validation for Nuclear Power Plants.

Detailed description of six signal processing modules are presented. These modules are listed below.

1. Generalized consistency checking for single variable (GCCS).

2. Generalized consistency checking for multiple variables (GCCM).

3. Process empirical modeling (PEM) for prediction.

4. Multi-dimensional process hypercube comparison (PHC).

5. Bias and noise detection (BND) module.

6. Univariate autoregression (UAR) modeling.

The signal validation system (SVS) consists of multiple and independent signal processing modules in a parallel architecture. Some of the existing methods have been improved and adapted to the present project. Other modules have been developed under this project and constitute original contribution to signal validation technology. These are GCCM, PEM, PHC and BND modules.

The overall objective of the university-industry research program at the University of Tennessee and its subcontractor, Combustion Engineering, Inc., was to develop and implement a comprehensive signal validation system for current power plants and future advanced reactors. All the signal validation modules were tested extensively using operational plant data from a Westinghouse four-loop PWR and from the EBR-II. The integrated system was implemented in a MicroVAX-II workstation. The SVS architecture and the system-independent feature of the signal 
validation modules incorporate sufficient flexibility for transferring this technology to fossil plants, National Production Reactors, and process industries. 


\title{
ACKNOWLEDGMENTS
}

The research and development was sponsored by the U.S. Department of Energy, Office of Energy Research, Nuclear Engineering research grant program. The continued assistance and suggestion provided by John W. Lewellen (technical monitor) and David M. Woodall (technical co-monitor) is greatly appreciated. We want to thank the DOE Assistance and Acquisition office, Argonne, $\mathbb{L}$, for the help during the grant period. The University of Tennessee extends special thanks to Northeast Utilities Services Corporation for their assistance, and to Argonne National Laboratory-West for the EBR-II data base. The cooperation provided by John I. Sackett and Ron W. King of ANL-West is greatly appreciated. The authors would like to acknowledge $\mathbf{E}$. Filstein for stimulating the hypercube signal validation methodology, based upon the work performed at Combustion Engineering, Inc., using a hypercube for data compression. Many thanks to the staff of the University of Tennessee Nuclear Engineering Department for their assistance in preparing the various reports during the grant period.

\section{DISCLAIMER}

\begin{abstract}
This report was prepared as an account of work sponsored by an agency of the United States Government. Neither the United States Government nor any agency thereof, nor any of their employees, makes any warranty, express or implied, or assumes any legal liability or responsibility for the accuracy, completeness, or usefulness of any information, apparatus, product, or process disclosed, or represents that its use would not infringe privately owned rights. Reference herein to any specific commercial product, process, or service by trade name, trademark, manufacturer, or otherwise does not necessarily constitute or imply its endorsement, recommendation, or favoring by the United States Government or any agency thereof. The views and opinions of authors expressed herein do not necessarily state or reflect those of the United States Government or any agency thereof.
\end{abstract}




\section{CONTENTS}

SECTION

PAGE

SUMMARY

ACKNOWLEDGMENTS $\ldots \ldots \ldots \ldots \ldots \ldots \ldots \ldots \ldots \ldots \ldots \ldots$ iii

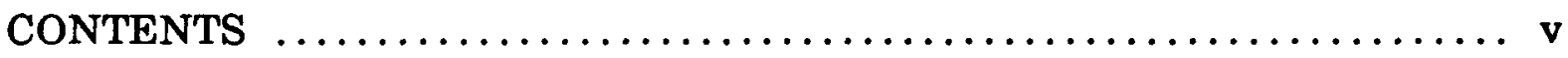

LIST OF ACRONYMS $\ldots \ldots \ldots \ldots \ldots \ldots \ldots \ldots \ldots \ldots \ldots \ldots \ldots \ldots \ldots$ ix

1. INTRODUCTION $\ldots \ldots \ldots \ldots \ldots \ldots \ldots \ldots \ldots \ldots \ldots \ldots \ldots \ldots \ldots \ldots \ldots \ldots$

2. GENERALIZED CONSISTENCY CHECKING OF SINGLE

VARIABLE (GCCS) WITH REDUNDANT MEASUREMENTS $\ldots \ldots \ldots \ldots 5$

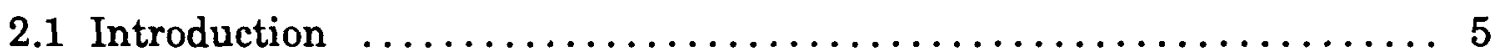

2.2 Generalized Consistency Checking Algorithm $\ldots \ldots \ldots \ldots \ldots \ldots \ldots 5$

2.3 The Sequential Probability Ratio Test (SPRT)

Algorithm $\ldots \ldots \ldots \ldots \ldots \ldots \ldots \ldots \ldots \ldots \ldots \ldots \ldots \ldots \ldots \ldots \ldots \ldots, 12$

2.4 Software System Incorporating GCC and

SPRT Procedures $\ldots . \ldots \ldots \ldots \ldots \ldots \ldots \ldots \ldots \ldots \ldots, 17$

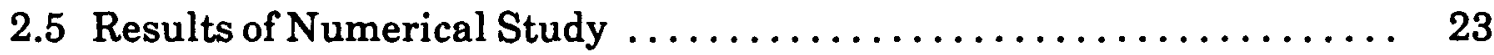

2.6 Application to Operational Data from a PWR $\ldots \ldots \ldots \ldots \ldots \ldots .27$

2.7 Computer Program Specifications $\ldots \ldots \ldots \ldots \ldots \ldots \ldots \ldots \ldots .28$

3. GENERALIZED CONSISTENCY CHECKING OF

MULTIVARIABLE REDUNDANT MEASUREMENTS (GCCM)

AND COMMON-MODE FAILURE DETECTION $\ldots \ldots \ldots \ldots \ldots \ldots \ldots, 59$

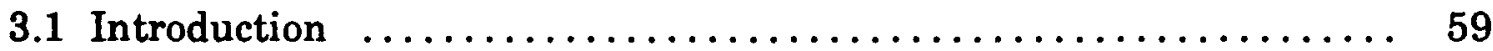

3.2 Outline of the GCCM Methodology $\ldots \ldots \ldots \ldots \ldots \ldots \ldots \ldots \ldots . \ldots 9$

3.3 Single Variable Consistency Checking (GCCS) ..............6 61

3.4 Intersignal Relationships $\ldots \ldots \ldots \ldots \ldots \ldots \ldots \ldots \ldots \ldots \ldots . \ldots \ldots$

3.5 Multivariate Consistency Checking Algorithm (GCCM) .........66

3.5.1 Summary of the GCCM Algorithm $. . \ldots \ldots \ldots \ldots \ldots \ldots, 63$

3.5.2 Description of the GCCM Algorithm $\ldots \ldots \ldots \ldots \ldots \ldots \ldots \ldots, 64$ 
3.6 Examples of Numerical Tests Performed Using the Multivariable GCC Algorithm ................................... 72

3.6.1 Implementation of the GCCM Algorithm ............. 73

3.6.2 Results and Interpretation of Numerical Experiments ...... 77

3.6.2.1 The Case of Partially Degraded

Redundant Signal Sets $\ldots \ldots \ldots \ldots \ldots \ldots \ldots \ldots, 77$

3.6.2.2 The Case of Partially and Totally Degraded

Redundant Signal Sets ..................... 84

4. AUTOMATED GNERATION OF NONLINEAR SYSTEM

CHARACTERIZATION

126

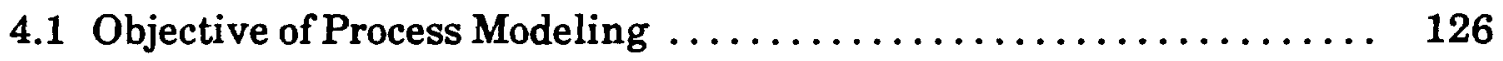

4.2 Outline of the Methodology $\ldots \ldots \ldots \ldots \ldots \ldots \ldots \ldots \ldots \ldots \ldots \ldots$

4.3 The Nonlinear Modeling Algorithm $\ldots \ldots \ldots \ldots \ldots \ldots \ldots \ldots, 128$

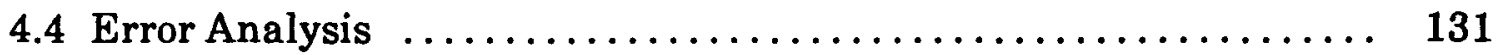

4.5 Results of Application to PWR Operational Data ............. 132

4.6 Results of Application to EBR-II Start-Up Data .............. 134

5. MULTI-DIMENSIONAL PROCESS HYPERCUBE FOR SIGNAL

VALIDATION $\ldots \ldots \ldots \ldots \ldots \ldots \ldots \ldots \ldots \ldots \ldots \ldots \ldots \ldots \ldots, 145$

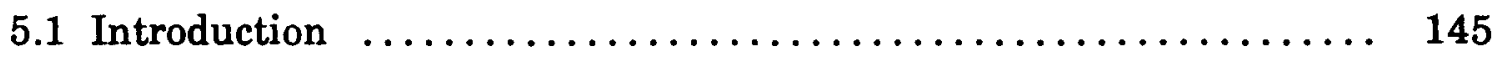

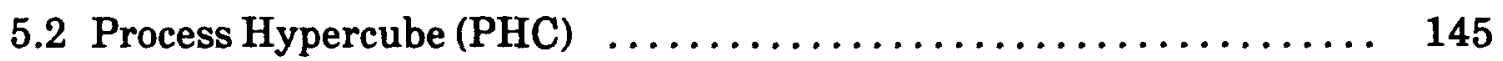

5.2.1 Basis of Approach $\ldots \ldots \ldots \ldots \ldots \ldots \ldots \ldots \ldots \ldots \ldots \ldots \ldots$

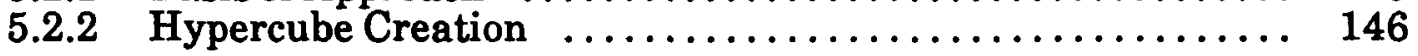

5.2.2.1 Hypercube Cell Size $\ldots \ldots \ldots \ldots \ldots \ldots \ldots \ldots \ldots, 147$

5.2.2.2 Redundant Signal Data Compression .......... 149

5.2.3 PHC Signal Validation $\ldots \ldots \ldots \ldots \ldots \ldots \ldots \ldots \ldots \ldots \ldots, 152$

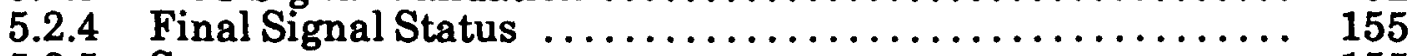

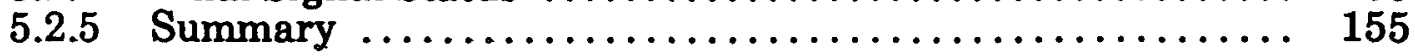

5.3 Implementation of Hypercube Technique $\ldots \ldots \ldots \ldots \ldots \ldots \ldots . \ldots \ldots$

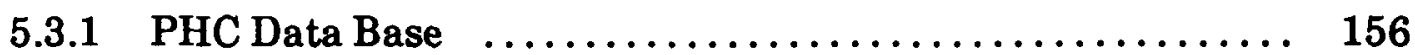

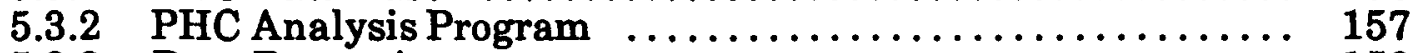

5.3.3 Data Processing $. . \ldots \ldots \ldots \ldots \ldots \ldots \ldots \ldots \ldots \ldots \ldots \ldots \ldots, 158$

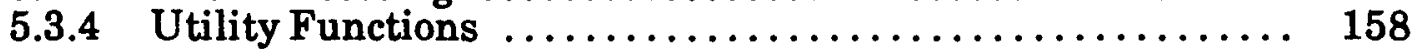

5.4 Data Analysis ............................... 159 
5.4.1 Populating the Hypercube $\ldots \ldots \ldots \ldots \ldots \ldots \ldots \ldots \ldots \ldots, 160$

5.4.2 Experimental Results $\ldots \ldots \ldots \ldots \ldots \ldots \ldots \ldots \ldots \ldots \ldots \ldots, 160$

5.4.3 PWR Data Analysis $\ldots \ldots \ldots \ldots \ldots \ldots \ldots \ldots \ldots \ldots \ldots, 161$

5.4.3.1 Consistent Power Signals $\ldots \ldots \ldots \ldots \ldots \ldots \ldots \ldots, 161$

5.4.3.2 Loss of Signal Example ..................... 162

5.4.3.3 Biased Flow Signal $\ldots \ldots \ldots \ldots \ldots \ldots \ldots \ldots \ldots \ldots, 163$

5.4.4 EBR-I Data Analysis $\ldots \ldots \ldots \ldots \ldots \ldots \ldots \ldots \ldots \ldots \ldots$

5.4.4.1 Lack of Redundancy $\ldots \ldots \ldots \ldots \ldots \ldots \ldots \ldots, 164$

5.4.4.2 Pseudo Redundancy $\ldots \ldots \ldots \ldots \ldots \ldots \ldots \ldots \ldots, 164$

5.4.5 Data Analysis Conclusions $\ldots \ldots \ldots \ldots \ldots \ldots \ldots \ldots \ldots, 165$

5.5 Remarks on the PHC Module $\ldots \ldots \ldots \ldots \ldots \ldots \ldots \ldots \ldots \ldots \ldots$

6. SIGNAL ANOMALY DETECTION MODULE $\ldots \ldots \ldots \ldots \ldots \ldots \ldots . \ldots . \ldots$

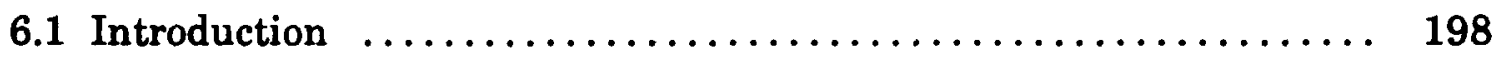

6.2 Methodology $\ldots \ldots \ldots \ldots \ldots \ldots \ldots \ldots \ldots \ldots \ldots \ldots \ldots \ldots \ldots \ldots, 199$

6.2.1 Anomaly Detection and Isolation ................. 199

6.2.2 Anomaly Characterization and Classification $\ldots \ldots \ldots \ldots \ldots, 202$

6.2.2.1 Modified CUSUM Algorithm .............. 203

6.2.2.2 Anomaly Classification $\ldots \ldots \ldots \ldots \ldots \ldots \ldots \ldots \ldots, 205$

6.3 Description of Computer Software System $\ldots \ldots \ldots \ldots \ldots \ldots 207$

6.4 Results of Application of the BND Module ............... 212

6.5 Remarks on the BND Module ........................ 214

7. MULTIVARIATE AUTOREGRESSION (MARS) MODELING FOR SENSOR AND PROCESS ANOMALY DETECTION AND

ISOLATTON $\ldots \ldots \ldots \ldots \ldots \ldots \ldots \ldots \ldots \ldots \ldots \ldots \ldots \ldots \ldots \ldots \ldots \ldots \ldots, 243$

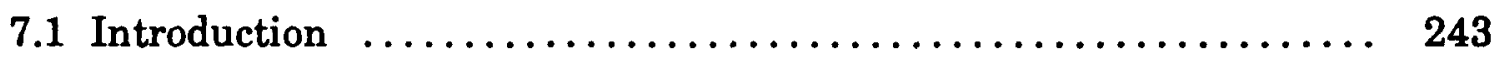

7.2 Multivariate Autoregressive Modeling of Process Variables ....... 243

7.3 Statistical Characteristics in the Frequency Domain .......... 246

7.4 Decomposition of Multivariate Dynamic System ............ 248

7.5 Application to Operating PWR Plant Noise Signals ........... 251

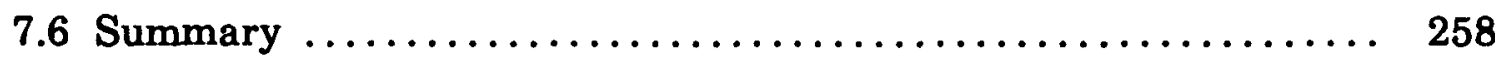


8. UNIVARIATE AUTOREGRESSION (UAR) TIME SERIES MODELING FOR SENSOR SURVEILLANCE

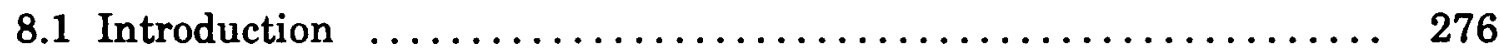

8.2 Determination of Univariate Autoregression Models ........... 276

8.3 Estimation of Frequency- and Time-Domain Signatures ......... 278

8.4 Example of Application to a PWR $\ldots \ldots \ldots \ldots \ldots \ldots \ldots \ldots \ldots . \ldots \ldots$

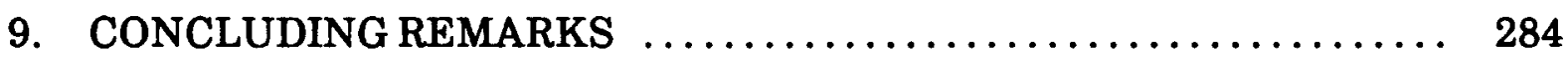

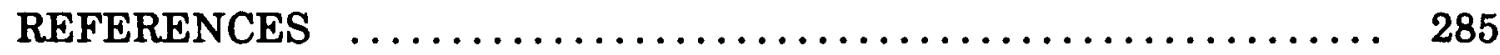




\section{LIST OF ACRONYMS}
ANL
Argonne National Laboratory.
BND
Bias and noise detection.
CE
Combustion Engineering, Inc.
CII
Cumulative inconsistency index.
CUSUM
Cumulative sum.
EBR-II
Experimental Breeder Reactor-II.
ESQ
Expert system for qualitative signal validation.
GCC
Generalized consistency checking.
GCCS
Single-variable generalized consistency checking.
GCCM
Multivariate generalized consistency checking.
MAR
Multivariate autoregression modeling.
MISO Multiple-input single-output model.
ORNL Oak Ridge National Laboratory.
PEM Process empirical modeling.
PHC Process Hypercube Comparison.
PWR Pressurized water reactor.
SE System executive.
SPRT Sequential probability ratio test.
STP Signal transmission path.
SVS Signal validation system.
UAR Univariate autoregression modeling. 


\section{Section 1}

\section{INTRODUCTION}

The approach for signal validation, instrument fault isolation and characterization incorporates some of the methods used in the past, and new techniques developed under this project [1]. The general architecture consists of parallel signal processing modules. Each of the modules performs a specific task. The current architecture consists of seven signal processing modules. There is no direct communication between the modules. Any cross information desired must be requested at the system executive level. Figure 1.1 shows the schematic of the signal validation system. A complete bibliography, including recent publications in fault detection, has been developed under this project and is given in Appendix A, Volume $1[2]$.

The system executive performs several tasks: sequencing of module operation, requisition for additional data, evaluation of signal validation information from the various modules, displaying instrument status to the operator, and the decision about the validity of the signal(s) under consideration. A higher level language is used for communication purposes. The central data base consists of plant data, subsystem models, past failure history, and instrument-related information.

The signal validation is performed at primarily steady-state or quasi steady-state plant operational conditions. However, some of the modules are capable of signal validation during transients. Wideband signal analysis is also included to monitor the fluctuating components of signals at steady-state operation. Process diagnostics (actuators, mechanical components, etc.) is not one of the objectives of the current program. But wideband frequency analysis and critical signal tracking methods implicitly contain information about the process behavior. 
The integrated signal validation system (SVS) is being implemented in a MicroVAX-II workstation. All the modules, except the expert system and hypercube modules, are also operational in an IBM-PC or compatible system. The Laboratory for Information and Systems Analysis, within the Nuclear Engineering Department of the University of Tennessee, consists of four VAX workstations and a 386 System. All the computers are networked using the ethernet, and have access to other computing facilities.

This report describes the following six signal validtion modules. All the modules have been tested extensively using both simulation data and power plant operational data.

1. Generalized consistency checking for single variable (GCCS) with redundant measurements.

2. Generalized consistency checking for multiple variable (GCCM) with application to common-mode failure detection.

3. Process empirical modeling (PEM) for prediction of one or more critical signals, and to monitor sensor degradation.

4. Multi-dimensional process hypercube comparison (PHC) for data compression and for tracking instrument and process behavior.

5. Bias and noise detection (BND) module for detecting and characterizing signal anomalies.

6. Multivariate autoregression (MAR) modeling of the fluctuating components of signals for process anomaly and sensor maloperation detection. 


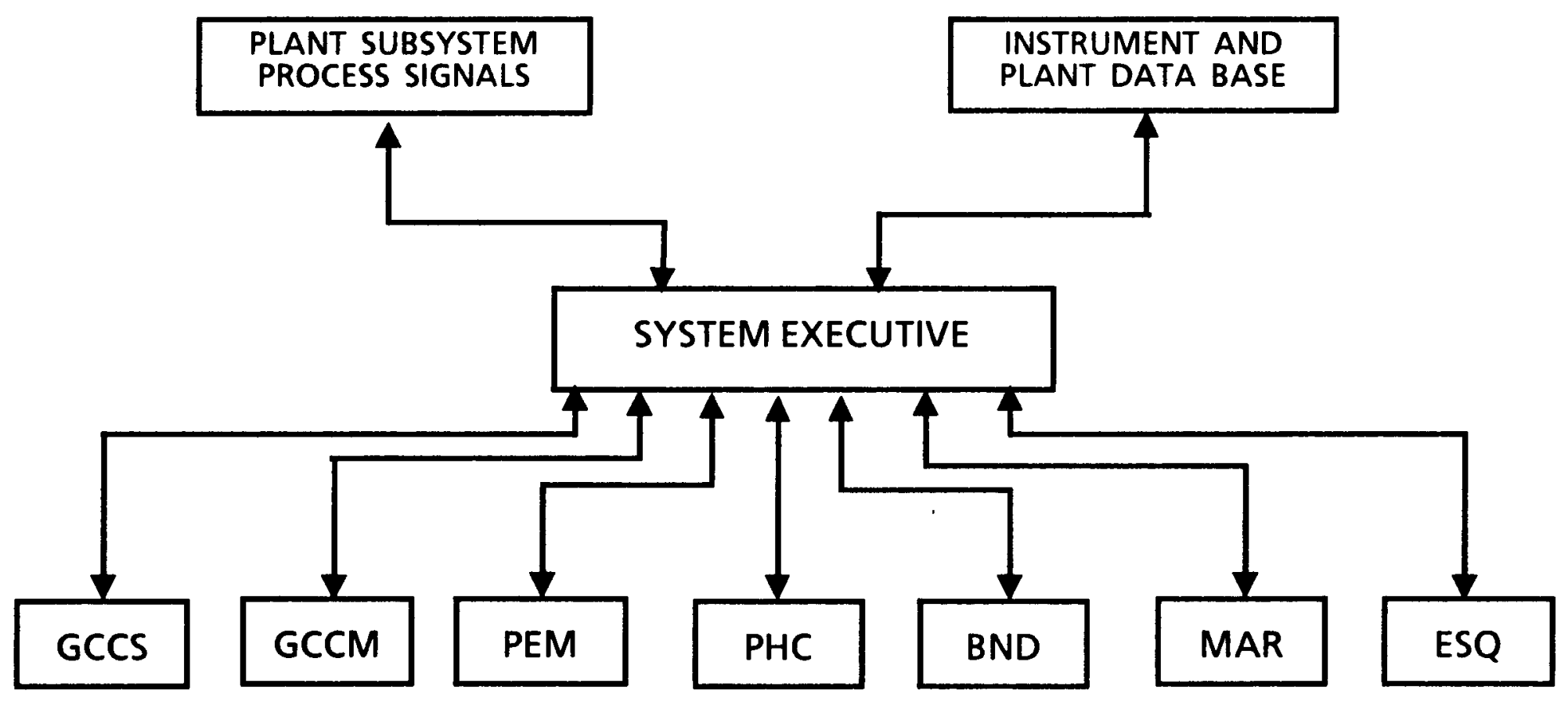

Figure 1.1. Schematic of the Signal Validation System (SVS) architecture showing seven signal validation modules. 
4

7. Univariate autoregression (UAR) modeling of signal fluctuations for sensor surveillance. This is a special case of the multivariate autoregression (MAR) modeling.

Detailed discussion of the above six modules are presented in Sects. 2 - 8, respectively. Each section contains a description of the method, implementational aspect, a brief description of the computer software (where necessary) and the results of application. Some concluding remarks and highlights of Volume 3 are given in Sect. 9. 


\section{Section 2}

\section{GENERAIIZED CONSIS'IENCY CHECKING OF SINGLE VARIABLE (GCCS) WI'TH REDUNDAN'T MEASUREMENTS}

\subsection{Introduction}

This section discusses the basic principles and recent developments of a signal validation software system utilizing redundant sensor readings. The purpose of this work is to develop a system of computer software for validating signals in nuclear power plants. The feasibility and performance of the above software system have been demonstrated in numerical tests, and will be discussed in this report.

The basis of the signal validation procedure is the use of two previously developed methods, the Generalized Consistency Check (GCC), $|3,4|$ and the Sequential Probability Ratio Test (SPRT)|5-71, and their combination in a Decision Estimater (D/E) unit. The software system discussed here consists of microcomputer-based data acquisition, data prequalification, signal validation (GCC \& SPR'T methods), and display modules. The information generated by the algorithms is displayed on the monitor in a tabular form, and a graphical display shows the changes as a function of time.

\subsection{Generalized Consistency Checking (GCC) Algorithm}

The GCC method is a systematic check of inconsistencies among redundant signals measuring the same process variable. The algorithm provides information about measurement inconsistencies at each sampling instant. After excluding the signals with maximum inconsistency indices, the best estimate at any time can be computed as a weighted average of the remaining signals. The procedure is then repeated for subsequent sampling instants. The algorithm does not make comparisons between sets of measurements at different times. Any two like measurements are defined to be inconsistent if the difference between their values is 
greater than a specified threshold value. This threshold value depends on the selected signal pair and is based on sensor tolerance or technical specifications. The inconsistency indices of the individual measurements and the best estimate for the given process variable will be determined as functions of sampling instants.

The availability of sufficient redundancy is an important requirement for this signal validation method. If only one signal is available the signal validation is limited to the observation of unusual behavior by checking the changes in the sensor time constant and signal-to-noise ratios. In case of duplex redundancy, the algorithm is capable of detecting a sensor failure, but not the identification of the failure itself. The triple redundancy provides the capability of detecting and identifying the sensor failure. The process variable can also be reconstructed in the form of weighted average after excluding the most inconsistent measurements.

To achieve the required levels of redundancy, a redundant array of like sensors may be used when available. If direct or hardware redundancy is not available, carefully validated and tuned analytical models may be used. The analytical redundancies are obtained from physical or empirical relationships that exist among variables in the system measured by dissimilar sensors. In case of physical models representing mass, energy or momentum balances, or system descriptions in the form of differential equations, the structure or the functional form of these relationships is fixed, only fitting the parameters to the particular case needs to be performed.

The empirical modeling of a system requires the knowledge of all important variables having significant effects on signals to be modeled. A flexible algorithm doing nonlinear modeling of steady-state set measurements has been developed and described in Sect. 4. The microcomputer based program fits optimal polynomial functions to the measured values recorded at different steady-state conditions. The 
identified nonlinear multivariate analytical system is used for predicting the signal state.

Another important requirement in the case of single $\mathrm{D} / \mathrm{E}$ module application is that the analytical models must be driven by preliminary validated sensor readings, or at least the reference model must be identified during "normal" operation. Figure 2.1 shows a schematic of $\mathrm{D} / \mathrm{E}$ and analytical measurement calculator modules for a single variable. For multivariate signal validation, the $\mathrm{D} / \mathrm{E}$ and the analytical measurement calculator units may be connected as a network of single variable validation modules. The input to the $\mathrm{D} / \mathrm{E}$ module are outputs of different sensors and/or analytical models. The output of a $\mathrm{D} / \mathrm{E}$ unit at a given time instant are the error messages to the user, the different error parameters (inconsistency and exclusion indices, SPRT parameters), and the estimate of the process variable based on the consistent subset of signals. The number of input to the $\mathrm{D} / \mathrm{E}$ unit (represented by "OR" gate) may vary and an estimate is still possible even after one or more of the input signals is degraded. However, a minimum of three signals is required to identify a faulty signal, and to obtain a reliable estimate. The analytical module in Fig. 2.1 is represented by an "AND" gate, because all of its input signals have to be available to calculate an analytical output. Besides the application of the GCC method to redundant signals measuring the same process variable, the method can be used for checking sensors not assumed to measure the same value. To demonstrate this point consider a reactor core equipped with in-core neutron detectors and assembly outlet thermocouples. The assembly-wise power distribution can be calculated by using either the neutron detector signal or the assembly outlet thermocouple readings for the fuel assemblies furnished with these detectors. Moreover, the three dimensional power distribution can be determined for the whole reactor core by a global interpolation, or by a least-squares fit. To this end, some 
preliminary knowledge specific to the particular reactor core is required. By this calculation, the three dimensional power distribution can be determined including all fuel assemblies regardless of the existence of detectors. In case of a fuel assembly equipped with neutron or temperature detectors, the assembly power given by a global fit and the assembly power based on the assembly's own instrumentation should have comparable values. To compute the ratios of the actual and the calculated power for all the instrumented fuel assemblies, the GCC algorithm can be used for a systematic search of degraded sensors. In a normal case these ratios as input signals to the GCC have values close to unity. The assemblies with ratios not equal to one, and the extent of the overall deviation can be identified by the GCC in a systematic and simple manner.

The first part of the algorithm determines the inconsistencies among a given set of measurements at time instant $\mathrm{k}$. This is based on comparing signals in pairs in the following manner. Suppose an error bound, $\eta_{i}$, has been assigned to each signal as an input parameter to the algorithm. If $m_{i}(k)$ and $m_{j}(k)$ form a pair of measurements at time instant $k$, the absolute value of their difference is compared with the sum of their error bounds. The pair of measurements is consistent if $|3,4|$

$$
\left|m_{i}(k) \cdot m_{j}(k)\right| \equiv \eta_{i}+\eta_{j}
$$

If a total of $\ell$ different measurements of the same process variable exist, after checking $\ell(\ell-1) / 2$ pairs, an error index, $\mathrm{I}_{\mathrm{i}}$, is assigned to every signal. The error indices of both signals are increased by one every time the given pair is inconsistent. The comparison is performed for all possible signal pairs. The error index varies between zero and $\ell-1$. Further management and isolation of faulty readings is based on: the values of maximum $\left(I_{\max }\right)$ and minimum $\left(I_{\min }\right)$ error indices, the number of measurements (signals) having the maximum, and the minimum error index, $\mathrm{N}_{\max }$ 
and $\mathrm{N}_{\min }$, respectively. This complex procedure is performed in the second part of the algorithm. The corresponding logical flow chart is shown in Fig. 2.2.

Depending on the values of $I_{\min }, I_{\max }, N_{\min }$ and $N_{\max }$, and the individual inconsistency indices, the best estimate for the process variable can be calculated directly, or only after repeating the whole reasoning with eliminations of the most faulty signals. If $I_{\max }=0$, all measurements are consistent and their average is the estimate. If $I_{\max }>0$, but $I_{\min }=0$, this means the measurements are partially consistent (no measurement is isolated as inconsistent), and the estimate is expressed as a weighted average. If $I_{\max }>0$ and $I_{\min }>0$, then the $N_{\max }$ will be isolated as faulty and excluded from further calculations. The whole procedure will be repeated with the remaining measurements in the next pass. If all the measurements are inconsistent, that is, $\mathrm{N}_{\max }=$ number of measurements in the current pass, then no estimate is possible on the basis of the current observation. The fault is detected, but not isolated. In this case, for steady-state or quasi-steadystate operations, the comparison between the previous estimate and the current signals may provide additional information. The estimate at the current sample may be calculated using only the signals consistent with the previous estimate. Using the inconsistency indices defined above, and some a priori weighting factors, $\{w i\}_{i=1}^{\ell}$ for each signal, the estimate of the measurements, $X^{*}(t)$ can be computed at every sampling instant:

$$
\mathrm{X}^{*}(\mathrm{t})=\frac{\sum_{\mathrm{i}=1}^{\mathrm{k}} \mathrm{w}_{\mathrm{i}}\left(\ell-1-\mathrm{I}_{\mathrm{i}}\right) \mathrm{m}_{\mathrm{i}}(\mathrm{t})}{\sum_{\mathrm{i}=1}^{\mathrm{k}} \mathrm{w}_{\mathrm{i}}\left(\ell-1-\mathrm{I}_{\mathrm{i}}\right)}
$$

where

$k$--- the remaining number of consistent signals at sampling instant $t$,

$\ell$..- the total number of signals,

$(\ell-k)$--- the number of excluded signals with inconsistency index 


$$
I_{i}=\max _{1} \leq j \leq \ell I_{j}
$$

The weighting factors $\left\{\mathrm{w}_{\mathrm{i}}\right\}_{\mathrm{i}=1}^{\ell}$ in Eq. (2.2) are defined arbitrarily. One possibility is to choose them to be inversely proportional to a power of the error bounds of the corresponding signals. Another procedure is to define the $w_{i}$ factors using the log likelihood ratio values given by the SPRT algorithm discussed in the next chapter. This would also be an appropriate way to include some feedback of the SPRT results to the GCC calculations. The way of defining the weighting factors in a run is arbitrary, and the user can select one of the options built in the algorithm.

The algorithm also checks the current measurements against the past value of the estimate $X^{*}(t-\Delta t)$. The j signals for which

$$
\left|m_{i}(t)-X^{*}(t-\Delta t)\right| \leq \eta_{i}, i=1, \ldots, j
$$

is satisfied are given higher priority, and are used to calculate another estimate for the current time instant based on only the current signal values consistent with the previous value of the estimate (and possibly with each other)

$$
\mathrm{X}^{*}(\mathrm{t})=\frac{\sum_{\mathrm{i}=1}^{\mathrm{j}} \mathrm{w}_{\mathrm{i}} \mathrm{m}_{\mathrm{i}}(\mathrm{t})}{\sum_{\mathrm{i}=1}^{\mathrm{j}} \mathrm{w}_{\mathrm{i}}}
$$

If no such values of $m_{i}$ exist $(j=0)$, no estimate can be calculated using this procedure. In case of steady-state or quasi-steady-state processes, the two estimates derived by two different ways should have similar values. Under unusual circumstances such as common-mode failures, or if the number of degraded signals is more than ( $(-1) / 2$, only the estimate given by Eq. (2.3) may be available, possibly including only one or two signals.

To demonstrate the performance of the logic outlined in the flow chart of Fig. 2.2, some typical failure combinations are explained in the following examples. Let five measurements $m_{1}, m_{2}, m_{3}, m_{4}$ and $m_{5}$ be available $(\ell=5)$, and consider the following cases: 


\section{Example 1:}

Suppose that $\mathrm{m}_{3}, \mathrm{~m}_{4}$ and $\mathrm{m}_{5}$ failed simultaneously, but not identically, i.e. $I_{1}=3, I_{2}=3, I_{3}=4, I_{4}=4$ and $I_{5}=4$. Since $I_{\min }=3>0, I_{\max }=4(=\ell-1)$ and $N_{\max }=3$ $<\ell=5$, the last three measurements having the maximum possible inconsistency index 4 are isolated from the set, and the inconsistency indices of the remaining two measurements decremented by $N_{\max }=3$, i.e. $I_{1}=0$ and $I_{2}=0$. The resulting estimate is based only on these two measurements in the form of a weighted average (see Eq. (2.2)).

Example 2:

Suppose that $m_{3}, m_{4}$ failed identically, and $m_{5}$ failed in a different way, i.e. $I_{1}=3, I_{2}=3, I_{3}=3, I_{4}=3$ and $I_{5}=4$. As in the previous case, $I_{\min }>0, I_{\max }=4(=\ell-1)$ and $N_{\max }=1<\ell=5$, the last measurement is isolated as degraded, and the inconsistency indices of the remaining four measurements $(k=4)$ are decremented by $N_{\max }=1$ in the next pass, i.e. $I_{1}=2, I_{2}=2, I_{3}=2$ and $I_{4}=2$. There are two consistent pairs $\left(m_{1}, m_{2}\right)$ and $\left(m_{3}, m_{4}\right)$ that are mutually inconsistent, and the recalculated parameters for the set of four signals are $0<I_{\max }=2<\mathrm{k}-1, \mathrm{I}_{\min }>0$ and $N_{\max }=k=4$. The degradation was detected, but faulty pair of signals cannot be isolated, and no estimate can be obtained. In case of quasisteady-state processes, an estimate can be computed at the current sample using only the signals consistent with the past estimate (see Eq. (2.3)).

Example 3:

Suppose that $\mathrm{m}_{4}$ and $\mathrm{m}_{5}$ failed simultaneously and identically, while the first three signals are partially inconsistent $\left(m_{1}\right.$ consistent with $m_{2}$ and $m_{3}$, but $m_{2}$ and $\mathrm{m}_{3}$ are not consistent), i.e. $\mathrm{I}_{1}=2, \mathrm{I}_{2}=3, \mathrm{I}_{3}=3, \mathrm{I}_{4}=3$ and $\mathrm{I}_{5}=3$. Since $\mathrm{I}_{\min }=2<0$, $I_{\max }=3<\ell-1, N_{\max }=4<\ell=5$ and $N_{\min }=1$, the last four measurements are isolated from the set with inconsistency indices 3 (less than the possible maximum, 4) and the inconsistency index of the remaining one measurement decremented by 
$N_{\max }=3$, i.e. $I_{1}=0$ and the measurement $m_{1}$ itself is the resulting estimate.

Example 4:

Suppose that $\mathrm{m}_{5}$ failed (inconsistent with all the other four signals), and the first four signals are partially consistent $\left(\mathrm{m}_{1}\right.$ consistent with $\mathrm{m}_{2}$ and $\mathrm{m}_{3}, \mathrm{~m}_{3}$ consistent with $m_{1}$ and $m_{4}$, but $m_{2}$ and $m_{4}$ are not consistent), i.e. $I_{1}=2, I_{2}=3, I_{3}=2, I_{4}=3$ and $I_{3}=4$. Since $I_{\min }=2>0, I_{\max }=4=\ell-1, N_{\max }=1$ and $N_{\min } n=2$, the last measurement is isolated from the set with inconsistency index 4 and the inconsistency indices of the remaining four measurements decremented by $\mathrm{N}_{\max }=1$. Only the first four measurements are considered in the next pass, where $\mathrm{m}_{2}$ and $\mathrm{m}_{4}$ were isolated with inconsistency indices 2 . In the next pass, the remaining two measurements $m_{1}$ and $m_{3}$ were consistent, and were used in the calculation of an estimate with zero inconsistency indices (see Eq. (2.2)).

The original version of the GCC method makes a decision at each sample based on information available at that instant only. A set of sensors may be inconsistent at a particular sampling instant due to noise spikes or pulses, and can be consistent for the subsequent samples. To overcome this problem a cumulative error index can be assigned to each sensor by increasing (decreasing) the index by one every time the sensor is inconsistent (cunsistent). When this index reaches a specified upper or lower bound, a decision is made that the sensor is in its degraded or normal state. This is not an optimal procedue since these bounds are fixed and the magnitude of a signal degradation is not utilized. An optimal procedure, based on specified missed and false alarm probabilities and on the actual degree of signal failures, is discussed in the next section.

\subsection{The Sequential Probability Ratio Test (SPRT) Algorithm}

The SPRT has the ability to sequentially check for sensor degradation, and to record the sensor degradation history. The SPRT makes decisions on the basis of 
cumulative information provided by the measuement history (Wald[8]) rather than based on a single observation. Contrary to the GCC method the SPRT does not make intersignal comparison or consistency checking among the signals. However, correlating the results of an individual SPRT applied to each signal independently may provide additional information on common cause failures usually not available by single signal analysis.

The SPRT is an optimal procedure in the sense that a minimum number of samples is required to make a decision based on specified missed and false alarm probabilities. These quantities provide a measure of confidence for the decision.

The SPRT' is applied to the difference between the sensor output value and the estimated value of the measured process variable. The latter will be approximated by the calculated estimate obtained as a result of the application of the GCC program. This estimate still has some uncertainty inherent to the GCC procedure, which may increase the false and missed alarm probabilities of the SPRT.

Let the measured value of a process variable $\mathrm{X}$ given by a particular sensor be $X\left(t_{n}\right)$ at time instant $t_{n}$, and let $X^{*}\left(t_{n}\right)$ be the estimate of $X$ computed by the GCC program. The measurement residual $s\left(t_{n}\right)=X\left(t_{n}\right)-X^{*}\left(t_{n}\right)$ is computed at each sample during normal operation in order to determine a mean $\mu_{0}$ and a variance $\sigma_{0}^{2}$. These define the Gaussian density function modeling the normal mode signal error

$$
P_{v}=p\left(s ; \mu_{0}, v_{0}^{2}\right)=\frac{1}{\sqrt{ } 2110_{0}^{2}} \exp \left(-\frac{\left(s-\mu_{0}\right)^{2}}{20_{0}^{2}}\right)
$$

For a normal sensor the mean of the error should be equal to zero, $\mu_{0}=0$. The sensor failure can be detected by a change in the mean value $\mu_{0}$, or a change in the variance $\sigma_{0}^{2}$. Failure thresholds in terms of mean value and variance are defined, and the corresponding Gaussian density functions $p_{1}=p\left(s ; \mu_{1}, \sigma^{2}\right)$ model the output 
statistics from degraded sensors. The present approach is based on the SPRT of the normal mode against an alternate degraded mode, assuming that both modes can be characterized by Gaussian distributions.

The basis for the SPRT lies in the recursive calculation of the logarithm of the likelihood ration (LLR) function $\lambda_{n}$, representing the degradation information of a sensor based on $n$ samples:

$$
\Lambda_{\mathrm{n}}=\ln \frac{\mathrm{p}\left(\mathrm{s}_{1}, \mathrm{~s}_{2}, \ldots, \mathrm{s}_{\mathrm{n}} \mid \mu_{1}, \mathrm{o}_{1}^{2}\right)}{\mathrm{p}\left(\mathrm{s}_{1}, \mathrm{~s}_{2}, \ldots, \mathrm{s}_{\mathrm{n}} \mid \mu_{0}, \mathrm{o}_{0}^{2}\right)}
$$

The logarithm of the likelihood ratio defined above will be updated at each sampling instant, substituting the new sensor error $s_{n+1}$ into the functional form of LLRs. Its value will be compared against two boundaries ( $\mathrm{A}<0$ and $\mathrm{B}>0$ ) derived from the specified error probabilities of false (a) and missed ( $\beta)$ alarms[11]

$$
\begin{aligned}
& A=\ln \left(\frac{\beta}{1-u}\right) \\
& B=\ln \left(\frac{1-\beta}{a}\right)
\end{aligned}
$$

For a normal sensor, the ratio would decrease and finally reach the specified bound $A<0$. Then the decision is made that the sensor is normal, and the ratio is initialized by setting it equal to zero. The continuous version of the increase (decrease) of the ratio for a degraded (normal) sensor can be represented by a stochastic diffusion process drifting between the two boundaries.

The sensor errors $\left(t_{n}\right)$ is assumed to be independent from sample to sample so that the joint density function in the expression for the LLR is obtained by multiplying the individual Gaussian density functions. Rewriting the defining equation for LLR, the recursive procedure becomes additive: 


$$
\lambda_{n}=\Lambda_{\mathrm{n}-1}+\ln \frac{\mathrm{p}\left(\mathrm{s}_{\mathrm{n}} \mid \mu_{1}, \mathrm{o}_{1}^{2}\right)}{\mathrm{p}\left(\mathrm{s}_{\mathrm{n}} \mid \mu_{0}, \mathrm{o}_{0}^{2}\right)}
$$

Distinguishing between bias (mean) degradation and excessive noise degradation, the general form of the LLR can be simplified. To check for only bias degradation $\left(\sigma_{1}=\sigma_{0}=\sigma\right)$, the expression for the LLR is reduced to the form [11]:

$$
\Lambda_{n}=\Lambda_{n-1}+\frac{\mu_{1}}{o^{2}}\left(s_{n}-\frac{\mu_{1}}{2}\right) .
$$

To detect for only excessive noise levels $\left(\mu_{1}=\mu_{0}=0\right)$ the LLR has the form

$$
\lambda_{n}=\lambda_{n-1}+\frac{1}{2}\left(\frac{1}{\sigma_{0}^{2}}-\frac{1}{\sigma_{1}^{2}}\right) s_{n}^{2}+\ln \frac{\sigma_{0}}{o_{1}} .
$$

These two recursive formulas were used in the SPRT program to detect bias and noise degradation, respectively. In case of bias degradation, the error series $s\left(t_{n}\right)$ was calculated for each redundant signal when the estimate $x^{*}\left(t_{n}\right)$ could be determined by the program GCC. The noise components of the signals must be separated from the DC components by high pass filters, and stored in a separate data file. This file serves as the in put data to the SPRT for noise detection.

While checking for bias degradation, instead of checking for both the normal mode and the degraded mode, an a priori assumption is made that a sensor is in the normal mode at the beginning of the SPRT. Consequently, a drift of theLLR parameter in the negative direction does not provide any additional information, since this is expected from a sensor in the normal state. A control action of resetting the LLR parameter to zero is applied, every time it is negative. The previously accumulated information is of no value. If the LLR drifts to values larger than zero, the sensor is more likely in the degraded mode, and no control action is applied in 
this case. The detection of a degraded signal needs more observations, thus the recursive calculation of the LLR parameter proceeds $[7,8]$.

For the one-sided case, a new upper bound is based on a specified mean time between the occurrence of false alarms and on the tolerance on mean bias and signal variance. These parameters represent a trade off between the time it takes to detect a degradation and the frequency of false alarms. The bounds $A$ and $B$ defined for the two-sided system were based on specified false alarm and missed alarm probabilities. In the one-side SPRT for detecting bias degradation, a specified mean time between the false alarms, T, were used to determine a degradation threshold. The relationship between the new bound $\mathrm{B}^{*}$ and the specified mean time between false alarms, $\mathrm{T}$, is given by $[7]$.

$$
\mathrm{T}=\frac{2 \mathrm{o}^{2}}{\mathrm{\mu}_{1}^{2}}\left(\mathrm{e}^{\mathrm{B}^{*}}-\mathrm{B}^{*}-1\right)
$$

For the case of large $T$, which is always worth maintaining, an approximate value for the bound $\mathrm{B}^{*}$ is given by

$$
\mathrm{B}^{*}=\ln \left(\frac{\mu_{1}^{2}}{2 \mathrm{o}^{2}} \mathrm{~T}\right)
$$

The detection performance $\tau(\mathrm{T})$ is defined as the mean detection time for a specific mean time $T$ between false alarms, and has the form $[7,8]$

$$
\mathrm{t}(\mathrm{T})=\frac{2 \mathrm{o}^{2}}{\mu_{1}^{2}}\left[\ln \left(\frac{\mu_{1}^{2}}{2 \mathrm{o}^{2}} \mathrm{~T}\right)-\frac{3}{2}\right)
$$

Maintaining the same specified mean time between false alarms, the following relationship was derived between the boundaries of the one- and two-sided systems $[7 \mid$ 


$$
e^{B^{*}}-B^{*}-1=A \frac{e^{B}-1}{e^{A}-1}-B
$$

where A and B are defined in Eqs. (2.6) and (2.7). If the LLR for bias degradation is computed for a time greater than the specified mean time between false alarms, and the LLR does not exceed the degradation bound, then the LLR is reinitialized to zero.

This one-sided approach developed for detecting bias degradation has several advantages.

- Checking the LLR parameter against the lower bound $A<0$ representing the normal state is not necessary.

- The extra time delay in detecting the degraded mode, due to the negative magnitude of the LLR accumulated under the normal mode, is eliminated when the control resetting to zero is applied. That is, if only the degradation test is performed, less observation time is required.

- If a sensor degradation occurs, the probability of its ultimate detection is one (the missed alarm probability is not defined).

In the software system discussed below, the SPRT either for bias or for noise degradation is optional, and is performed after the GCC part of the program is completed.

\subsection{Software System Incorporating GCC and SPRT Procedures}

The off-line application of the GCCSPRT program requires that the data files containing the prerecorded digital signals (both for the actual values and the noise components) have a specific form. This data file should contain all the available direct and analytical redundant measurements to be validated. 
To initialize the GCCSPRT program the user must respond to some interactive prompts from the keyboard. Each prompt and the proper response is discussed in detail below.

"ENTER THE NAME OF MEASUREMENTT DATA FILE:"

This input requires the name of the data file containing both the direct redundant signals and the analytical signals, if any. Each record contains up to 16 real number (four bytes each) representing simultaneous samples of the redundant signals at a sampling instant.

"NUMBER OF' SIGNALS IN 'THE DA'TA FILE (INTEGER):"

The number of signals analyzed, that is, the number of values in each record. "NUMBER OF POINTS/SIGNAL:"

The number of samples per signal. This determines the number of records in the data file, and is limited by the memory size of the computer. "ENTER 1 IF PROCESS NOISE DATA:"

If the data file contains noise signals, the program will perform the SPRT analysis for noise degradation. Otherwise, it is skipped and so are the following two prompts.

"ENTER NOISE DATA FILÉ NAME:"

The structure of the noise data file is the same as that of the redundant signals, so we should have as many noise signals and samples as the number of redundant measurements.

"ENTER SPECIFIED FALSE AND MISSED ALARMS PROBABILITIES FOR NOISE DEGRADATION (REAL):" 
This prompt requires the false and missed alarm probabilities for SPRT for noise degradation (suggested value is 0.001 for both).

"ENTER TIME STEPS IN SEC BETWEEN SAMPLES (REAL):"

The input specifies the time period between subsequent samples. "NUMBER OF SAMPLES TO SKIP PERIODICALLY:"

This allows the user to skip samples if the original sampling rate was too high. It is an integer number larger than or equal to zero.

"ENTER SPECIFIED MEAN TIME IN SEC BETWEEN FALSE ALARMS FOR BIAS DEGRADATION (REAL):"

This input specifies the false alarm criterion for SPRT for bias degradation. The missed alarm probability is zero in the modified (one-sided) SPRT. To get values of the false alarm probability of the order of $0.001,10,000$ time steps is recommended for the above input.

"ENTER"

- ERROR BOUND,

- MAXIMUM ACCEPTABLE BIAS MAGNITUDE,

- NORMAL STANDARD DEVIATION

- MAXIMUM ACCEPTABLE STANDARD DEVIATION FOR EACH SIGNAL:"

These parameters specify the normal and the maximum acceptable signal error magnitudes and variances for each signal: The first parameter is used in the GCC algorithm, the second is used in the SPRT algorithm for bias degradation, the third is necessary in both SPRTs for bias and noise degradation, while the fourth parameter is used only in case of SPRT for detecting noise degradation. 
"ENTER 1 FOR ON LINE TABLE DISPLAY ON THE SCREEN:"

As an option, the display generated during the execution of the GCC program can be seen on the screen. The tabular form contains the resulting output parameters and error messages for five signals. The information appearing on the screen is discussed in this chapter. The table is updated for each analyzed sample.

"ENTER 1 FOR PRINTING OUT RESULTING DIRECTIONS:"

As another option, the results of the GCC and SPRT algorithm can be printed on a line printer as a function of the sampling instants analyzed. A sample output is shown in Figs. 2.4-2.19.

The results generated during the execution of the algorithm GCCSPRT are stored in a data file named SVSTORAGE.DAT. This will be used by the second program of the batch file named SVPLOT to creat plots of time dependent calculated parameters. These include estimates of the process variable, the inconsistency and exclusion indices for each signal as a function of time, and the sequential probability ratio functions for noise and bias degradation. These time dependent functions provide an overall descriptiun of the consistency of the redundant measurements over the analyzed time period. There is an option to display the results on the screen as they are generated. The table appears on the screen and contains several variables related to signal validation. It is updated at every sample. Also, the corresponding failure messages generated by the GCC and the SPRT programs related to the status of signals at the given time instant will appear on the screen. Some typical failure messages for different outcomes are shown below.

- "All sensors are consistent and used in estimation"

- "All sensors are used in estimation with different weights"

- "Number of partially consistent measurements" 
- "All measurements are inconsistent!"

- "Possible common mode failure!"

- "Excluded signals with high (low) reading:"

- "Signals inconsistent with the past estimate:"

- "Signals consistent with the past estimate:"

- "Signals experienced bias (noise) failure:"

- "No bias (noise) failure was detected"

- "Only one signal remained!"

These messages are updated when new failures appear or when they are no longer valid.

The following parameters are displayed and updated on the screen at every sample.

- The calculated estimate as a weighted average of the redundant measurements. Any time the average cannot be calculated due to the strong inconsistencies among the measurements, the estimate will be set to its previous value.

- The current sample number or the sampling time instant. There is an option to skip samples.

- The value of each sensor reading which serves as input to the GCCSPRT program for the given sampling time.

- The inconsistency index of each sensor generated by the GCC part of the program. The failure messages displayed at the top of the table corresponds to these error index values. The last two messages are based on the SPRT calculation for the cases of noise and bias type degradations.

- The bound against which the value of LLRs are compared to check for bias degradation. This threshold value is calculated using the predefined average 
mean time between false alarms, and the mean and variance assigned to the degraded status.

- The LLR value for each sensor as calculated by the SPRT applied to bias type degradation. A sensur is declared to have bias degradation if this value exceeds the above threshold (one-sided SPRT).

- The upper and lower bounds against which the value of LLRs are checked to determine excess noise degradation. These bounds are based on the specified false and missed alarm probabilities.

- The LLR value for each sensor as calculated by the SPRT applied to noise type degradation. A sensor will be declared to have noise degradation if this value exceeds the upper bound and will be declared to be normal if the value is below the lower bound.

These parameters and the corresponding failure messages will be displayed as they are generated for each sample. Some sample tables displayed on the screen are shown in Tables 2.1 through 2.8. These tabulations were generated during the sample run discussed in the next chapter, and were directly dumped from the screen to the printer.

The GCCSPRT algorithm is considered as a decision/estimator (D/E) unit of the process of validating redundant (direct or analytical) signals, measuring the same process variable. In the case of validating several variables measured by sets of redundant signals, it is necessary to build a network of $\mathrm{D} / \mathrm{E}$ and analytical measurement calculator units, where the different decision/estimator units and analytical measurement calculator units are interconnected. In case of multivariate signal validation, properly implemented analytical relationships can allow the detection of common cause sensor faults or anomalies in nonsensor plant components. 


\subsection{Results of Numerical Study}

To test the ability of the signal validation program to detect and isolate signal faults, extensive numerical experiments were performed. Some typical results are presented in this chapter. A numerical simulation is used to generate five measurements having different kinds of degradations. Excessive noise level and drift of different magnitudes in the mean values of signals were introduced simultaneously. The plots of the simulated input measurements are shown in Fig. 2.3. The performance parameter set used in the signal validation algorithm are specified as follows:

- The missed and false alarm probabilities for noise SPRT are 0.01 .

- The aveage mean time between false alarms for bias SPRT was chosen to be 10,000 samples.

- The tolerance for the consistency check is specified to be $3^{\circ} \mathrm{F}$ for each signal.

- The corresponding tolerance for the bias SPRT is taken to be $4^{\circ} \mathrm{F}$.

- The normal and the degraded noise variance for the noise SPRT were specified to be $\sigma^{2}{ }_{1}=1^{\circ} \mathrm{F}^{2}$ and $\mathrm{o}^{2}{ }_{1}=4^{\circ} \mathrm{F}^{2}$.

The output functions such as signal estimate, log likelihood ratios, inconsistency and exclusion indices were calculated for each signal as functions of time. The estimate of the signal as computed by the GCC is shown in Fig. 2.4. The effect of degraded signals on the estimate is not significant, and is much smaller than the magnitude of the original degradation of individual signals.

The LLR functions for checking bias degradation are shown in Figs. 2.5 through 2.9. The plots show the values of LLR calculated after being reset to zero. The LLR is set equal to zero whenever it is negative (control is applied) or it exceeds its upper threshold (a failure is detected). The LLR is then recomputed for the next sample. These recomputed values are plotted at each sample. The LLR functions remain at 
about -7.6 from time zero until about 10 sec. indicating that all five signals are normal. When the signals start to degrade at $10 \mathrm{sec}$. with different extent, the LLR functions start to increase in the positive direction.

The number of samples necessary to detect the bias failure by the SPRT decreased as the bias magnitude for the different signals increased (see Figs. 2.5 through 2.9). First, signal \#5 was identified by the SPRT after 2 samples to have undergone a bias degradation, as is illustrated in Table 2.1, then the signal \#4 was isolated after 6 samples (see Table 2.3). The first detection of bias degradation in signals \# 2 and \#3 was after 8 and 18 samples, respectively (see Tables 2.4 and 2.7). The repeated detection of bias degradation in case of signals \#2 and \#3 is due to resetting to zero after the LLR exceeds the degradation threshold, and due to their relatively small bias error. In case of the unbiased signal (\#1), the SPRT did not indicate a bias error.

The results of a GCC analysis depend only on the error bounds specified for each signal, and in an optimal case, the GCC gives results consistent with those of the SPRT analysis. For the given case, the inconsistency and exclusion indices are plotted as functions of samples in Figs. 2.15 through 2.19. The GCC first excluded signal \# 5 with error index 3 after 6 samples, signal \# 4 was excluded after 10 samples with error index 3 (see Tables 2.3 and 2.5). Signals \#3 and \#2 were excluded at samples 18 and 16 respectively, with inconsistency indices 3 and 2 , respectively (see Tables 2.7 and 2.6). The isolated signals have zero weights in the computation of the signal estimate. Signal \#1 was consistent over the entire analyzed time period, and it was used at each sampling instant in the calculation of the signal estimate. Note that the criterion for excluding a faulty signal does not depend only on whether or not its inconsistency index has maximum value (number of signals minus one). The exclusion of one particular signal also depends on the inconsistency indices of others through a complex logic (see the flow chart in Fig. 
2.2). In the abuve example, signals were identified to be inconsistent with the others, and were excluded from the average, having inconsistency indices ranging from 2 to 4. The marker symbols in Figs. 2.16 through 2.19 indicate the time instants and the inconsistency indices at which the given signal was excluded.

Usually, degradations of signals influence the estimate (weighted average calculated by the GCC), which in turn causes the LLRs to increase, even for the normal signals. This increase is much smaller than in case of degraded signals and less than the degradation threshold. When the degradation was large enough, the GCC excluded the degraded signals from the calculation of the estimate and the LLR of the normal signal started to return to its negative normal value. The fluctuations in the LLR of the normal signal (\#1) is due to the noisy degraded signals causing fluctuations in the inconsistency indices, and consequently in the estimate. Note that the LLR of the normal signal in Fig. 2.5 did not reach the degradation threshold and no false alarm was given.

The SPRT for checking noise degradation was applied to the fluctuating components of the signals. Besides the bias degradation, excessive white noise representing noise degradation was superimposed on signals \#4 and \#5.

Unlike for the bias degradation case, in the normal state, the LLRs for the noise case decrease until the lower bound $A$ is crossed, at which point it is reset to zero, as shown in Figs. 2.10 through 2.14. The decrease with constant slope is due to the constant term $\ln \left(\sigma_{0} / \sigma_{1}\right)$ in the recursive expression of LLR (Eq. 2.9). Recall that no control action is used in the SPRT as applied to detecting noise degradation. The LLR computed for the noise degradation responded to the noise degradation in signals \#4 and \#5. However, the degradation threshold belonging to the 0.01 false and missed alarm probabilities, was exceeded after 3 and 23 samples, respectively (see Tables 2.2 and 2.8 , and Figs. $2.13,2.14$ ). The change in the normal trend 
(decreasing LLR values) indicates the presence of an excessive noise level. The LLRs for the degraded signals were reinitialized, and the recursive calculation was restarted at a zero value.

At 40 sec. all the bias and noise error components were switched off, and the LLRs checking for bias or noise degradation returned to their normal values. Also, all the inconsistency indices became zero.

The numerical test discussed above represents only a particular (however, not very extreme) failure combination for a given set of performance parameters. Further tests showed that beyond a certain level of inconsistency the algorithm cannot give a reasonable estimate. It can only indicate that total inconsistency among the signals occurred. For a less degraded signal set, the performance of the fault detection and signal reconstruction will depend on the choice of the performance parameter set for the particular application. One should select the above parameters so that the outputs and warning messages of the GCC and the SPRT are consistent with respect to each other. The choice of extreme values for the parameter set may result in the failure of the algorithm.

The results of the consistency check, and the calculated estimate will depend strongly on the error bounds defined for each signal. By choosing small error bounds, the algorithm may find that all signals are inconsistent and no estimate can be calculated. Consequently, the SPRT for checking bias degradation canot be performed. On the other hand, if the error bounds were defined to be too big compared to the expected deviation between signals, the degraded signals will not be excluded from the weighted average and the estimate will be incorrect.

Besides the actual deviation between signals, the success of detecting bias degradation by the SPRT also depends on the particular value of the maximum allowable deviation of the mean value, and on the normal noise variance specified for 
the given process. These parameters should be based on the knowledge of the given process and sensor characteristics. Also, some trial runs with various sets of performance parameters must be made in advance for off-line applications.

Despite the sample-by-sample comparison during the run, the final decision about the status of analyzed signals should be based on the results acquired over a period of time. The time dependent output functions, such as SPRT functions for bias and noise degradation, inconsistency and exclusion indices calculated for a certain length of data, will serve as in put information for further qualitative reasoning using AI methods.

An earlier version of the signal validation algorithm was applied to experimental data from a hot air loop, and detection of bias, noise and pulse type errors were successfully performed during both steady-state and transient conditions 17 .

\subsection{Application to Operational Data from a PWR}

An example of the application of single variable consistency checking is described using plant operational data from a Westinghouse four-loop PWR. Figures $2.20 \mathrm{a}-2.20 \mathrm{~d}$ show four redundant measurements of the cold leg temperature. The mutual consistency among the signals is evaluated using a tolerance of 2 deg. $F\left(\eta_{i}\right.$ values in Eq. (2.1)). The inconsistency indices of the four measurements are plotted in Figs. 2.21a - 2.21d. Measurement No. 2 has an inconsistency index equal to the maximum possible value of three at a few measurements points where this sensor output is excluded from the set. The signal estimate is calculated using all the four measurements (weighted according to their inconsistnecy indices), and is shown in Fig. 2.22. The sequential probability ratio test (SPRT) indices for the four measurements are plotted in Figs. 2.23a - 2.23d. The degradation threshold index is a function of the mean time between false alarms and the maximum acceptable 
mean bias degradation value; in the example these are in minutes and deg. F, respectively. The SPRT values are less than the degradation threshold for signals 1 , 3 and 4 , and exceed the threshold at a few measurement points in the case of signal No. 2. This case study and others illustrate the application of the GCC algorithm to actual plant measurements.

\subsection{Computer Program Specifications}

The algorithm GCCSPRT is written in Microsoft FORTRAN 77 V 3.31 and has been linked and compiled using an IBM AT compatible PC with an 80287 math coprocessor.

In the current version of the program, five redundant signals with corresponding noise data files can be analyzed. The data file containing the output parameters calculated by the program GCCSPRT has the following structure: the

first record (record length is equal to 4 bytes) contains the calculated estimate for the first sample analyzed, the next five records contain the LLR parameters computed for bias degradation, and for all the five signals. The next five records contain the five LLR parameters computed for noise degradation. The next five records contain the inconsistency indices, and the next five records give the exclusion indices of the five redundant signals (the exclusion index for a given sampling time is 1 if the signal is excluded from the computation of the estimate as a result of GCC, and is equal to zero if the signal value is included in the computation). The above structure is repeated for all sampling instants. The output data file containing the above parameter set for all sampling time, is created by the GCCSPRT program with the name SVSTORE.DAT, and it will be read and plotted by the second program of the batch file, SVPLOT. 
TABLE 2.1

Partially consistent measurements. Bias error in signal No. 5 is detected.

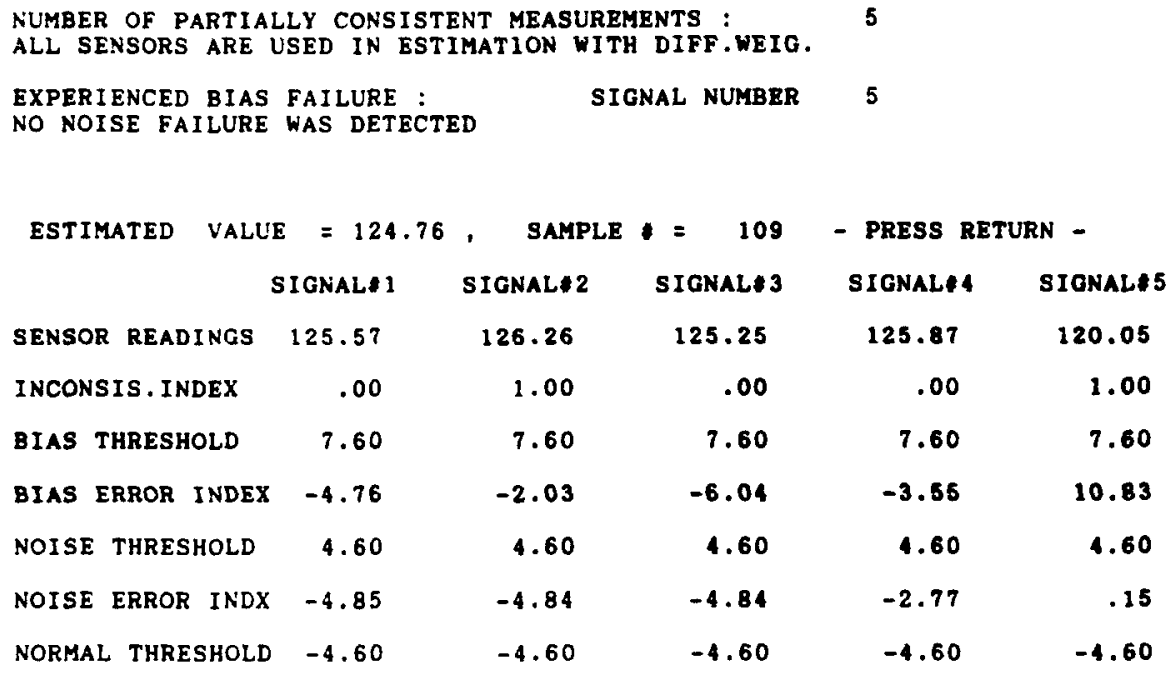

TABLE 2.2

Consistent measurements. Noise error of signal No.4 is detected.

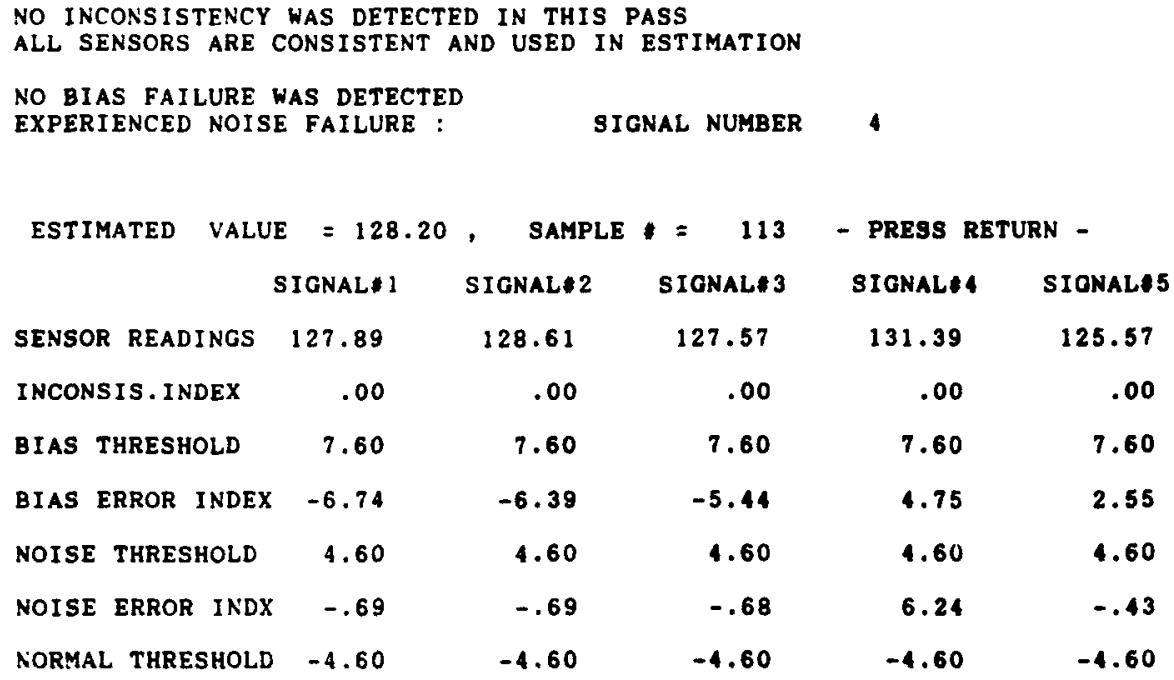


TABLE 2.3

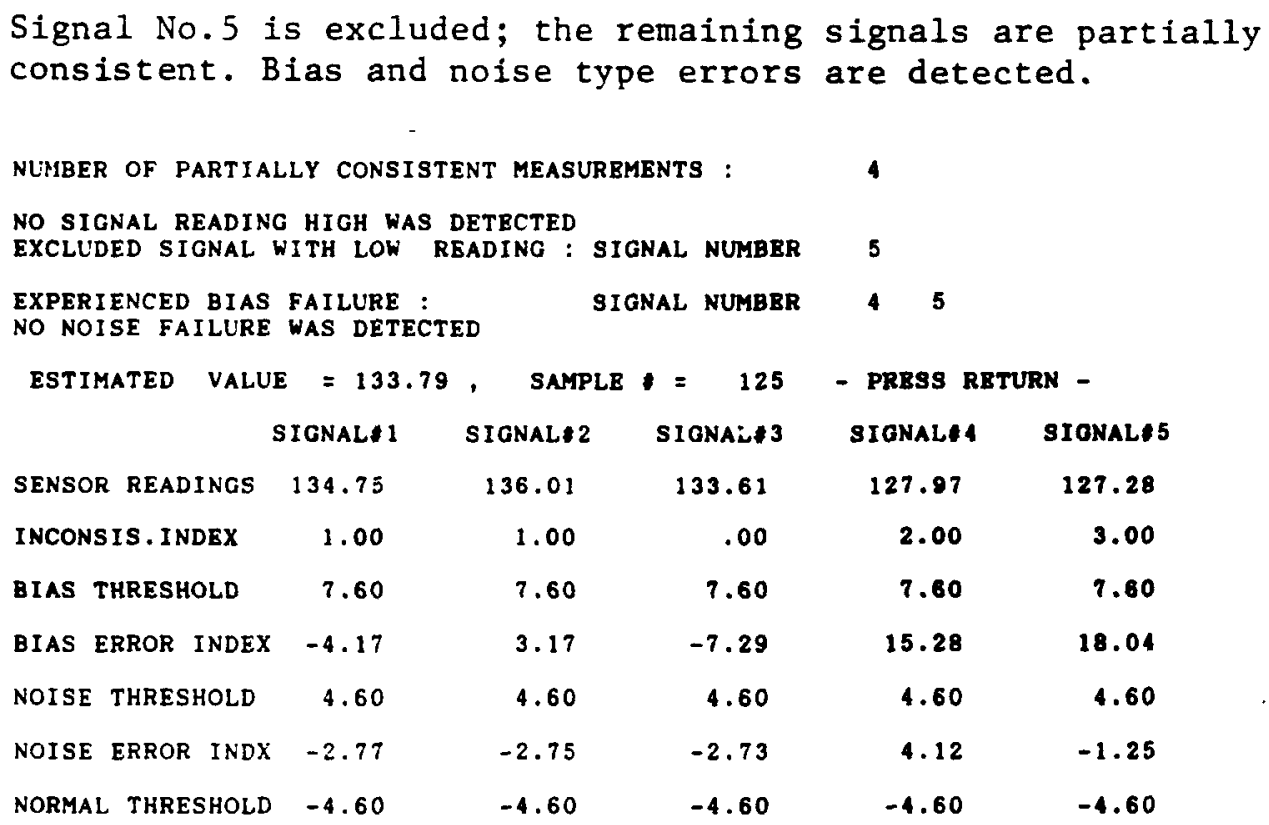

TABLE 2.4

Partially consistent measurements. Bias errors are detected.

NUMBER OF PARTIALLY CONSISTENT MEASUREMENTS : ALL SENSORS ARE USED IN ESTIMATION WITH DIFF. WEIG.

EXPERIENCED BIAS FAILURE :

SIGNAL NUMBER

5

NO NOISE FAILURE WAS DETECTED

ESTIMATED VALUE $=136.95$, SAMPLE $=133$ - PRESS RETURN -

SIGNAL\#1 SIGNAL:2 SIGNAL\#3 SIGNAL\&4 SIONAL\$5

$\begin{array}{llllll}\text { INCONSIS.INDEX } & 1.00 & 2.00 & .00 & 1.00 & 2.00\end{array}$

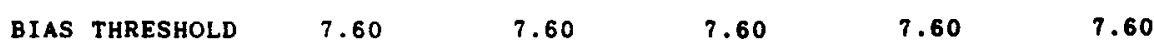

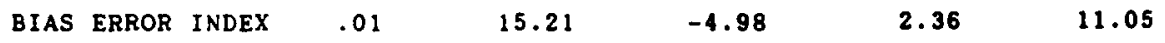

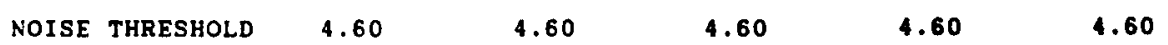

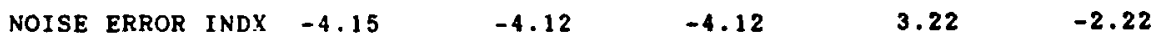

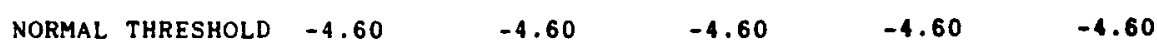


TABLE 2.5

Signals No. 4 and 5 are excluded. Their bias errors are also detected at the same time.

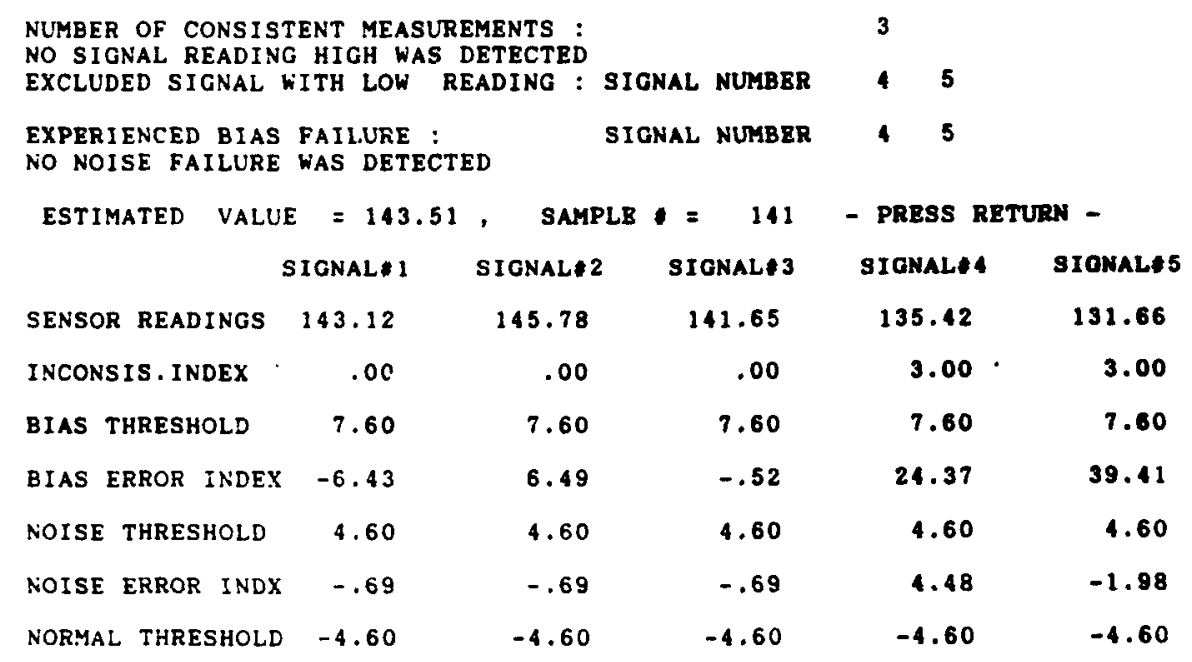

TABLE 2.6

Signals No.2, 4 and 5 are excluded. Their bias errors are also detected at the same time.

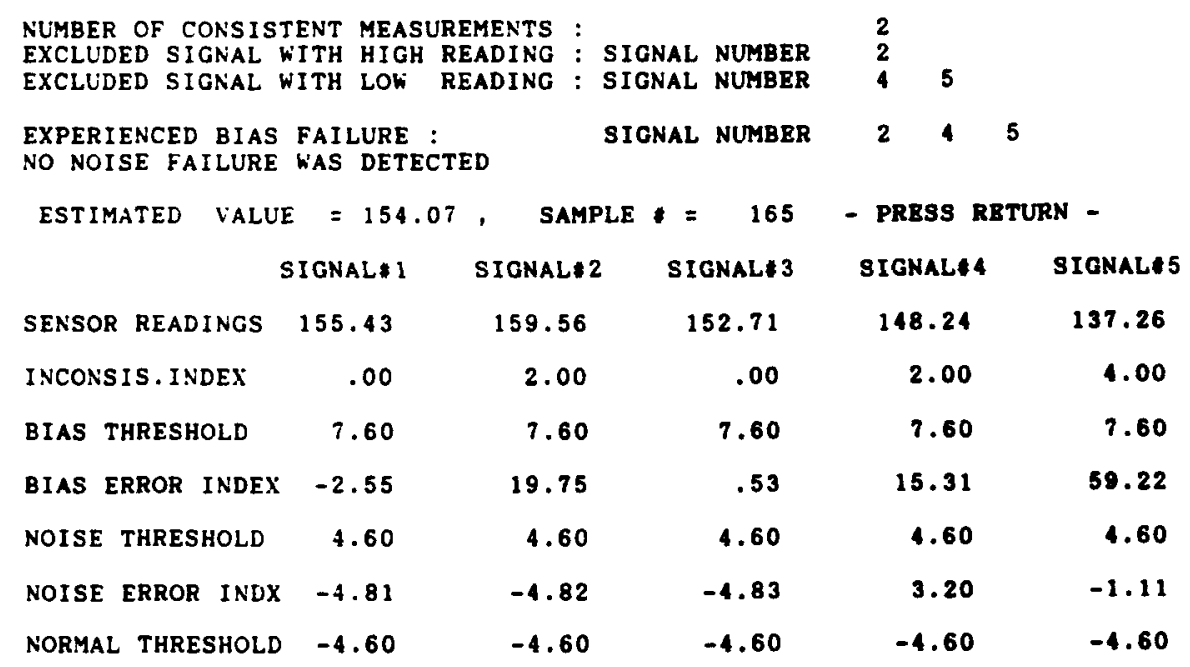


TABLE 2.7

Only signal No.l is used, all other signals are excluded, and their bias errors are detected.

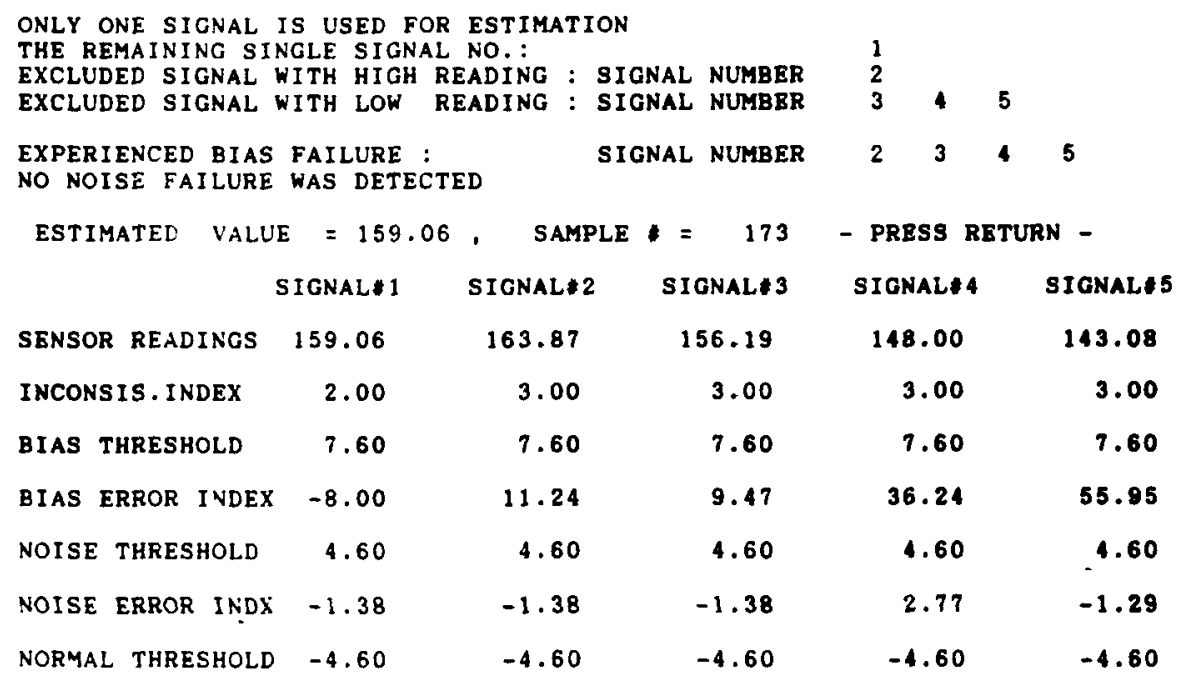

TABLE 2.8

Only signals No.l and 3 are used for estimation. The bias error of the excluded signals, as well as, the noise error of signal No. 5 are detected.

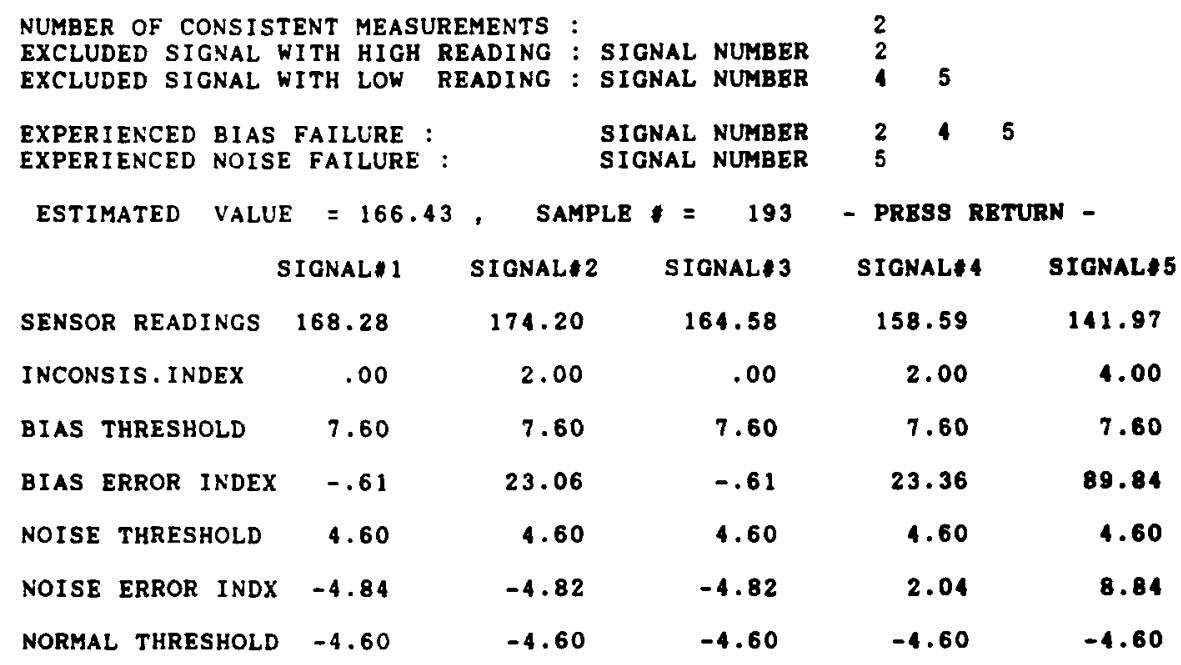




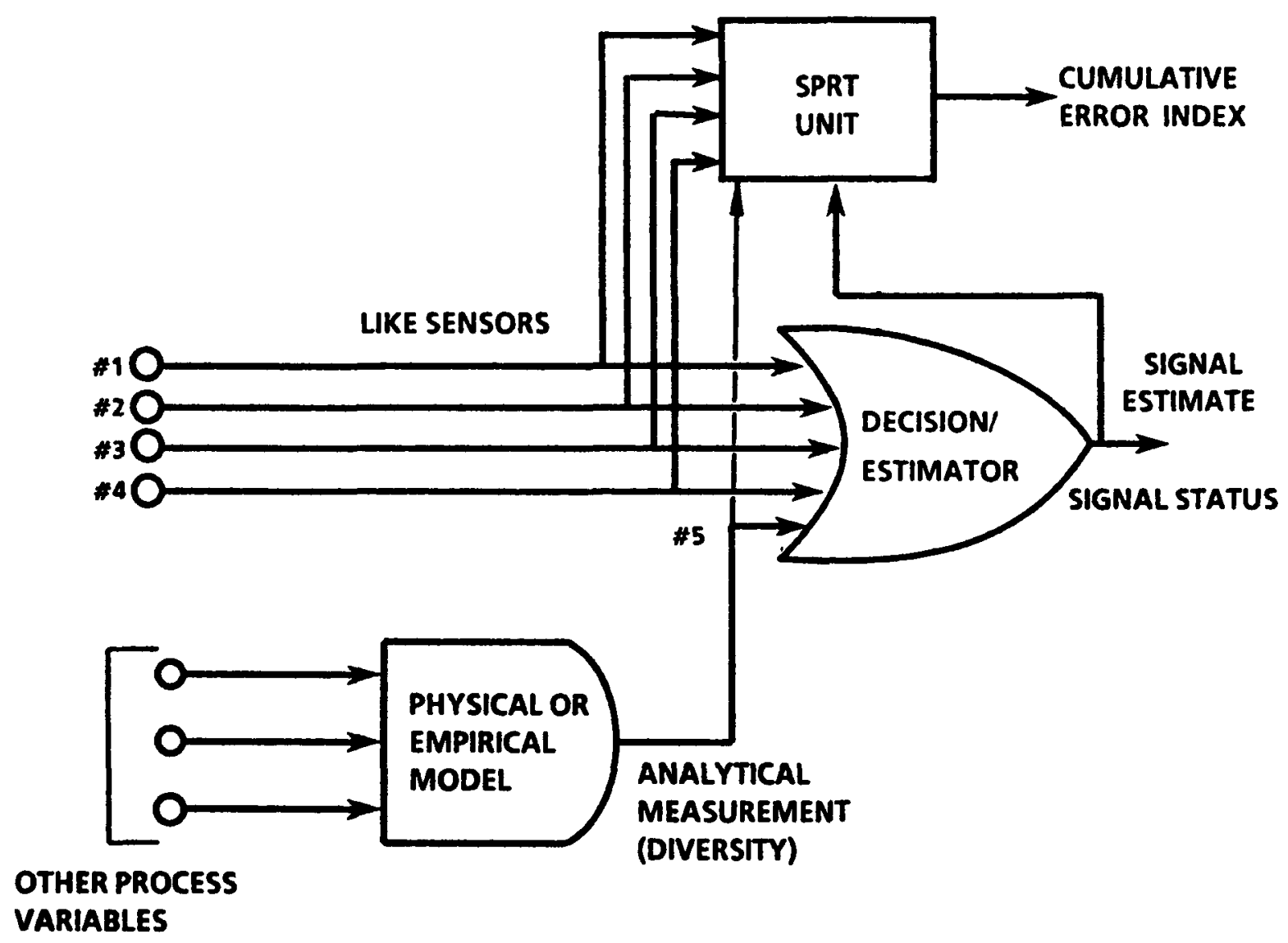

Figure 2.1. Generalized consistency checking module for single variable (GCCS) showing the decision/estimator and the sequential probability ratio test (SPRT) units. 


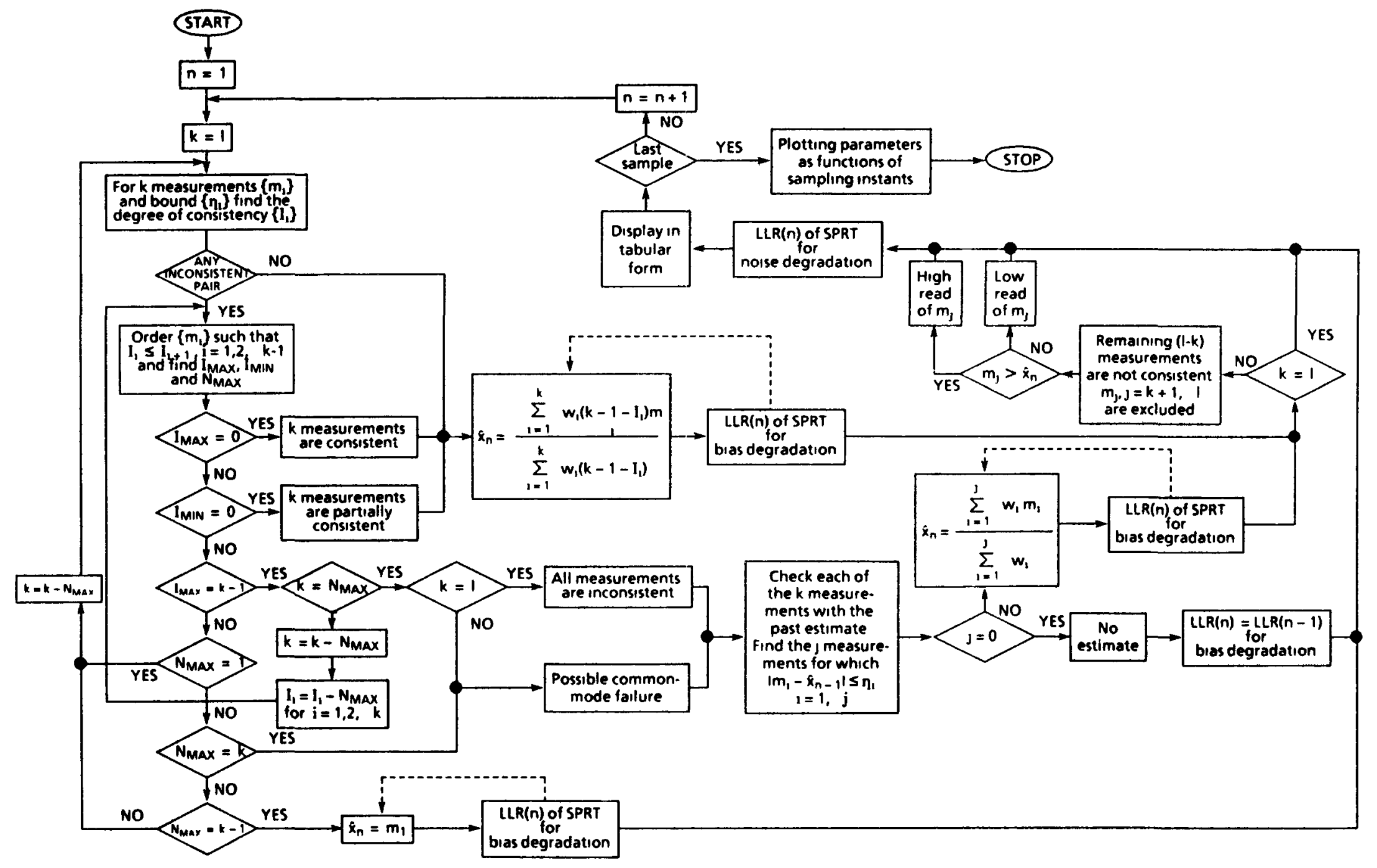

Figure 2.2. Signal validation logic flow chart for GCC - SPRT module. 


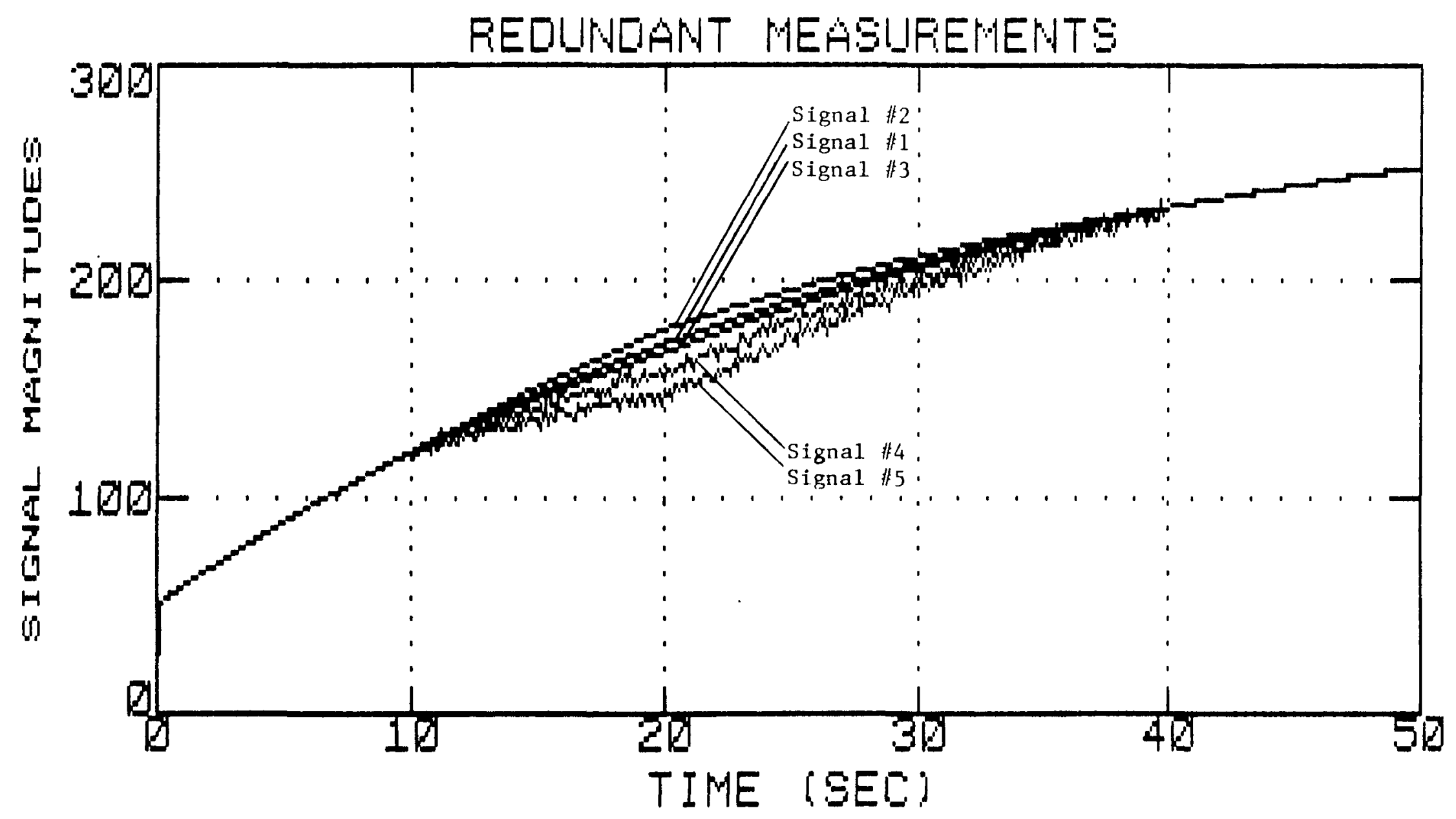

Figure 2.3. Five redundant temperature (deg F) signals with bias, noise, or both errors in various signals. 


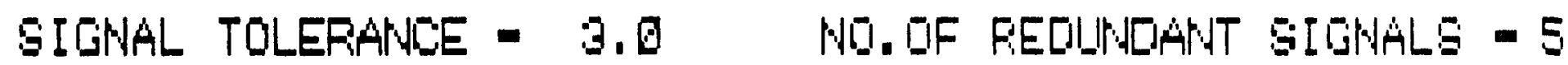

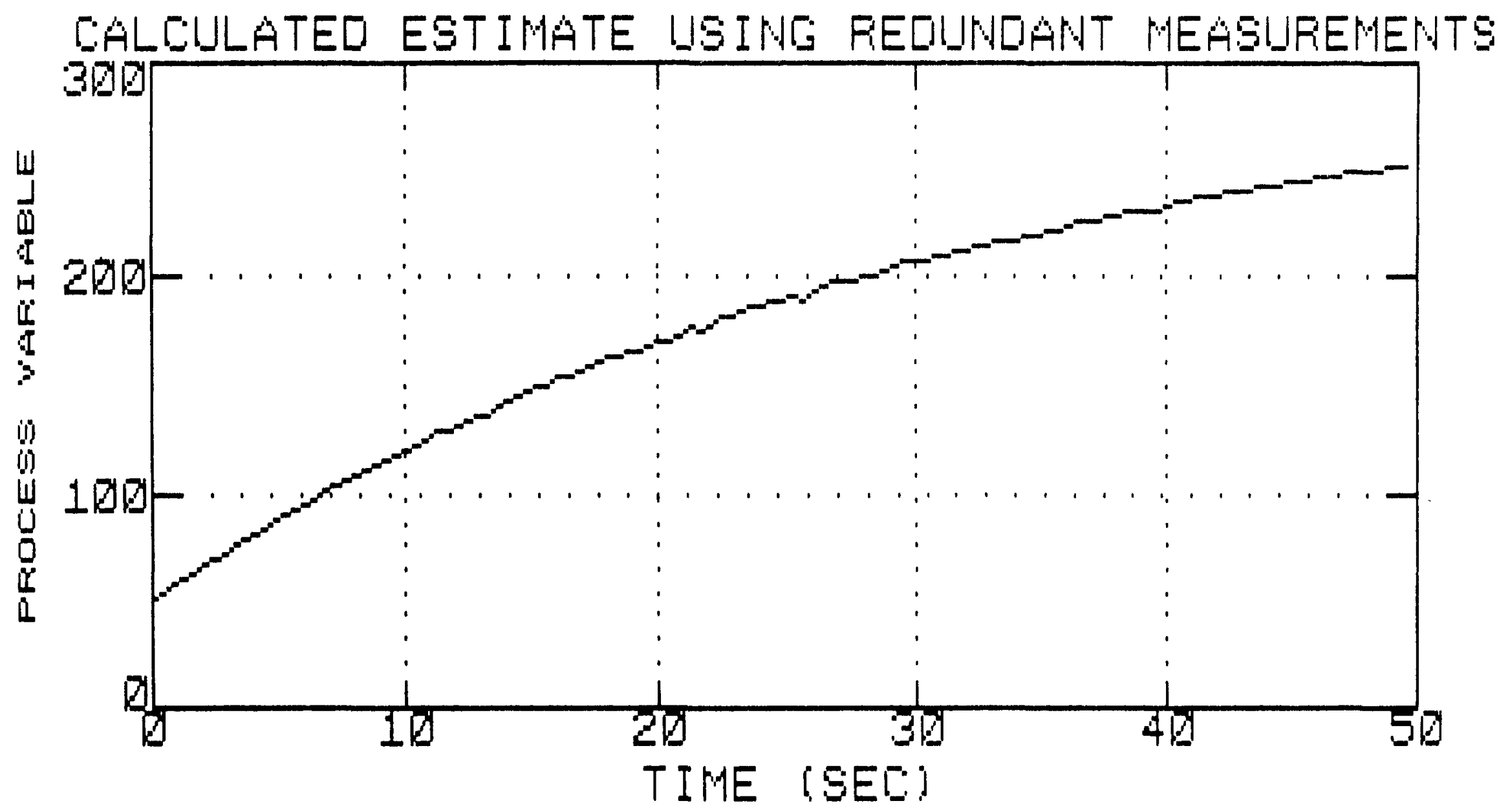

Figure 2.4. The estimate of the process variable based on the five redundant signals. 


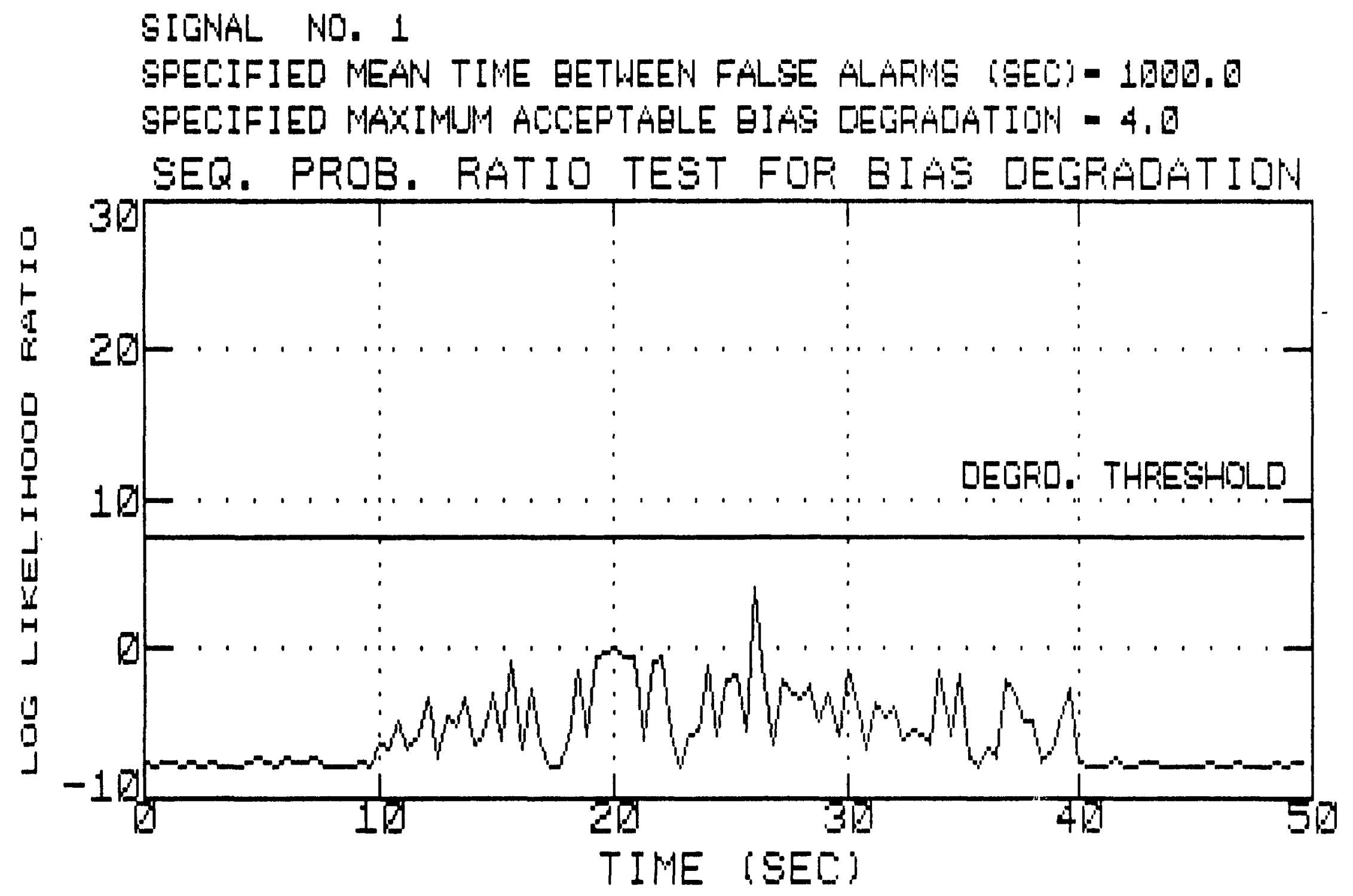

Figure 2.5. Log likelihood ratio of the sequential probability ratio test for bias error in signal No. 1 . 


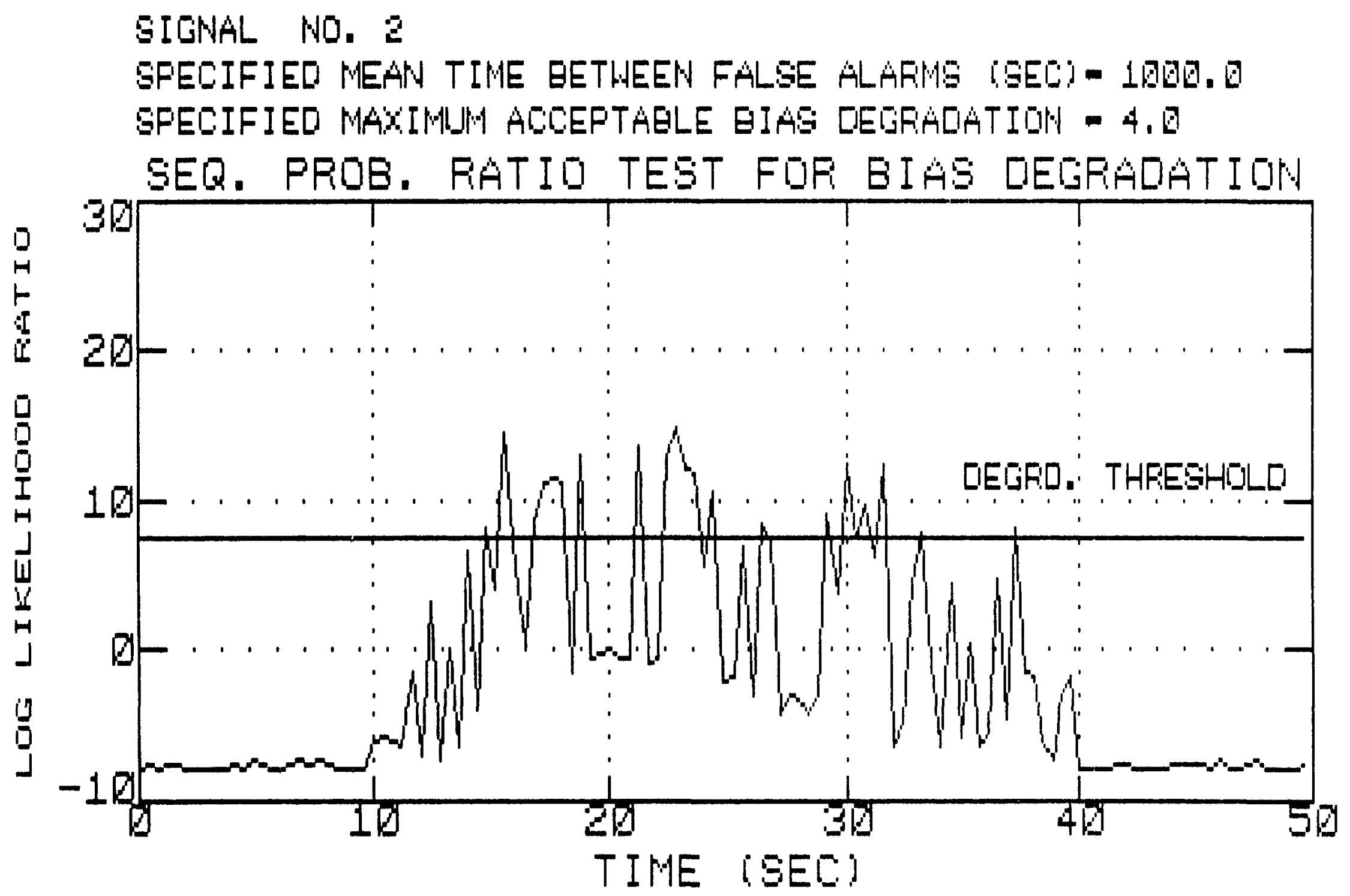

Figure 2.6. Log likelihood ratio of the sequential probability ratio test for bias error in signal No. 2 . 
SICNAL NO. 3

SFECIFIED MEAN TIME BETHEEN FALSE ALAFMS CSECI- 1000.0

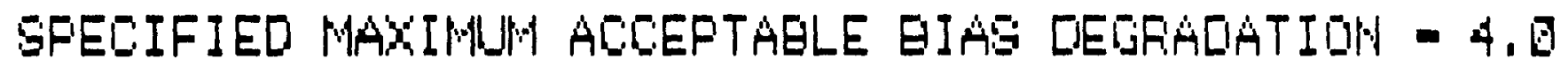

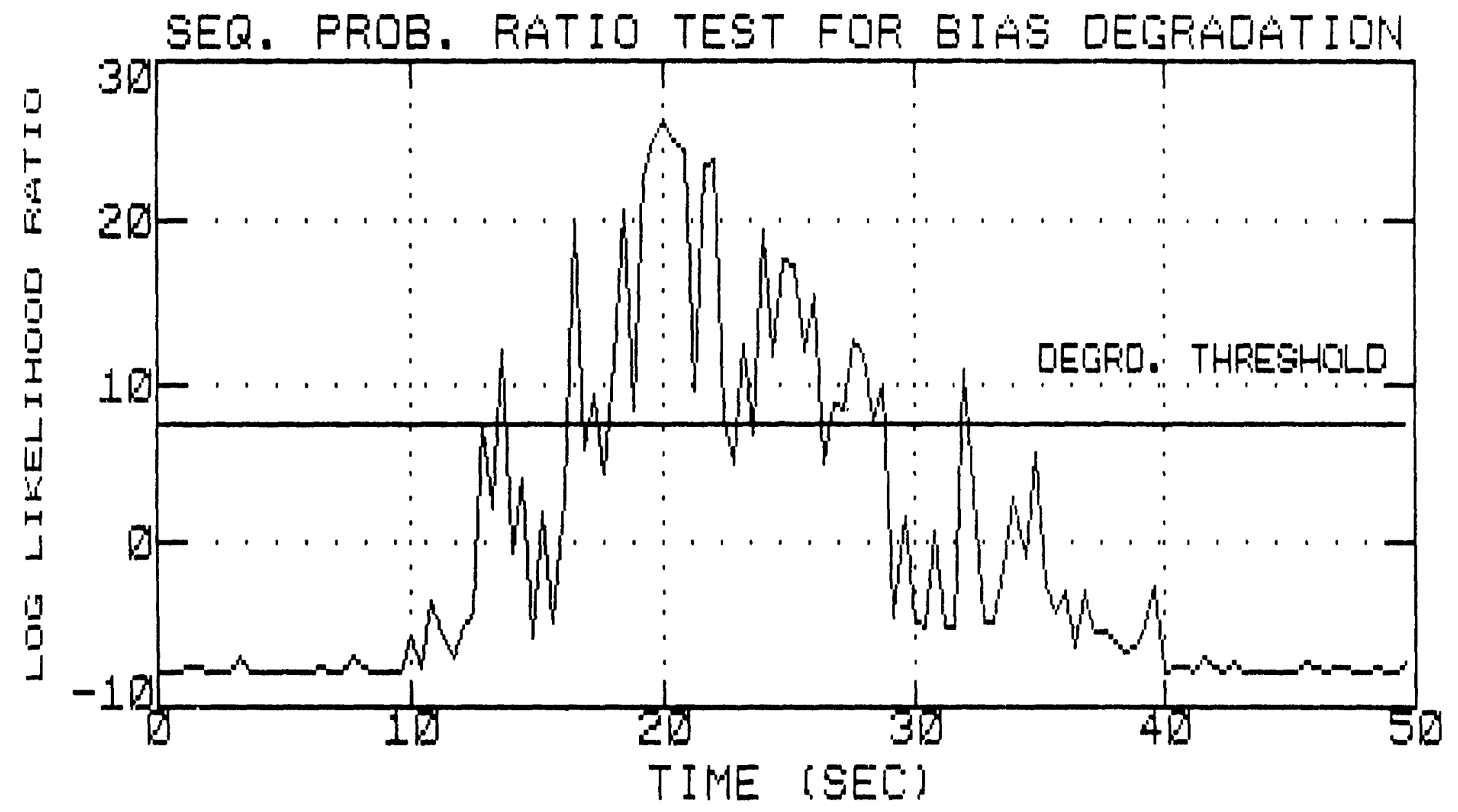

Figure 2.7. Log likelihood ratio of the sequential probability ratio test for bias error in signal No. 3 . 


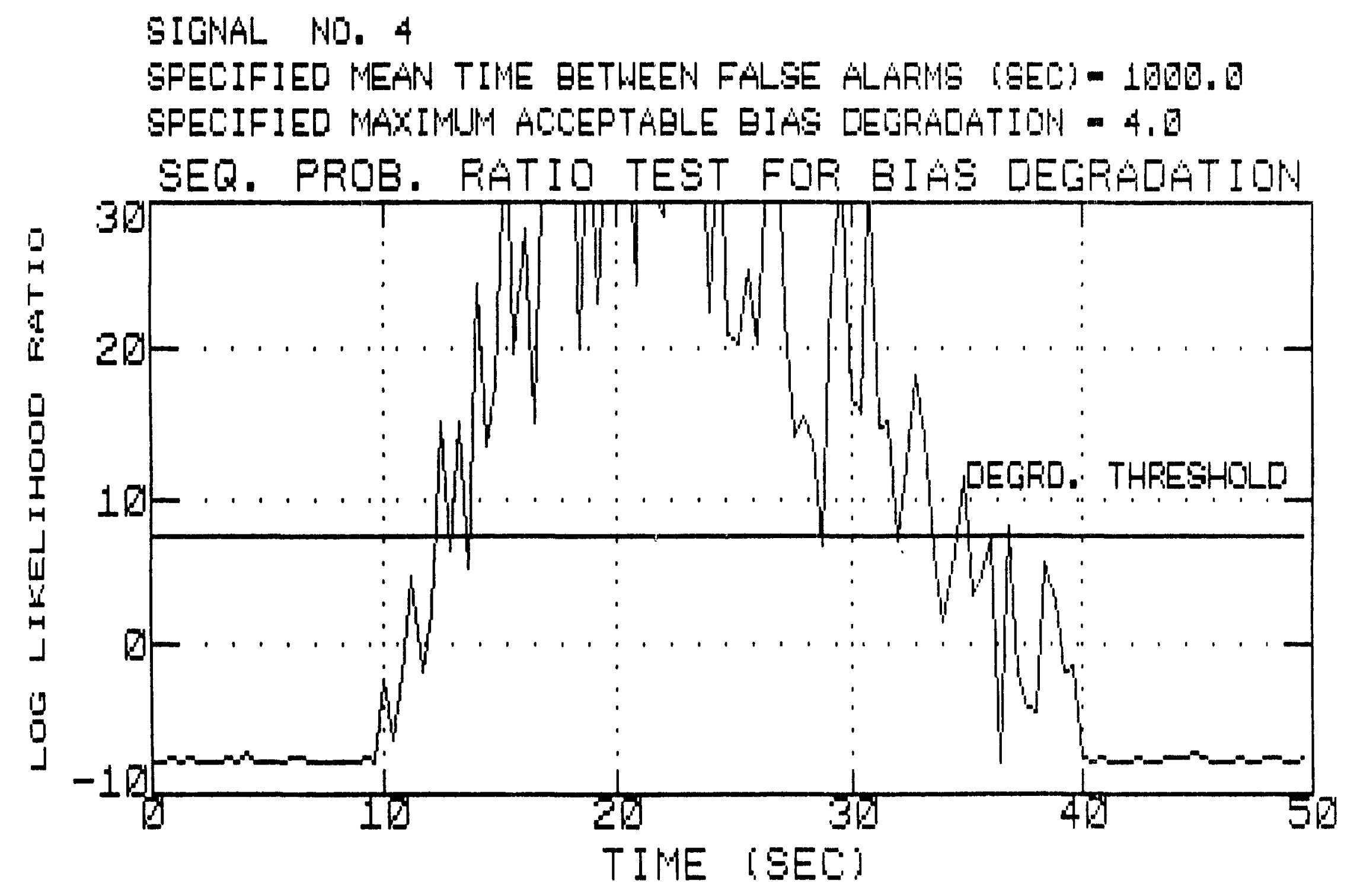

Figure 2.8. Log likelihood ratio of the sequential probability ratio test for bias error in signal No. 4 . 
SIGNAL NO. 5

SPECIFIED MEAN TIME EETWEEN FALSE ALARMS (GEC) - 1000.0 SPECIFIED MAXIMUM ACCEPTAELE GIAS DECFALATION - 4,0 SEQ. PROB, RATIO TEST FOR BIAS DELRADATIDN

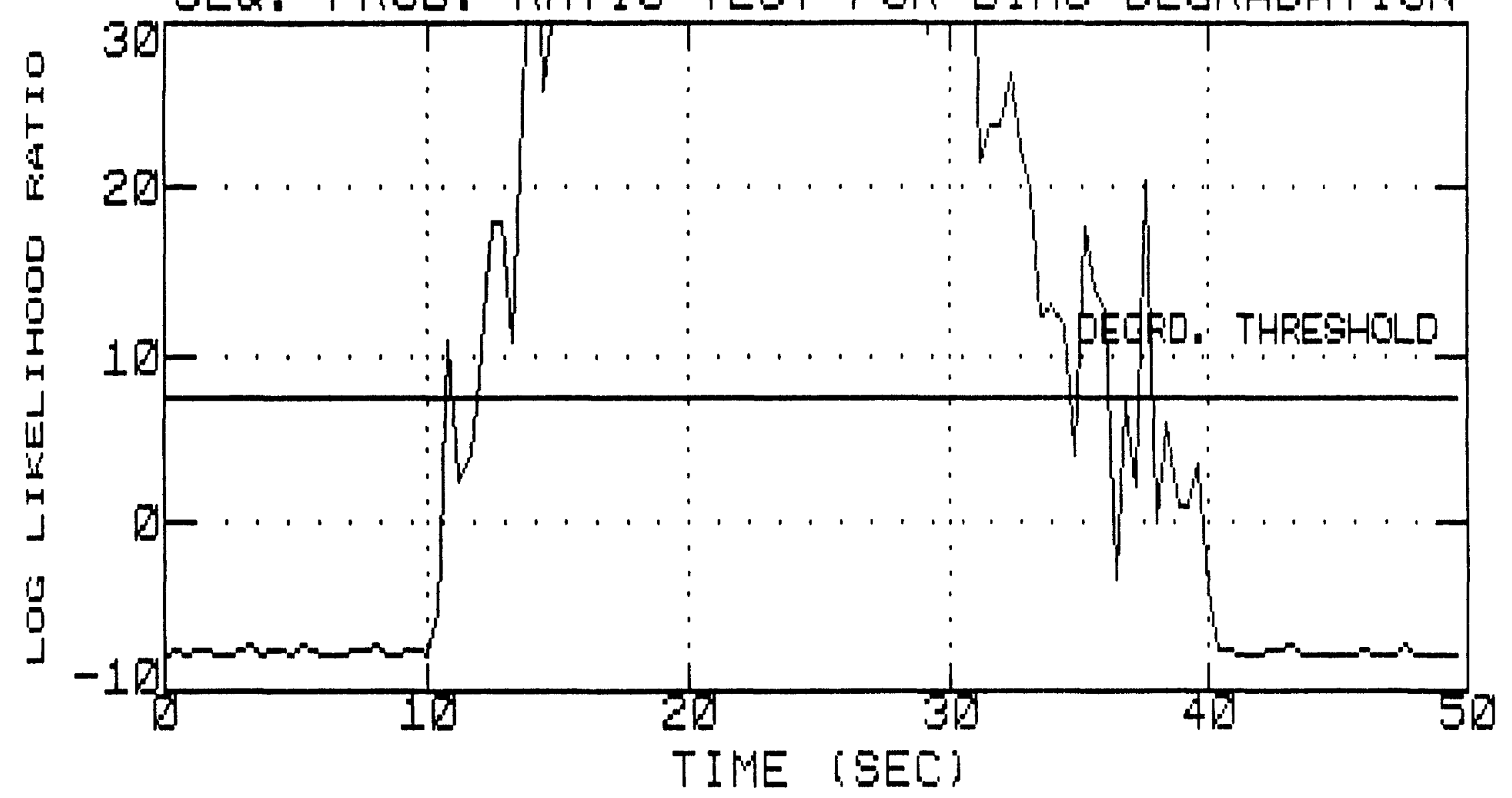

Figure 2.9. Log lokelihood ratio of the sequential probability ratio test for bias error in signal No. 5 . 


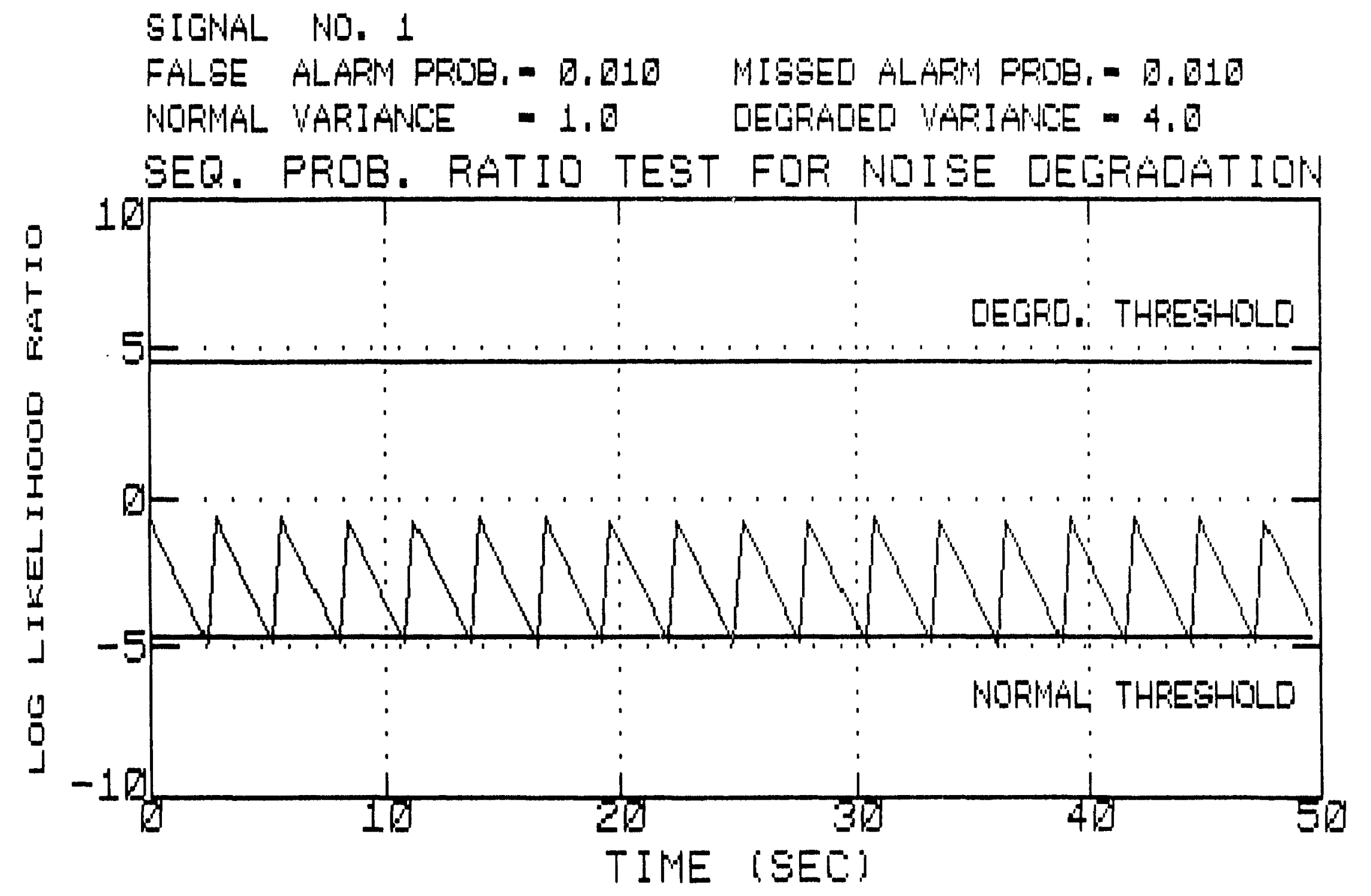

Figure 2.10. Log likelihood ratio of the sequential probability ratio test for noise error in signal No. 1. 
SICNAL NO. 2

FALSE ALARM PROB, - 0.010 MISSEC ALAFH FFOE, - O.010

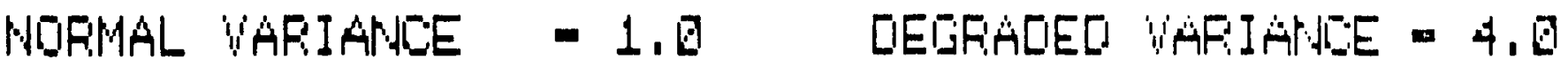

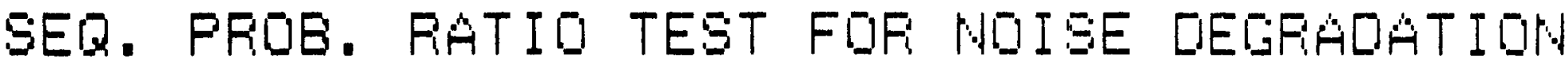

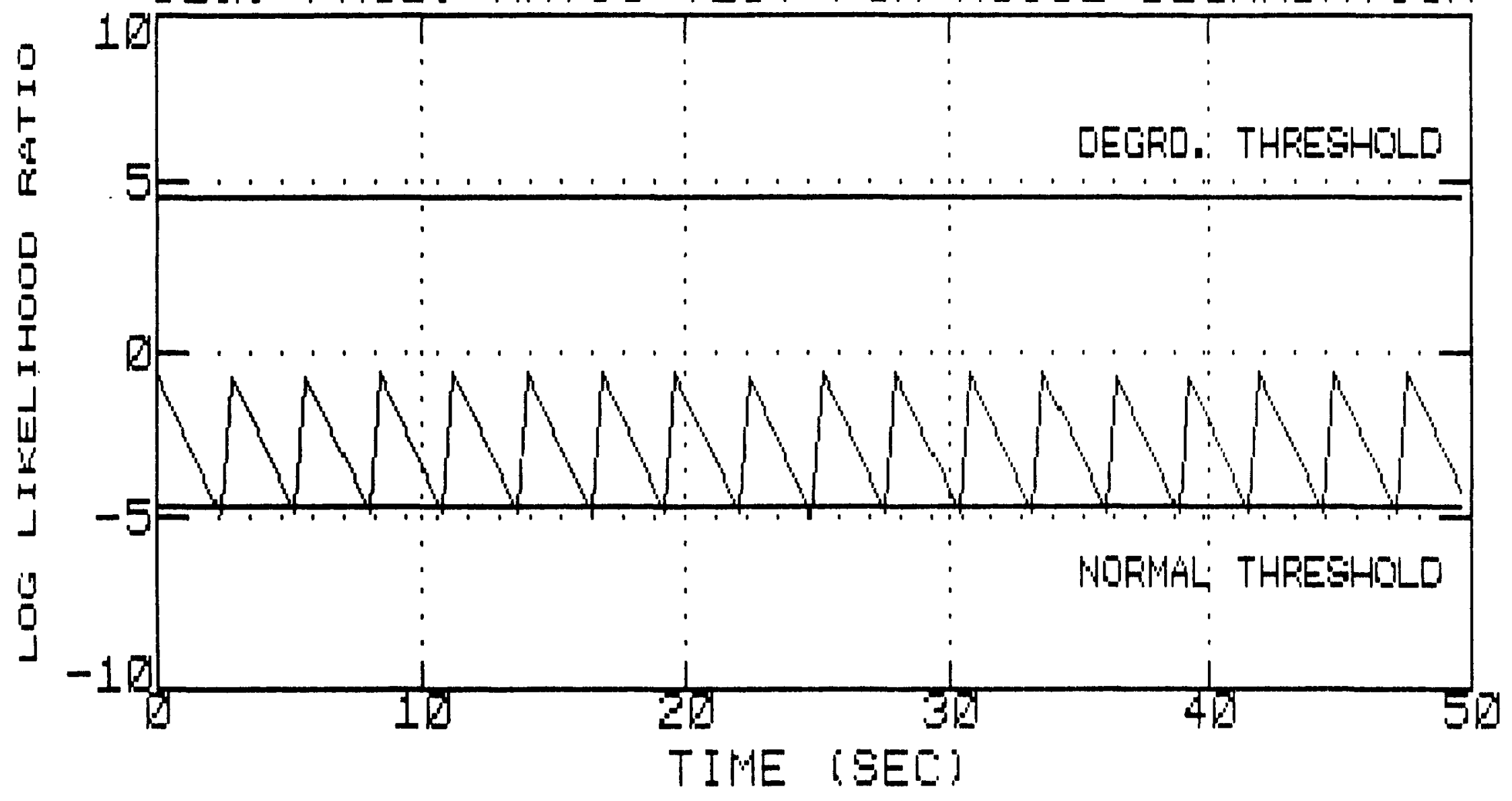

Figure 2.11. Log likelihood ratio of the sequential probability ratio test for noise error in signal No. 2 . 


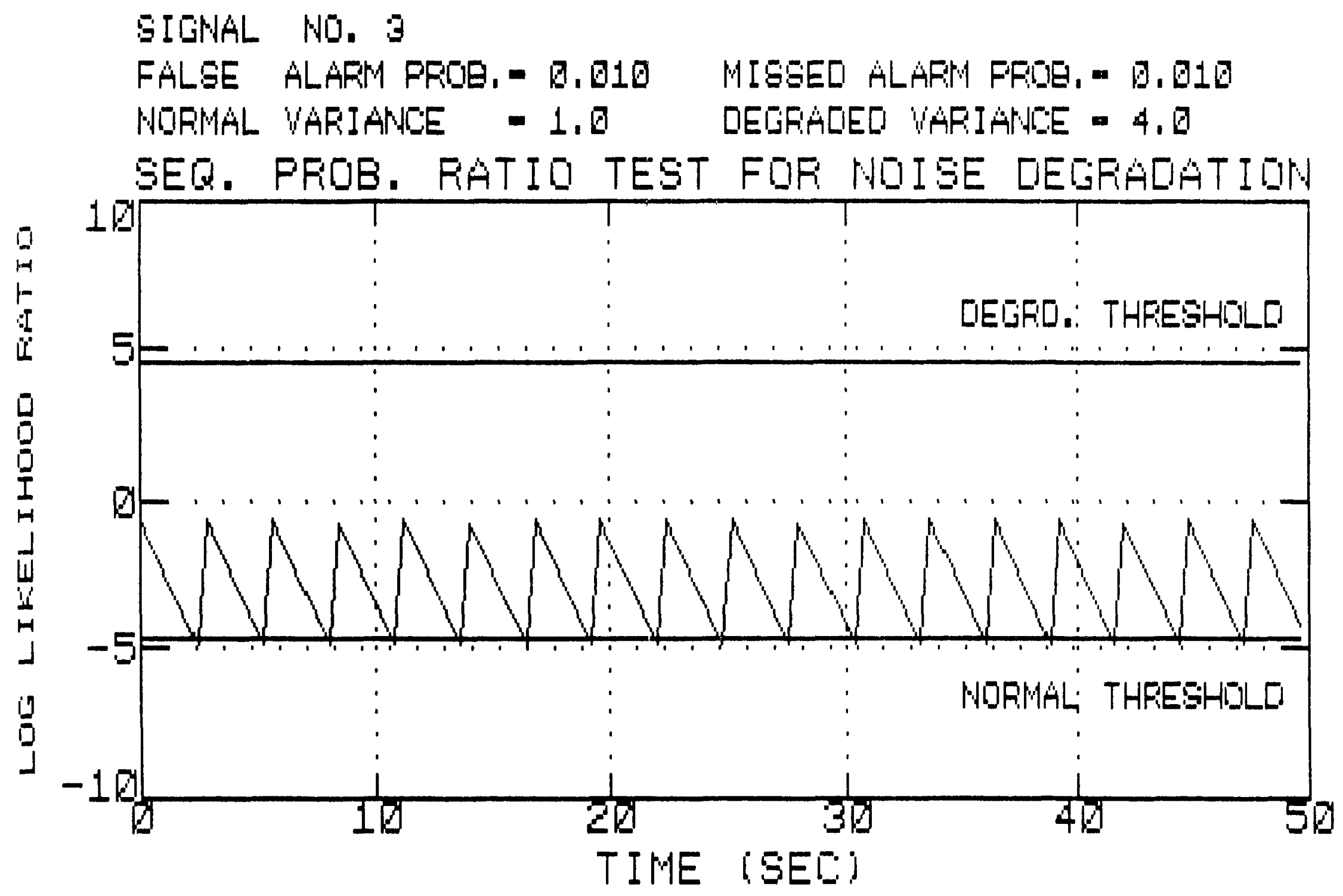

Figure 2.12. Log likelihood ratio of the sequential probability ratio test for noise error in signal No. 3 . 


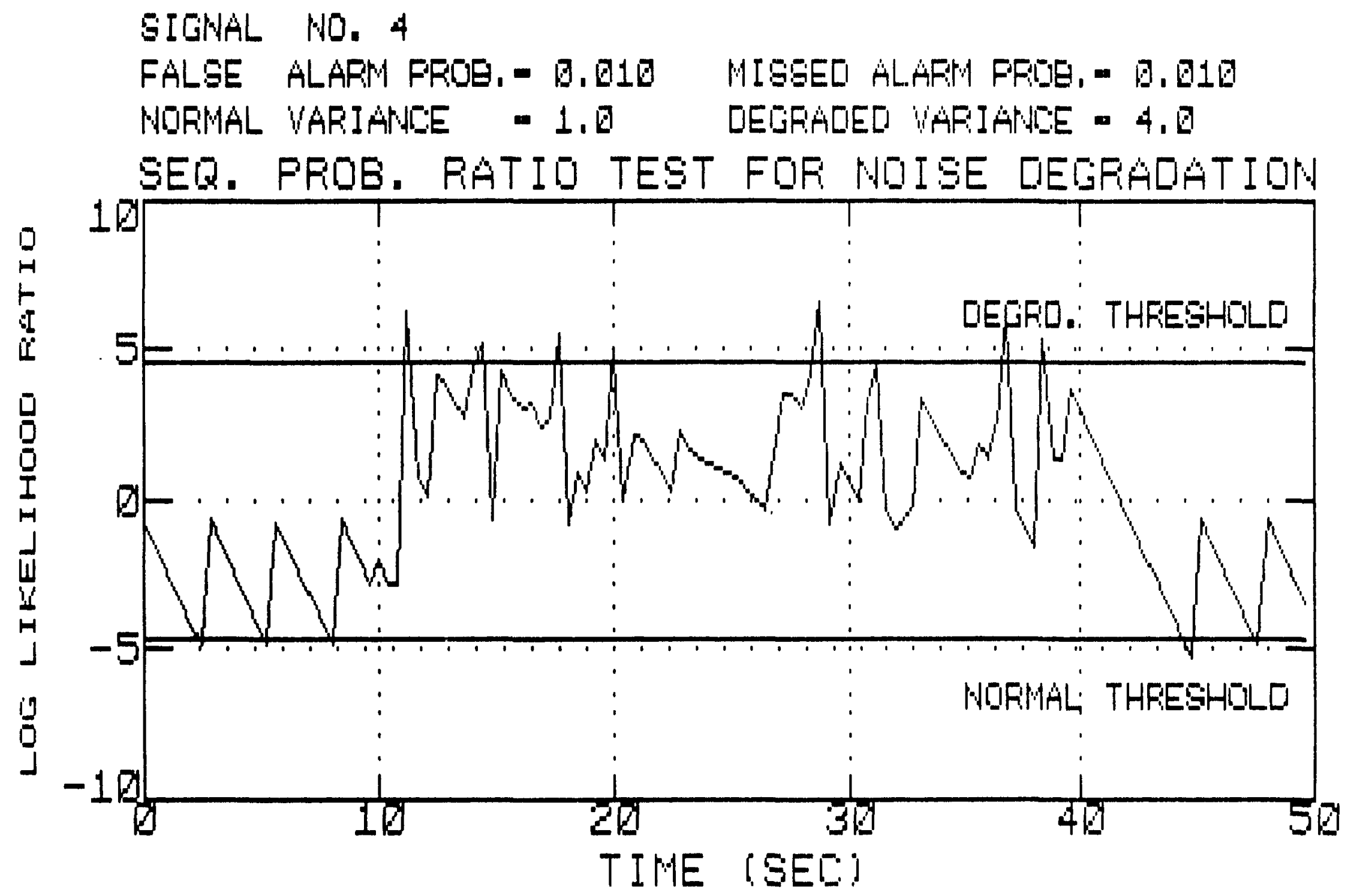

Figure 2.13. Log likelihaod ratio of the sequential probability ratio test for noise error in signal No.4. 


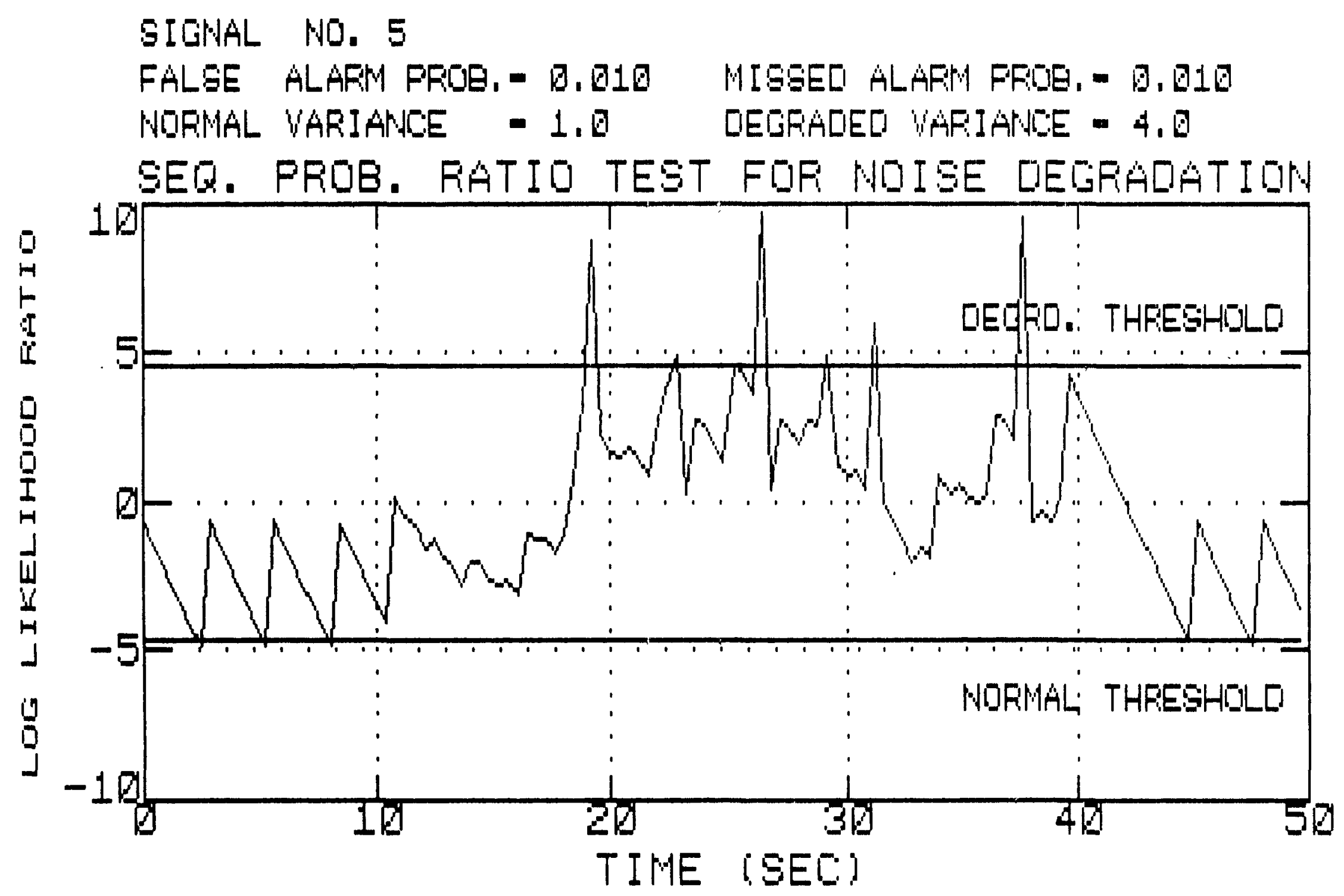

Figure 2.14. Log likelihood ratio of the sequential probability ratio test far noise error in signal No. 5 . 
SICINAL NO. 1

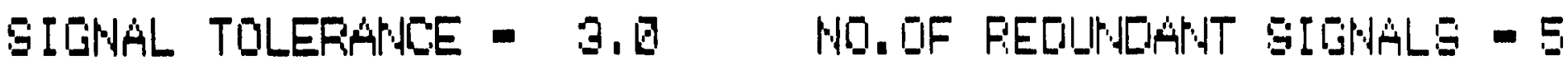

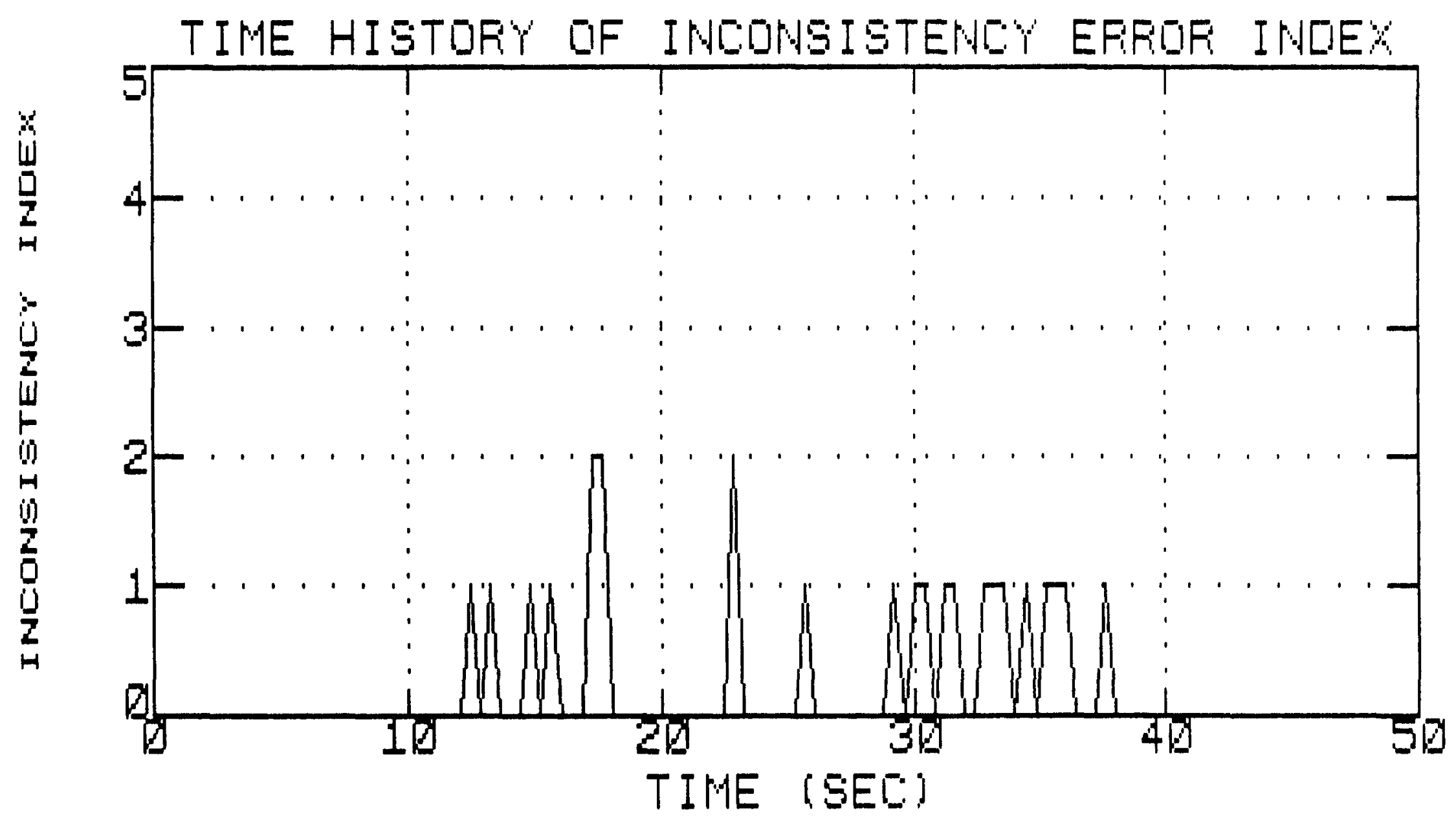

Figure 2.15. Inconsistency indices for signal No.2. No samples were exc1uded. 
GIONAL NO. 2

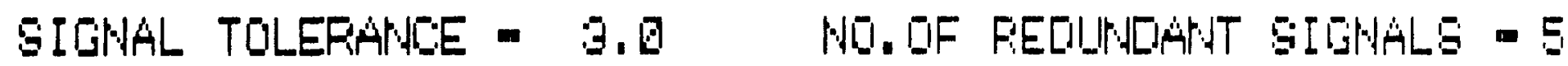

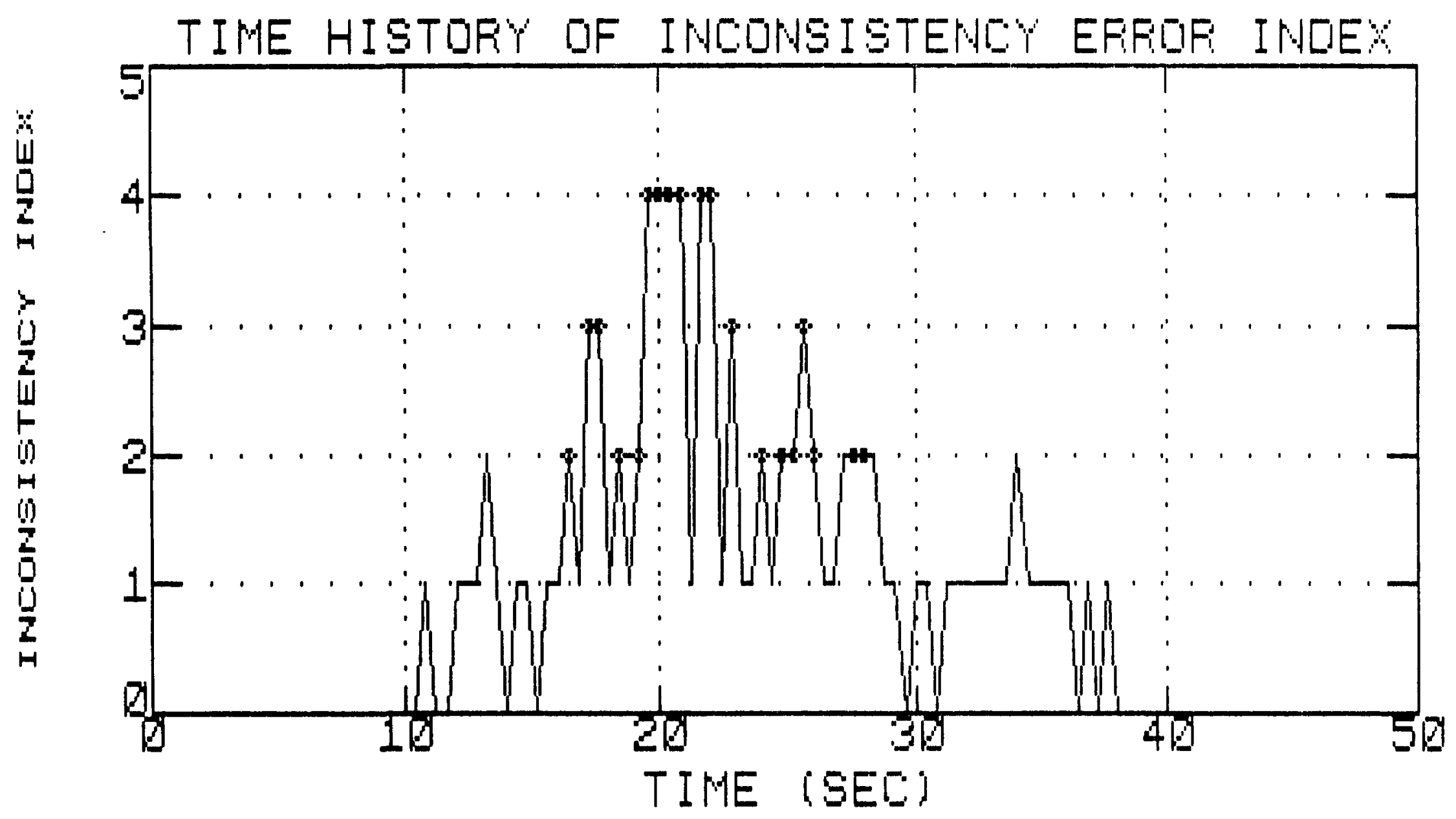

Figure 2.16. Inconsistency indices for signal No.2. The samples at which signal No.2 is excluded are represented by cross marker on the graph. 
SICNAL NO. 3

SICNAL TOLERANCE - 3.0 NO. OF REDILULANT GICHALS - 5

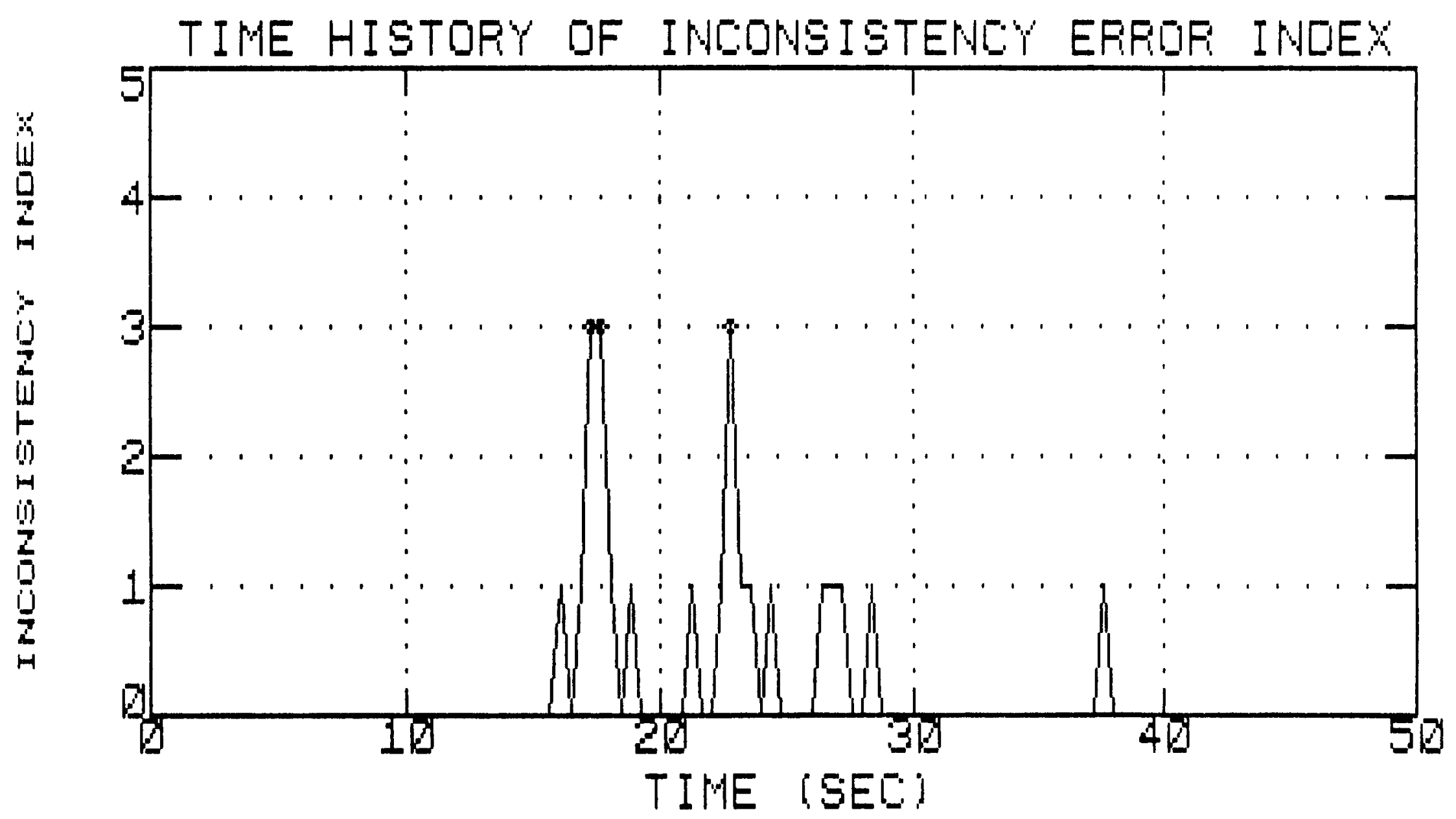

Figure 2.17. Inconsistency indices for signal No.3. The samples at which signal No.3 is excluded are represented by cross marker on the graph. 


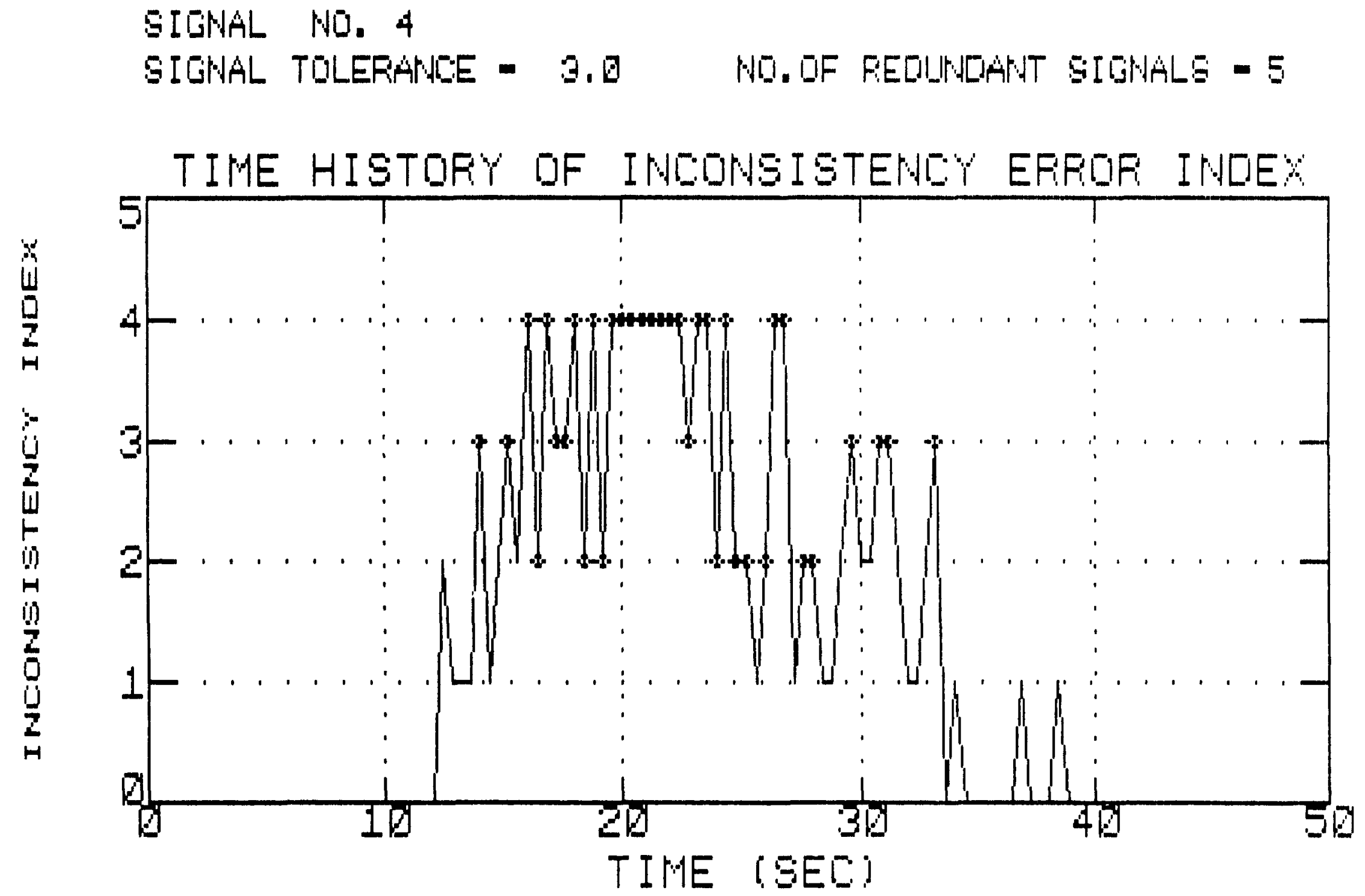

Figure 2.18. Inconsistency indices for signal No.4. The samples at which signal No.4 is excluded are represented by cross marker on the graph. 


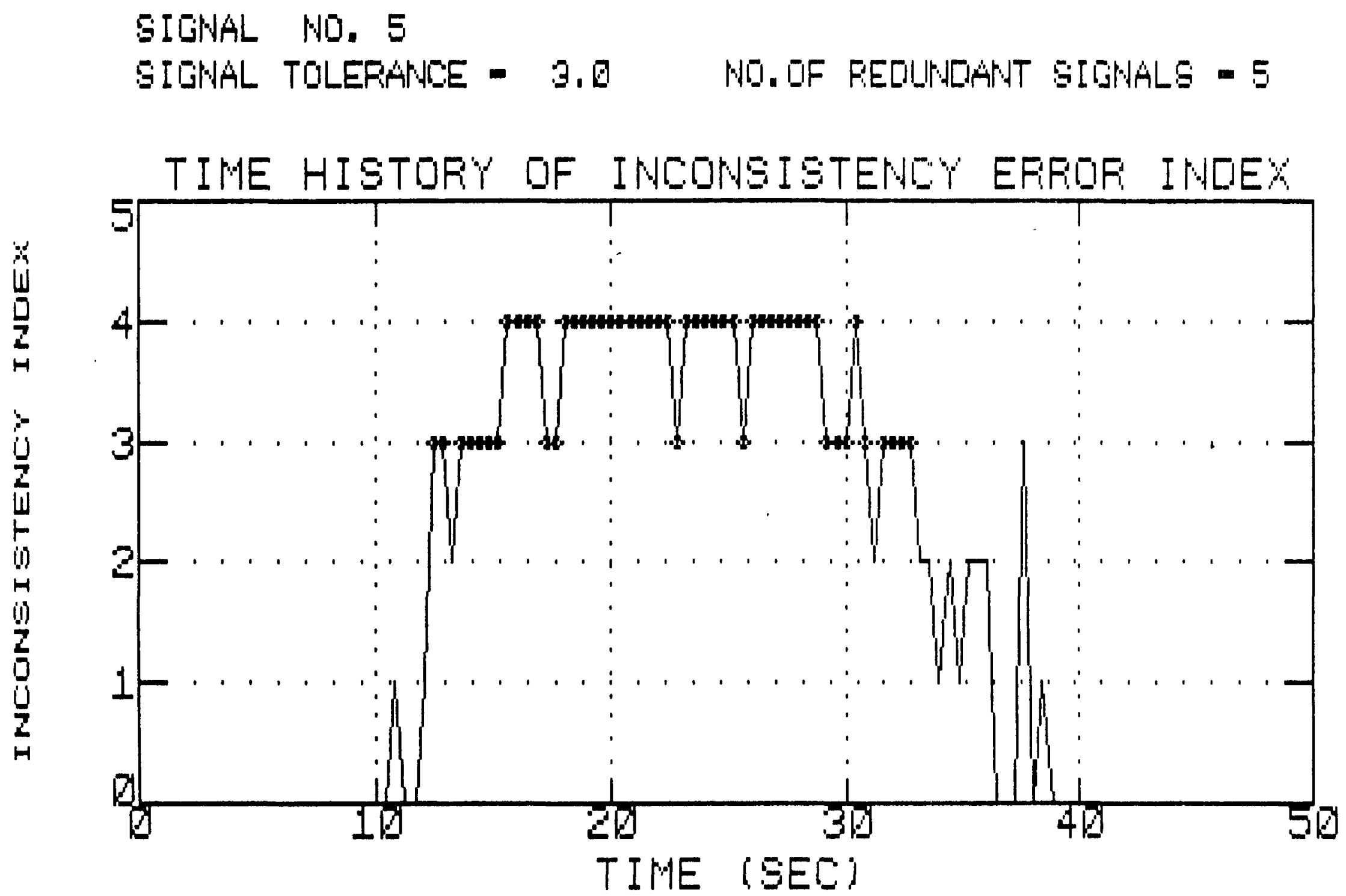

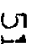

Figure 2.196 Inconsistency indices for signal No.5. The samples at which signal No.5 is excluded are represented by cross markers on the graph. 


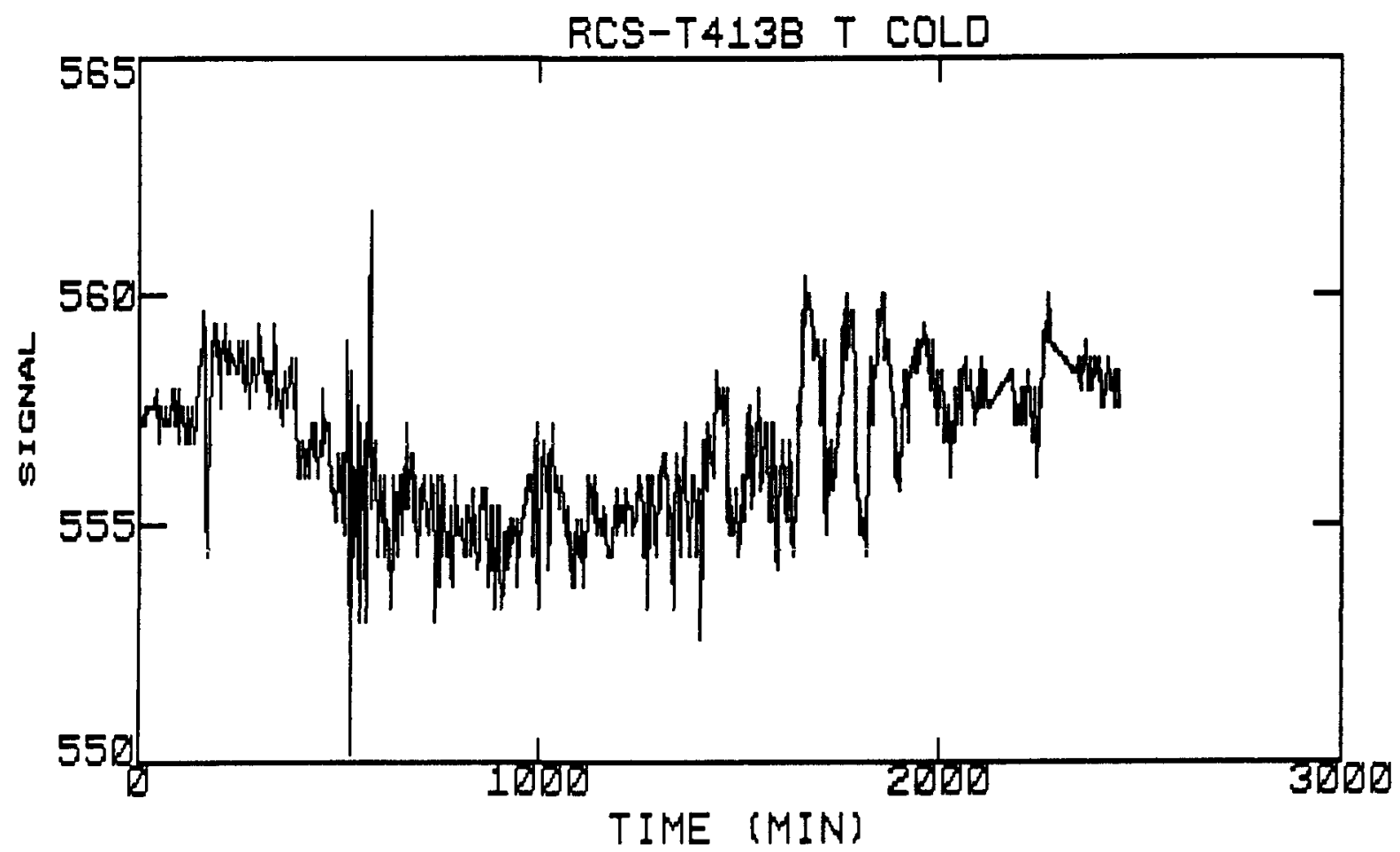

Figure 2.20a. Operational PWR start-up data. Cold leg temperature, measurement No. 1.

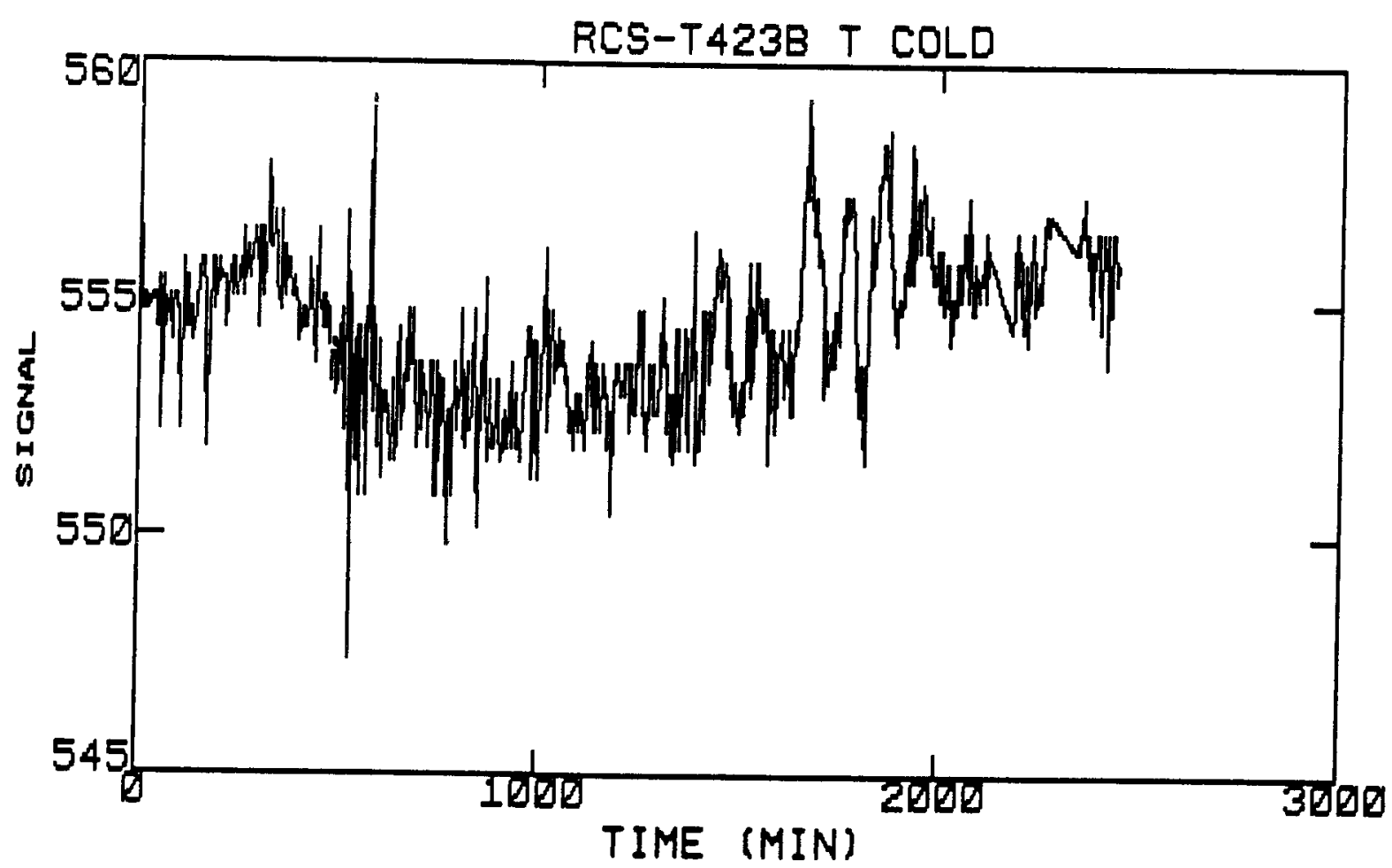

Figure 2.20b. Operational PWR start-up data

Cold leg temperature, measurement No. 2 . 


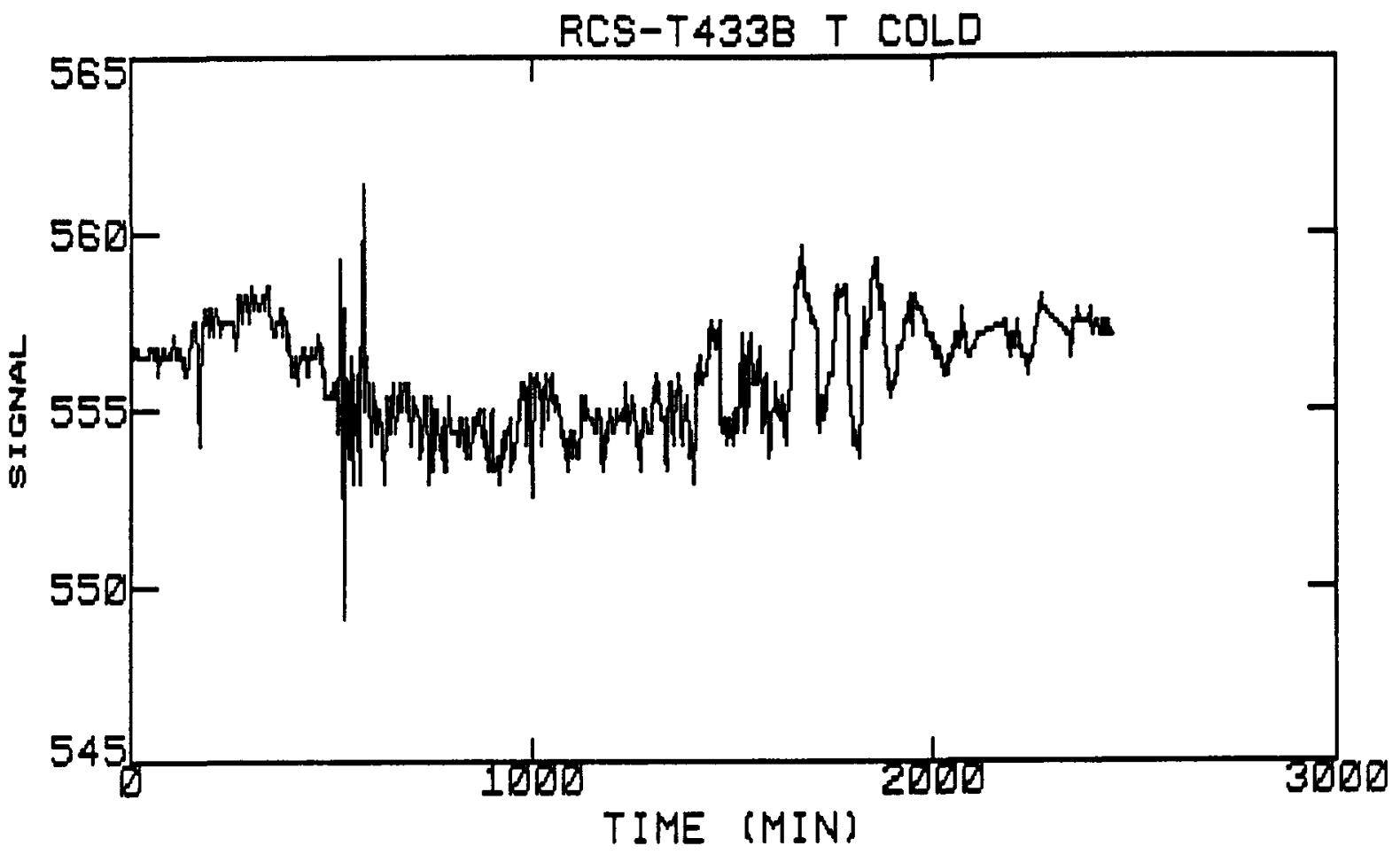

Figure 2.20c-operational PWR start-up data.

Cold leg temperature, measurement No. 3 .

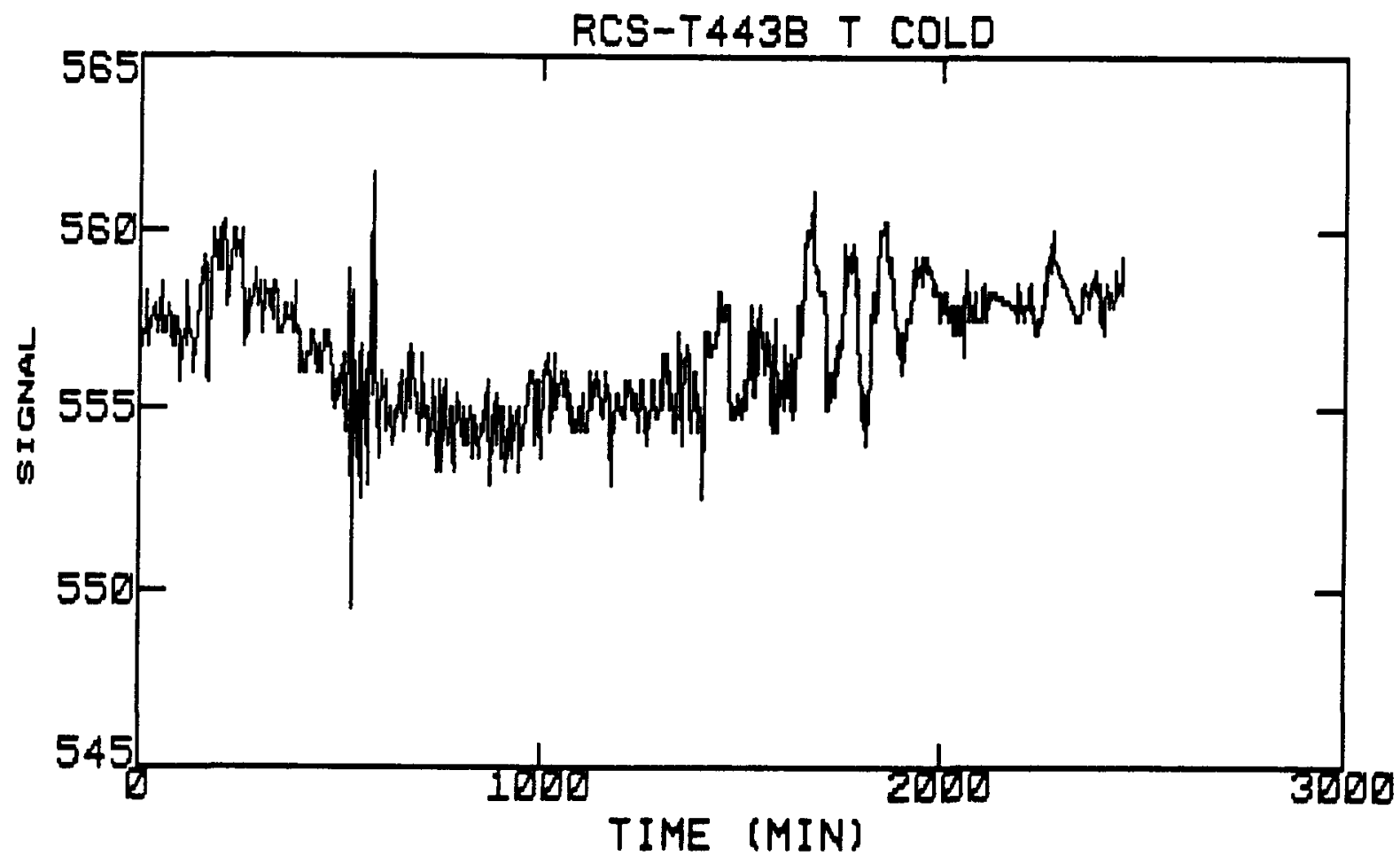

Figure 2.20ã. Operational PWR start-up data.

Cold leg temperature, Measurement No. 4. 
SIGNAL NO. 1

SIGNAL TOLERANCE - $2.0 \mathrm{~F}$ NO. OF REDUNOANT SIGNALS - 4

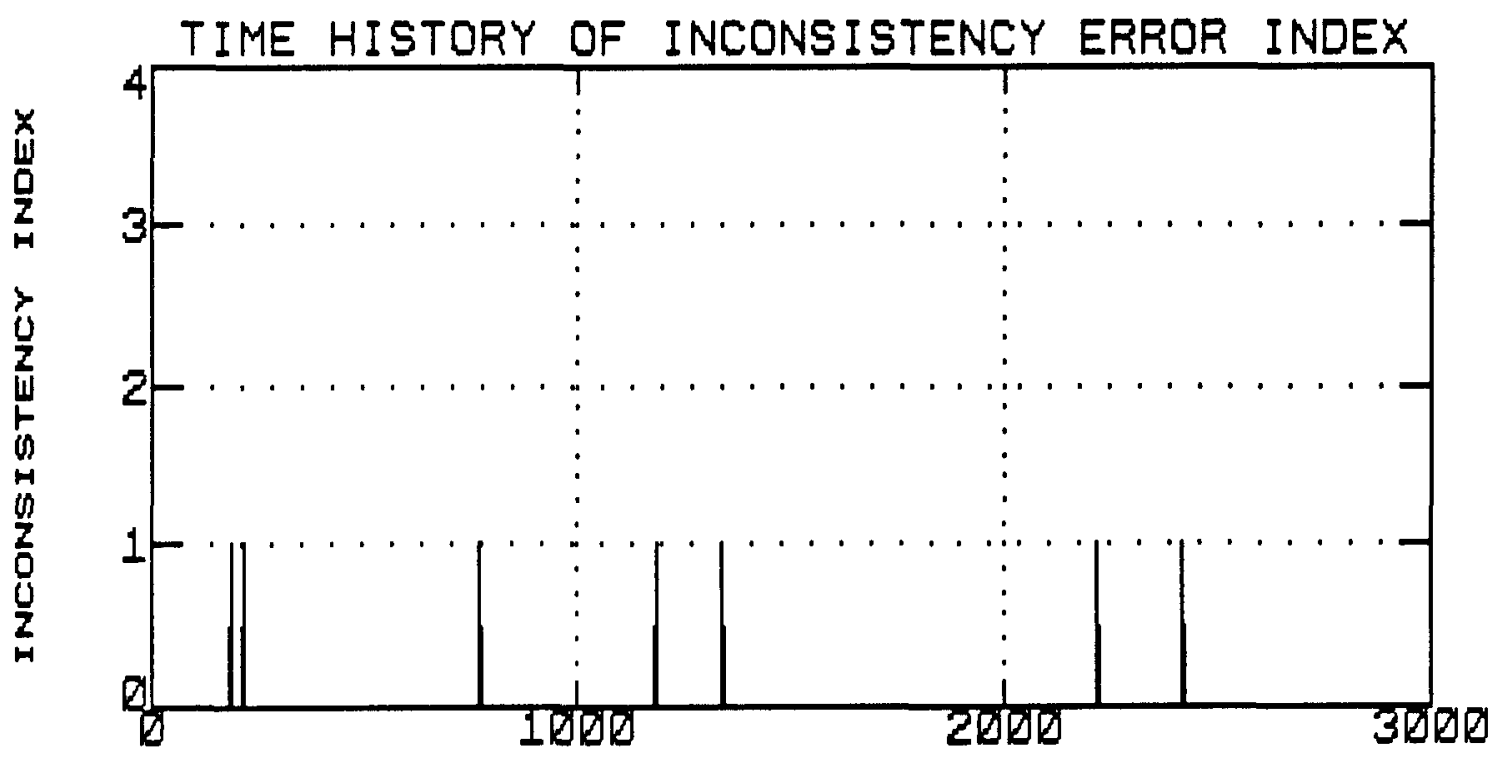

TIME (MIN)

Figure 2.21a. Inconsistency index for cold leg temperature signal, measurement No. 1 .

SIGNAL NO. 2

SIGNAL TOLERANCE - $2.0 \mathrm{~F}$ NO. OF REDUNDANT SIGNALS - 4

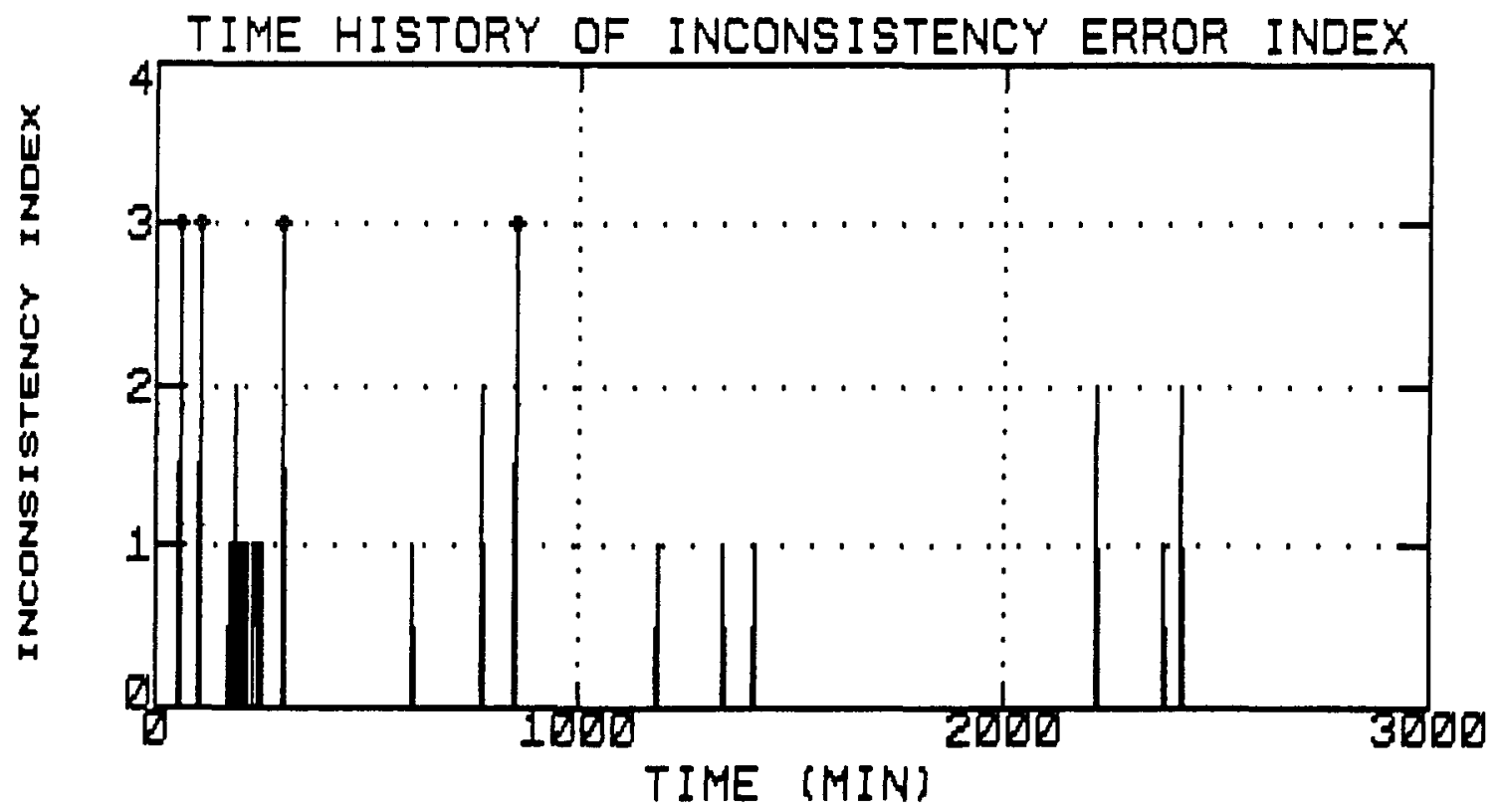

Figure $2.21 \mathrm{~b}$. Inconsistency index for cold leg temperature signal, measurement No. 2 . 
SIGNAL NO. 3

SIGNAL TOLERANCE - $2.0 \mathrm{~F}$ NO. OF REDUNDANT SIGNALS - 4

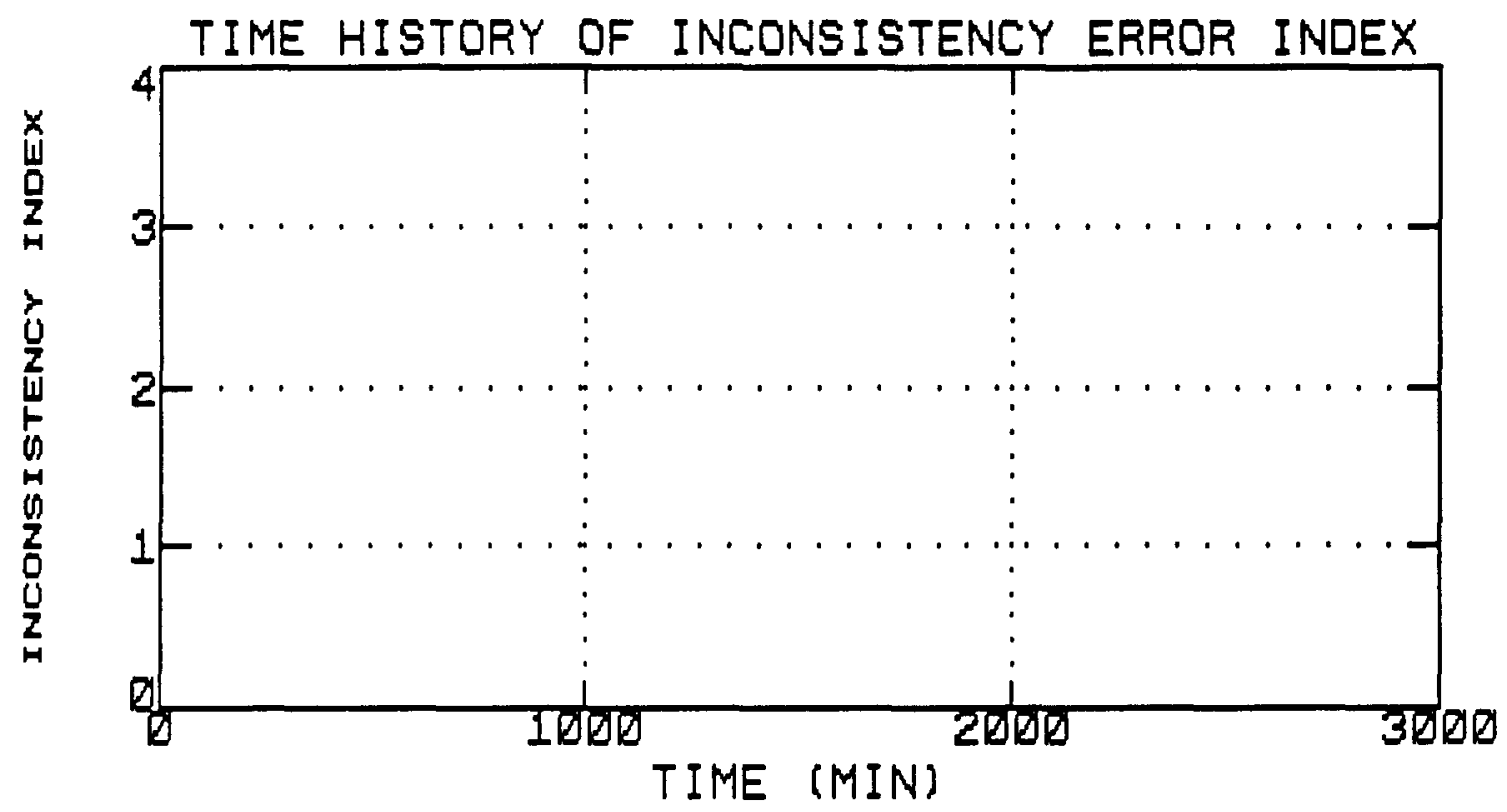

Figure $2.21 \mathrm{c}$. Inconsistency index for cold leg temperature signal, measurement No. 3.

SIGNAL NO. 4

SIGNAL TOLERANCE - $2.0 \mathrm{~F}$ NO. OF REDUNDANT SIGNALS - 4

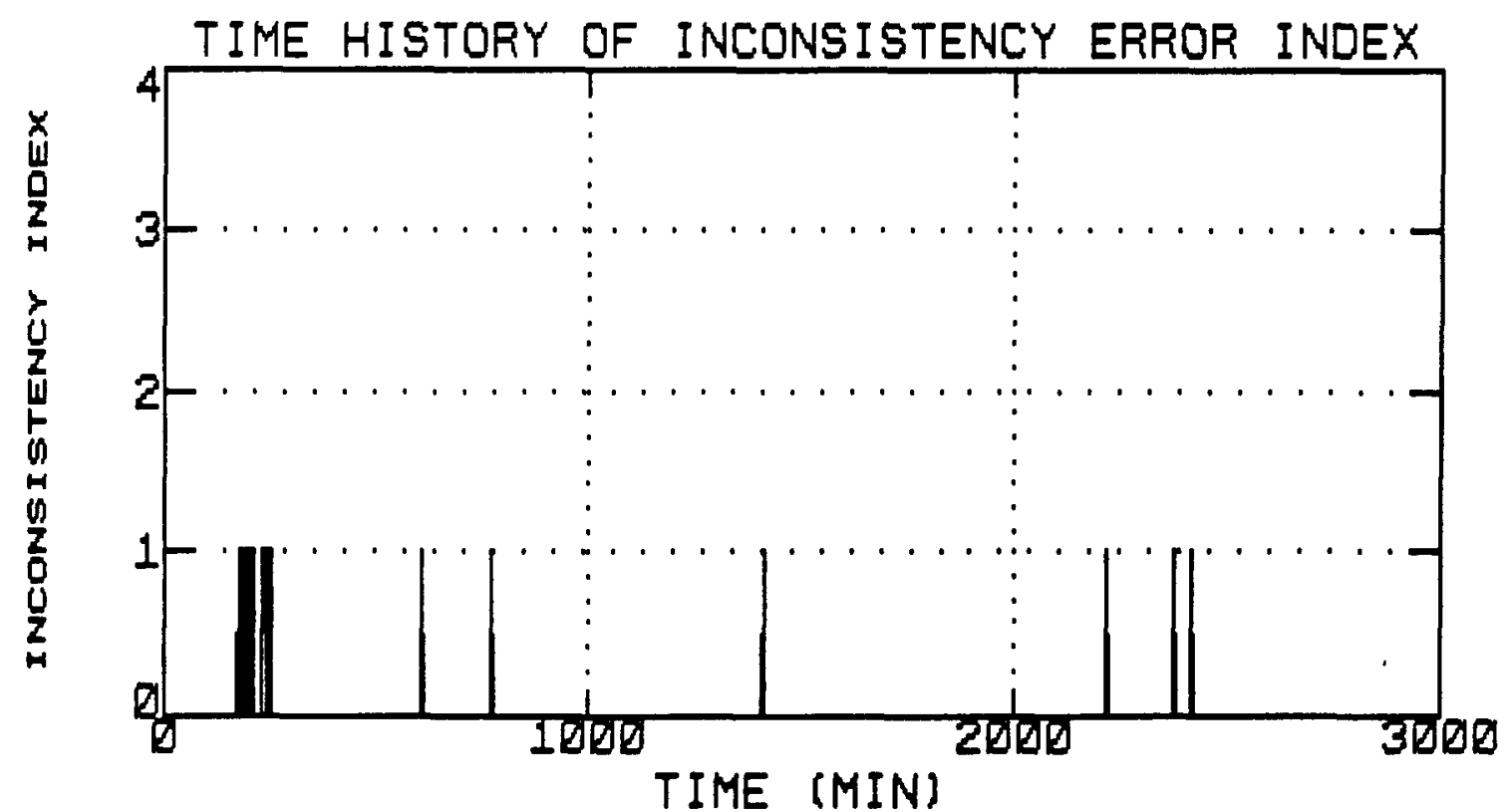

Figure $2.21 \mathrm{~d}$. Inconsistency index for cold leg temperature signal, measurement No. 4 . 
SIGNAL TOLERANCE - $2.0 \mathrm{~F}$ NO. OF REDUNDANT SIGNALS - 4

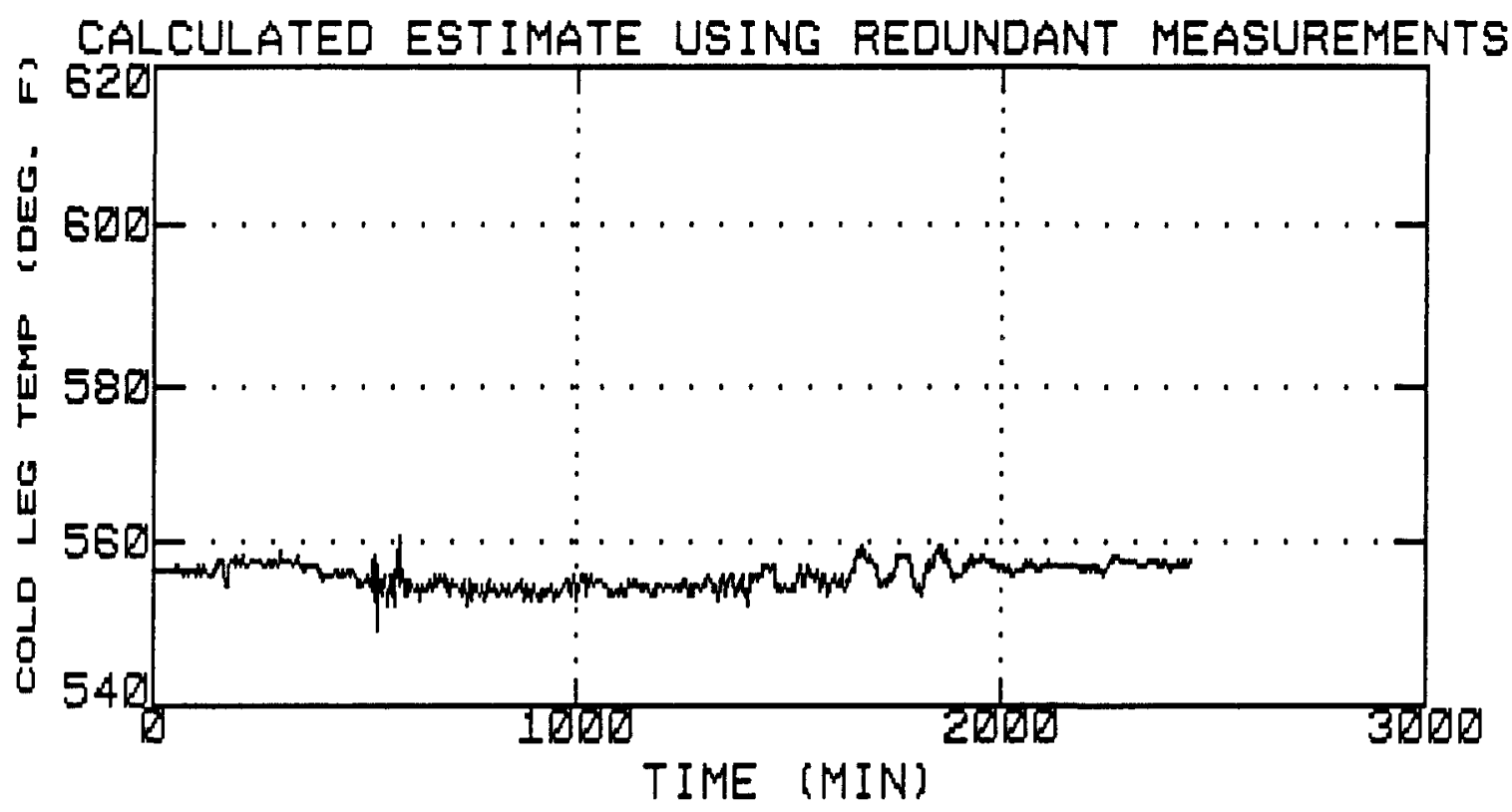

Figure 2.22. Estimated value of the cold leg temperature signal using all the four redundant measurements. The estimation is a weighted average of the four measurements according to their inconsistency indices. 
SIGNAL NO. 1

SPECIFIED MEAN TIME BETWEEN FALSE ALARME (GEC) - 100.0 SPECIFIED MAXIMUM ACCEPTABLE BIAS DEGRADATION - $3.0 \mathrm{~F}$

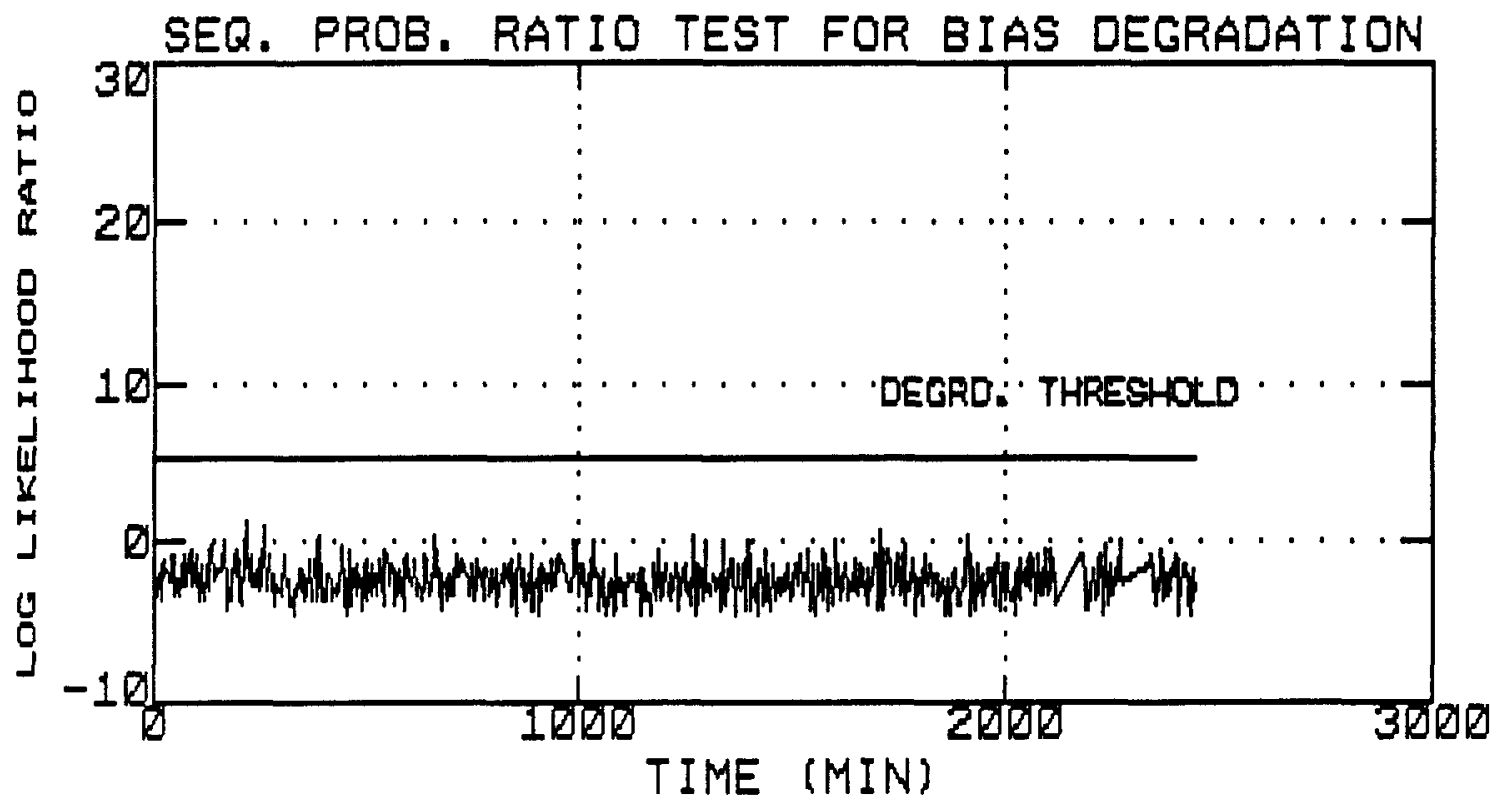

Figure. $2.23 \mathrm{a}$. SPRT index for cold leg temperature signal, measurement No. 1 .

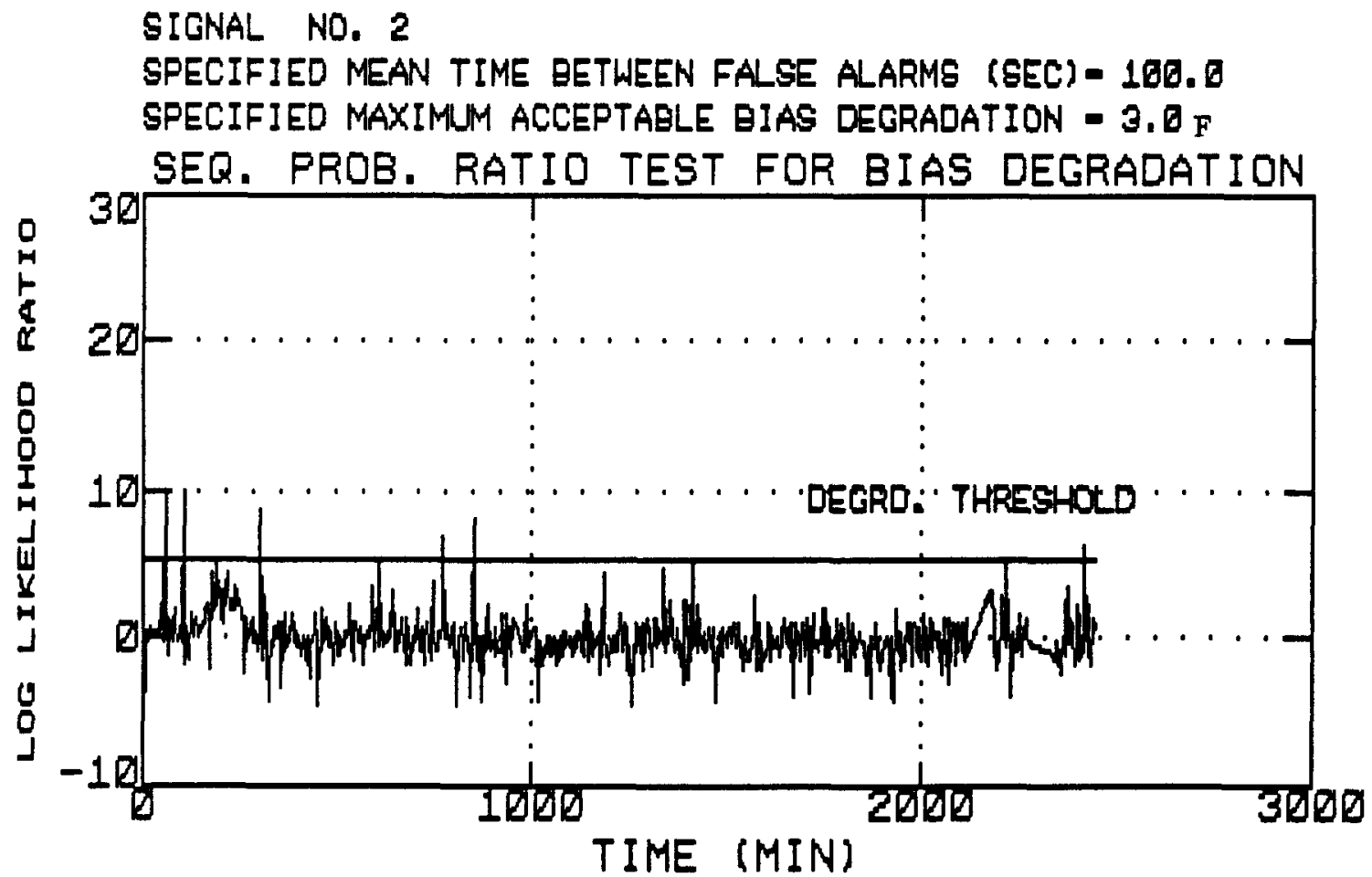

Figure 2.23b. SPRT index for cold leg temperature signal, measurement No. 2. 
SIGNAL NO. 3

SPECIFIED MEAN TIME BETWEEN FALSE ALARMS (SEC) - 160.0 SPECIFIED MAXIMUM ACCEPTAELE GIAS DECRADATION - $3.0 \mathrm{~F}$ SEQ. PROB. RATIO TEST FOR BIAS DEGRADATIDN

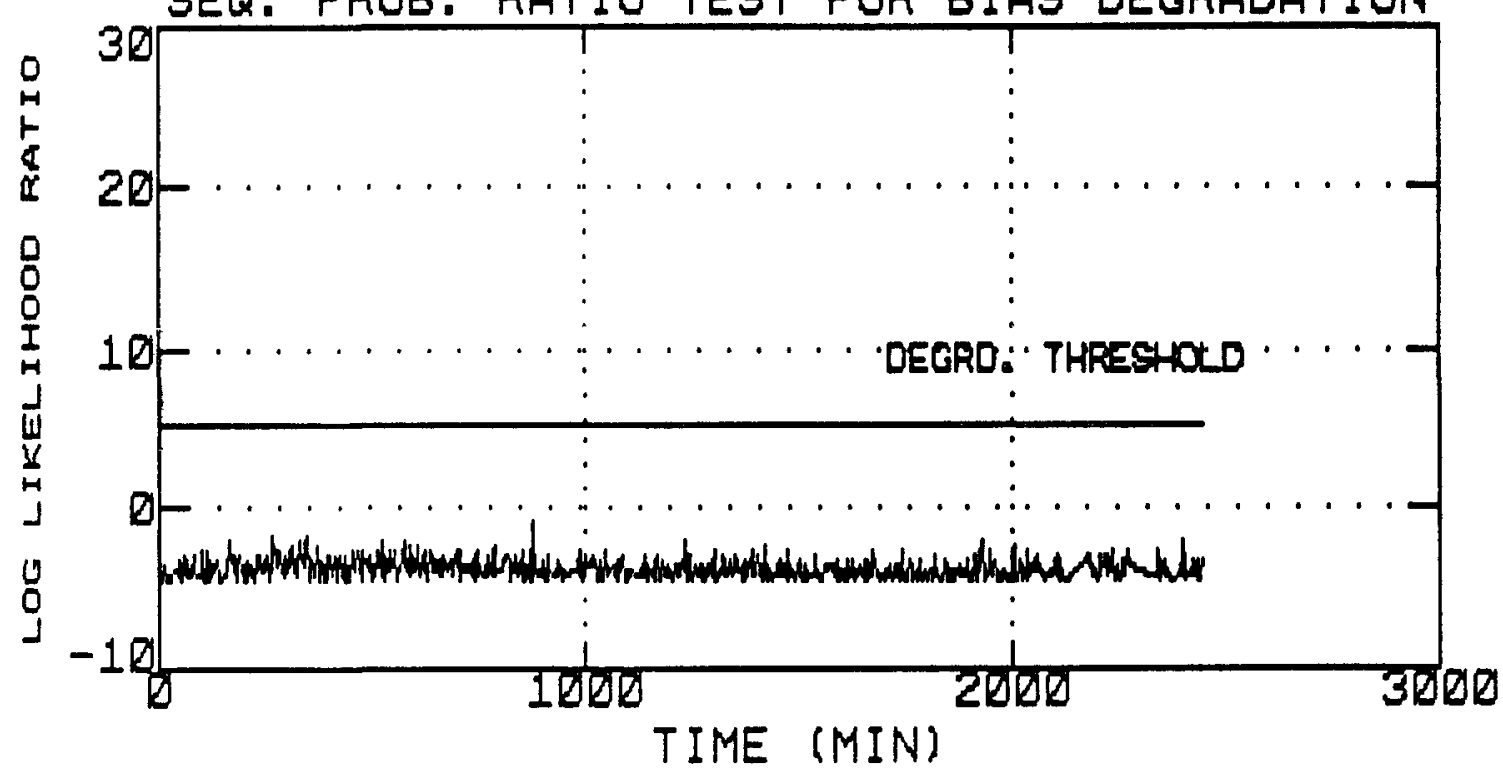

Figure 2.23c. SPRT index for cold leg temoerature, measurement No. 3.

SIGNAL NO. 4

SPECIFJED MEAN TIME BETWEEN FALSE ALARMS (SEC) - 100.0

SPECIFIED MAXIMUM ACCEPTAQLE QIAS DEGRADATION - 3.0F

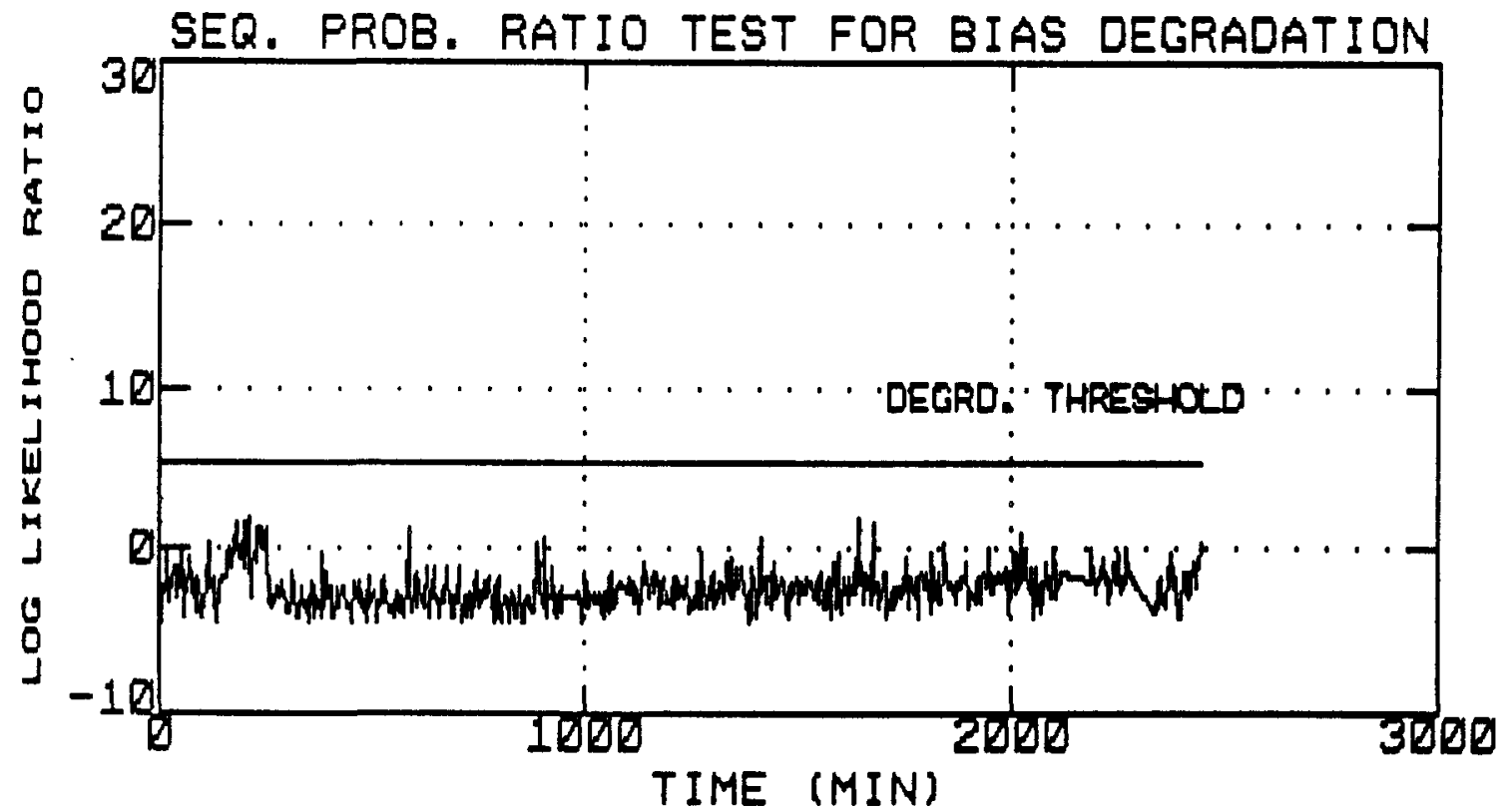

Figure 2.23d. SPRT index for cold leg temperature, measurement No. 4. 


\section{Section 3}

\section{GENERALIZED CONSISTENCY CHECKING OF MULTIVARIABLE REIUNDAN'T MEASUREMENTS (GCCM) AND COMMON-MODE FAILURE DETECTION}

\subsection{Introduction}

The generalized consistency checking (GCC) of a single process variable with redundant measurements involves pairwise comparison of the measurements, and uses a threshold, based on which any two like signals are declared to be mutually consistent or inconsistent [9]. In addition to the direct measurements, one or more analytical measurements may be used to increase the reliability of decision making. The analytical output requires a model (physical or empirical) relating the variable of interest to other dependent variables. In addition, valid measurements of these input signals are necessary to estimate the analytical measurement. In this case the validation of input signals should be performed. Furthermore, it is often difficult to detect the existence of common-mode failure of redundant measurements (sensors) without additional information. For these reasons the single variable GCC algorithm is extended to multivariable signal consistency checking (GCCM). The objectives of the GCCM module are

(1) to separate sensor fault (bias, decalibration) from normal changes in the system, and

(2) to detect and isolate common-mode sensor failures (indicating correlation in sensor failures).

\subsection{Outline of the GCCM Methodology}

It is assumed that each process variable is measured by several redundant signals. The normal behavior of the subsystem under consideration must be established during a learning period by the nonlinear data-driven modeling 
procedure (see Sect. 4 for details). The multivariable consistency checking procedure may be applied to any subsystem characterized by redundant measurements of a set of dependent process variables. The following definitions are used for the purpose of clarification.

(a) Variable -- a state variable representing the dynamics of a system.

(b) Measurement -- output of an instrument channel at a given time instant.

(c) Sample -- a redundant set of measurements of all variables at a given time instant.

(d) Prediction (analytical measurement) -- value of the process variable estimated by a model and measurements of other dependent variables.

The GCCM algorithm consists of a systematic comparison of redundant sensor measurements and their predicted values at each time sample. The input to the empirical models are the other process variables, which are also represented by redundant measurements. Whenever a direct measurement and its predicted value are inconsistent, the cumulative inconsistency index (CII) of both the direct measurements of the selected output variable and the input measurements used for prediction will be increased by one. Based on the CII a complex logic has to be developed to isolate the faulty signals. The simultaneous checking has to be developed to isolate the faulty signals. The simultaneous checking within one redundant measurement set, and the cross-checking among redundant measurement sets of dissimilar process variables, results in an algorithm capable of detecting and isolating bias and calibration errors, and the more complex commonmode failures.

The multivariable GCC algorithm (GCCM) consists of a network of singlevariable GCC modules in parallel. The GCCM algorithm has several levels. The final decision for a given time instant is made only when all possible comparisons at 
the lower levels have been performed. The entire consistency checking is made for each multivariable sample, and a decision is made on the status of each redundant measurement at every discrete time step. The purpose of this repetitive comparison is to exploit the redundant information contained in (1) the measurement redundancy (direct), and (2) the modeling of the relationship among process variables (characterized by empirical models). The consistency checking of multiple variables, each with a set of redundant measurements, starts at the single variable GCC level. A schematic of the multivariate signal validation module (GCCM) is shown in Fig. 3.1.

\subsection{Single Variable Consistency Checking (GCCS) Algorithm}

The GCCS algorithm $[9]$ is a systematic checking of inconsistencies among redundant measurements of one process variable. At time instant $k$, any two like measurements $m_{i}(k)$ and $m_{j}(k)$ are said to be consistent with each other if

$$
\left|m_{i}(k)-m_{j}(k)\right| \leq \eta_{i}+\eta_{j}
$$

where $\eta_{i}$ is based on the instrument tolerance and operating experience. If Eq. (3.1) is not satisfied then $m_{i}(k)$ and $m_{j}(k)$ are said to be inconsistent with each other. The inconsistency indices of the individual measurements and the best estimate of the variable are determined for each sample. The measurements with the maximum number of inconsistency indices are excluded from the consistent set, and a weighted average of the remaining measurements is determined. If there is disagreement among all the redundant measurements, the algorithm flags an error message.

Figure 3.2 is a schematic of the single variable GCC module. It consists of a decision/estimator unit and a sequential probability ratio test (SPRT) unit. The 
SPRT is useful for detecting slow degradation in the signal behavior and can be used to detect incipient failures.

\subsection{Intersignal Relationships}

Consider a subsystem characterized by the variables $X=\left(x_{1}, x_{2}, \ldots, x_{N V A R}\right)$ where NVAR is the number of variables. The physical relationship among these variables is necessary in order to isolate faulty measurements using the GCCM module. These relationships are characterized by the general nonlinear empirical models, and a set of multiple-input single-output (MISO) models are generated, one for each variable. This requires a learning phase during which steady-state measurements are made, and models are developed to characterize various operating regions. The MISO model for signal $x_{k}$ has the form

$$
\begin{gathered}
x_{\ell}=C_{\ell U}+\sum_{i=1}^{\text {NTERM }} C_{\ell t} \phi_{\ell \ell}(X)=\psi_{\ell}\left(x_{1}, x_{2}, \ldots, x_{\ell-1}, x_{\ell+1}, \ldots, x_{\text {NVAR }}\right) \\
\ell=1,2, \ldots, \text { NVAR }
\end{gathered}
$$

where $\left\{c_{0}, c \ell_{1}, \ldots, c_{\ell M}\right\}$ is a vector of coefficients, and $\left\{\Phi \ell_{i}(X)\right\}$ are nonlinear polynomial terms. Equation (2.2) represents a time-invariant model, and may be used to predict the variable $x_{\ell}$ given $\left\{x_{1}, x_{2}, \ldots, x_{\ell-1}, x_{\ell+1}, \ldots, x_{N V A R}\right\}$. The datadriven modeling module identifies the appropriate functional forms $\{\Phi \ell\}$.

The modeling algorithm excludes those input variables that do not influence the behavior of the output variable. In general, the number of input variables necessary to model an output (see Eq. [3.2]) is less than or equal to (NVAR-1). Care must be exercised in establishing the data-driven models. A detailed study is necessary to determine physically realizable models such that the process changes are reflected accurately in the models. Thus the data base for use by the GCCM 
module contains the following information. For the given number of signals NVAR, a maximum of NOFMOD nonlinear MISO models, (NOFMOD $\leq$ NVAR) in the form of cross products of input variables. The model accuracy and the range of model validity is also given.

Denote the functional forms of the models by

$$
x_{\ell}=\Phi_{\ell}\left(x_{1}, x_{2}, \ldots, x_{\ell-1}, x_{\ell+1}, \ldots, x_{n}\right), \quad \ell=1,2, \ldots, \text { NOFMOD. }
$$

The models are stored in the form of cross products of input variables.

\subsection{Multivariate Consistency Checking Algorithm (GCCM)}

\subsubsection{Summary of the GCCM Algorithm}

For a given signal validation consider the direct redundant measurements (for example, variable A in Fig. 3.1) of a signal and its predicted value from the corresponding model. Note that each of the input variables to the model has a set of redundant measurements. Consider the direct measurements of signal $\mathrm{A}$, and its predicted value. If any direct measurement and its predicted value are inconsistent, then increase the cumulative inconsistency index (CII) of the direct measurement and all the input measurements to the model by one. Repeat this for all the direct measurements of variable $\mathrm{A}$, and calculate the $\mathrm{CIIs}$ for all the redundant measurements of each participating input variable. A bad input measurement will have the maximum possible $\mathrm{CI}$ regardless of the status of other redundant input measurements.

The basic assumption in the above analysis is that if one of the input measurements deviates from the correct value, its effect will show up in the model prediction, thus resulting in the inconsistency between the predicted value and the direct measurement of the signal. The same conclusion is reached when the predicted value is correct, but the measured (direct) values of the output variable are 
faulty. In some instances more than one faulty input signal may result in a "correct" predicted value (due to error cancellation). Since these cases require very special correlated deviations in the input and output measurements, they will likely be detected in the next comparison based on the empirical model of another variable.

Once the GCCM has identified the bad measurements at a given sample time, we can go back to the local GCC level and check whether at this level the same measurements have been declared as anomalous. A case in point occurs when a majority of the direct redundant measurements have failed by a common mode. Here the single variable GCC will declare them as inconsistent. Such common-mode failures can be easily detected by the GCCM algorithm. If the results of GCCS and GCCM algorithms differ, then the signal estimate is recalculated. A complex logic has been developed to validate all the variables simultaneously at any time instant.

\subsubsection{Description of the GCCM Algorithm}

Let NVAR be the number of variables describing a subsystem. Let $N R(k)$, $k=1,2, \ldots, N V A R$ be the number of direct redundant measurements of variable $x_{k}$. Let

$$
x(k, i), \quad k=1,2, \ldots, N \text { NAR; } \quad i=1,2, \ldots, N R(k)
$$

be the $\mathrm{i}$-th redundant measurement of variable $\mathbf{x}_{\mathbf{k}}$. Using the given multiple-input single-output model corresponding to output variable $x(k)$, and substituting the $i_{1}$-th measurement of input variable $x\left(k_{1}\right)$, the $i_{2}$-th measurement of input variable $x\left(k_{2}\right)$, . .. and the $i_{n_{k}}$-th measurement of input variable $x\left(k_{n_{k}}\right)$, the predicted output value $x \operatorname{pred}(k, i)$ is

$$
\mathbf{x}_{\text {pred }}(k, i)=\phi_{k}\left\{x\left(k_{1}, i_{1}\right), x\left(k_{2}, i_{2}\right), x\left(k_{3}, i_{3}\right), \ldots, x\left(k_{n_{k}}, i_{n_{k}}\right)\right\}
$$

where $k \in$ OUTVAR, and the indices $\left(k_{1}, k_{2}, k_{3}, \ldots, k_{n_{k}}\right) \in(1,2,3, \ldots, N V A R), n_{k}<$ NVAR represent a subset of a vailable process variables. The set denoted by 
OUTVAR contains the output variables of the NOFMOD number of MISO models. The input variable selection has been performed by the nonlinear empirical modeling module.

The index $\mathrm{k}$ on the left hand side of Eq. (3.5) identifies the predicted process variable, while the index $i$ denotes the $i$-th predicted value, and also identified the given selection of direct input measurements for model $\phi_{k}$ on which the prediction is based:

$$
i=\left(i_{1}, i_{2}, i_{3}, \ldots, i_{n_{k}}\right)
$$

where the choices for the included input variables are

$$
\begin{aligned}
& i_{1} \in\left(1,2,3, \ldots, N R\left(k_{1}\right)\right), \\
& i_{2} \in\left(1,2,3, \ldots, N R\left(k_{2}\right)\right),
\end{aligned}
$$

and

$$
i_{n_{k}} \in\left(1,2,3, \ldots, N R\left(k_{n_{h}}\right)\right) .
$$

Selecting the MISO model for output variable $\mathrm{x}(\mathrm{k})$, the total number of computed outputs taking all possible input measurement combinations is

$$
\operatorname{NOUT}(k)=\prod_{1=1}^{n_{h}} N R\left(h_{1}\right)
$$

where the parameter set $\left(k_{1}, k_{2}, k_{3}, \ldots, k_{n_{k}}\right)$ represents the input variables of the given $M I S O(k)$ model for output variable $x(k)$.

The NOUT(k) number of predicted values of variable $x(k)$ calculated in Eq. (3.7) are compared against the $N R(k)$ number of direct measurements of the same variable. If the difference between the predicted and measured values is greater than a model- and measurement-dependent preset value $\Delta x(k)$, the inconsistency indices (IIs) of all participating direct signals will be increased by one. If

$$
\mid \operatorname{xpred}(k, i)-\left(x\left(k, i_{k}\right) \mid>\Delta x(k)\right.
$$


where the possible values of index $i$ are listed in Eq. (3.6), and $i_{k} \in(1,2,3, \ldots$, $\mathrm{NR}(\mathrm{k})$ ), then for the redundant signals of the output variable of model MISO(k)

$$
\Pi^{M I S O(k)}\left(k, i_{k}\right)=\Pi^{M I S O(k)}\left(k, i_{k}\right)+1
$$

and for the redundant signals of the input variables

$$
I^{M I S O(k)}\left(k, i_{k_{i}}\right)=\Pi^{M I S O(k)}\left(k, i_{k_{i}}\right)+1
$$

where $i_{k_{i}} \in\left(1,2,3, \ldots, N R\left(k_{i}\right)\right)$. The inconsistency indices of variables not included in the model MISO $(\mathrm{k})$ are zero.

The maximum possible inconsistency index of the redundant measurements of the output variable $x(k)$ originated from the model $\mathrm{MISO}(k)$ comparison is equal to the total number of computed outputs as given in Eq. (3.7):

$$
\operatorname{IMAX}^{\mathrm{MISO}(\mathrm{k})}(\mathrm{k})=\operatorname{NOUT}(k)
$$

The maximum possible inconsistency indices of the redundant measurements of the participating input variables originating from the model MISO(k) comparison are

$$
\operatorname{IIMAX} \operatorname{MISO(k)}_{\left(k_{i}\right)=N R(k)} \prod_{\substack{\ell=1 \\ \ell \neq i}}^{n k} N R\left(k_{\ell}\right)
$$

where $N R(k)$ is the degree of signal redundancy of the output variable $x(k)$.

A faulty measurement of the input variable $x\left(k_{i}\right)$ will have the maximum inconsistency index IIMAX ${ }^{M I S O}\left(k^{\prime}\right)\left(k_{i}\right)$ regardless of the status of other redundant input measurements. This is based on the assumption that any computed output based on an input measurement set containing the above incorrect measurement value will be inconsistent with all the direct measurements of the given output variable. 
Repeating the above calculations for all the available MISO models of the given variable set, the cumulative inconsistency index is defined for each redundant signal by adding up their corresponding inconsistency indices originating from inconsistency checking through different MISO models:

$$
\operatorname{CII}\left(\ell, i_{1}\right)=\sum_{k \in \text { OUTVAR }} 11^{\text {MISO }(k)}\left(\ell, i_{1}\right)
$$

The maximum possible cumulative inconsistency index for variable $\ell$ is

$$
\operatorname{CIIMAX}(\ell)=\sum_{k \in O U T V A R} \operatorname{IIMAX} X^{M \operatorname{ISO}(k)}(\ell)
$$

In summary $\mathrm{CII}\left(\ell, \mathrm{i}_{\ell}\right)$ is the calculated total inconsistency index obtained from the comparison of direct measurements with predicted values for all the models, and the contribution where signal $x \ell$ appears either as an input or as an output. The maximum value CIIMAX $(\ell)$ is fixed for variable $\ell$.

If after the multi-model comparison, the $\mathrm{CII}$ of a measurement is equal to the above corresponding maximum index CIIMAX, the signal is declared to be faulty and excluded from the redundant measurement set at the given time instant. In other words, in order to exclude a faulty signal of a variable, it has to fail the consistency tests of all MISO models containing the variable in question. In general, in order to exclude a signal, it is not enough only to compare its actual and maximum possible CIIs. The number of MISO models containing the given variable (either as their output or input) must also be considered. Obviously, redundant signals of a variable not included in any MISO model cannot be validated using the GCCM. Redundant signals of a variable with maximum possible CIIs still can be normal if the variable is included only in one MISO model and there is at least another variable with completely faulty redundant measurement set in the given MISO model with the corresponding maximum CIs. With increasing number of MISO models containing a given variable, the chance for misinterpretation is reduced. The 
final decision about the signal status over a batch of multivariable redundant data samples should depend on the model redundancy and model sensitivity to each process variable.

The cumulative inconsistency index of a correct signal is usually not equal to zero, but is supposed to be minimum compared with those of the other redundant, and perhaps partially or totally faulty signals. After removing the redundant measurements declared to be degraded, the CIIs of the remaining redundant signals are recalculated. The actual CIIs may be higher due to other partial inconsistencies between signals not declared to be faulty. Let us choose a variable $x\left(k_{j}\right)$ whose reduced $\mathrm{CI}$ is to be calculated, and a MISO model which contains the variable as an input to MISO(k). Let us denote the number of detected bad measurements of a variable $\mathrm{x}(\ell)$ out of its $\mathrm{NR}(\ell)$ redundant measurements with $\mathrm{NB}(\ell), \ell=1,2,3, \ldots$, NVAR.

The partial inconsistency index of variable $x\left(k_{i}\right)$ caused by the detected faulty signals in model MISO $(k)$ where the variable $x\left(k_{i}\right)$ is an input variable

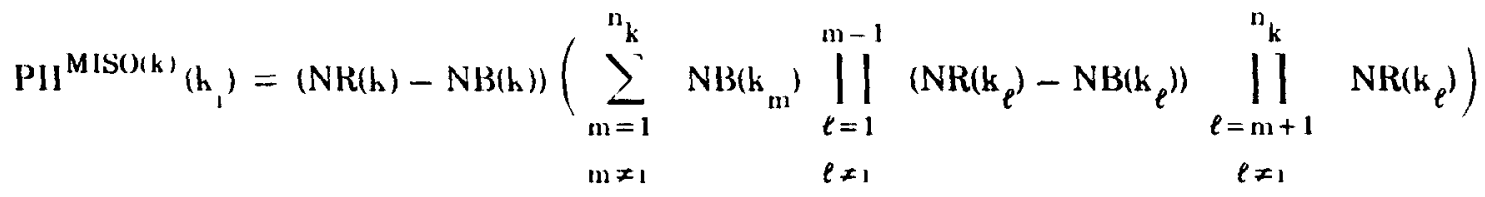

$$
\begin{aligned}
& +N B(h) \prod_{\substack{\ell=1 \\
\ell \neq 1}}^{n_{k}}\left(N R\left(k_{\ell}\right)-N B\left(k_{\ell}\right)\right)
\end{aligned}
$$

where

$\mathrm{n}_{\mathrm{k}}$ is the number of in put variables to the model MISO(k), $\mathrm{NR}\left(\mathrm{k}_{\ell}\right)$ is the degree of redundancy for the input variable $\mathrm{x}\left(\mathrm{k}_{\ell}\right), \ell=1,2,3, \ldots$, $\mathrm{n}_{\mathrm{k}}$, 
$\mathrm{NB}\left(\mathrm{k}_{\ell}\right)$ is the number of detected bad measurements of the input variable $\mathrm{x}\left(\mathrm{k}_{\ell}\right)$, $\ell=1,2,3, \ldots, \mathrm{n}_{\mathrm{k}}$

$N R(k)$ is the degree of redundancy for the MISO output variable $x(k)$,

$\mathrm{NB}(\mathrm{k})$ is the number of detected bad measurements of the MISO output variable $\mathbf{x}(\mathbf{k})$.

The partial inconsistency index of variable $\mathbf{x}\left(\mathrm{k}_{\mathrm{i}}\right)$ caused by the detected faulty signals in model $\operatorname{MSO}\left(\mathrm{k}_{\mathbf{j}}\right)$ where the variable $\mathbf{x}\left(\mathrm{k}_{\mathbf{j}}\right)$ is the output variable

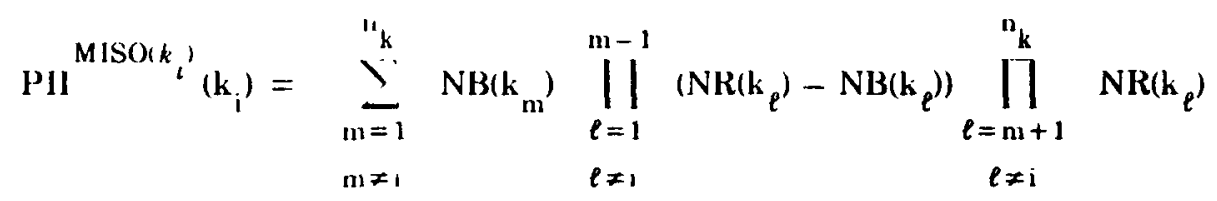

For the given variable $x\left(k_{i}\right)$ the total number of CUs caused by detected faulty signals is calculated by summing up the PIIs in Eqs. (3.13) and (3.14) for all the MISO models containing the given variable $\mathbf{x}\left(\mathbf{k}_{\mathrm{i}}\right)$ either as input or output variable. After reducing the CIls by the above PIIs for each redundant signal, a signal having no partial inconsistencies will have zero CII. The other signals having non-zero reduced CIIs indicate the effects of partial inconsistencies between the remaining signals.

Figure 3.1 is a schematic of the multivariable consistency checking logic. The set of single variable consistency checking units give the estimate of each variable and the consistency status of the redundant measurement sets for the given time sample based on the separate redundant measurement sets. In the next step the multivariable signal comparison is performed using analytical signal predictions based on the stored empirical MISO models. Each MISO output variable is predicted using all possible combinations of the corresponding input variables to the selected MISO models. Going through all the models and input variable sets, the cumulative 
inconsistency indices are calculated based on the comparison of the measured and the predicted values of the given variable. The final decision for the given time sample is based on these cumulative inconsistency indices, and on the results of single variable GCC as well. The sequential probability ratio test for every signal and for the given time instant is performed once the GCC-based decision was made. Then the whole process will be repeated for the next time sample. After processing a batch of multivariable time samples we can estimate the status of each redundant measurement.

The problem of error cancellation or compensation is related to the problem of the sensitivity of the identified model to the uncertainties in the current input measurements to be validated. The basic assumption in the above procedure is that if one of the input measurements deviates from its correct value due to sensor error, its effect will show up in the computed output values, causing inconsistency between the measured and predicted output values. The same inconsistency can be reached if the input measurements and the corresponding predicted output have the correct values but the direct output measurement is incorrect due to sensor fault. In both cases the cumulative inconsistency indices of all participating direct measurements (including the direct output measurement) will be increased by one. While an incorrect output measurement can cause inconsistency directly, the deviation of an input signal has to be transferred through the MISO model to the predicted output value. This means that an incorrect reading of an input measurement can be detected by comparing the computed and measured output values, only if the given MISO model is sensitive enough to that input variable. A further problem is that if there are more than one faulty signals among the input measurements, their contribution to the predicted output value may be cancelled. These cases are less likely to occur, but if they do, they will be declared incorrectly as normal 
measurements for the given MISO model. Since these cases require very special deviations in the input and output measurements, they will likely be detected in the next pass based on another MISO model.

Once the multivariable consistency checking algorithm identifies the bad measurements for the given time sample, we can go back to the single variable GCC level and check if the single and multivariable GCC logic selected the same measurements as faulty. In certain cases the single variable GCC cannot give the correct answer due to limited available information. One example is if the majority of the redundant measurements fail simultaneously and identically. In this case the single variable GCC will select the identically failed measurements to be correct based on majority, and consequently the signal estimate will be incorrect. Applying only the single variable GCC logic, there is no way to correctly judge this situation. That is why the multivariable GCC logic could be important in detecting correlated, common-mode or identical failures in the redundant measurement set. Also, using the information given by the multivariable GCC we can calculate the signal estimate for each variable at the given time instant. The signal estimate is computed primarily based on the direct redundant measurements as a weighted average, where the weighting factor is the ratio between the $\mathrm{CI}$ and the maximum possible CII. If all the redundant signal values of a variable are incorrect, and consequently excluded from the measurement set, the only way to estimate the correct value of the variable is to use its corresponsing MISO model and the best direct measurements of the input variables. If no MISO model exists for the given variable, or if no estimate can be calculated for all the necessary input variables, then no estimate can be given for the variable in question. In this case, one possible solution to get the estimate is to interpolate between the estimates of the same variable at several different time instants. 
The questions of the error compensation and model sensitivity, as well as the problem of establishing a sufficient number of accurate empirical MISO models, need further work and numerical experiments. Also, the empirical modeling algorithm has to generate the information the multivariable GCC algorithm needs in a proper form and with the required accuracy.

Once the emprical models and their confidence levels are identified, they are used for measurement prediction. The accuracy of the predicted values depends not only on the model accuracy but the uncertainties of the input measurements used for prediction. The propagation of the input measurement uncertainties to the predicted value needs an extensive analytical and numerical computation. Since the multivariable consistency checking procedure relies on a systematic comparison of the deviation between redundant and predicted measurements and their corresponding signal tolerance, it is extremely important to get an accurate process prediction together with its confidence level. Since the empirical models give a status representation of the intersignal relationships (no time variable or process memory are involved in the models), the algorithm is not applicable to transient measurements in its present form.

\subsection{Examples of Numerical Tests Performed using the Multivariable GCC Algorithm}

Several examples of results from two numerical experiments are shown in this section in order to demonstrate and explain the operation of the GCCM algorithm. The input information to the GCCM analysis, like MISO models used for the test, and the series of simulated multivariable redundant measurements, which have to be validated are shown. Also the results of the GCCM analysis, the calculated CIs, and the estimates of the variables as functions of sample numbers are discussed and shown. After processing a batch of multivariable samples, the GCCM algorithm 
gives the final evaluation of signal confidence in terms of overall goodness percentages of signals characterizing the analyzed data length. Functions and tables generated as results of the GCCM algorithm are discussed. The numerical cases to be discussed represent actual applications of the consistency checking and fault detection algorithm in redundant signal sets of related process variables. Analyses of partially or fully degraded redundant signal sets are presented.

\subsubsection{Implementation of the GCCM Algorithm}

In the first step the program reads in a set of MISO models generated and selected by the PEM module, or generated by a simulation program in the present numerical test, for the given variable set. One of the input files to the GCCM program, called MISOMODx.xxx contains the following information about the empirical MISO models to be used in the GCCM program:

NVAR -- the number of variables included in the set.

NOFMOD -. (NOFMOD $\leq$ NVAR) the number of MISO models generated and selected by the PEM module.

- The steady-state measurement range for each variable used for establishing the MISO models in the PEM module.

- IDOUT(k) -- the identification number of the k-th MISO model output variable $(\mathrm{k}=1,2, \ldots$, NOFMOD).

$\operatorname{NTERM}(k)$-- the number of terms in the polynomial form of the $k$-th MISO model.

- CONST(k) - the constant terms in the polynomial form of the k-th MISO model. 
- $\quad A C C U R(k)$-- the prediction accuracy of the $k$-th MISO model in percentage.

- $\operatorname{COEFF}(k, i)$-- the coefficient of the $i$-th term in the polynomial form of the $k$-th MISO model $(i=1,2, \ldots$, NTERM(k)).

- $\quad \operatorname{NPOWER}(k, i, j)$-- the power of variable $j$ in the $i$-th term in the polynomial form of the $k$-th MISO model $(j=1,2, \ldots, N V A R)$.

In real application, this information is generated by the PEM module based on a set of steady-state multivariable measurements during a learning period. In the present numerical experiment the above data file is created synthetically.

After printing out the information fromthe file MISOMODx.xxx for checking, the next subroutine called SIGSLCT selects the input and output variables, and determines the model redundancy of each variable (model redundancy of a given variables $=$ number of MISO models containing the given variable either as one of the input variables, or as the output variable of the MISO model).

The next subroutine called MAXPCI determines the maximum possible cumulative inconsistency indices of each variable based on the structure of the given MISO models, and on the signal redundancy levels of each participating variable. Also, the subroutine calculates the maximum possible inconsistency indices which can be assigned to a given variable as being an input, or an output, considering all the NOFMOD number of MISO models. The maximum possible CII of a variable depends only on the redundance levels of the other variables taking part in the MISO model(s), and it is independent of the redundancy level of the given variable itself.

The next subroutine called SENSIT calculates the sensitivity of the output variable to its input variables for each considered MISO model. The relative contribution of the input variables to the value of the output variable in the given 
measurement range determines the detectability of degraded measurements of the input variables.

After these precalculations based on MISO model information, the program starts the sample-by-sample evaluation of the current redundant measurements to be validated. The redundant measurements of all variables at the given sample are read in from the data file MRDMEASx.xxx. The following subroutines are used in sequence for every multivariable sample:

- Subroutine INCONS detects the inconsistencies among the redundant measurements of the output variable of a given MISO model and their predicted values calculated using all possible combinations of the redundant measurements of input variables. The predicted and the measured MISO output values are declared to be inconsistent if the absolute value of their difference is greater than the sum of the predefined prediction and measurement errors. This procedure is repeated for all MISO models considered. Whenever an inconsistency is detected, the ClIs of every participating output and input measurement are increased by one. At the end of the measurement processing, $\mathrm{C} \Pi$ values will be given to every redundant measurement.

- Based on the above CIIs and the model redundancies of variables, the subroutine called EVALUATR identifies the measurements to be excluded. The criterion for exclusion is the following. The measurement has to have its maximum possible $\mathrm{CI}$, and its model redundancy is bigger than one. If the model redundancy of the measurement with the maximum possible $\mathrm{CI}$ is only one, the sufficient condition for excluding the measurement is that there should not be another variable in the same MISO model with totally degraded redundant measurement set. If there 
is another variable in the same MISO model with all its redundant measurements degraded, the former measurement having its maximum possible $\mathrm{CII}$ and model redundancy equal to one is not declared to be degraded, and it is not excluded. Once the degraded measurements are identified and excluded, the CIIs of the remaining measurements are recalculated without including the degraded measurements.

- The next subroutine called ESTIM gives the best possible estimate of each variable based on only their redundant measurements and their calculated CIIs. The estimates are weighted averages of redundant measurements with weighting coefficients inversely proportional to the corresponding CIIs. If all the redundant measurementsof a MISO model output variable are degraded, the predicted values of the MISO output variable is considered as the estimate of the variable at the given sample. In this case the program selects the input measurements with minimum CIIs to predict the output value of the MISO model. If all the redundant measurements of a variable which is not an output variable of any MISO model are degraded, its estimates cannot be calculated. The program considers the variable estimates at the previous sample as the current estimate.

After repeating the above procedure for every multivariable redundant sample, the subroutine called OAVOTE gives the confidence or goodness percentage of every redundant signal based on the processing of the whole batch of samples. This number characterizes the overall consistency of the signals during the evaluated sample period. 
The subroutines called DATAPLOT, CUSPLOT and ESTMPLOT plot out the values of multivariable redundant signals, the calculated CIIs for each signal and the estimates of every variable, respectively, as functions of sample numbers.

\subsubsection{Results and Interpretation of Numerical Experiments}

\subsubsection{The Case of Partially Degraded Redundant Signal Sets}

In the following some details of the performed numerical experiments are discussed. The MISO models of the first example are shown in Table 3.1. In this given case we have five process variables with four or five redundant signals. Three MISO models were selected out of the possible five to use in the GCCM procedure (or generated by the PEM module in a real case), and the corresponding MISO output variables are the variables No. 1, 3 and 5 , respectively. The variable range over which the MISO models were established during the PEM learning period is also shown. Table 3.2 shows the following calculated parameters for every MISO model: (a) the relative contribution of the constant term to the predicted value, (b) the relative prediction error in percentages, and $(c)$ the relative contribution of the uncertainty of the input variables to the uncertainty of the predicted (output) variable. These latter parameters give the sensitivity of the MISO output variable to the input variables. Faulty measurements of an input variable with high relative contribution (with high output sensitivity to the given input variable) can likely be detected. Degraded measurements of other input variables with low relative contribution to the output variable must have bigger deviation in order to cause inconsistencies between the measured and predicted output values, consequently their detectability is lower. All these parameters are calculated by the subroutine called SENSIT based on the three MISO models stored in the input file 
MISOMOD1.002, and on the variable range in which the MUSO models were established.

The second input file to the GCCM analysis is the actual multivariable samples of redundant signals of the included process variables. The actual measured mulivariable samples, which are to be validated, are stored in a separate measurement file called MRDMEAS1.002. In the given numerical example we have fifty samples of redundant signals of five variables. The program reads in each sample separately, then calculates the CIIs and makes local decisions about the signal consistency at each multivariable redundant sample. The fifty samples of redundant signal values on which the GCCM operated in the first numerical test are shown in Figs. 3.3a through 3.3e. The curves with different markers represent the redundant signals of the given variable. The normal and consistent status of the redundant signals is given by the starting constant values of each variable. Variable Nos. 1, 2 and 4 have consistent redundant signals, slightly deviating in the range of samples 20 and 40, then having identically consistent values at sample No. 50 .

The following bias type faults are artificially introduced in certain signals of two variables:

(a) The last three signals of variable 3 have ramp bias error, increasing to sample No. 25, and then decreasing to the correct value at sample No. 50 . The first two signals of variable 3 have the correct constant values for all fifty samples (Fig. 3.3c).

(b) The last two signals of variable 5 have an increasing ramp deviation starting from sample No. 13. The first two signals of variable 5 have the correct constant values for all fifty samples (Fig. 3.3e). 
The aim of applying the GCCM algorithm in the numerical test is to detect the known faulty measurements, even if, at certain samples, the majority of the redundant signals of one or two variables are identically biased, and also to give the best estimate of each variable at each sample.

There are two important issues which highly influence the success of the GCCM analysis, and can be studied through numerical experiments. One is the model redundancies of the variables; that is, the number of different MISO models containing a given variable either as one of the input variables, or as the output variable. In an extreme case, when a given variable is not part of any MISO model at all, the variable cannot be validated by the GCCM procedure. Variables included only in one MISO model might be validated depending on the actual case (e.g., the sensitivity of the predicted value to the given variable, if it is an input variable). The higher the model redundancy of a variable, the higher is the confidence of GCCM decision with respect to the given variable. The final decision about the status of a given signal cannot be based entirely on the calculated CIs, but also, the above mentioned signal sensitivity and model redundancy must be taken into account. Besides the CIIs calculated for every signal and at each sample, the final output of the GCCM procedure is a confidence number characterizing the status of a given signal over the processed sample set.

In the following we will discuss the results given by the GCCM applied to the input data shown in Figs. 3.3a through 3.3e. At each sample the GCCM algorithm computes the CIIs of every redundant signal and an estimate for each variable.

- The redundant measurements of the five variables at sample No. 1 are shown in Table 3.3 along with the corresponding measurement uncertainties. Since the numerical example was generated so that all the redundant measurements of all variables are consistent with each other 
and with the predicted values at sample No. 1 , the CIIs are all equal to zero for each measurement.

- In the subsequent samples a slow bias was introduced in certain signals, and some non-zero CIIs appeared at sample No. 5; still they are much lower than the maximum possible $\mathrm{CH}$ for the redundant signals of the given variables (see Table 3.4)。

- At sample No. 8, the CII of the third signal of variable 3 reached its maximum possible CI limit (Table 3.5); and since the model redundancy of the given variable is 2 (variable 3 is one of the inputs in the first MISO model, and is the output of the second MISO model), the measurement was declared to be faulty and was excluded from the redundant set. The new CIls recalculated after the exclusion can also be seen in Table 3.5. The lower part of Table 3.5 indicates the excluded measurements showing their model redundancy, and indication by zeros for the non-excluded measurements.

- At sample No. 10, the last three measurements of variable 3 are identified as degraded measurements and excluded from the set (see Table 3.6). The recalculated CIIs for the other measurements are equal to zero, which means that the causes of the initial inconsistencies are isolated, and the remaining measurements give a fully consistent set after exclusion.

- Due to the additional slowly increasing ramp bias introduced in the last two signals of variable 5 , they reached their maximum possible CIls, first the third signal at sample No. 15 , then the fourth signal of variable 5 at the next sample ('T'ables 3.7 and 3.8). Since their model redundancy is also equal to 2 (variable 5 is an input in the second MISO model, and is 
the output of the third MISO model), they were isolated as faulty signals and excluded from the redundant signal set. Note that at sample No. 16 the identically deviating faulty signals of variables 3 and 5 were detected and excluded simultaneously. The capability of the GCCM algorithm for simultaneous detection of correlated or common-mode failures is based on the intersignal comparison using the cross-information modeled by the empirical MISO models. This capability does exist even if the majority, or as we shall see, the whole set of the redundant signals of the given variable is degraded.

- As it can be seen in Fig. 3.3c, the three identically faulty signals of variable 3 started to drift back to their correct values at sample No. 25 . At sample No. 44 all the above three signals have CMs less than their maximum possible CIs (Table 3.9), consequently they are not excluded from the redundant signal set. The signals are still deviated from their correct values causing partial inconsistency among the compared variables. In this transition region from the degraded status to the normal one, where the CIs of the previously excluded signals are still high but less than their possible maximum CIs, the CIls of the other signals are not reduced as they were previously, since no total inconsistency is found among the previously degraded signals of variable 3. During the transition period the good signals are penalized due to the remaining partial inconsistencies, and their CIIs increased due to the absence of signal exclusion and the subsequent $\mathrm{CH}$ recalculation. The two degraded signals of variable 5 are still excluded, and the CIs of signals being in the same MISO model(s) with the above degraded signals are reduced by the appropriate value. 
- As the values of the previously faulty signals of variable 3 are nearing their correct values, the CUs of all related signals decrease, except that of the increasingly deviating two signals of variable 5 . At sample No. 47, all normal signals have zero CIIs recalculated after excluding the two faulty signals of variable 5 (Table 3.10 ).

After processing all the fifty available multisignal samples, a final evaluation of the redundant signals is given in Table 3.11. The confidence or goodness of each signal is based on the comparison of their actual and maximum possible $\mathrm{CH}$ values, and is given in percentages. The high percentages of the known good signals (approx. 93\%), and the low values (approx. 25\%) for the signals with known changing deviation during the analyzed period give an acceptable estimation of overall signal status. Note that these percentages are results of a conservative estimation of signal confidence according to the rule that "all measurements used in a MISO model are penalized if inconsistency between the model prediction and the measured values is detected."

The CIl functions calculated over the fifty samples are shown in Figs. 3.4a through 3.4e. The curves with different markers represent the $\mathrm{CHs}$ of the redundant signals of given variables. These $\mathrm{CI}$ values are calculated at each sample independently. No intersample comparison or cumulative error calculation is involved. The following gives a discussion of the $\mathrm{C}$ I functions.

- At the first four samples no inconsistencies were detected. The slow deviation of the last three signals of variable 3 reached the inconsistency limit causing non-zero $\mathrm{CII}$ values for all the participating signals. In the case of variables 1,2 and 4, clear transition regions can be seen at sample Nos. 5 through 10, and 41 through 47 (see Figs. 3.4a, 3.4b and 3.4d). This is a distorting effect of the non-excluded, partially consistent redundant 
measurements of variable 3 with high, but below the threshold deviation causing high, but not maximum CIIs. A similar transition period can be observed in the CII functions of the normal signals of variables 2, 3 and 4 with relatively high CII values at samples 13 through 15 . This is caused by the transition of signals 3 and 4 of variable 5 from the normal status in to the degraded status starting at sample No. 13 (see Fig. 3.3e). In the numerical test all the signals of the variables 1,2 and 4 have normal values for the whule data length (see Figs. 3.3a, 3.3b and 3.3d); still they have relatively high CIIs in the above mentioned transition periods. This is due to the basic rule that if inconsistency between the measured and the predicted MISO output values is detected, all the participating measurements, including the normal ones, will be penalized by increasing their CIls by one. Based on this rule, the GCCM finally gives a conservative estimate of the signal status at the end of multisample processing.

- The gradually deviating signals of variables 3 and 5 were excluded when the corresponding Clls reached the maximum possible values. Both variables have a model redundancy of 2 (number of MISO models containing the given variable). The remaining normal signals of the above two variables have low CПs, except in the discussed transition periods.

The estimated correct signal values for all the given variables are shown in Figs. 3.5a through 3.5e. They are calculated at each sample separately using a weighted average of redundant measurements, where the weighting factors are inversely proportional to the CII values. The excluded measurements are not taken in the average. If all the redundant measurements of a variable were degraded at a 
certain sample, the estimate of this variable would be given by the corresponding MISO model prediction using the measurements of the input variables having minimum CIIs. If the given variable with totally degraded redundant measurement set is not the output of one of the MISO models, the estimate cannot be determined at the given sample. The algorithm will take the estimate at the previous sample as the current estimate. As can be seen in Figs. 3.5a through 3.5e, the GCCM algorithm gave the correct constant values as estimates for all the five variables at all the fifty samples, including the cases when the majority of the redundant measurements of one or two variables were degraded.

\subsubsection{The Case of Partially and Totally Degraded Redundant Signal Sets}

In the second numerical test we used the same signal and variable set, and the same empirical MISO model set and measurement samples of redundant signals as we used in the first numerical test, except variable 3 , where now all the four redundant signals will experience an identical slow ramp increase up to sample No. 25 , then a similar slow ramp decrease beyond sample No. 25, reaching their normal initial value at sample No. 50. 'This identical and simultaneous degradation of the whole redundant signal set of a variable represents a very extreme and difficult situation of common-mode failures.

The actual measured multivariable samples, which are to be validated, are stored in the measurement file MRDMEAS2.002. The fifty samples of redundant signal values to be analyzed in the second numerical test are shown in Figs. 3.6a through 3.6e. Again the curves with different markers represent the redundant signals of the given variable. The normal value of the redundant signals is given by the starting constant values of each variable.

In the following we will discuss the results of the GCCM analysis applied to the measurement data shown in Figs. 3.6a through 3.6e. 
- The redundant measurements of the five variables at sample No. 1 are identical to those in the first numerical example discussed above, and are shown in Table 3.3 along with the corresponding measurement uncertainties. All the redundant measurements of all variables are consistent with each other and with the predicted values at sample No. 1 , so the CIIs are all equal to zero for each measurement.

- In the subsequent samples a slow and almost identical bias was introduced in all the four redundant signals of variable 3 , representing common-mode degradation. Increasing CHs appeared starting at sample No. 5 (Table 3.12), and at sample No. 8 the CIs of signal Nos. 2 and 3 of variable 3 reached their maximum possible CII limits (Table 3.13) as expected. However, due to the differences in varying sensitivities discussed above, signal No. 1 of variable 1 with normal reading also reached its maximum possible CII. Since only one MISO model contains the variable No. 1 (as the output variable of the first MISO model), there is no other MISO model which can be used to prove that variable No. 1 has correct redundant measurements. At the same time, since no other variable with totally degraded redundant measurement set exists, the signal No. 4 of variable No. 1 is excluded at this sample based on having its maximum possible $\mathrm{CII}$, even though it has the correct constant value (see Fig. 3.6a or Table 3.13). Also the deviating redundant signals of variable No. 3 with maximum possible CIIs are excluded (the model redundancy of the given variable is 2). The new recalculated CIs of the remaining signals can also be seen in Table 3.13. Table 3.13 also indicates the excluded measurements showing their model redundancy, and indicated by zeroes for the non-excluded measurements. 
- At sample No. 10 all the five measurements of variable No. 3 reached their maximum possible CШs (see Table 3.14). Since their model redundancy is higher than one, they were excluded (common-mode degradation of the whole redundant measurement set); consequently, the measurements of variable No. 1 with their maximum possible CIIs and model redundancy equal to one were not excluded. The remaining measurement set is fully consistent.

- As the ramp bias introduced in the last two signals of variable 5 increases, they reached their maximum possible CIIs at sample No. 16 (Table 3.15). Since the model redundancy of variable No. 5 is equal to 2 , they were isolated as faulty signals and excluded from the redundant signal set. Note that at sample No. 16 the identically deviating faulty signals of both variables 3 and 5 were correctly detected and excluded. The GCCM algorithm gives the same result through sample No. 41.

- The five identically faulty signals of variable 3 started to drift back to their correct value at sample No. 25. During the transition period of variable No. 3 from the degraded status to the normal one, the algorithm misjudges the measurements of the correct variable No. 1. At sample No. 42 , the last two signals of the previously degraded variable No. 3 have CIIs less than their maximum possible CIIs (Table 3.16), changing their fully excluded redundant signal set into a partially excluded one. Consequently two redundant measurements of variable No. 1 having the maximum possible Clls of one hundred are excluded.

- At sample No. 44 only the two degraded signals of variable No. 5 with increasing bias are excluded, and the CHs of signals being in the same MISO model(s) with the above two degraded signals are reduced by the 
appropriate value. The signals of variable No. 3 with decreasing bias still deviate from their correct values causing partial inconsistency among the compared variables. In this transition region of variable No. 3 from the degraded status to the normal one, the CHs of the signals of other related variables are not reduced and this results in relatively high $\mathrm{CI}$ values (see Table 3.17).

- As the values of the previously faulty signal set of variable 3 are nearing their correct values, the CIIs of all related signals decrease, except that of the increasingly deviating two signals of variable 5. At sample No. 47, all normal signals have zero CIIs recalculated after excluding the two faulty signals of variable 5 (Table 3.18 ).

After processing all the fifty available multisignal samples, a final evaluation of the redundant signals is given in Table 3.19. The confidence of each signal is given in percentages. As expected, all the five signals of variable No. 3 with changing deviation during the analyzed period have low confidence value (approx. $24 \%$ ). The good signals have high confidence value (approx. 92\%), except the signals of variable No. 1 (approx. 87\%) excluded during the transition periods. As in the case of the first discussed numerical test, these percentages give a conservative estimation of signal confidence.

The CII functions calculated over the fifty samples are shown in Figs. 3.7a through 3.7e. The curves with different markers represent the CIIs of the redundant signals of given variables. The increasing effects of signal transition on the CIIs of normal signals can be clearly observed.

The estimated values for all the five variables are shown in Figs. 3.8a through 3.8e. They are calculated at each sample separately using a weighted average of redundant measurements, where the weighting factors are inversely proportional to 
the CII values. The estimate of variable No. 3 in Fig. $3.8 \mathrm{c}$ has the same ramp bias as the five redundant signals until the point where all the five redundant signals were excluded. When the estimate of the variable is calculated at samples taken after the full degradation, it is given by the prediction of MISO model No. 2 using the measurements of the input variables having minimum Cחs. As can be seen in Figs. 3.8a through 3.8e, the GCCM algorithm gives approximately the correct estimates of all the five variables over the fifty samples, including the cases of totally degraded redundant signal set.

Application of the GCCM module to operational data from a Westinghouse four-loop PWR is described in Ref. [25]. 
TABLE 3.1

MISO Models (Empirical Models) Used for Predicting Variables 1, 3 and 5 in the Numerical Experiments.

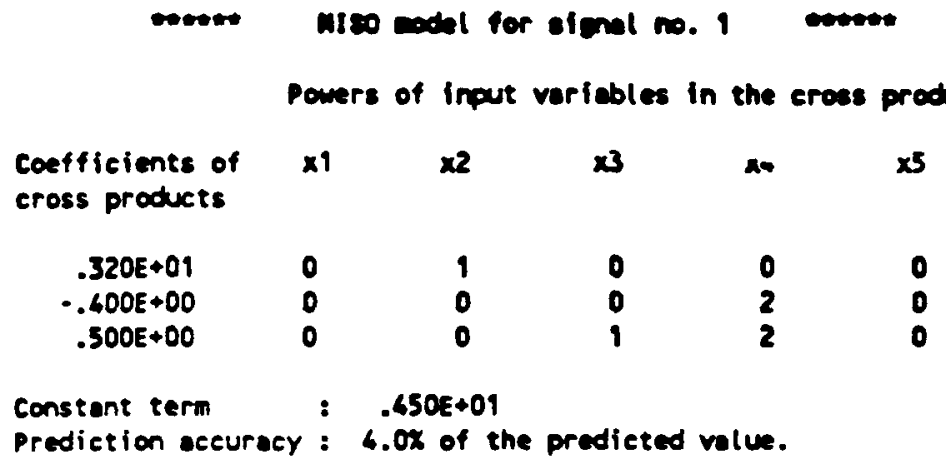

menar MIso model for signal no. 3 metret Powers of input veriebles in the eross products

$\begin{array}{lccccc}\begin{array}{l}\text { Coefficients of } \\ \text { cross products }\end{array} & \times 1 & \times 2 & \times 3 & \times 6 & \times 5 \\ -230 E+01 & 0 & 0 & 0 & 1 & 0 \\ -110 E+01 & 0 & 2 & 0 & 0 & 0 \\ .200 E+00 & 0 & 0 & 0 & 2 & 1 \\ -.100 E-01 & 0 & 0 & 0 & 0 & 4 \\ \text { Constant term } & : \quad .510 E+01 & & \\ \text { Prediction occuracy : 4.0x of the predicted value. }\end{array}$

"..."." MIso model for signal no. 5 Powers of input variables in the cross products

$\begin{array}{cccccc}\begin{array}{c}\text { Coefficients of } \\ \text { cross products }\end{array} & x 1 & x 2 & x 3 & x 4 & \times 5 \\ .500 E+00 & 0 & 1 & 0 & 0 & 0 \\ .200 E+00 & 0 & 1 & 0 & 1 & 0 \\ -.500 E-02 & 0 & 0 & 0 & 3 & 0\end{array}$

Constent term : .310 $6+01$

Prediction accuracy : $\mathbf{5 . 0 x}$ of the predicted value.

Modeled steady state range of variables :

Variab. 1 Variab. 2 Variab. 3 Varicb. 4 Variab. 5

Fran: .636E+02 .160E+01 . 130E+02 .210E+01 .362E+01

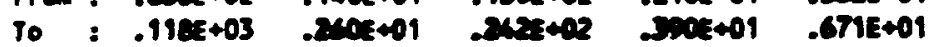


TABLE 3.2

Sensitivities of the Calculated MISO Models.

model no.1: arput voricble no.: 1

Relotive contribution of the const.tern to the output : . $\$ \% 401 \%$

Relative modeling error in predicted value:.60E\$01X

Relative input error contribution to the output error :

$\begin{array}{lll}\text { Input variable } 2 & \ldots & .8 x E+00 x \\ \text { Input variable } 3 & \ldots & .13 E+01 x \\ \text { Input variable } 4 & -. & .22 E+02 x\end{array}$

Model no.2: Output vericble no.: 3

Reletive contribution of the const.term to the output : .27E*02x

Relative codet ing error in predicted velue : .60:+01x

Relotive input error contribution to the output error :

Input veriable $2 \quad \ldots \quad .59 E+01 x$

Input variable $4 \quad \ldots \quad .71 E+01 x$

Input variable $5 \quad \ldots \quad-.18 E+02 x$

Model no.3: Output variable no.: 5

Relative contribution of the const.term to the output : . .6E+02X

Relative modeling error in pradicted velue : .50E+01X

Relative input error contribution to the output error:

$\begin{array}{lll}\text { Inpust variable } 2 & \ldots & .27 E+01 \% \\ \text { Inout voriable } 4 & \ldots & .15 E+01 \%\end{array}$

TABLE 3.3

The Fully Consistent Redundant Measurement Set at Sample Number 1.

\begin{tabular}{|c|c|c|c|c|c|}
\hline \multirow[t]{2}{*}{ 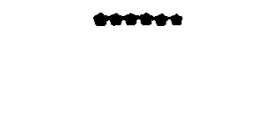 } & \multicolumn{3}{|c|}{ nectendent enecurements of exple no. } & 1 & conerese \\
\hline & signat 1 & signal 2 & sienal 3 & Signal 4 & sienal 5 \\
\hline $\begin{array}{l}\text { Variable no. } 1 \text { : } \\
\text { Variable no. } 2 \text { : } \\
\text { Voriable no. } 3 \text { : } \\
\text { Variable no. } 4 \text { : } \\
\text { Variable no. } 5 \text { : }\end{array}$ & $\begin{array}{l}.909 E+02 \\
.200 E+01 \\
.186 E+02 \\
.300 E+01 \\
.516 E+01\end{array}$ & $\begin{array}{l}.909 E+02 \\
.200 E+01 \\
.186 E+02 \\
.300 E+01 \\
.516 E+01\end{array}$ & $\begin{array}{l}.909 E+02 \\
.200 E+01 \\
.186 E+02 \\
.300 E+01 \\
.516 E+01\end{array}$ & $\begin{array}{l}.90 \% E+02 \\
.200 E+01 \\
.186 E+02 \\
.300 E+01 \\
.516 E+01\end{array}$ & $\begin{array}{l}.186 E+02 \\
.300 E+01\end{array}$ \\
\hline \multicolumn{6}{|c|}{ Error bounds of mesured velues : } \\
\hline $\begin{array}{l}\text { variable no. } 1 \text { : } \\
\text { variable no. } 2 \\
\text { variable no. } 3 \text { : } \\
\text { variable no. } 4 \text { : } \\
\text { variable no. } 5 \text { : }\end{array}$ & $\begin{array}{l}.455 E+00 \\
.100 E-01 \\
.929 E-01 \\
.150 E-01 \\
.258 E-01\end{array}$ & $\begin{array}{l}.455 E+00 \\
.100 E-01 \\
.929 E-01 \\
.150 E-01 \\
.258 E-01\end{array}$ & $\begin{array}{l}.455 E+00 \\
.100 E-01 \\
.929 E-01 \\
.150 E-01 \\
.258 E-01\end{array}$ & $\begin{array}{l}.655 E+00 \\
.100 E-01 \\
.929 E-01 \\
.150 E-01 \\
.258 E-01\end{array}$ & $\begin{array}{l}.92 \%-01 \\
.150 z-01\end{array}$ \\
\hline
\end{tabular}

sig.1 sig.2 sig.3 sig.4 $\quad 5 i g .5$

Variable 1:

Variable 2:

mx.posible $=100$,

$m x$.possible $=220$.

ectupl =

cetual :

anx.possible $=160$, cetul =

Variable 3 :

mx.posstble $=176$,

ectul :

Variable 5 :

nx.postble = 120,

ecturl =

0
0
0
0
0

$\begin{array}{llll}0 & 0 & 0 & \\ 0 & 0 & 0 & \\ 0 & 0 & 0 & 0 \\ 0 & 0 & 0 & 0 \\ 0 & 0 & 0 & \end{array}$


TABLE 3.4

Partially Consistent Redundant Measurement Set at Sample No. 5. No Measurement was excluded.

\begin{tabular}{|c|c|c|c|c|c|}
\hline \multirow[t]{2}{*}{ menes } & \multicolumn{3}{|c|}{ Rectundant mosurements of eaple no. } & 5 & 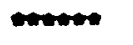 \\
\hline & sienal 1 & stenal 2 & sient 3 & sienal 4 & slenal 5 \\
\hline $\begin{array}{l}\text { Variable no. } 1 \\
\text { Vericble no. } 2 \\
\text { Variable no. } 3 \\
\text { Varibble no. } 4 \\
\text { Varicble no. } 5\end{array}$ & $\begin{array}{l}.909 E+02 \\
.200 E+01 \\
.186 E+02 \\
.300 E+01 \\
.516 E+01\end{array}$ & $\begin{array}{l}.911 E+02 \\
.200 E+01 \\
.186 E+02 \\
.301 E+01 \\
.518 E+01\end{array}$ & $\begin{array}{l}.913 E+02 \\
.201 E+01 \\
.194 E+02 \\
.301 E+01 \\
.519 E+01\end{array}$ & $\begin{array}{l}.007 E+02 \\
.200 E+01 \\
.193 E+02 \\
.29 E+01 \\
.515 E+01\end{array}$ & $\begin{array}{l}.192 E+02 \\
.20 \% E+01\end{array}$ \\
\hline
\end{tabular}

Inconsistency indices of the redundant measuraments of

$510.9 \quad 5 i g .2 \quad 5 i g .3 \quad 5 i g .4 \quad 8 i g .5$

\begin{tabular}{|c|c|c|c|c|c|c|}
\hline $\begin{array}{l}\text { Veriable 1: } \\
\text { Veriable 2: } \\
\text { Variable 3: } \\
\text { Veriable 4: } \\
\text { Varimle } 5:\end{array}$ & $\begin{array}{l}\text { max.possible }=100, \\
m x . \text { possible }=220 \\
m x . \text { possible }=160 \\
\text { max.possible }=176,\end{array}$ & $\begin{array}{l}\text { ectual } \\
\text { ectual } \\
\text { ectual } \\
\text { ectual } \\
\text { ectuil }\end{array}$ & $\begin{array}{r}=4 \\
=\quad 16 \\
=\quad 0 \\
=\quad 8 \\
=\quad 9\end{array}$ & $\begin{array}{r}0 \\
12 \\
0 \\
2 \\
13\end{array}$ & $\begin{array}{r}0 \\
11 \\
67 \\
8 \\
22\end{array}$ & 1 \\
\hline
\end{tabular}


TABLE 3.5

Identification of Degraded Measurment at Sample No. 8; 'This is the Third Signal of Variable No. 3. The CII's, Recalculated After Excluding the Faulty Signal, are also shown.

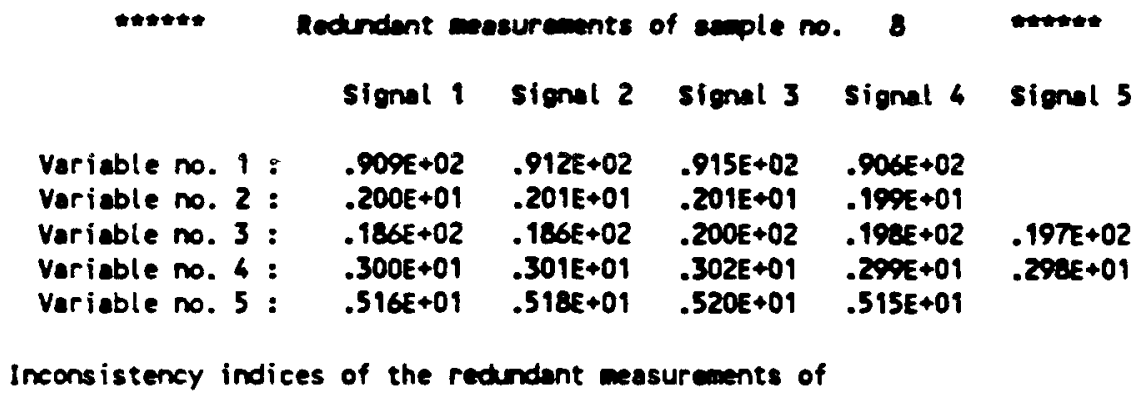

sig.t sig.2 sig.3 sig.4 sig.5

\begin{tabular}{|c|c|c|c|c|c|c|}
\hline $\begin{array}{l}\text { Variable 1: } \\
\text { Variable 2: } \\
\text { Variable 3: } \\
\text { Variable 4: } \\
\text { yariable 5: }\end{array}$ & $\begin{array}{l}\max . \text { possible }=100, \\
\operatorname{mx} . \text { possible }=220, \\
\max . \text { possible }=160, \\
\operatorname{mx} . \text { possible }=176, \\
\max \text {.poss ible }=120\end{array}$ & $\begin{array}{l}\text { ectual } \\
\text { ectual } \\
\text { ectual } \\
\text { ectual } \\
\text { ectug! }\end{array}$ & $\begin{array}{l}=56 \\
=114 \\
=0 \\
=96 \\
=\quad 12\end{array}$ & $\begin{array}{r}52 \\
114 \\
0 \\
96 \\
60\end{array}$ & $\begin{array}{r}67 \\
114 \\
160 \\
86 \\
60\end{array}$ & $\begin{array}{r}60 \\
113 \\
151 \\
91 \\
60\end{array}$ \\
\hline
\end{tabular}

Inconsistency indices of redundant measurements of the ramining variables after excluding the faulty variables detected in the previous pass :

sig.1 sig.2 sig.3 sig.4 sig.5

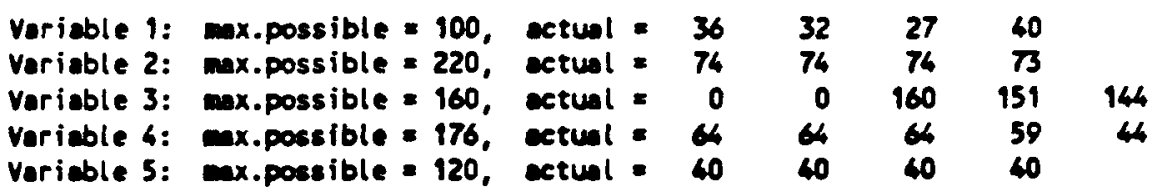

Identified degreded masurements:

Excluded enesuraments of vericble no.1 : Exeluded Easurments of variable no.2 : Excluded mesurements of veriable no.3 : Excluded measurements of vericble no.4 : Excluded mesurements of vericble no.s :

$\begin{array}{lllll}0 & 0 & 0 & 0 & \\ 0 & 0 & 0 & 0 & \\ 0 & 0 & 2 & 0 & 0 \\ 0 & 0 & 0 & 0 & 0 \\ 0 & 0 & 0 & 0 & \end{array}$


TABLE 3.6

Results at Sample No. 10. After Identifying and Excluding the Three Degraded Measurements of Variable No. 3 at Sample No. 10, the Remaining Redundant Measurement Set is Fully Consistent.

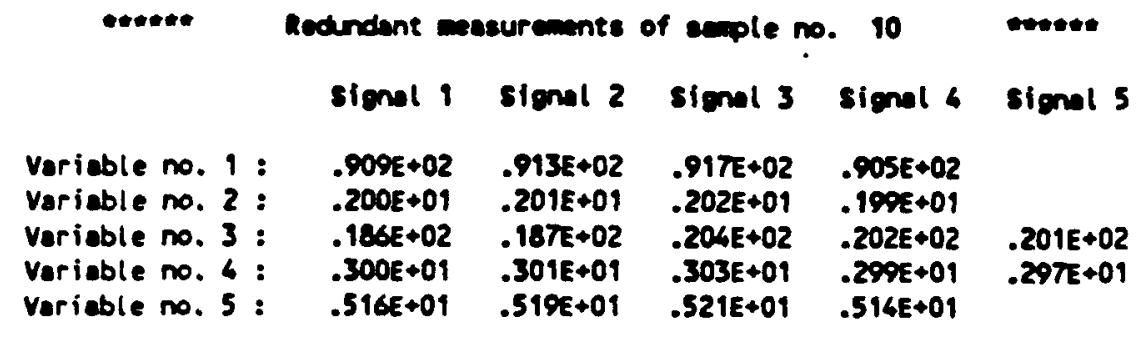

Inconsistency indices of the redundent mesurcuents of

Sig.1 Sig.2 sig.3 Sio.6 810.5

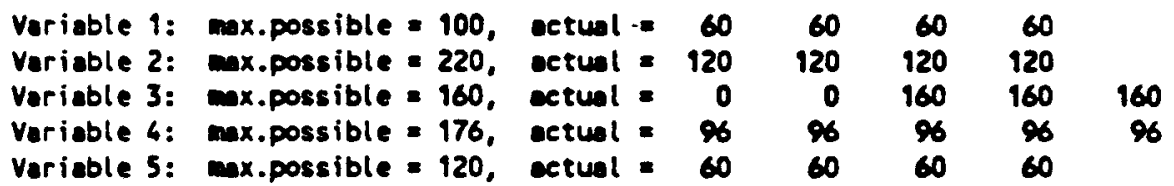

Inconsistency indices of redundant wesurements of the remining veriebles

after excluding the feulty variables detected in the previous pess :

sig.t sig.2 $\quad 5 i g .3 \quad s i g .6 \quad s i g .5$

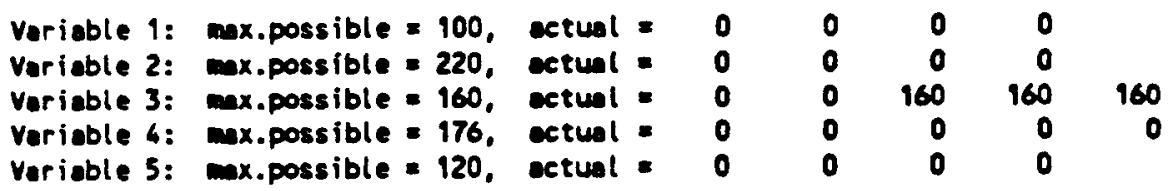

Identified degreded mesuraments :

Excluded mesurements of variable no.1 Excluded measurements of veriable no.2 : Excluded measurements of veriable no.3 : Excluded mesurcments of variable no.4: Excluded measurements of varieble no.5:

$\begin{array}{lllll}0 & 0 & 0 & 0 & \\ 0 & 0 & 0 & 0 & \\ 0 & 0 & 2 & 2 & 2 \\ 0 & 0 & 0 & 0 & 0 \\ 0 & 0 & 0 & 0 & \end{array}$


TABLE 3.7

The Iegraded Measurements of Two Different Variables are Identified and Excluded Simultaneously at Sample No. 15.

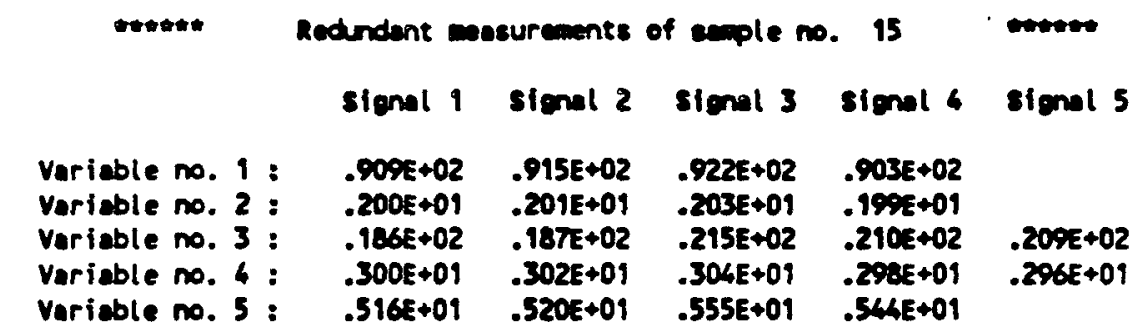

Inconsistency indices of the redundent mesurcments of

sig.1 sig.2 sig.3 sig.4 sig.5

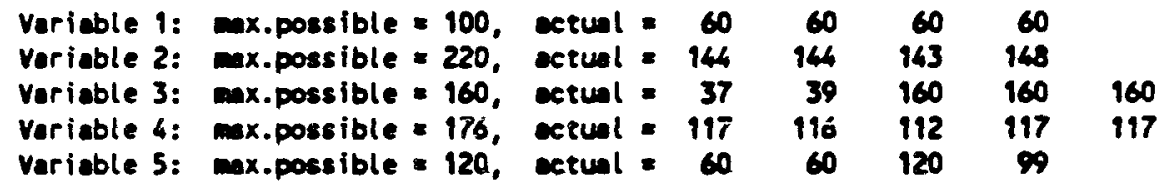

inconsistancy irdices of redundant masurewents of the remining veriables after excluding the faulty variables detected in the previous pass :

sig.1 sig.2 sig.3 $5 i g .4 \quad$ sig.s

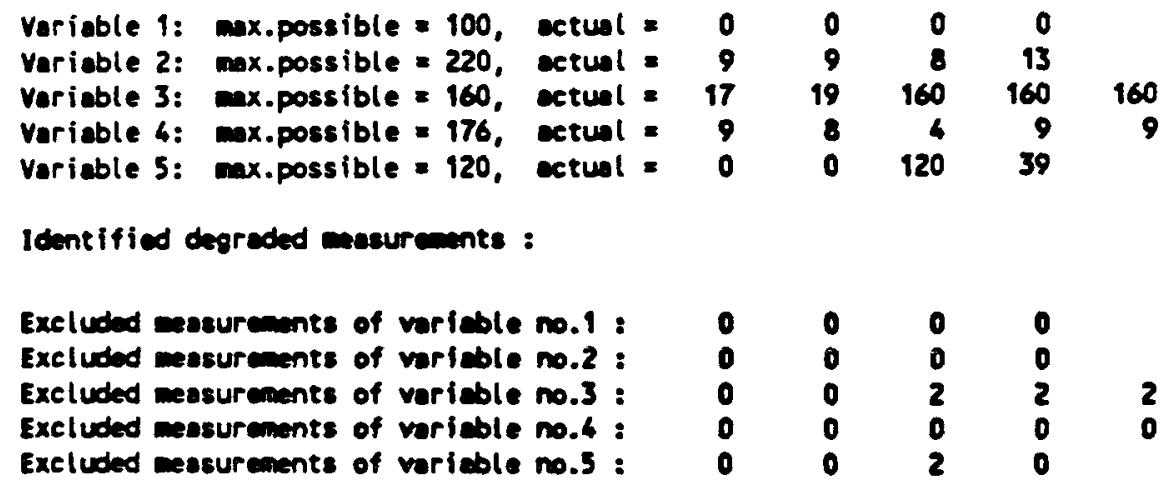


TABLE 3.8

The Detection of Multiple Degradations in the Measurement of Variables No. 3 and No. 5 at Sample No. 16. After Exclusion of these Two Signals, The Remaining Measurements are Fully Consistent.

\begin{tabular}{|c|c|c|c|c|c|}
\hline \multirow[t]{2}{*}{ 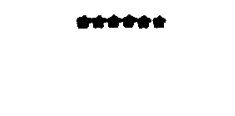 } & \multicolumn{4}{|c|}{ Rectundent meseurements of seple no. } & (1) \\
\hline & signal 1 & slanal 2 & $\sin a l 3$ & sienal 4 & signel 5 \\
\hline $\begin{array}{l}\text { Variable no. } 1 \text { : } \\
\text { varibble no. } 2 \\
\text { variable no. } 3 \text { : } \\
\text { Variable no. } 6 \text { : } \\
\text { variable no. } 5 \text { : }\end{array}$ & $\begin{array}{l}.909 E+02 \\
.200 E+01 \\
.186 E+02 \\
.300 E+01 \\
.516 E+01\end{array}$ & $\begin{array}{l}.916 E+02 \\
.202 E+01 \\
.18 \pi E+02 \\
.302 E+01 \\
.520 E+01\end{array}$ & $\begin{array}{l}.023 E+02 \\
.203 E+01 \\
.217 E+02 \\
.305 E+01 \\
.566 E+01\end{array}$ & $\begin{array}{l}.902 E+02 \\
.19 \% E+01 \\
.212 E+02 \\
.292 E+01 \\
.554 E+01\end{array}$ & $\begin{array}{l}.210 E+02 \\
.295 E+01\end{array}$ \\
\hline
\end{tabular}

Inconsistency indices of the redundant measurements of

sig. $\operatorname{sig.2}$ sig.3 sig.4 sig.5

\begin{tabular}{|c|c|c|c|c|c|c|}
\hline $\begin{array}{l}\text { Variable 1: } \\
\text { Variable 2: } \\
\text { Variable 3: } \\
\text { Variable 4: } \\
\text { Variable 5: }\end{array}$ & $\begin{array}{l}\text { max.possible }=100, \\
\text { max.possible }=220, \\
\text { mx.possible }=160, \\
\text { m.possible }=176,\end{array}$ & $\begin{array}{l}\text { eetual } \\
\text { ectual } \\
\text { ectual } \\
\text { ectual } \\
\text { ectun! }\end{array}$ & $\begin{array}{l}=60 \\
=150 \\
=\quad 40 \\
=120 \\
=\quad 60\end{array}$ & $\begin{array}{r}60 \\
150 \\
40 \\
120 \\
60\end{array}$ & $\begin{array}{r}60 \\
150 \\
160 \\
120 \\
120\end{array}$ & $\begin{array}{r}60 \\
150 \\
160 \\
120 \\
120\end{array}$ \\
\hline
\end{tabular}

Inconsistency indices of redundant mesurcments of the ramining variebles ofter excluding the foulty variables detected in the previous pass :

sig.1 sig.2 sig.3 sig.4 sig.5

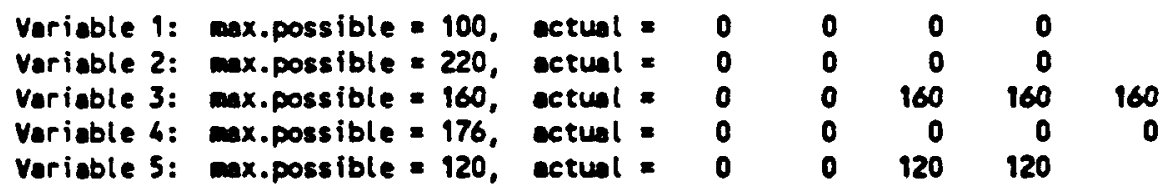

Identified degreded messurements :

Excluded measurements of veriable no.9 : Excluded measurements of veriable no.2 : Excluded measurements of vorieble no.3 : Exeluded measurements of veriable no.4: Excluded measurements of varieble no.5 :

$\begin{array}{lllll}0 & 0 & 0 & 0 & \\ 0 & 0 & 0 & 0 & \\ 0 & 0 & 2 & 2 & 2 \\ 0 & 0 & 0 & 0 & 0 \\ 0 & 0 & 2 & 2 & \end{array}$




\section{TABLE 3.9}

At Sample No. 44, Only Variable No. 5 Has Faulty Measurements. The Remaining Measurements Show Partial Degradation.

\begin{tabular}{|c|c|c|c|c|c|}
\hline \multirow[t]{2}{*}{ mtrtete } & \multicolumn{4}{|c|}{ Redundant mesureants of sesple no. } & 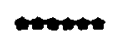 \\
\hline & sienal 1 & sional 2 & stenal 3 & sienal 4 & stenal 5 \\
\hline $\begin{array}{l}\text { Variable no. } 1 \text { : } \\
\text { Variable no. } 2 \text { : } \\
\text { Variable no. } 3 \text { : } \\
\text { Variable no. } 4 \text { : } \\
\text { Variable no. } 5 \text { : }\end{array}$ & $\begin{array}{l}.909 E+02 \\
.200 E+01 \\
.186 E+02 \\
.300 E+01 \\
.516 E+01\end{array}$ & $\begin{array}{l}.912 E+02 \\
.201 E+01 \\
.186 E+02 \\
.301 E+01 \\
.518 E+01\end{array}$ & $\begin{array}{l}.915 E+02 \\
.201 E+01 \\
.192 E+02 \\
.302 E+01 \\
.852 E+01\end{array}$ & $\begin{array}{l}.906 E+02 \\
.19 \% E+01 \\
.196 E+02 \\
.299 E+01 \\
.245 E+01\end{array}$ & $\begin{array}{l}.19 e c+02 \\
.292 x+01\end{array}$ \\
\hline
\end{tabular}

Inconsistency indices of the redundant measurements of

sig.1 sig.2 $s i g .3 \quad s i g .6 \quad s i g .5$

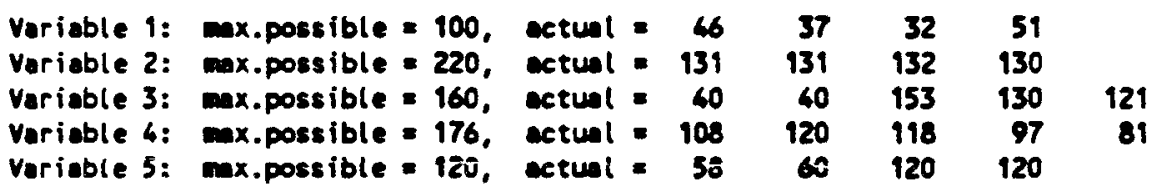

Inconsistency indices of redendant easurments of the ramining variables after excluding she faulty variables detected in the previous pass :

sig.1 sig.2 sig.3 sig.4 sig.5

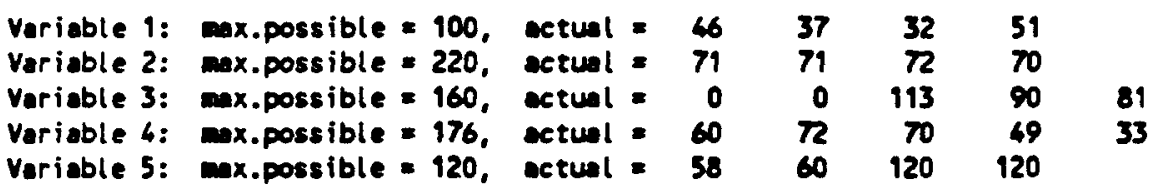

Identified degraded esesurcments :

Excluded measurements of variable no. 1 : Excluded measurements of variable no.2 : Excluded messurements of veriable no.3 : Excluded measurements of variable no.4 : Excluded messurements of varieble no.5:

$\begin{array}{lllll}0 & 0 & 0 & 0 & \\ 0 & 0 & 0 & 0 & \\ 0 & 0 & 0 & 0 & 0 \\ 0 & 0 & 0 & 0 & 0 \\ 0 & 0 & 2 & 2 & \end{array}$


TABIE 3.10

After Excluding the Last Two Measurements of Variable No. 5 at Sample No. 47, the Remaining Measurements are Fully Consistent.

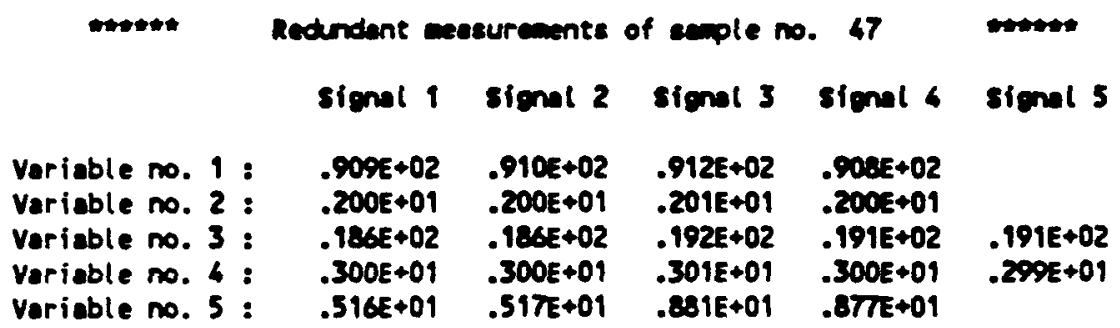

Inconsistency indices of the redundant mesurements of-

\section{sig.1 sig.2 sig.3 sig.4 sig.5}

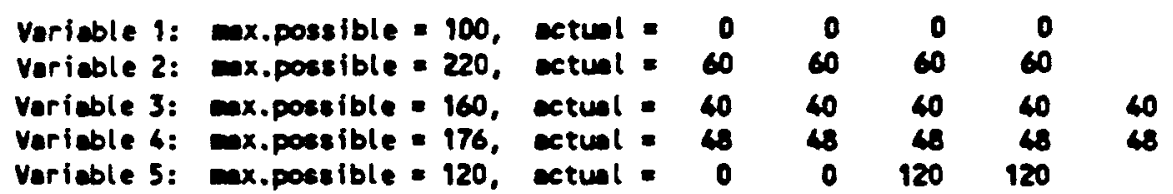

Inconsistency Indices of redundant mesuranents of the ramining verisbles after excluding the foulty variables detected in the previous pase:

sig.1 sig.2 sig.3 sig.4 sig.5

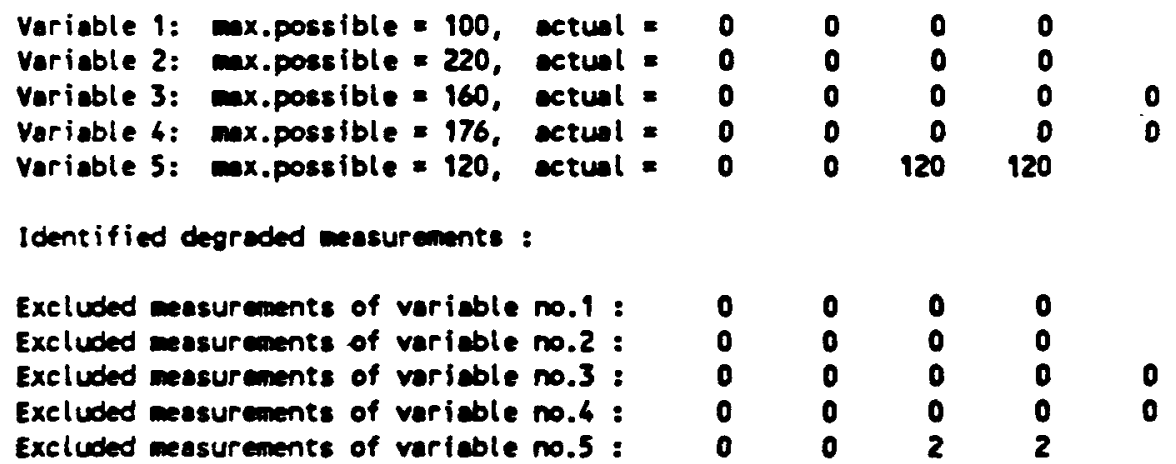


TABLE 3.11

The Overall Confidence Factors of Each Signal Calculated
Over 50 Samples.

\begin{tabular}{|c|c|c|c|c|c|}
\hline & Signal 1 & signal 2 & signal 3 & signal 4 & Signal 5 \\
\hline $\begin{array}{l}\text { Variable no. } 1 \text { : } \\
\text { Variable no. } 2 \text { : } \\
\text { Variable no. } 3 \text { : } \\
\text { Variable no. } 4 \text { : } \\
\text { Variable no. } 5:\end{array}$ & $\begin{array}{l}93.40 \% \\
93.76 \% \\
97.46 \% \\
94.56 \% \\
93.57 \%\end{array}$ & $\begin{array}{l}94.16 \% \\
94.02 \% \\
96.91 \% \\
94.25 \% \\
92.40 \%\end{array}$ & $\begin{array}{l}93.60 \% \\
94.11 \% \\
21.06 \% \\
92.66 \% \\
23.45 \%\end{array}$ & $\begin{array}{l}91.40 \% \\
93.46 \% \\
24.86 \% \\
94.60 \% \\
26.27 \%\end{array}$ & $\begin{array}{l}25.91 \% \\
93.13 \%\end{array}$ \\
\hline
\end{tabular}

'TABLE 3.12

Second Numerical Experiment. Partially Consistent Redundant Measurements at Sample No. 5 Showing Their Values and CII's.

\begin{tabular}{|c|c|c|c|c|c|}
\hline \multirow[t]{2}{*}{$t * t+t$} & \multicolumn{2}{|c|}{ Redundant measurenents } & of seiple no. & 5 & \multirow{2}{*}{ signal 5} \\
\hline & signal 1 & signal 2 & signal 3 & signal 4 & \\
\hline $\begin{array}{l}\text { Variable no. } 1 \\
\text { Variable no. } 2\end{array}$ & $\begin{array}{l}.909 E+02 \\
.200 E+01\end{array}$ & $\begin{array}{l}.911 E+02 \\
.200 E+01\end{array}$ & $\begin{array}{l}.913 E+02 \\
.201 E+01\end{array}$ & $\begin{array}{l}.907 E+02 \\
.200 E+01\end{array}$ & \\
\hline Variable no. 3 : & $.193 E+02$ & $.194 E+02$ & $.194 E+02$ & $.193 E+02$ & $.192 E+02$ \\
\hline $\begin{array}{l}\text { Variable no. } 4 \\
\text { Variable no. } 5\end{array}$ & $\begin{array}{l}.300 E+09 \\
.516 E+01\end{array}$ & $\begin{array}{l}.301 E+01 \\
.518 E+01\end{array}$ & $\begin{array}{l}.301 E+01 \\
.519 E+01\end{array}$ & $\begin{array}{l}.299 E+01 \\
.515 E+01\end{array}$ & $.299 E+01$ \\
\hline
\end{tabular}

Inconsistency indices of the redundant measurements of

sig.1 sig.2 sig.3 sig.4 sig.5

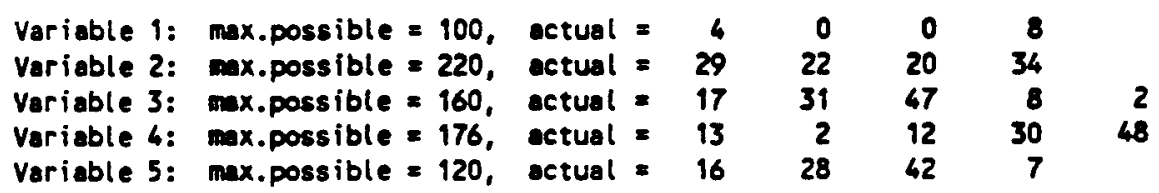




\section{TABLE 3.13}

Some Measurements of the Gradually Degrading Variable No. 3 Were Excluded at Sample No. 8. Also, One Measurement of Variable No. 1, with Model Redundancy Equal to One, Was Excluded Incorrectly. The Remaining Measurement Set is Partially Consistent.

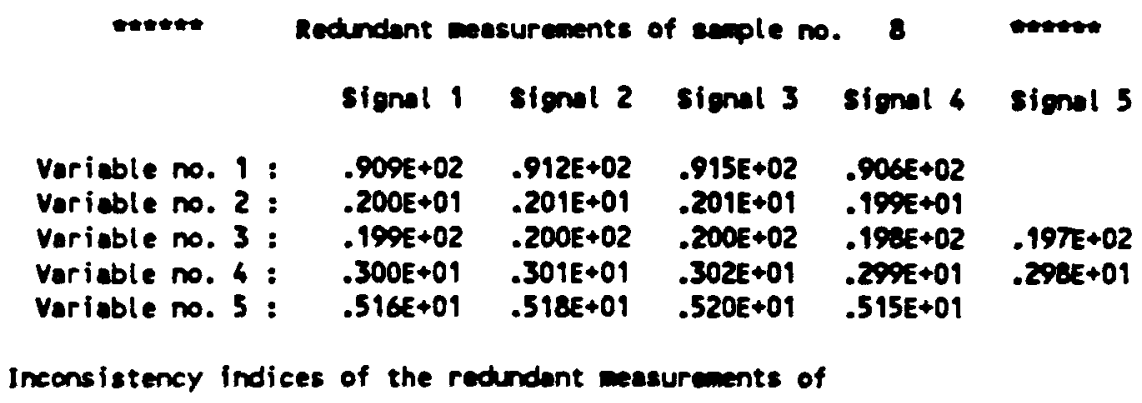

$\operatorname{sig} .1 \operatorname{sig} .2 \operatorname{sig} .3 \operatorname{sig} .4 \operatorname{sig} .5$

\begin{tabular}{|c|c|c|c|c|c|c|}
\hline $\begin{array}{l}\text { Veriable 1: } \\
\text { variable 2: } \\
\text { Voriable 3: } \\
\text { voriable 4: }\end{array}$ & $\begin{array}{l}m x . \text { possible }=100, \\
m x . \text { possible }=220, \\
m x . \text { possible }=160, \\
m x . \text { possible }=176, \\
m x \text {. possible }=120\end{array}$ & $\begin{array}{l}\text { ectual } \\
\text { ectual } \\
\text { ectual } \\
\text { ectual } \\
\text { ectus! }\end{array}$ & $\begin{array}{l}=96 \\
=193 \\
=156 \\
=160 \\
=100\end{array}$ & $\begin{array}{r}92 \\
193 \\
160 \\
160 \\
100\end{array}$ & $\begin{array}{r}83 \\
193 \\
160 \\
160 \\
100\end{array}$ & $\begin{array}{l}100 \\
192 \\
151 \\
155 \\
100\end{array}$ \\
\hline
\end{tabular}

Inconsistency indices of redundant measurements of the ramining variables ofter excluding the foulty variables detected in the previaus pass :

$$
\operatorname{sig.1} \operatorname{sig} .2 \operatorname{sig} .3 \operatorname{sig} .4 \operatorname{sig} .5
$$

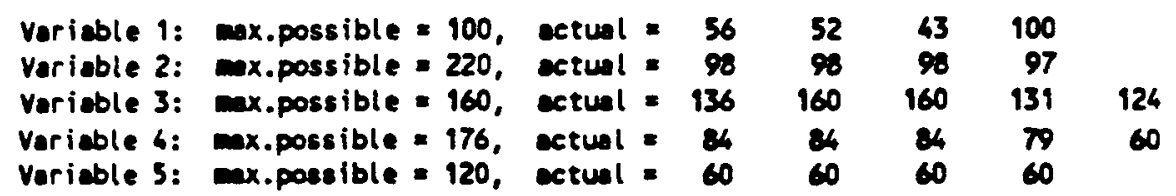

Identified degreded mesurements :

Excluded measurunents of variable no.1: Excluded measurements of varieble no.2 : Excluded measurements of variable no.3 : Excluded measurements of variable no.4: Excluded measurements of variable no.5 :

$\begin{array}{lllll}0 & 0 & 0 & 1 & \\ 0 & 0 & 0 & 0 & \\ 0 & 2 & 2 & 0 & 0 \\ 0 & 0 & 0 & 0 & 0 \\ 0 & 0 & 0 & 0 & \end{array}$


TABILE 3.14

All the Five Measurements of Variable No. 3 Have Reached Their Maximum Possible CII's at Sample No. 10, and Are Excluded from the Set. The Remaining Measurement Set, Including Variable No. 1 Which Was Previously Declared to Be Faulty, Is Fully Consistent.

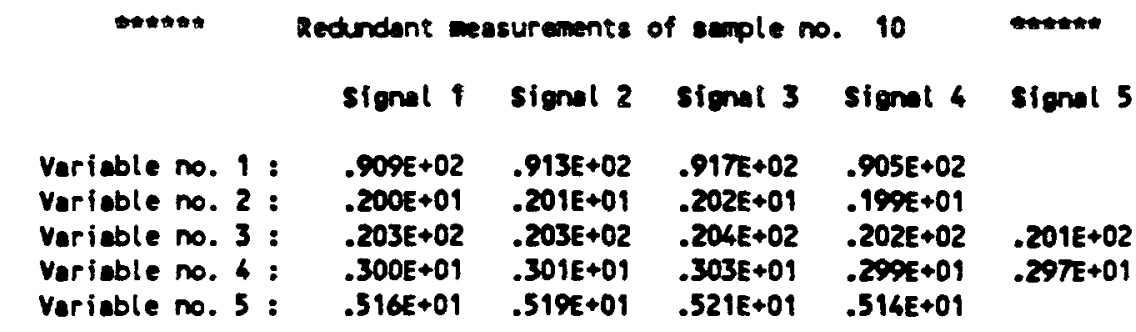

Inconsistency Indices of the redundant measurements of

sig.1 sig.2 sig.3 sig.h sig.5

Voribble 1: max.possible $=100$, ectual $=\begin{array}{llll}100 & 100 \quad 100 \quad 100\end{array}$

veriable 2: $m x$.possible $=220$, cetul $=200 \quad 200 \quad 200$

Verioble 3: max.possible $=160$, cctual $=160 \quad 160 \quad 160 \quad 160 \quad 160$

Voriable 4: ax.possible = 176, ectual = $\begin{array}{lllll}160 & 160 & 160 & 160 & 160\end{array}$

varieble 5: mx.possible = 120, ectul $=100 \quad 100 \quad 100100$

Inconsistency Indices of redundant mesurements of the remining variables after excluding the foulty variobles detected in the previous pass :

sig.1 sig.2 $\operatorname{sig.3} \quad$ sig.4 $\quad$ sig.s

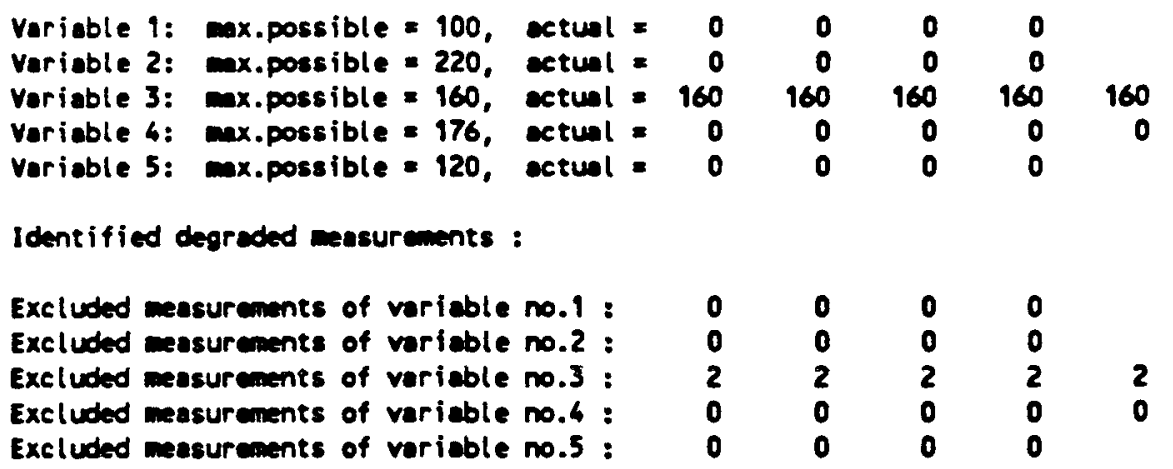

The entire redundant mesurement set of varieble no. 3 is degraded.

Its estimete is based on the prediction of MIso model no. 2 
TABLE 3.15

The Identically Changing Faulty Measurements of Variables No. 3 and No. 5 are Correctly Excluded at Sample No. 16. The Remaining Measurement Set is Fully Consistent.

\begin{tabular}{|c|c|c|c|c|c|}
\hline \multirow[t]{2}{*}{ minte } & \multicolumn{4}{|c|}{ Rectundent Esesurements of semple no. } & 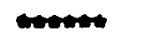 \\
\hline & sienal 1 & signol 2 & stenat 3 & stenal 4 & sienal 5 \\
\hline $\begin{array}{l}\text { Variable no. } 1 \\
\text { Variable no. } 2 \\
\text { Variable no. } 3 \text { : } \\
\text { Variable no. } 6 \\
\text { Variable no. } 5 \text { : }\end{array}$ & $\begin{array}{l}.909 E+02 \\
.200 E+01 \\
.214 E+02 \\
.300 E+01 \\
.516 E+01\end{array}$ & $\begin{array}{l}.916 E+02 \\
.202 E+01 \\
.215 E+02 \\
.302 E+01 \\
.520 E+01\end{array}$ & $\begin{array}{l}.923 E+02 \\
.203 E+01 \\
.21 \pi E+02 \\
.305 E+01 \\
.566 E+01\end{array}$ & $\begin{array}{l}.902 E+02 \\
.199 E+01 \\
.212 E+02 \\
.290 E+01 \\
.554 E+01\end{array}$ & $\begin{array}{l}.210 E+02 \\
.295 E+01\end{array}$ \\
\hline
\end{tabular}

Inconsistency indices of the redundent measurements of

$\operatorname{sig.1} \operatorname{sig} .2 \operatorname{sig} .3 \quad \operatorname{sig} .4 \quad \operatorname{sig} .5$

Veriable 1: mx.possible $=100$, ectul $=100 \quad 100 \quad 100 \quad 100$

Variable 2: mx.possible $=220$, ectual $=\begin{array}{llll}210 & 210 & 210 & 210\end{array}$

Veriable 3: max.possible $=160$, actual $=\begin{array}{lllll}160 & 160 & 160 & 160 & 160\end{array}$

Voriable 4: max.possible = 176, ectual = $\begin{array}{lllll}168 & 168 & 168 & 168 & 168\end{array}$

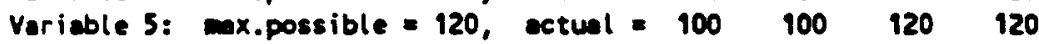

Inconsistency indices of redundant measurements of the remoining variables ofter excluding the foulty voriables detected in the previous pass :

sig.t sig.2 sig.3 sig.t sig.5

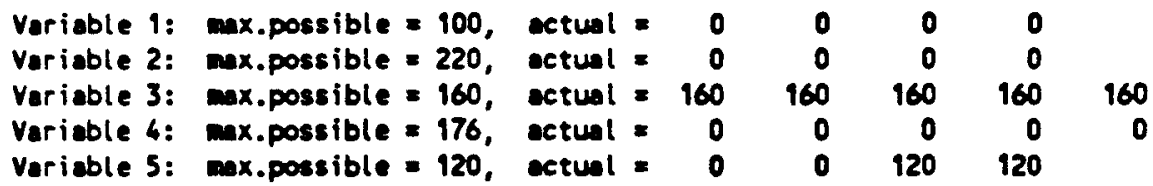

Identified degraded easurements :

Excluded measuranents of veriable no.1 : 00000

Excluded measurements of variable no.2: 000000

Excluded measurements of variable no.3 : 2212

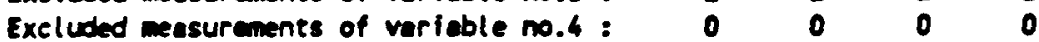

Excluded mesurments of variable no.5 : $\quad 0 \quad 0 \quad 2$

The entire redundent mesurement set of variable no. 3 is degraded.

Its estimate is based on the prediction of Miso model no. 2 
TABLE 3.16

The Partially Excluded Signal Sets, and the Partially Consistent Signals are Recorded at Sample No. 42 During a Transient Period.

\begin{tabular}{|c|c|c|c|c|c|}
\hline \multirow[t]{2}{*}{ menese } & \multicolumn{3}{|c|}{ Redundant exesurements of semple no. } & - 42 & 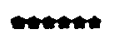 \\
\hline & signal 1 & signal 2 & signal 3 & sienal 4 & Sienal 5 \\
\hline $\begin{array}{l}\text { Variable no. } 1 \text { : } \\
\text { Variable no. } 2 \text { : } \\
\text { veriable no. } 3 \text { : } \\
\text { veriable no. } 4 \text { : } \\
\text { veriable no. } 5 \text { : }\end{array}$ & $\begin{array}{l}.909 E+02 \\
.200 E+01 \\
.201 E+02 \\
.300 E+01 \\
.516 E+01\end{array}$ & $\begin{array}{l}.913 E+02 \\
.201 E+01 \\
.201 E+02 \\
.301 E+01 \\
.519 E+01\end{array}$ & $\begin{array}{l}.916 E+02 \\
.202 E+01 \\
.202 E+02 \\
.302 E+01 \\
.833 E+01\end{array}$ & $\begin{array}{l}.905 E+02 \\
.199 E+01 \\
.200 E+02 \\
.299 E+01 \\
.823 E+01\end{array}$ & $\begin{array}{l}.199 E+02 \\
.290 E+01\end{array}$ \\
\hline
\end{tabular}

sig.1 sig.2 sig.3 sig.4 sig.s

Veriable 1: max.possible $=100$, ectul $=\begin{array}{llll}100 & 97 & 92 & 100\end{array}$

Variable 2: max.possible $=220$, sctual $=207 \quad 207 \quad 200 \quad 207$

Variable 3: max.possible $=160$, ectual $=\begin{array}{llllll}160 & 160 & 160 & 156 & 153\end{array}$

veriable 4: max.possible = 176, ectul = $\begin{array}{lllll}168 & 168 & 168 & 168 & 157\end{array}$

Veriable 5: max.possible = 120, ectual $=\begin{array}{llll}100 & 100 & 120 & 120\end{array}$

Inconsistency indices of redundant mesurenents of the ramining voriables efter exeluding the foulty variables detected in the previous poss :

$\operatorname{sig} .1 \operatorname{sig} .2 \operatorname{sig} .3 \operatorname{sig} .4 \operatorname{sig} .5$

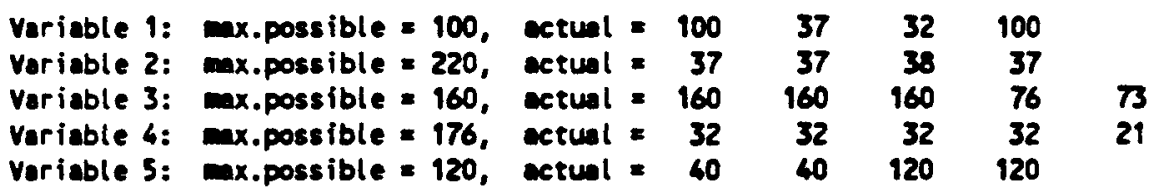

Identified degraded measureasents :

Excluded measurements of variable no.1 Excluded measurements of variable no.2 : Excluded measurements of verieble no.3 : Excluded meesurements of variable no.4: Excluded measurements of variable no.5:

$\begin{array}{lllll}1 & 0 & 0 & 1 & \\ 0 & 0 & 0 & 0 & \\ 2 & 2 & 2 & 0 & 0 \\ 0 & 0 & 0 & 0 & 0 \\ 0 & 0 & 2 & 2 & \end{array}$


TABLE 3.17

The Correctly Excluded Measurements at Sample No. 44. The Remaining Measurement Set is Partially Consistent.

\begin{tabular}{|c|c|c|c|c|c|}
\hline \multirow[t]{2}{*}{ (1) } & \multicolumn{4}{|c|}{ Redendent esesuremente of exple no. } & גדימריט \\
\hline & sienal 1 & Stenal 2 & $\sin 3$ & sienal 6 & sienal 5 \\
\hline $\begin{array}{l}\text { Variable no. } 1 \\
\text { Variable no. } 2 \\
\text { Variable no. } 3 \\
\text { Veriable no. } 6 \\
\text { Variable no. } 5\end{array}$ & $\begin{array}{l}.000 E+02 \\
.200 E+01 \\
.197 E+02 \\
.300 E+01 \\
.516 E+01\end{array}$ & $\begin{array}{l}.912 E+02 \\
.201 E+01 \\
.1908+02 \\
.301 E+01 \\
.518 E+01\end{array}$ & $\begin{array}{l}.915 E+02 \\
.201 E+01 \\
.198 E+02 \\
.302 E+01 \\
.052 E+01\end{array}$ & $\begin{array}{l}.906 E+02 \\
.199 E+01 \\
.196 E+02 \\
.29 \%+01 \\
.045 E+01\end{array}$ & $\begin{array}{l}.196 E+02 \\
.20 \% E+01\end{array}$ \\
\hline
\end{tabular}

Inconsistency indices of the rectundent masurements of

sig.t sig.2 sig.3 sig.4 sig.5

Variable 1: mx.possible = 100, ectul = $81 \quad 66 \quad 56 \quad 91$

Variable 2: mx.possible = 220, ectul $=183 \quad 183 \quad 185 \quad 181$

Voriable 3: Ex.possible = 160, ectual = $141 \quad 167 \quad 153 \quad 130 \quad 121$

Voriable 6: max.possible $=176$, ectul $=156 \quad 168 \quad 168 \quad 134 \quad 108$

Variable 5: mx.possible = 120, ectul = 98 $100 \quad 120120$

Inconsistency indices of redundant messurements of the remaining voricbles ofter excluding the faulty veriables detected in the previous pass:

sig.1 sig.2 519.3 sig.4 sig.s

Variable 1: max.possible = 100, ectul = $81 \quad 66 \quad 5691$

Variable 2: $m$ x.possible $=220$, ectul $=123 \quad 123 \quad 125 \quad 121$

Veriable 3: mx.possible = 160, ectul $=101 \quad 107 \quad 113 \quad 90 \quad 81$

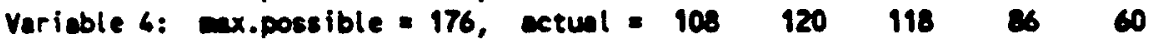

Voricble 5: mx.possible = 120, ectul = 90100120120

Identified degredad measurements :

Excluded mesurements of vericble no.1 : Excluded exesurments of voricble no.2 : Excluded mesurments of vericble no.3 : Excludad mesurcments of vericble no.6: Excluded anesuremente of vericble no.s :

$\begin{array}{lllll}0 & 0 & 0 & 0 & \\ 0 & 0 & 0 & 0 & \\ 0 & 0 & 0 & 0 & 0 \\ 0 & 0 & 0 & 0 & 0 \\ 0 & 0 & 2 & 2 & \end{array}$


TABLE 3.18

The Correctly Excluded Measurements at Sample No. 47.

The Remaining Measurement Set is Fully Consistent.

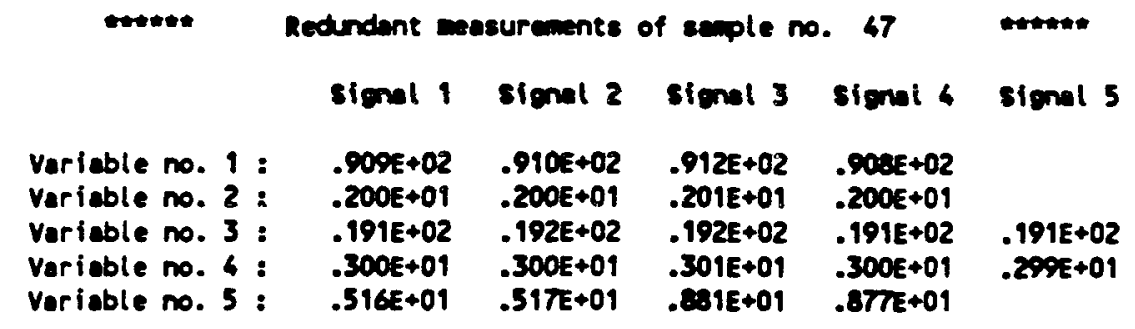

Inconsistency indices of the redundant measurements of

$\operatorname{sig} .1 \operatorname{sig.2} \operatorname{sig} .3 \operatorname{sig} .4 \operatorname{sig} .5$

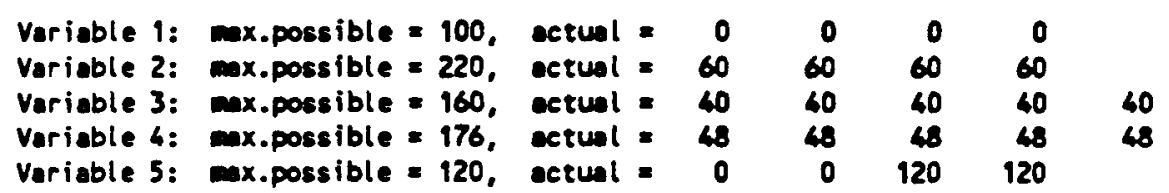

Inconsistency indices of rectudant measurements of the ranoining variables after excluding the faulty variables detected in the previous pass :

sig.1 sig.2 sig.3 sig.4 sig.5

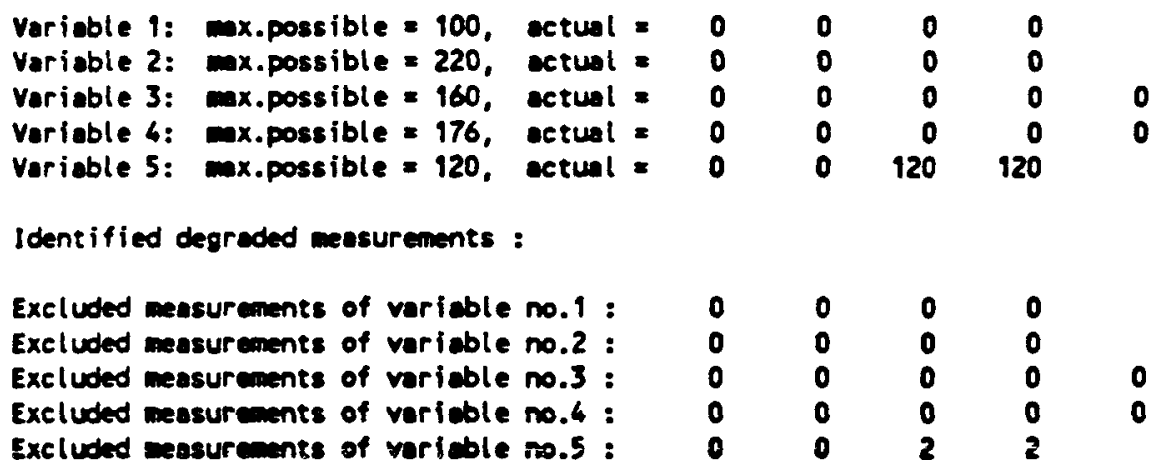


TABLE 3.19

'The Overall Confidence Factors at Each Signal Calculated over 50 Samples in the Second Numerical Example

H*t* Results of the multivariate consistency checking wm*

The goodness of the individual signals based on the given 50 samples:

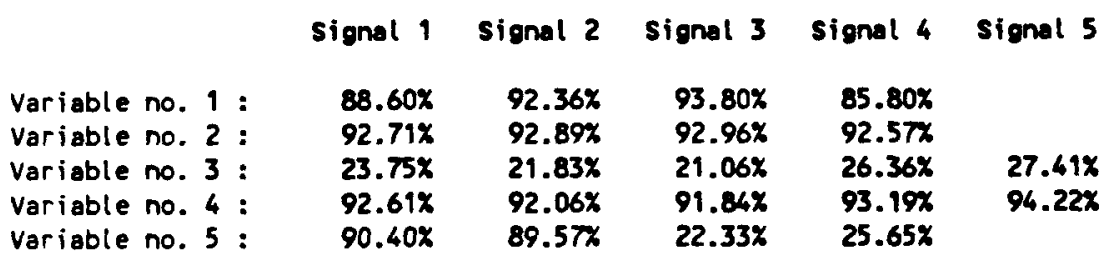




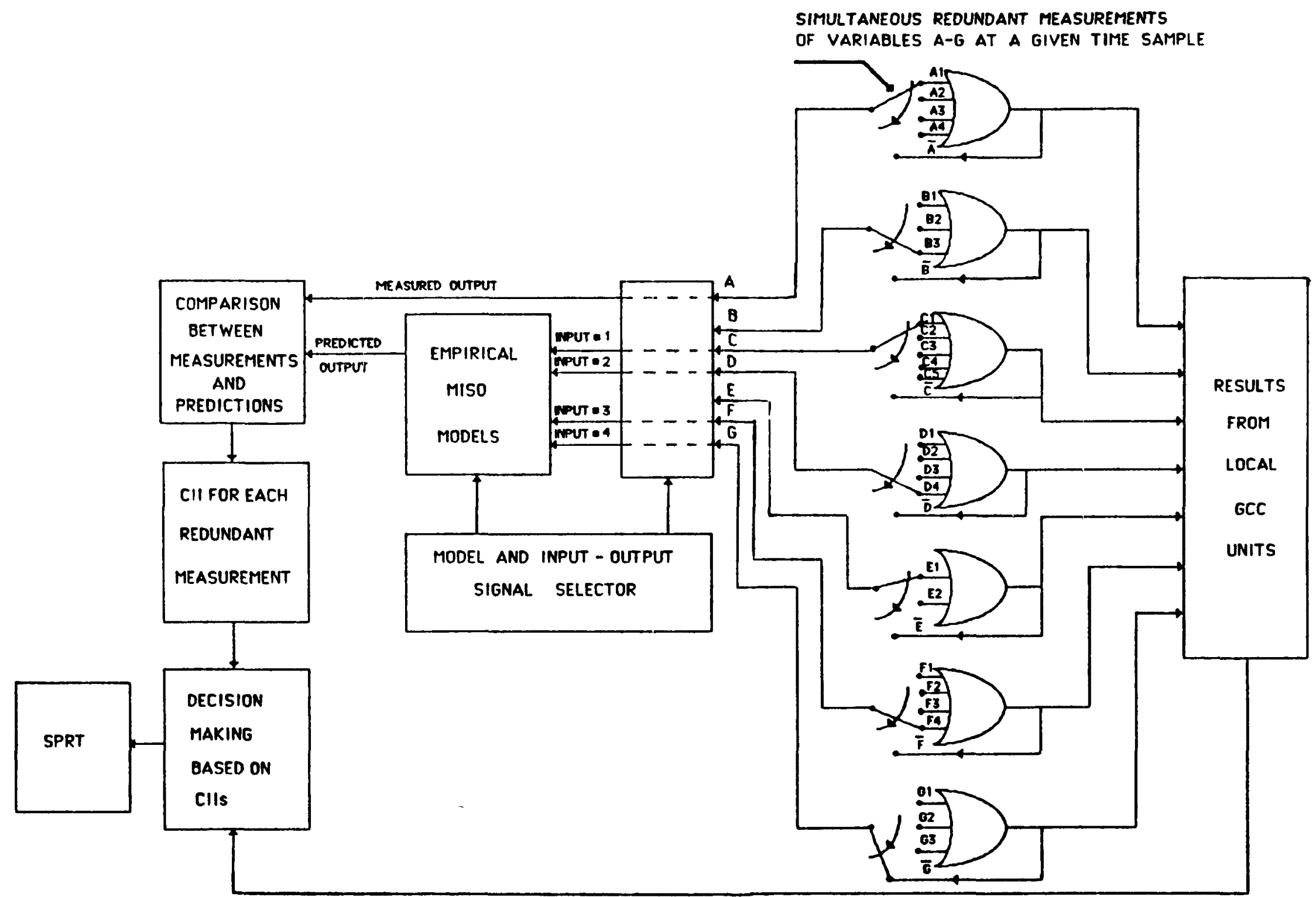

Figure 3.1. GCCM Module Showing Intersignal Comparison Through a Set of Empirical Models and a Network of Single-Variable Generalized Consistency Checking Modules. 


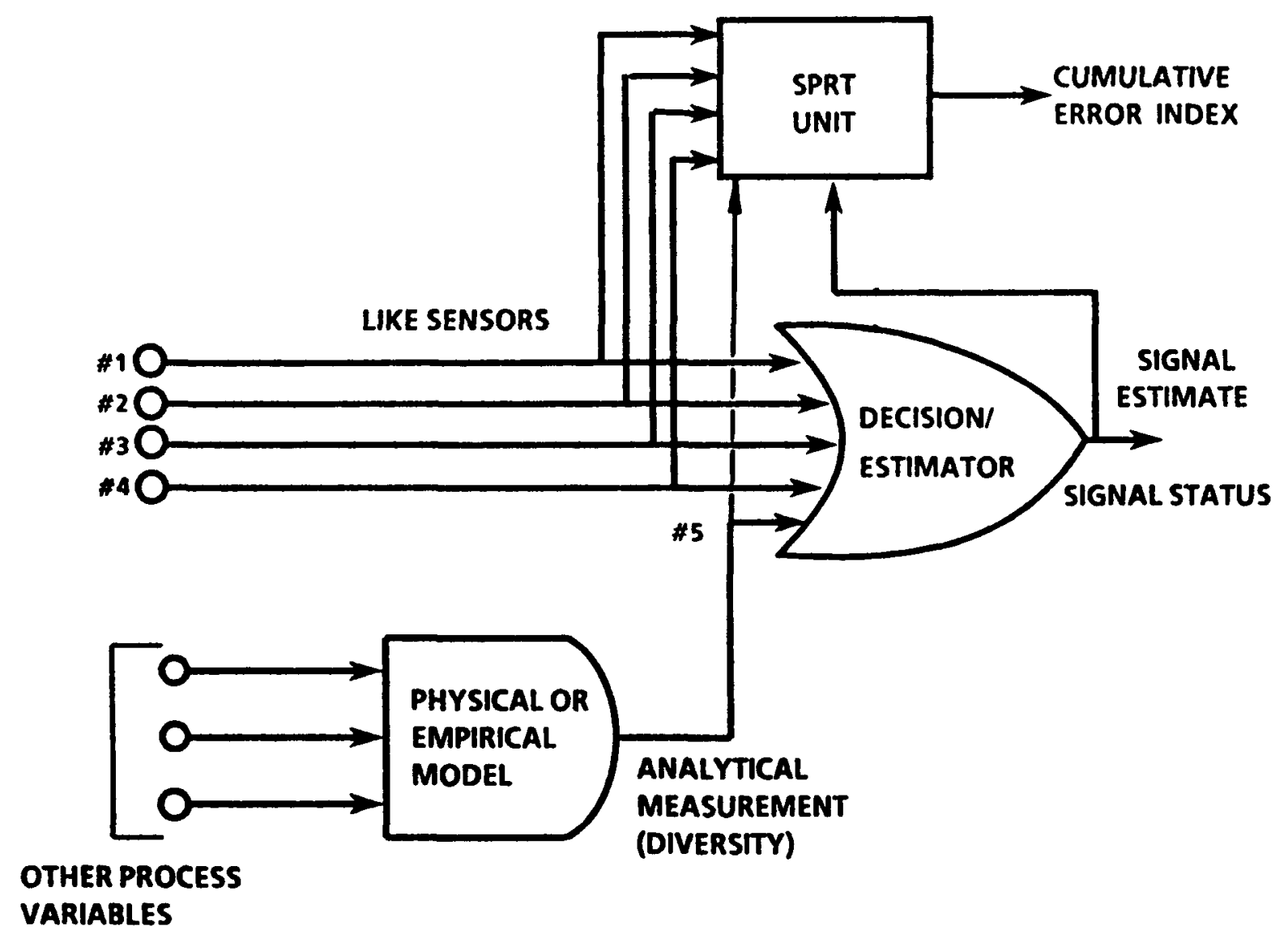

Figure 3.2. Single-Variable Generalized Consistency Checking Module, Showing the Decision/Estimator and the Sequential Probability Ratio Test Unit. 
VARIABLE NO.1

REDUNDANCY LEVE - 4

NO. OF MJSO MOOZLS CONTAINING THIS VARIABLE - 1

TOTAL NUMEER OF VARIABLES -

REDUNDANT MEASUREMENTS

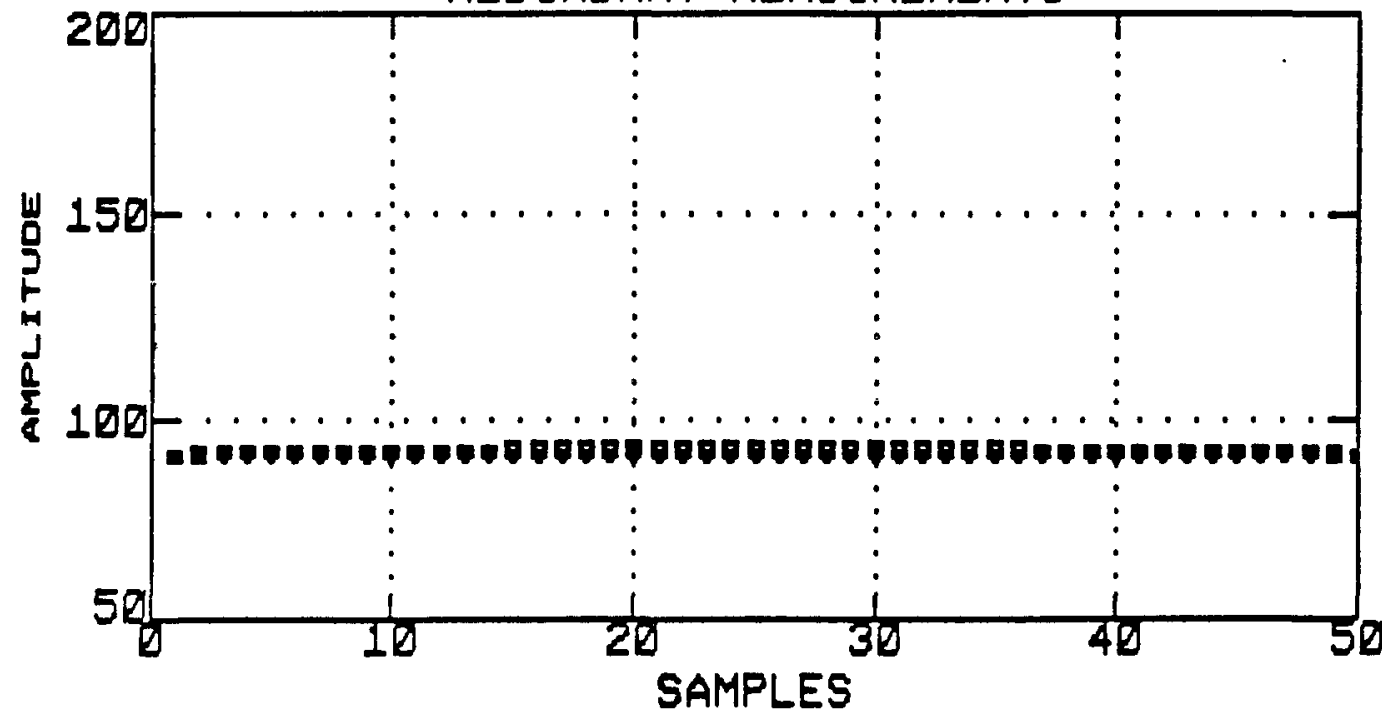

Figure 3.3a. 50 Samples of the Four Redundant Measurments of Variable No. 1 Used in the First Numerical Experiment.

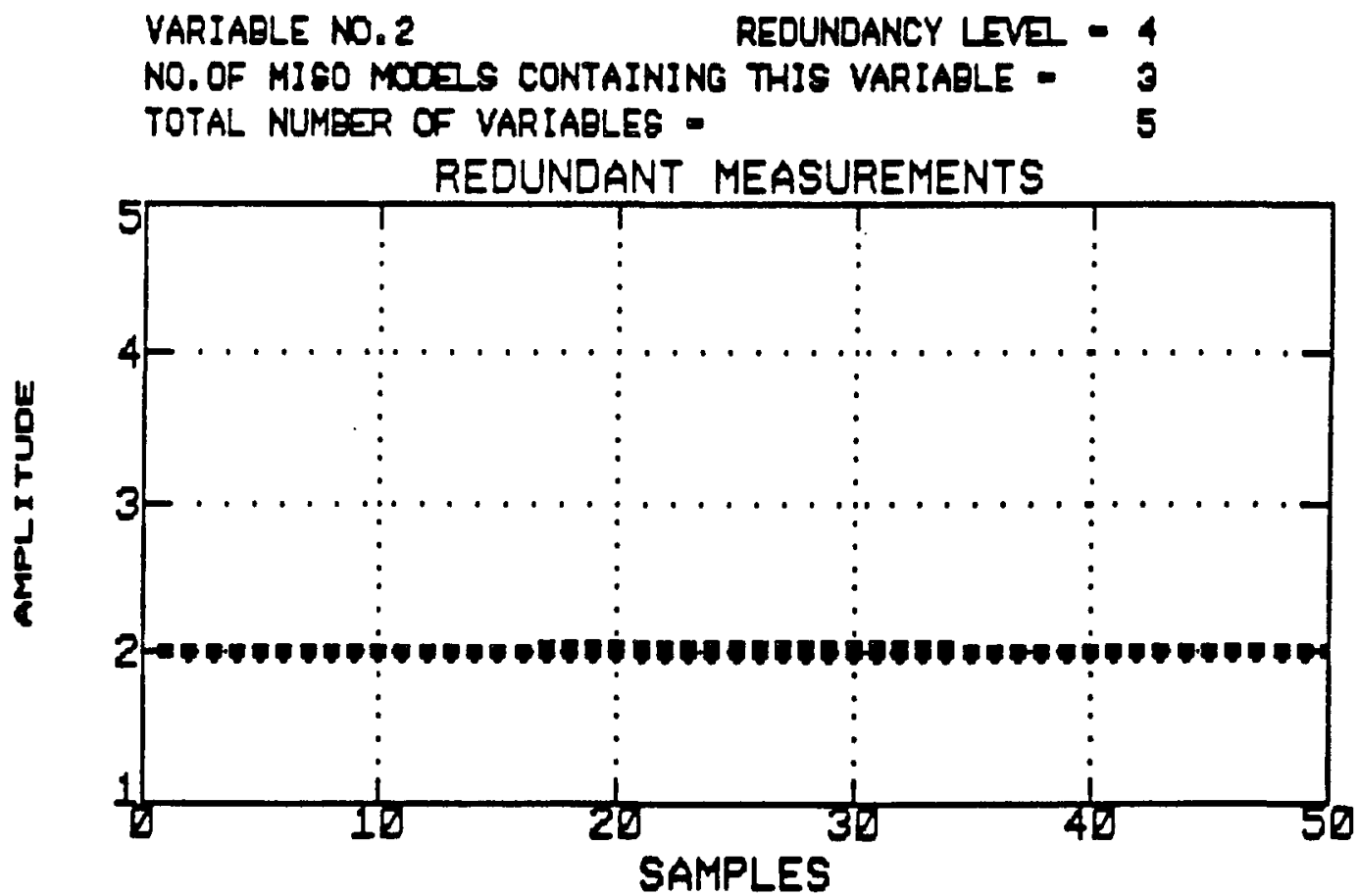

Figure 3.3b. 50 Samples of Four Redundant Measurements of Variable No. 2 Used in the First Numerical Experiment. 
VARIABLE NO.3

REOUNOANCY LEV - 5

NO. OF MISO MOOJS CONTAINING THIS VARIAOLE - 2

TOTAL NUMEER OF VARIABLES -

REDUNDANT MEASUREMENTS

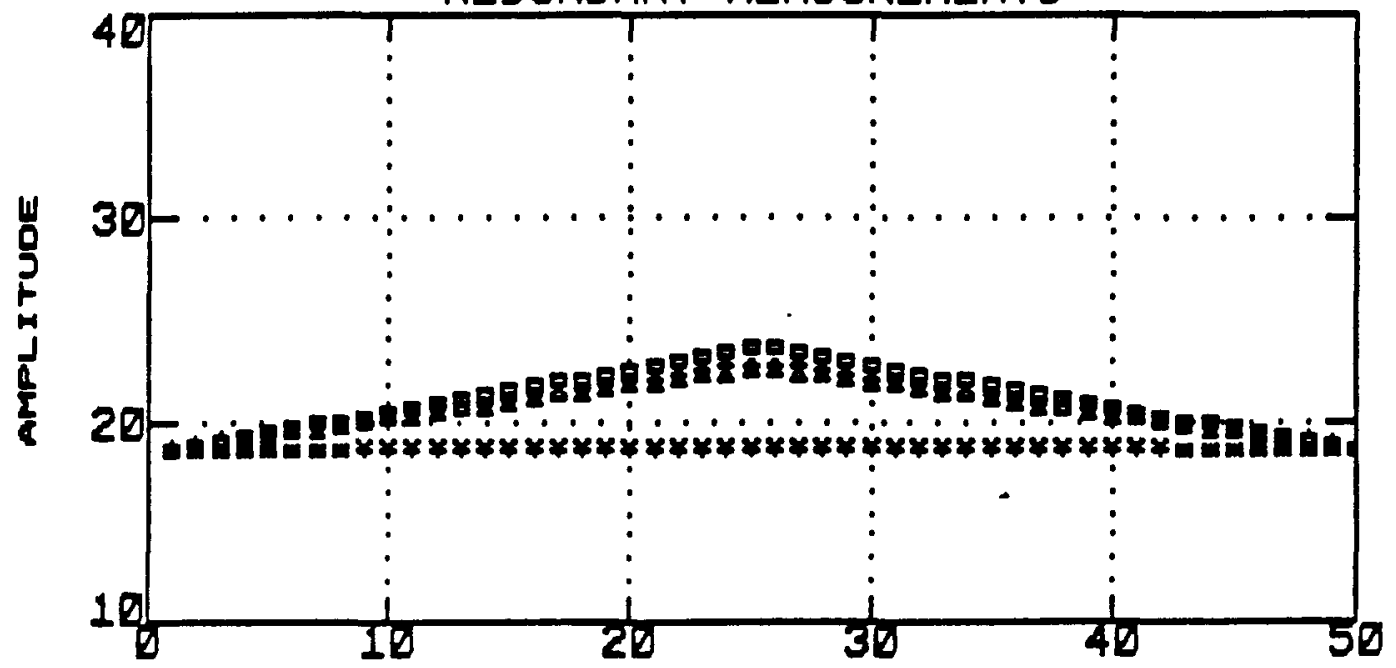

Figure 3.3c. 50 Samples of Five Redundant Measurements of Variable No. 3 Used in the First Numerical Experiment. Identical Ramp Deviations in Signals 3, 4 and 5 Were Introduced.

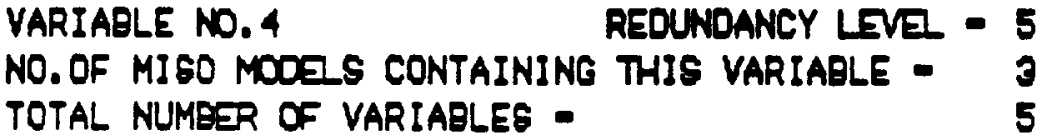
TOTAL NUMEER OF VARIABLES -

REDUNDANT MEASUREMENTS

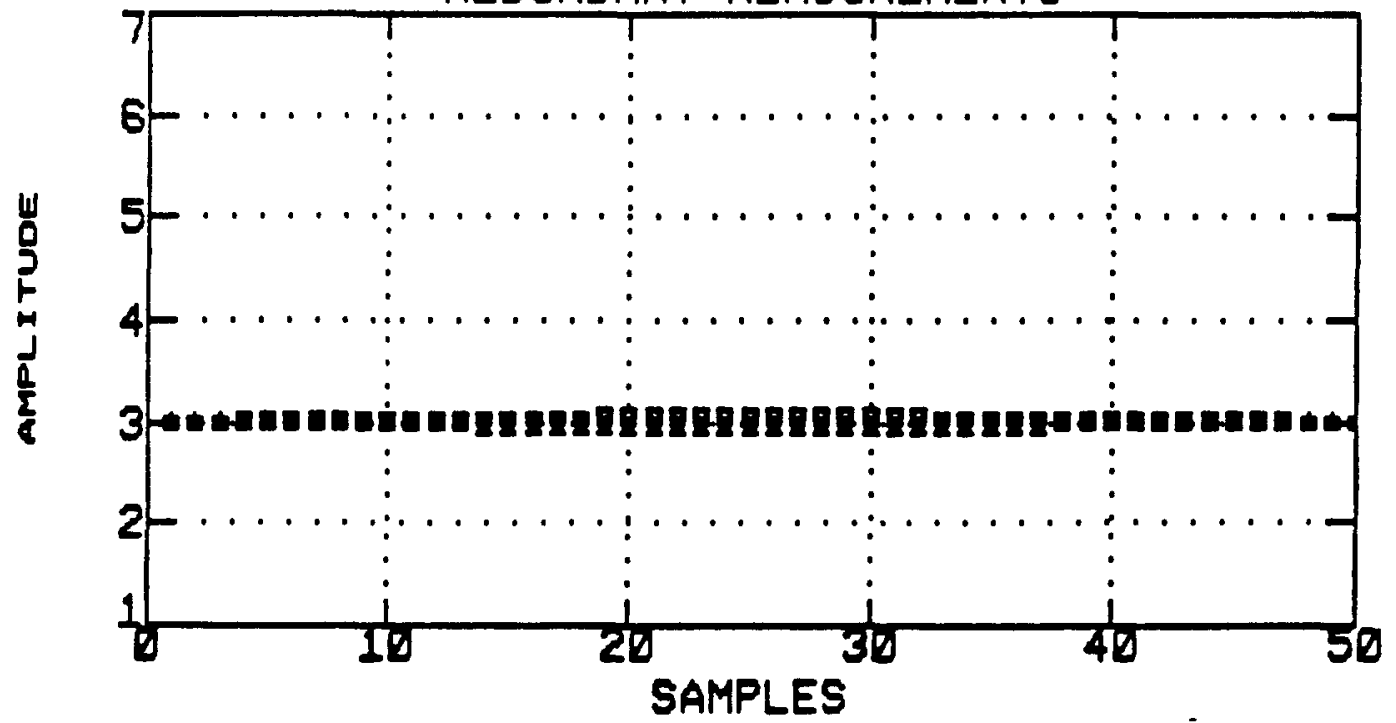

Figure 3.3d. 50 Samples of Five Redundant Measurements of Variable No. 4 Used in the First Numerical Experiment. 
VARIABLE NO.5

REDUNOANCY LEVI - 4

NO. OF MISO MOOES CONTAINING THIS VARIADLE - 2

TOTAL NUMBER OF VARIABLES - 5

REDUNDANT MEASUREMENTS

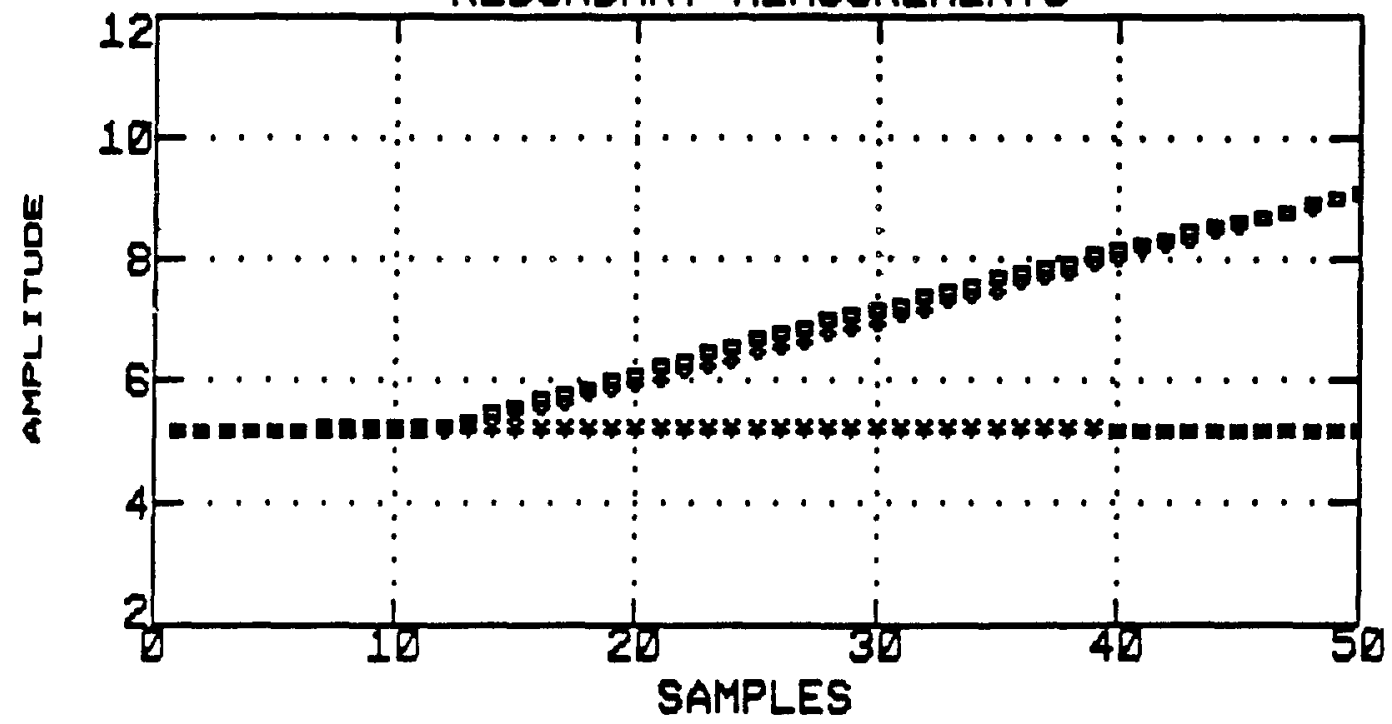

Figure 3.3e. 50 Samples of Four Redundant Measurements of Variable No. 5 Used in the First Numerical Experiment. Identical Ramp Deviations in Signals 3 and 4 Were Introduced. 
VARIAELE NO. 1

REDUNDANCY LEVE - 4

NO. OF MISO MOOES CONTAINING THIS VARIAQLE - 1

TOTAL NUMEER OF VARIABLES -

CUMULATIVE INCONSISTENCY INDICES (CII)

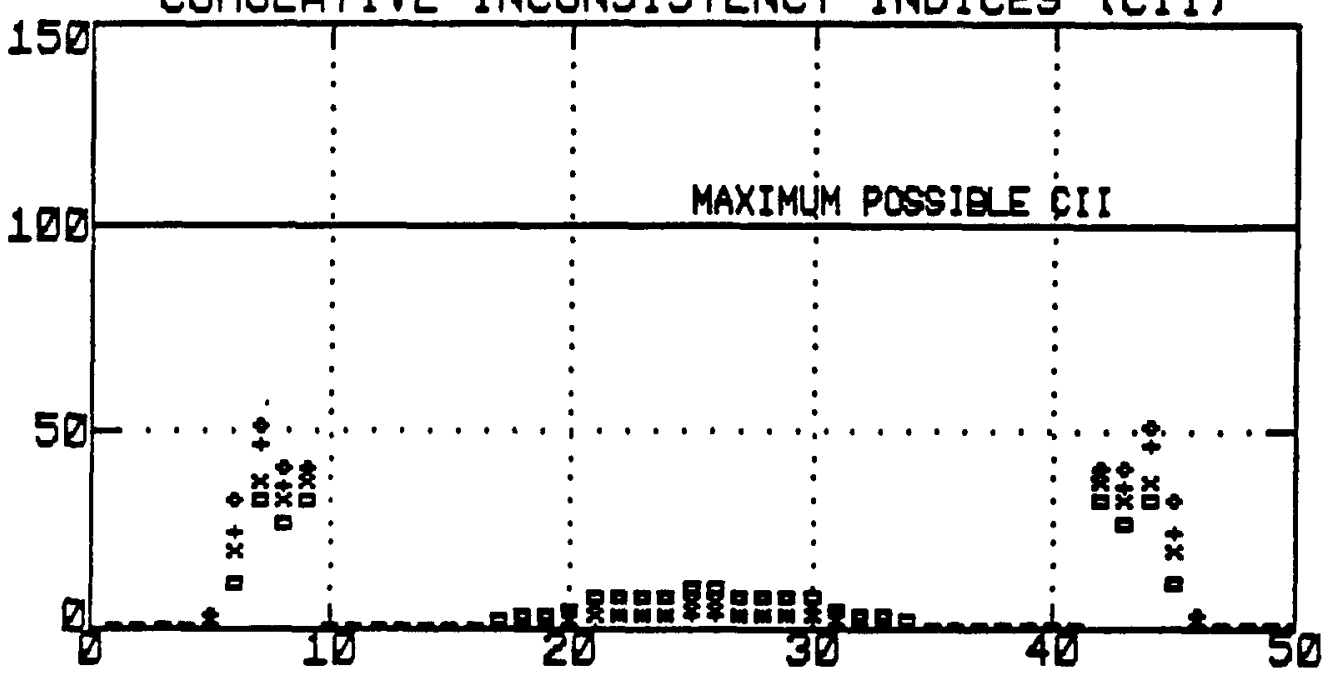

Figure 3.4a. Cumulative Inconsistency Indices (CII) of the Four Redundant Signals of Variable No. 1 Calculated in the First Numerical Experiment. No Degraded Signal Was Detected.

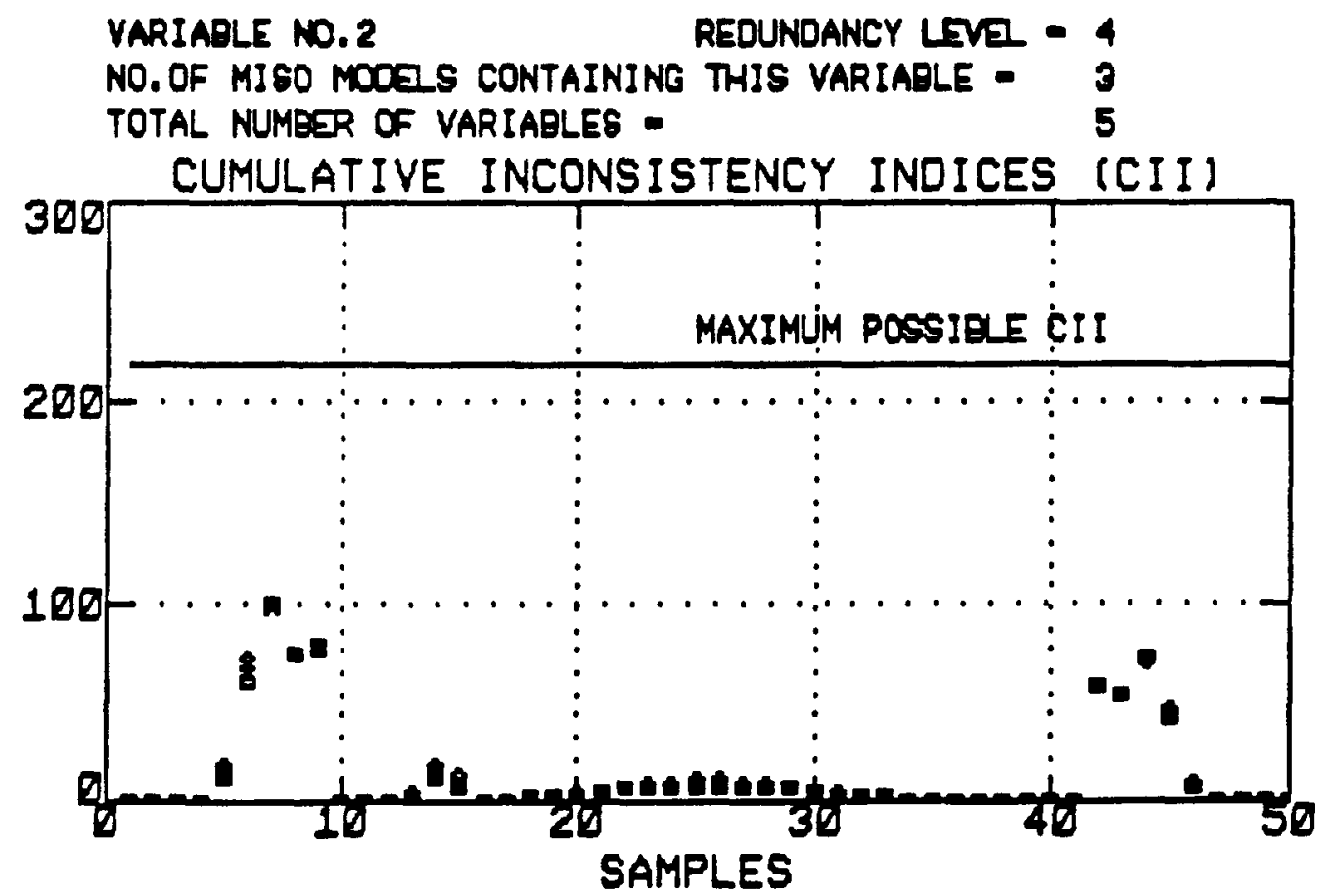

Figure 3.4b. Cumulative Inconsistency Indices (CII) of the Four Redundant Signals of Variable No. 2. 
VARIADLE N0.3 REOUNOANCY LEV - 8

NO. DF MISO MODZS CONTAINING THIS VARLADLE - 2

TOTAL NUMEER OF VARIAQLEG - 5

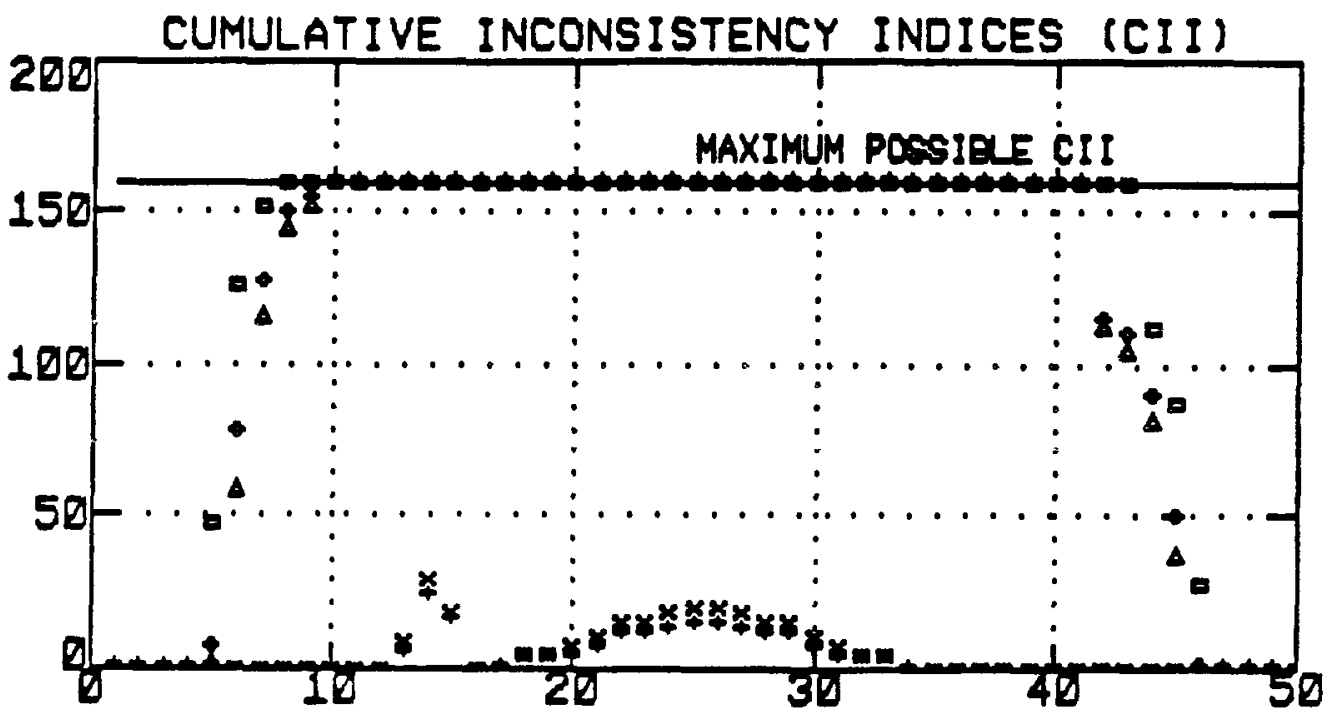

Figure 3.4c. Cumulative Inconsistency Indices (CU) of the Five Redundant Signals of Variable No. 3 Calculated in the First Numerical Experiment. Signals 3, 4 and 5 have Maximum CI's in the Range 10-40 Samples. These are Identified and Excluded as Degraded
Signals.

VARIAQLE NO. 4 NO. OF MISO MOOZ S CONTAINING THIS VARTAQLE TOTAL NUMEER OF VARIABLES REDUNDANCY LVIZ - 5
THIS VARIAGLE - 3

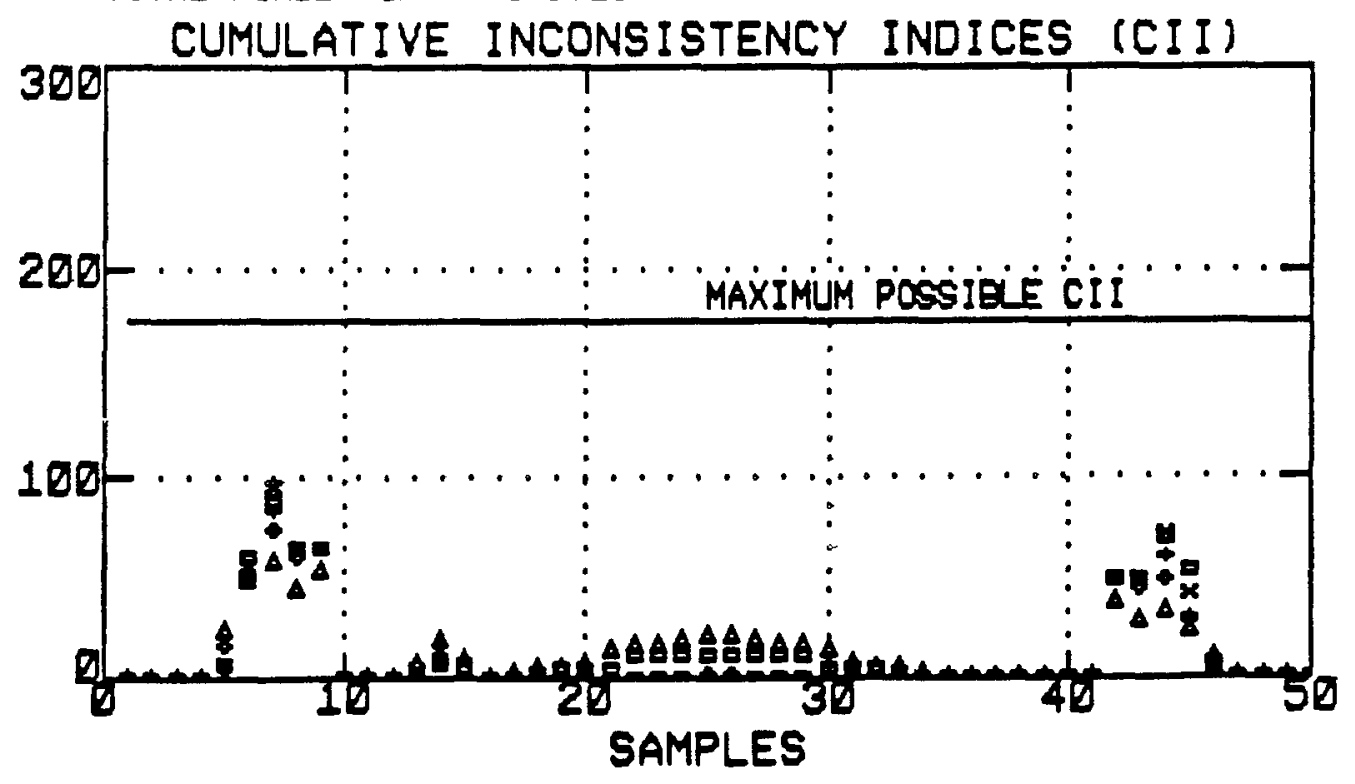

Figure 3.4d. Cumulative Inconsistency Indices (CI) of the Five Redundant Signals of Variable No. 4. No Faulty Signals.. 
VARIABLE NO.5

REDUNOANCY LEVZ - 4

NO. OF MI60 MOOESS CONTAINING THIS VARIADLE - 2

TOTAL NUMESR OF VARIAELES -

\section{5}

CUMULATIVE INCONSISTENCY INDICES (CII)

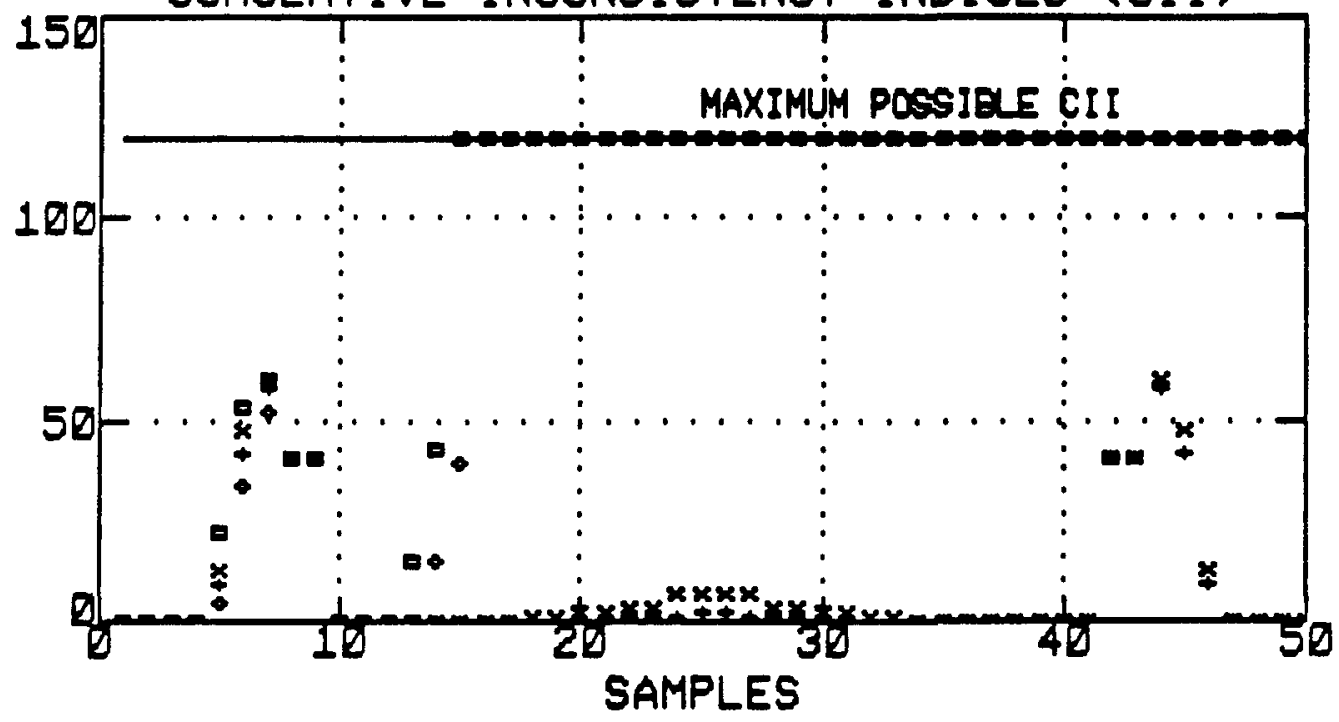

Figure 3.4e. Cumulative Inconsistency Indices of the Four Redundant Measurements of Variable No. 5 Calculated in the First Numerical Experiment. Signals 3 and 4 have Maximum Cn's Starting at Sample No. 16. These are Identified and Excluded as Degraded Signals. 
VARIAQLE NO.1

REDUNDANCY LVVZ - 4

NO. OF MISO MOOELS CONTAINING THIS VARIAPLE - 1

TOTAL NUMEER OF VARIABLES -

SIGNAL ESTIMATE

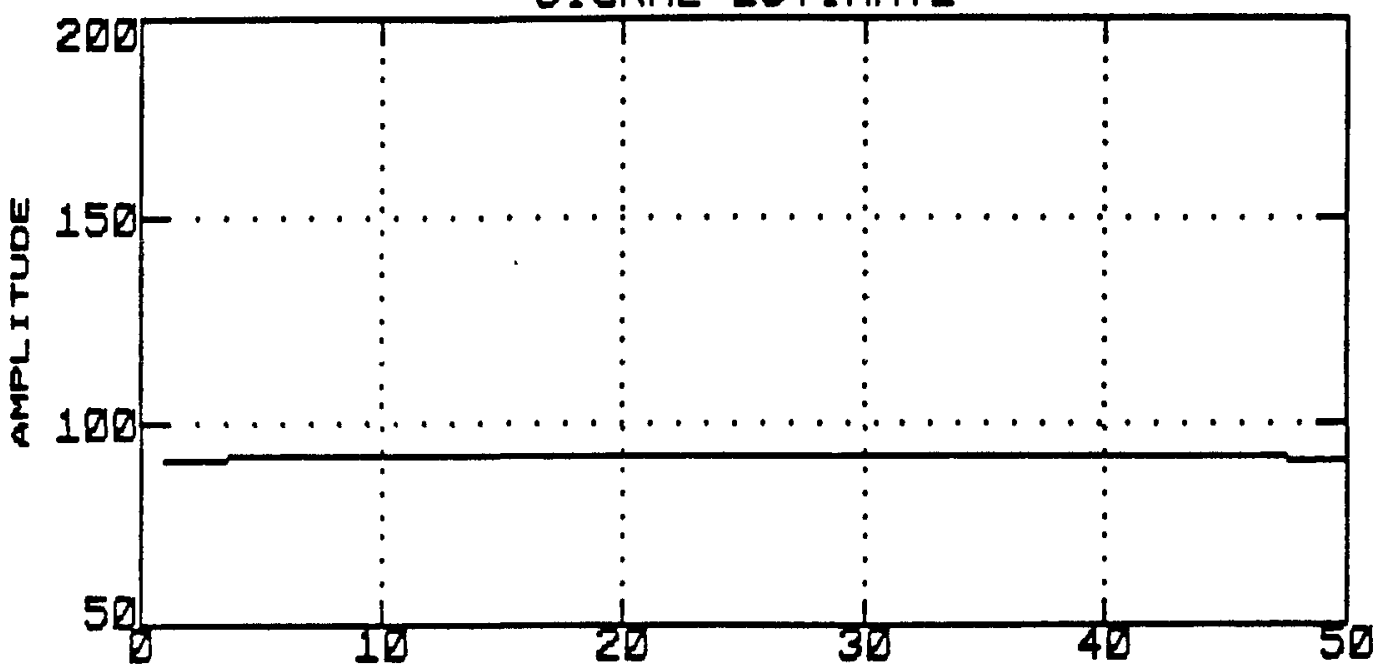

Figure 3.5a. Estimate of variable No. 1 based on the weighted average of its four redundant measurements.

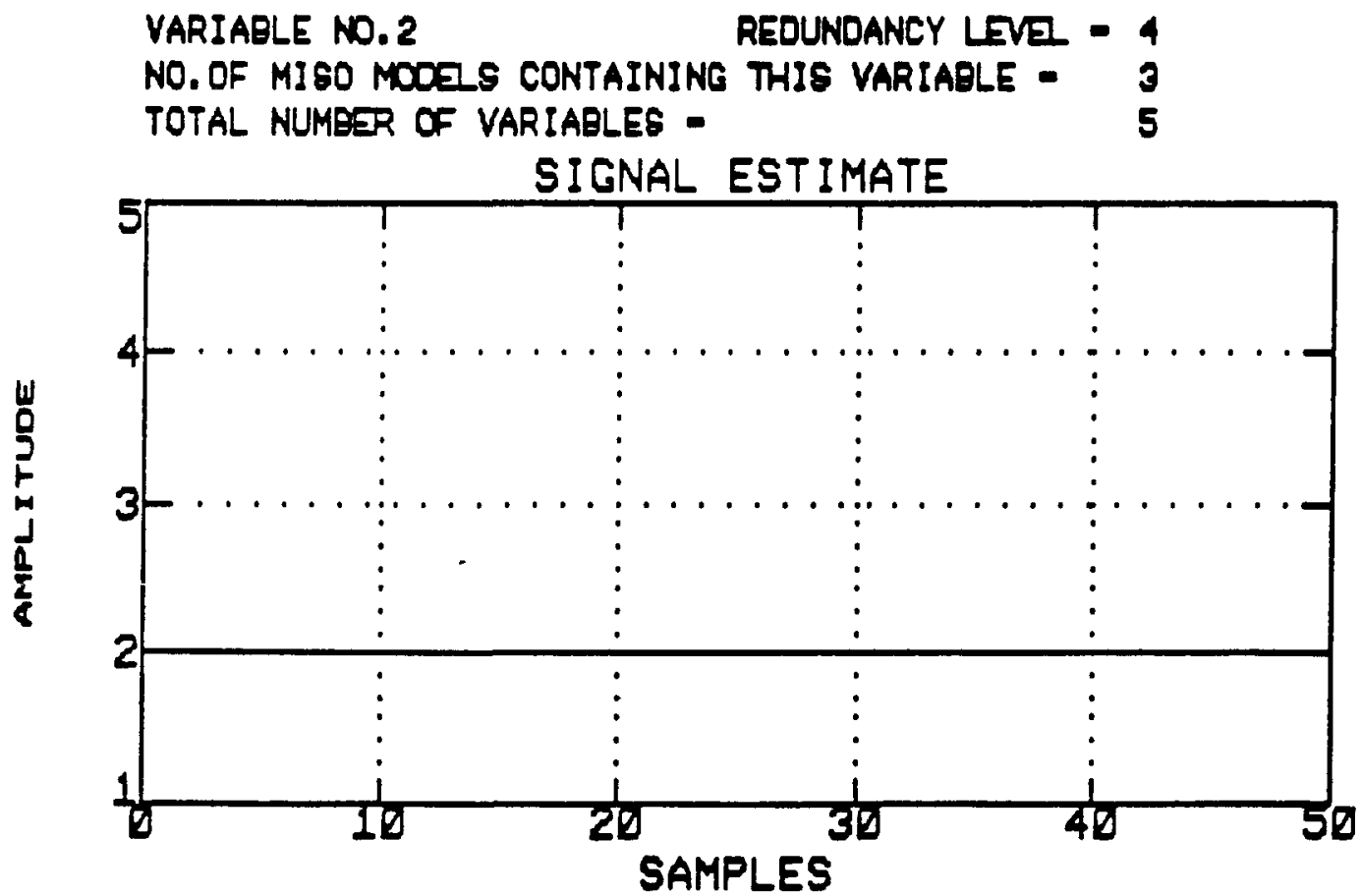

Figure 3.5b. Estimate of variable No. 2 based on the weighted average of its four redundant measurements. 


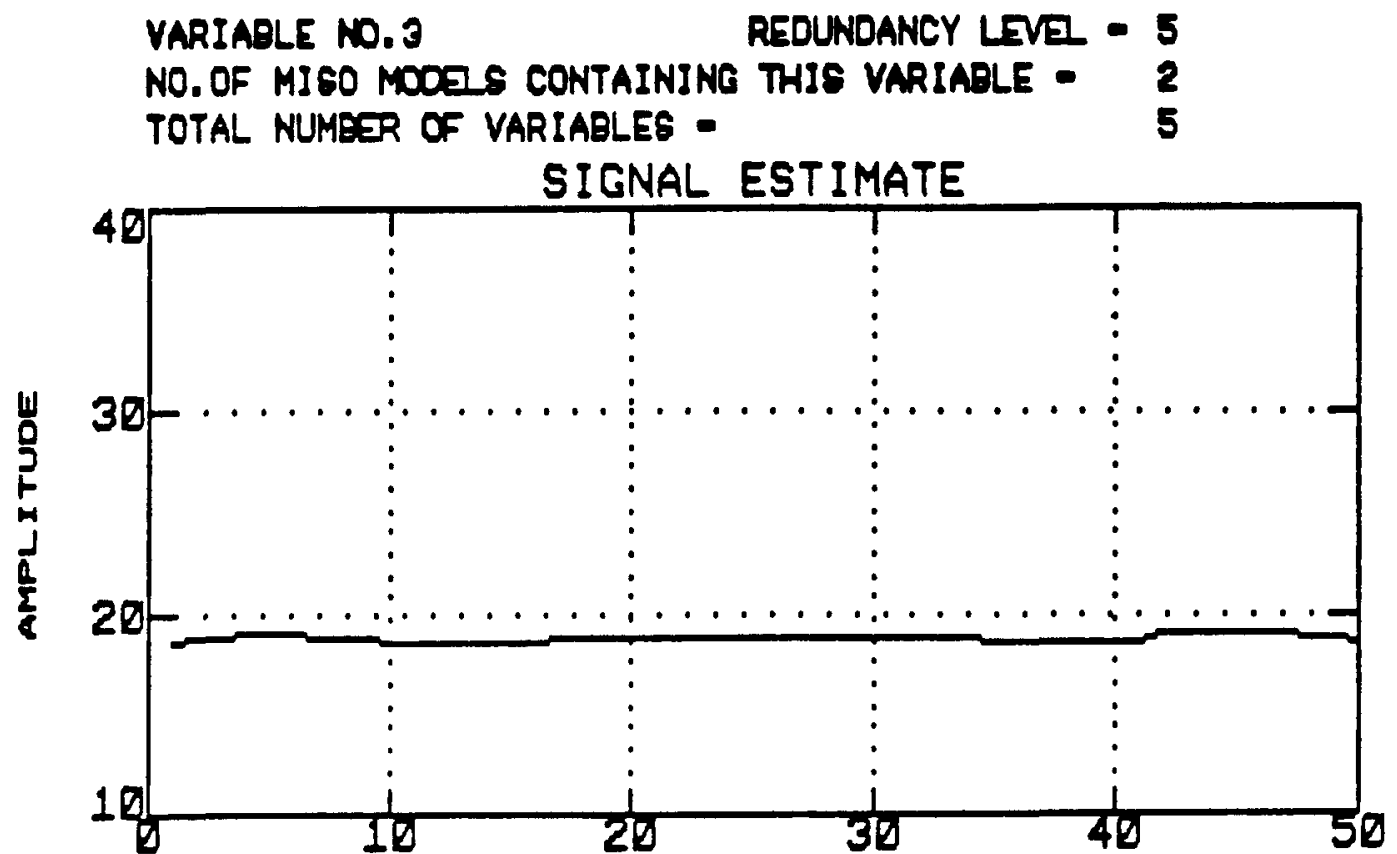

Figure 3.5c. Estimate of variable No. 3 based on the weighted average of its five redundant measurements.

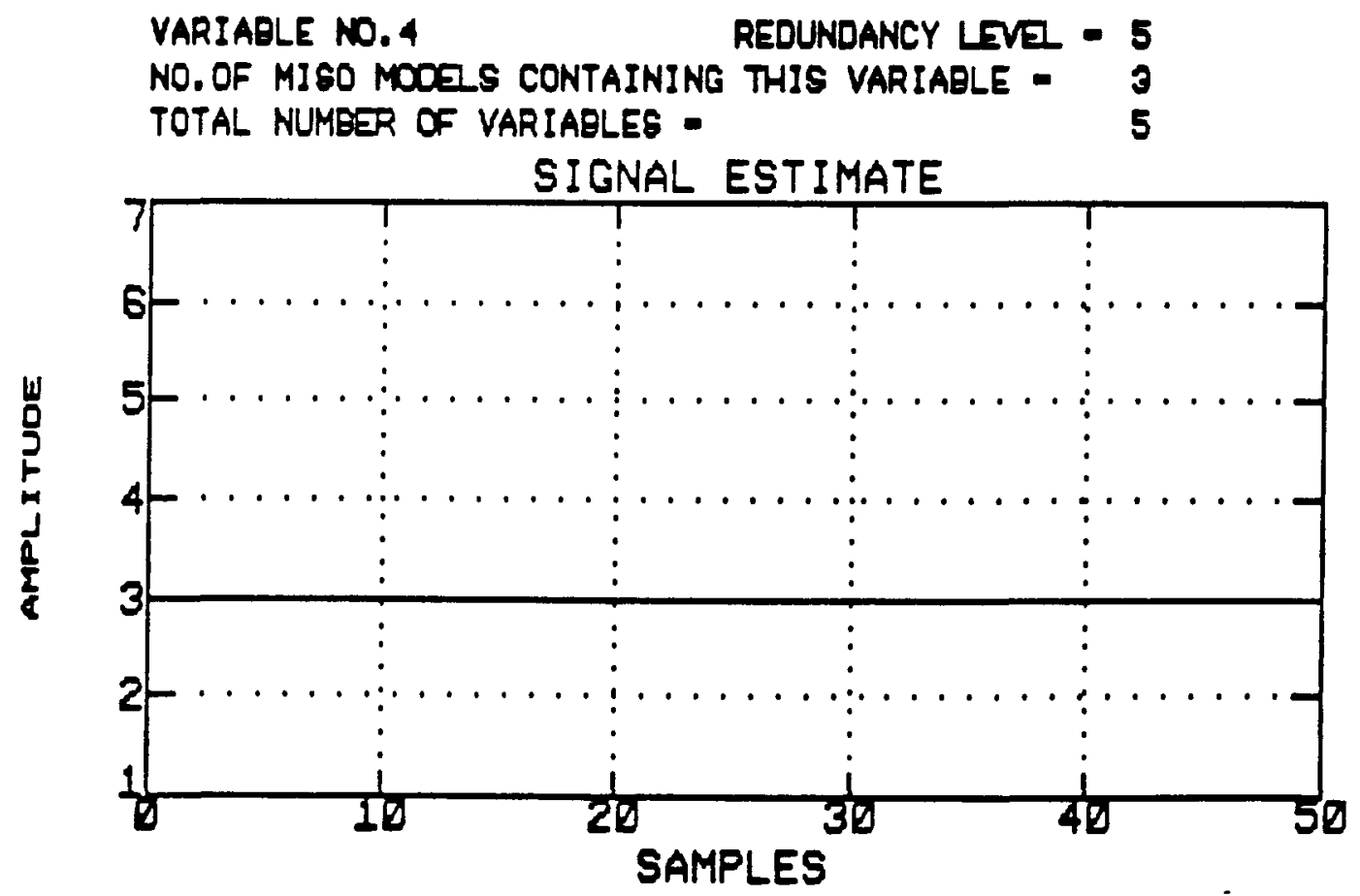

Figure 3.5d. Estimate of variable No. 4 based on the weighted average of its five redundant measurements. 


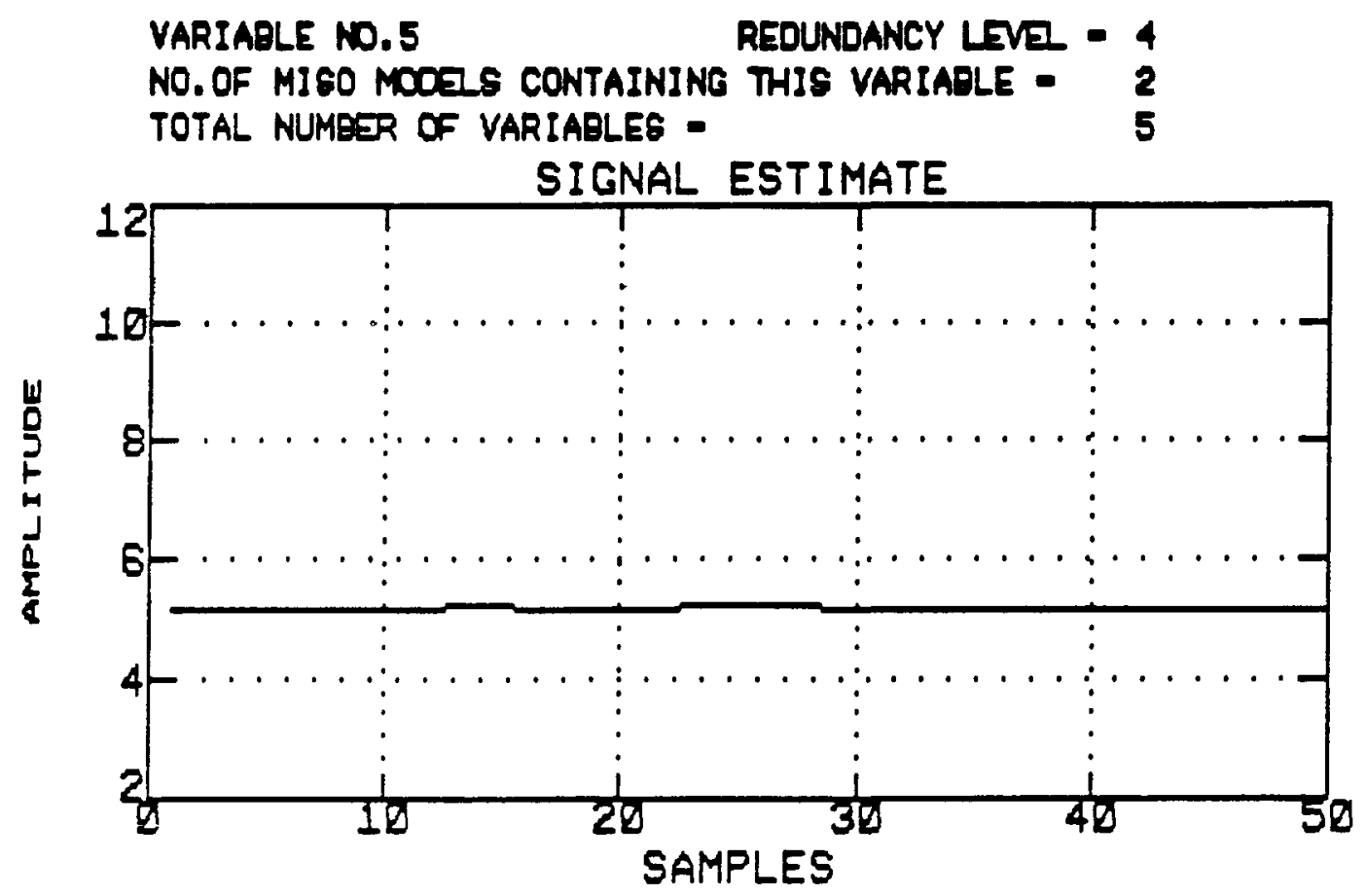

Figure 3.5e. Estimate of variable No. 5 based on the weighted average of its four redundant measurements. 
VARIAOLE NO.1 REDUNDANCY LEVI - 4

NO. OF MISO MOOZLS CONTAINING THIS VARIAOLE - 1

TOTAL NUMESR OF VARIAELEG -

REDUNDANT MEASUREMENTS

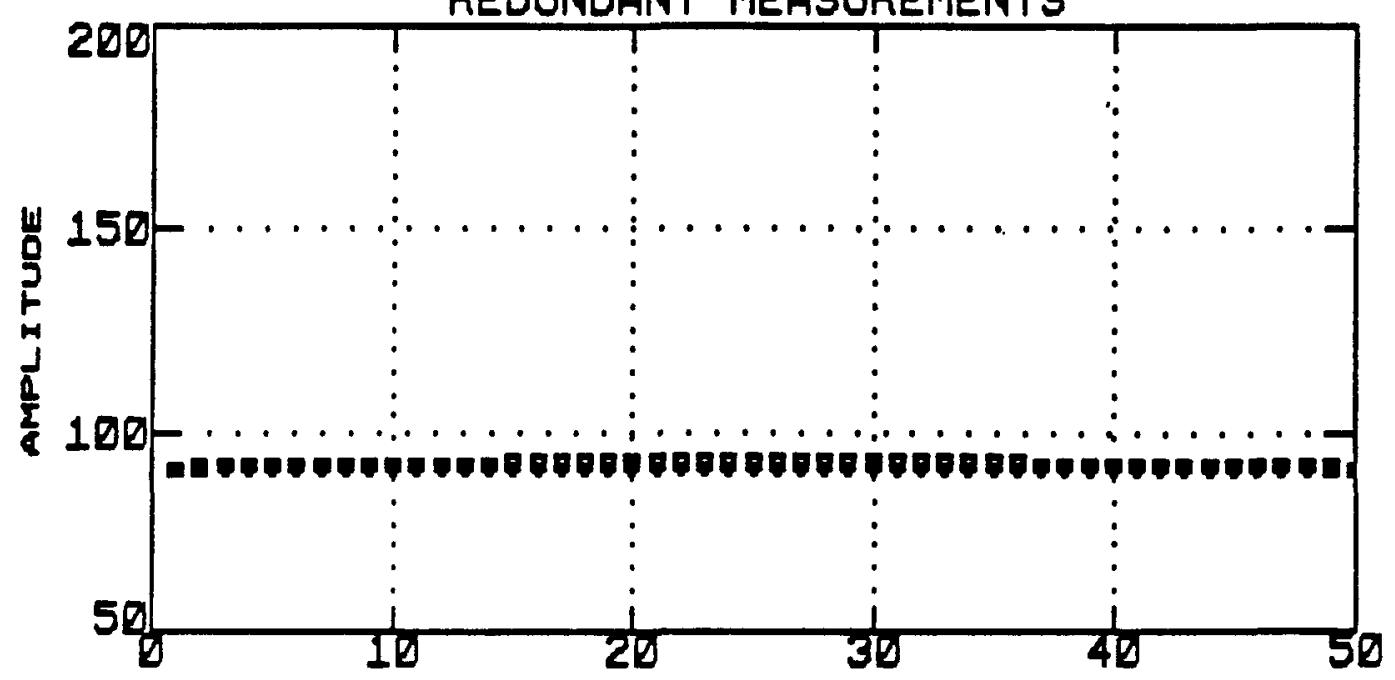

Figure 3.6a. 50 samples of the four redundnat measurements of variable No 1 used in the second numerical experiment.

VARIABLE NO.2

REDUNDANCY LEVE - 4

NO. OF MISO MOOELS CONTAINING THIS VARIABLE - 3

TOTAL NUMBER OF VARIAGLES -

\section{REDUNDANT MEASUREMENTS}

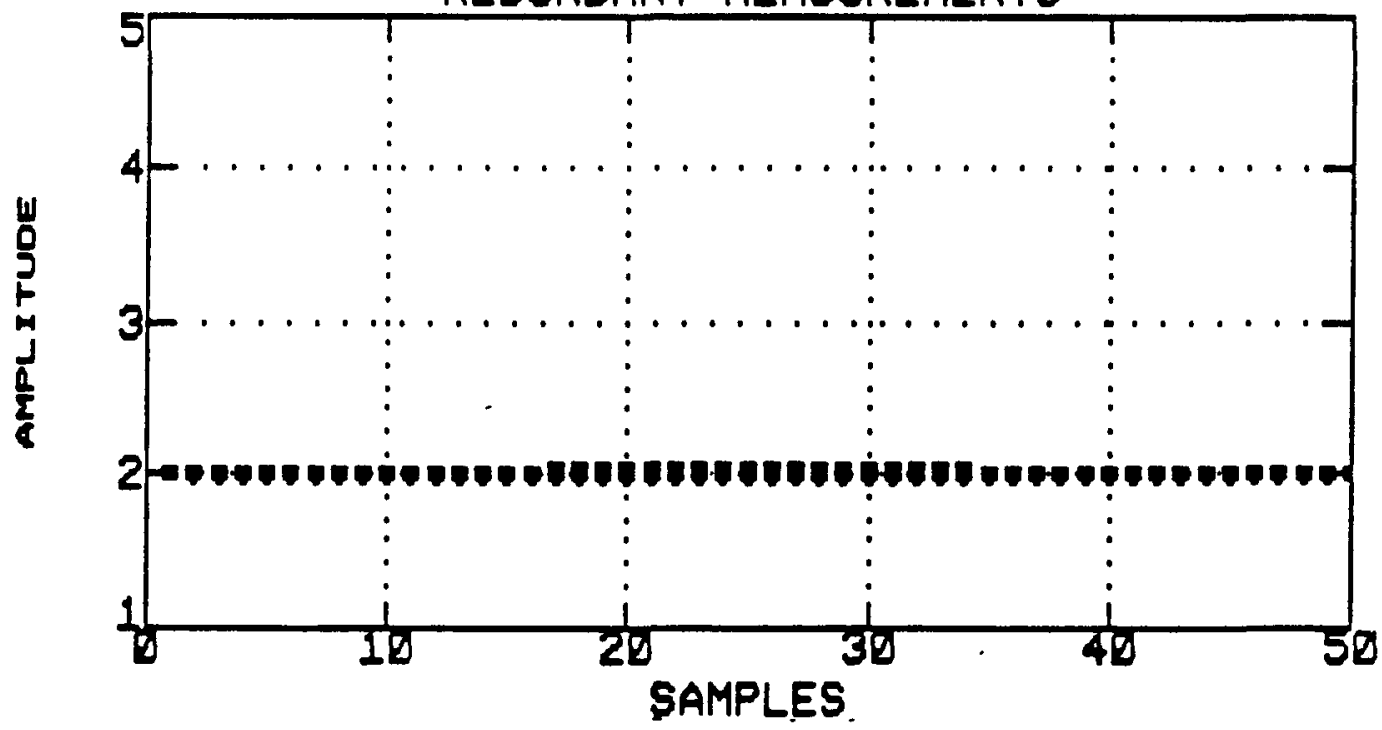

Figure 3.6b. 50 samples of the four redundnat measurements of variable No 2 used in the second numerical experiment. 
VARIAOLE NO.3 REDUNDANCY LEVI - 5

NO. OF MISO MOOES CONTAINING THIS VARIABLE - 2

TOTAL NUMBER OF VARIABLES -

REDUNDANT MEASUREMENTS

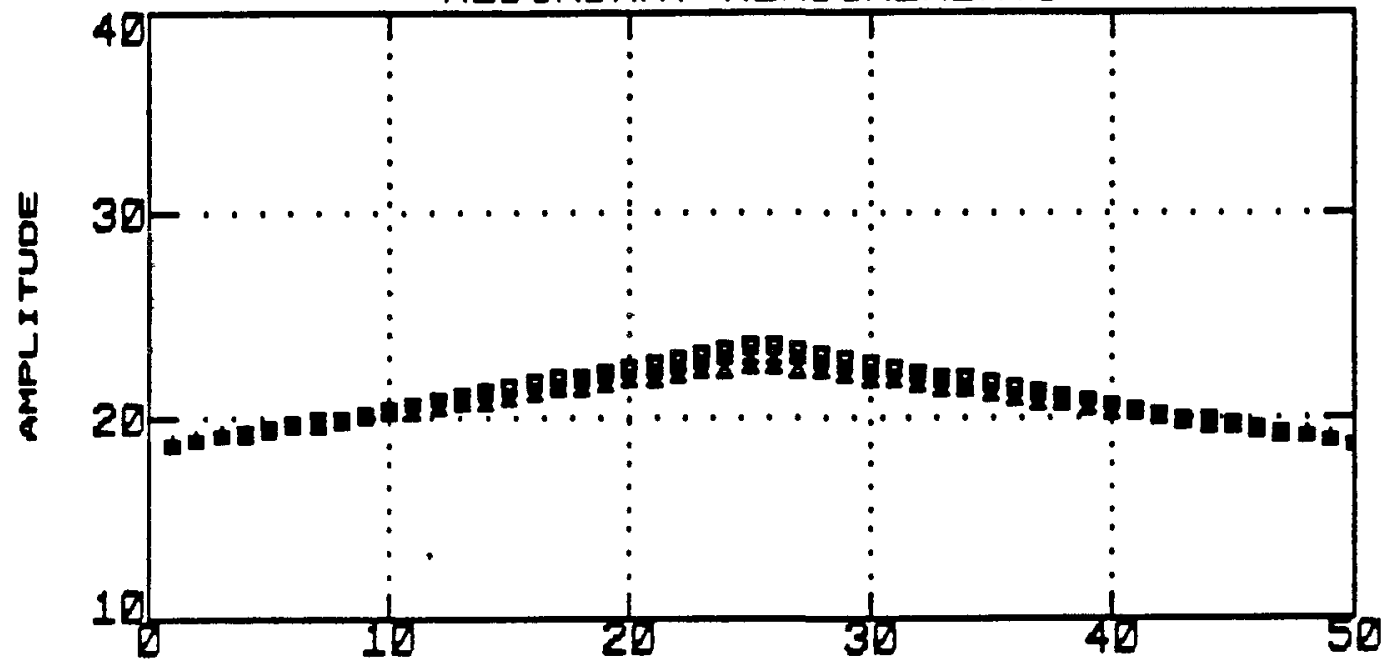

Figure 3.6c. 50 samples of the five redundnat measurements of variable No 3 used in the second numerical experiment. All the five signals have identical ramp deviation from the nurmal constant value.

VARIABLE NO. 4

REDUNDANCY LEVE - 5

NO. OF MISO MOOELS CONTAINING THIS VARIAELE - 3

TOTAL NUMEER OF VARIABLES -

REDUNDANT MEASUREMENTS

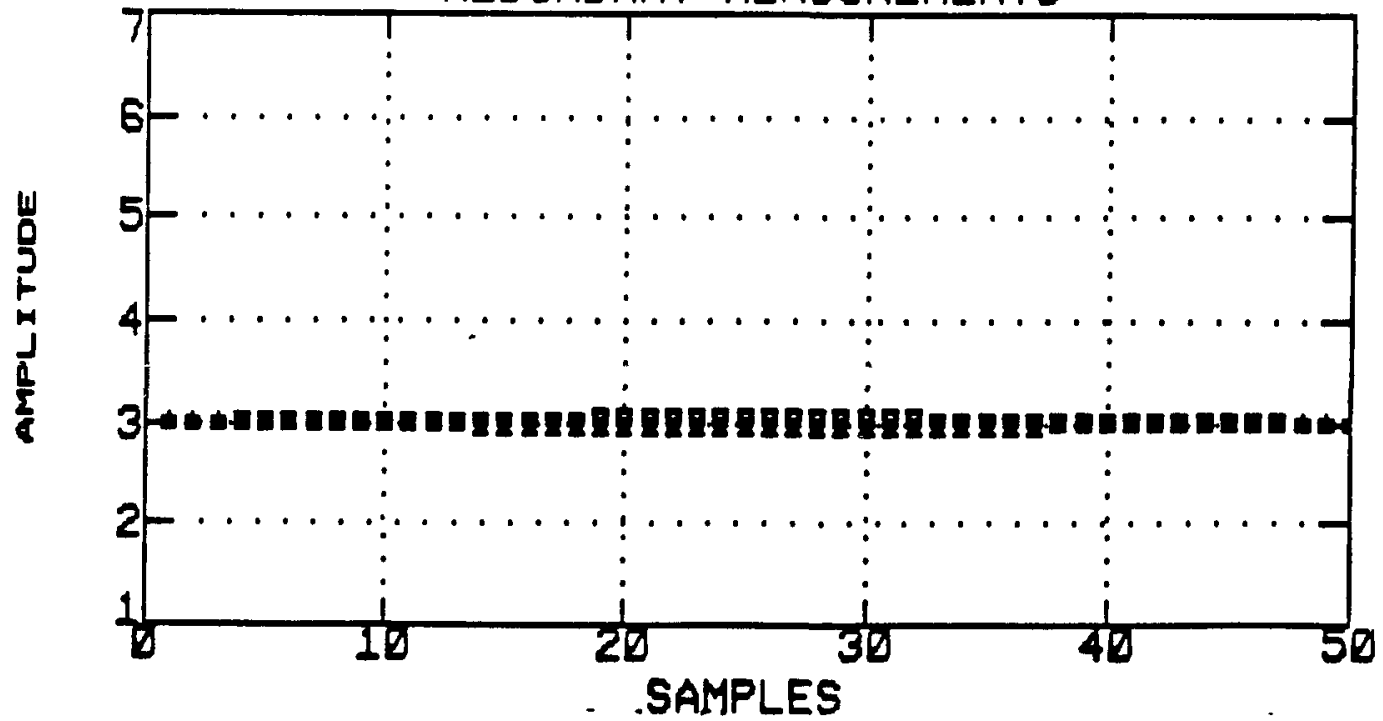

Figure 3.6d. 50 samples of the five redundnat measurements of variable No 4 used in the second numerical experiment. 
VARIABLE NO. 5

REDUNDANCY LEVE - 4

NO. OF MIGO MOOELS CONTAINING THIS VARIABLE - 2

TOTAL NUMEER OF VARLABLES -

5

REDUNDANT MEASUREMENTS

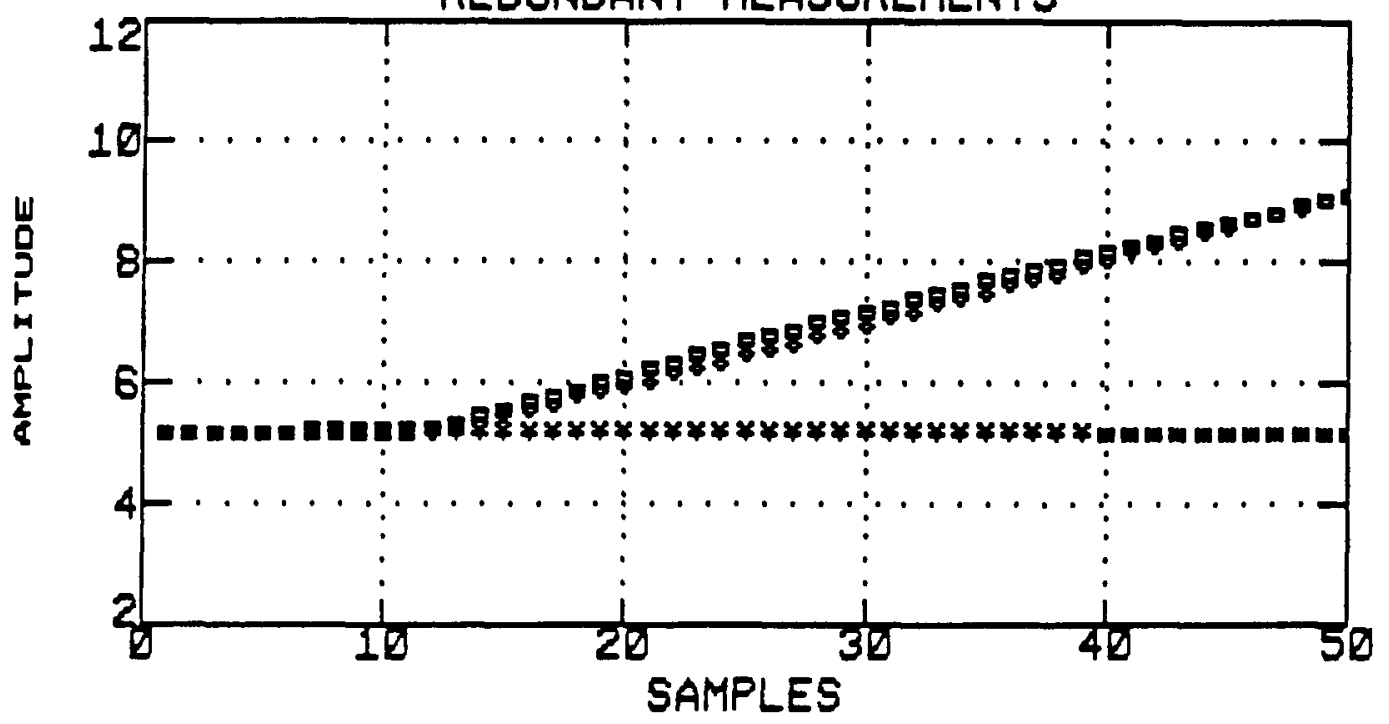

Figure 3.6t. 50 samples of the five redundant measurements of variable No 5 used in the second numerical experiment. Signals 3 and 4 have identical ramp deviation from the normal constant value, beginning at sample No. 12. 


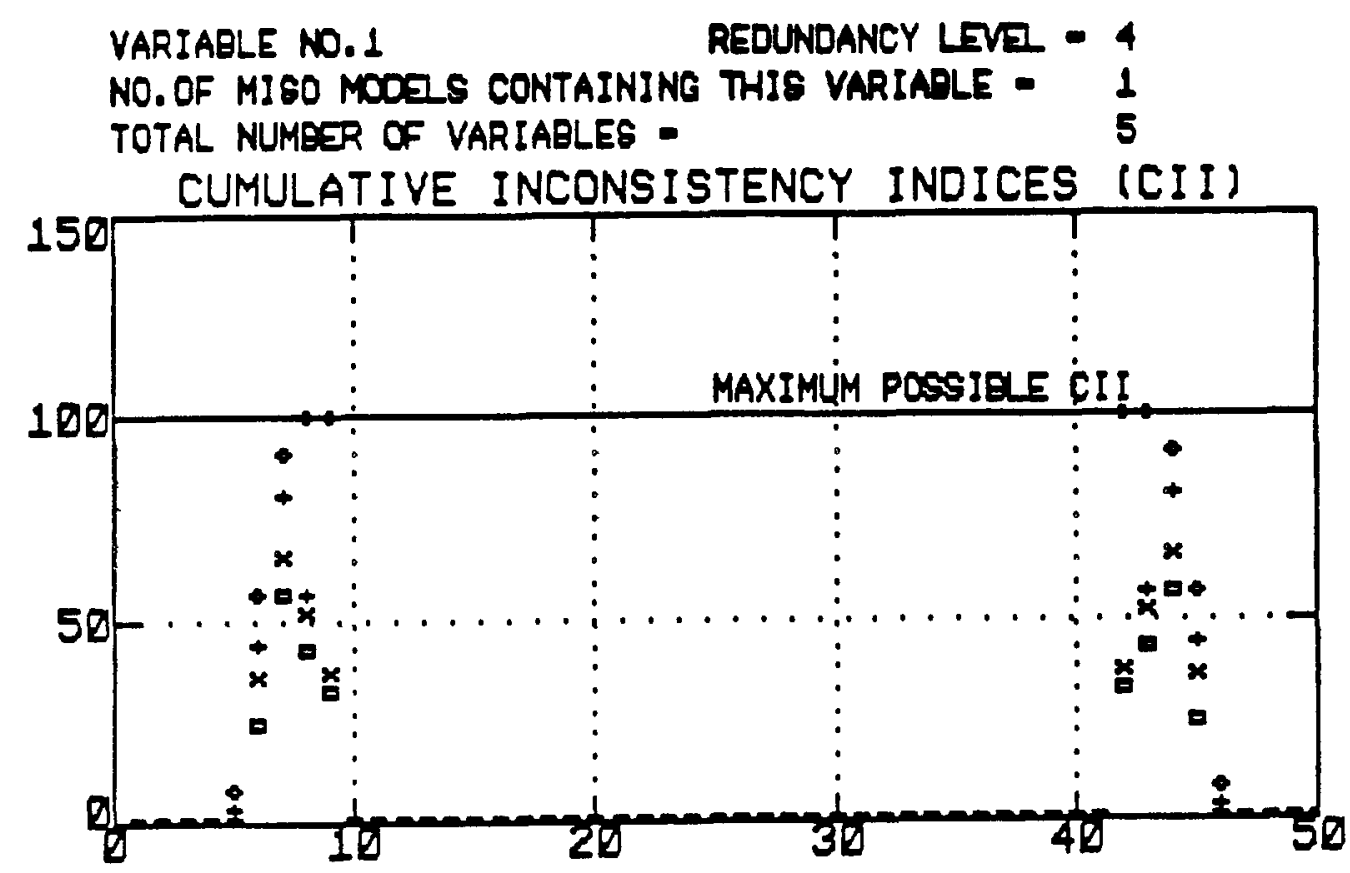

Figure 3.7a. Cumulative inconsistency indices of the four redundant measurements of variable No. 1 calculated in the second numerical experiment.

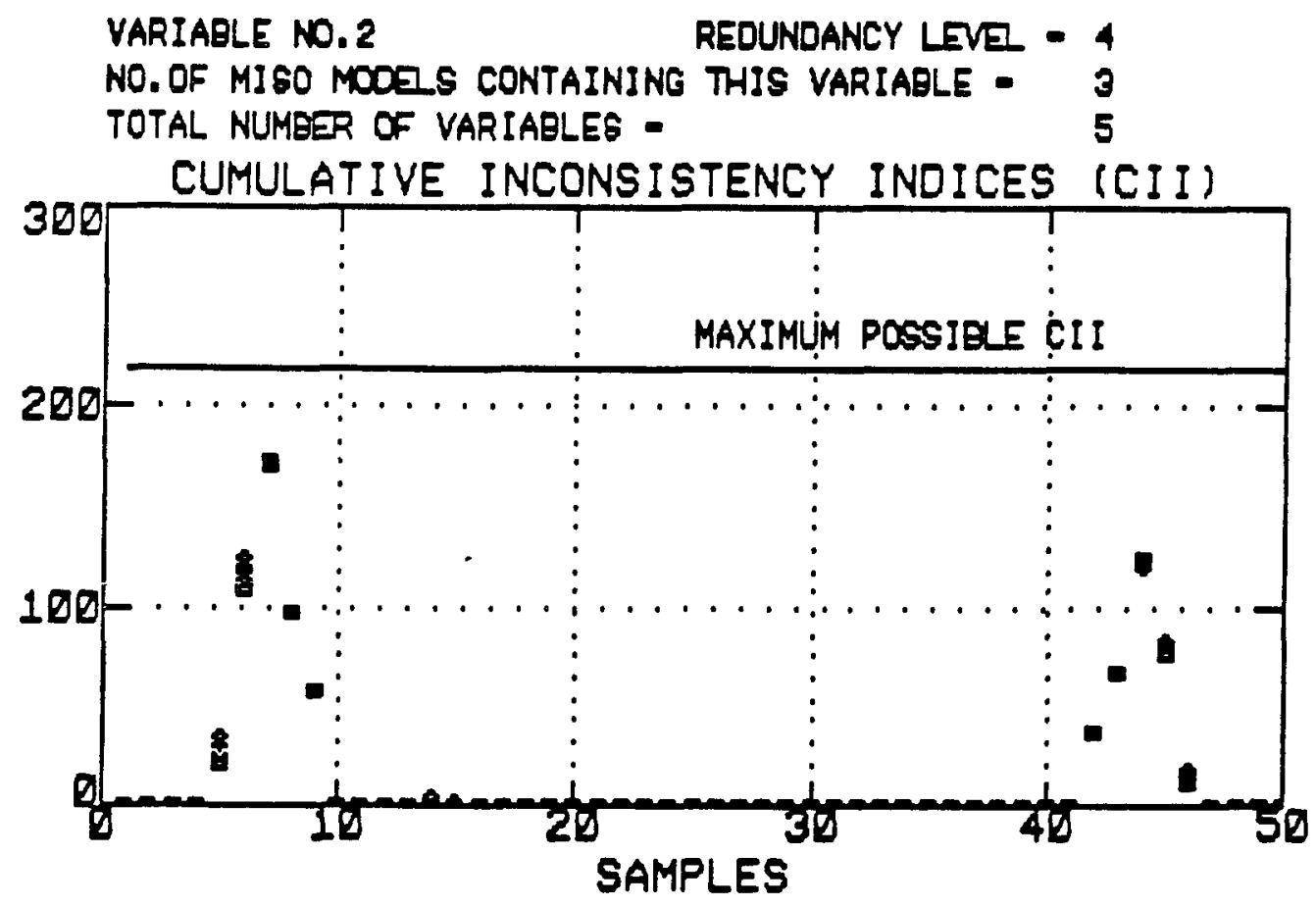

Figure 3.7b. Cumulative inconsistency indices of the four redundant signals of variable No. 2. 
VARIABLE NO.3 REDUNDANCY LEVE - -5
NO.OF MIGO MOOES CONTAINING THIS VARIADLE - 2
TOTAL NUMESR OF VARIABLES -

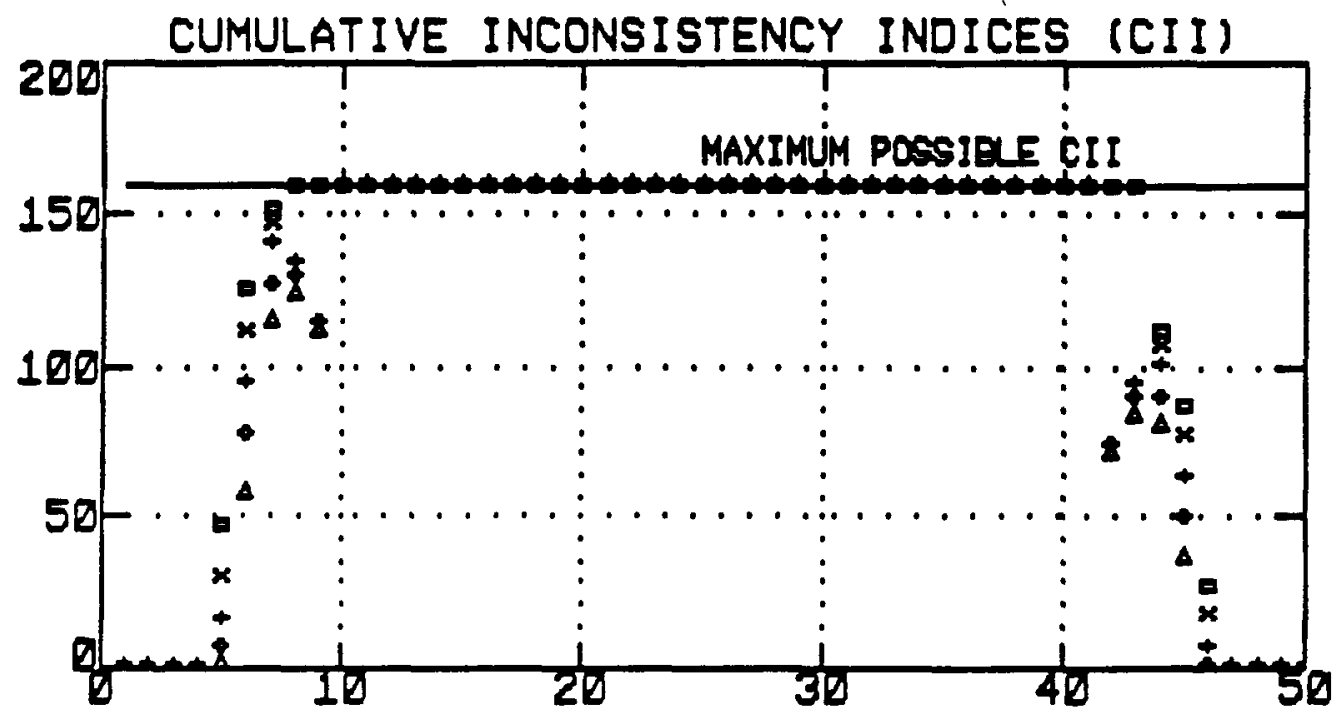

Figure 3.7 . Cumulative incunsistency indices of the five redundant measurements of variable No. 3 calculated in the second numerical experiment. All of the five signals have maximum CII's in the range $10-40$ samples. These are identified and excluded as degraded signals.

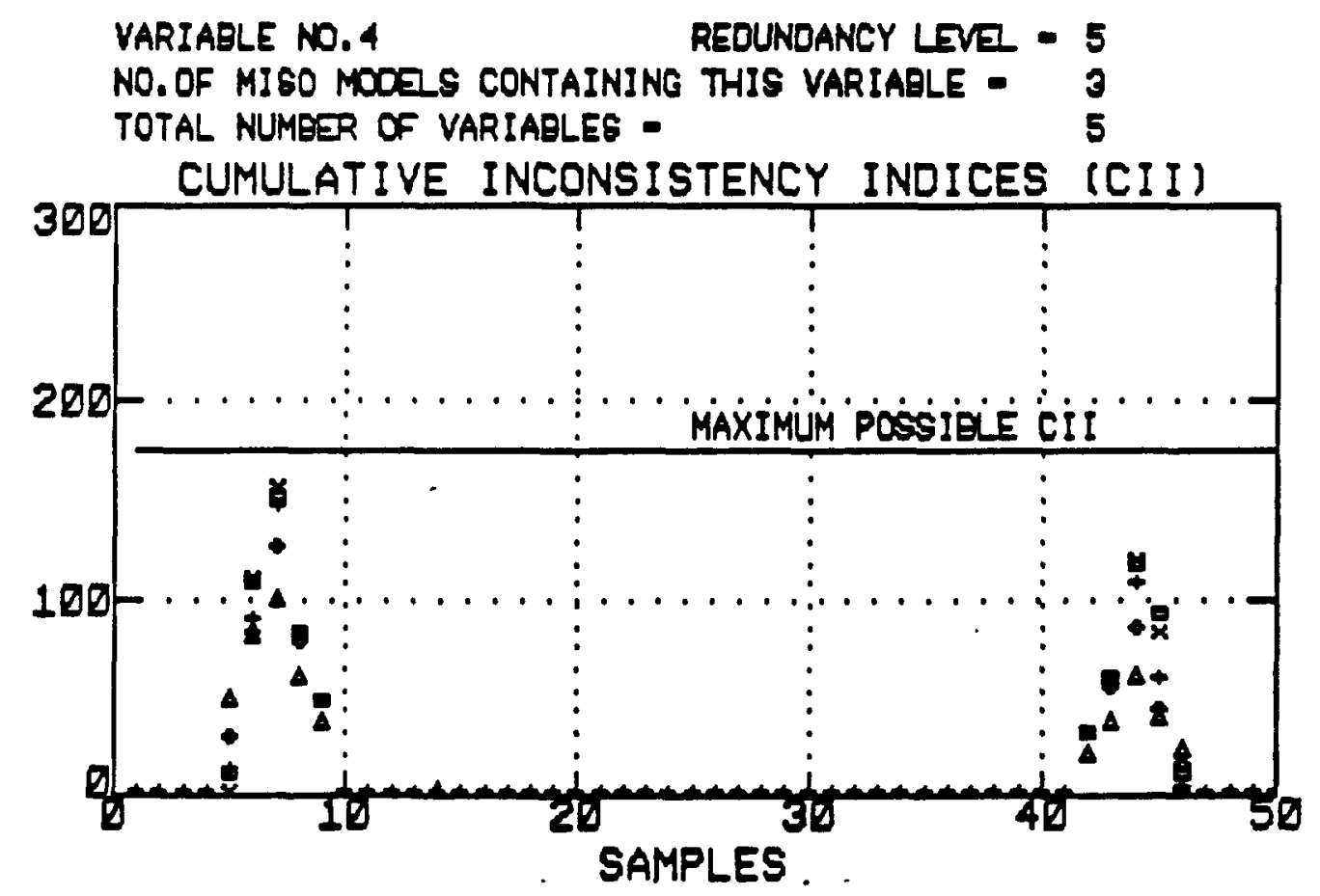

Figure 3.7d. Cumulative inconsistency indices of the five redundant signals of variable No. 4. 


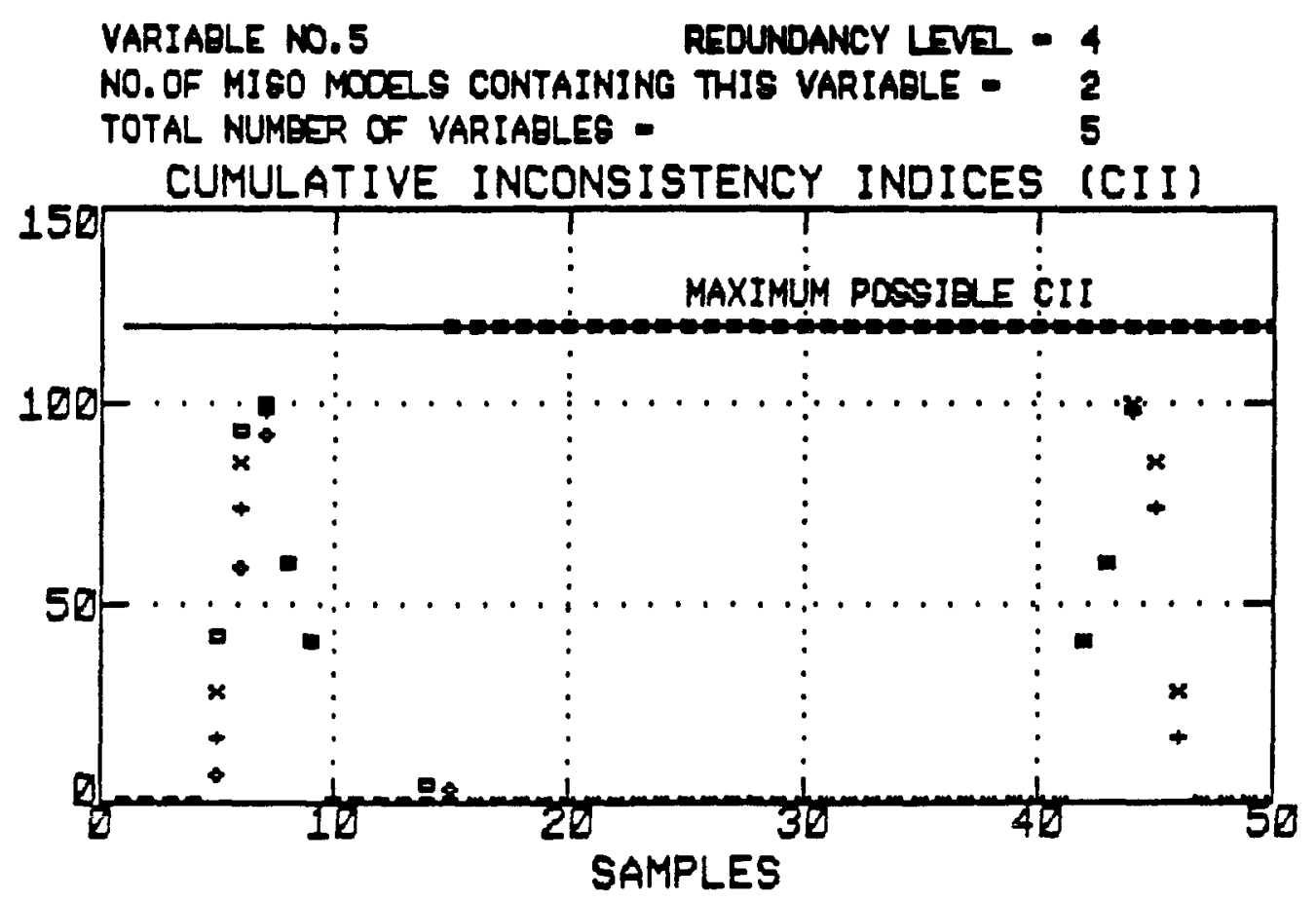

Figure 3.7e. Cumulative inconsistency indices of the four redundant measurements of variable $\mathrm{Nu} .5$ calculated in the second numerical experiment. Signals 3 and 4 have maximum CII's beginning at sample No. 16. They were identified and excluded as degraded signals. 
VARIABLE NO.1

REDUNDANCY LEVE - 4

NO. OF MIGO MOOES CONTAINING THIS VARIAOLE - 1

TOTAL NUMEER OF VARIAELES -

5

SIGNAL ESTIMATE

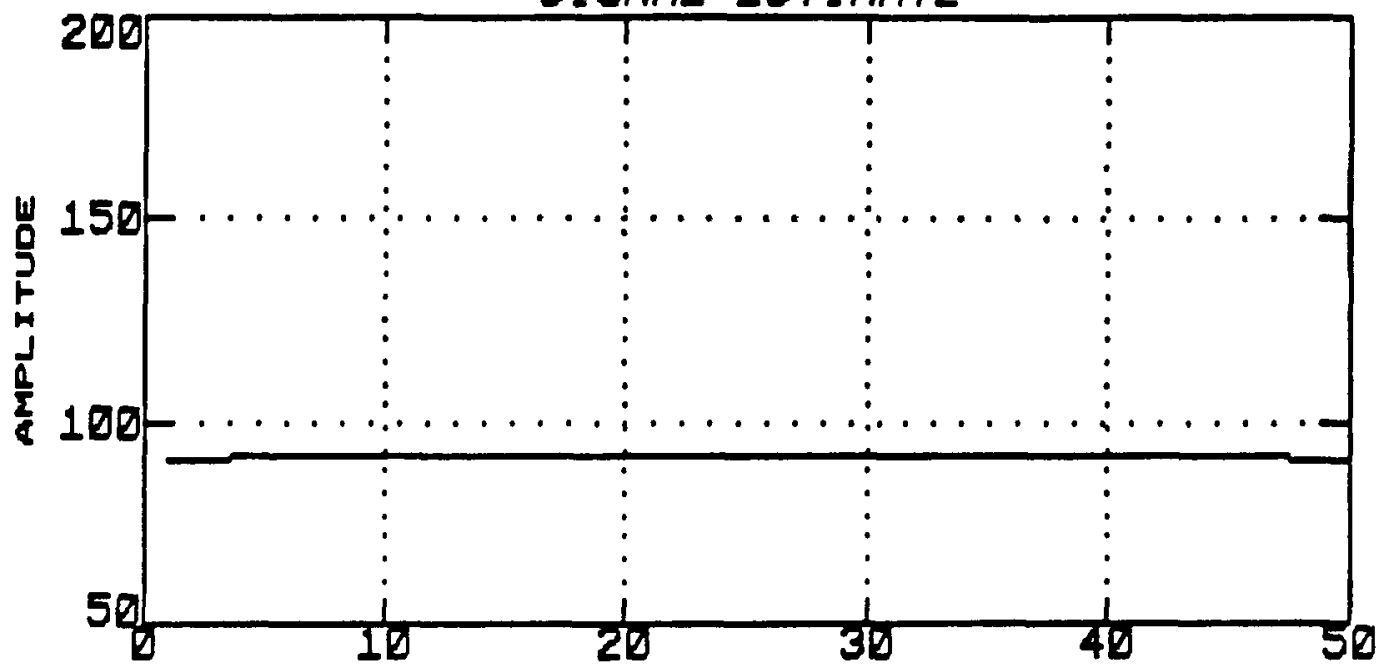

Figure 3.8a. Estimate of variable No. 1 based on the weighted average of its four redundant measurements.

VARIABLE NO.2

REDUNDANCY LEVI - 4

NO. OF MISO MOOEIS CONTAINING THIS VARIABLE - 3

TOTAL NUMBER OF VARIABLES -

SIGNAL ESTIMATE

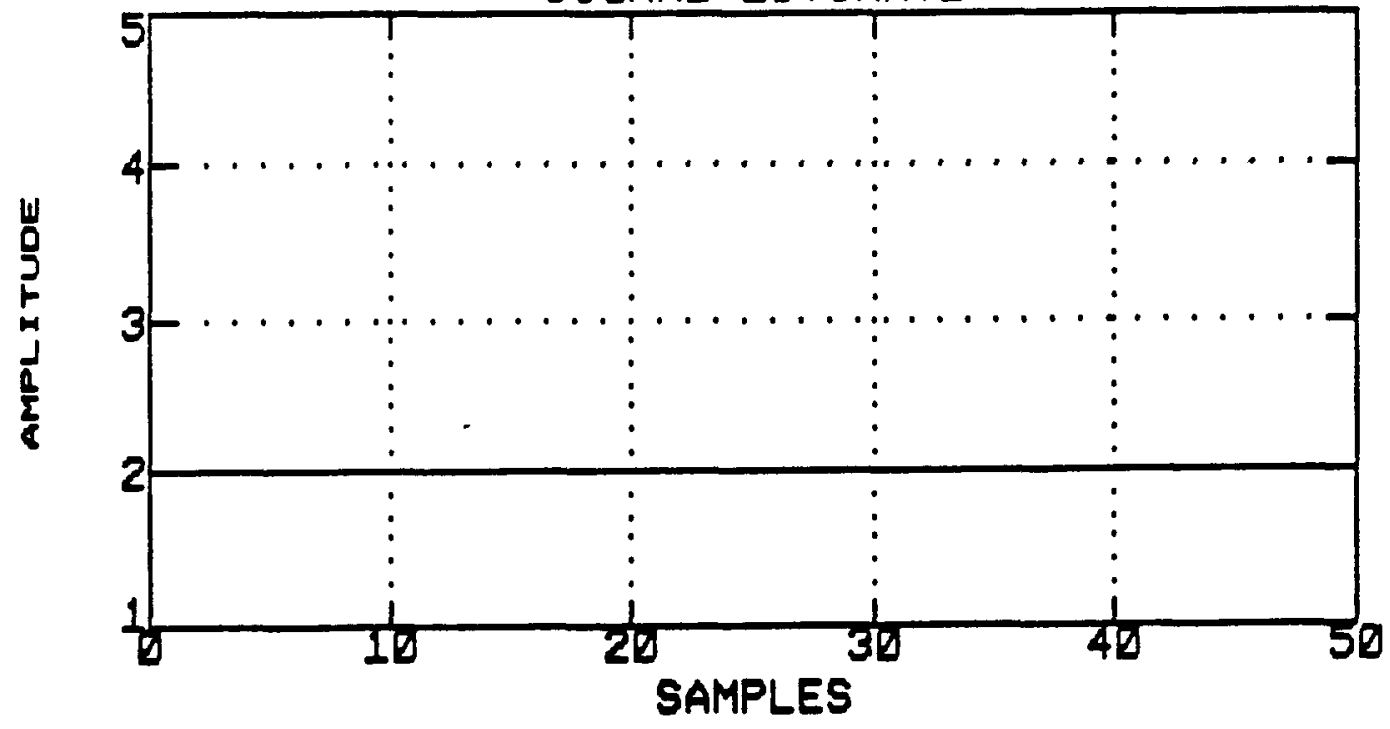

Figure 3.8b. Estimate of variable No. 2 based on the weighted average of its four redundant measurements. 


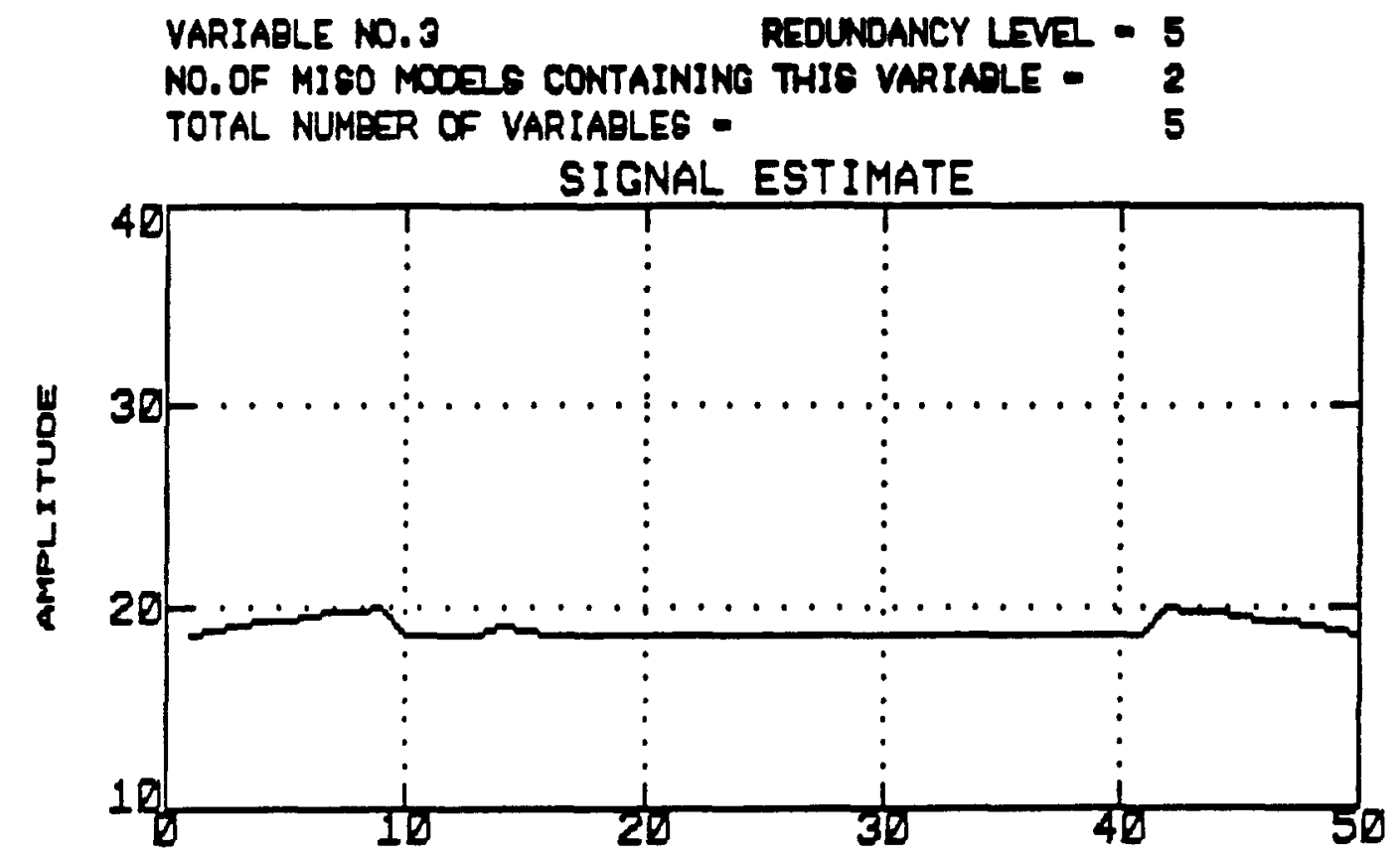

Figure 3.8c. Estimate of variable No. 3. In the range 1-9 samples and in the range 42-50 samples, the estimate is based on the five redundant measurements of variable No. 3. In the range of samples 10-41, where all the five signals have degraded, the estimate is based on the MISO model prediction.

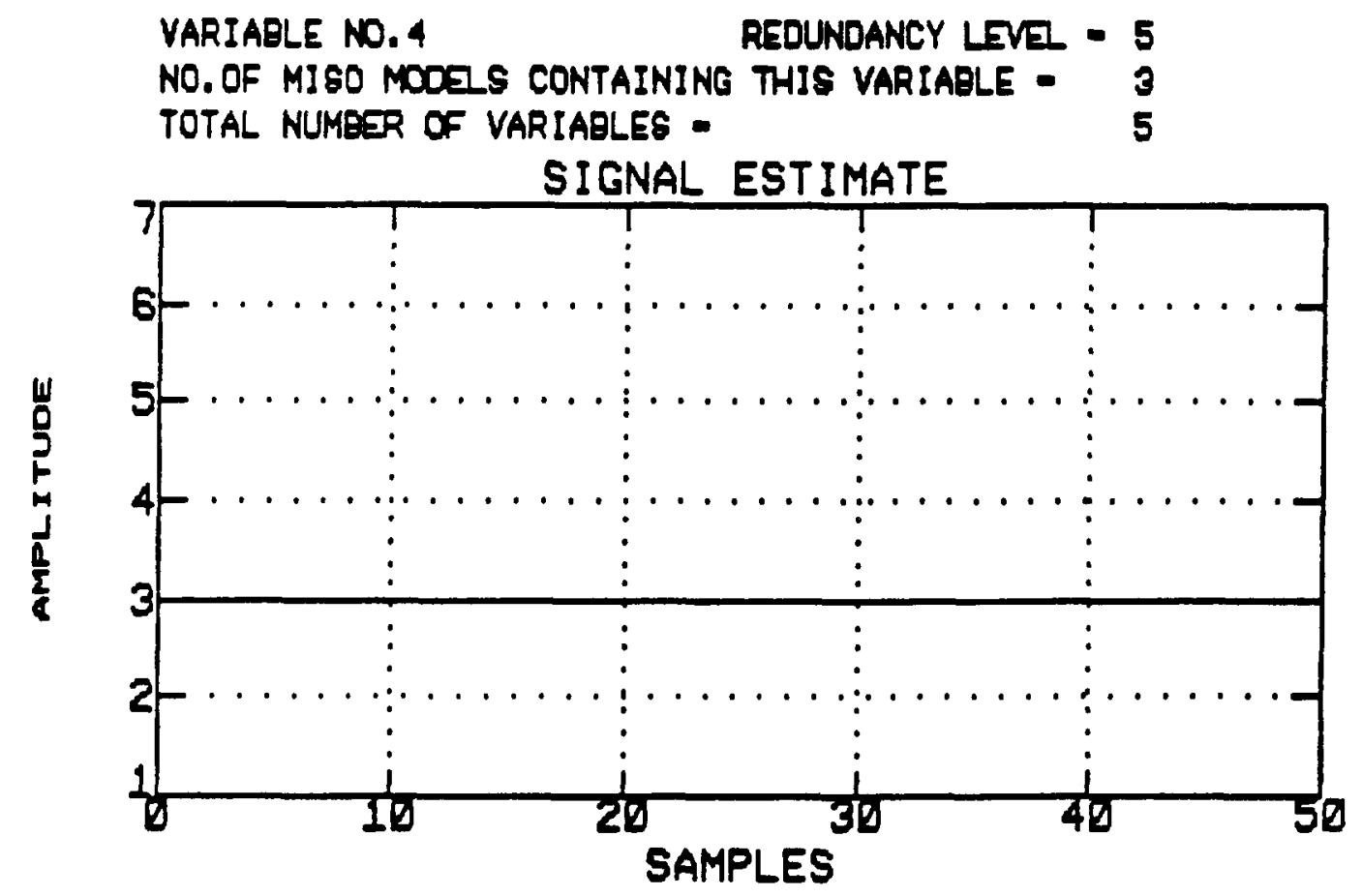

Figure 3.8d. Estimate of variable No. 4 based on the weighted average of its five redundant measurements. 


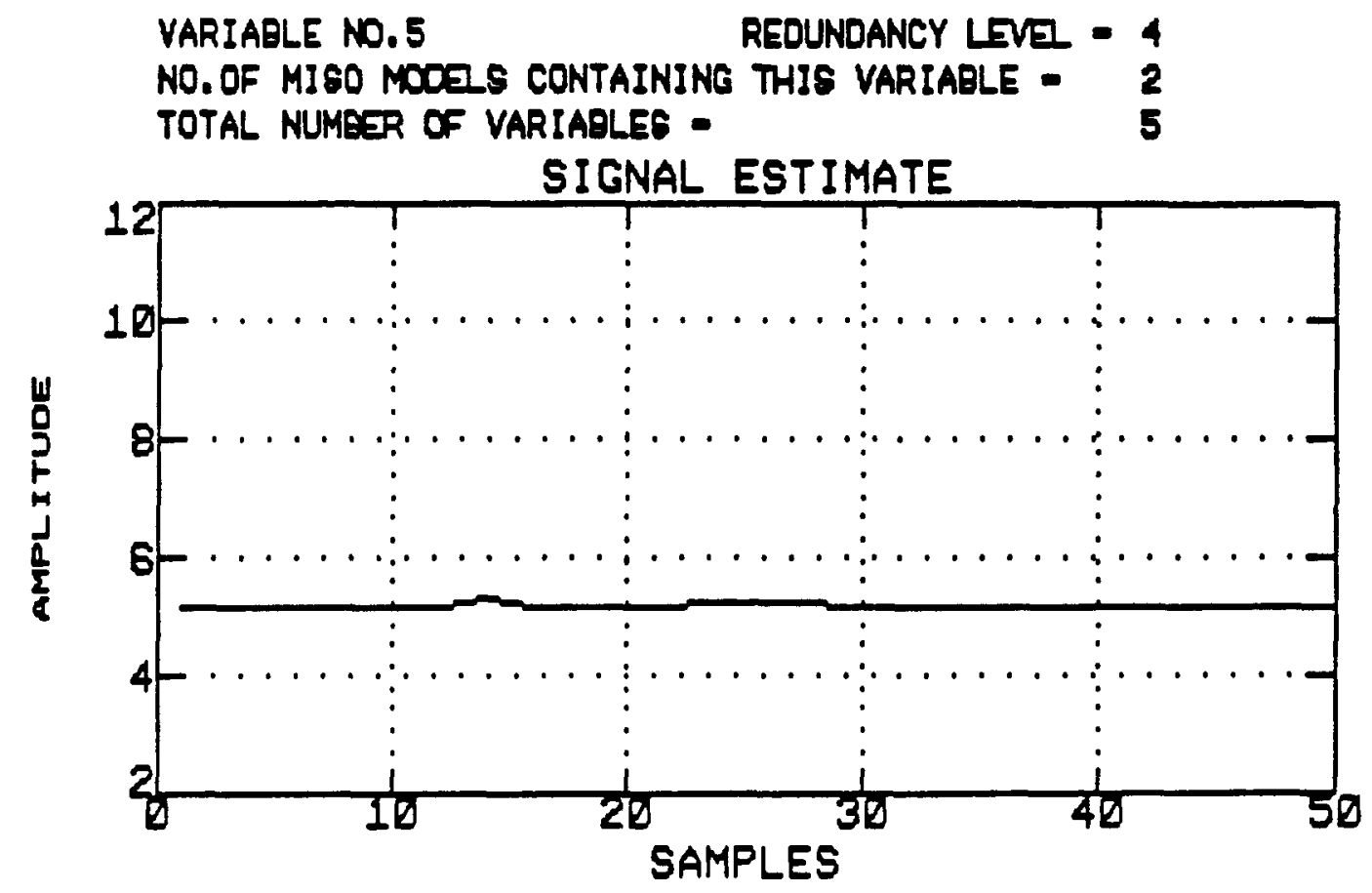

Figure 3.8e. Estimate of variable No. 5 based on the weighted average of its four redundant measurements. 


\section{Section 4}

\section{AUTOMA'TEI) GENERATION OF NONIINEAR SYSTEM CHARACTERIZATION}

\subsection{Objective of Process Modeling}

The prediction of process variables can be achieved either by physical modeling or by empirical modeling of a plant subsystem. We have developed an automated procedure for generating process empirical models (PEM) using operational data [10]. Independent prediction of critical signals is required for consistency checking of instrument outputs, for their degradation monitoring, and for isolating commonmode failures. Steady-state empirical models can be developed using data from different steady-state conditions, or using a large amount of data from the same steady-state operation. The latter requires sufficient variations in the signal levels. In actual applications it may be necessary to generate several empirical models, one for each operating regime. New results of application to PWR operational data is presented 111 .

\subsection{Outline of the Methodology}

The general form of the data-driven empirical modeling developed under this project is given by (see Fig. 4.1)

$$
y=C_{0}+\sum_{i=1}^{N} C_{1} \Phi_{i}(X)
$$

where $y$ is the variable to be predicted, $X=\left\{x_{1}, x_{2}, \ldots, x_{m}\right\}$ is the set of input variables that influences the behavior of $y$, and $\left\{C_{0}, C_{1}, \ldots, C_{N}\right\}$ is a set of constant coefficients. $\left\{\phi_{\mathrm{i}}, \mathrm{i}=1,2, \ldots, \mathrm{N}\right\}$ are nonlinear polynomial terms. Desrochers and Mohseni[12] developed a method of nonlinear polynomial fitting. We have adopted 
the general approach given in this paper and developed a new algorithm[13]. The following aspects of the development are our contribution to this approach.

(1) A complete software package to handle data, optimize model selection, and predict sensor output.

(2) Application to different industrial processes to determine the validity of the method.

(3) Choice of operating regions to determine best minimum-term models.

(4) A more accurate model by including a constant term in Eq. (3.1) and the coefficient estimation at the end of the overall optimization procedure.

Our study showed that some of the conclusions in Ref. [12] were not fully valid. It was claimed that the algorithm in Ref. [12] would generate the actual model of polynomial order $\mathrm{n}$, at the end of $\mathrm{N}$ steps. But a method need not exactly generate the actual model. The point is to obtain the best predictive estimation. For example, the optimal $(\mathrm{N}+1)$ term model may not include all the terms of the optimal $\mathrm{N}$-term model. Computing the $\left\{\mathrm{C}_{\mathrm{i}}\right\}$ sequence as the model terms are selected will not result in the same sequence if it is computed at the end of optimization.

The analytical measurement or prediction of a critical signal $y$ as a function of related variables in a subsystem, during steady-state or quasi steady-state operating condition, is given by

$$
y=f(X)=f\left(x_{1}, x_{2}, \ldots, x_{m}\right)
$$

This is shown schematically in Fig. 3.1. No assumption is made that the variables $\mathrm{X}$ are independent of $y$.

Our objective is to generate a minimum-term, optimal model from measurements $\left(y_{1}, y_{2}, \ldots, y_{L}, X(1), X(2), \ldots, X(L)\right)$, where $L$ is the number of data points. The PEM module algorithm generates optimal minimum-term nonlinear 
models such that the prediction error is a minimum for a specified maximum polynomial of order $n$ of $\phi_{i}(X)$. The algorithm performs optimal model selection by scanning optimal models of all polynomial orders $r \leq n$. The best model is then selected from this set. The algorithm is fully automated with minimum user interface.

We will present a detailed algorithm to determine an optimal model and consider the following aspects.

(1) Selection of the polynomial order and the number of terms $\mathrm{N}$ in the empirical model.

(2) Scaling of variables in order to avoid numerical instability.

(3) Error propagation due to the fitting procedure and measurement uncertainties.

\subsection{The Nonlinear Modeling Algorithm}

The general nonlinear steady-state system predictor has the form

$$
y=C_{0}+\sum_{i=1}^{N} C_{i} \phi_{i}(X)
$$

After choosing the functionals $\phi_{i}(X)$, the coefficients $\left(C_{0}, C_{1}, \ldots, C_{N}\right)$ are calculated using the least-squares procedure. Each $\phi_{i}$ is a nonlinear term or cross product of the components of $X$. The number of possible cross product terms is a function of the polynomial order and the number of components in X. In order to handle the nonlinear models, and to reduce the number of terms, or even the order of the model, several theories have been developed[14]. Among them the algorithm proposed by Desrochers and Mohsenil 12l is found to be useful for our applications. Their algorithm is not generally optimal as stated in Ref. [12]. 
We want to determine a minimum-term fit of the form of Eq. (4.4), without evaluating all possible cross product terms. The basic principle of the algorithm is given below. Let $L$ be the number of steady-state measurements of $y$ and $X$, and $M$ be the number of possible cross products. Using each cross product we can calculate $M$ vectors, each of dimension $\mathrm{L}$.

$$
\mathrm{V}(\mathrm{i})=\left\{\phi_{\mathrm{i}}(\mathrm{X}(\mathrm{k}))\right\}
$$

$\mathrm{i}=1,2, \ldots, \mathrm{M} ; \mathrm{k}=1,2, \ldots, \mathrm{L} . \mathrm{X}(\mathrm{k})$ is the measurement at time $\mathrm{k} . \Phi_{\mathrm{i}}$ is the $\mathrm{i}$-th cross product. Thus, we have an L-dimensional Euclidean space, with $\mathbf{M}$ vectors in this space. In this space the scalar signal y is represented as an L-dimensional vector. Our goal is to select $N$ vectors, $N<M$, to give an optimal fit in the leastsquares sense.

The computer algorithm follows the basic mathematical steps similar to those described in Ref. [12].

STEP 1. Determine the cross-product term closest to the vector $\mathrm{YL}$. This will provide us with one-term model. Calculate the projection matrices

$$
P(i)=\frac{\left[V^{J}(i)\right]\left[V^{j}(i)\right]^{T}}{\left[V^{j}(i)\right]^{T}\left[V^{j}(i)\right\rfloor}, i=1,2, \ldots M
$$

where $\mathrm{j}$ is the loop counter.

STEP 2. Project the actual output vector YL to different directions and calculate the scalar length.

$$
Y L_{(i)}=P(i) Y L j
$$

and

$$
R(i)=[Y L j(i)]^{T}[Y L j(i)]
$$


STEP 3. Find the largest scalar length from among R(i) and identify the cross product term based on the largest scalar length as the next term in the model.

STEP 4. If we need more terms, project all the remaining vectors onto the subspace orthogonal to the vector we have selected. $\mathrm{YL}$ is also projected on to this subspace. The cross product term that best approximates the error in $\mathrm{YL}_{\mathrm{L}}$ is selected as above. Calculate the projection matrix corresponding to the selected term, and project the remaining vector onto the space orthogonal to the vector selected previously.

$$
P_{(u k)}=\frac{\left[V^{j}(i k)\right]\left[V^{j}(i k)\right]^{T}}{\left[V^{j}(i k)\right]^{T}\left[V^{j}(i k)\right]}
$$

and

$$
\begin{gathered}
V^{j+1(i)}=M^{j} V^{j}(i), i=1,2, \ldots, M \\
i \neq i \ell, \ell=1,2, \ldots, j .
\end{gathered}
$$

where

$$
M^{j}=1-\sum_{\ell=1}^{j} P\left(i_{\ell}\right)
$$

STEP 5. Project the output vector on to this subspace, check its length and select the next best term.

$$
Y^{L}+1(i)=M^{j} Y^{L j}(i)
$$

Determine the error. Stop, or go to STEP 1.

Once the polynomial terms are selected the coefficients $\left(\mathrm{C}_{0}, \mathrm{C}_{1}, \ldots, \mathrm{C}_{\mathrm{N}}\right)$ are determined from the linear least-squares solution

$$
\left(\mathrm{C}_{0}, \mathrm{C}_{1}, \ldots, \mathrm{C}_{\mathrm{N}}\right) \sum_{\mathrm{k}=1}^{\mathrm{M}}\left[\mathrm{y}(\mathrm{k})-\mathrm{C}_{0}-\sum_{\mathrm{i}=1}^{\mathrm{N}} \mathrm{C}_{\mathrm{i}} \phi_{\mathrm{i}}(\mathrm{X}(\mathrm{k}))\right)^{2}
$$


$\underline{\text { Remarks: }}$

1. The order in which the terms $\phi_{\mathrm{i}}(\mathrm{X})$ are selected does not influence the final modeling error.

2. The optimal N-term model is achieved without testing all possible polynomial terms for a given maximum polynomial order $\mathrm{n}$, and for a given number of signals.

3. A signal $x_{i}$ may be excluded from the model if its influence on signal $y$ is negligible.

A complete software system has been developed and is implemented in an IBMPC (or compatible computer) and in the VAX workstation.

\subsection{Error Analysis}

An error analysis of output prediction is developed, and includes both modeling error and measurement error. For a given model fit, the overall fractional prediction error, $\varepsilon_{\mathrm{p}}$, is defined by

$$
\dddot{v}_{p}=\frac{1}{l} \sum_{k=1}^{L}\left|\frac{y_{i n}(k)-y_{p}(k)}{y_{p}(k)}\right|^{2}
$$

where

$$
\begin{aligned}
& y_{m}(k)=\text { measured value of } y \text { at time instant } k \\
& y_{p}(k)=\text { predicted value of } y \text { at time instant } k \\
& L
\end{aligned}
$$

The actual standard deviation of the prediction error for a given time instant $k$ is then estimated as

$$
o_{\mathrm{pk}}=\varepsilon_{\mathrm{p}} \cdot \mathrm{y}_{\mathrm{p}}(\mathrm{k})
$$


The measurement error is estimated as follows. Consider the functional form

$$
y=f\left(x_{1}, x_{2}, \ldots, x_{m}\right)
$$

The first variation of $\mathrm{y}$ at time instant $\mathrm{k}$ is given by

$$
\delta y(k)=\sum_{i=1}^{m} \frac{\partial f}{\partial x_{i}} \delta x_{i}(k)
$$

Assuming that the variations are uncorrelated, the variance of $y$ due to uncertainties in $\left\{x_{i}\right\}$ is estimated as

$$
\delta y^{2}=\sum_{i=1}^{m}\left|\frac{\partial f}{\partial x_{i}}\right|_{X(k)}^{2} \partial x_{i}^{2}
$$

Thus the measurement error standard deviation is given by

$$
o_{m h}-\left\{\sum_{i=1}^{m}\left|\frac{\partial f}{\partial x_{i}}\right|^{2} x(k) o_{i k}^{2}\right\}^{\frac{1}{2}}
$$

where $\mathrm{k}$ denotes the time instant $\mathrm{k}$, and $\left\{\sigma_{\mathrm{ik}}\right\}$ is the measurement error of $\mathrm{x}_{\mathrm{i}}$ at time instant $k$. Then the total error is given by

$$
\sigma_{T k}=\sigma_{p k}+\sigma_{m k}
$$

\subsection{Results of Application to PWR Operational I)ata}

About 2000 minutes of data, during startup of a typical Westinghouse four-loop PWR, were acquired. The data base consists of the following signals.

Description

Power range monitors

Pressurizer level

Pressurizer pressure

Reactor coolant system flow rate

( 3 signals from each loop)
Number of Signals

4

3

3

12 
RCS hot leg temperature $\quad 4$

RCS cold leg temperature 4

Steam generator feedwater flow rate $\quad 8$

(2 signals from each loop)

$\begin{array}{ll}\text { Steam generator level } & 16\end{array}$

(3 narrow range and 1 wide range from each loop)

$\begin{array}{ll}\text { Steam generator pressure } & 12\end{array}$

( 3 from each loop)

The process empirical modeling approach has been used as an independent and diverse mthod for estimating one or more process variables. This estimate may be compared with direct sensor redundancy. The GCCM module utilizes this estimate extensively for comparison to isolate common-mode failures and to identify simultaneous failure of several instrument channels. Four examples of empirical modeling are summarized here. For a description of data see Sect. 3.

\section{Application 1}

Four-loop Westinghouse PWR (start-up data)

Reactor Power $=f\left(\right.$ cold leg temperature $\left(x_{1}\right)$, hot leg temperature $\left.\left(\mathbf{x}_{2}\right)\right)$.

$$
\begin{array}{ll}
\text { Power }(\%)=\quad & 18178.25+0.002544 \mathrm{x}_{2}^{2}-64.498 \mathrm{x}_{1} \\
& +0.056761 \mathrm{x}_{1}^{2}-1.17943 \mathrm{x}_{2} .
\end{array}
$$

Figure 4.3 shows the comparison between the measured signal and the predicted value of power and has an average error of $0.56 \%$. The reactor coolant system flow rate is constant during the start-up, and does not directly contribute to the empirical model; hence this signal is excluded from the input signal set. 


\section{Application 2}

Four-loop Westinghouse PWR (start-up data)

Pressurizer level $=\mathbf{f}\left(\right.$ Reactor Power $\left(\mathrm{x}_{1}\right)$, Pressurizer Pressure $\left(\mathbf{x}_{2}\right)$,

Cold leg temperature $\left(\mathrm{x}_{3}\right)$,

hot leg temperature $\left.\left(\mathbf{x}_{4}\right)\right)$

Pressurizer level $(\%)=-722.978+0.736539 \mathrm{x}_{4}$

$$
\begin{aligned}
& -0.068457 \mathrm{x}_{1}-0.008616 \mathrm{x}_{2} \\
& +0.640089 \mathrm{x}_{3} .
\end{aligned}
$$

Figure 4.4 shows the comparison between measured signal and the predicted value of pressurizer level with an average error of $0.34 \%$. Note that charging pump flow, spray flow and let-down flow are not considered here (were not available) and are assumed to be constant in this case.

\subsection{Results of Application to EBR-II Start-Up Data}

\section{Application 1}

Experimental Breeder Reactor-II (start-up data)

Reactor Power $=\mathbf{f}\left(\right.$ Inlet Plenum Temp $\left(\mathbf{x}_{1}\right)$, Upper Plenum Temp ( $\left.\mathbf{x}_{2}\right)$, Secondary Sodium Flow Rate $\left.\left(\mathbf{x}_{3}\right)\right)$.

Power $(\%)=\quad 297.817+0.184929 x_{3}-0.081698 x_{1}$

$+0.506694 x_{2}$.

Figure 4.5 shows the comparison between the measured signal and the predicted value of power with an average error of $1.09 \%$ 


\section{Application 2}

Experimental Breeder Reactor-II (start-up data)

IHX Secondary Sodium Outlet Temperature $=$

f(Upper Plenum Temp ( $\left.\mathbf{x}_{1}\right)$, IHX Primary Na Outlet Temp $\left.\left(\mathbf{x}_{2}\right)\right)$

IHX Secondary Sodium Outlet Temperature $(\operatorname{deg} F)=$

$$
\begin{aligned}
& 4978.046+0.009978 x_{1} x_{2}-4.1883 x_{1} \\
& -0.001201 x_{1} 2-8.065 x_{2} .
\end{aligned}
$$

Figure 4.6 shows that the comparison between measured signal and the predicted value of intermediate heat exchanger (IHX) secondary sodium outlet temperature with an average error of $0.81 \%$.

In all the above cases a single model is used to characterize the entire operating range (based on power). In general it may be necessary to have different models for different operating regimes. 


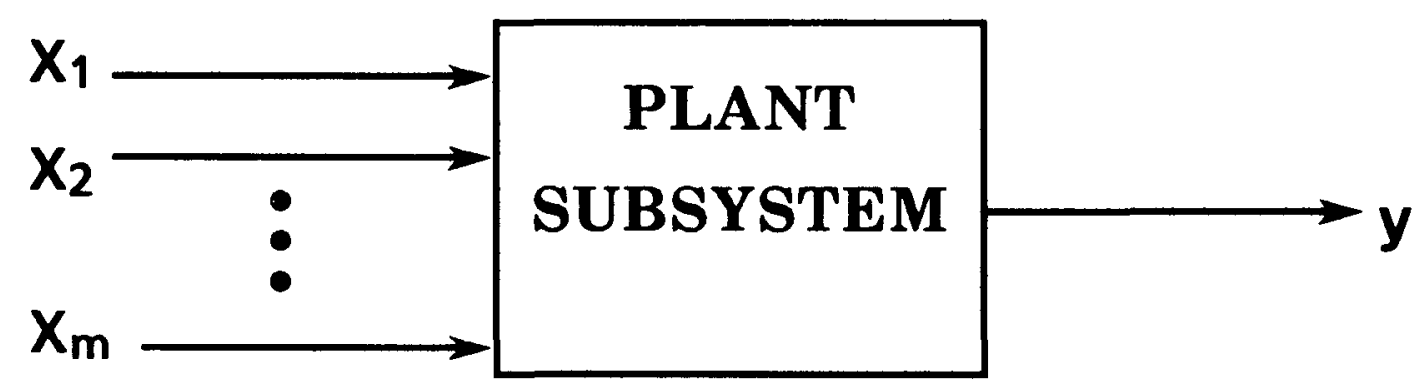

$$
y=c_{0}+\sum_{i=1}^{N} c_{i} o_{i}(x)
$$

Figure 4.1. Process empirical modeling showing input-output relationship. 


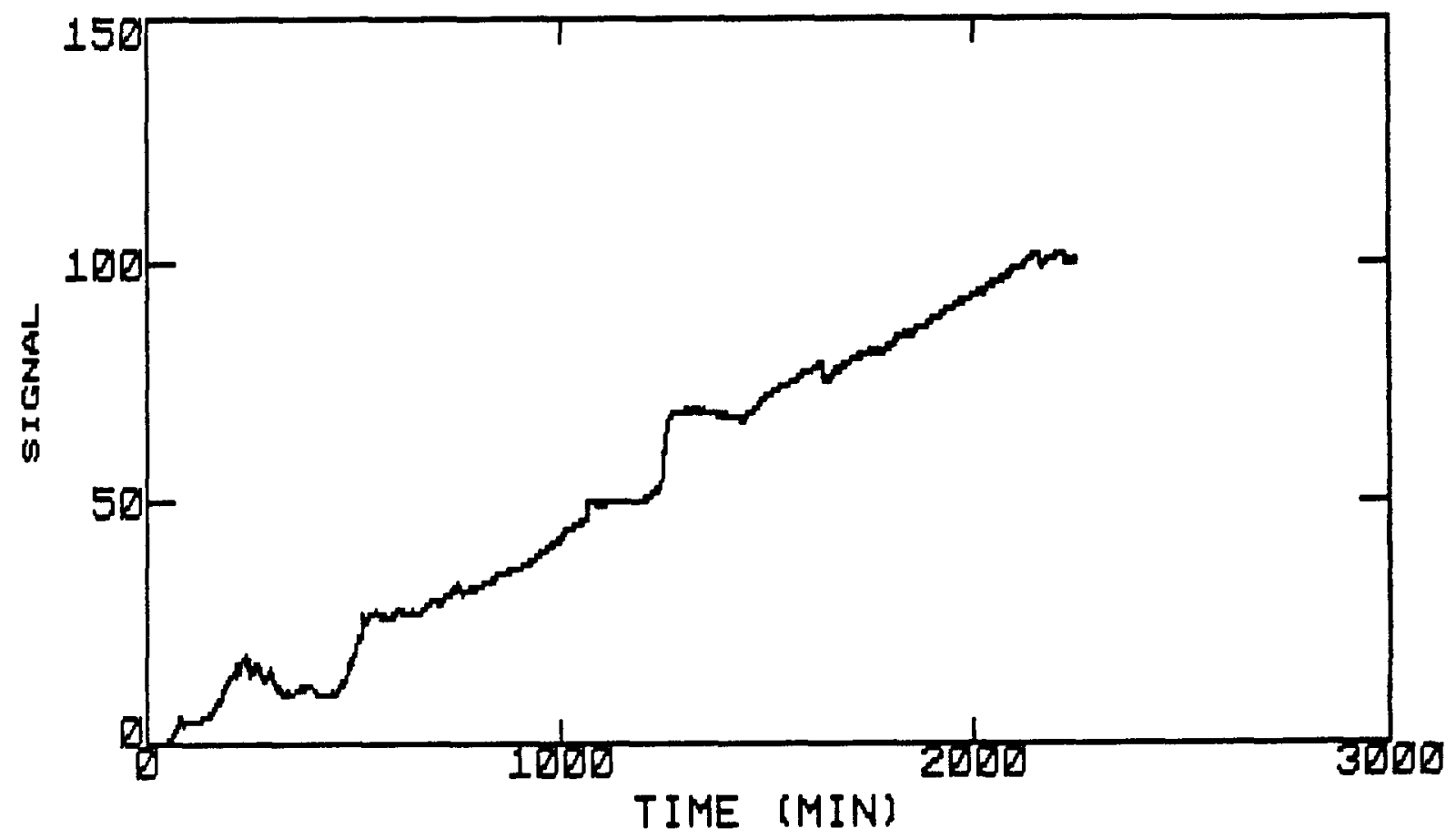

Figure 4.2a. Reactor power (\%) signal during start-up of a Westinghouse four-loop PWR. 


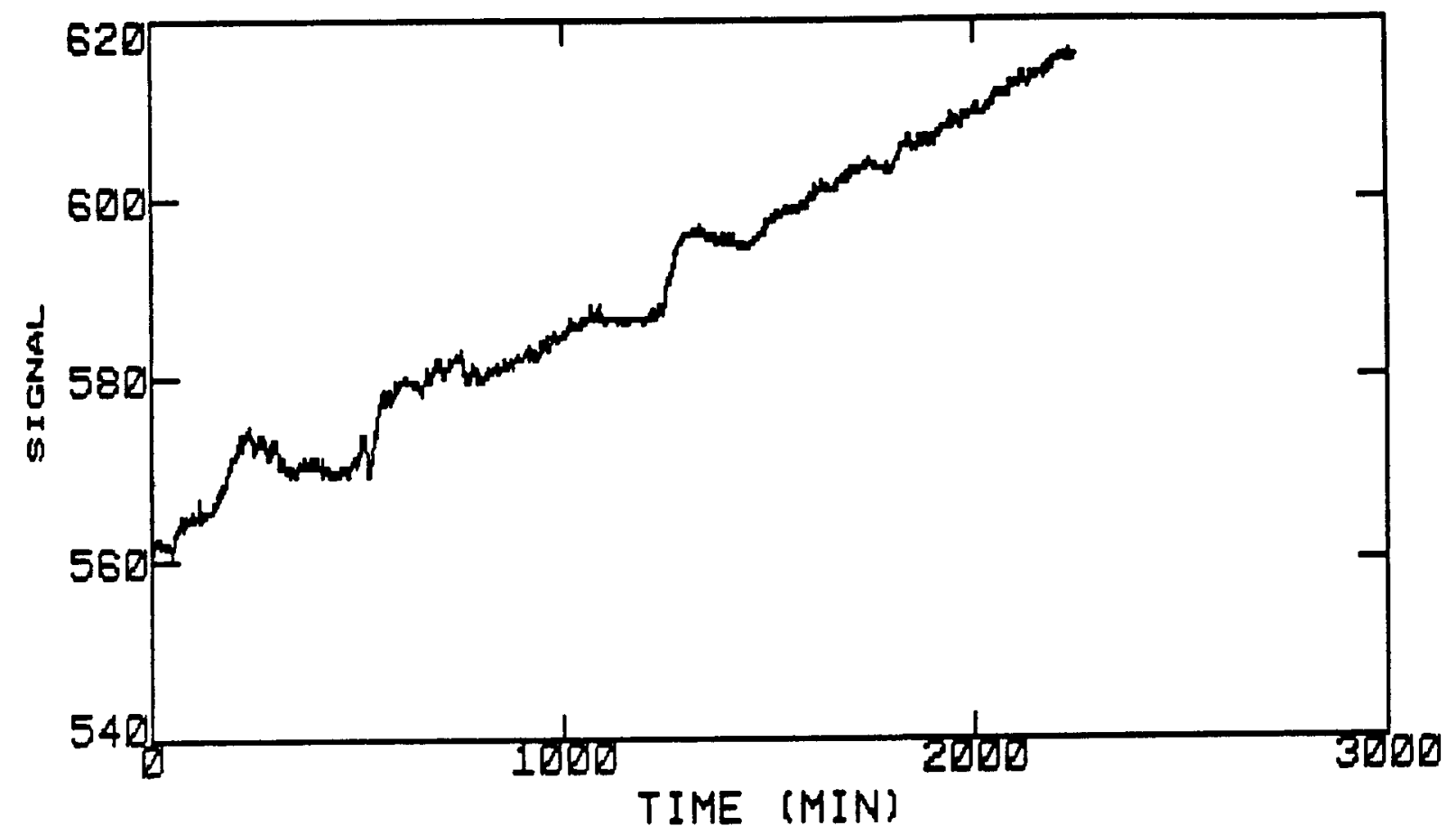

Figure 4.2b. Hot leg temperature (F) signal during start-up of a Westinghouse four-loop PWR.

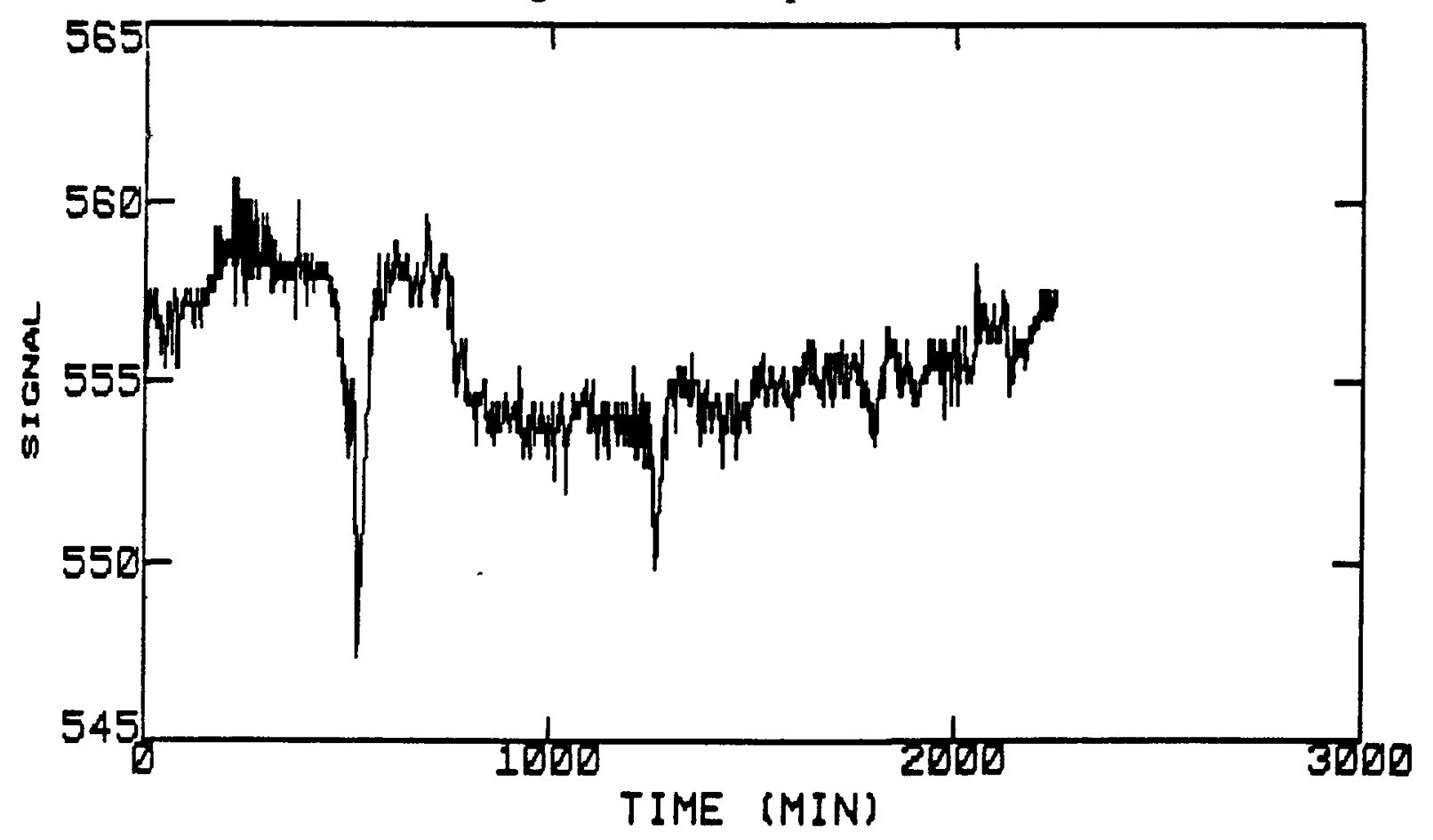

Figure 4.2c. Cold leg temperature (F) signal during start-up of a Westinghouse four-loop PWR. 


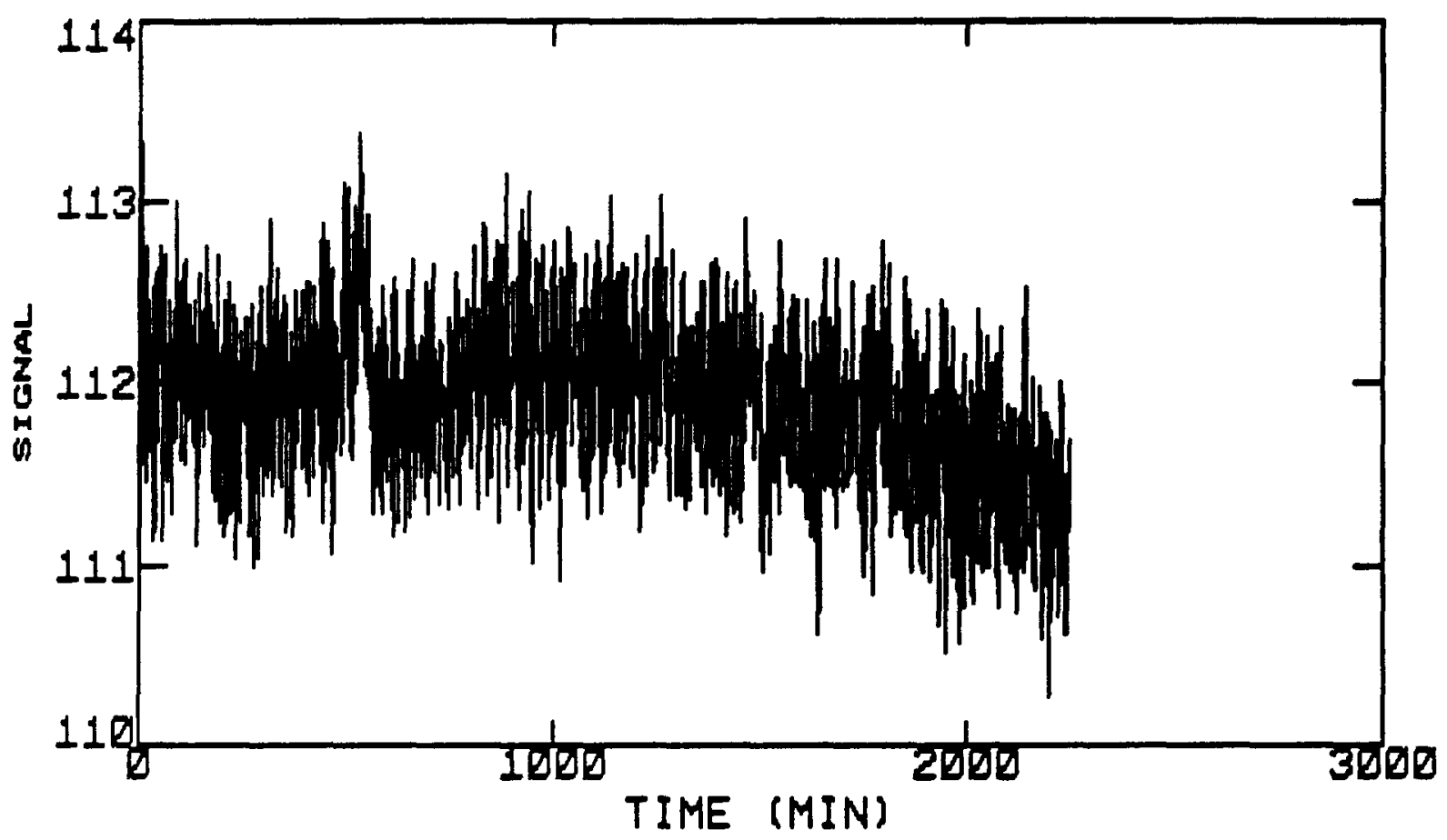

Figure 4.2d. Reactor coolant system flowrate (gpmx103) signal during start-up of a Westinghouse four-loop PWR.

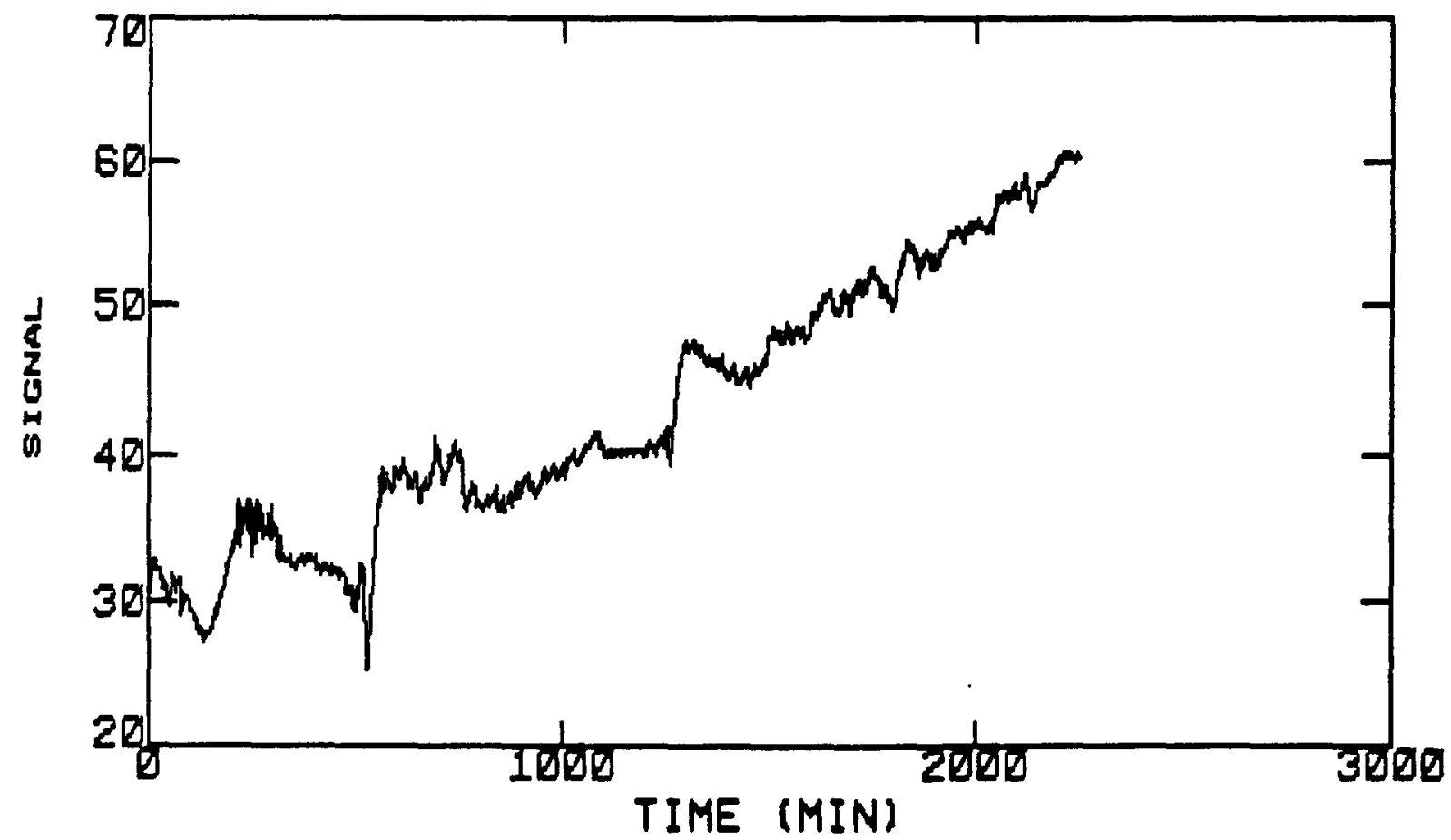

Figure 4.2e. Pressurizer level signal during start-up of a Westinghouse four-loop PWR. (\%) 


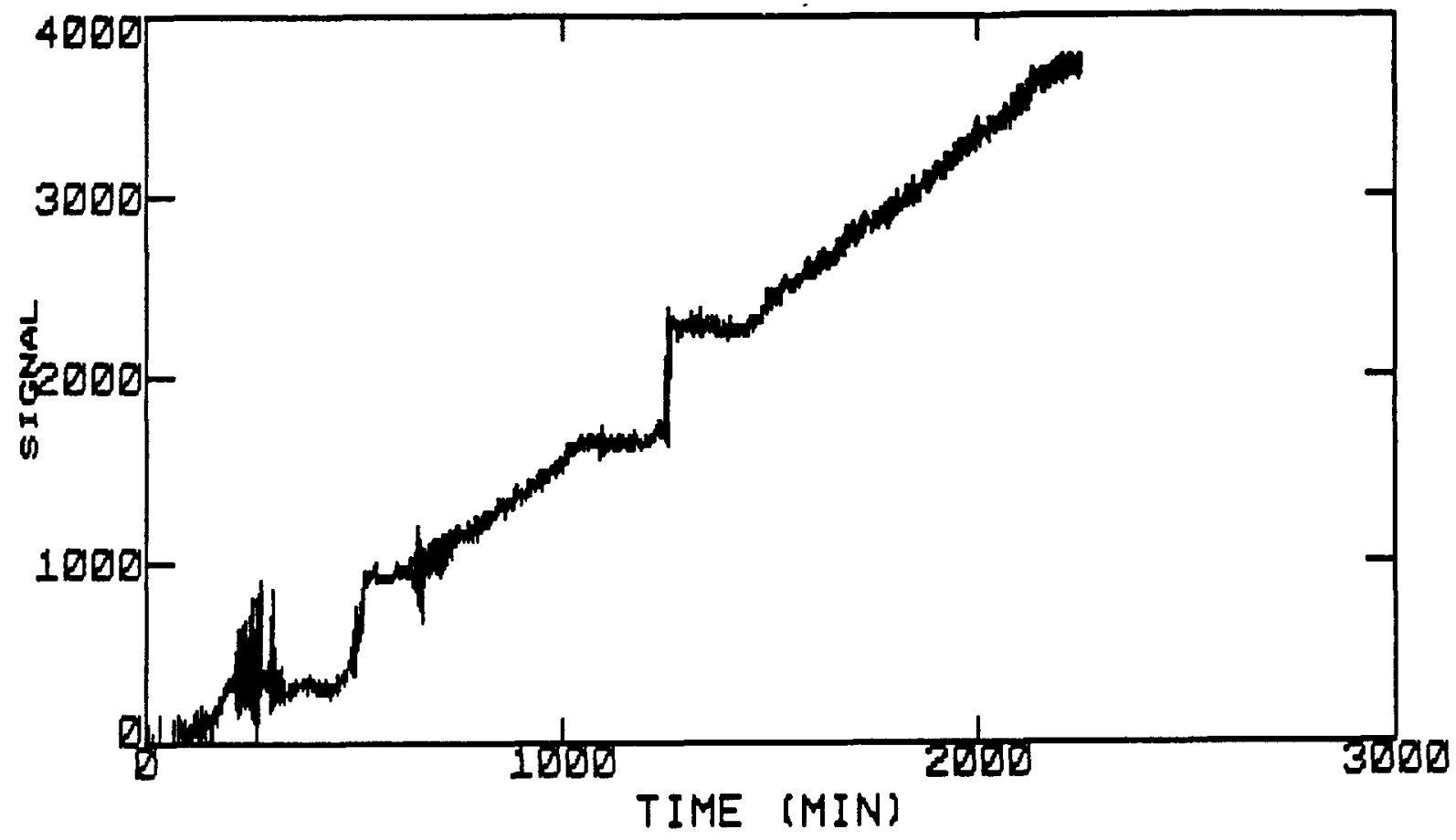

Figure 4.2f. Steam generator feedwater flowrate $\left(\mathrm{lbm} / \mathrm{hr} \times 10^{3}\right)$ signal during start-up of a Westinghouse four-loop PWR.

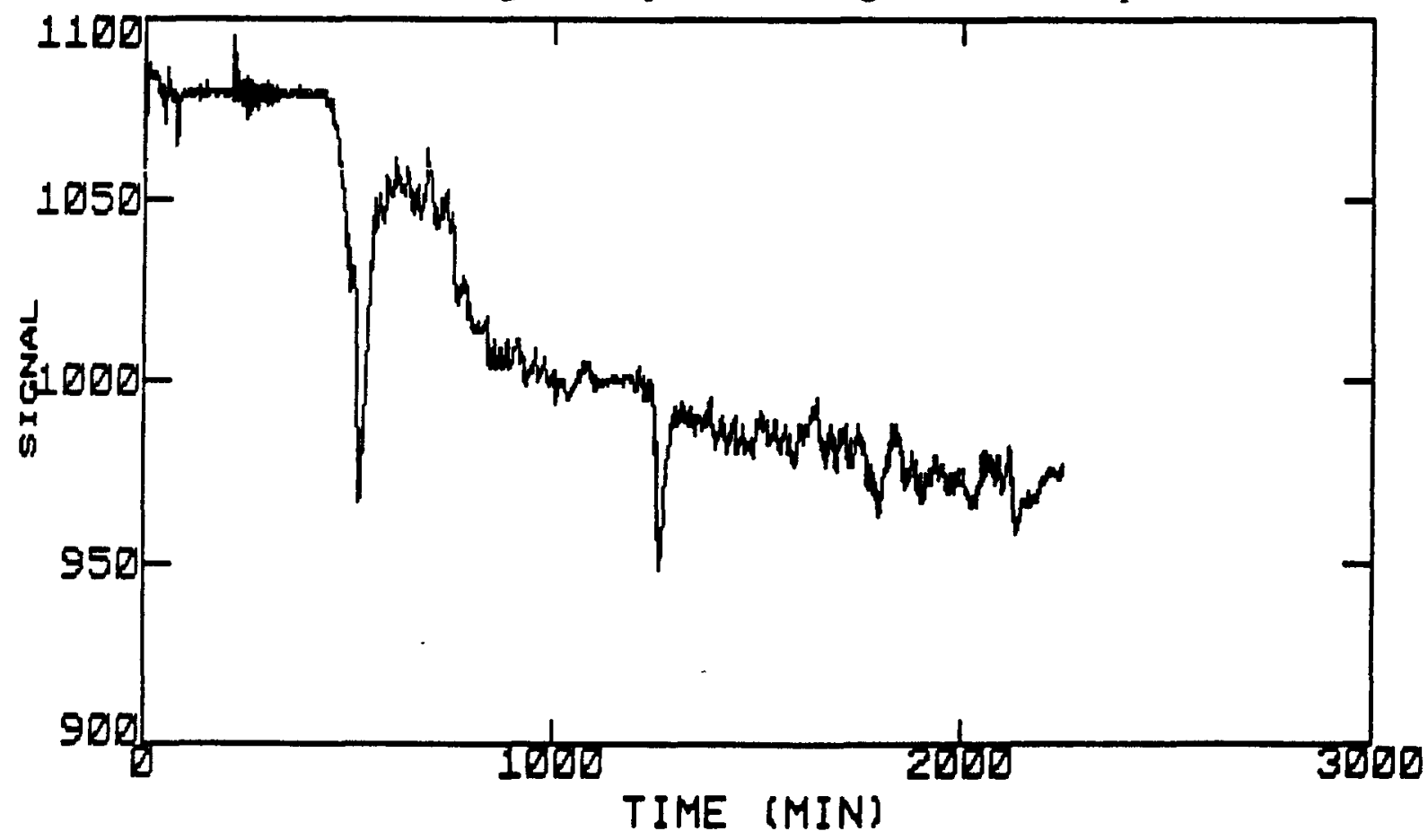

Figure 4.2g. Steam pressure (lb/in2) signal during start-up of a Westinghouse four-loop PWR. (\%) 


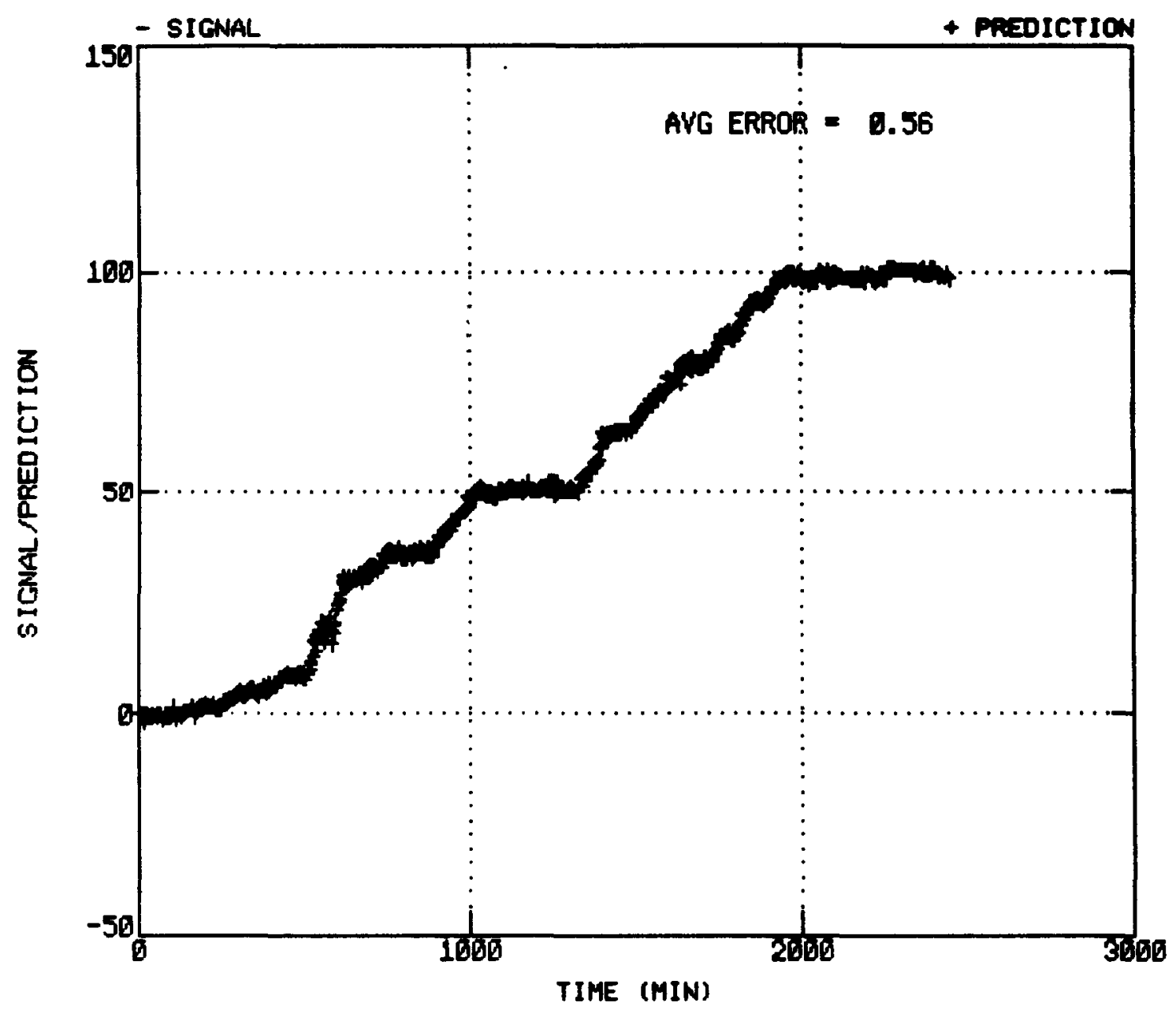

Figure 4.3. Cumparison of Measured and Predicted Reactor Power Signal in a PWR. 


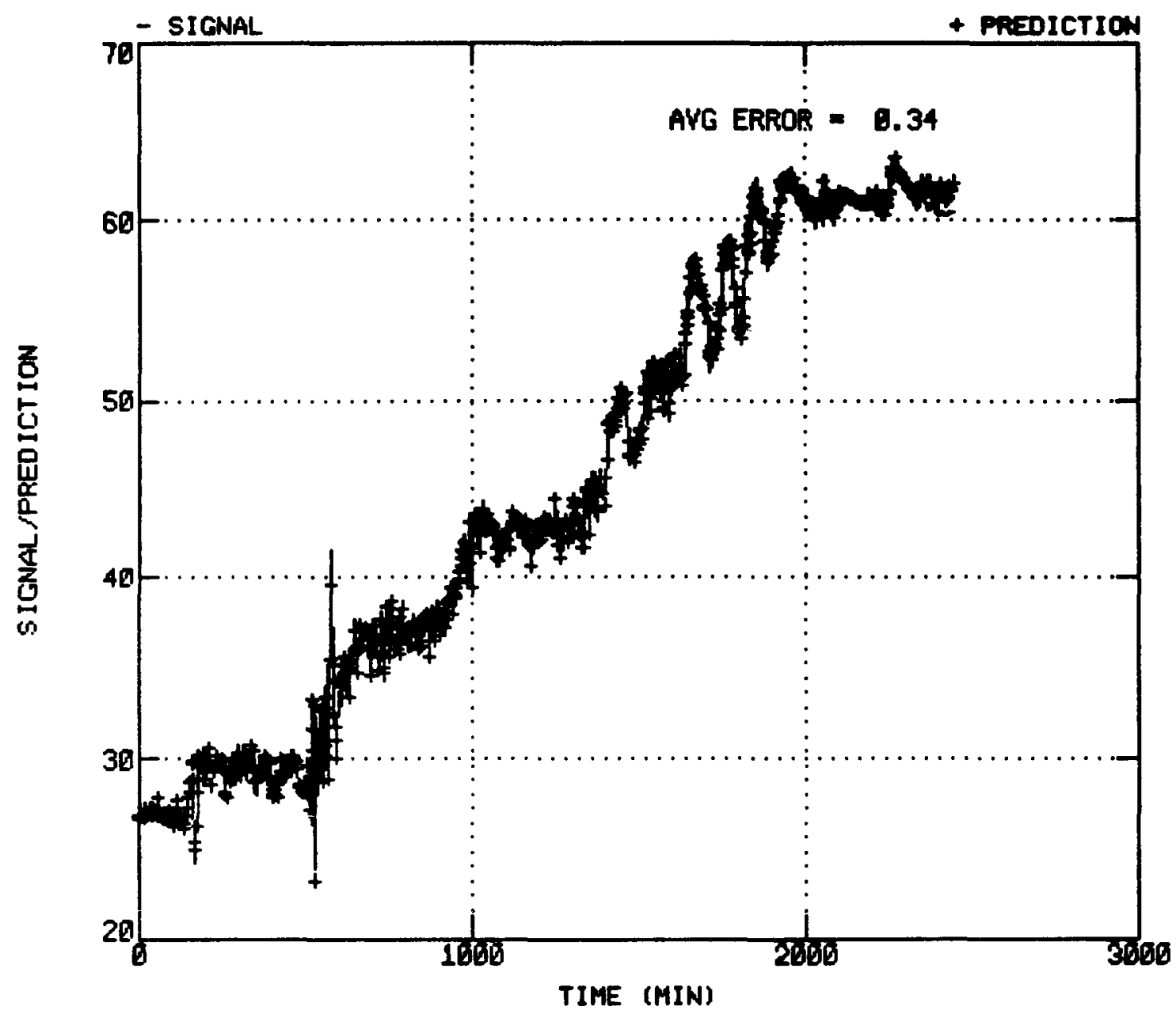

Figure 4.4. Comparison of Measured and Predicted Pressurizer Level Signal in a PWR. 


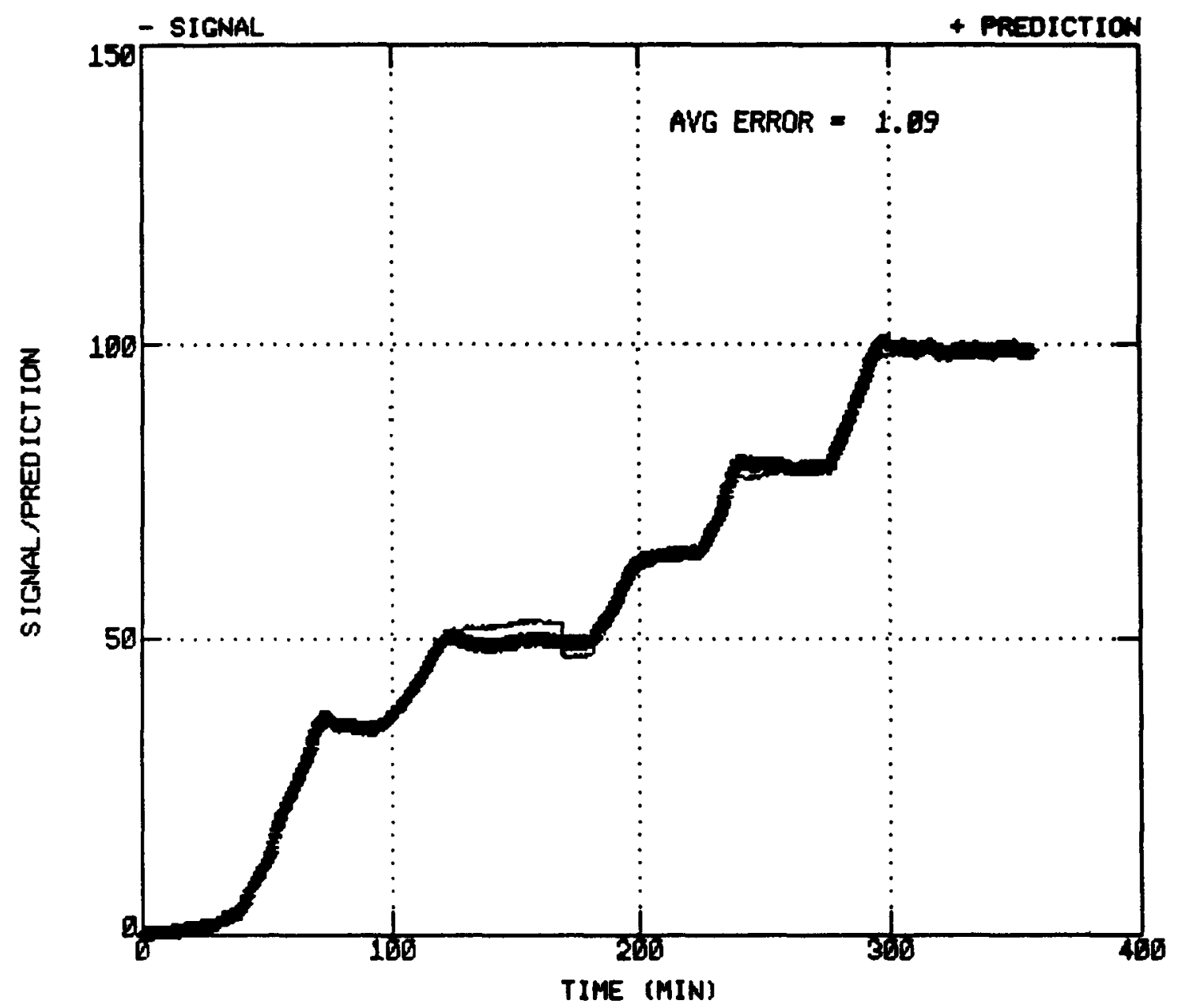

Figure 4.5. Comparison of Measured and Predicted Power Signal in the EBR-II. 


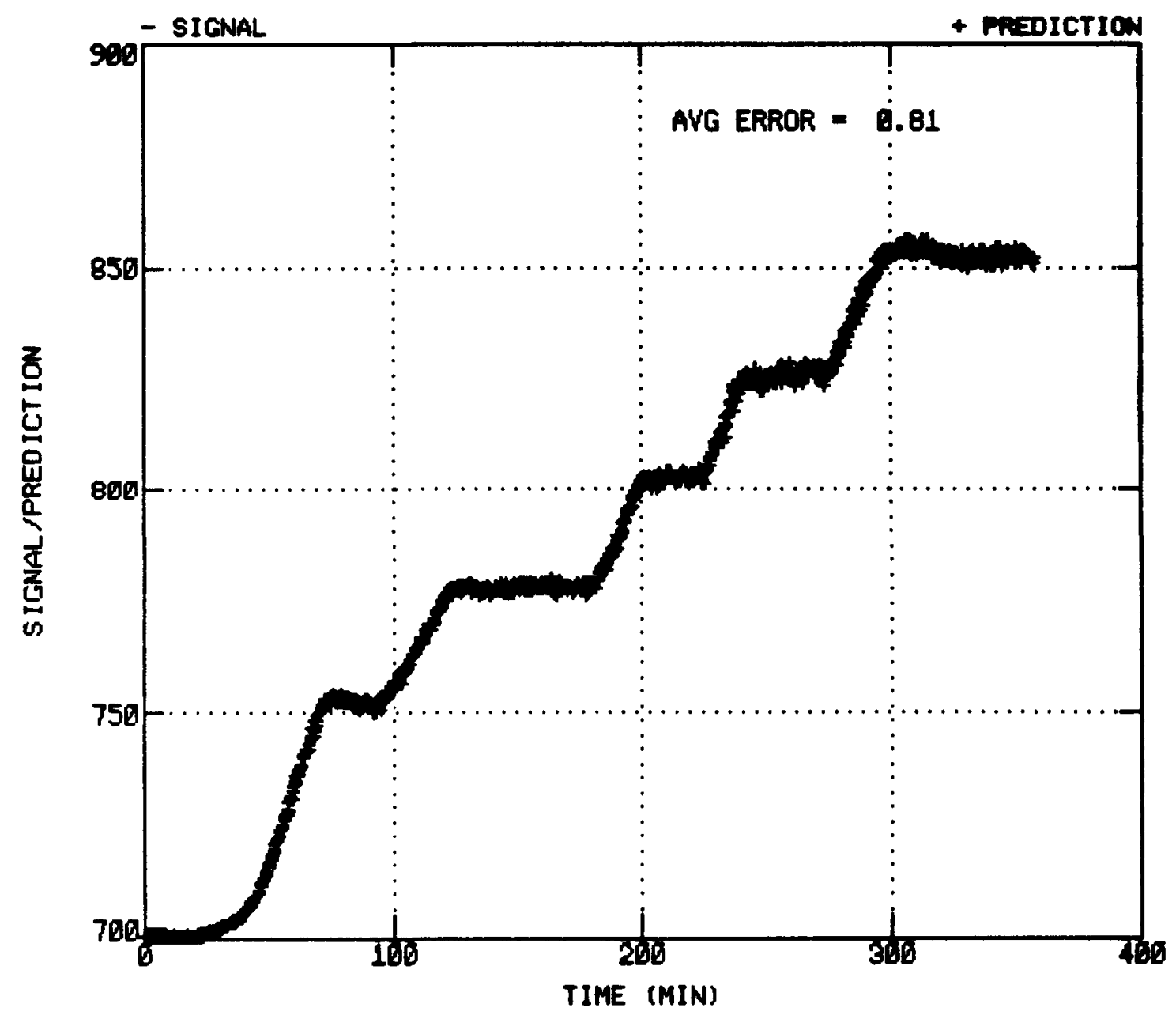

Figure 4.6. Comparison of Measured and Predicted IHX Secondary Outlet Temperature Signal in the EBR-II. 


\section{Section 5}

\section{MUITI-DIMENSIONAL PROCESS HYPERCUBE FOR SIGNAL VALIDATION}

\subsection{Introduction}

This section describes the development of a new technique for performing signal validation and process monitoring. The technique, called the process hypercube comparison (PHC), learns the valid operating states of a process to which newly observed data are compared and faulty signals identified. The application of the hypercube signal validation technique to operational data from a commercial pressurized water reactor (PWR) and the Experimental Breeder Reactor I (EBR-II) is also reported $|13|$.

\subsection{Process Hypercube Comparison (PHC)}

A new methodology for signal validation was originated during this research. This new technique basically "learns" the operating states of a system. Once learned, subsequently observed process states are compared against the learned domain. The states having prior existence are declared normal and the signals deemed valid. An evaluation is made on those states which have not been previously observed to identify the abnormal signal(s). This methodology, termed the process hypercube comparison ( $\mathrm{PHC}$ ), drives its name from the fact that the learned process states are stored in a hypercube data structure.

\subsubsection{Basis of Approach}

Separation of process changes and signal changes constitutes a significant obstacle to reliable signal validation. That is, process changes can easily "trick" certain methodologies into a determination of signal failure (or non-failure). An ideal method for signal validation would be a detailed model of the process (plant) whereby a determination could be made as to whether the indicated process conditions, as well as the operating path taken, constitute a valid operating regime 
or not. However, this ideal method is neither feasible nor realistic. An approximation to the ideal process qualifier can, however, be developed. Rather than model the process to determine the valid operating conditions, the process measurements will provide a definition of the valid operating regime.

The concept behind signal validation using the $\mathrm{PHC}$ is somewhat similar to an empirical modeling approach. However, rather than model one process variable using a selected group of related process variables, the PHC makes a global (plantwide) comparison of the process variables. Those conditions judged normal are retained for future reference. A determination can be made as to the normality of future conditions (and the validity of the corresponding signals providing the conditions) based upon this data base of values.

\subsubsection{Hypercube Creation}

A hypercube data structure is used to create a history of the valid process conditions. The hypercube is just an efficient method for data storage and compression. In essence the hypercube contains a multi-dimensional histogram of the process conditions. This hypercube data storage is a generic method which could be applied to any type process such as the human body, the financial market or an automobile engine.

The first task is the creation and population of the hypercube. The creation of the hypercube must be a continuously ongoing procedure. The addition of new normal operating conditiuns must be a continuous process since the plant may be operating at $100 \%$ power for some time after which the plant is reduced in power to $90 \%$. This is definitely a valid operating condition but one which has not existed before. A data set which contains data points taken over the entire operating range is most useful for creating the hypercube. Research using plant start-up data has shown good results. 
The PHC is first trained off-line using data representing the given operating range. The hypercube is created and each sampled data point is stored. The

frequency of occurrence for each data point (process state) is updated. Those process states which appear infrequently may be removed by scanning the entire PHC and noting the frequency of each state. A flow chart of the hypercube creation phase is given in Figure 5.1.

\subsubsection{Hypercube Cell Size}

During the creation and utilization of the PHC, a prime consideration which must constantly be remembered is that there is a maximum number of potential hypercube cells which may be created. This is limited by the largest unsigned integer number which may be represented by a given computer that is, for a 32-bit machine: 4,294,967,295 cells. The following equation gives the potential number of hypercube memory cells needed given the total number of variables and the number of intervals for each variable.

$$
N_{v}=\prod_{i=1}^{N_{v}} N_{s} \text { (i) }
$$

where

$\mathrm{N}_{\mathrm{c}}$ = number of potential hypercube memory cells,

$\mathrm{N}_{\mathrm{v}}$ = number of process variables to store,

$\mathrm{N}_{\mathrm{s}}$ = number of intervals in each process variable. 


\section{Generic Example}

Assume that there are three variables to analyze $\left(N_{v}=3\right)$. Further, that there are 10, 5 and 8 intervals for the first, second and third variables respectively. The number of potential hypercube cells is

$$
N_{\mathrm{c}}=10 * 5 * 8=400
$$

The hypercube cell size (or resolution) is a function of the interval widths selected for each variable. One of the primary issues which must be addressed concerning the hypercube memory structure is the optimal width of the hypercube intervals. Small intervals describe the different operating conditions in more detail but require a larger number of potential hypercube cells. Once again, the potential number of cells assumes a limiting role.

\section{$\underline{\text { Nuclear Plant Example }}$}

Now as an example, consider that a hypercube is to be created for a nuclear power plant, specifically a PWR, based on the following process variables:
Hot Leg Temperature
Reactor Power
Cold Leg Temperature
Steam Pressure
Reactor Coolant Flow
Steam Flow
Reactor Coolant Pressure
Feedwater Temperature
Pressurizer Level
Feedwater Flow
Pressurizer Pressure
Steam Generator Level

This gives a total of twelve variables. Assume that each variable is divided into 2 intervals. This results in a total of 4096 cells. Now assume that there are 3 and 4 intervals for each variable; this yields a total of 531,441 and 16,777,216 cells, respectively. 
Redundant measurements therefore are reduced to a single value using a compact consistency checking scheme. This scheme is different from the generalized consistency checking (GCC)algorithm described in Sect. 2.. An inconsistency index is calculated using a distance measure based on the signal tolerance. This separation index is computed as

$$
s_{j}=\sum_{k \neq j}^{N} \frac{\left|m_{j}-m_{k}\right|}{\varepsilon}
$$

where

$$
\begin{aligned}
& \mathrm{S}_{j}=\text { inconsistency separation index for signal } \mathbf{j} \\
& \mathrm{m}_{\mathrm{j}}=\text { measured value for signal } \mathbf{j} \\
& \varepsilon=\text { tolerance ( } \leq \text { interval width) for the signals, } \\
& \mathrm{N}=\text { number of redundant signals in the group. }
\end{aligned}
$$

Separation indices are compared and the signals(s) with the largest inconsistency index are declared anomalous. The separation index is recomputed for the remaining signals. The procedure is repeated until either the separation index is zero or there are no signals.

Those signals with zero separation are averaged for an estimated value or the entire group of original signals is used in a weighted average to compute an estimate. The weighting factors are inversely proportional to their most recently computed inconsistency separation index. The weighted average is computed from

$$
\Theta=\frac{\sum_{j=1}^{N} m_{j} w_{j}}{\sum_{j=1}^{N} w_{j}}
$$


Figure 5.2 is a plot of the number of potential hypercube cells as a function of the number of variables and an equal number of intervals for each variable. One can quickly see that the number of variables and intervals must be minimized as much as possible without losing valuable information. With the large number of monitored process variables in a nuclear power plant, the number of cells in the hypercube can be extremely large as shown in the above example.

The number of variables and the cell resolution (width) must be considered along with the importance placed upon each of the process variables. The purpose of the signal validation also plays a role in this selection. That is, for detecting shifts in calibration, the interval width must be kept relatively small. For routine plant operation, intervals at infrequently-encountered power levels may be omitted. For signal validation during possible transient conditions, the intervals must cover the entire operating regime. An additional point to consider is that some variables, such as reactor coolant system (RCS) flow, RCS pressure and pressurizer pressure assume a nearly constant level from $0 \%$ to $100 \%$ power. Keeping the above limiting factors in mind, the variables to be validated are selected and the operating range over which a history is to be kept is chosen. Lastly, the resolution (width) of the intervals for each variable is selected.

\subsubsection{Redundant Signal Data Compression}

In the case where several redundant signals of a given variable exist, it may be infeasible to store each signal individually. Rather, a single estimate for the entire redundant signal group should be used. A check must be performed to ensure that the entire group lies within one interval width of each other. From this fact alone it is easy to see that the smallest interval width ever used need be no smaller than the signal tolerance. 
where

$\Theta=$ variable estimate for the signal group,

$\mathrm{m}_{\mathrm{j}}=$ measured value of signal $\mathrm{j}$,

$w_{j}=$ weighting factor of signal $j$,

$=1.0-\left(S_{j} / \Sigma S_{k}\right) \quad[j=1, \ldots N]$.

The variable estimates from each redundant group are then used to compute the location of the process state in the hypercube. The interval number for each variable is computed first from

$$
I_{k}=\frac{\Theta_{k}-a_{k}}{\delta_{k}}
$$

where

$I_{k}=$ interval number for variable $\mathrm{k}\left(\mathrm{I}_{\mathrm{k}}=0,1, \ldots \mathbf{M}_{\mathrm{k}}-1\right)$,

$\Theta_{\mathrm{k}}=$ variable $\mathrm{k}$ estimate,

$a_{k}=$ selected lower limit for variable $\mathbf{k}$,

$\delta_{k}=$ interval width for variable $\mathbf{k}$

$=\left(\beta_{k}-a_{k}\right) / M_{k}$,

$\beta_{\mathrm{k}}=$ selected upper limit for variable $\mathrm{k}$,

$\mathbf{M}_{\mathrm{k}}=$ maximum number of intervals for variable $\mathrm{k}$.

The key used to uniquely identify each process state is computed from

$$
\text { Key }=\sum_{k=1}^{N}\left|l_{k} * \prod_{j=0}^{k-1} M_{j}\right|
$$

with $M_{0}=1$. The use of the hypercube requires that only the occupied cells be stored. If the hypercube data compression were not used, storage of the process 
states could easily require 16 megabytes of memory. This is the motivating factor for using the hypercube rather than a simple multi-dimensional array.

\subsubsection{PHC Signal Validation}

The hypercube cannot only determine whether or not a particular process state has existed before, but can also be used to identify abnormal signal(s). Once the hypercube has been created, signal validation may then be performed on future data by comparing the newly observed process states to those previously observed. The PHC is queried at each data sample to make this determination. In order to prevent false alarms, a block of data is used in determining whether or not a given signal is anomalous.

Signal validation is accomplished by alternating which signal(s) are suspected of being in error. By looking at the PHC, the abnormal signal(s) may be identified. The computational effort required for this determination rises as the following increase: (1) the total number of variables stored in the hypercube, (2) the number of potential hypercube cells, and (3) the number of suspected abnormal signals. A flow chart is given in Figure 5.3. Shown in the figure is a new declaration, that of abnormal process. The process is declared abnormal when several variables are found to be abnormal. The assumption made here is that a catastrophic signal failure is highly unlikely.

\section{PHC Example \#1}

For example, consider a system in which only three variables $(x, y, z)$ are monitored. (Most examples are restricted to three-dimensional cases for the benefit of the reader.) Suppose that each variable is divided into five intervals. And further suppose that the cells on the diagonal of the cube describing all the possible states are the only ones occupied. These cells would be numbered $(1,1,1),(2,2,2), \ldots,(5,5,5)$. 
Now during the observation of this process, a new unobserved state is seen numbered $(1,1,5)$ and labeled $(A)$ on Figure 5.4. It is obvious that both the $\mathrm{x}$ and $\mathrm{y}$ have been observed together before in this combination but that $\mathrm{z}$ was observed in a different state (i.e. $z=1$ instead of $z=5$ ). Thus the true state is probably $(1,1,1)$ and variable $z$ is in error. From this conclusion, it can also be surmised that the correct value of $z$ lies in the range of the first interval.

The above example has also demonstrated an additional feature of the PHC, that is, in the event a signal is identified as incorrect, an estimate of the actual process variable may be provided. This estimate is accurate to within $\pm \delta_{\mathrm{k}} / 2$ (half the variable interval width). When the hypercube assumes a nearly linear behavior, the PHC can identify at most $N_{v}-2$ signals as being anomalous, where $N_{v}$ is the total number of process variables stored in the PHC. Depending on the overall shape of the $\mathrm{PHC}$ in the multi-dimensional space, a different number of maximum abnormal signals may be identifiable. The limiting criteria is whether an observed data point is inside or outside of an enclosed region of the hypercube. The following example illustrates this limitation.

\section{$\underline{\text { PHC Example \#2 }}$}

Note that in Figure 5.5 there are two different two-dimensional hypercubes. Figure 5.5a shows a linearly behaving relationship between the two variables. It is easy to see that an abnormal state is easily identified, however it is impossible to distinguish which variable is in error. In Figure $5.5 \mathrm{~b}$ the two variables assume a nonlinear relationship and depending on the location of the newly observed state, a specific variable may or may not be noted as abnormal. In location (3) the abnormal variable cannot be identified, while at location (4) the abnormal variable may be identified as $y$. 


\section{$\underline{\text { PHC Example \#3 }}$}

Returning to Figure 5.4, another example in which the abnormal signal cannot be distinguished is given. At location $(5,2,1)$, labeled (B), the combination of $\mathrm{x}, \mathrm{y}$ and $\mathrm{z}$ has not existed before. Further analysis shows that neither can the combination of any two variables be found in the learned hypercube.

The hypercube is a multi-dimensional histogram and is essentially a body in a multi-dimensional space. It is important to recognize that the search for abnormal signal(s) does not always correspond to the shortest distance to the surface of the hypercube.

\section{$\underline{\text { PHC Example \#4 }}$}

Returning to the first example (Figure 5.4) of above, note that the distance between the learned state $(1,1,1)$ and the newly observed state $(A)$ is 4 units. The nearest cell $(3,3,3)$ however is only $\sqrt{\mathbf{1 2}}=3.46$ units away. Thus it is shown that the nearest observed state is not necessarily the true state. This aspect is accounted for in the PHC signal validation algorithm.

Although the hypercube signal validation is somewhat simple in theory, the implementation of the algorithm is not a trivial task, primarily because of the multidimensional space which must be searched in determing the anomalous signal(s). The computational effort required to make this analysis is directly proportional to the number of variables stored in the hypercube. There is a geometric increase in computing time needed as the number of postulated signals in error increases. 


\subsubsection{Final Signal Status}

The PHC methodology provides three basic results from its analysis:

1. An inconsistency separation index.

2. An indication of variable abnormality.

3. An estimate of the process variable.

From the above results, a final determination of the status of individual signals is made. In the simplest case, when a signal is consistent with its redundants and the variable is found in the hypercube, the signal is deemed accurate. A little more thought is required for the other possibilities. Figure 5.6 gives the procedure for determining the final signal status based upon the signal's consistency test results and its variable group results. As previously stated, in order to avoid false alarms a block of data samples are analyzed to furnish a final indication of signal validity.

\subsubsection{Summary}

A process hypercube comparison ( $\mathrm{PHC}$ ) has been implemented for the purpose of signal validation. A hypercube is created during a learning period, after which the hypercube may be used to detect abnormal signal(s). An estimate of a variable may be provided in the event an abnormality is detected. A simple consistency checking scheme is used to reduce a group of redundant measurements to a single estimate. 


\subsection{Implementation of Hypercube Technique}

The hypercube signal validation methodology has been implemented in a VAX workstation using the $\mathrm{C}$ programming language. The process hypercube comparison (PHC) module algorithm and theory development were discussed in Sect. 5.2. The PHC module detects consistency failure using a combination of the inconsistency separation index and the variable exclusion status results previously described.

\subsubsection{PHC Data Base}

The PHC module has a system parameter file to store various constants which can be changed later (rather than putting these constants in the text of the program itself). Such parameters are tailored towards a specific process or plant. These parameters include the thresholds of each signal for the detectable consistency failure mode.

The PHC module retains several parameter files for reference:

1. KEYINDEX.PHC - the process state keys are kept here to indicate the process states that were observed during the learning period.

2. FREQUENCY.PHC - the number of times that a particular process state occurred is saved here.

3. SIGNALS.PHC - the information describing signal relationships is stored here. This information includes signals in the redundant groups, variable and group identifications.

4. INTERVALS.PHC - the upper and lower limits and the number of intervals for each variable as specified by the user are retained here. 
The PHC outputs three different results files:

1. INDICES.PHC - the inconsistency separation indices found by the PHC algorithm are stored here with the number of signals in each redundant group.

2. ESTIMATE.PHC - the best estimate from either the consistency checking or the hypercube algorithm, as appropriate, is saved here along with the source of the estimate (the specific algorithm).

3. VARIABLE.PHC - the results of the hypercube analysis for each signal group is kept here.

Each of these three results can be plotted for the user to view.

\subsubsection{PHC Analysis Program}

The main hypercube program (HYPER) is used to create (learn) the process hypercube. HYPER is alsu used as the stand-alone signal validation program. Data from more than one data set may be added to the hypercube. Once the hypercube has been created, infrequently appearing cells may be removed (cleaned-up). The two files FREQUENCY.PHC and KEYINDEX.PHC are created from this overall learning procedure.

Before population of the hypercube however, two files must be created by the user to inform the HYPER program as to the contents of the input data file (or file on which signal validation is to be performed). The first ASCI file is INTERVALS.PHC which contains the number of variables on the first line. This is followed by an input line for each variable containing the variable lower and upper limit, and the number of intervals between these two limits. 
The last input file (SIGNALS.PHC) contains the information describing the actual signal arrangements according to the following format:

\{NGROUPS - Number of signal groups\}

\{NVAR - Number of variables not in a loop (e.g. Power)\}

\{NONVAR - Number of variable in a loop (e.g. RCS Flow)\}

[Repeat the following for each signal group ( $I=1$, NGROUPS)]

\{IDGROUP(I) - Group identification number,

NREDUN(I) - Number of redundant signals in this group,

'TOLER(I) - Signal tolerance for each group

$\{$ MCHAN(I,J);J = 1, NREDUN(I) - List of signals in the group\}.

\subsubsection{Data Processing}

The data processing is initiated by executing the HYPER program from the directory in which the data and parameter files exist. Note that the results files are also placed in this directory. Data processing will stop at the end of each data block to allow the user the opportunity to view the results of that block. Signal validation is then continued through each block in succession.

\subsubsection{Utility Functions}

Certain basic utility function are provided at the top level of the HYPER menu system. These options for data processing and results display include:

1. Evaluation of data using the PHC signal validation methodology.

2. Creation and population of a new hypercube.

3. Addition of new data to an existing hypercube.

4. Plotting of the inconsistency separation index.

5. Plotting of the hypercube variable estimate.

6. Plotting of the hypercube variable status. 
7. Viewing the final signal status for a data block.

8. Display of the hypercube features (ranges and interval widths of the variables).

9. Listing of the hypercube cells and frequencies.

10. Removal of hypercube cells appearing infrequently.

11. Changing of the background results display level.

During data processing, intermediate computational results from the PHC program are displayed in a separate window. The amount of intermediate results shown may be controlled by the user. One of four levels may be selected.

1. No results.

2. Minimal results - which displays only the resultant confidence factors for each signal.

3. Detailed results - which provides intermediate computational results.

4. Debug level - which is basically used in debugging for viewing all the step of the data processing.

The results view level can be altered at any time during data processing.

\subsection{Data Analysis}

This section presents results of the hypercube signal validation technique applied to operational data from both a commercial Westinghouse pressurized water reactor (PWR) plant and the Experimental Breeder Reactor II (EBR-II). The process data were taken during a plant start-up procedure. Results from the PHC module showing examples of both valid and anomalous signals are presented. It must be remembered that data with signal failure is not routinely available. 


\subsubsection{Populating the Hypercube}

The hypercube range (upper and lower limit) is selected along with the number of intervals. The selected values for the PWR and EBR-I data are given in Tables 5.1 and 5.2, respectively. The experimental results from the creation of the two hypercubes is shown in Table 5.3. The important result to note in this table is that the number of potential cells is very large while the actual number of occupied cells is relatively small. Alsu a comparison of the hypercube before and after the removal (clean-up) of infrequently appearing states shows a considerable difference in samples. The number of cells drops by more than half while the number of samples is decreased by only ten percent in the PWR case. This outcome provides definitive proof of the efficiency of the hypercube.

\subsubsection{Experimental Results}

A sampling of the results provided by the $\mathrm{PHC}$ is given at this point to familiarize the reader with the PHC output. More complete results from the application of the PHC are presented later in this section. Figure 5.1 gives a printout from the PHC after finding 3 of 11 signals to be abnormal. The anomalous signals are identified along with an estimate of the true process conditions.

An example plot of each of the primary hypercube results is given in Figs. 5.8 5.10. The PHC inconsistency index (Figure 5.8) shows that only one signal (RCSP458) was consistent with respect to the rest of its redundant group at all time instances. The other signals were excluded from the variable estimate at those places marked with an asterisk. A validated variable estimate from the PHC evaluation is given in Figure 5.9. Lastly, the variable status is presented in Figure 5.10. The variable status shows the $\mathrm{PHC}$ results for a particular variable. The $y-$ axis can indicate that the variable was found or normal (zero), the process was 
abnormal (one) or the variable was not found in the hypercube (two). If the signal is beyond the selected range for the hypercube, an asterisk is plotted along with the fact that the variable was not found.

The PHC module is capable of reporting consistency (signal level) failure results. The consistency checking algorithm found several cases of signal anomaly. The technique was shown to work well even in changing process (transient) situations. Only difference in sensor response time (or relative location) should cause erroneous results between steady-state and transient process conditions.

\subsubsection{PWR Data Analysis}

This section presents results of the hypercube signal validation technique applied to operational data from a commercial Westinghouse pressurized water reactor (PWR) plant. The PWR process data were taken during a plant start-up procedure. Results from each module showing examples of both valid and anomalous signals are presented.

\subsubsection{Consistent Power Signals}

The first results are from the consistency checking portion of the PHC analysis. A case of consistent signals are the four power signals shown in Figure 5.11. Figure 5.12 shows an example of the inconsistency indices computed for the four redundant power signals by the PHC module. The graphs are blank (that is the inconsistency index is zero) which indicates that the signals are consistent with respect to one another. The variable status results from the $\mathrm{PHC}$ analysis showed the variable indications to be found in the learned hypercube at all time instants.

During changing process conditions it is not unusual for some limited number of inconsistencies to be detected. Other signals have shown cases in which the signal was found to be inconsistent a few times, but which should not result in a premature 
decision of signal failure. This illustrates the importance of analysis on a block-byblock basis to prevent false alarms.

\subsubsection{Loss of Signal Example}

The second example of consistency failure detection involves a group of redundant signals in which one signal is lost for a short time period. Data from three redundant steam generator pressure signals are given in Figure 5.13. It is obvious at first glance that the signal MSS-P516 was lost around $t=1000$ minutes. The PHC inconsistency indices (Figure 5.14) for this signal also show the omission of the signal. Note the values of the inconsistency indices. The $\mathrm{PHC}$ indices indicate a relative measure of the degree of error in the signal. At the worst point, the signal is one-hundred tolerance bands from the other two signals. This PHC inconsistency separation index provides not only an indication of anomaly, but also the degree of error in a signal is readily available. The closely grouped asterisks on the plot of the inconsistency indices indicate that this signal was excluded from the variable estimate for the redundant group during the loss of signal event.

One of the touted benefits of signal validation is the computation of a best estimate of the process variable. Figure 5.15 shows the best estimates for the steam generator pressure. The graph also includes, for comparison, estimates from three other signal validation techniques under development in this project - single variable generalized consistency checking (SGCC), multivariable generalized consistency checking (MGCC) and process empirical modeling (PEM). Comparison of the estimate and the raw data of Figure 5.13 shows the omission of the faulty signal from the estimate during the loss of the signal. This validated estimate of the process conditions is the signal of choice for display to an operator and as input to a control or safety system. 


\subsubsection{Biased Flow Signal}

Next an example of bias in a RCS flow signal is presented as related to consistency testing. Figure 5.16 indicates that one signal (RCS-F435) is biased about $15 \%$ (percent is the measured units for RCS flow) from the other two flow signals. The resultant inconsistency indices from the PHC module are shown in Figure 5.17. The signal is excluded from the estimate so often, that the plotted asterisks appear as a solid line. What is important to note here is the rejection of the faulty measurement from the variable estimate.

\subsubsection{EBR-II Data Analysis}

This section presents results of the PHC methodology applied to operational data from the Experimental Breeder Reactor II (EBR-II) plant. Results from the module showing examples of both valid and anomalous signals are presented. Recall that data with signal failure is not routinely available and the module may not detect failure of any type.

Some of the results for the EBR-II data are similar to those of the PWR data and are not repeated. There were instances of momentary signal loss, spikes and other phenomena previously observed in the PWR data. This section attempts to show examples of new anomalies.

The advantage of the PWR data was its abundance of redundant signals. Some EBR-II signals are not truly redundant. For example the evaporator (EV) steam outlet temperature signals are actually located at the outlet of the seven individual evaporators. This type of redundancy is referred to here as "pseudo redundancy." Advantages of the EBR-II data were the greater number of data signals and data samples. Most of the EBR-II signals were temperature 
measurements whereas the PWR data had a mixture of temperature, pressure, level and flow indicators.

\subsubsection{Lack of Redundancy}

The availability of only two redundant signals for some process variables played havoc with the consistency checking algorithm in a couple of cases. Figure 5.18 shows the pair of intermediate heat exchanger (IHX) secondary inlet temperature signals. The top signal (R2-TC-546AR) is at a normal operating value; the bottom signal (R2-RT-533BB) is at a zero level. This second sensor is known to be bad. The PHC inconsistency indices are given in Figure 5.19. The PHC consistency checking scheme is unable to differentiate between the good and bad signal. However, the PHC determined that the variable indication was incorrect (Figure 5.20) and a PHC estimate was subsequently calculated. The estimates from the various signal validation techniques are shown in Figure 5.21. The estimate provided by the PHC is phenomenally better than that of the SGCC module because of the use of the learned process conditions stored in the hypercube. The PHC estimate is not as accurate as that for the PEM and MGCC due to the PHC interval width (resolution).

\subsubsection{Pseudo Redundancy}

As mentioned above, one of the drawbacks of the EBR-II data is its lack of redundancy. To compensate for this, "pseudo" redundant signals were combined in to a single variable group. For example, there is one evaporator (EV) steam outlet temperature signal located at the exit of each of the seven evaporators. These seven signals were therefore combined into a single "redundant "group. In the event one of the evaporators is isolated, it is entirely probably that faulty indications regarding the signal status will be reached by the $\mathrm{PHC}$ from the inter-signal comparisons. 
The measured data for the first and second superheater (SU) sodium inlet temperature signals are shown in Figures 5.22 and 5.23, respectively. The inlet temperatures are at $635^{\circ} \mathrm{F}$ and $685^{\circ} \mathrm{F}$ for the two superheaters. The PHC inconsistency indices for four of the five signals are given in Figure 5.24. Since the temperatures of the superheaters are different, the signals from the first superheater are excluded by the $\mathrm{PHC}$ because they represent a minority (only two of five) redundants in the group. Thus it would be best to separate the superheater signals in to two different variable groups in this case, even though the first group will have only two redundant signals in it (there should be a minimum of three signals for each redundant group). The PHC results generally declare the first two signals to be faulty.

Another case in which pseudo redundancy did not fare well is for the two superheater (SU) steam inlet temperature signals. The measured data for these two signals are shown in Figure 5.25. In this instance, there is only one signal for each superheater, so that one must use pseudo redundancy or nothing. The module estimates are given in Figure 5.26. From the graph the $\mathrm{PHC}$ variable estimate computation method is shown to be superior to the SGCC method. The key to this is that the PHC inconsistency indices of Figure 5.27 are equivalent. Thus neither signal is excluded and both are used in computing an estimate.

Incidentally, the above two cases have shown bad results using the "pseudo" redundancy. However on the whole, the use of pseudo redundancy proved to be a good approach.

\subsubsection{Data Analysis Conclusions}

There were several good examples for the consistency failure detection. One of the differences between the PWR data versus that of the EBR-II data is its 
characteristic of having several secondary loops. The possibility of different process conditions in the different loops resulted in the PHC technique being re-written in such a manner as to account for this occurrence. For instance, the hypercube methodology checks each secondary loop independently with those process conditions in the primary system, which are common to all secondary loops (e.g. reactor power). The data analysis results have shown the PHC methodology to successfully perform signal validation on both the PWR and the EBR-II data. The PHC is a good technique for a global analysis of the plant signals, but is not as well suited for resolving small signal deviations from the normal.

\subsection{Remarks on the PHC Module}

This research on the development and improvement of signal validation technology has resulted in the origination of a new technique for performing signal validation. The process hypercube comparison (PHC) "learns" the valid operating states of a process to which newly observed data points may be compared and faulty signals identified. This technique (module) has also been consolidated into a modular comprehensive signal validation system (SVS). Application of the signal validation technique to operational data from a commercial pressurized water reactor (PWR) and the Experimental Breeder Reactor II (EBR-II) has also been presented. The PHC has proven to be an excellent technique for performing signal validation based upon a global analysis of the plant process states, but it is not well suited for a microscopic examination of the process signals.

Future research should concentrate upon the application of the PHC signal validation methodology to more operational plant data. This increased experience will provide a greater fundamental trust and acceptance of the methodology capabilities and provide the necessary insight into making further improvements in the technique. 
The PHC methodology might well be applied to noise level failure detection also. The PHC noise level validator could be implemented on a sample-by-sample basis in which the data processing would be identical to that used for the signal level processing described in this report except that the signal noise is compared and stored rather than the d.c. signal level. A PHC noise validator could also be based on block-by-block data processing in which the root-mean-square (RMS) noise level for the block is utilized for comparison and storage. 
TABLE 5.1

PWR Hypercube Specifications

\begin{tabular}{|c|c|c|c|c|}
\hline Variable & $\begin{array}{l}\text { Lower } \\
\text { Limit }\end{array}$ & $\begin{array}{l}\text { Upper } \\
\text { Limit }\end{array}$ & $\begin{array}{l}\text { Number of } \\
\text { Intervals }\end{array}$ & $\begin{array}{l}\text { Width of } \\
\text { Interval }\end{array}$ \\
\hline Power $(\%)$ & 0 & 100 & 20 & 5 \\
\hline PRZR Level (\%) & 25 & 75 & 5 & 10 \\
\hline PRZR Pressure ( psia ) & 2200 & 2300 & 4 & 25 \\
\hline Cold Leg 'Temp. $\left({ }^{\circ} \mathrm{F}\right)$ & 530 & 580 & 5 & 10 \\
\hline Hot Leg 'Temp. $\left({ }^{\circ} \mathrm{F}\right)$ & 530 & 630 & 10 & 10 \\
\hline RCS Flow Rate (\%) & 70 & 120 & 5 & 10 \\
\hline RCS Pressure ( psia) & 2150 & 2350 & 4 & 50 \\
\hline Feedwater Flow ( kpph) & 0 & 3900 & 10 & 390 \\
\hline SG Level $(\%)$ & 25 & 75 & 5 & 10 \\
\hline Steam Pressure (psig) & 900 & 1150 & 5 & 50 \\
\hline SG Steam Flow ( ) & 70 & 120 & 5 & 10 \\
\hline \multicolumn{3}{|c|}{ Number of Potential Cells: } & $500,000,000$ & \\
\hline
\end{tabular}


TABLE 5.2

EBR-II Hypercube Specifications

\begin{tabular}{lrccc}
\hline Variable & $\begin{array}{c}\text { Lower } \\
\text { Limit }\end{array}$ & $\begin{array}{c}\text { Upper } \\
\text { Limit }\end{array}$ & $\begin{array}{c}\text { Number of } \\
\text { Intervals }\end{array}$ & $\begin{array}{c}\text { Width of } \\
\text { Interval }\end{array}$ \\
\hline Power $(\%)$ & 0 & 100 & 10 & 10 \\
Inlet Plenum Temp $\left({ }^{\circ} \mathrm{F}\right)$ & 690 & 710 & 2 & 10 \\
Upper Plenum Temp $\left({ }^{\circ} \mathrm{F}\right)$ & 700 & 875 & 5 & 35 \\
IHX Primary Outlet $\left({ }^{\circ} \mathrm{F}\right)$ & 700 & 720 & 2 & 10 \\
IHX Secondary Outlet $\left({ }^{\circ} \mathrm{F}\right)$ & 690 & 865 & 5 & 35 \\
SU Na Inlet Temp $\left({ }^{\circ} \mathrm{F}\right)$ & 620 & 860 & 4 & 60 \\
SU Na Outlet Temp $\left({ }^{\circ} \mathrm{F}\right)$ & 600 & 780 & 4 & 45 \\
EV Na Outlet Temp $\left({ }^{\circ} \mathrm{F}\right)$ & 540 & 600 & 3 & 20 \\
IHX Secondary Inlet $\left({ }^{\circ} \mathrm{F}\right)$ & 540 & 600 & 3 & 20 \\
Secondary Na Flow $(\%)$ & 0 & 100 & 5 & 20 \\
Drum FW Flow $(1 \mathrm{bm} / \mathrm{hr})$ & 0 & $3 \times 105$ & 5 & 60,000 \\
Drum Feedwater Temp $\left({ }^{\circ} \mathrm{F}\right)$ & 355 & 555 & 4 & 50 \\
EV Feedwater Temp $\left({ }^{\circ} \mathrm{F}\right)$ & 530 & 590 & 3 & 20 \\
EV Steam Outlet Temp $\left({ }^{\circ} \mathrm{F}\right)$ & 535 & 595 & 3 & 20 \\
SU Steam Inlet Temp $\left({ }^{\circ} \mathrm{F}\right)$ & 560 & 650 & 3 & 30 \\
SU Steam Outlet Temp $\left({ }^{\circ} \mathrm{F}\right)$ & 600 & 825 & 5 & 45 \\
\cline { 3 - 5 } Number of Potential Cells: & & $1,944,000,000$ & \\
\hline \hline
\end{tabular}


TABLE 5.3

Hypercube Experimental Results

Result

Data Set

$\frac{\text { Data Set }}{\text { PWR }}$

Number of Signals

Number of signal Groups

Number of Loops

Number of Variables

Number of Potential Cells

Number of Occupied Cells

Before Clean-up Operation

Frequency Range of Cells

Before Clean-up Operation

Total Number of Samples

Before Clean-up Operation

Number of Occupied Cells

After Clean-up Operation

Frequency Range of Cells After Clean-up Operation

Total Number of Samples

After Clean-up Operation
77

26

4

11

$5 \times 108$

363

73

$1-367$

$1-667$

3117

3440

155

61

$3-367$

3- 667

2848

3424 


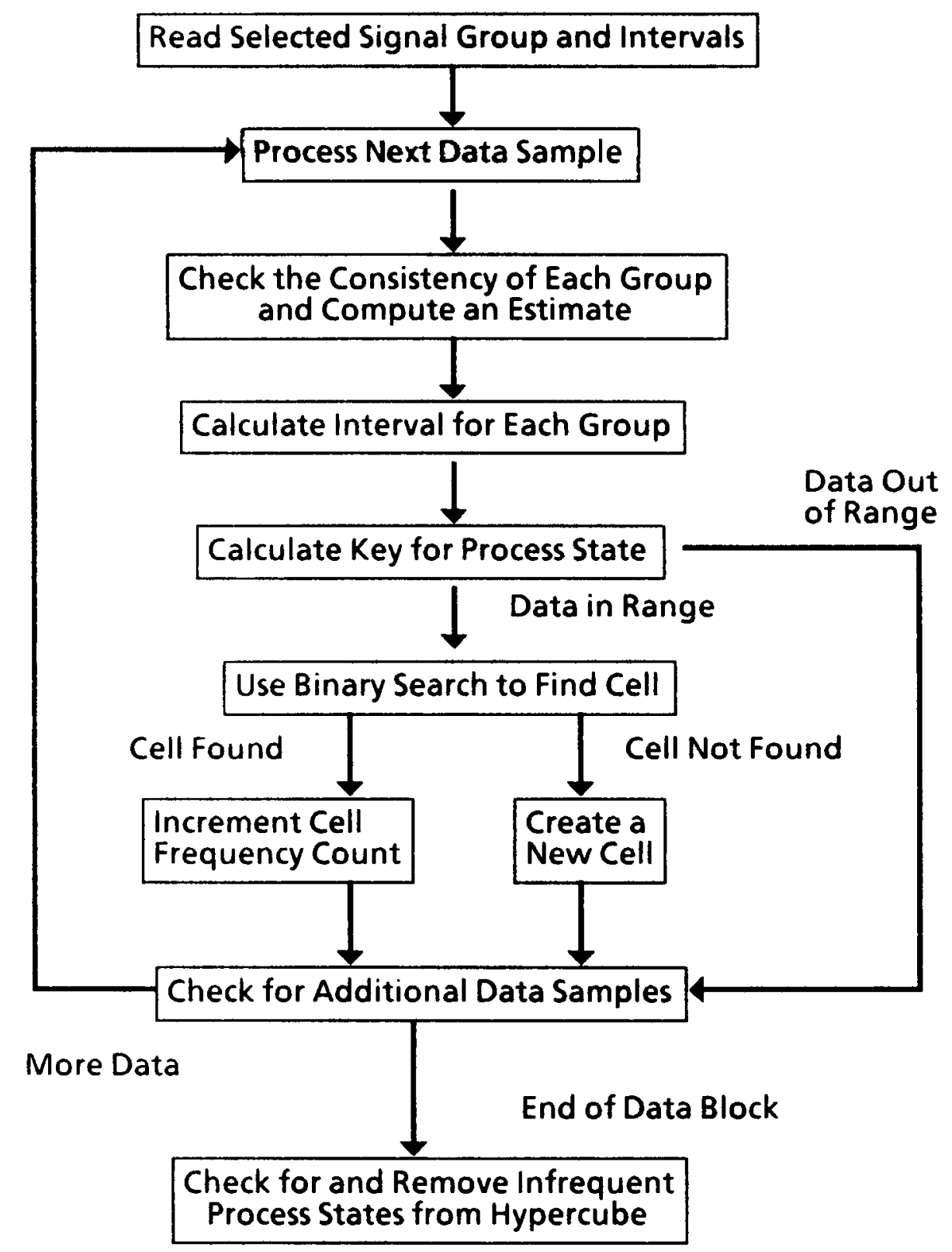

Figure 5.1. Schematic for Creating Hypercube. 


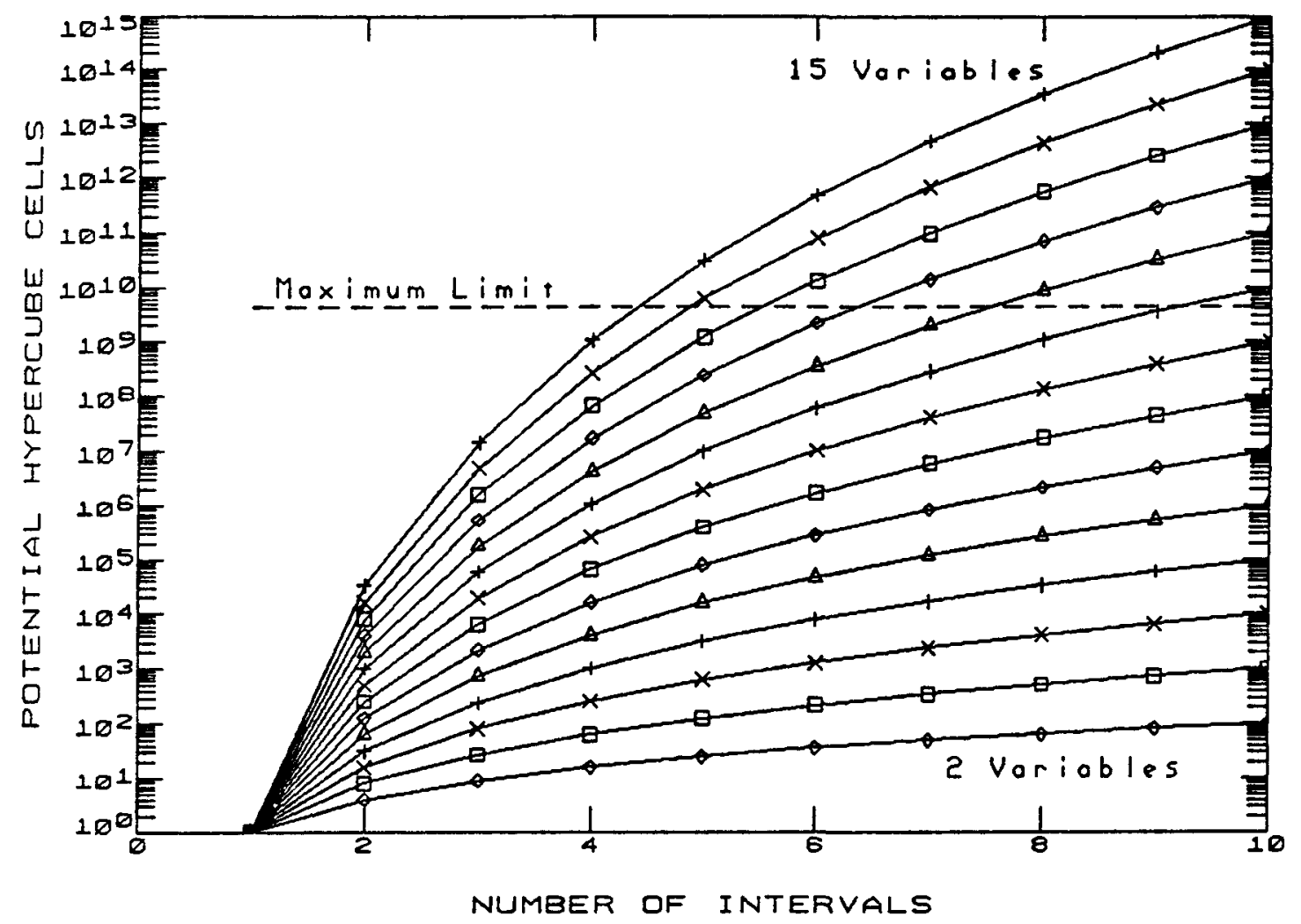

Figure 5.2. Number of Potential Hypercube Cells as a Function of the Number of Variables. 


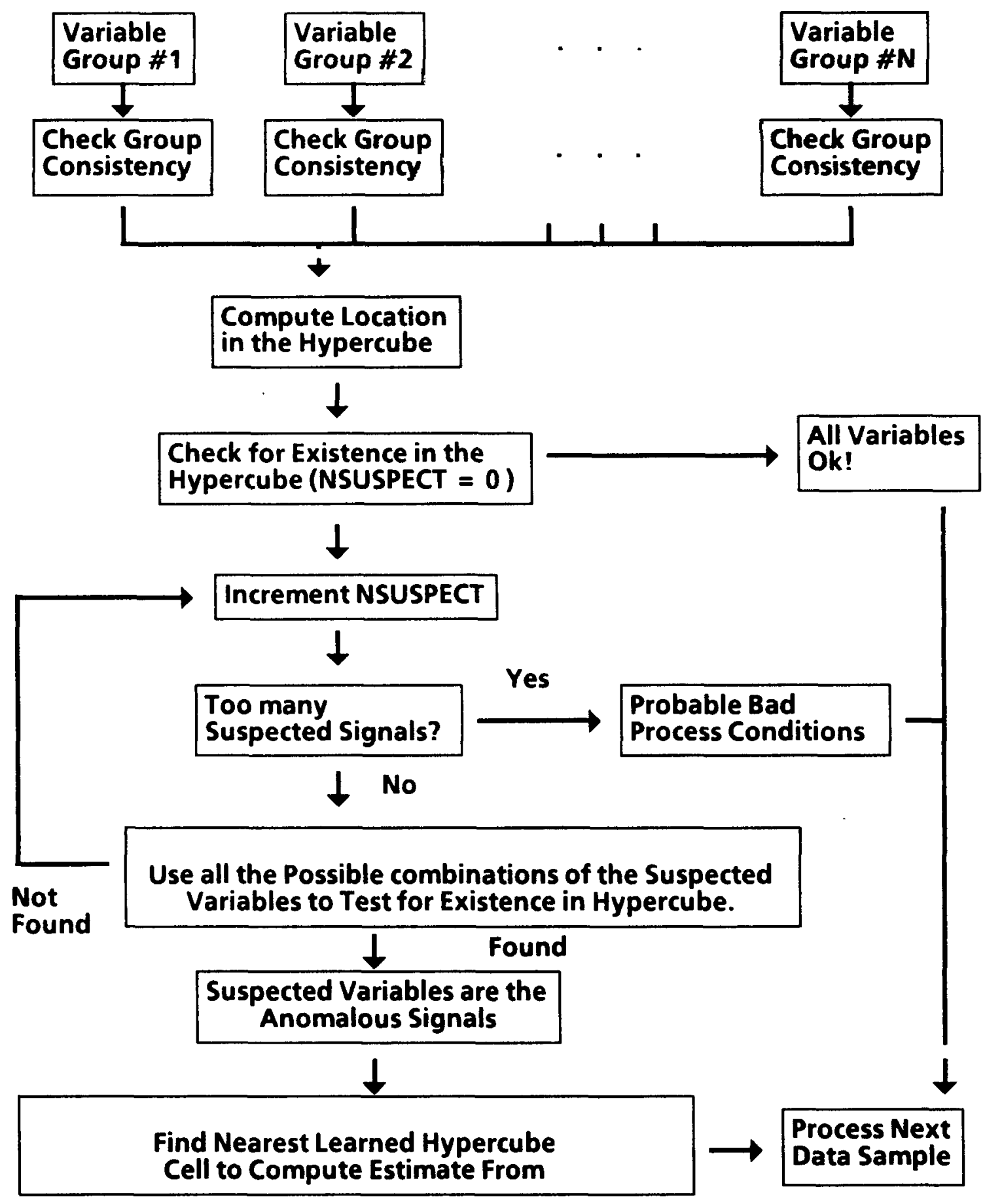

Figure 5.3. Flow Chart of Hypercube Methodology. 


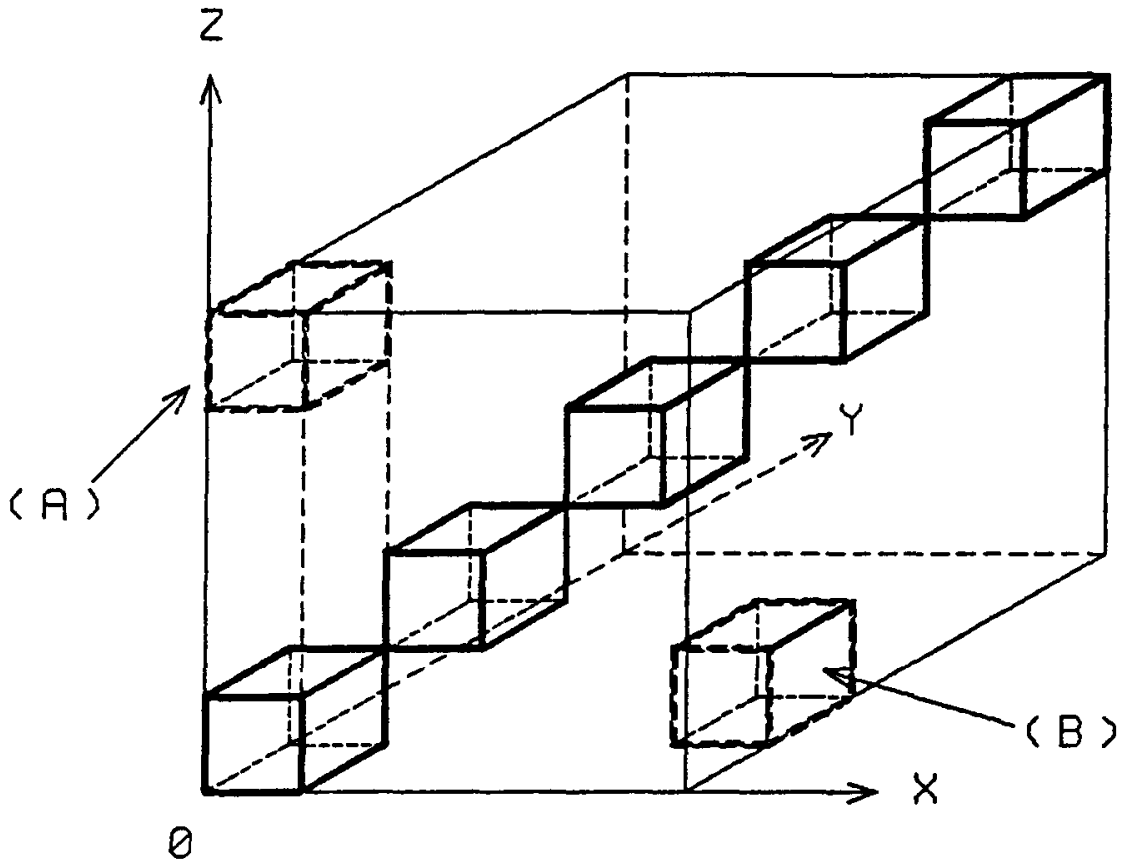

Figure 5.4. Three-dimensional Hypercube Example. 


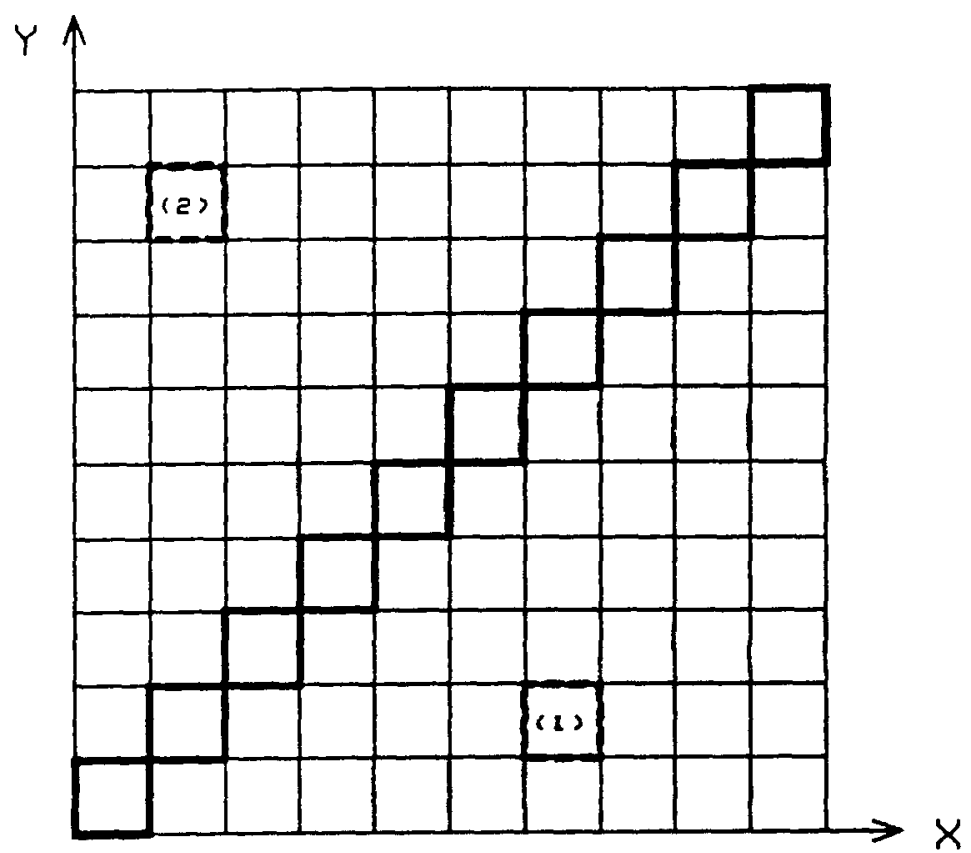

Figure 5.5a

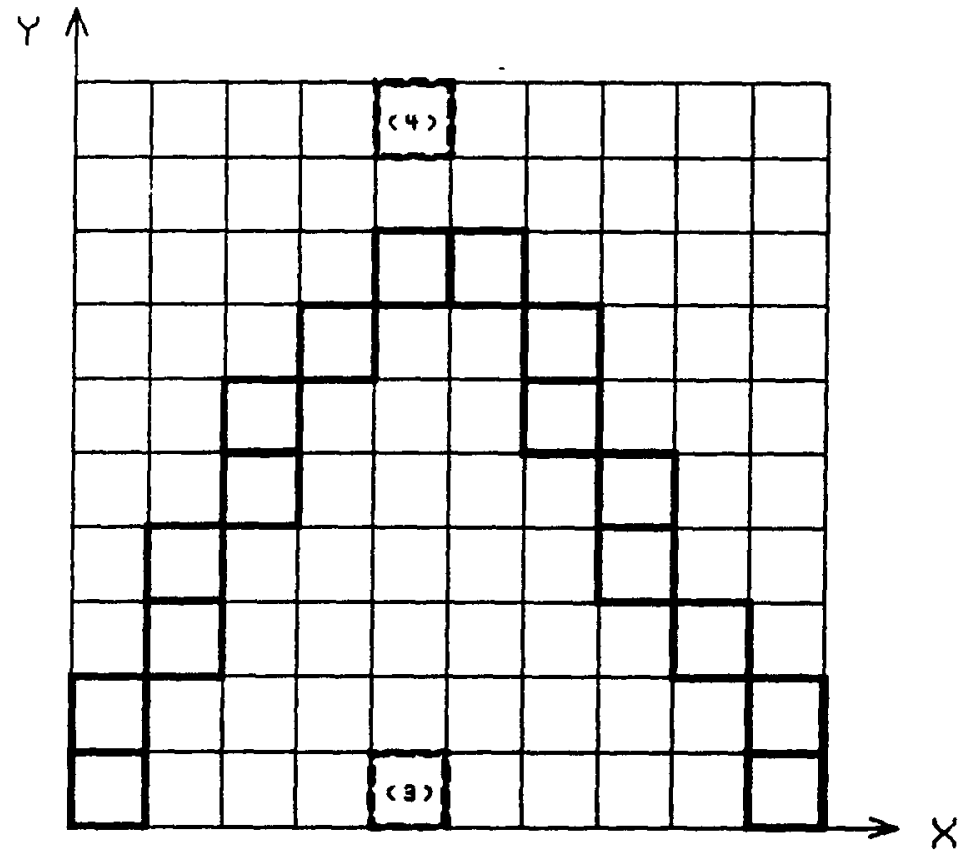

Figure 5.5b

Figure 5.5. Two-dimensional Hypercube Example. 


\begin{tabular}{|c|c|c|c|}
\hline $\begin{array}{c}\text { Decision } \\
\text { Criteria } \\
\text { for Each } \\
\text { Individual } \\
\text { Signal }\end{array}$ & $\begin{array}{c}\text { Signal's } \\
\text { Variable } \\
\text { Group Found } \\
\text { in the } \\
\text { Hypercube }\end{array}$ & $\begin{array}{c}\text { Process } \\
\text { Abnormality } \\
\text { Detected } \\
\text { by the } \\
\text { Hypercube }\end{array}$ & $\begin{array}{c}\text { Signal's } \\
\text { Variable } \\
\text { Group NOT } \\
\text { Found in the } \\
\text { Hypercube }\end{array}$ \\
\hline $\begin{array}{c}\text { Signal } \\
\text { Consistent } \\
\text { wrt Group }\end{array}$ & $\begin{array}{c}\text { Signal } \\
\text { Accurate }\end{array}$ & $\begin{array}{c}\text { Signal } \\
\text { Probably } \\
\text { Accurate }\end{array}$ & $\begin{array}{c}\text { Signal } \\
\text { Possibly } \\
\text { Failed }\end{array}$ \\
\hline $\begin{array}{c}\text { Signal } \\
\text { Partially } \\
\text { Consistent } \\
\text { wrt Group }\end{array}$ & $\begin{array}{c}\text { Signal } \\
\text { Marginal }\end{array}$ & $\begin{array}{c}\text { Signal } \\
\text { Status } \\
\text { Indeter- } \\
\text { minate }\end{array}$ & $\begin{array}{c}\text { Signal } \\
\text { Probably } \\
\text { Failed }\end{array}$ \\
\hline $\begin{array}{c}\text { Signal } \\
\text { Inconsistent } \\
\text { wrt Group }\end{array}$ & Fignal & $\begin{array}{c}\text { Signal } \\
\text { Fuestionable }\end{array}$ & $\begin{array}{c}\text { Signal } \\
\text { Possibly } \\
\text { Accurate }\end{array}$ \\
\hline
\end{tabular}

Figure 5.6. Hypercube Decision Criteria. 
Process state absent from learned hypercube domain!

The anomalous process variables are 3,6 and 8 .

$\begin{array}{lrrrr}\text { Variable \# } 1 \text {, Measurement: } & 1.89 & & \\ \text { Variable \# 2, Measurement: } & 25.99 & & \\ \text { Variable \# 3, Measurement: } & 2250.00 & \text { Estimated: } & 2237.50 \\ \text { Variable \# 4, Measurement: } & 554.80 & & \\ \text { Variable \# 5, Measurement: } & 559.30 & & \\ \text { Variable \# 6, Measurement: } & 2251.50 & \text { Estimated: } & 2225.00 \\ \text { Variable \# } & \text { 7, Measurement: } & 27.95 & & \\ \text { Variable \# 8, Measurement: } & 55.42 & \text { Estimated: } & 50.00 \\ \text { Variable \# 9, Measurement: } & 1069.67 & & \\ \text { Variable \# 10, Measurement: } & 108.93 & & \\ \text { Variable \# 11, Measurement: } & 110.00 & & \end{array}$

Figure 5.7. Printout from Hypercube Signal Validation. 


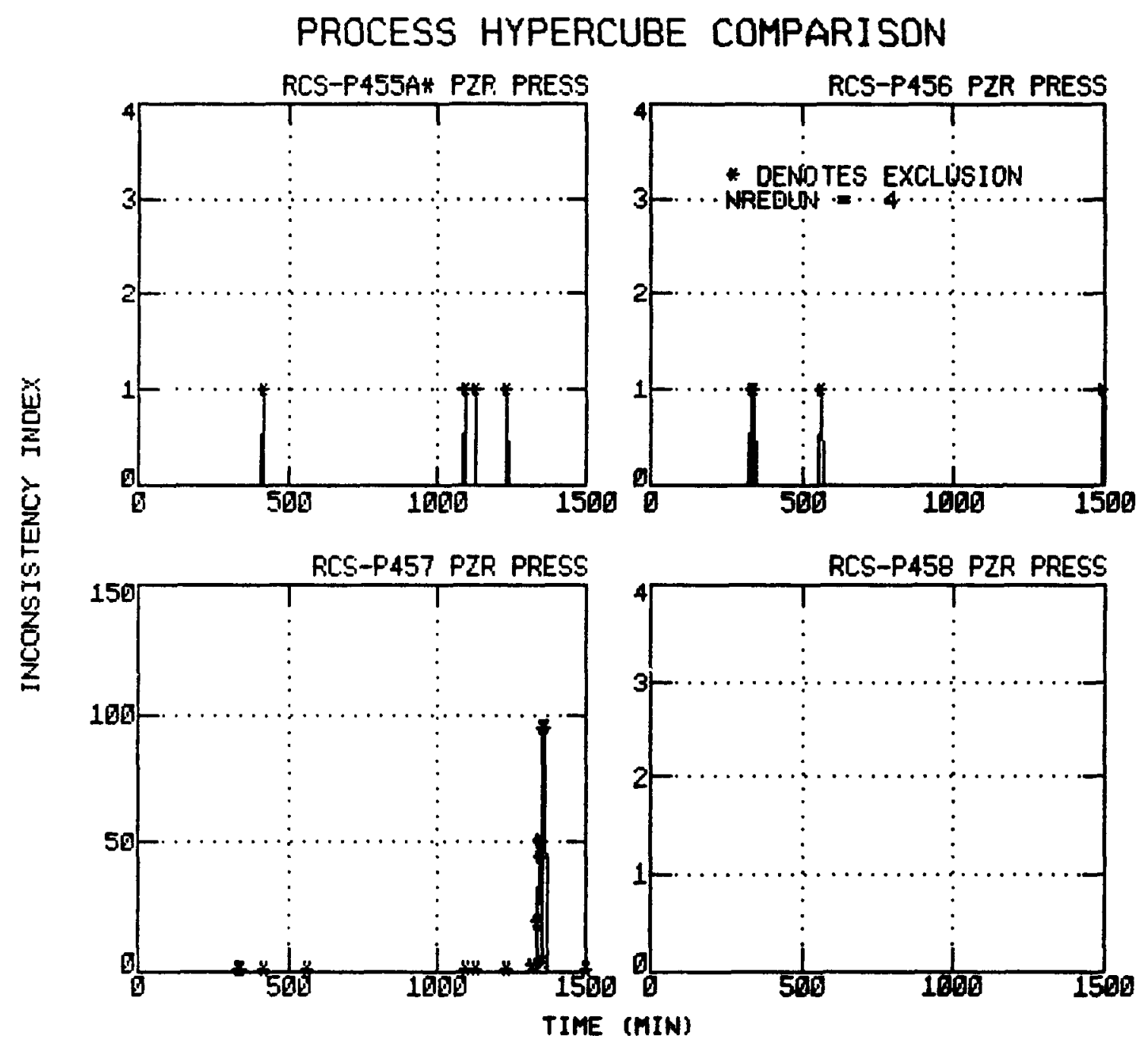

Figure 5.8. PHC Inconsistency Separation Index. 


\section{PROCESS HYPERCUBE COMPARISON}

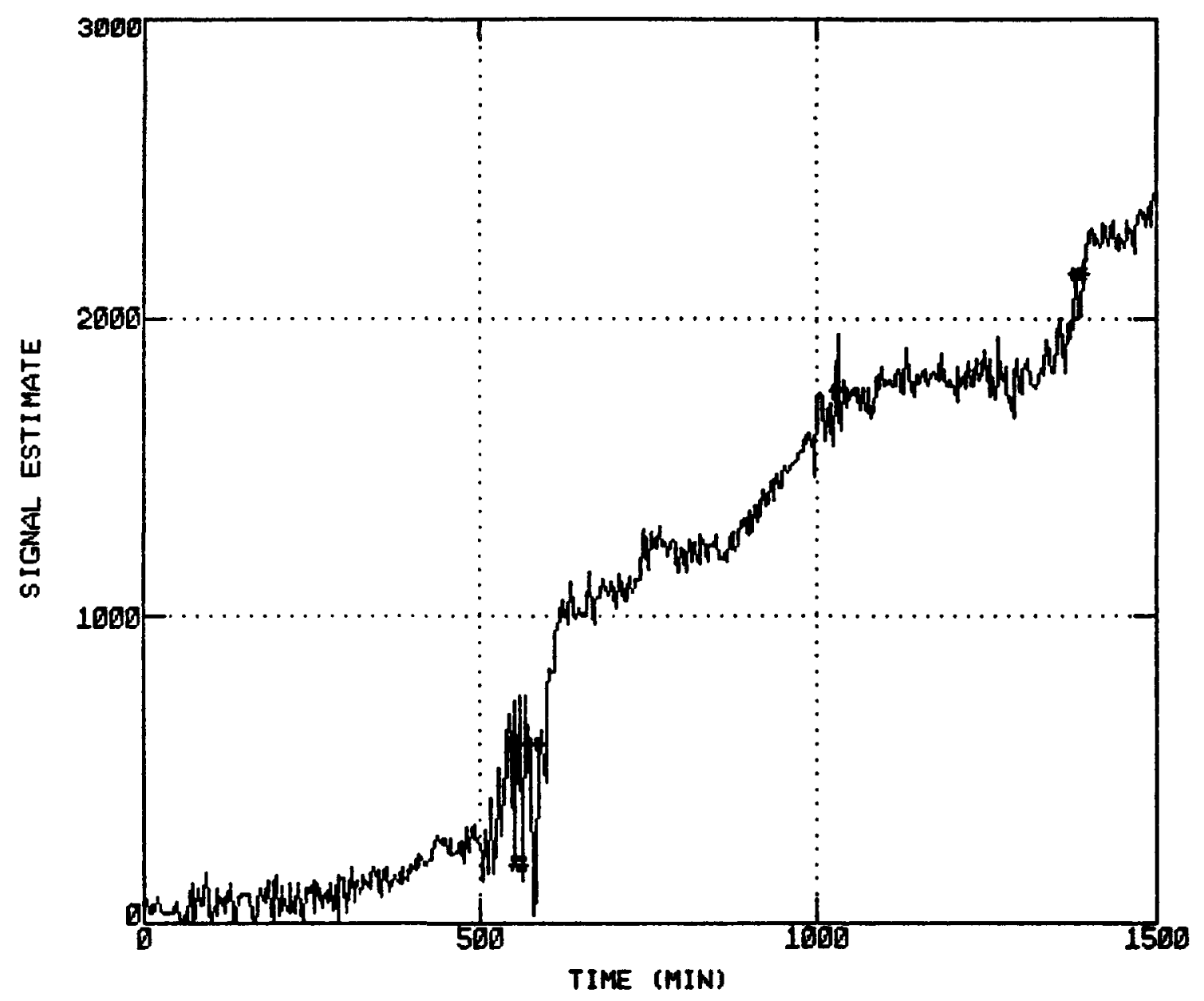

Figure 5.9. PHC Variable Estimate. 


\section{PROCESS HYPERCUBE COMPARISON}

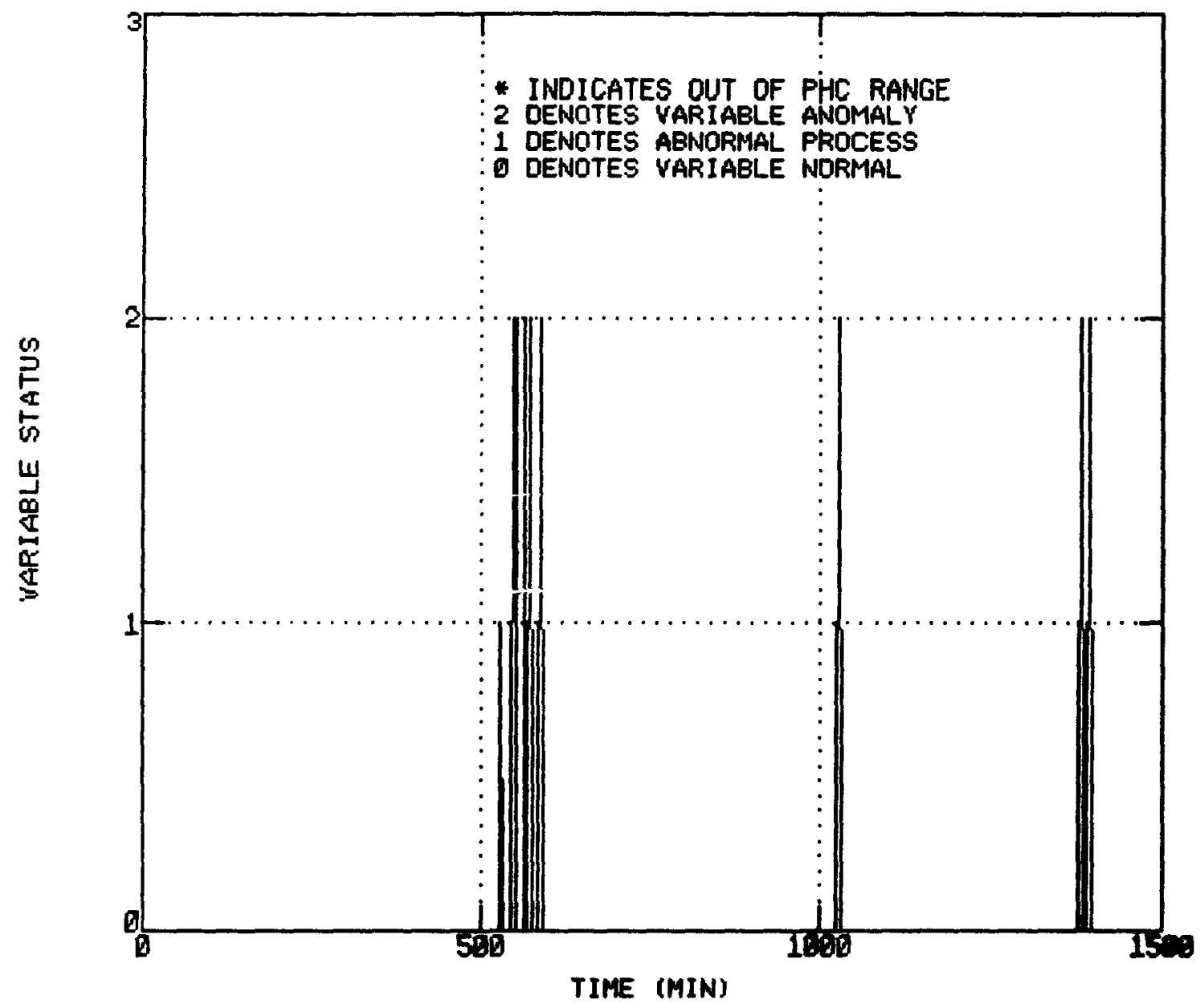

Figure 5.10. PHC Variable Status. 


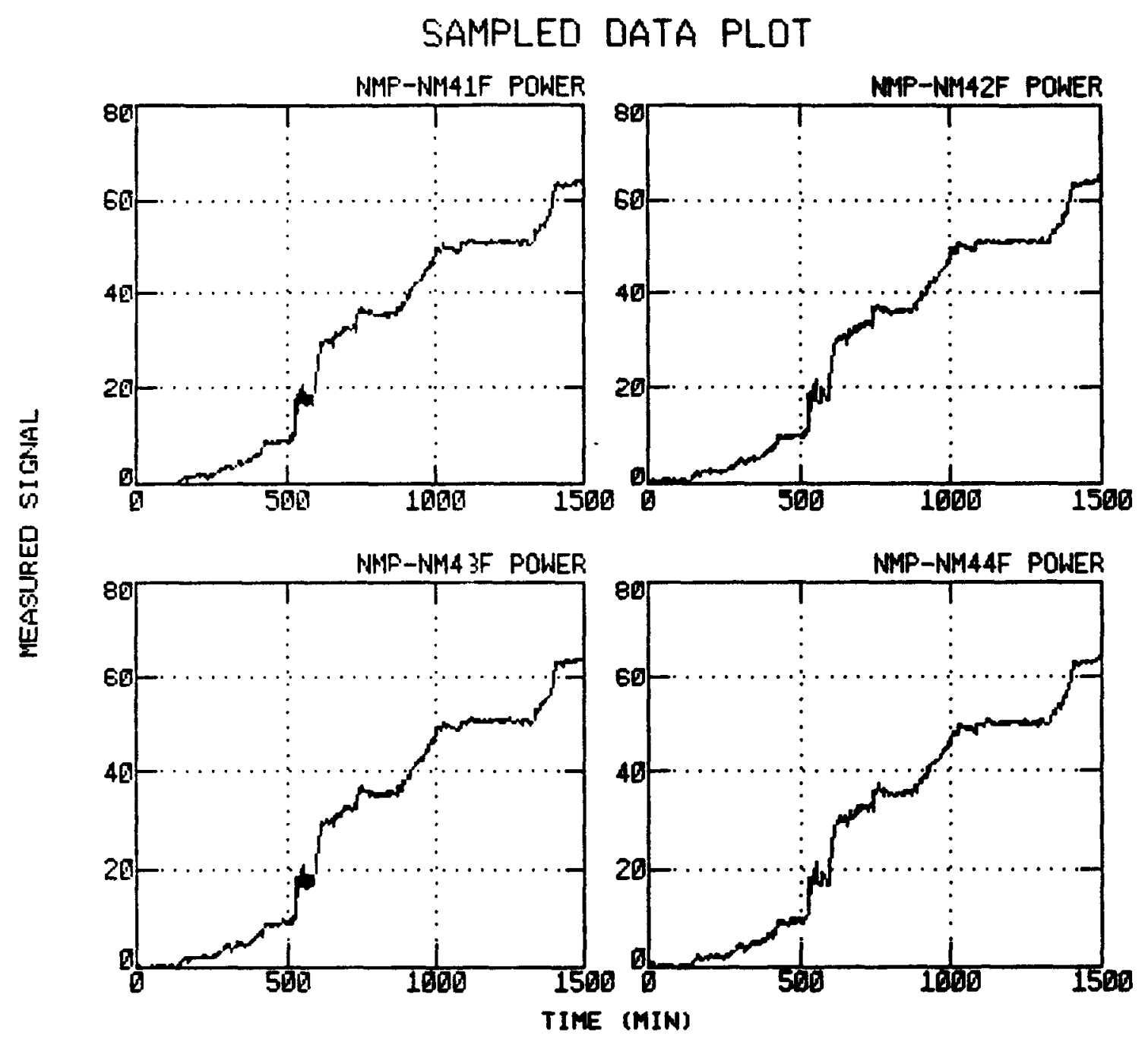

Figure 5.11. Measured Data for the PWR Power Signals. 


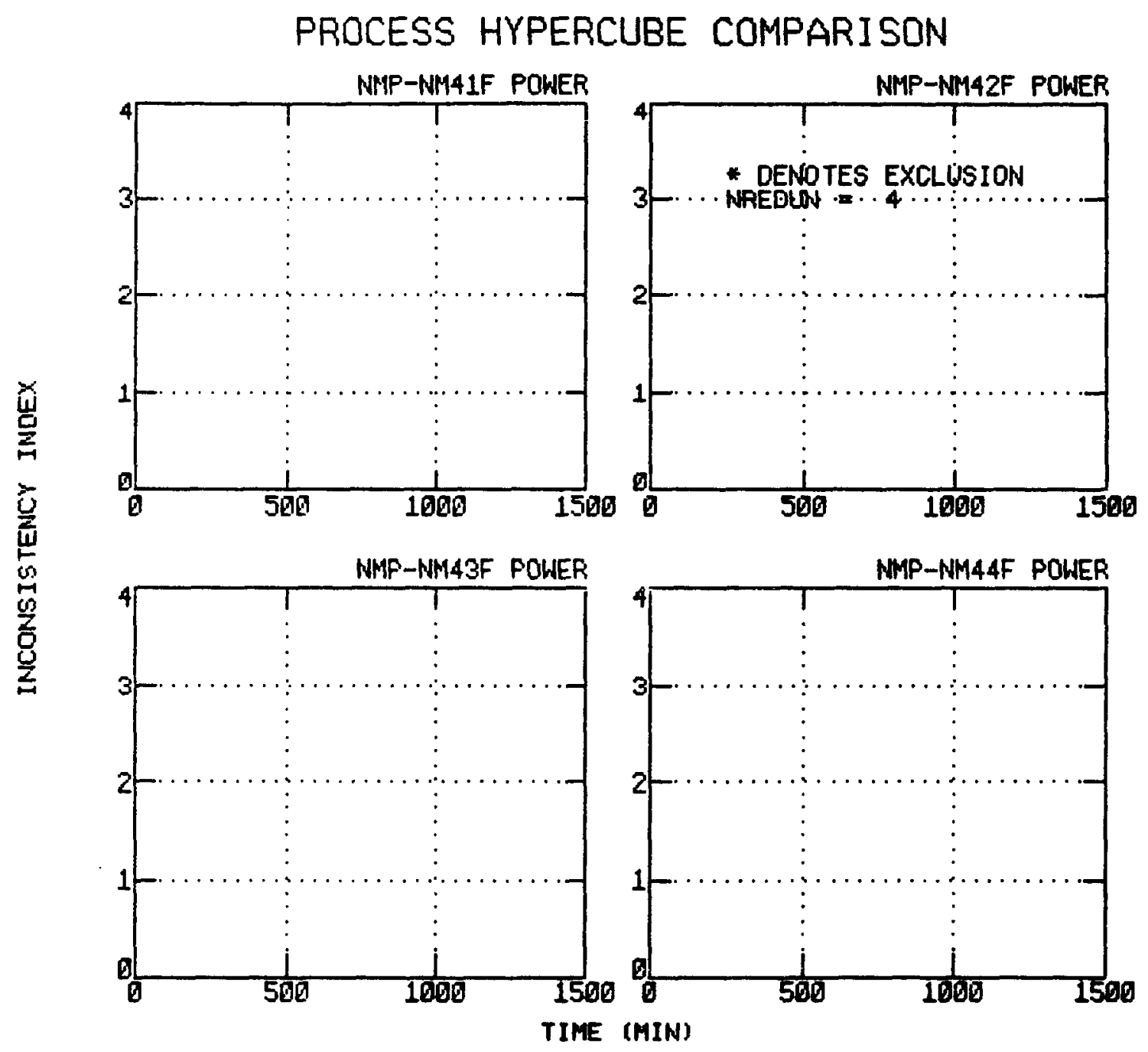

Figure 5.12. PHC Inconsistency Indices for the PWR Power Signals. 


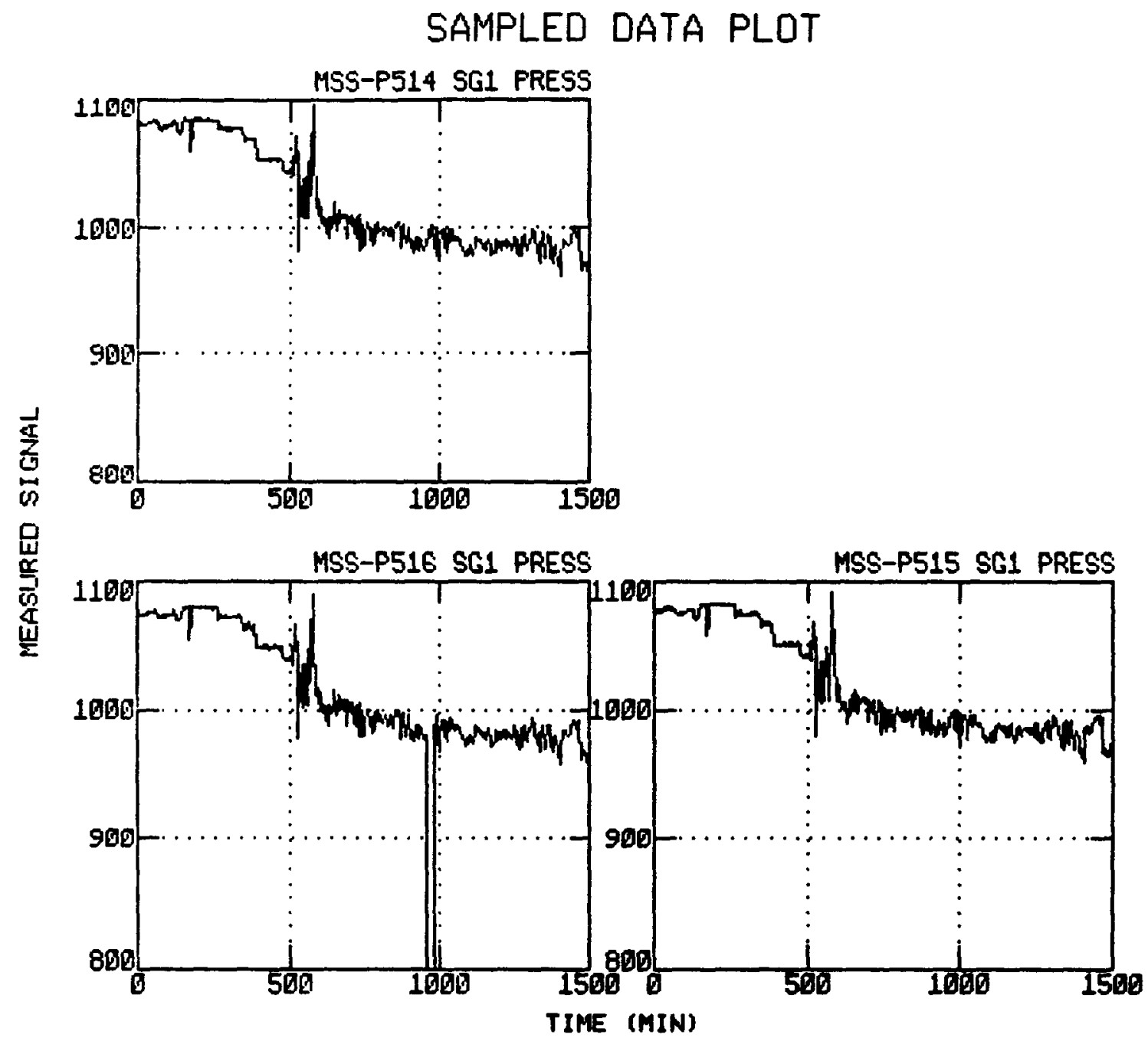

Figure 5.13. Measured Data for Three Redundant PWR Steam Generator Steam Pressure Signals. 


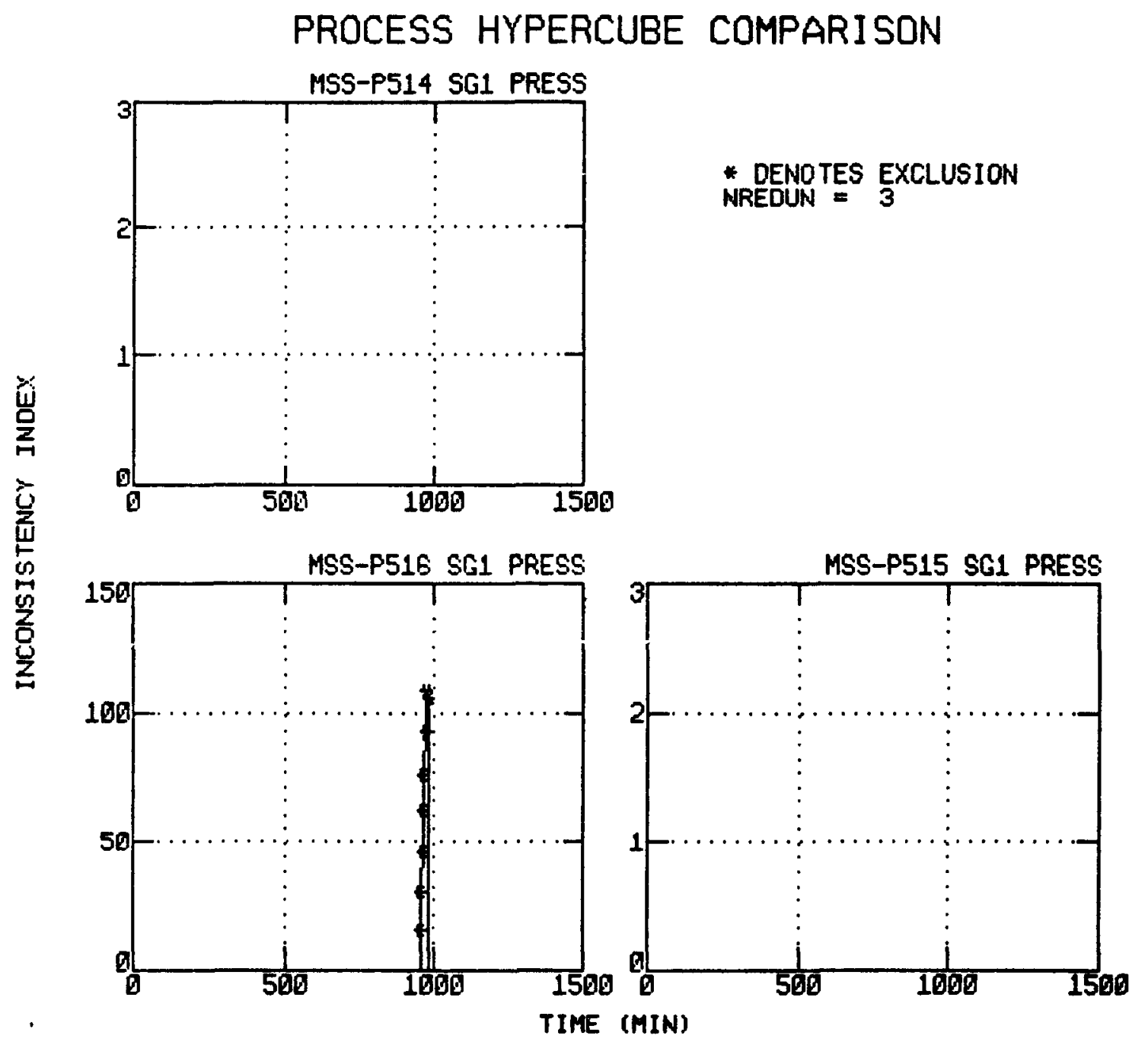

Figure 5.14. PHC Inconsistency Indices for the PWR Steam Generator Steam Pressure Signals. 


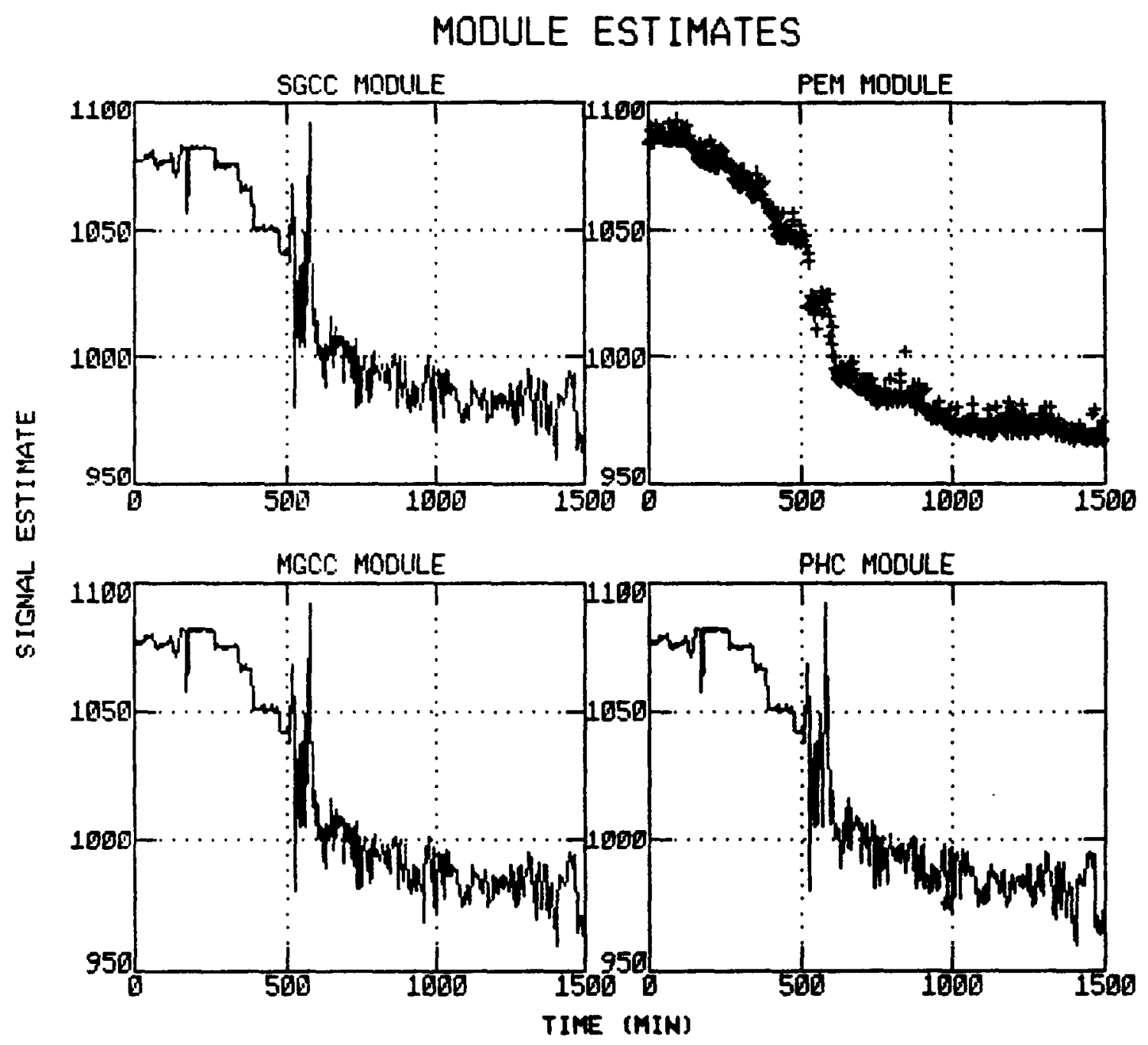

Figure 5.15. PWR Steam Generator Steam Pressure Estimates. 


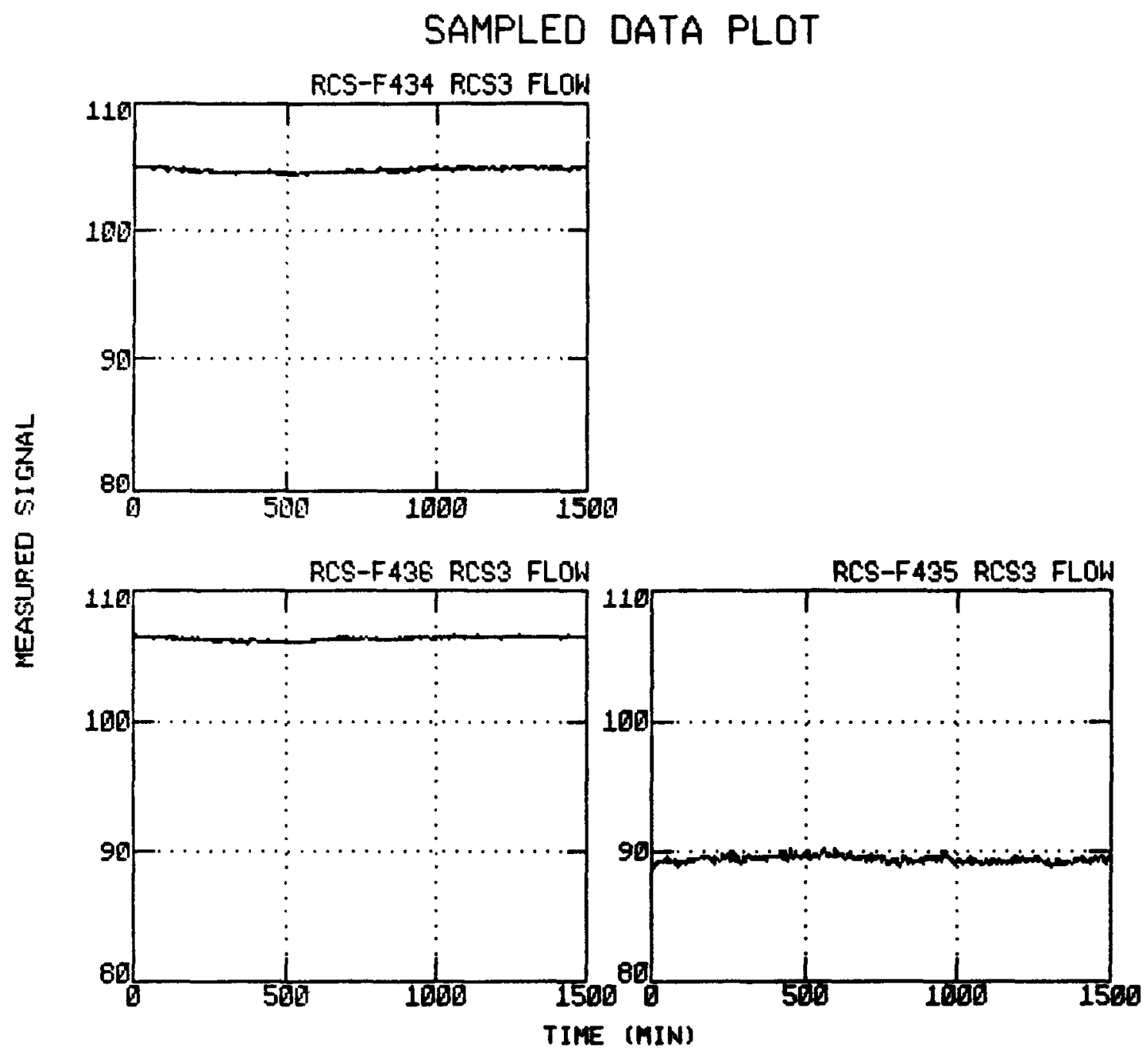

Figure 5.16. Measured Data for Three Redundant PWR RCS Flow Signals. 


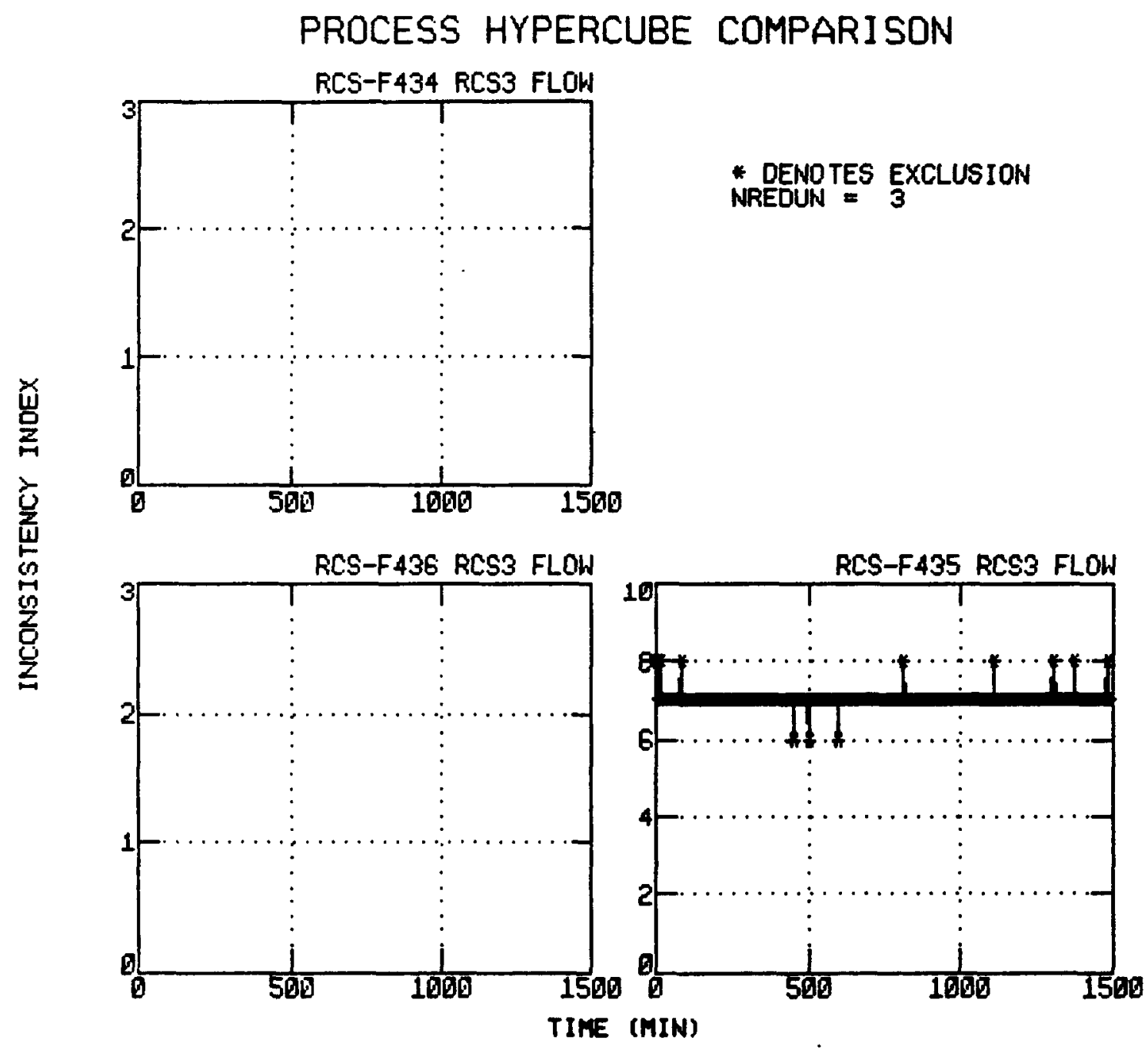

Figure 5.17. PHC Inconsistency Indices for the PWR RCS Flow Signals. 


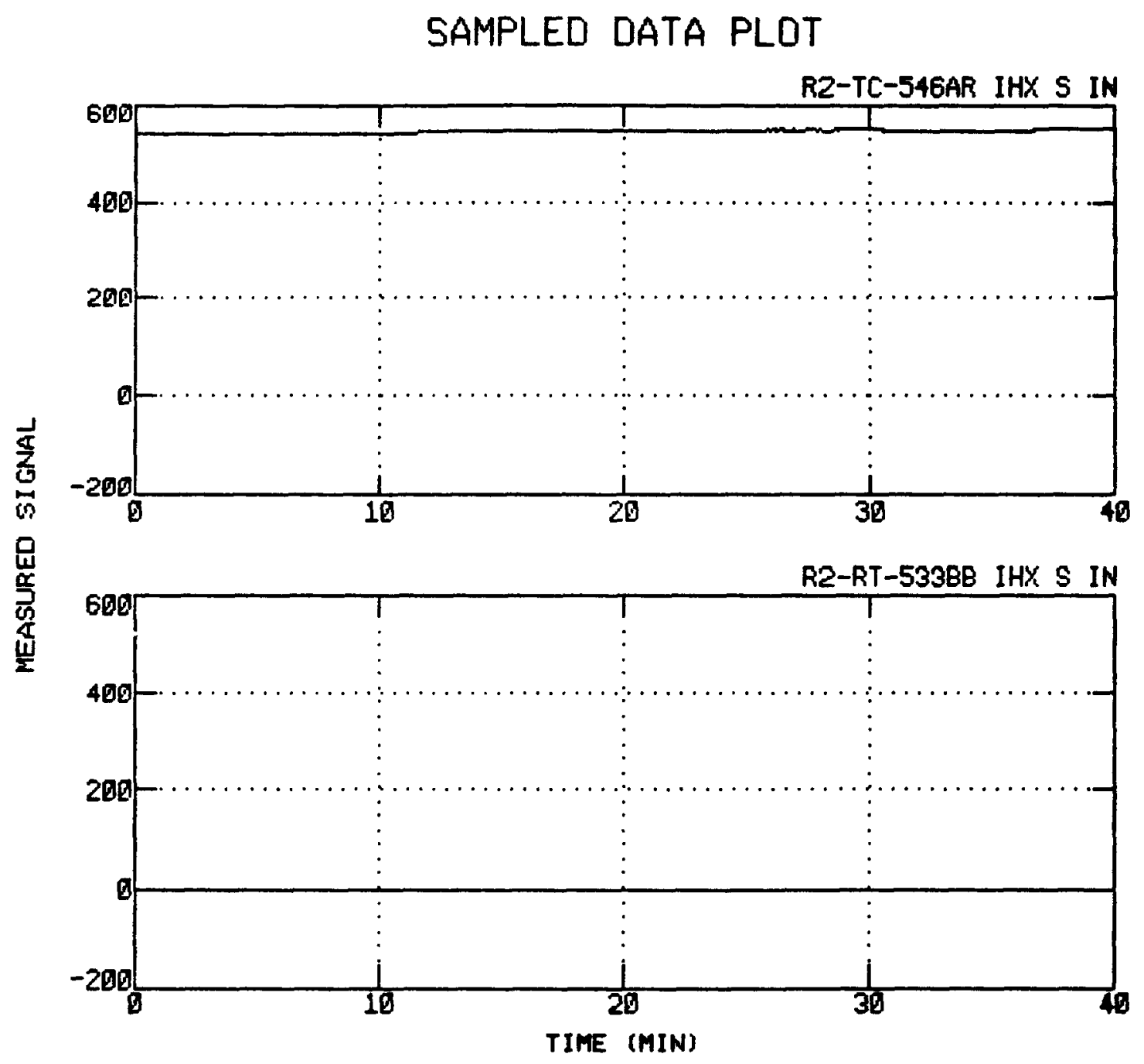

Figure 5.18. Measured Data for the IHX Secondary Inlet Temperature Signals. 


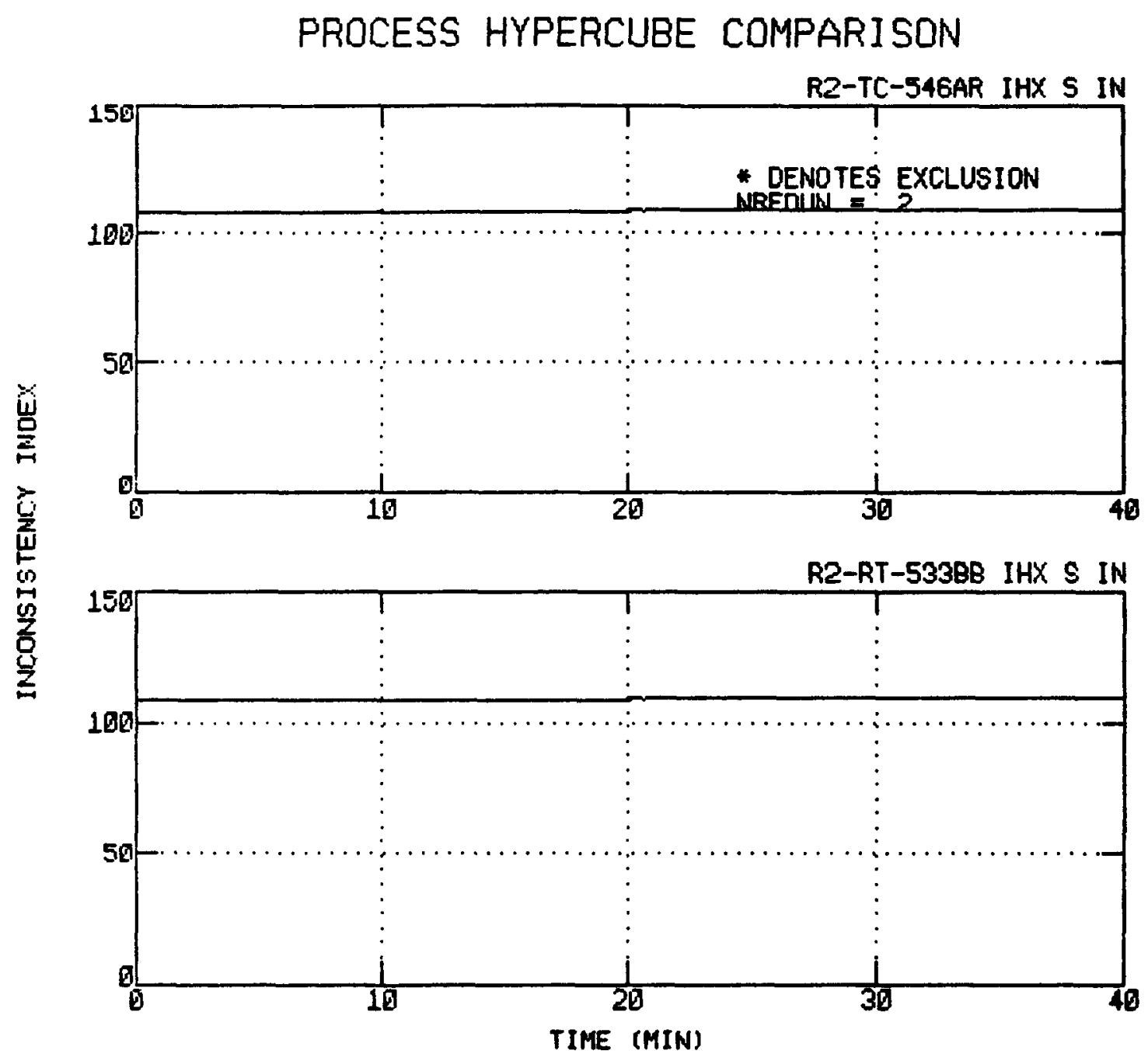

Figure 5.19. PHC Inconsistency Indices for the IHX Secondary Inlet Temperature Signals. 


\section{PROCESS HYPERCUBE COMPARISON}

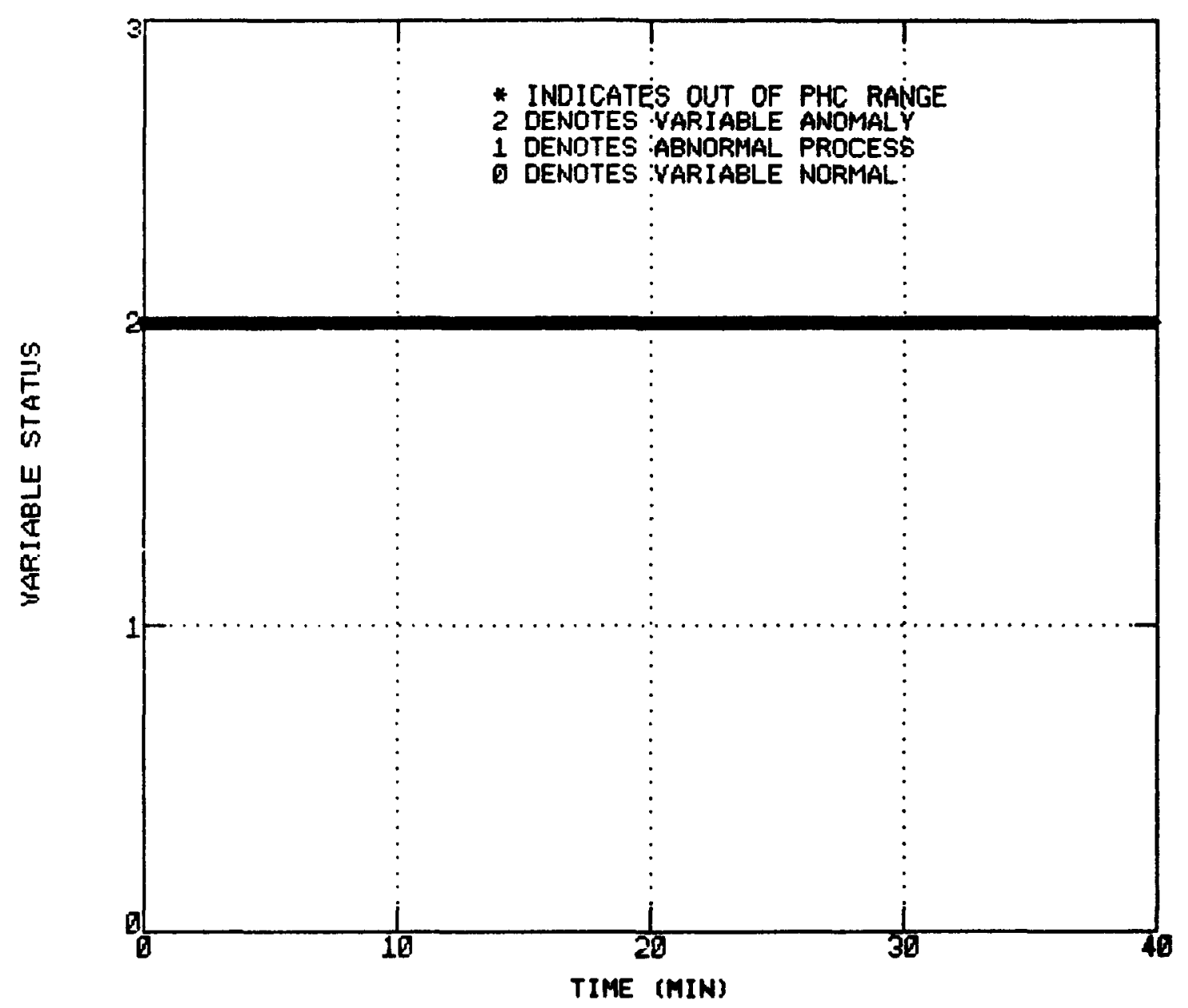

Figure 5.20. PHC Variable Status for the IHX Secondary Inlet Temperature. 


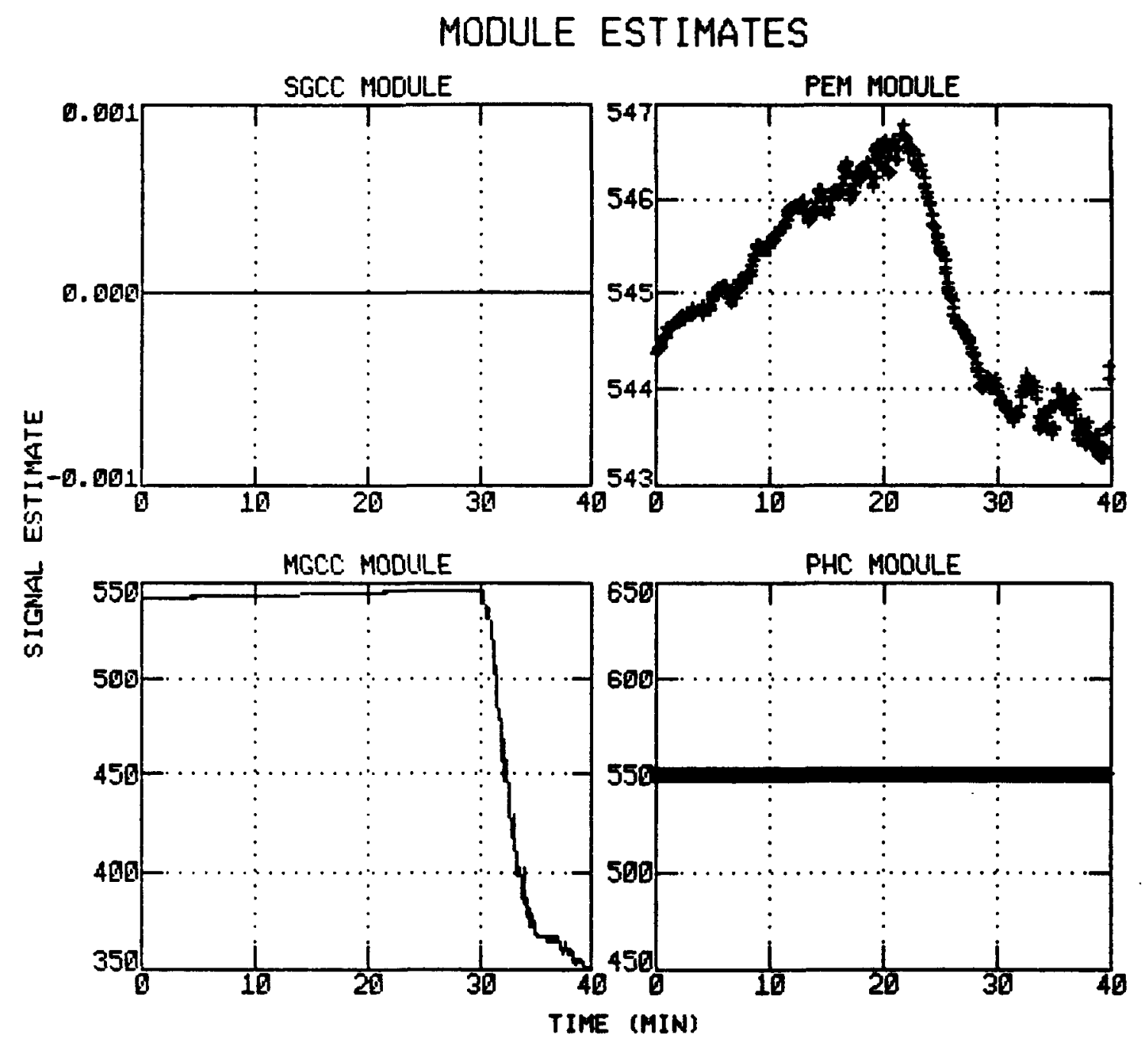

Figure 5.21. IHX Secondary Inlet Temperature Estimates. 


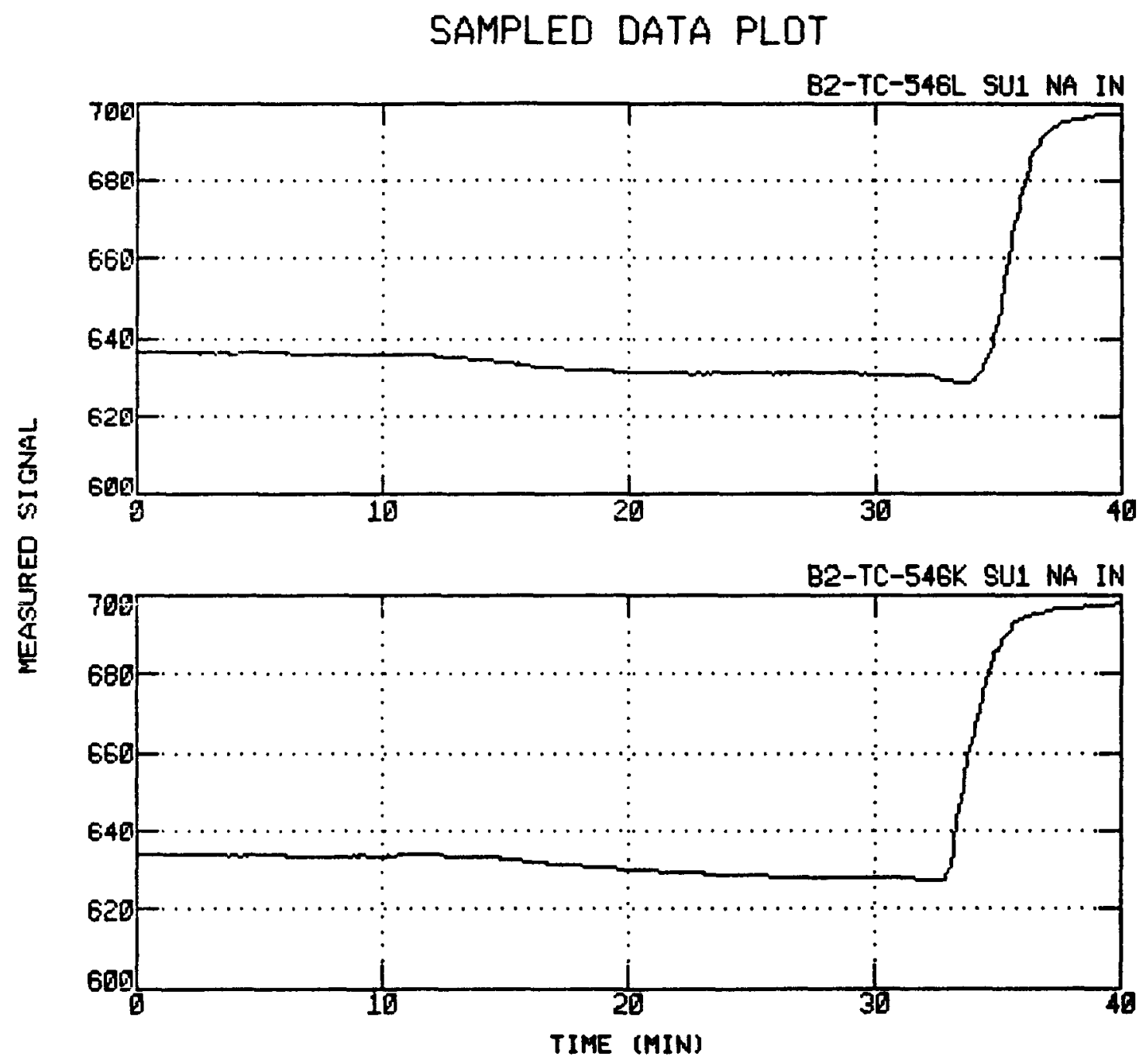

Figure 5.22. Measured Data for the First Superheater Sodium Inlet Temperature Signals. 


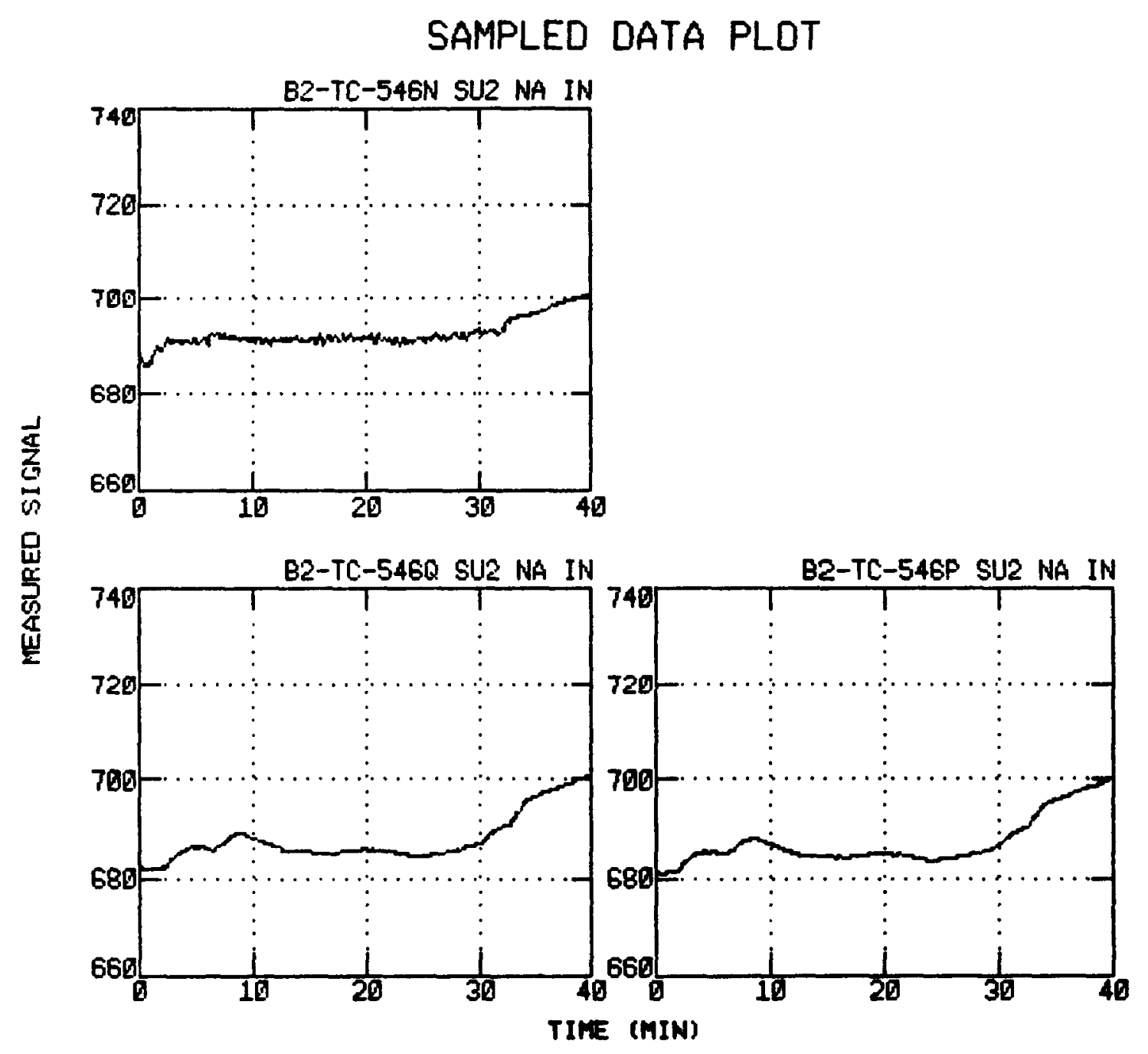

Figure 5.23. Measured Data for the Second Superheater Sodium Inlet Temperature Signals. 


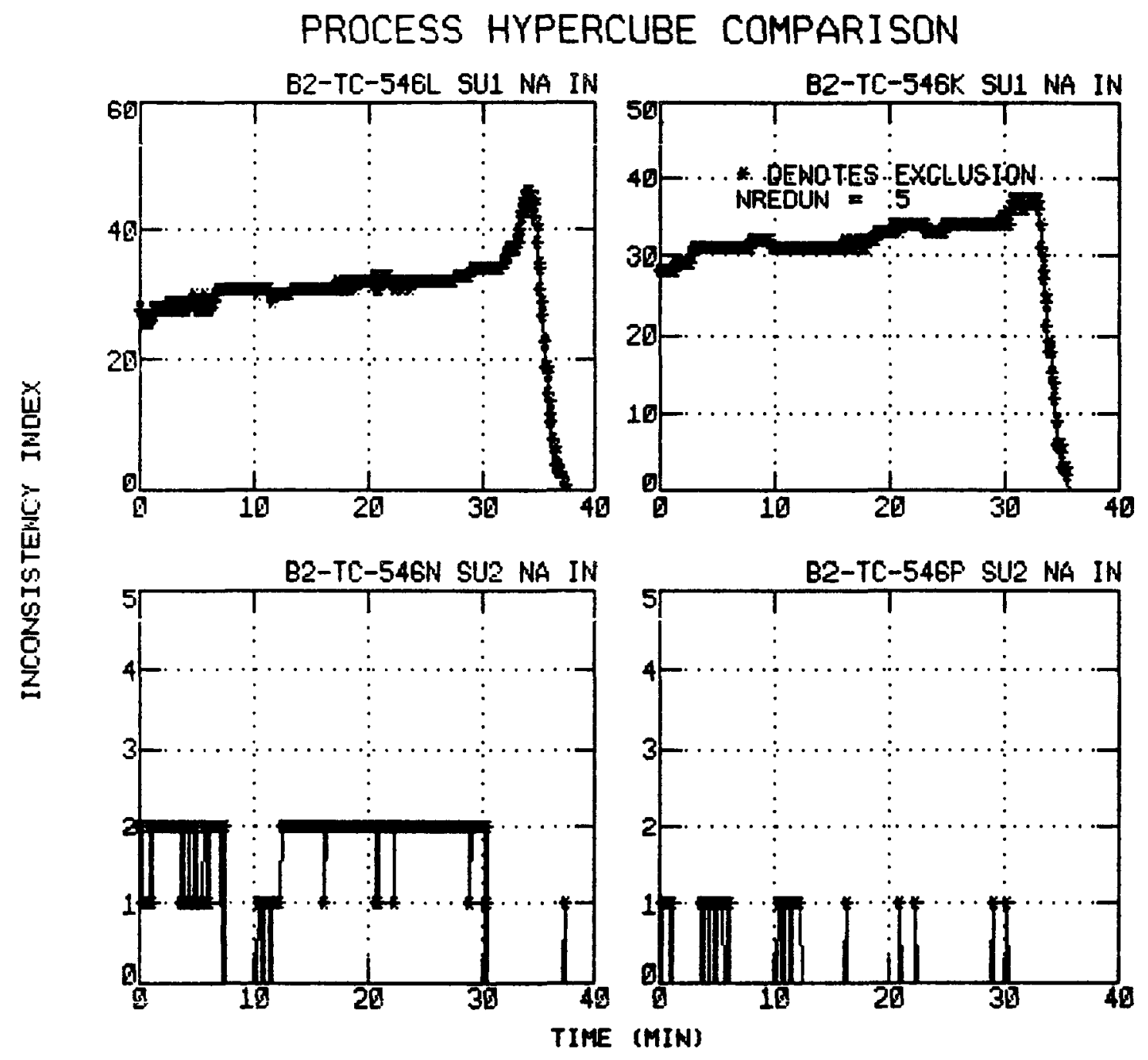

Figure 5.24. PHC Inconsistency Indices for the Superheater Sodium Inlet Temperature Signals. 
SAMPLED DATA PLOT

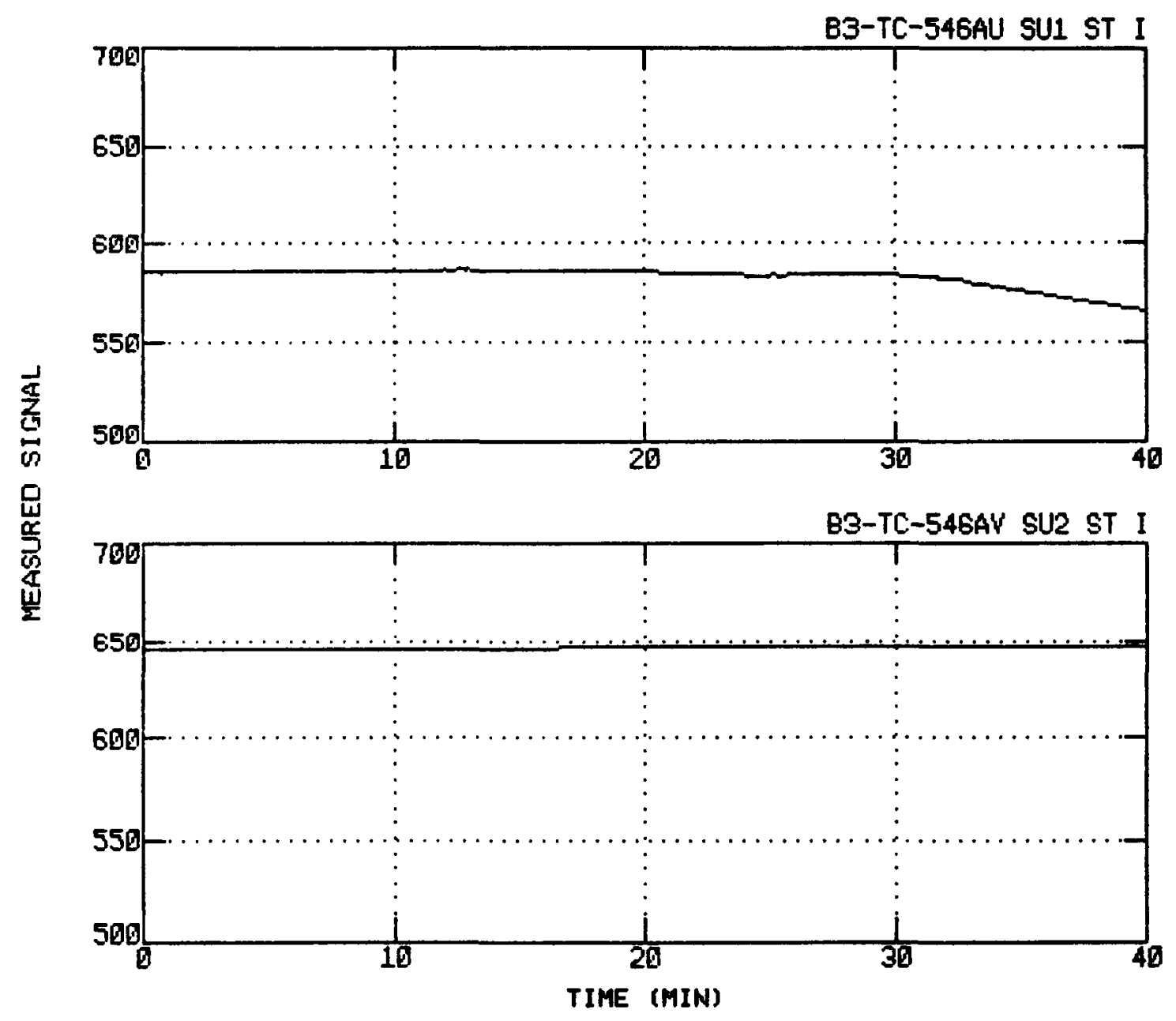

Figure 5.25. Measured Data for the Superheater Steam Inlet Temperature Signals. 


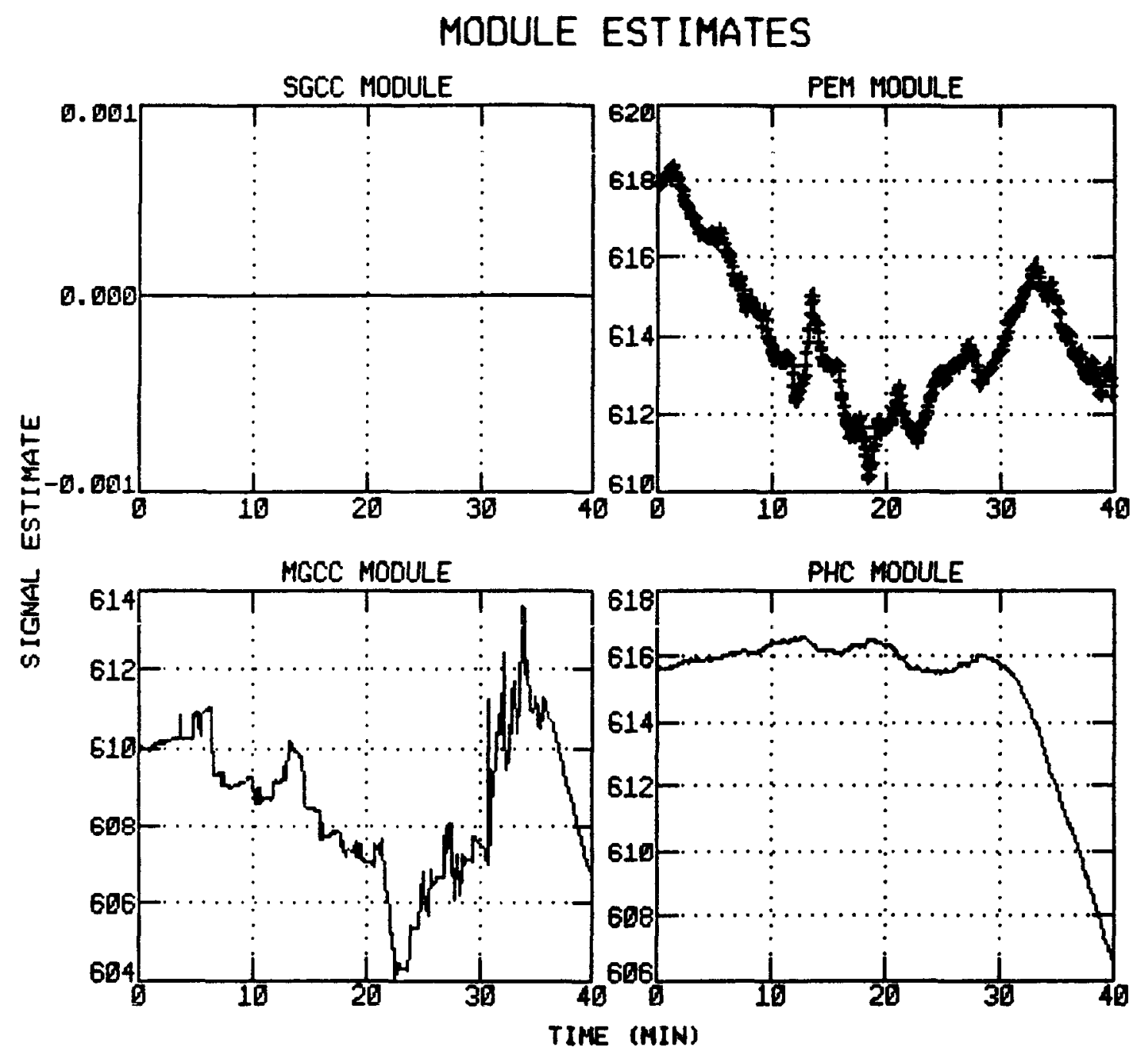

Figure 5.26. Superheater Steam Inlet Temperature Estimates. 
PROCESS HYPERCUBE COMPARISON

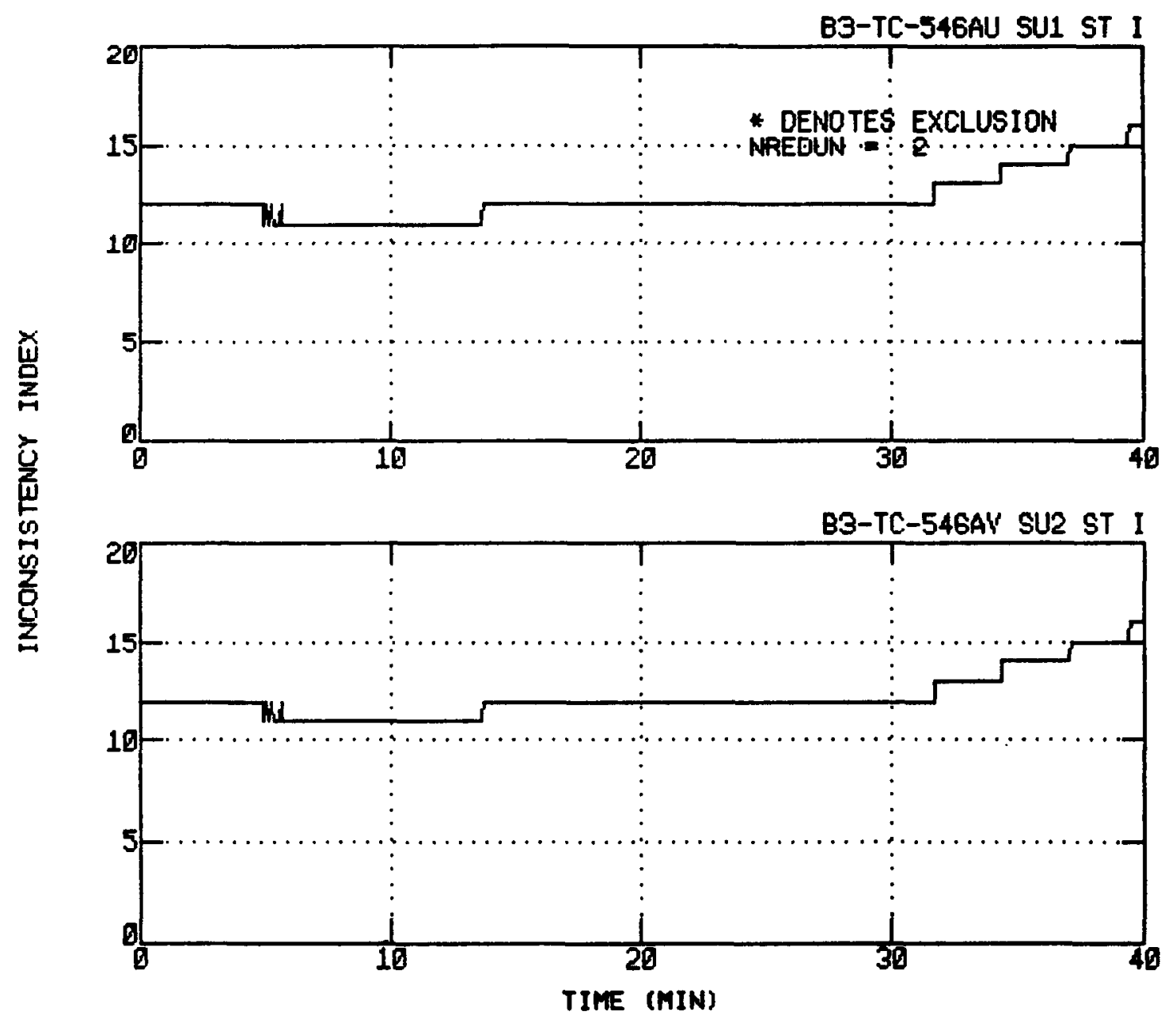

Figure 5.27. PHC Inconsistency Indices for the Superheater Steam Inlet Temperature Signals. 


\section{Section 6 \\ SIGNAL, ANOMALY DETECTION MODULE (BNI)) \\ 6.1. Introduction}

A signal recorded from process instrumentation is said to have an anomaly, if during steady-state operation, the deviation in the level of the signal, its root-meansquare (RMS) value, or its statistical distribution exceeds a preset tolerance. This deviation could be an unacceptable increase or a decrease in the quantity being monitored. Anomaly in a signal may be characterized by wideband or singlefrequency noise, bias error, pulse-type error, nonsymmetric distribution, or a change in the signal bandwidth. Various signatures can be easily computed from data samples and compared against specified threshold values. In real processes, pulses can appear with different time widths, and at different rates of change of the signal. Thus, in characterizing an anomaly as a pulse-type, the fastest pulse width is constrained by the signal sampling interval. For example, if a signal is sampled at $100 \mathrm{~Hz}$, we will not be able to detect pulses occurring at $\mathrm{kHz}$ rates. Discussion with utility and Combustion Engineering personnel indicated that it is not practical to detect pulses having a narrow time width.

A new module has been developed to detect, isolate and characterize possible anomalies in plant signals. 'The bias and noise detection (BNI)) module performs the computations at three levels. These are (1) anomaly detection, (2) sensor isolation, and (3) anomaly characterization. Several time-domain quantities are calculated to identify a signal anomaly. These are mean value, standard deviation, amplitude probability density function (APDF), skewness, flatness or kurtosis, level crossings, and others. The possible presence of a common anomaly in signals is determined in the second level. Anomaly characterization such as the instant of anomaly occurrence, duration of anomaly, and classification into bias, pulse or noise-type is 
made in level three using a modification of the cumulative sum (CUSUM) method 114]. The user has the option of checking the various indices against their nominal values, or the option of anomaly detection and characterization when an anomaly is suspected in a signall 15].

The methodology is presented in Sect. 6.2. The description of the computer algorithm is given in Sect. 6.3. Section 6.4 presents a discussion of the results of application of the BND module. Some concluding remarks are given in Sect. 6.5. The BND module has been tested using both simulated data and operational plant data.

\subsection{Methodology}

\subsubsection{Anomaly Detection and Isolation}

The detection of abnormal behavior in a particular signal is carried out by the analysis of quantities that represent the actual status of the corresponding instrument output. These quantities are then compared with nominal values to flag a sensor as being suspicious. 'The calculated signatures represent the sensor in three different domains: deterministic, statistical and frequency. The estimation of each quantity is presented below.

Let $\left(\mathrm{x}_{1}, \mathrm{x}_{2}, \ldots, \mathrm{x}_{\mathrm{N}}\right)$ be $\mathrm{N}$ samples of a signal $\mathrm{x}(\mathrm{t})$. This defines a block of data.

A. Mean value

The mean value is calculated as

$$
m=\frac{1}{N} \sum_{k=1}^{N} x_{k}
$$

B. Root-mean-square (RMS) value

The RMS value is given by

$$
\operatorname{RMS}=\left(\frac{1}{N} \sum_{k=1}^{N} \lambda_{k}^{2}\right)^{1 / 2}
$$


C. Standard deviation

$$
0=\left(\mathrm{RMS}^{2}-\mathrm{m}^{2}\right)^{\mathrm{I} / 2}
$$

D. Amplitude probability density function (APDF)

The amplitude probability density function is characterized by (in most cases) the mean value, variance $\left(0^{2}\right)$, skewness and the flatness (or kurtosis) of the signal.

in order to detect the presence of nonsymmetric behavior, a higher central moment, the skewness, is calculated. This is defined by [16]

$$
\mathrm{G}_{1}=\frac{\mu_{3}}{\mathrm{o}^{3}}
$$

where

$$
\begin{aligned}
& \mu_{3}=\text { the third central moment, } \\
& \sigma=\text { the standard deviation, }
\end{aligned}
$$

and it is evaluated as

$$
a_{1}-\left\{\frac{1}{N} \sum_{k=1}^{N}\left(x_{k}-m\right)^{3}\right\} / o^{3}
$$

For a Gaussian or a symmetric APDF, $\mathrm{G}_{1}$ is zero.

For checking the periodicity or a nonGaussian distribution, the fourth moment or the flatness is calculated. This is defined by [16]

$$
\mathrm{G}_{2}=\frac{\mu_{4}}{3 \mathrm{o}^{4}}
$$

where

$\mu_{4}$ is the fourth central moment and it is calculated as

$$
\mathrm{G}_{2,}=\left\{\frac{1}{\mathrm{~N}} \sum_{\mathrm{k}=1}^{\mathrm{N}}\left(\mathrm{x}_{\mathrm{k}}-\mathrm{m}\right)^{4}\right\} \frac{1}{\left(3 \mathrm{o}^{4}\right)}
$$


For a Gaussian APDF, $G_{2}$ is equal to 1 , and for a uniform distribution $\mathrm{G}_{2}$ is equal to 0.6 .

E. Zero crossings

In order to detect changes in the signal bandwidth, the number of level crossings around the signal DC value is calculated. The level crossing rate (or the zero crossing rate) may be related to the signal time constant. For a second order system, where the two time constants are proportional to each other, it has been shown [17] that

$$
\mathrm{t}=\frac{\mathrm{C}}{\mathrm{N}_{\mathrm{a}}} \text {, }
$$

where

$\tau=$ the signal time constant,

$\mathrm{C}=$ a proportionality constant,

$\mathrm{N}_{\mathrm{zc}}=$ the number of zero (DC level) crossings per unit time (sec).

The isolation of a suspicious sensor is performed by comparing the signatures defined above with nominal values. These nominal values are either specified by the user or learned by the program. Intervals of goodness are created using these nominal values and the corresponding tolerances are set for each signal and feature.

Any sensor for which the calculated quantity falls outside the allowable interval is considered suspect. Once these sensors have been isolated, an analysis of cross information from multiple signals is performed to check the presence of common anomalies. A flow chart showing a simplified version of the detection, isolation and cross analysis method is given in Fig. 6.1.

Other parameters are also calculated even though they are not used at this stage for detection. These may represent abnormal status of the sensor and shuuld be 
studied for each particular system, included as part of the decision process. These quantities are associated with the first derivative or the rate of change of the original signal and are calculated using the forward finite difference

$$
\operatorname{ROC}_{k}=\frac{\lambda_{h 11}-\lambda_{k}}{\Delta t},
$$

where

$$
\begin{aligned}
& \operatorname{ROC}_{k}=\text { the rate of change at sample instant } k, \\
& x_{k+1}=\text { the value of the signal at sample instant } k+1, \\
& x_{k}=\text { the value of the signal at the sample instant } k, \\
& \Delta t=\text { time interval between consecutive samples. }
\end{aligned}
$$

Using the $\mathrm{ROC}_{\mathrm{k}}$ values for a block of data, its $\mathrm{RMS}$ value, variance, amplitude probability density function, skewness, flatness and the number of zero crossings are calculated. The algorithm displays these quantities, and compares them against specified nominal values.

At this stage, the BNI) module displays the signal status to the operator, and an anomaly status is sent to the system executive. If desired, signal anomaly characterization will be made in the next step.

\subsubsection{Anomaly Characterization and Classification}

Once a sensor has been isolated as suspicious, the anomaly characterization and classification as to the type of anomaly are performed. The characterization is made using a modified cumulative sum (CUSUM) algorithm. The classification takes into consideration time duration, and relations among mean, peak and rms values of the signal. 


\subsubsection{Modified CUSUM Algorithm}

The cumulative sum (CUSUM) approach of Page and Hinkleyl14] is used as the basic method for fault detection. The problem is to detect the change in the mean value of a sequence of independent Gaussian random variables. Let $\left\{x_{n}\right\}_{n}$ be a sequence of observations defined by

$$
x_{n}=m_{n}+e_{n}
$$

where $e_{n}$ is a white noise process with variance $o^{2}$ and

$$
m_{11}=\left\{\begin{array}{l}
m_{0} \text { if } n<r-1 \\
m_{1} \text { if } n>r
\end{array}\right.
$$

where $r$ is the instant at which the signal changes its level.

The procedure to compute the cumulative sum for detecting an increase in the mean value is as follows.

$$
\begin{aligned}
U_{0} & =0 \\
U_{h} & =\sum_{j=1}^{k}\left(x_{j}-m_{v}-v_{m} / 2\right) \\
U_{m ! n} & =\min _{0<j<k} U_{k}
\end{aligned}
$$

provide the alarm when

$$
\mathrm{U}_{\mathrm{k}}-\mathrm{U}_{\mathrm{nun}}>\mathrm{H}
$$

Both $\mathrm{H}$ and $\mathrm{v}_{\mathrm{m}}$. are positive values to be determined. $\mathrm{v}_{\mathrm{m}}$ is called the reference value, and is the maximum variation that we want to allow in the mean value of the signal, and $\mathrm{H}$ is the decision interval given by [18]

$$
11=-\frac{o}{v_{m} / 2} \ln \alpha
$$

where $a$ is the probability of false alarms. The effect of subtracting the reference value in the summation is to produce a downward slope of the CUSUM, while the 
signal is inside the level of acceptance (see Figs. 6.2 and 6.3). If the signal exceeds the reference value, a positive tendency persists in the CUSUM graph. As soon as the actual CUSUM exceeds its minimum by more than a specified amount, $H$, an alarm is triggered.

The time instant of the jump can be estimated by either the instant $T_{i}$ at which the alarm is given or the instant $T_{m}$ at which $U_{k}$ has its minimum value $U_{\min }$. We will define $T_{m}$ as the time instant at which a change in the level of the signal occurs. If a decrease in the signal level is to be tested at the same time, another detector will be used simultaneously. This is defined as follows.

$$
\begin{gathered}
S_{0}=0 \\
S_{k}-\sum_{j=1}^{h}\left(y_{j}-m_{0}+v_{m} / 2\right) \\
S_{1 n, d s}=\max _{0<j<h} S_{h}
\end{gathered}
$$

and provide the alarm when

$$
S_{\text {md }}-S_{h}>H
$$

An example of variation in the CUSUM algorithm is shown in Fig. 6.4.

Once the jump is detected, the CUSUM $U_{h}\left(\right.$ or $\left.S_{h}\right)$ continues to change with a positive (or negative) slope, until it attains a maximum value $U_{\max }$ (or minimum $S_{\min }$ ) (see Fig. 6.3). At that point, the tendency changes again to negative (positive), indicating that the signal is now inside the reference interval $\mathrm{v}_{\mathrm{m}}$, and it has returned to normal values. If we set a new alarm as

$$
\begin{gathered}
U_{m \text { ma }}-U_{h}>H \\
\text { or } \\
S_{h}-S_{m I n}>H
\end{gathered}
$$


it is possible to determine the duration ( $\left.\mathrm{T}_{\mathrm{d}}\right)$ of the anomaly according to

$$
\begin{gathered}
\mathrm{T}_{\mathrm{d}}=\mathrm{T}\left(\mathrm{U}_{\max }\right)-\mathrm{T}\left(\mathrm{U}_{\min }\right) \\
\text { or } \\
\mathrm{T}_{\mathrm{d}}=\mathrm{T}\left(\mathrm{S}_{\min }\right)-\mathrm{T}\left(\mathrm{S}_{\max }\right)
\end{gathered}
$$

Thus, with some modifications of the original CUSUM algorithm, we are able to determine the instant at which the anomaly begins as well as its time duration.

It is evident that one of the important advantages of Page-Hinkley test, besides its simplicity, is its capability to archive past history of the observed process in terms of $U_{\min }\left(S_{\max }\right)$. In most practical cases, the processing is done by computing blocks of data with fixed length $N$, so the value of $U_{0}\left(S_{0}\right)$ at the beginning of each block is not zero (except for the first block) in order to keep this feature. In this case we reset these values to

$$
\begin{aligned}
\mathrm{U}_{0}= & \mathrm{U}_{\mathrm{N}}-\mathrm{U}_{\min } \\
& \text { and } \\
\mathrm{S}_{0}= & \mathrm{S}_{\max }-\mathrm{S}_{\mathrm{N}}
\end{aligned}
$$

A flow chart showing the logic of the modified CUSUM algorithm is shown in Fig. 6.5.

\subsubsection{Anomaly Classification}

The classification is performed for the following types of anomalies.

- Jump or persistent change in the mean value.

- Pulse or spurious changes in the signal level.

- Sinusoidal noise. 
A first classification is made taking into account the time duration $T_{d}$ of the anomaly calculated by the mudified CUSUM algorithm. In this sense, any anomaly lasting for more than a threshold time $T_{h}$ is classified as a jump. This threshold time $\mathrm{T}_{\mathrm{h}}$ must be set in advance fur each particular sensor taking into account the sensor bandwidth. If $T_{d}$ is less than ' $T_{h}$, then the following analysis is performed.

- Calculate the actual MEAN value of the entire block, and subtract this frum the data.

- Calculate the average peak value of the entire data block.

- Calculate the RMS value of a sinusoidal-type signal as 0.707 times the average peak value.

- Calculate the actual RMS value of the entire block.

- If the actual RMS value is within a specified fraction of the RMS value of a sinusoidal-type signal, then the anomaly is classified as a SINUSOIDAL.

- If the actual RMS value is less than a fraction of the RMS value of a sinusoidal-type signal, then the anomaly is classified as a PULSE. A pulse-type anomaly has a small RMS value compared to that of a sinusoidal-type anomaly.

- If the above tests are not satisfied, then the anomaly is declared to be a JUMP. The signal has a sustained change in its level.

The decisions limits must be set by the user. 


\subsection{Description of Computer Software System}

The software is organized in two main programs. Each program calls different subroutines. All the programs and subroutines are written in the FORTRAN language. The Microsoft FORTRAN 77 Compiler V3.3 was used to compile the source code, and the corresponding linker to produce the final form of the programs. The package SASLGRAF was used to generate graphical representation of results.

The first program called INPUTDAT is responsible for generating a file TEMP.DAT containing information about the data file to be processed and the nominal values for the quantities defined in Sect. 6.2.1 for each particular signal stored in the data file. The nominal values can either be entered by the user from the keyboard, or can be learned by the program. When this initial step is completed a second program called JPNMAIN is invoked. This program reads both the TEMP.DAT file and the data file containing the signals to be processed, and creates a CUS.DAT file in which the results of the modified CUSUM algorithm are stored. Figure 6.6 shows an overall lugic chart of the program-system described above.

The program JPNMAIN performs all the calculations and makes decisions about the status of each signal. The program calls the following subroutines: READ, APDF, CUSUM and PLO'T. A logic flow chart of JPNMAIN is shown in Fig. 6.7.

A description of each prompt and the user response are presented below.

1. "ENTER NAME OF 'IIIL INPUT IDA'TA FILL"

This input requires the name of the data file containing the signals to be processed. Each record in this data file contains up to six different measurements.

2. "ENTER NO. OF SIGNALS IN THIS DATA FILE, NSIGNL =" This input requires the number of signals in each record. 
3. "ENTER IDATA $=1$ IF INTEGER DATA FILE, IDATA $=2$ IF FLOATING POINT

DATE FILE, IDATA $=$ "

IDATA $=1$ indicates that the data file contains integer values and IDATA $=2$ indicates that the data file contains real values. If IDATA $=1$ the following will appear.

4. "ENTER NO. OF BITS OF THE ANALOG TO DIGITAL CONVERTER, NBITS $="$

This input requires the number of bits of the analog-to-digital converter (ADC) used for data sampling.

5. "ENTER VOLTAGE RANGE OF THE ANALOG-TO-DIGITAL CONVERTER, VOLRAN $="$

This input requires the range, in volts, used by the ADC. This value along with that entered in the previous prompt are used to convert the integer stored in the data file to real numbers using

$$
x_{k}=1_{x k} * \frac{\text { VOLRAN }}{2^{\text {NBITS }}},
$$

where $\mathbf{x}_{\mathrm{k}}$ is a real number,

$\mathrm{I}_{\mathrm{xh}}$ is an integer number.

6. "ENTER NO. OF BLOCKS TO PROCESS, NBLOCK = "

For practical purposes, the processing is done by using a fixed number of data points, designating this as a block. Thus the input requires the number of blocks to be processed.

7. "ENTER NO. OF POINTS PER BLOCK TO ANALYZE (LT. 1000), $\mathrm{N}=$ " This input calls for the length of each block. The maximum length allowed is 1000 points. 
8. "ENTER NO. OF POIN'IS TO SKIP, NSKIP" = "

This in put requires the number of points that the user wants to skip at the beginning of each data block.

9. "ENTER NO. OF BINS, NBIN. ="

NBIN is the number of intervals for computing amplitude probability density function.

10. "ENTER SAMPLE INTERVAL (SEC), DELT = "

DELT is the time interval between consecutive samples (seconds). This is the inverse of the sampling frequency.

11. "ENTER IFLAG $=1$ IF YOU WANT TO LEARN NOMINAL VALUES, IFLAG $="$

If the answer is 1 then the program will call the LEARNING subroutine in order to calculate the nominal values of the following quantitites: $\mathrm{DC}$ value, root-mean-squared value, standard deviation, and time constant. The learning will be performed for all the signals stored in the data file and the following information will be requested.

12. "FOR LEARNING ENTER NO. OF BLOCKS, NBLCK1 = "

This in put is the number of blocks to be evaluated during the learning process. The length of each block will be equal to the value entered in 7 .

If the answer to 10 is different from 1 the following will be requested for all the signals stored in the datafile:

13. "ENTER FOR SIGNAL NO.1

NOMINAL MEAN VALUE $="$ 
14. "ENTER FOR SIGNAL NO.I

NOMINAL STANDARD DEVIATION = "

15. "ENTER FOR SIGNAL NO.1

NOMINAL TIME CONSTANT $($ SEC $)=$

The inputs 13 to 15 require the nominal values of the mean value, standard deviation and time constant respectively, corresponding to the signal number I in the data file. I varies from 1 to NSIGNL which is the value entered in 2.

16. "WOULD YOU LIKE TO PERFORM FULL ANALYSIS (IFULL=1) OR ONLY NUMERICAL (IFULL $=0)$ ? IFULL $="$

If the answer is IFULL $=0$ only the quantities defined in Sect. 6.2 .1 will be calculated and no further analysis will be carried out. If the answer is IFULL $=1$, the entire calculation, including anomaly classification will be performed. For IFULL $=1$ the following questions must be answered.

17. "ENTER FOR SIGNAL NO. 1

PROBABILITY OF FALSE ALARMS IN ANOMALY DETECTION =" This input requires the probability of false alarms, $a$, for performing the modified CUSUM algorithm described in Sect. 6.2.2.1.

18. "ENTER FOR SIGNAL NO. 1

TOLERANCE, IN PERCENT, FOR THE MEAN VALUE ="

This input calls for the tolerance, in percent, allowed in the mean value for the 1-th signal in the data file. This value will be used in the modified CUSUM algorithm in order $t$ callulate the reference value $v_{m}$ as

$$
v_{m}=\frac{1) C T O 1}{100} * \text { DCNOM }
$$

where 
DCNOM is the nominal DC value entered in 13 or calculated by the LEARNING subroutine, DCTOL is the tolerance in the DC value. The same value DCTOL will generate the goodness interval to be used in signal anomaly detection and isolation.

19. "ENTER FOR SIGNAL NO.I

UPPER LIMIT FOR STANDARD DEVIATION ULSTD (FACTOR) $=$ LOWER LIMIT FOR S'TANDARD DEVIATION LLSTD $($ FACTOR $)="$

This input requires the bounds using the actual standard deviation of the l-th signal. Both standard deviations greater than the upper limit and smaller than the lower limit are considered as abnormal. The bounds are set as

$$
\begin{aligned}
& \text { S'IDMX }(1)=\operatorname{STDNOM}(1) * \operatorname{ULSTD}(1) \\
& \text { S'IDMN }(1)=\operatorname{STDNOM}(1) * \operatorname{LLSTD}(1)
\end{aligned}
$$

where

STDMX = the maximum STD value allowed, STDNOM = the nominal STD value (answer to 14),

ULSTD = the upper linit, a factor of STD (answer to 19), STDMN = the minimum STD value allowed, LLSTD $=$ the lower limit, a factor of STD (answer to 19).

20. "ENTER FOR SIGNAL NO.1

NOMINAL TIME CONS'TANT $($ SEC $)="$

This input calls for the numinal time constant of the 1-th signal stored in the data file. This is going to be used in the faulty sensor detection and isolation process.

An example of the described prompts and corresponding responses are shown in Table 6.1. All the information entered and/or calculated by the LEARNING 
subroutine will be stored in the file TEMP.DAT and will be read by the program JPNMAIN. This program performs the detection, isolation, characterization and classification process.

\subsection{Results of Application of the BNI) Mlodule}

The anomaly detection algorithm was applied to four different types of simulated data. The first was a normal signal, the second was a signal with pulsetype anomaly, the third was a signal with single frequency sinusoidal noise, and the fourth was a signal with changing levels. Sample plots of each signal are shown in Figs. 6.-8a, 9a, 10a and $11 \mathrm{a}$, respectively. All the signals have a nominal mean value equal to 200 , and nominal standard deviation equal to 2 . The number of points per block is 1000 , and tulerances and other parameters used in the analysis are given in Table 6.1.

The numerical results, corresponding to the calculated quantities defined in Sect. 6.2.1 are shown in Tables 6.2-6.5 for each of the four signals. They compare very well with the expected results. The signals with an anomaly have a higher standard deviation compared to the normal signal. The value of skewness is close to zero for thuse signals for which the anomaly is symmetric with respect to the mean value (normal, sinusoidal and jump-type). The skewness is large and is approximately two for pulse-type anomaly. The flatness value is close to one for the Gaussian cast (normal signal) and close to 0.6 for periodic cases (periodic jump and sinusoidal signals). If the signal is stationary, we found that as many as 10,000 points should be used for these statistical calculations.

The fault isolation and cross analysis results are shown in Table 6.6. All the signals have mean values within the allowable (specified) range of $\pm 2.5 \%$ of the nominal mean value. The signal with sinusoidal-type error has a standard deviation greater than 2 times the nominal value. The range of allowable change in the signal 
time constant is $\pm 10 \%$ of the nominal value. Signals with sinusoidal noise and periodically changing level have increased time constants, because of adding lowfrequency components. Table 6.8 summarizes the results at the end of the processing of all data blocks.

The characterization and classification of the anomalies are summarized in Table 6.7. A binary function characterizing the signal anomaly is defined as follows.

$$
\begin{aligned}
& \mathrm{B}(\mathrm{t})=1 \quad \text { ("positive" anomaly) } \\
& \mathrm{B}(\mathrm{t})=0 \quad(\text { no anomaly } \quad) \\
& B(l)=-1 \quad \text { ("negative" anomaly) }
\end{aligned}
$$

"Positive" anomaly refers to an increase relative to the mean value, and "negative" anomaly refers to a decrease relative to the mean value. Figures 6.-9b, $10 \mathrm{~b}$ and $11 \mathrm{~b}$ show the function $\mathrm{B}(\mathrm{t})$ for each of the signals that show abnormal behavior and Table 6.7 summarizes the results from the anomaly classification process for each signal. The number of pulses or jumps are calculated using the CUSUM algorithm. In addition, the anomaly is identified using the time duration of the anomaly, the reference value, and the probability of false alarms.

Plots of the amplitude probability density functions for both amplitude and rate of change are shown in Figs. $6.8 \mathrm{~b}$ and $6.8 \mathrm{c}$ for the normal case; $6.9 \mathrm{c}$ and $6.9 \mathrm{~d}$ for the signal with pulse-type anomaly; $6.10 \mathrm{c}$ and $6.10 \mathrm{~d}$ for the signal with sinusoidaltype anomaly; and $6.11 \mathrm{c}$ and $6.11 \mathrm{~d}$ for the signal with jump-type anomaly. Whenever the signal contains a periodic-type anomaly, the amplitude probability density (APD) shows a bimodal distribution. The APD functions of rate of change signals show a symmetric behavior. In practice it is necessary to use a large number of samples, (in the 10,000 range) and up to 200 bins for APD calculation. 
The BND module is also applied to the PWR plant operational data. Figure 6.12a is a sample plot of the pressurizer pressure signal during start-up. The BND module suggests a possible pulse-type anomaly in the signal as shown in Fig. 6.12b. The APD functions of the signal and its rate of change are plotted in Figs. 6.13a and $6.13 \mathrm{~b}$, respectively. Figure $6.14 \mathrm{a}$ is a sample plot of the cold leg temperature signal during start-up. The binary error indices are shown in Fig. 6.14b, and indicate a possible pulse-type (one sample) and bias-type anomalies (duration greater than one sample) in the signal. The corresponding APD functions of the signal and its rate of change are plotted in Figs. 6.15a and 6.15b respectively. The bimodal characteristic of the APD may indicate a low-level fixed-frequency fluctuation in the signal. The "anomalies" indicated above are caused by the nature of the start-up transient and there are no persistent errors exhibited in the signals.

\subsection{Remarks on the BND Module}

The algorithm for signal anomaly detection performs a quick comparison of the actual signal indices with their nominal values. If necessary, a detailed characterization and fault isolation may be made. 'The application of this module to a plant subsystem requires a learning phase to determine the normal behavior of the signals. The choice of the modified CUSUM algorithm for anomaly detection performed very well with the improvements incorporated in our algorithm. The success of the characterization depends on the values of $\mathrm{v}_{\mathrm{m}}$ and $\mathrm{H}$, which are related to the probability of false alarms and time delays in the detection. The simplicity and low computing power of the modified CUSUM algorithm makes it attractive for on-line processing.

The detection of pulse-type anomaly is limited by the data sampling rate. Pulses with duration less than twice the sampling interval cannot be detected effectively. Discussion with utility and Combustion Engineering personnel 
indicated that it is not practical to detect pulses having a narrow time width. If the detection of extremely narrow time width pulses is necessary, then a pulse-counting analog device must be used.

The bias and noise detection (BND) module may be used on-line to provide a preliminary indication of suspicious signal behavior with minimum user interface. 
TABLE 6.1

Computer Monitor Prompts Showing the Required Input Data. The Numerical Values are Entered by the User.

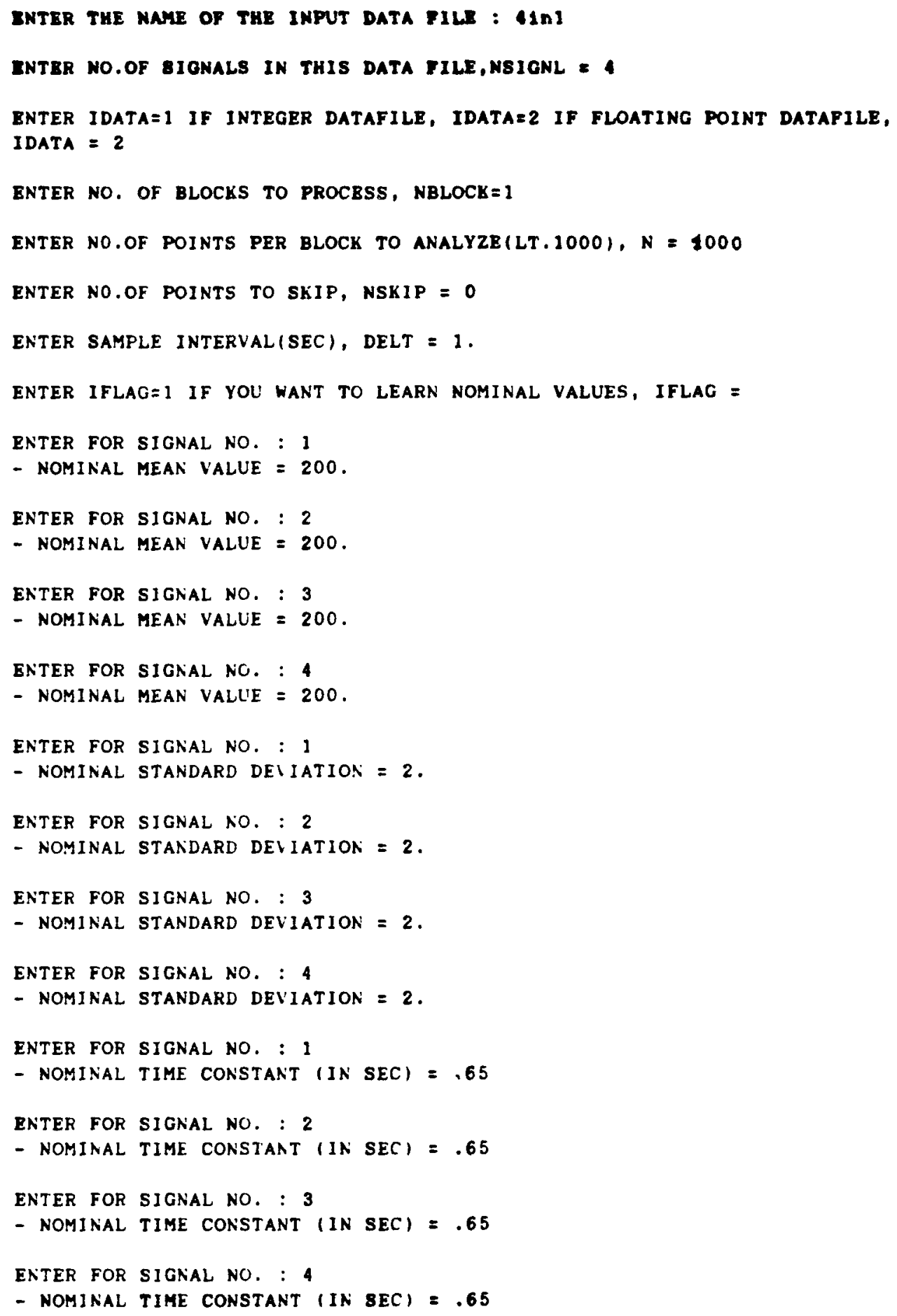




\section{TABLE 6.1 (continued)}

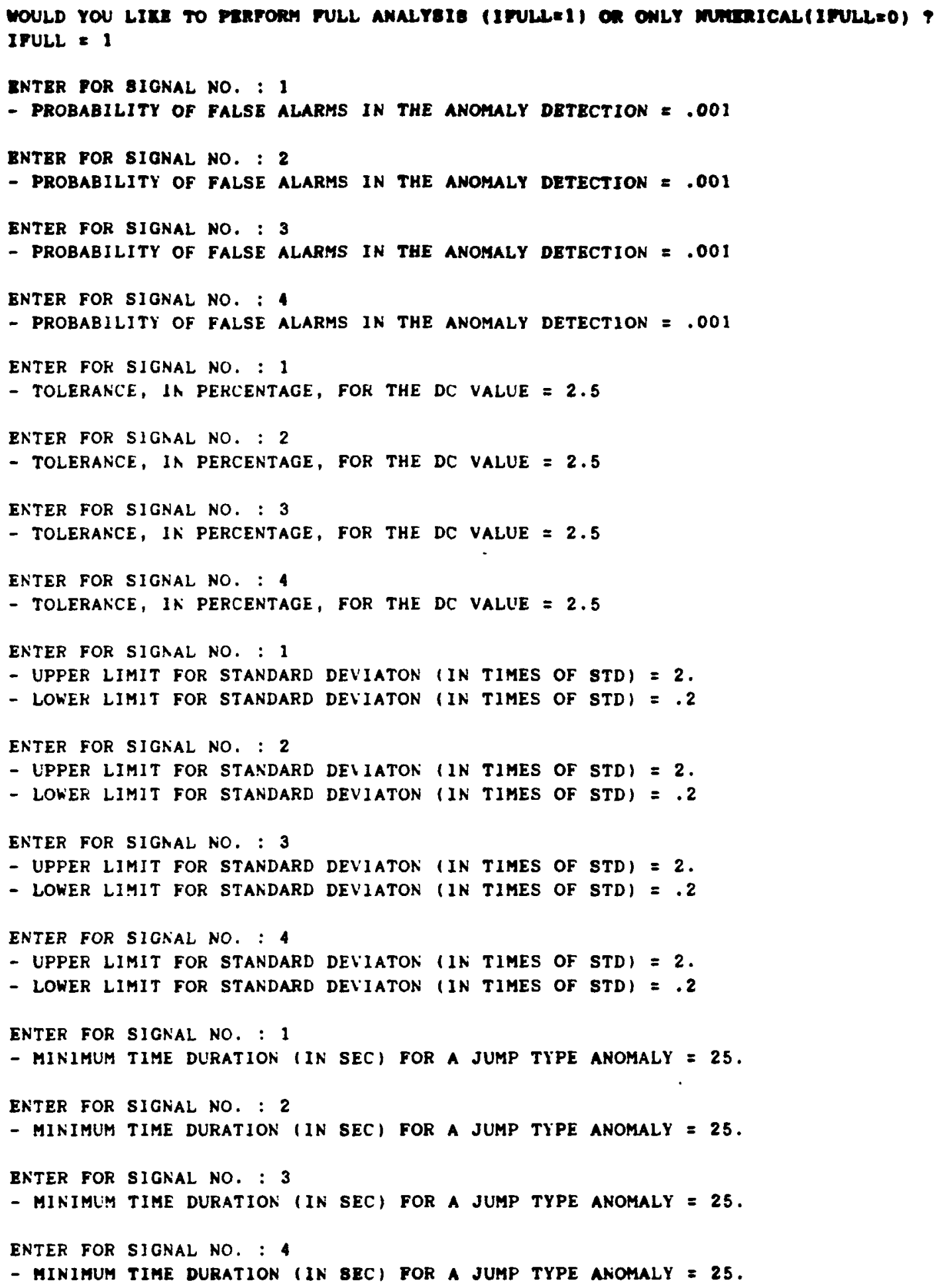


TABIE 6.2

Summary of Statistical Quantities for the First Signal in the Data File 4IN1. Normal Signal

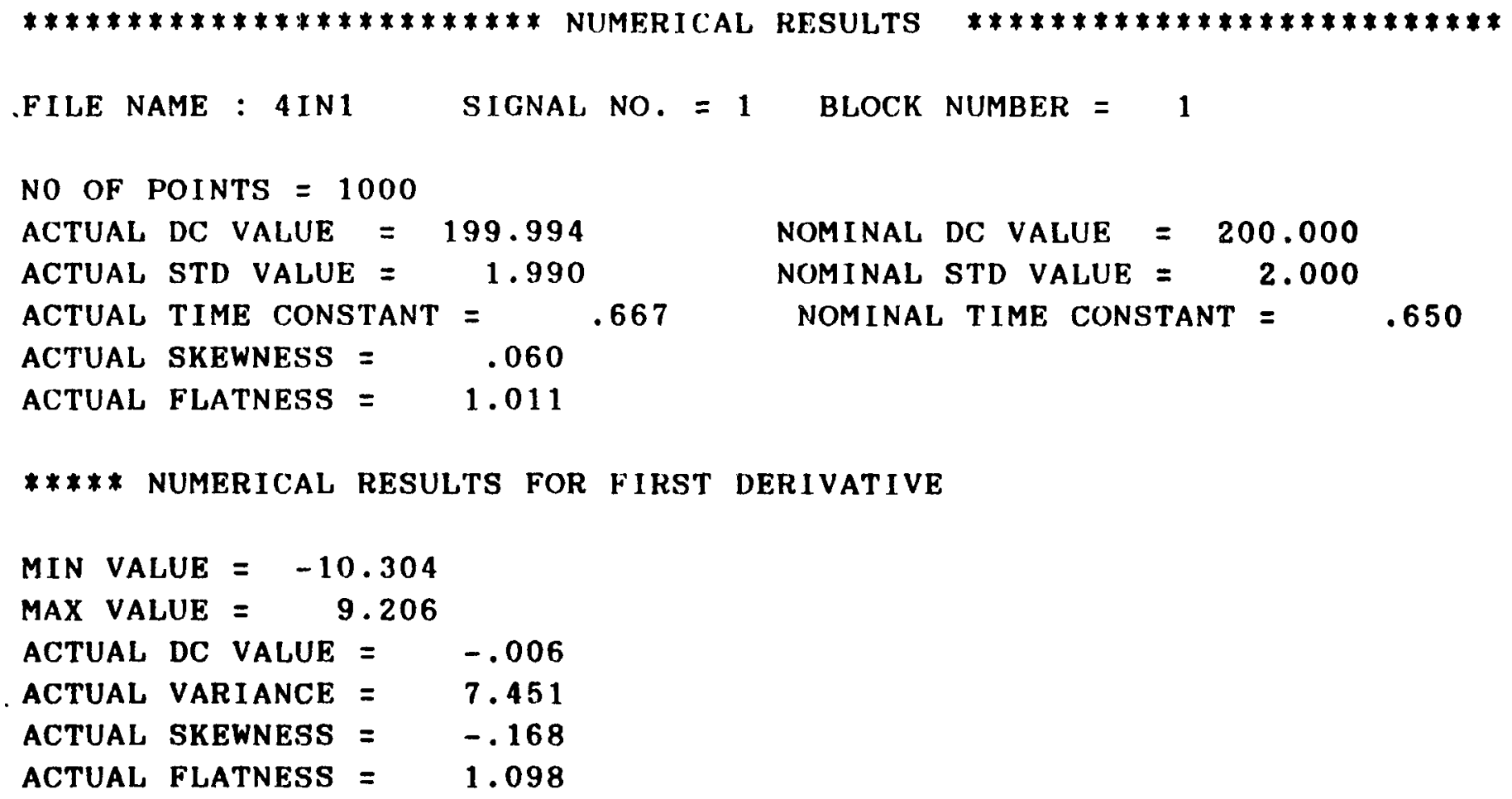




\section{TABLE 6.3}

Summary of Statistical Quantities for the Second Signal in the Data File 4IN1. Pulse-Type Anomaly.

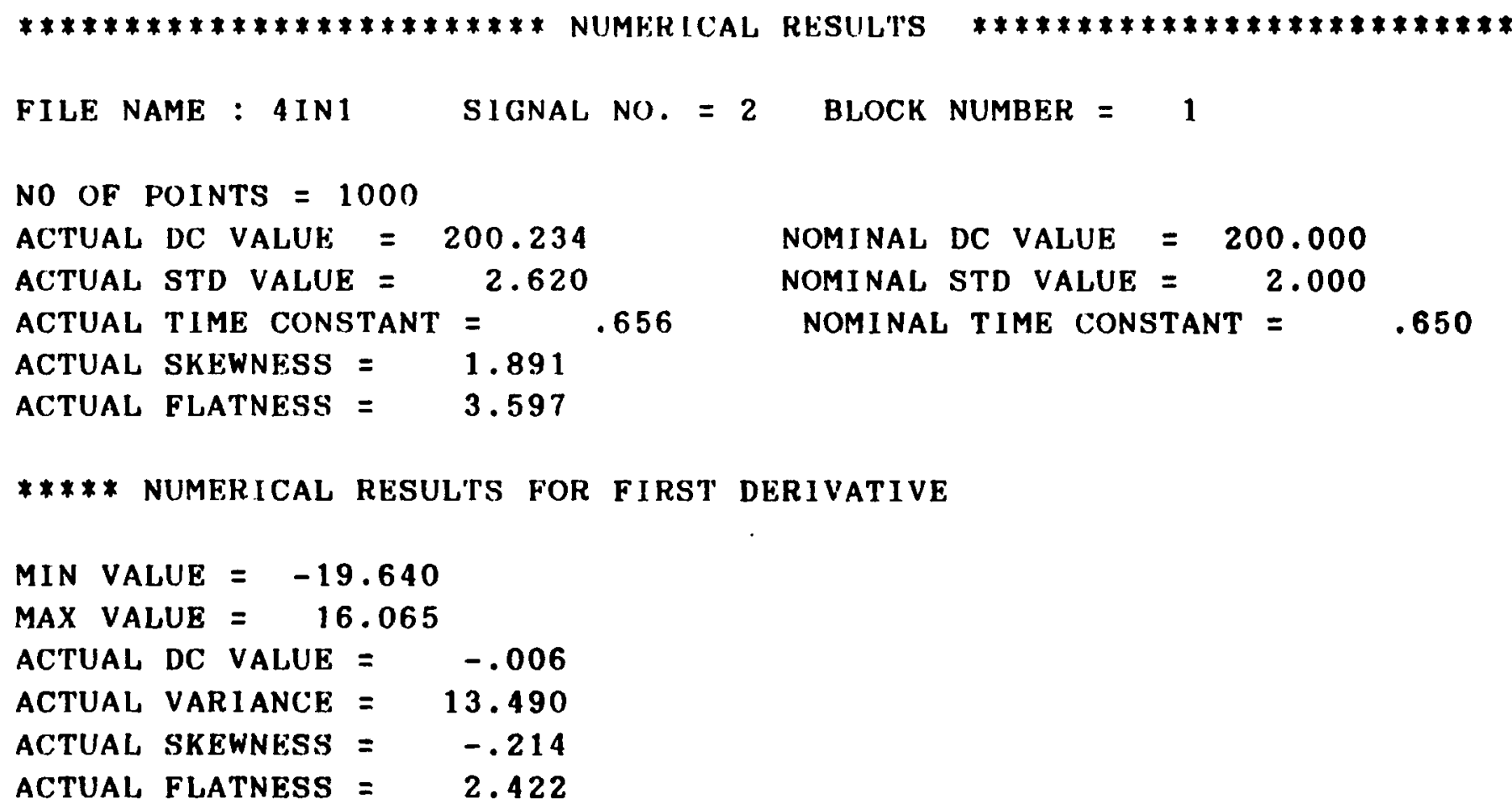

MIN VALUE $=-19.640$

MAX VALUE $=16.065$

ACTUAL DC VALUE $=-\mathbf{0 0 6}$

ACTUAL VARIANCE $=13.490$

ACTUAL SKEWNESS $=-.214$

ACTUAL FLATNESS $=2.422$

********************************************************************* 


\section{TABIE 6.4}

Summary of Statistical Quantities for the Third Signal in the Data File 4IN1. Sinusoidal-Type Anomaly.

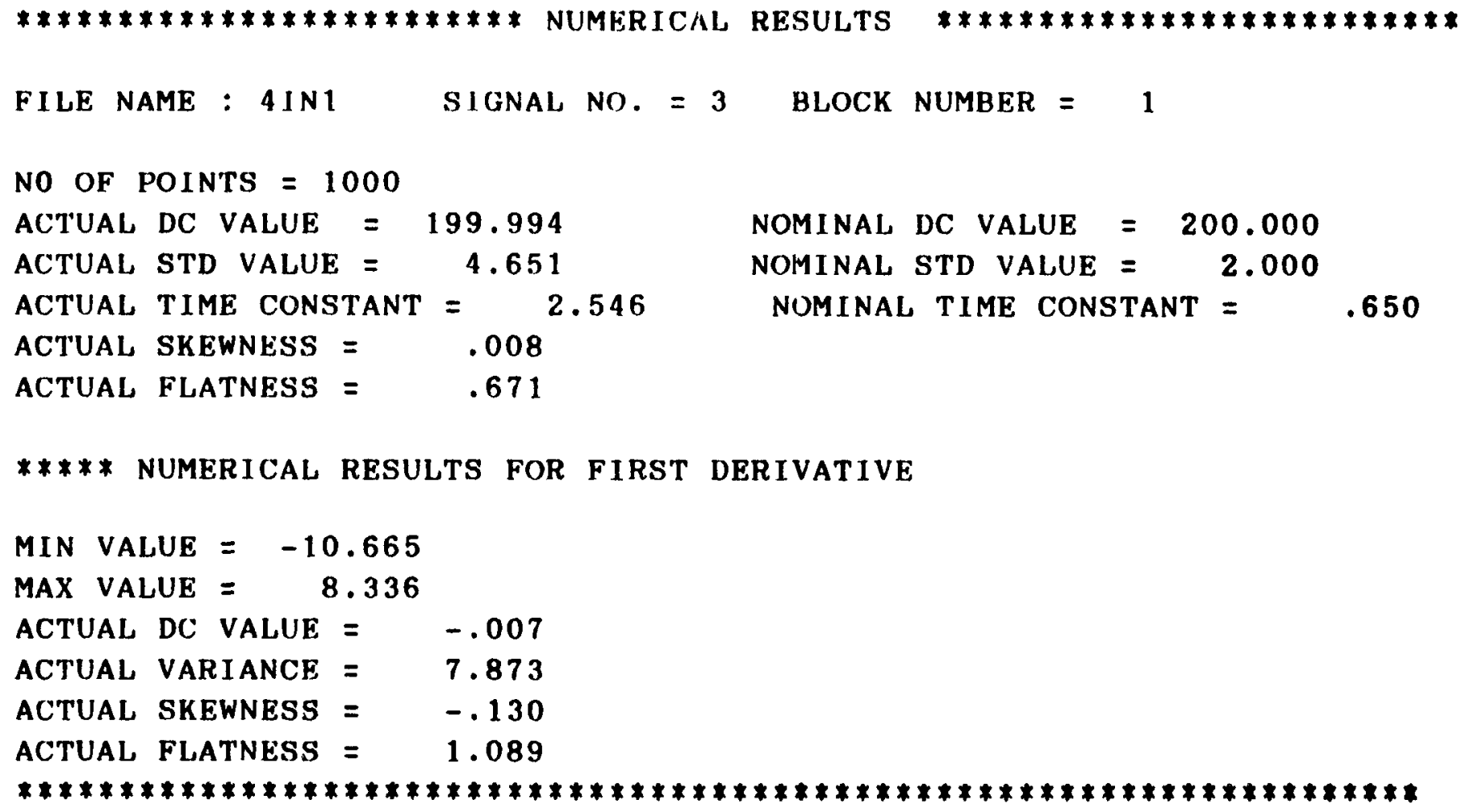




\section{TABLE 6.5}

Summary of Statistical Quantities for the Fourth Signal in the Data File 4IN1. Jump-Type (bias) Anomaly.

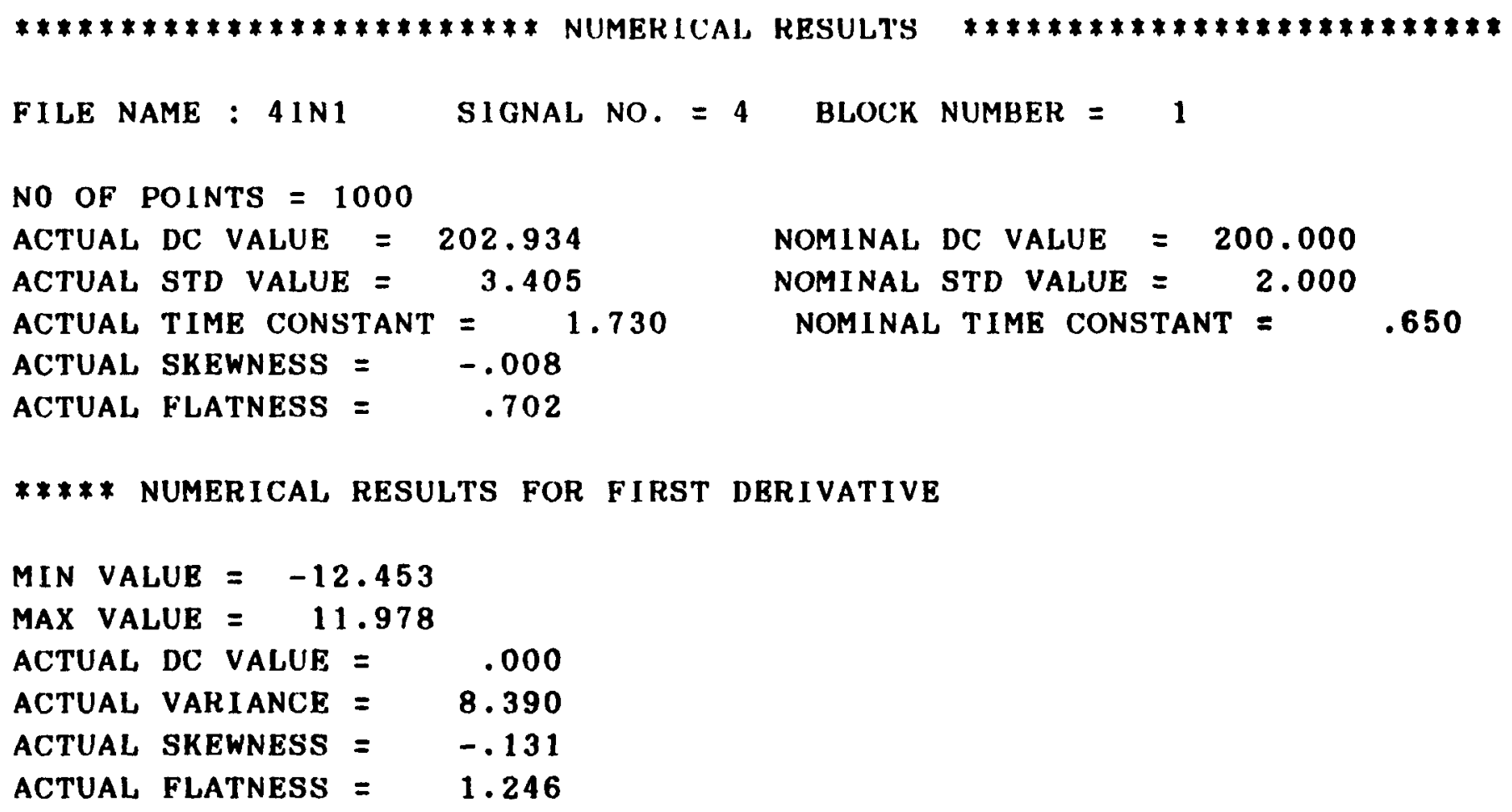




\section{TABLE 6.6}

Results of Cross Analysis of Possible Anomaly Among the Four Signals. This is a Short Summary of Signal Anomaly Display to the User.

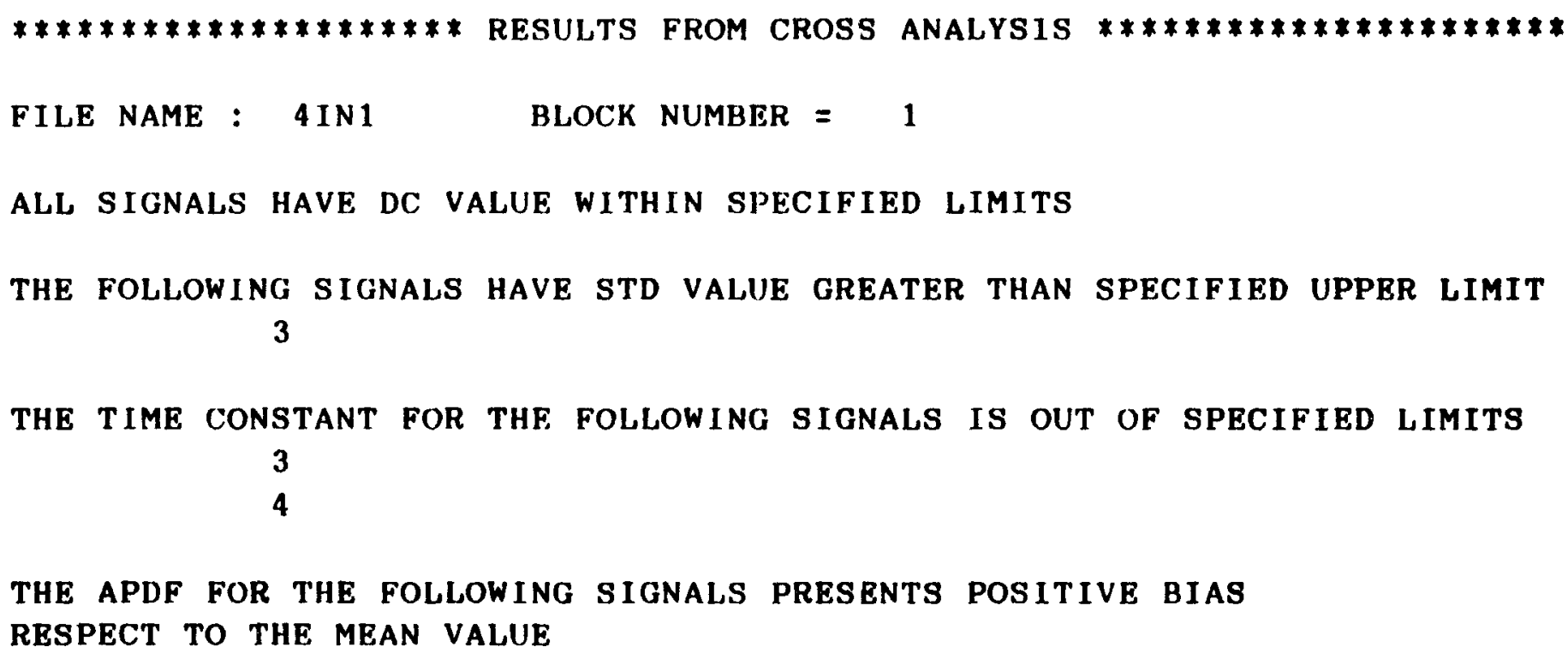


TABLE 6.7

Summary of CUSUM Analysis and Signal Anomaly Identification

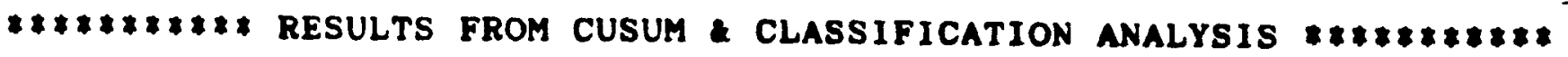

FILE NAME = 4INI SIGNAL NO. $=1$ BLOCK NO. $=1$

NO. OF POSSIBLE JUMPS $=0$

NO. OF POSSIBLE PULSES OR SINUSOIDAL TYPE ANOMALY $=0$

NO ANOMALY has been detected

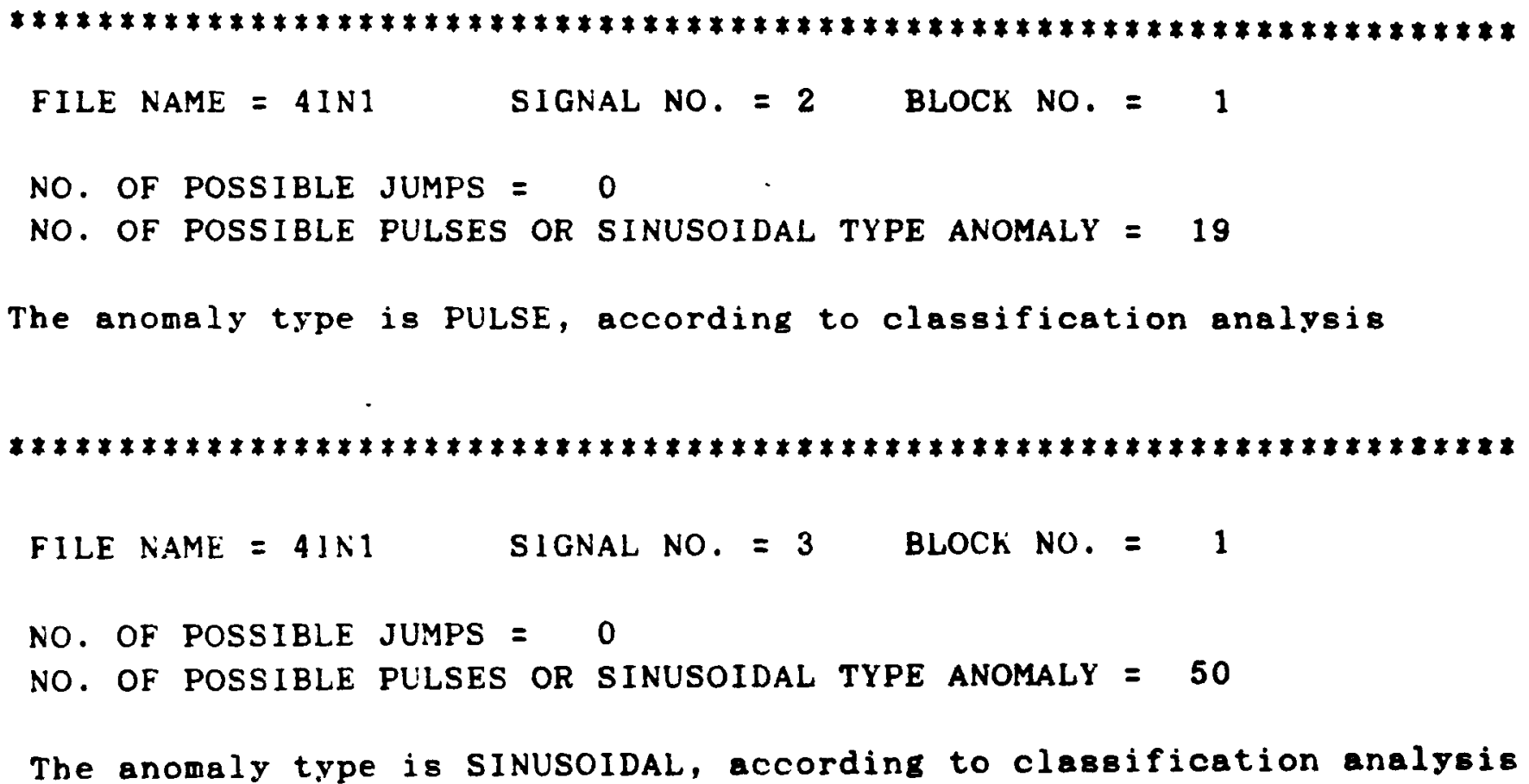

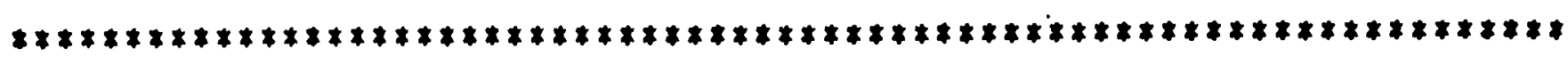

FILE NAME $=4$ IN 1 SIGNAL NO. $=4$ BLOCK NO. $=1$

NO. OF POSSIBLE JUMPS $=8$

NO. OF POSSIBLE PULSES OR SINUSOIDAL TYPE ANOMALY $=2$

The anomaly type is JUMP, according to classification analysis 


\section{TABIEE 6.8}

Summary of Results for all the Data Blocks Used in the Analysis

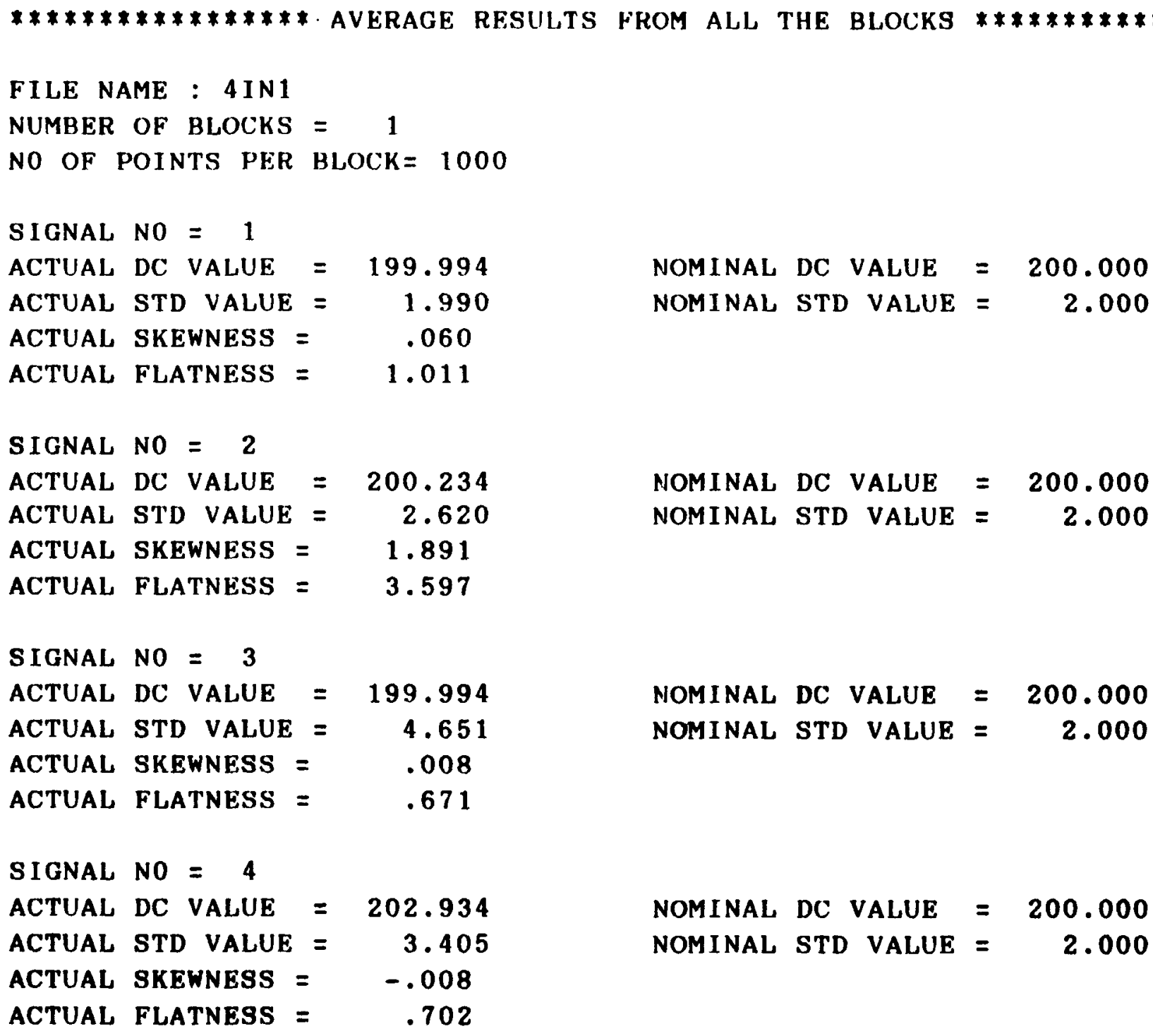

NOMINAL DC VALUE $=200.000$

NOMINAL STD VALUE $=2.000$

NOMINAL DC VALUE $=200.000$

NOMINAL STD VALUE $=2.000$

NOMINAL DC VALUE $=200.000$

NOMINAL STD VALUE $=2.000$

NOMINAL DC VALUE $=200.000$

NOMINAL STD VALUE $=2.000$ 


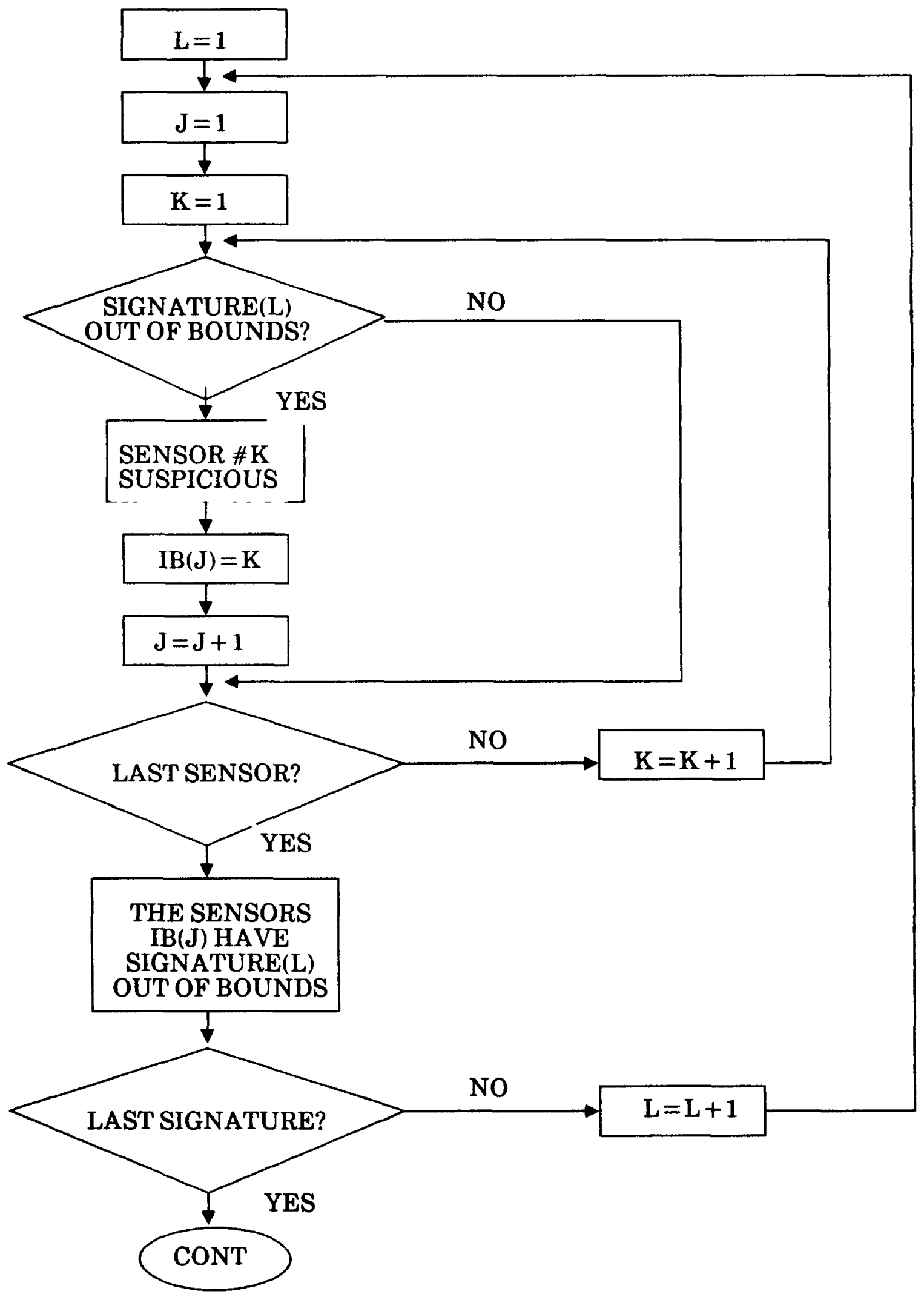

Figure 6.1 Flow chart showing the anomaly detection and cross analysis. 


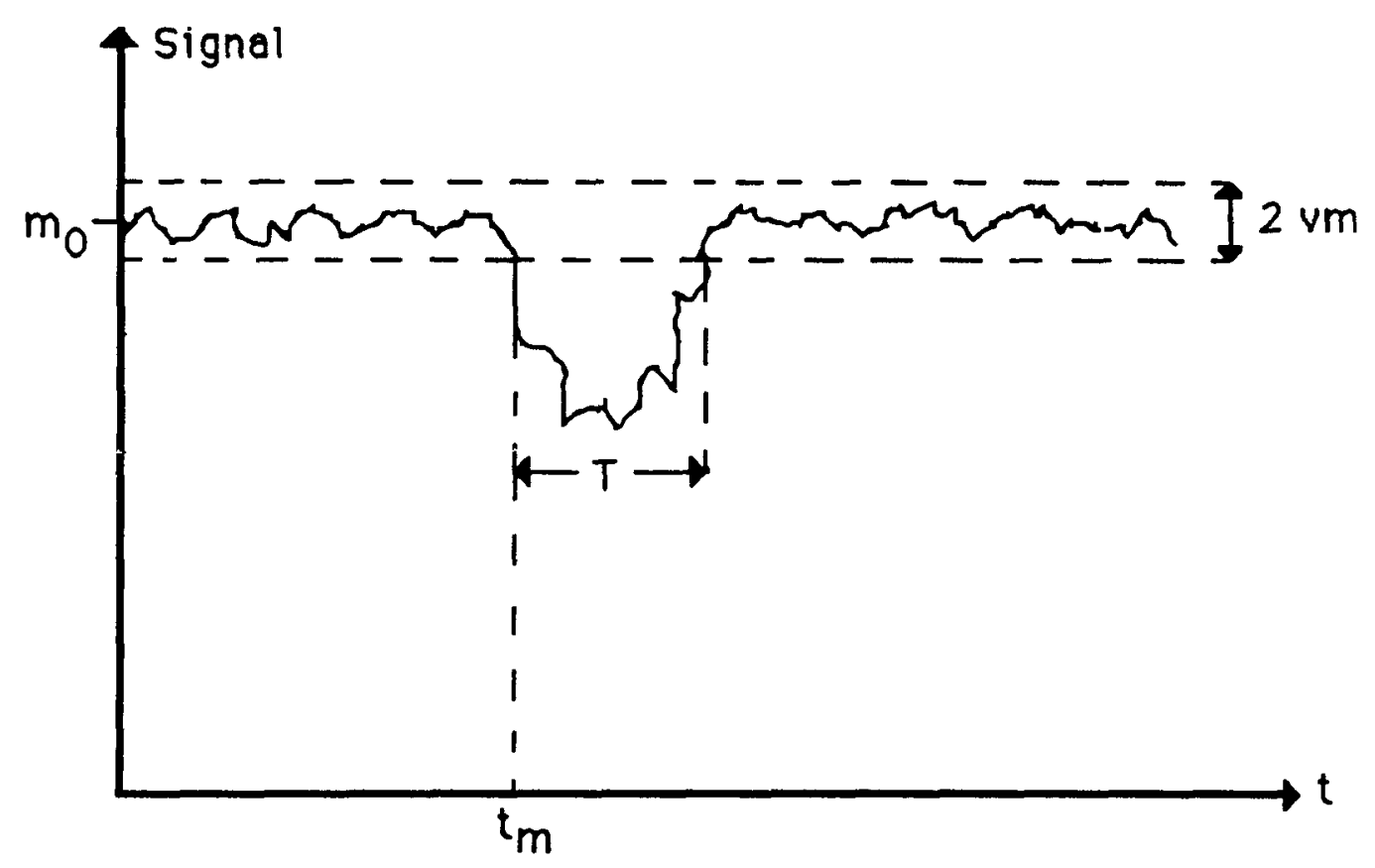

Figure 6.2. A Test Signal with "Negative" Jump

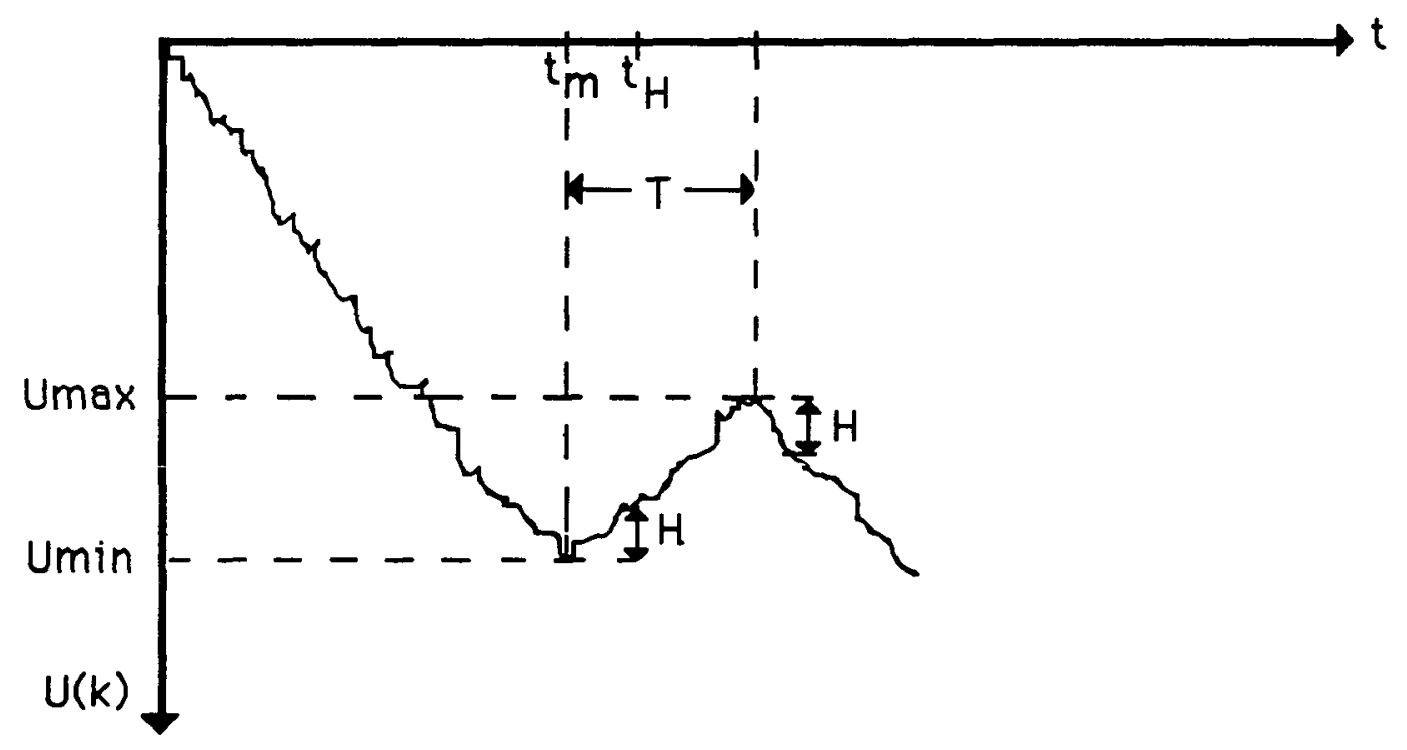

Figure 6.3. Illustration of the Cumulative Sum (CUSUM) for a Signal with "Negative" Jump. The Various Parameters are shown in the Figure. 


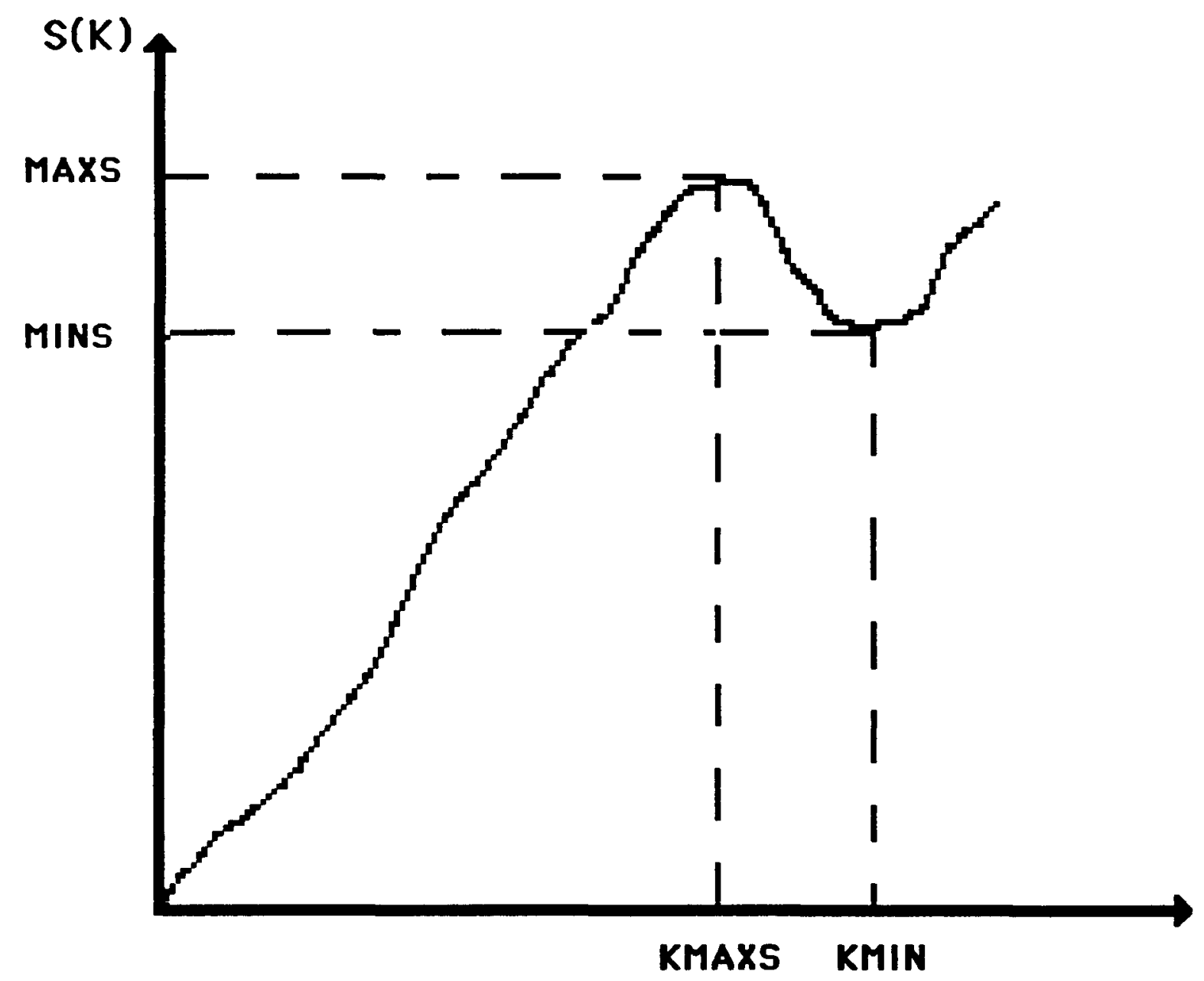

Figure 6.4. Illustration of the Cumulative Sum (CUSUM) for a Signal with "Positive" Jump. The Various Parameters are shown in the Figure. 


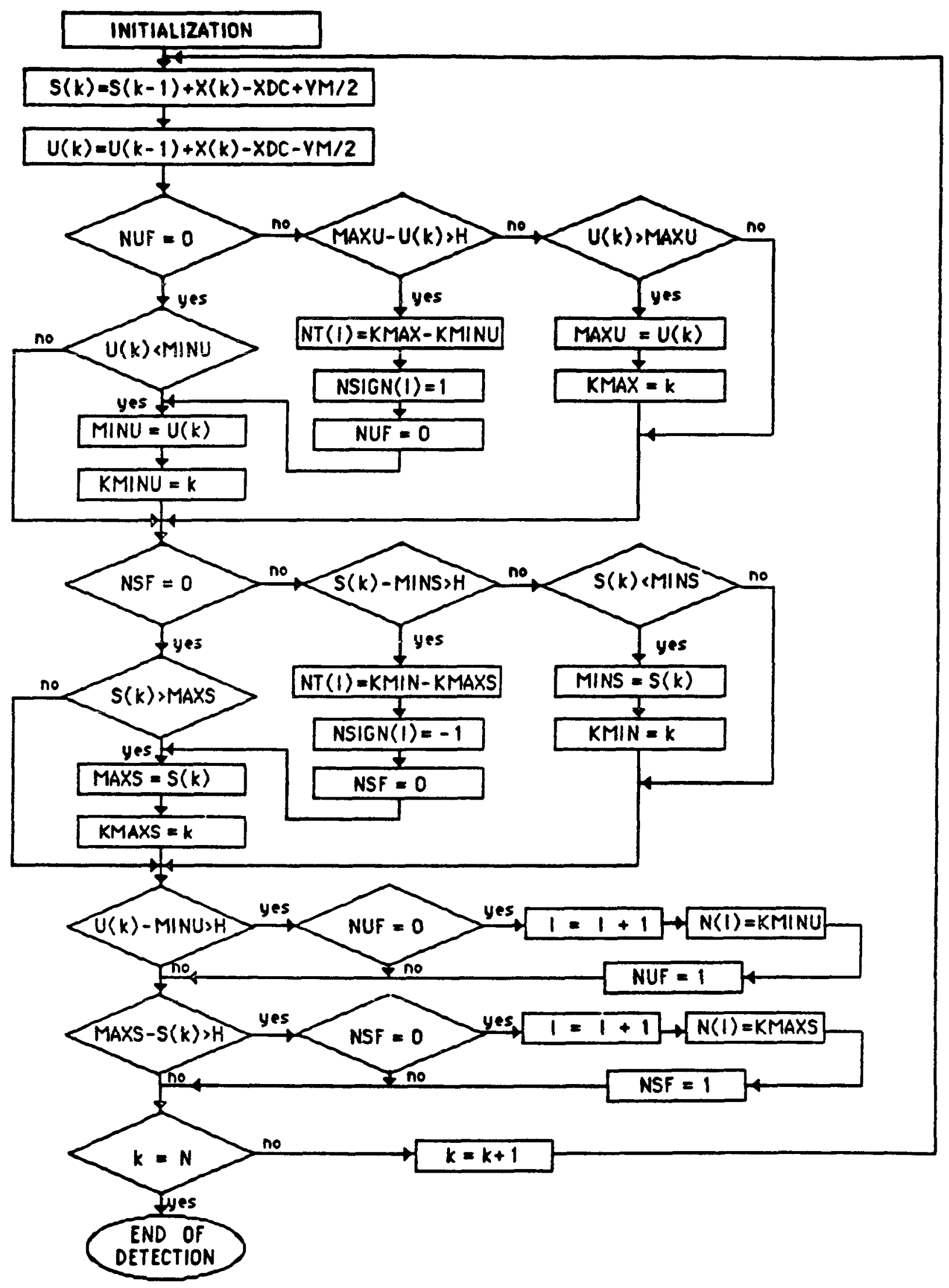

Figure 6.5. Flow Chart Showing the Modified CUSUM Algorithm. 


\section{PROGRAMS}

\section{FILES}

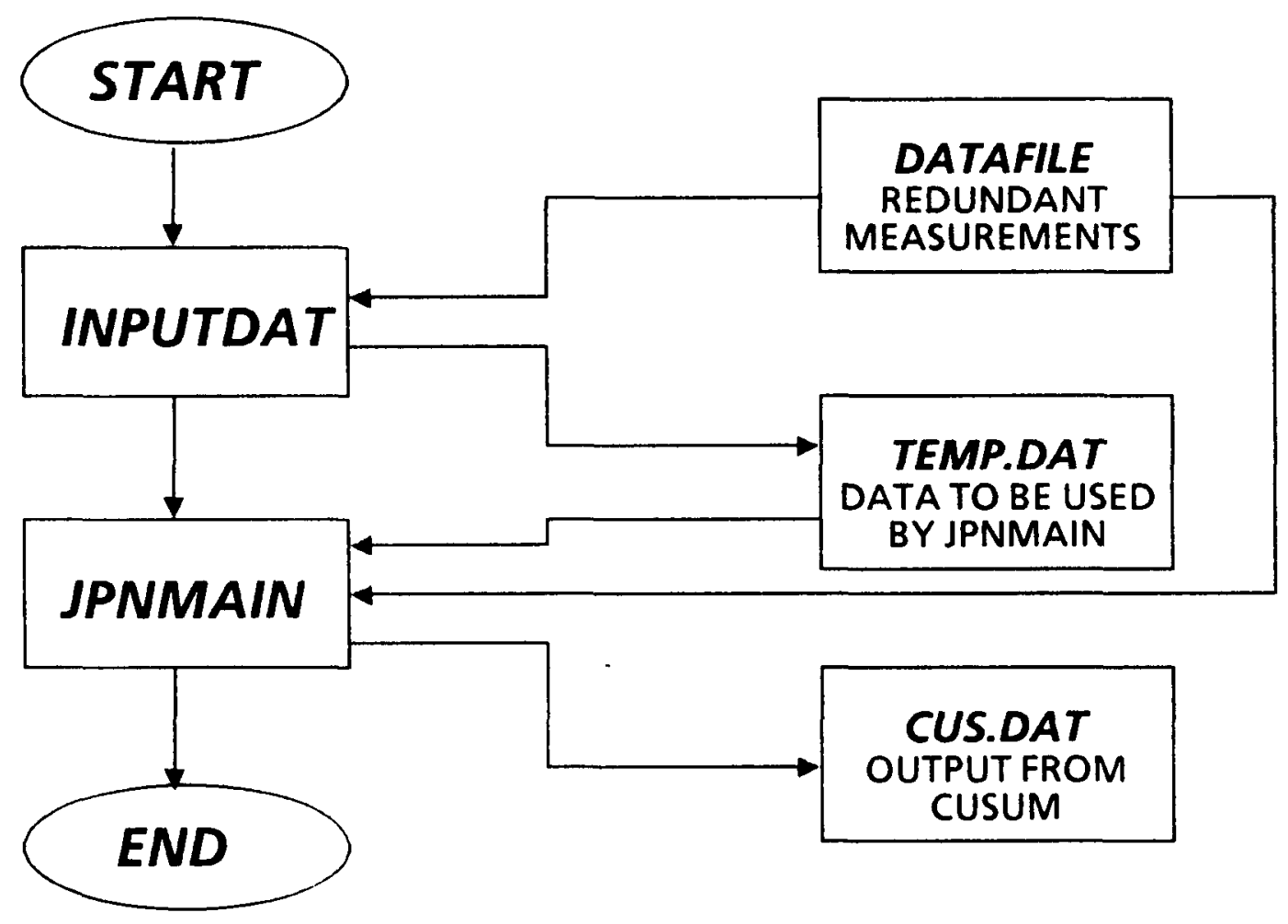

Figure 6.6 The overall logic flow chart of the anomaly detection software system. 


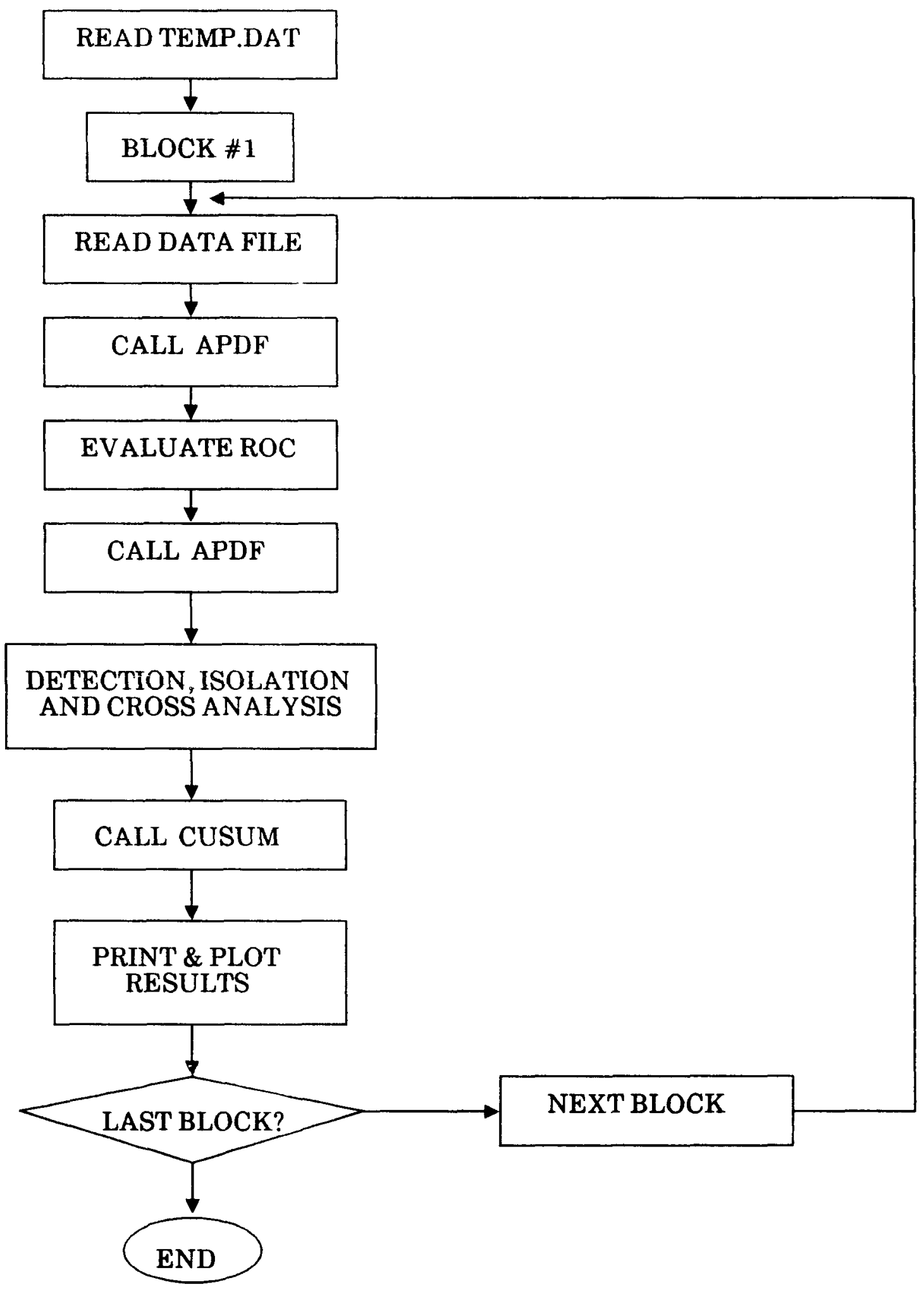

Figure 6.7 The flow chart showing the various steps in the program JPNMAIN. 


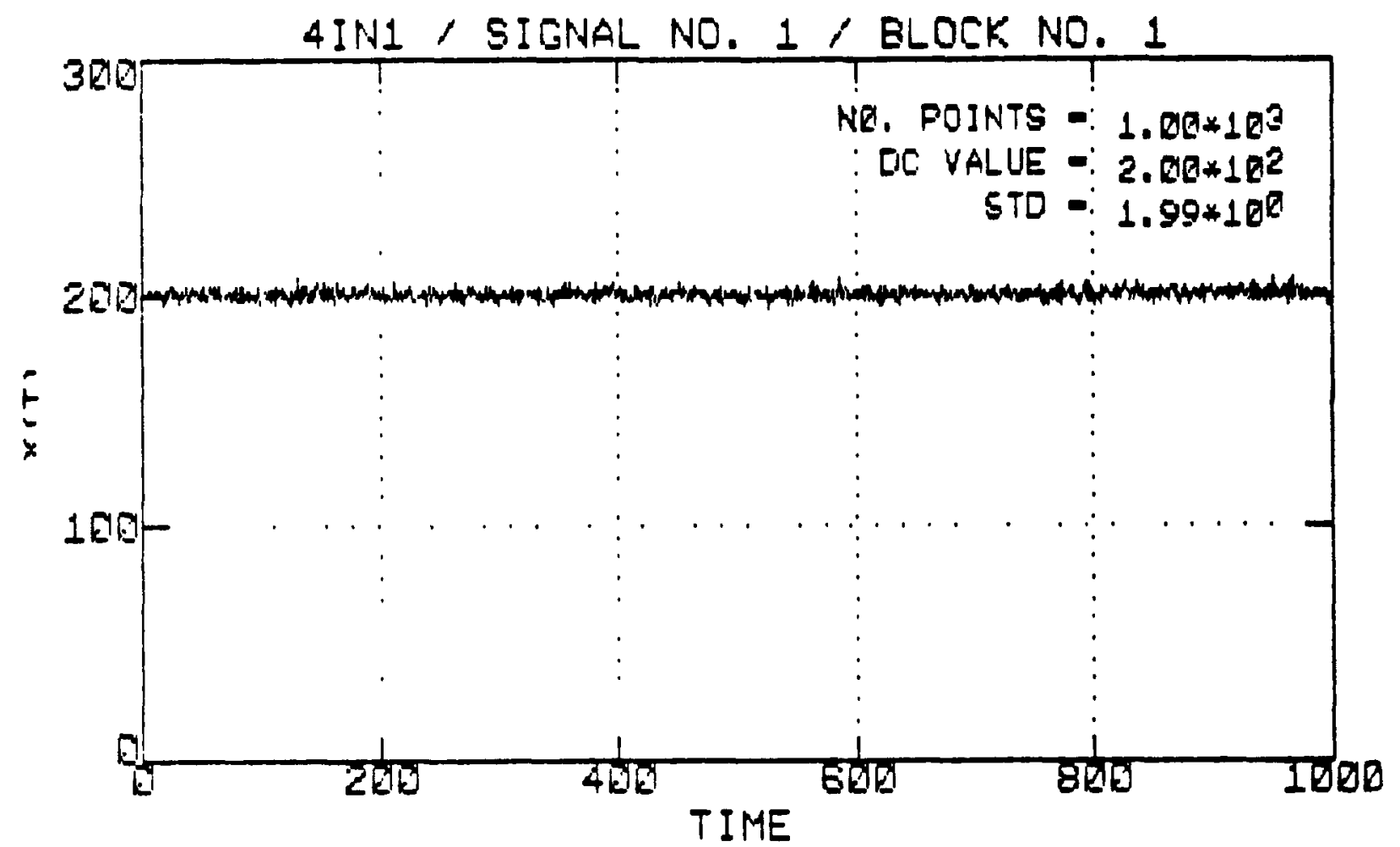

Figure 6.8a. A Simulated Test Signal with No Anomalies.

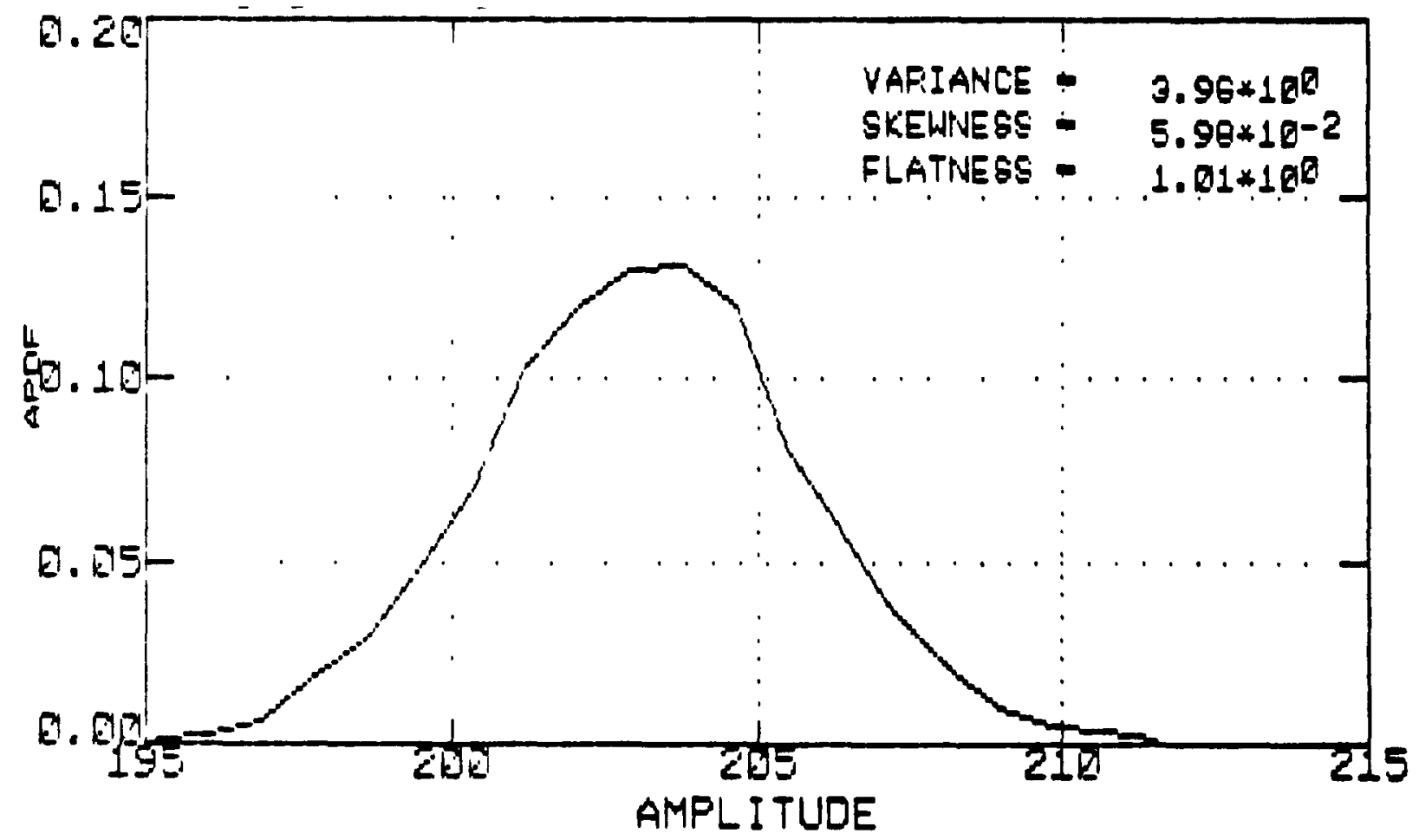

Figure 6.8b. The Amplitude Probability Density Function of the Signal Shown in Fig. 5.8a. 


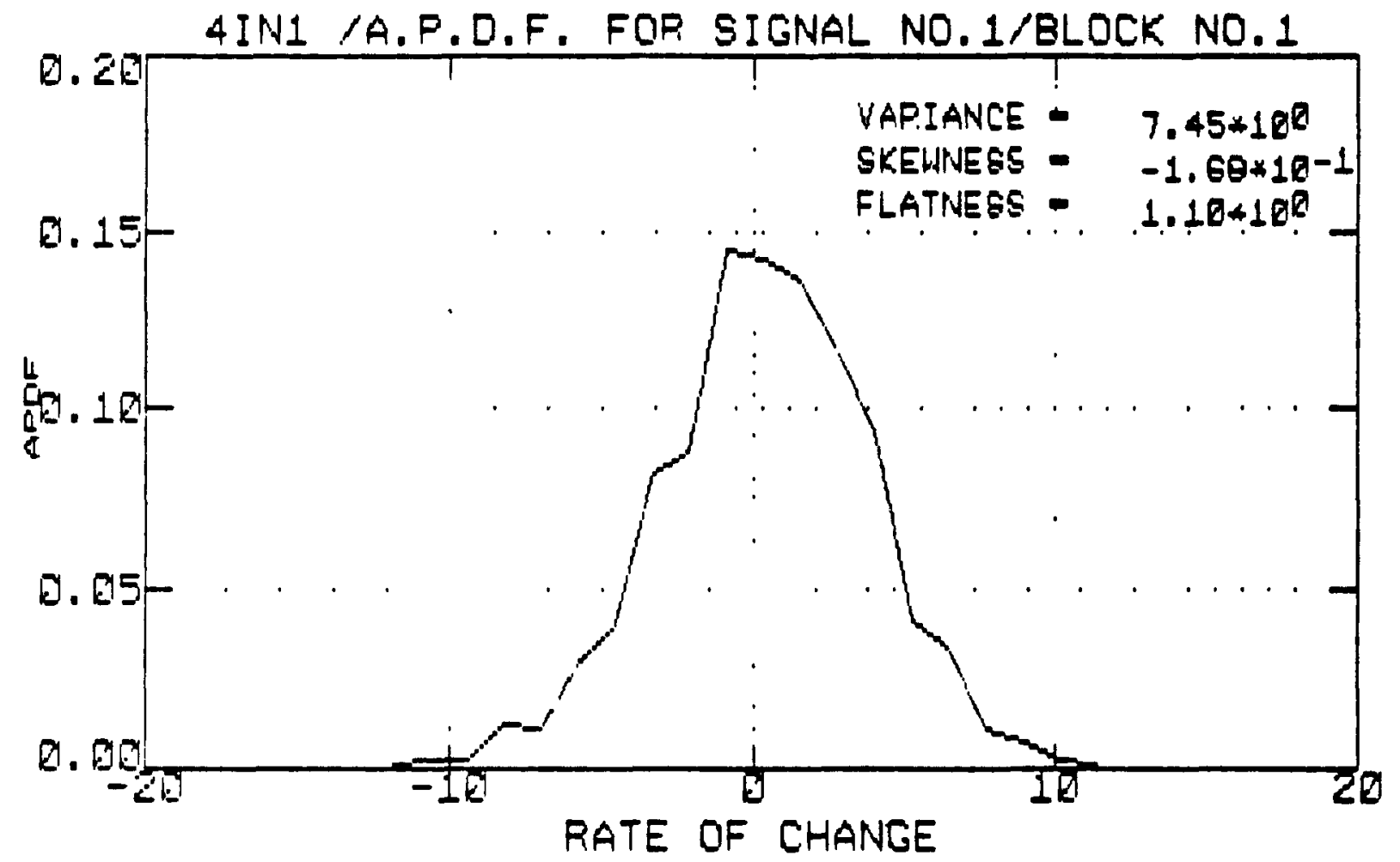

Figure 6.8c. The Amplitude Probability Density Function of the Rate of Change of the Signal Shown in Fig. 5.8a. 


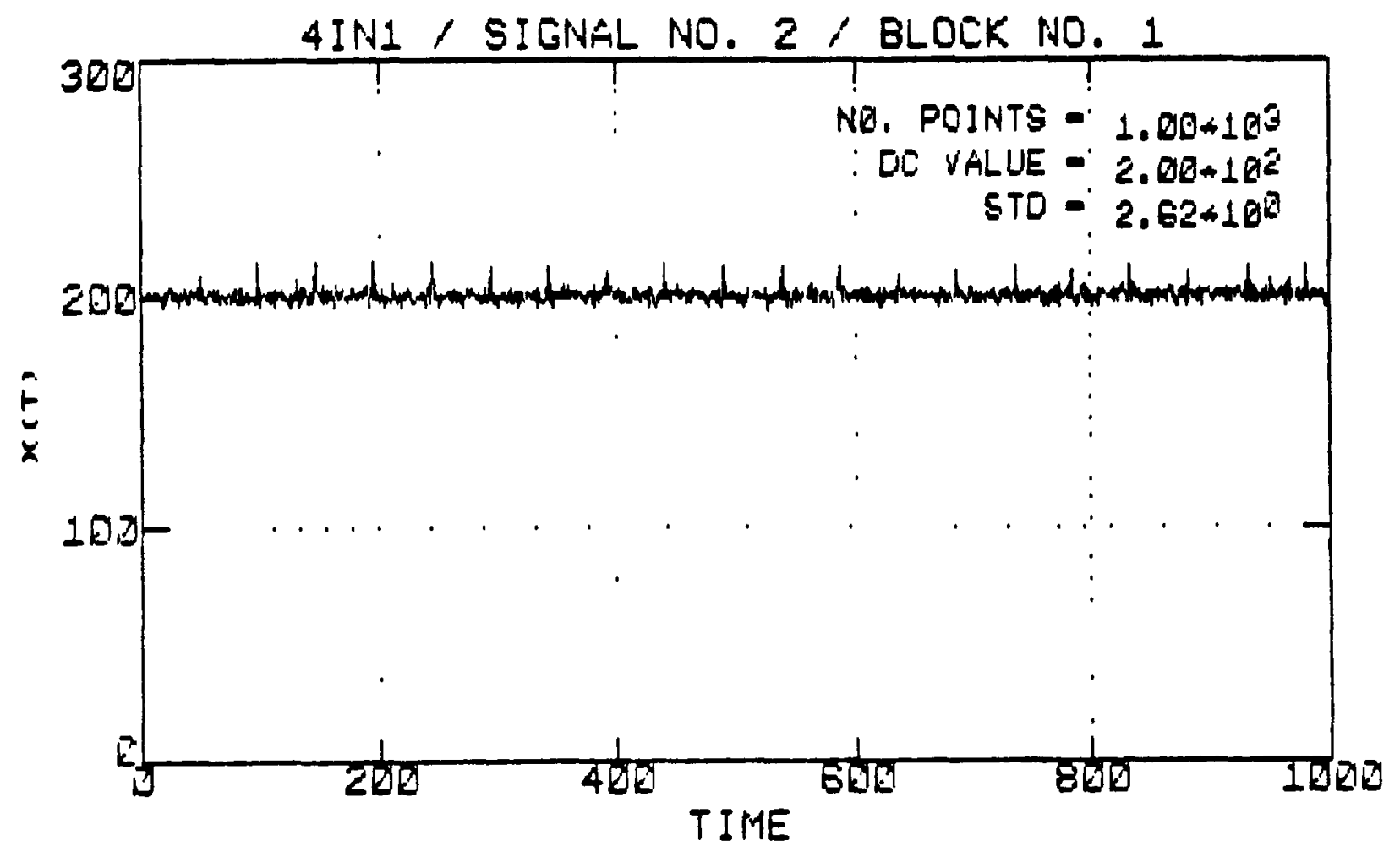

Figure 6.9a. A Simulated Test Signal with Pulse-Type Anomaly.

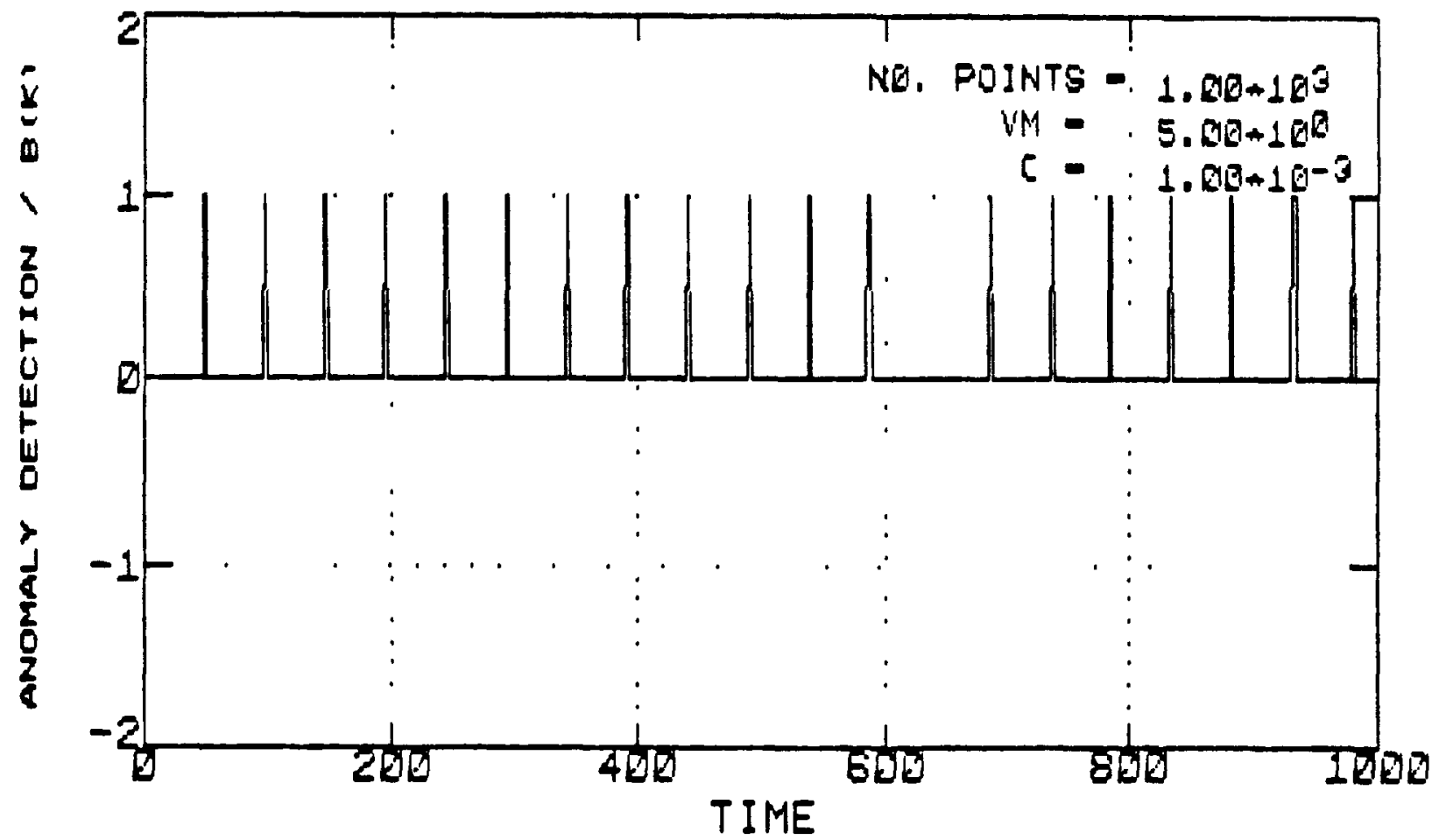

Figure 6.9b. Anomaly Detection (Binary Error Index) Using the Modified CUSUM Algorithm for the Signal Shown in Fig. 5.9a. 


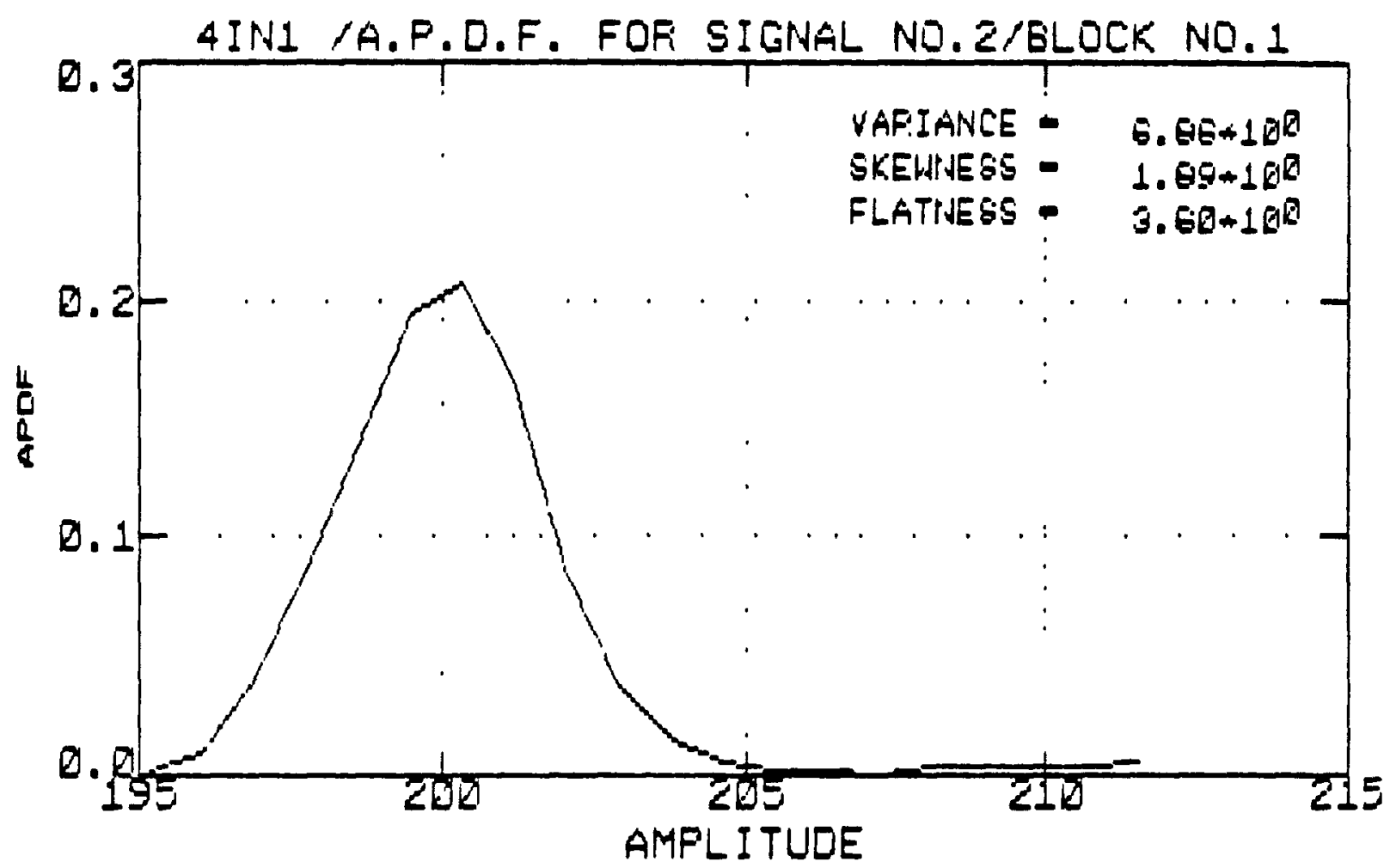

Figure 6.9c. The Amplıtude Probability Density Function of the Signal Shown in Fig. 5.9a.

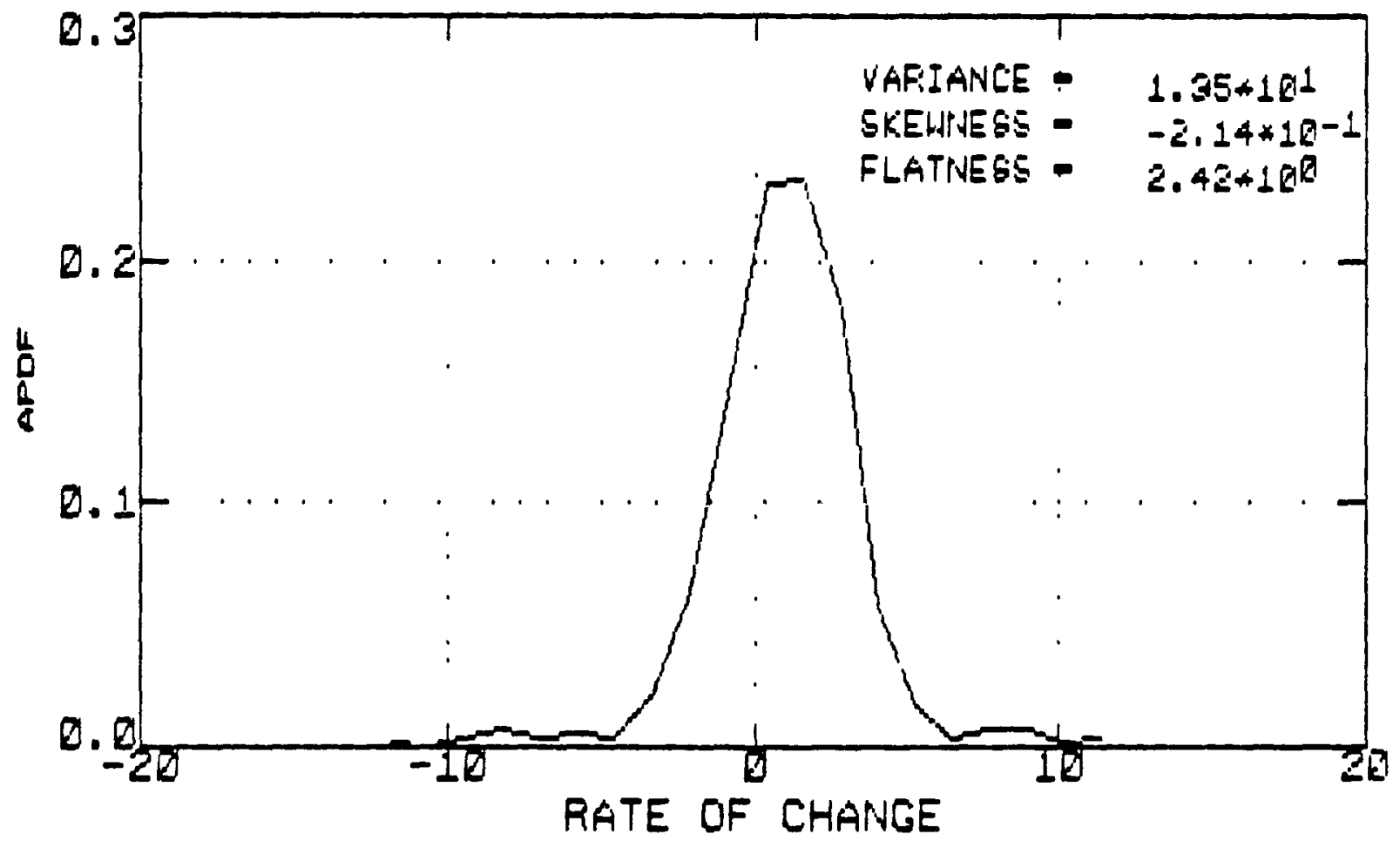

Figure 6.9d. The Amplitude Probability Density Function of the Rate of Change of the Signal Shown in Fig. 5.9a. 


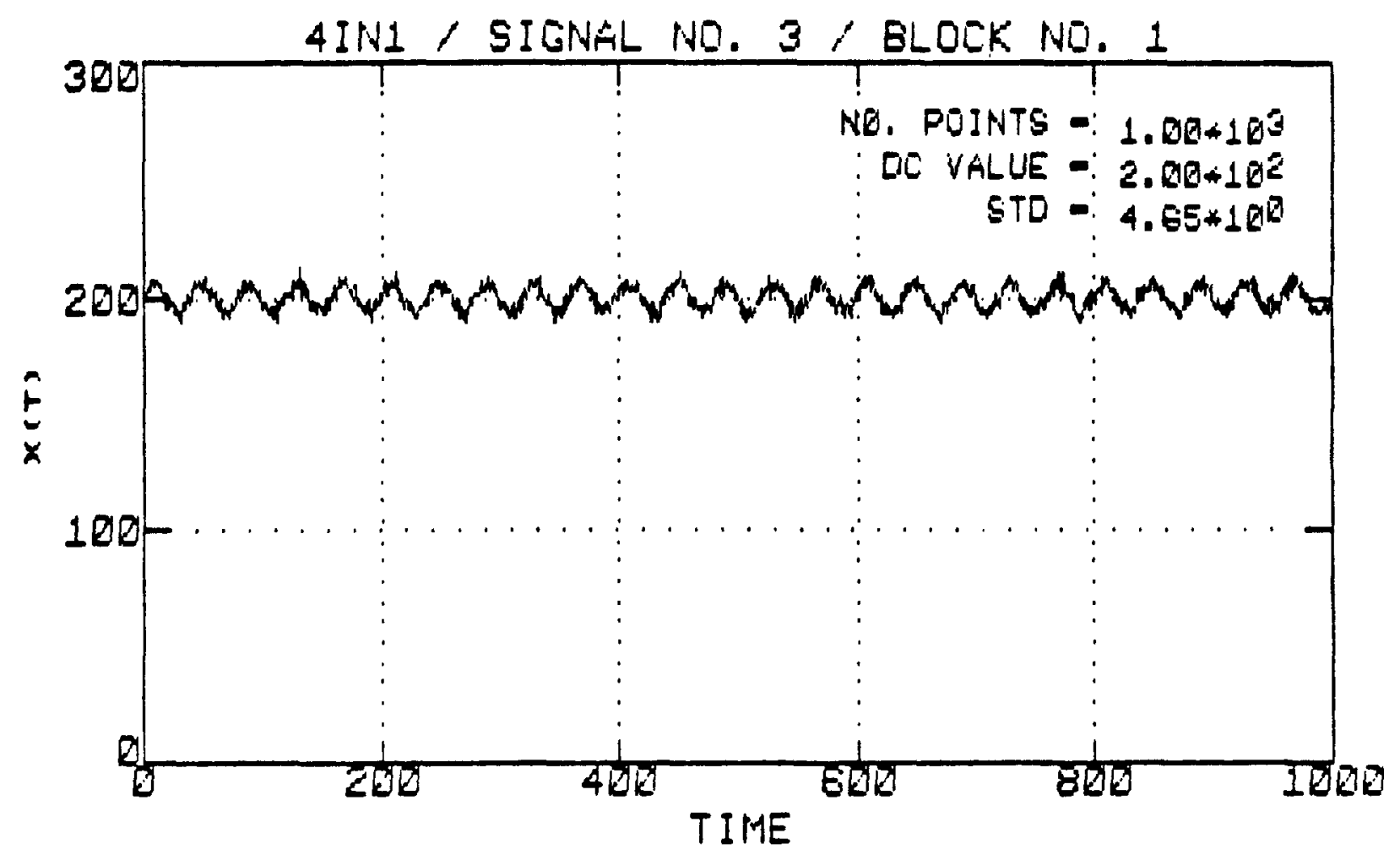

Figure 6.10a.. A Simulated Test Signal with Sinusoidal-'Type Anomaly.

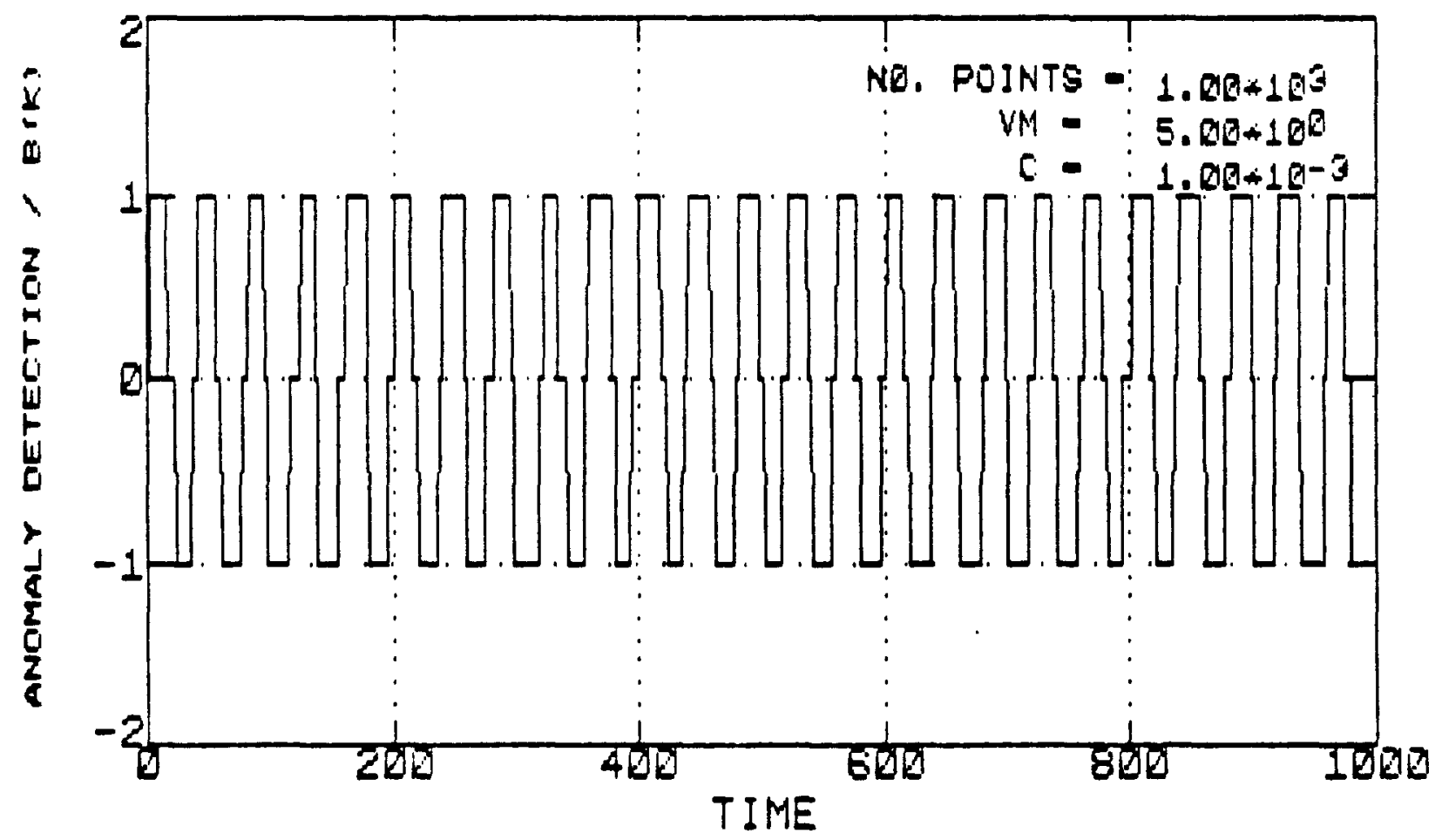

Figure 6.10b. Anomaly Detection (Bipolar Error Index) Using the Modified CUSUM Algorithm for the Signal Shown in Fig. 5.10a. 


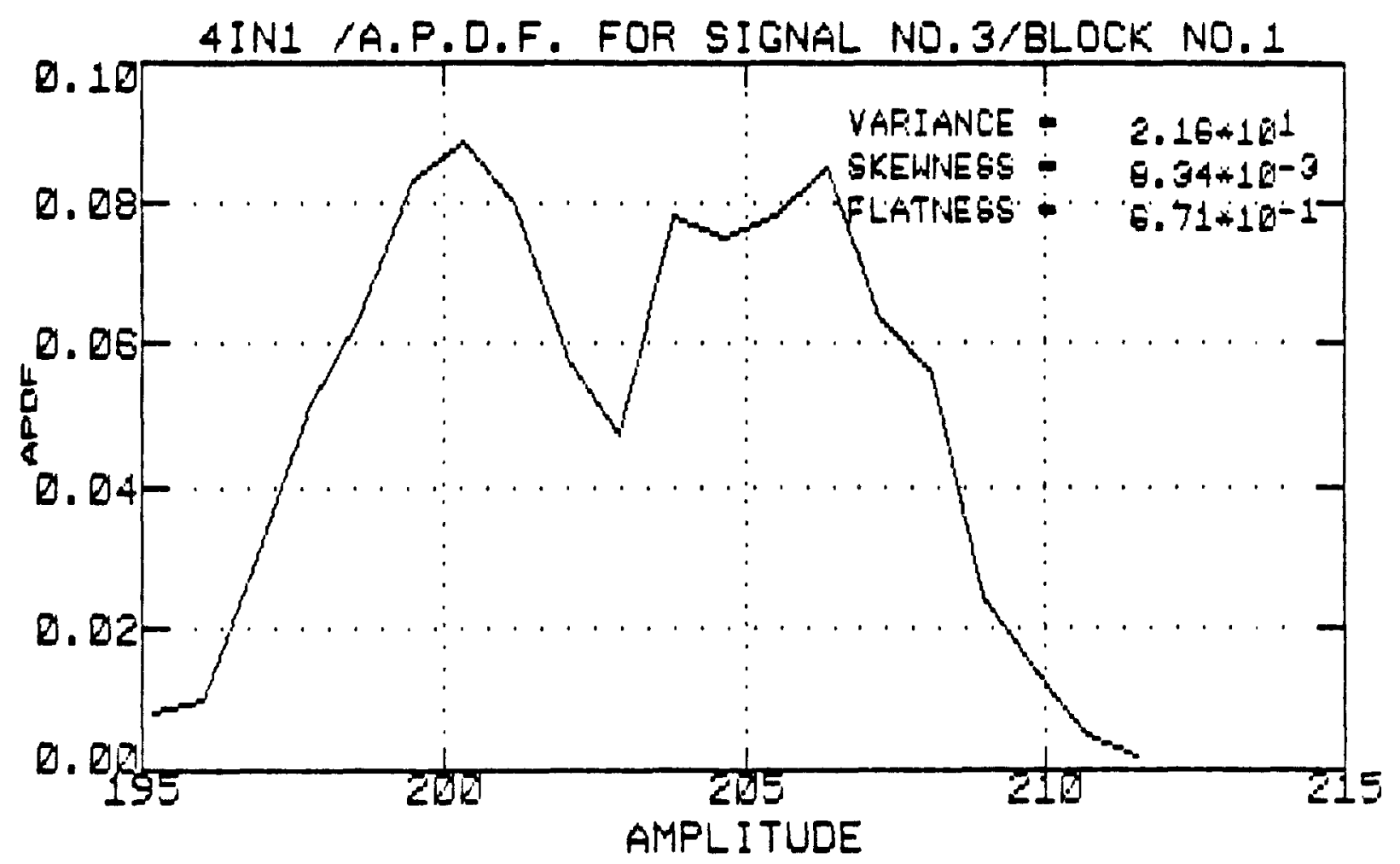

Figure 6.10c. Amplitude Probability Density Function of the Signal Shown in Fig. 5.10a.

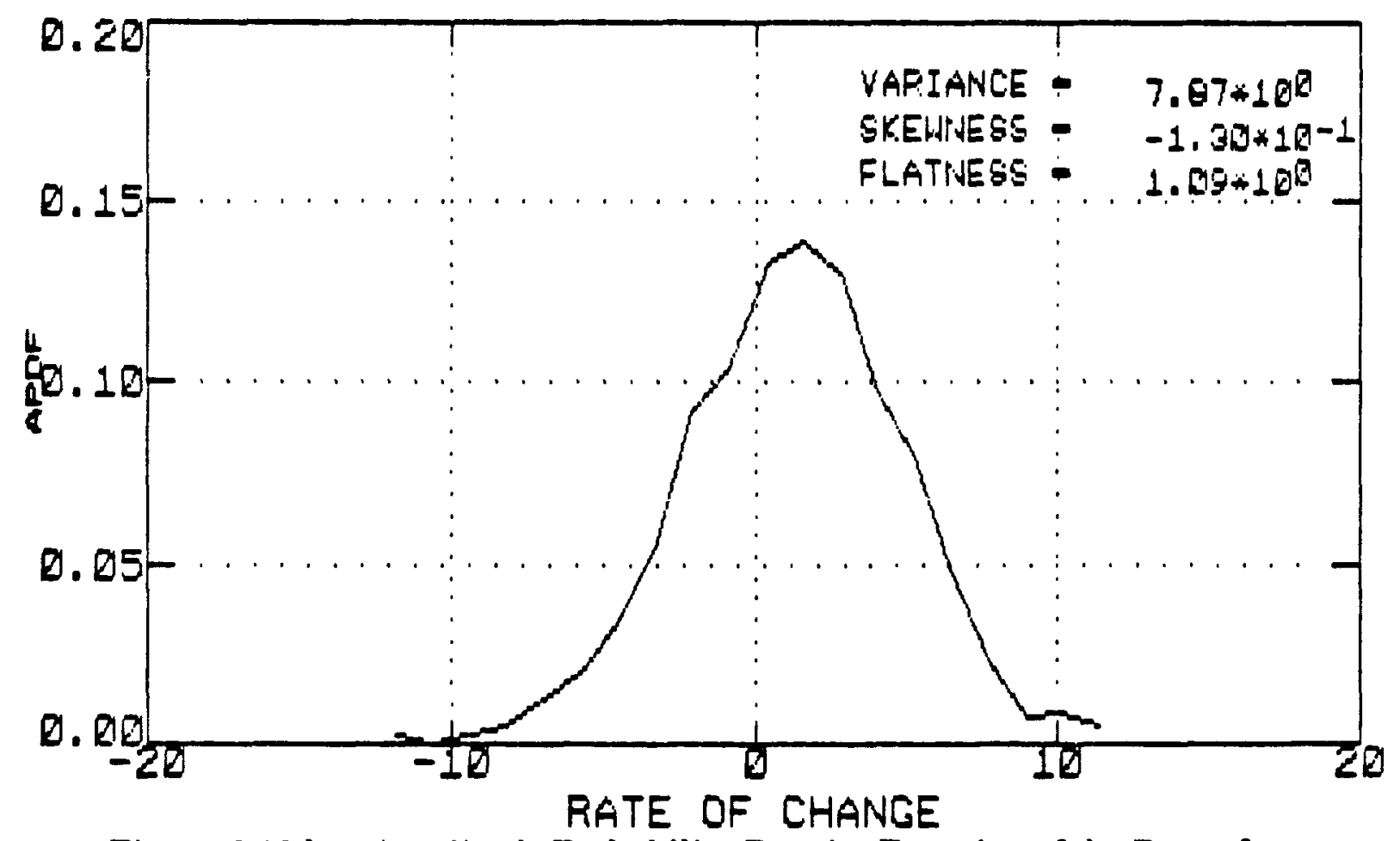

Figure 6.10d. Amplitude Probability Density Function of the Rate of Change of the Signal Shown in Fig. 5.10a. 


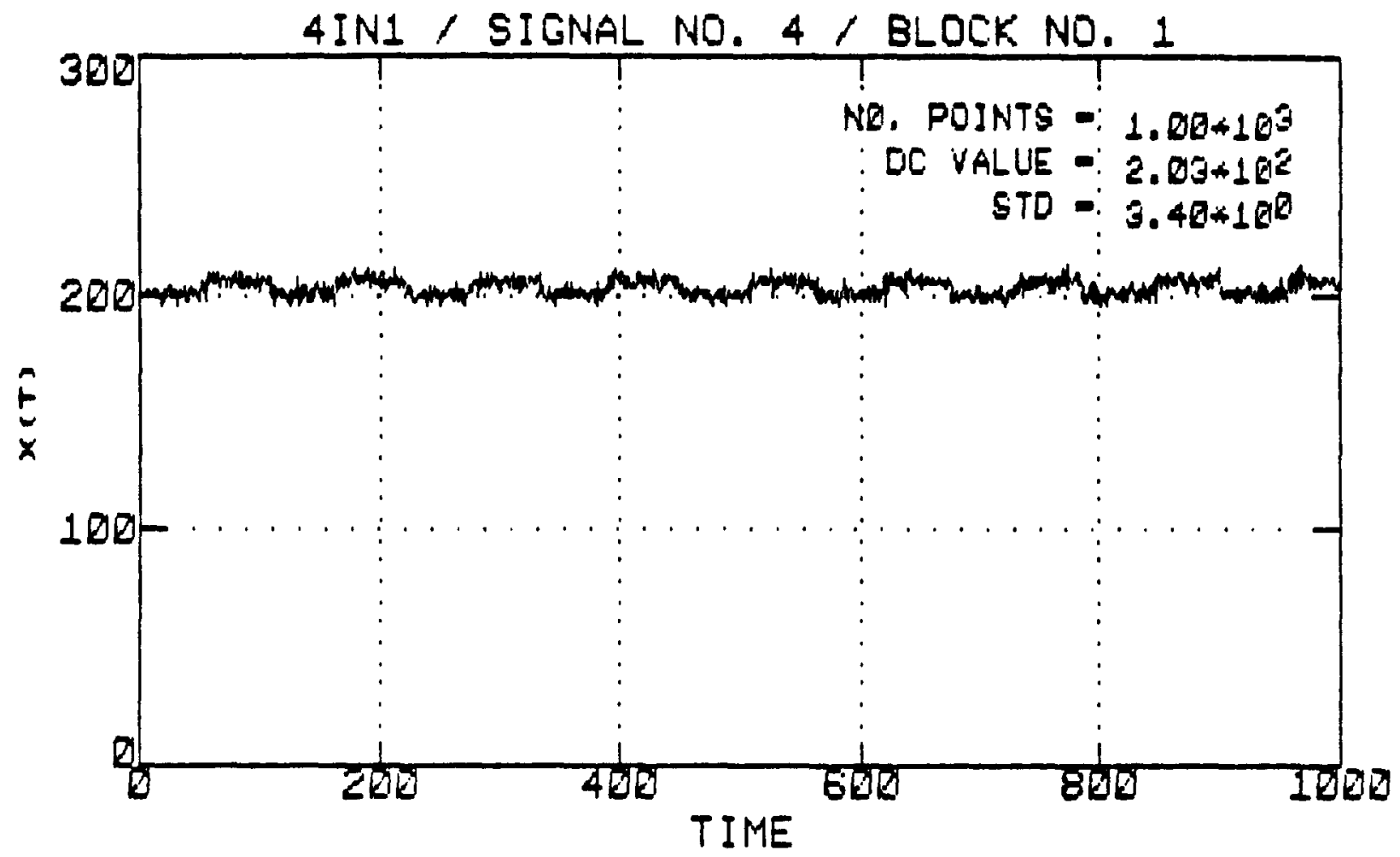

Figure 6.11a. A Simulated Test Signal with Jump-Type Anomaly.

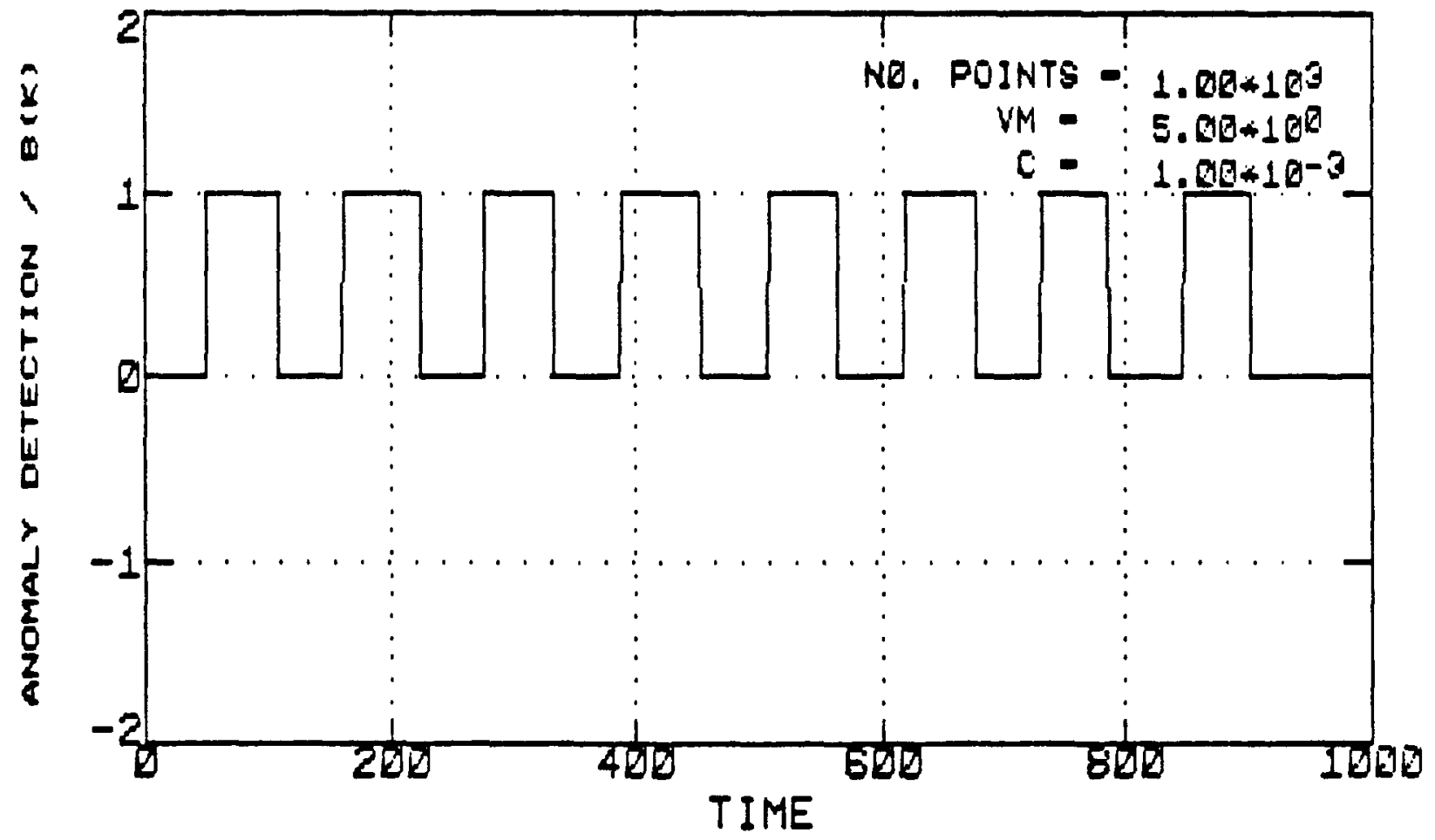

Figure 6.11b. Anomaly Detection (Binary Error Index) Using the Modified CUSUM Algorithm for the Signal Shown in Fig. 5.11a.. 


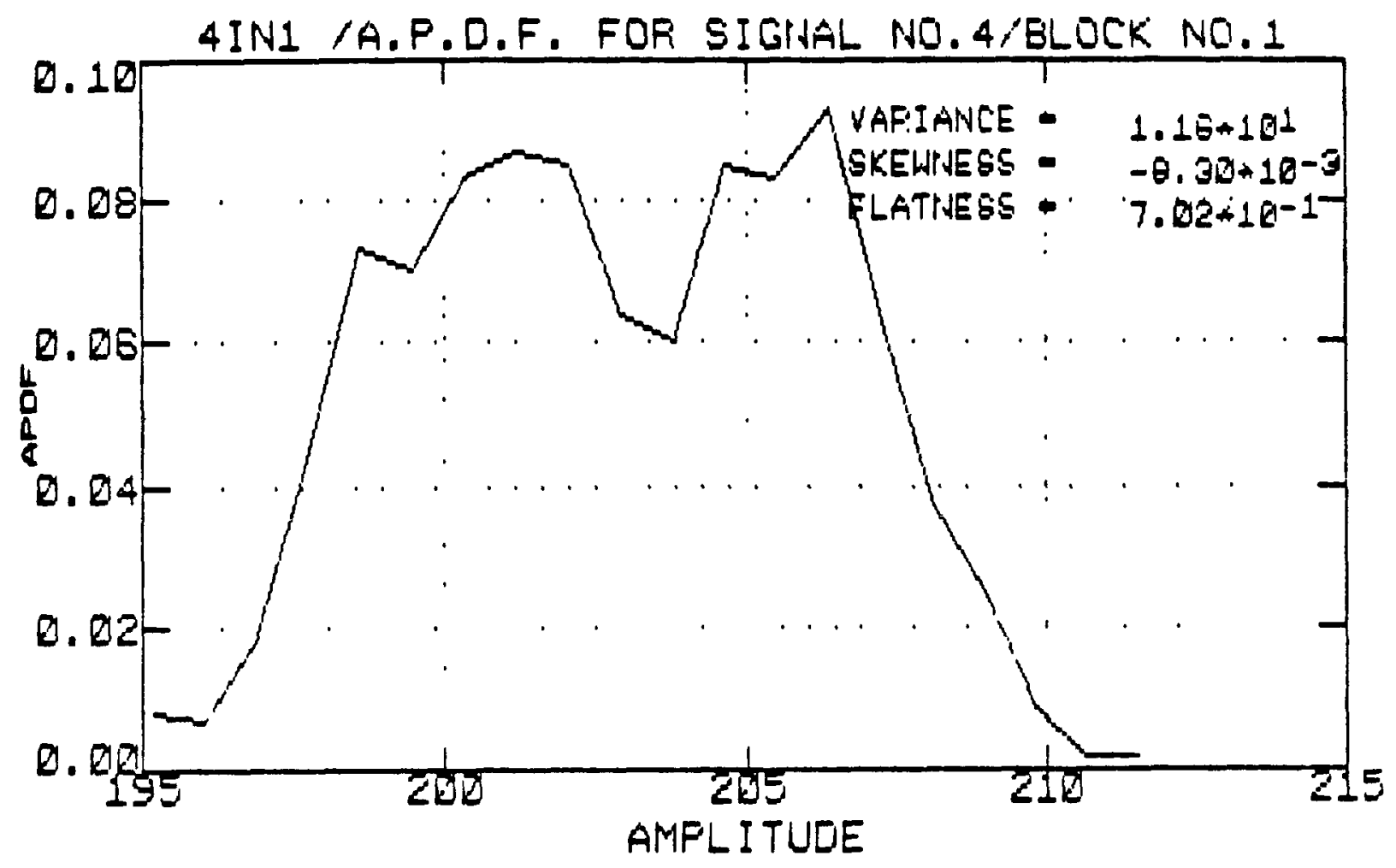

Figure 6.11c. Amplitude Probability Density Function of the Signal Shown in Fig. 5.1 1a.

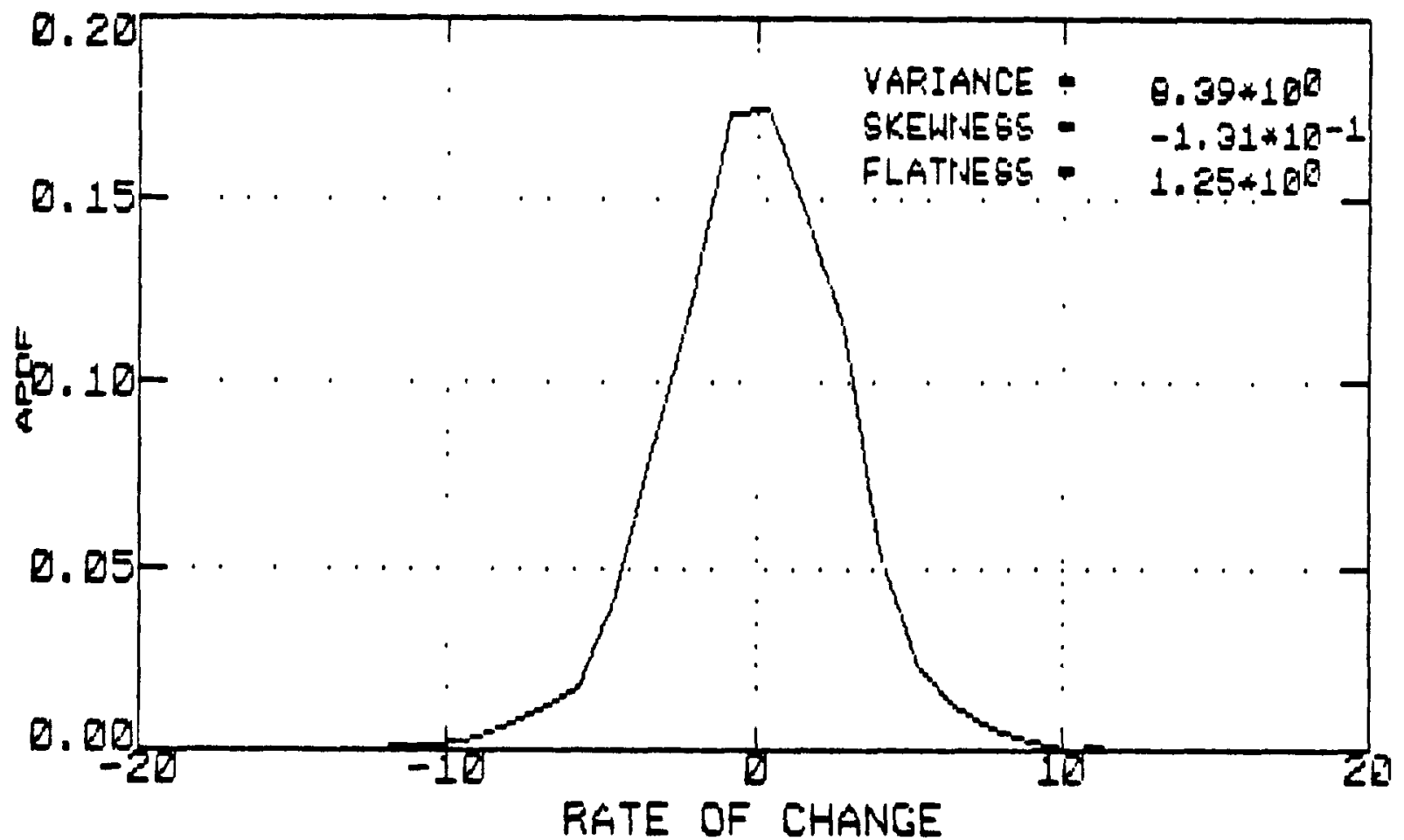

Figure 6.11d. Amplitude Probability Density Function of the Rate of Change of the Signal Shown in Fig. 5.11a. 
RCS-P455A* PZR PRESS

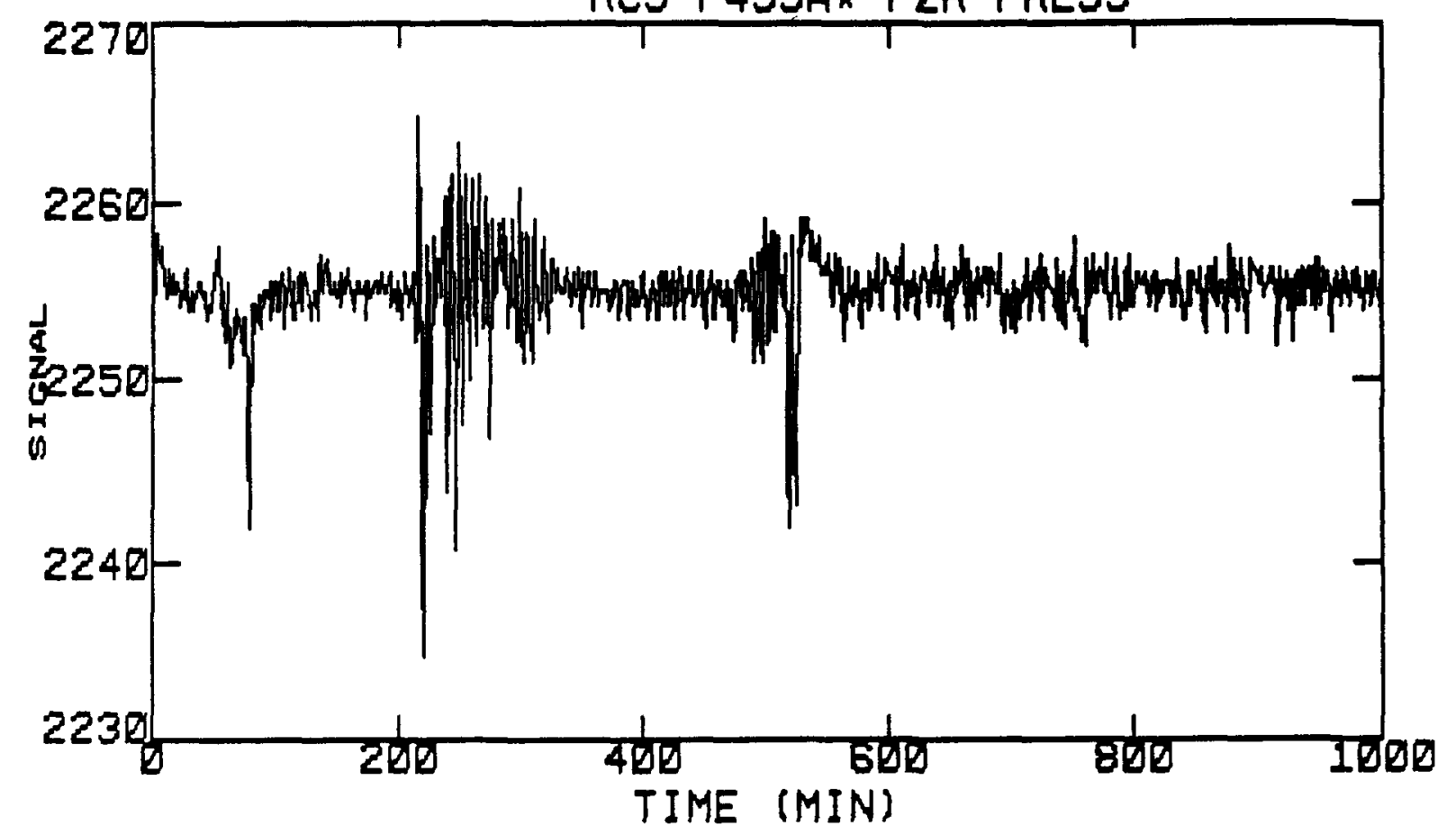

Figure 6.12a. Pressurizer Pressure (1b/in2) signal During Start-Up of a Westinghouse Four-Loop PWR.

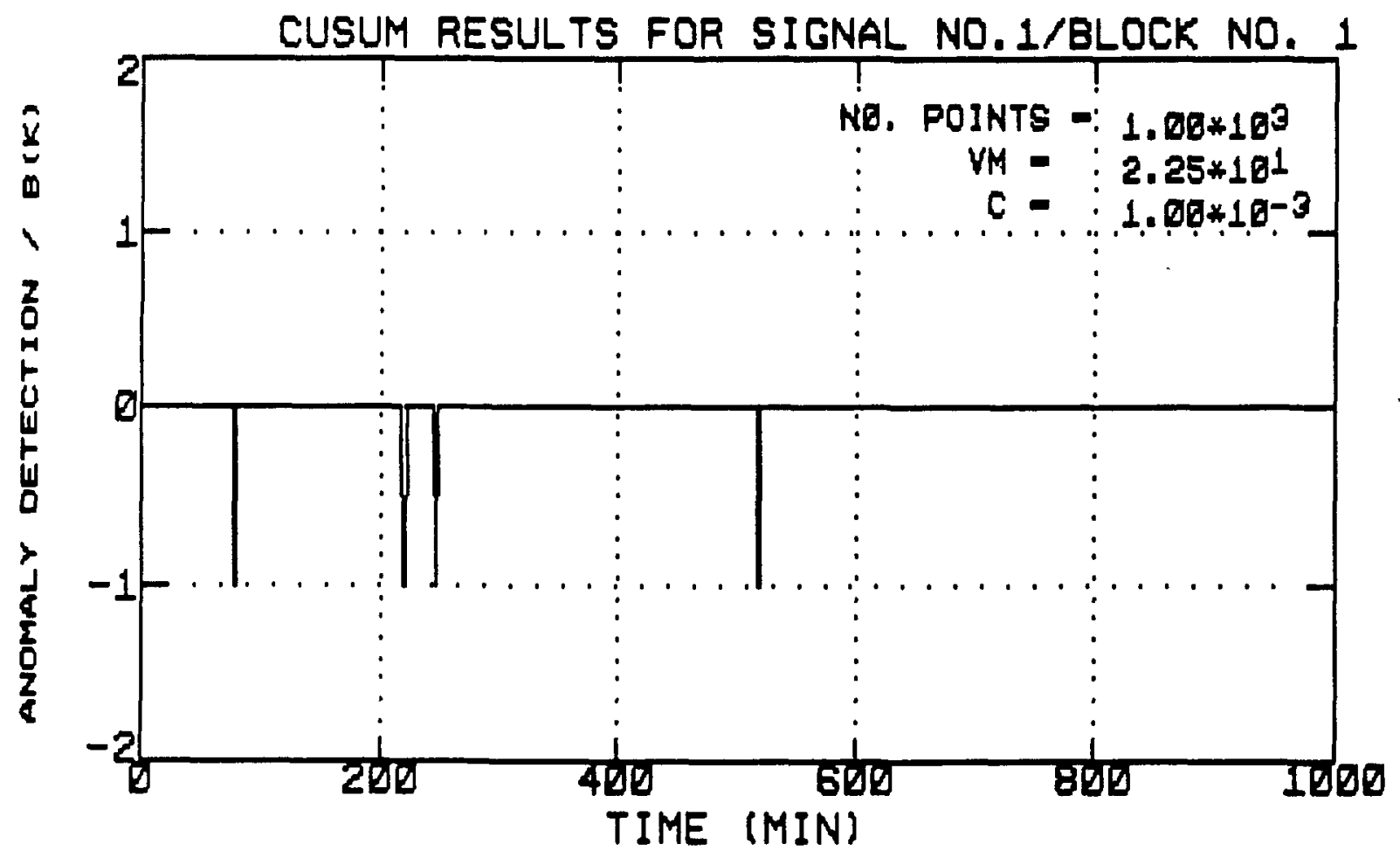

Figure 6.12b. Binary Anomaly Index for the Signal Shown in Fig. 5.12a Indicating Variations in the Signal Level. 


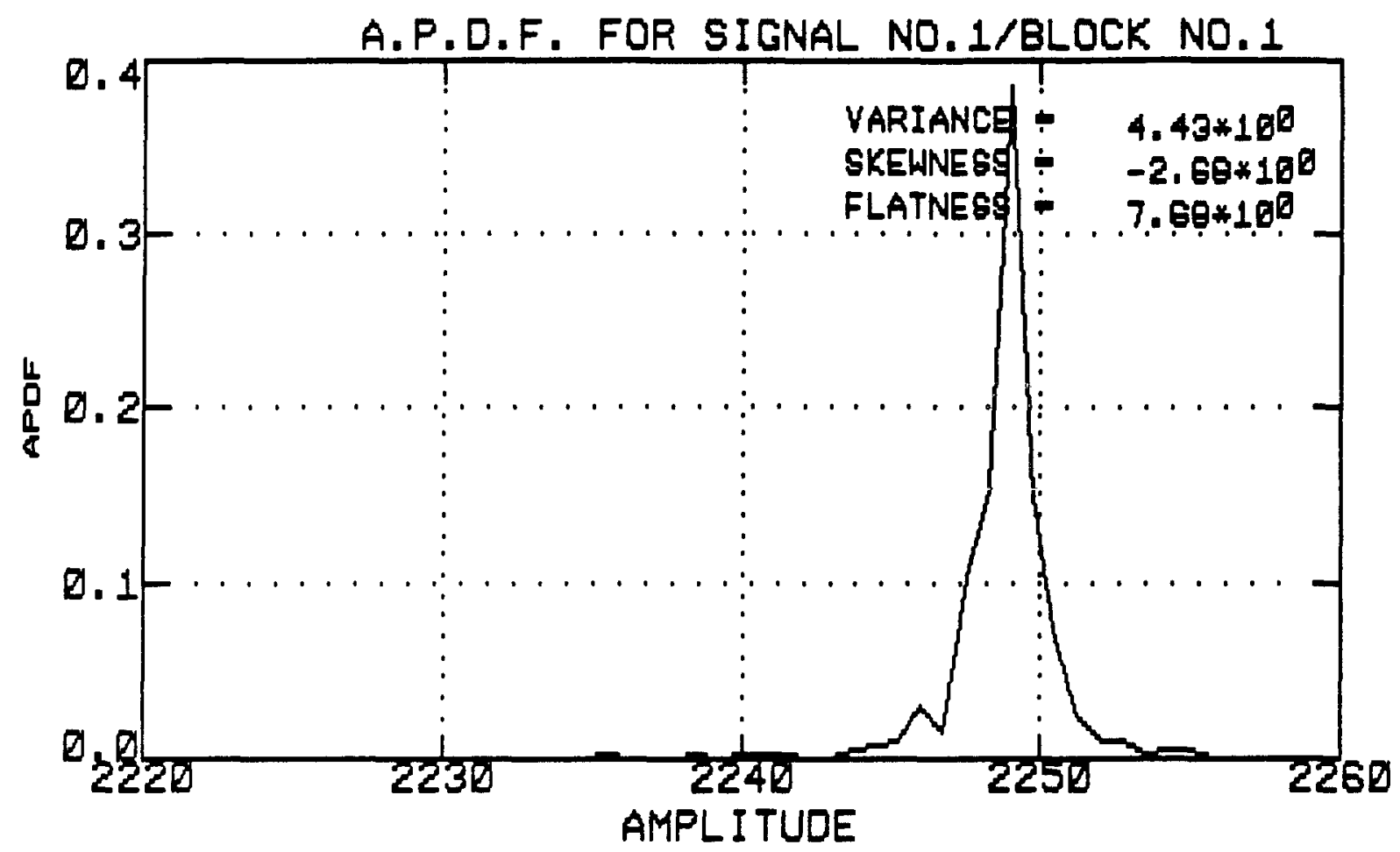

Figure 6.13a. Amplitude Probability Density Function of the Signal Shown in Fig. 5.12a (Pressurizer Pressure).

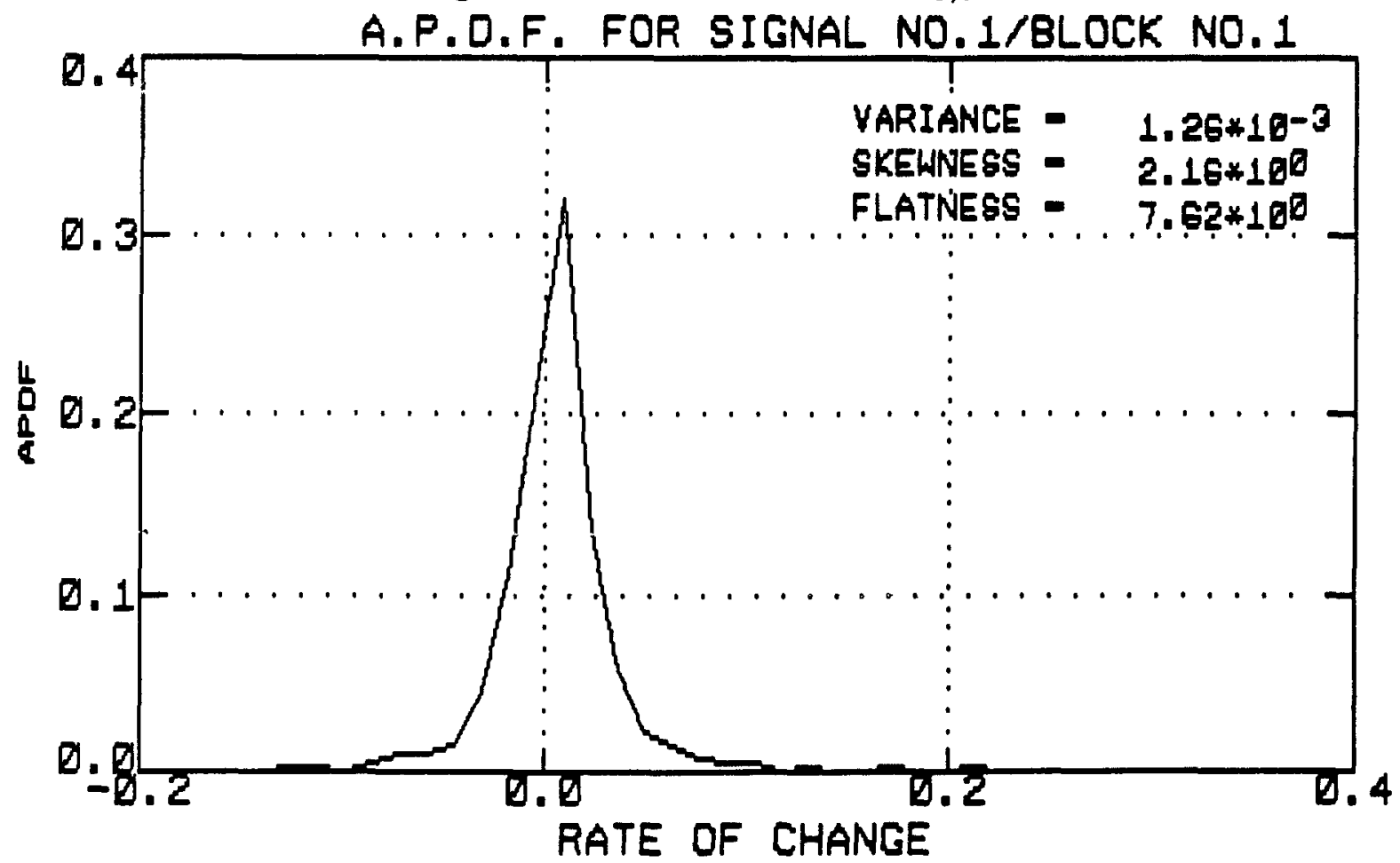

Figure 6.13b. Amplitude Probability Density Function of the Rate of Change of the Signal (Pressurizer Pressure) Shown in Fig. $5.12 \mathrm{a}$. 


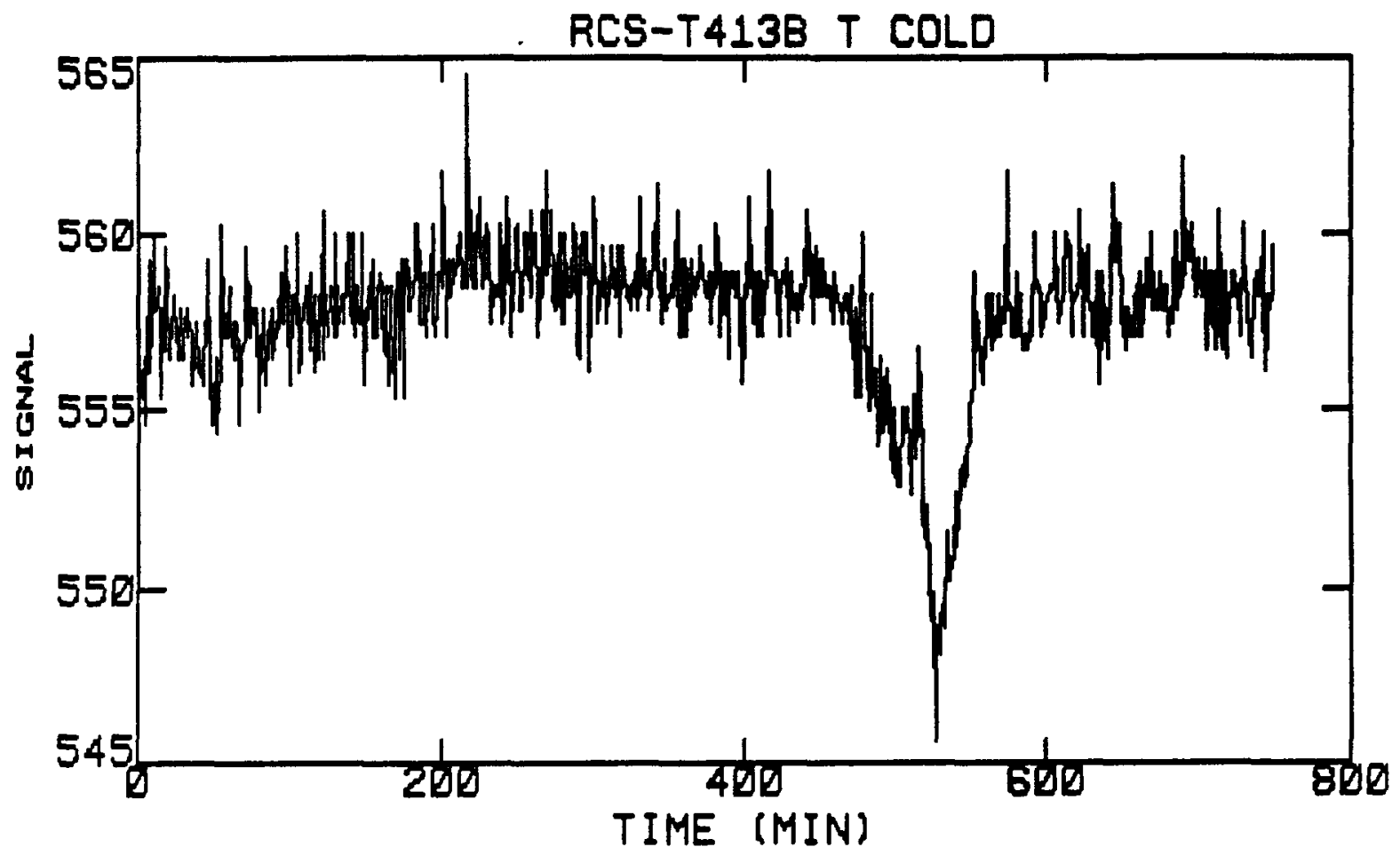

Figure 6.14a. Cold Leg Temperature (F) Signal During Start-Up of a Westinghouse Four-Loop PWR.

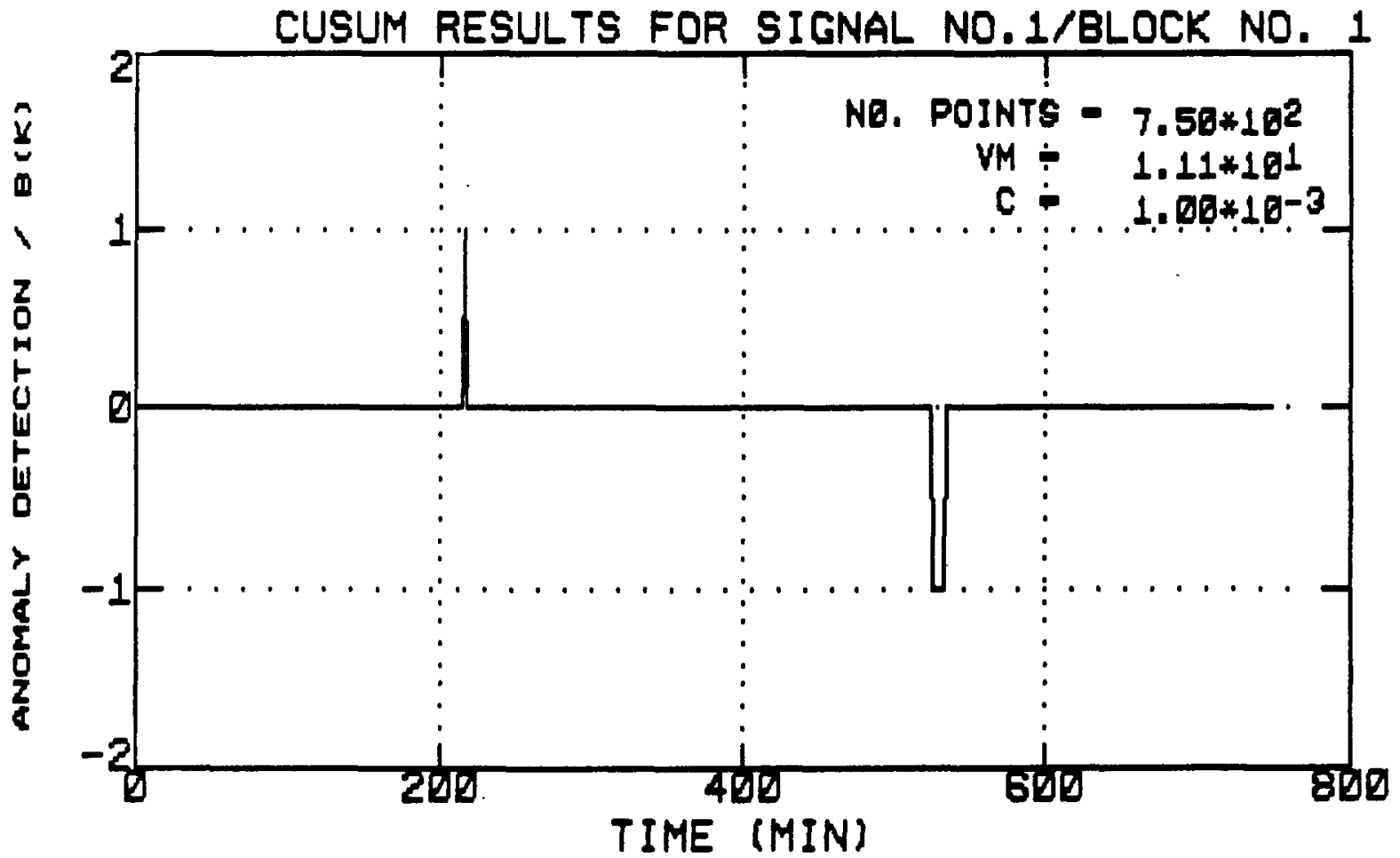

Figure 6.14b. Binary Anomaly Index for the Cold Leg Temperature Signal Shown in Fig. 5.14a Indicating Variations in the Signal Level. 


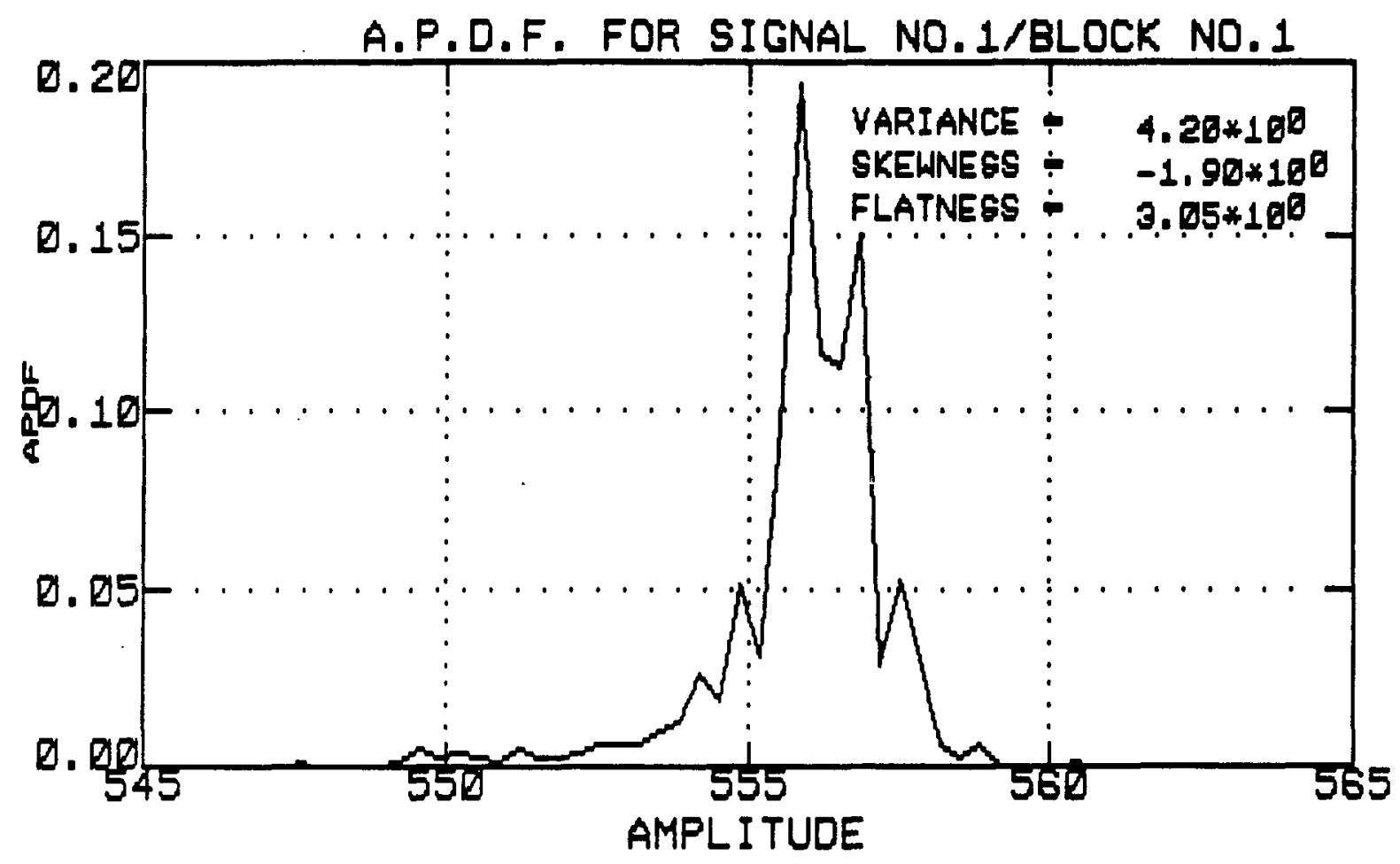

Figure 6.15a. Amplitude Probability Function of the Cold Leg Temperature Signal Shown in Fig. 5.14a.

A.P.D.F. FOR SIGNAL NO.1/BLOCK NO.1

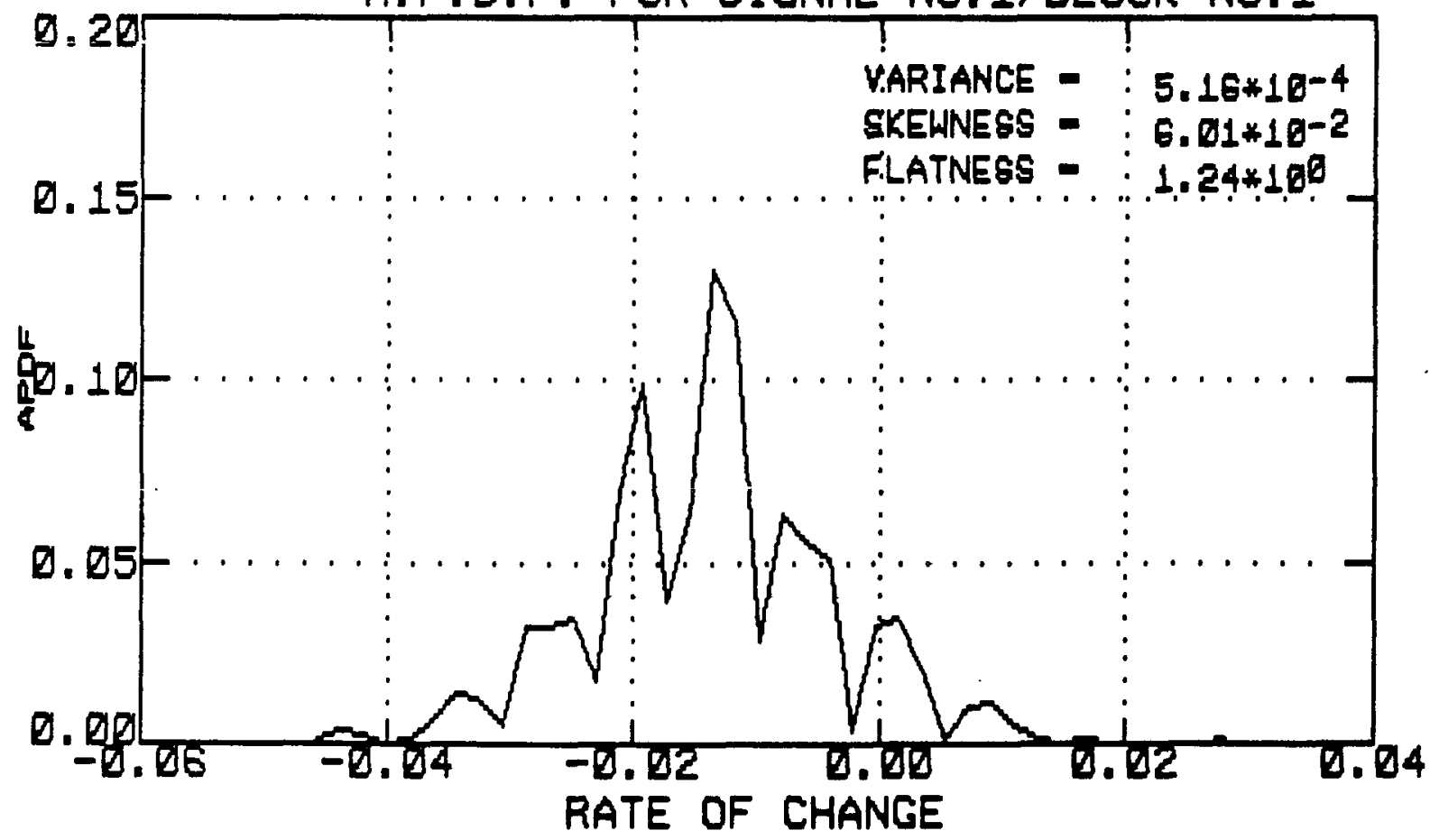

Figure 6.15b. Amplitude Probability Density Function of the Rate of Change of the Cold Leg Temperature Signal Shown in Fig. $5.14 \mathrm{a}$. 


\section{Section 7}

\section{MULTIVARIA'TE AU'TOREGRESSION (MAR) MODELING FOR SENSOR ANI) PROCESS ANOMALY DETECTION AND ISOLATION}

\subsection{Introduction}

Multivariate analysis of the fluctuating components of power reactor operating signals is useful for plant diagnostics, for isolating process and sensor anomalies, and for automated plant monitoring. In order to develop a reliable procedure, we improved upun the previously established techniques for empirical modeling of fluctuation signals in power reactors. Application of the complete algorithm to operational data from the Loss-of-Fluid-Test (LOFT) reactor showed that earlier conjectures (based on physical modeling) regarding the perturbation sources in a Pressurized Water Reactor (PWR) affecting coolant temperature and neutron power fluctuations can be systematically explained. This advanced methodology has important implications regarding plant diagnostics, and system or sensor anomaly isolation.

\subsection{Multivariate Autoregressive Modeling of Process Signals}

Our primary interest is in establishing direct cause-and-effect relationships among the measured signals using frequency dependent statistical functions. One way of creating these frequency functions is to fit an assumed low order linear model to the measured set of signals, and then to transform the identified model into the frequency domain. The multivariate autoregressive time series modeling was selected to perform this task. The aim is to estimate the parameters of the assumed model using the available data samples (or the correlation matrices estimated from the data). Once the model parameters were estimated, the desired frequency 
features can be determined by substituting the estimated model parameters into their analytical forms derived from the model.

The multivariate autoregressive (MAR) modeling of the digitized set of stationary measurement vectors is described by[18]

$$
X(1)=\sum_{i=1}^{n} A(i) X(t-i \Delta t)+V(t)
$$

where $X(t)=\left(x_{1}(t), x_{2}(t), \ldots, x_{m}(t)\right)$ represents the measurement signal vector at time instant $t$, and $\Delta t$ is the sampling interval. The measured $m$-dimensional random sequence $X(t)$ has zero mean and finite variance (i.e. they are the noise components of the measured process signals, digitized after removing the mean values of stationary signals). Due to the assumption of Eq.(7.1), the value of a given noise signal in vector $\mathrm{X}(\mathrm{t})$ at the time instant $\mathrm{t}$ depends on its past values and the past values of the other measured signals as well. The matrices $\mathrm{A}(1), \mathrm{A}(2), \ldots, \mathrm{A}(\mathrm{n})$ are the (mxm) MAR coefficient matrices. They describe the coupling among the signals and the overall memory of the system (that is, the weights of contributions of past values to the current measurement vector).

The m-dimensional white Gaussian noise vector $V(t)$ represents the instantaneous contribution to each individual signal which cannot be derived from intersignal transmissions. The components of vector $\mathrm{V}(\mathrm{t})$ may be correlated (that is, the off-diagonal elements of matric $\Sigma, \sigma_{i j}$ are not necessarily equal to zero). In the above mathematical construction (Eq. 7.1), the $V(t)$ term can be interpreted in physical sense as the external driving noise source of the equivalent multivariate linear system.

The first step is to determine the unknown $A$ and $\Sigma$ matrices (i.e. to fit the model represented by Eq.(7.1) using the estimated correlation matrices of the given set of 
measurement vectors. 'The MAR coefficient and noise covariance matrices are estimated by solving the set of matrix Yule-Walker equations, derived from the defining equation Eq.(7.1). It describes the linear relationship between the MAR model matrices and the measurement-based correlation matrices.

$$
\begin{aligned}
& C(k)=\sum_{i-1}^{n} A(i) C(k-i), k=1,2, \ldots, n \\
& C(0)-\sum_{i-1}^{n} A(i) C(-i)+\Sigma
\end{aligned}
$$

where the biased estimator of the correlation matrices $C(k), k=0,1, \ldots, n$ of measurement vectors were used. The diagonal elements of matrix $C(k)$ are the estimated autocorrelation functions at time lag $\mathrm{k}$, while the off-diagonal elements represent the corresponding cross correlation functions at time lag $k$. The MAR model identified by solving the above linear equations is equivalent to the original measured system, at least at the level of the correlation matrices of the first $\mathbf{n}$ time lags (because Eqs. (7.2a,b) are satisfied).

The above set of matrix equations can be solved by a computationally efficient algorithm, which is recursive with respect to increasing MAR model order|14]. The calculation of successively higher order MAR matrices proceeds until an optimal model order $(n)$ is defined (that is, until the determinant of the covariance matrix of the driving noise sourse, $\Sigma$ is reduced to a desired value). Also, several other criteria (FPE, AIC) were used for selection of optimal MAR model order, but experienced judgement is still required. The optimal model order may also depend on the signautre to be estimated by the MAR model. The recursive algorithm also provides the MAR matrices for all the lower order MAR models, so the effect of choosing different model orders on the results can be studied easily. 


\subsection{Statistical Characteristics in the Frequency Domain}

Once the optimal MAR order is established, that is, the $A(1), A(2), \ldots A(n)$ and $\Sigma$ matrices are determined, the model is transformed to the frequency domain and several signatures are calculated for the individual signals or signal pairs. These are:

a. auto- and cross power spectral density functions,

b. ordinary coherence $(\mathrm{COH})$ and partial coherence $(\mathrm{PCOH})$, and the corresponding phase functions,

c. ordinary and partial noise source contribution ratio functions (NSCR and PNSCR),

d. transfer functions among the measured signals giving the frequency response from one signal to another.

The spectral matrix of the measurement vector can be derived from the multivariate autoregressive assumption (Eq.7.1.), and it will have the form

$$
S_{X X}(f)=H(f)^{-1} \Sigma H^{*}(f)^{1} \Delta t
$$

where $\quad I(f)=1-\sum_{i=1}^{n} A(i) \exp (-j 2 n f i \Delta t)$ is an $(\mathrm{mxm})$ complex matrix constructed from the MAR matrices. The spectral matrix contains the auto power spectral density (APSD) functions of the signals in its diagonal elements, and the off-diagonal elements define the corresponding cross power spectral density (CPSD) functions.

The ordinary coherence function between signals $x i$ and $x j$ is defined as

$$
\mathrm{COH}_{1 J}(f)=\frac{\left|S_{1 j}(f)\right|^{2}}{S_{i 1}(f) S_{j j}(f)}
$$


The $\mathrm{COH}_{\mathrm{ij}}(f)$ function has value between zero and unity, and indicates the commonality - as a function of frequency - of the two signals including the effects of all possible signal transmission paths (STP) connecting the two signals.

The corresponding phase shift between the two signals as a function of frequency is defined as

$$
\operatorname{PHASE}_{1 J}(n)=\operatorname{artg}\left\{\frac{\left.\operatorname{Im} \mid S_{i j}(t)\right]}{\operatorname{Re}\left[S_{i j}(n)\right]}\right\}
$$

The ordinary noise source contribution ratio from the driving noise source of signal $x_{j}$ to the auto power spectral density of signal $x_{i}$ is defined as

$$
\operatorname{NSCR}_{I J}(f)=\frac{\left\{\left\{\left.H\left(f^{-1}\right\}_{1 j}\right|^{2} o_{H}\right.\right.}{S_{11}(n)} \Delta t
$$

where $\sigma_{\mathrm{jj}}$ is the jth diagonal element of the matrix $\Sigma$. The matrix $\mathrm{H}(\mathrm{f})^{-1}$ contains all the signal transmission path (STP) transfer functions that may exist between any two given signals. The NSCR analysis will characterize the resulting global coupling among the measured process variables. The ith signal's auto spectrum $\mathrm{S}_{\mathrm{ii}}(f)$ in the denominator also contains the contributions from the off-diagonal elements of the covariance matrix $\Sigma$. In many cases the off-diagonal elements are assumed to have negligible values compared with the diagonal values (assumption of uncorrelated driving noise components); consequently the sum of the individual NSCR functions for any given signal is equal to one by definition. In our case the deviation of this sum from unity, as a function of frequency, will indicate the goodness of the selected signal combination and model order for the given frequency range. These frequency functions represent the global contribution of one signal to the other through all possible signal transmission paths between the signals $i$ and $j$. Thus they cannot characterize the direct relationship, or the direct STP of specified signal pairs. 


\subsection{Decomposition of Multivariate Dynamic System}

In order to get the individual transfer functions connecting the measured variables by eliminiating the effect of the remaining variables, the following multivariate linear system modeling based on MAR-modeling was introduced[19, 20]:

$$
X(f)=G(f) X(f)+N(f)
$$

where $G(f)$ is the transfer matrix, $G_{i j}(f)$ is the $(i, j)$ th element, and zeros in the diagonal elements. $G_{i j}(f)$ can be expressed using the MAR-matrices by rearranging Eq. (7.1)

$$
G_{1 j}(t)=-\frac{H_{13}(f)}{H_{11}(f)} \text { and } G_{11}(f)=0
$$

The $(\mathrm{i}, \mathrm{j})$ element of the spectral matrix of the inherent noise source $N(f)$ is

$$
u_{1 j}(n)=E\left\{N_{1}\left(n N_{j}^{*}(n)\right\}=\frac{o_{1 j}}{H_{11}(n) H_{j}^{*}(f)}\right.
$$

which defines the auto- and cross-spectral density functions of the residual inherent noise field as a result of the multivariate signal decomposition.

Using the system decomposition defined by Eq. (7.7), the auto power density function of each signal can be decomposed into contributions from the other signals' noise sources and from its own inherent noise source. Also, the cross power spectral density function of two given signals can be split into two parts: (a) direct correlation between the two signals due to contribution of one signal noise source to the other signal through the corresponding transfer functions (terms $G_{i j}(f) Q_{j i j}(f)$ and $\mathrm{G}_{\mathrm{ji}}(\mathrm{f}) \mathrm{Q}_{\mathrm{ii}}(\mathrm{f})$ ), and (b) contributions from the correlated inherent noise sources (terms $Q_{i j}(f)$ and $\left.Q_{j i}(f)\right)$. The spectral properties of the resulting inherent noise field were also calculated (APSD, CPSD, COH and PHASE functions), and plotted together with the corresponding frequency signatures of measured noise signals. 


\section{Partial Coherence and Partial Noise Source Contribution Ratio Functions}

Constructing the transfer matrix $G$ and the inherent noise source spectral matrix $Q$ from the MAR model, the partial coherence function between signals xi and $x \mathrm{j}$ is defined as follows:

$$
\begin{gathered}
\operatorname{PCOHij}(f)= \\
\frac{1 Q_{i j}+G_{i j} Q_{i j}+G_{j i}^{*} Q_{i i}+G_{i j} G_{j i}^{*} Q_{i j} \mid}{\left(Q_{i i}+I G_{i j}{ }^{2} Q_{i j}+2 \operatorname{Re}\left[G_{i j} Q_{i j}^{*}\right]\right)\left(Q_{i j}+\mid G_{j i}{ }^{2} Q_{i j}+2 \operatorname{Re}\left[G_{j i} Q_{j i}^{*}\right]\right)}
\end{gathered}
$$

where the partial CPSD of the two signals is defined in the numerator, and the partial APSDs are defined in the denominator, excluding the effects of all other signals and allowing only the direct transmission between the two given signals.

The corresponding phase shift between the two signals excluding the effects of others is defined by the real and imaginary parts of the partial CPSD function:

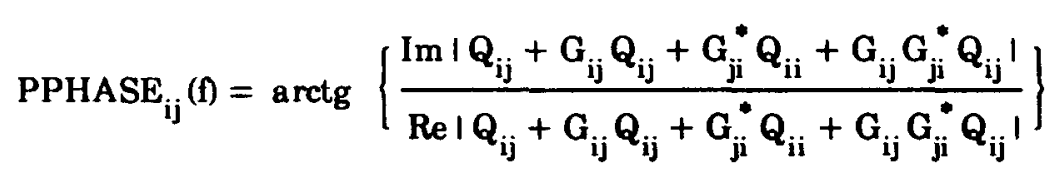

Keeping only the terms connecting the given two signals directly, the partial noise source contribution ratio function from signal $j$ to signal $i$ has the form:

$$
\operatorname{PNSCR}_{i j}(f)=\frac{\left|G_{i j}(f)\right|^{2} Q_{i j}(f)}{\left.Q_{i j}(f)+\left|G_{i j}(f)\right|^{2} Q_{i j}(f)+2 \operatorname{Re}\left[G_{i j}(f) Q_{i j}^{*}(f)\right]\right)} \text { for } i \neq j
$$

and the self-contribution of signal i from its own noise source:

$$
\operatorname{PNSCR}_{\mathrm{ij}}(f)=\frac{Q_{\mathrm{ij}}(f)}{Q_{\mathrm{ii}}(f)+\left|G_{\mathrm{ij}}(f)\right|^{2} Q_{\mathrm{ij}}(f)+2 \operatorname{Re}\left[G_{\mathrm{ij}}(f) Q_{\mathrm{ij}}^{*}(f]\right)}
$$

The partial coherence and the partial noise source contribution ratios relate any two signals directly by excluding the influence of other signals. This procedure is essential to minimize the misinterpretation of the cause-and-effect relationship. 
In this context, it must be stated that the selection of a proper combination of the signals is very important, and can be achieved by physical considerations of the system and preliminary experimentation. The effect of hidden or unmeasured process variables (not included in the MAR modeling) are implicitly involved in the system decomposition. All the effects of unmeasured signals on the measured signal set are represented by the inherent noise com-ponents, and are specific to the given signal combination. Thus, physically significant process variables, unmeasurable or accidentally excluded from the STP analysis, can cause false STPs among the signals analyzed.

Note that the $\operatorname{PNSCR}_{i j}(f)$ function becomes zero if there is no direct transfer from signal $\mathrm{j}$ to signal $\mathrm{i}$, while the $\mathrm{PCOH}_{\mathrm{ij}}$ function still has some non zero value for the case of $G_{i j}(f)=G_{j i}(f)=0$ due to the contribution of correlated inherent noise components $Q_{i j}(f)$. In the case of uncorrelated noise sources $\left(Q_{i j}(f)=0\right)$, and without feedback from signal $i$ to signal $j\left(G_{j i}(f)=0\right)$, these two functions become identical.

The comparison of the above ordinary and their corresponding partial frequency functions provide information on noise generation and propagation mechanism, and on the cause-and-effect relationships among the selected signals in a multivariate dynamic system. The information contained in the above frequency-dependent statistical functions (or in the systematic comparison of these functions) can be translated into characteristics of actual physical processes specific to the given system (for example, axially propagating coolant density fluctuations, fuel to coolant heat transfer, pressure standing waves, pump rotation, etc.).

Besides creating a baseline pattern for normal system operation (containing statistical features sensitive enough to changes in the system), the above STP analysis can also be used for extracting process related technical parameters (such as, velocity of coolant flow, heat transfer coefficient, detector response time, pump 
speed). This method is also a potential candidate for performing automated reactor diagnostics and sensor fault monitoring.

\subsection{Application to Operating PWR Plant Noise Signals}

The above analysis was applied to process noise signals recorded during steadystate operation at four different power levels $(25 \%, 50 \%, 75 \%$ and $100 \%)$ in the Lossof-Fluid-Test (LOFT) reactor. The schematic of the reactor is shown in Fig. 7.1. The analog data acquisition of the noise process signals was performed by the Instrumentation \& Controls Division of the Oak Ridge National Laboratory, while the digitization and signal processing were carried out at the Nuclear Engineering Department of the University of Tennessee, using an IBM PC. The optimal model and the corresponding frequency-dependent functions were calculated in the frequency range $0-5 \mathrm{~Hz}$ and $0-25 \mathrm{~Hz}$, using data lengths of $1500 \mathrm{sec}$ and $300 \mathrm{sec}$, respectively (15000 data point per signal). The high-pass filters were set at $0.05 \mathrm{~Hz}$ for both cases, while the low-pass filters were set at $4 \mathrm{~Hz}$ and $20 \mathrm{~Hz}$, respectively. A maximum model order of $n=40$ was used for various combinations of the following process signals:

- in-core neutron noise signals (NE-5D8-11, 27, 44 and 63),

- core-exit thermocouple (TC) noise signals (TE- 5UP-3 and TE-2UP-5),

- noise signals of differential pressure transducers across the primary coolant pumps (PDE-PC-1), across the steam generator (PDE-PC-2), and across the reactor core (PDE-PC-6).

A brief description of the results in terms of spectral properties of individual signals (APSD of the measured process variables and that of their computed inherent noise sources) and the cross-information of signal pairs ( $\mathrm{COH}, \mathrm{PCOH}$, NSCR and PNSCR) are be summarized in the following sections. 


\section{Results of the STP Analysis in the Frequency Range 0-5 Hz}

In each of the following cases, no feedback from the second signal to the first one was observed in the frequency range 0-5 Hz (i.e. $\operatorname{NSCR}_{12}(f)=\operatorname{PNSCR}_{12}(f)=0$ ) making the analysis and interpretation easy. The partial coherence $\mathrm{PCOH}_{12}(f)$ and the partial noise source contribution ratio functions from the first to the second signal $\mathrm{PNSCR}_{21}(f)$ are identical. The relationship between the coherence and partial coherence functions, as well as the noise source contribution ratio functions, varied from case to case, indicating the various strength of signal coupling. For all the signal pairs described in this chapter, the phase shift was linear as a function of frequency, over a certain frequency range (typically $0-4 \mathrm{~Hz}$ ) with a positive slope indicating the presence of propagating effects from the first signal to the second. The phase plot starts either from zero degree or from -180 degree, depending on the analyzed signal pair. This also gives information on the sign of cross correlation function of the two signais. In the following we will discuss some concrete results of the STP analysis in terms of pairwise comparison of the measured signals.

- The APSD functions of four measured noise signals (solid line), and those of the corresponding inherent noise sources or residual noise components (dashed line) are plotted in Fig. 7.2. The difference between the signal APSD and the APSD of inherent noise component indicates the effect of other signals (that is, contributions from other signal noise sources).

- Comparison of noise signals pump $\Delta p$ and core $\Delta p$ (Fig. 7.3). Approximately $80 \%$ of the core $\Delta p$ fluctuations in the frequency range $0-4 \mathrm{~Hz}$ originate from the pump $\Delta p$ fluctuations. The phase shift between them starts from - 180 deg at zero frequency and is linear as a function of frequency up to $4 \mathrm{~Hz}$. The time delay between the pump $\Delta p$ and core $\Delta p$ inferred from the phase slope is $0.125 \mathrm{sec}$. This result was the same for all the four analyzed power 
levels. The coherence and phase functions of the inherent noise components of the pump $\Delta p$ and core $\Delta p$ (frequency independent!) are plotted by dashed lines. Actually the coherence is zero, which means that the noise sources of the two given signals are independent of each other.

- Comparison of noise signals pump $\Delta p$ and in-core neutron flux (Fig. 7.4): The $\mathrm{COH}$ and $\mathrm{NSCR}_{21}$ functions are identical and have higher values than their corresponding partial functions, but the latter are still high. The direct transmission path from the pump $\Delta p$ to the in-core neutron noise is significant. The $\mathrm{COH}$ and $\mathrm{PCOH}$ functions have similar phase plots linear up to $4 \mathrm{~Hz}$ with time delay $0.38 \mathrm{sec}$ inferred from the slope. There is no phase shift between the two signals at zero frequency. The inherent noise components of the two signals are independent.

- Comparison of noise signals pump $\Delta \mathrm{p}$ and core-exit coolant temperature (Fig.7.5): The $\mathrm{COH}$ and $\mathrm{NSCR}_{21}$ functions are identical, and the phase is linear up to $2 \mathrm{~Hz}$ starting from $-180 \mathrm{deg}$. at zero frequency. The time delay inferred from the slope is $0.625 \mathrm{sec}$. The corresponding partial functions $\left(\mathrm{PCOH}_{\text {and }} \mathrm{PNSCR}_{12}\right)$ also have identical functional shape and have low values (less than 10\%) indicating that there should be an indirect signal transmission path from pump $\Delta \mathrm{p}$ to core-exit temperature through other process variables. Another explanation would be the correlated inherent noise components, but for the given case the coherence of the inherent noise components is zero (no dashed line can be seen besides the signals' coherence function).

- Comparison of noise signals core $\Delta p$ and in-core neutron flux (Fig.7.6): High coherence $(80 \%)$ and low partial coherence $(20 \%)$ with similar phase plot in the range $0-4 \mathrm{~Hz}$ was observed. The $\mathrm{NSCR}_{21}$ is very low while the $\mathrm{PNSCR}_{21}$ 
function (identical with the $\mathrm{PCOH}$ function) has somewhat higher values $(20 \%)$. The time delay between the two signals is $0.25 \mathrm{sec}$. (inferred from the linear phase plot over the frequency range $0-2 \mathrm{~Hz}$ ). The two noise signals have independent inherent noise components.

- Comparison of noise signals core $\Delta p$ and core-exit temperature (Fig. 7.7): The cuherence is about $50 \%$ while the partial coherence is almost zero over the same frequency range. Very low NSCR and PNSCR functions were detected in both signal flow directions indicating the lack of direct signal coupling. The high global conherence implies the effect of a third signal connecting the given two signals. The time delay between the two signals inferred from the linear phase related to the global coherence is 0.5 sec.

- Comparison of noise signals in-core neutron flux and core-exit coolant temperature (Fig. 7.8): High coherence (60\%), smaller partial coherence $(20 \%)$, and low $\mathrm{NSCR}_{21}$ were observed in the frequency range $0-2.5 \mathrm{~Hz}$. The $\mathrm{COH}$ and $\mathrm{PCOH}$ functions have similar phase plots, being linear in the above frequency range, starting from-180 deg. at zero frequency. The inferred time delay is $0.25 \mathrm{sec}$.

The sum of the noise contribution ratio functions for each of the four noise signals is shown in Fig. 7.9. By the definition in Eq. (3.6) of the NSCR functions (each has positive value), their sum is exactly equal to one if the residual noise components in the MAR model are independent (i.e. the covariance matrix $\Sigma$ is diagonal). Figure 3.9 indicates that for the given signal combination, the MAR model order was correct in the given frequency range.

By including more signals (steam generator $\Delta \mathrm{p}, \mathrm{PDE}-\mathrm{PC}-2$ ) and another coreexit thermocouple (TE-2UP-3) in the analysis, further physical understanding of the process is possible. 
- Comparison of noise signals pump $\Delta \mathrm{p}$ and steam generator $\Delta \mathrm{p}$ (Fig. 7.10): The $\mathrm{COH}$ and $\mathrm{PCOH}$ functions are identical with high values (70\%) over the range $(0-4 \mathrm{~Hz}$, and with identical linear phase plots. They are in phase at zero frequency. The noise inferred time delay between the two signals is 0.13 sec. The $\mathrm{NSCR}_{21}$ and $\mathrm{PNSCR}_{21}$ functions are identical with the functional shape of the coherence function. All these results indicate the dominance of the direct signal transmission path from the pump $\Delta p$ fluctuation to the steam generator $\Delta \mathrm{p}$ fluctuation.

- Comparison of noise signals steam generator $\Delta p$ and core $\Delta p$ (Fig. 7.11): The $\mathrm{COH}$ function of the two signals is relatively high in the range $0-3 \mathrm{~Hz}$, and shows constant out-of-phase relationship. The $\mathrm{PCOH}$ function characterizing the direct coupling has low value (below 10\%), and has zero phase shift. This indicates that the direct STP between the two signals and the STPs through other signals create opposite phase behavior, and the latter dominates the resultant relationship between the two signals. The fact that both NSCR and PNSCR functions in both directions are zero supports the independency of the two signals. The reason why their coherence function is still high is that they have a common driving source, the pump $\Delta p$ fluctuation. The corresponding inherent noise components are not quite independent. Their coherence is constant as a function of frequency - about $5 \%$ - shown by the dashed line, and they are in-phase. As a consequence of having high coherence and out-of-phase behavior between the core $\Delta \mathrm{p}$ and the steam generator $\Delta \mathrm{p}$, the latter also has high coherence and linear phase with the other signals -in-core neutron noise and core-exit coolant temperature noise. However, no direct STP was detected between the steam generator $\Delta \mathrm{p}$ and any other process signal. 
- Comparison of noise signals pump $\Delta \mathrm{p}$ and two different core-exit thermocouples located at different radial and axial positions. TheAPSD functions of the two core-exit TCs (solid line) and those of their inherent noise components (dashed line) are shown in Fig. 7.12. The difference between the signal and inherent APSD values implies the significance and the frequency range of contributions from other signals. The pairwise comparison is shown in Figs. 7.5 and 7.13. The corresponding $\mathrm{COH}$ and NSCR21 functions are identical, and the phase is linear upto $2.5 \mathrm{~Hz}$ and 1.5 $\mathrm{Hz}$, for the thermocouple TE-5UP-3 and TE-2UP-5, respectively. The phase starts from -180 deg at zero frequency for both cases. The time delays inferred from the phase slopes are $0.625 \mathrm{sec}$ and $1.0 \mathrm{sec}$, respectively. The difference in the delay times is due to the different axial locations of the two thermocouples, implying the propagation nature of the transmitting mechanism. The corresponding partial functions ( $\mathrm{PCOH}$ and $\left.\mathrm{PNSCR}_{12}\right)$ have low values, indicating the weakness of the direct STP from pump $\Delta p$ to the two different core-exit TC signals. As we saw earlier the corresponding inherent noise components are uncorrelated, consequently there must be a third transmitting process, or other processes to produce the pronounced global coherences and linear phase relationship.

- Comparison of noise signals in-core neutron flux and two different core-exit thermocouples (TE-5UP-3 and TE-2UP-5) located at different radial and axial positions (Figs. 7.8 and 7.14): Th coherence functions are relatively high in the frequency range $0-2.5 \mathrm{~Hz}$ and $0-1.5 \mathrm{~Hz}$, respectively. The phase plots are linear in the above range, starting from -180 deg at zero frequency. The inferred time delays are different, $0.25 \mathrm{sec}$ and 0.51 , respectively. The $\mathrm{COH}$ function between the two TC signals has a non zero 
value in the frequency range $0-1.5 \mathrm{~Hz}$ (see Fig. 7.15), and the phase function is linea starting from zero at zero frequency (the two TCs are inphase at low frequencies). The time delay between the TC signals, inferred from the phase slope is 0.26 sec. The zero PCOH, NSCR and PNSCR functions in both directions indicate the lack of direct signal transmission path between the two thermocouples.

The results in this section were shown as pairwise comparisons, although they were acquired from multivariate simultaneous analysis. Figure 7.6 summarizes certain aspects of the multivariate analysis in the form of a signal transmission path diagram (STPD). The STPDs having high and almost identical $\mathrm{COH}$ and $\mathrm{PCOH}$ values represent strong direct coupling between the two signals in the indicated directions. The transit time between the two signals inferred from the slope of the linear phase is shown in the figure. Also, the phase shift at zero frequency is displayed. The analysis was performed for $100 \%$ power operation. The STP map for a lower power level $(25 \%, 50 \%$ and $75 \%)$ was basically identical with the one in Fig. 7.16.

Unlike the two-signal Fourier analysis, the MAR-based STP analysis can reveal the inter-signal relationships of multivariate dynamic systems by processing all the recorded signals simultaneously (signal "peeling" technique). The process fluctuation generated by the noise source of one signal can propagate to other signals through the process. This dynamic structure is characterized by the MAR-based signal flow network of a multivariate stochastic process in a systematic manner. The above statistical features also reflect the possible correlation among the inherent noise components. 


\subsection{Summary}

The following conclusions are obtained from the above STP analysis:

a. The primary driving noise source in the frequency range $0-4 \mathrm{~Hz}$ is the fluctuation of the differential pressure measured across the primary coolant pump.

b. Strong signal flow from the pump $\Delta p$ fluctuation to the core $\Delta p$ fluctuation $(80 \%)$ and to the steam generator $\Delta p$ fluctuation $(70 \%)$ was found with opposite phase behavior.

c. There is direct signal flow from the pump $\Delta p$ fluctuation to the in-core neutron flux fluctuation (70\%) which may be due to the pressure fluctuation-induced coolant density fluctuation. As we saw, this signal transmission path plays an important role in the pump $\Delta \mathrm{p}$ and core-exit TC coupling.

d. All the three measured $\Delta \mathrm{p}$ noise signals have high coherence with the coreexit coolant temperature noise. However, the corresponding direct signal transmission paths are very weak. For all the three cases the transmitting process is the in-core neutron flux fluctuation.

e. The transit time between the in-core neutron noise and the core-exit coolant temperature noise does not depend on the axial position of the in-core neutron detectors (pont-kinetic neutronic behavior). However, there was a significant difference in the transit times related to core-exit TCs located at different elevations above the core (the axial separating distance between the two analyzed thermocouples is $13^{\prime \prime)}$.The typical transit time values are $0.51 \mathrm{sec}$ and $0.25 \mathrm{sec}$ for the thermocouples TE-2UP-5 and TC-5UP-3, respectively.

f. Despite the relatively high coherence, there is no direct STP between the two thermocouples. The zero PCOH and PNSCR functions indicate that there is 
no direct, physical coolant propagation from one TC to the other. The high coherence between them comes from the in-core neutron and core-exit temperature coupling. The linear phase shift between the two TCs isdue to the linear phase relationship between the in-core neutron noise and the individual core-exit TC signals. The noise-inferred transit time between the two TCs $(0.26 \mathrm{sec})$ is equal to the difference between the corresponding neutron-temperature transit times $(0.51 \mathrm{sec}$ and $0.25 \mathrm{sec}$, the higher value belongs to the thermocouple TE-2UP-5 located at the higher elevation). Using this transit time difference and the axial distance between the two TCs, the estimated average coolant velocity in the upper plenum region is $4.16 \mathrm{ft} / \mathrm{sec}$. This coincides fairly well with the design value.

The results of the application to the LOFT reactorprocess data demonstrate the feasibility of applyingsuch techniques to commercial NPPs. By monitoring various STP-related signatures, diagnostics of system or sensor anomalies may be performed. The application of this technique in operating NPPs may be automated by information management using an AI-based expert system. 


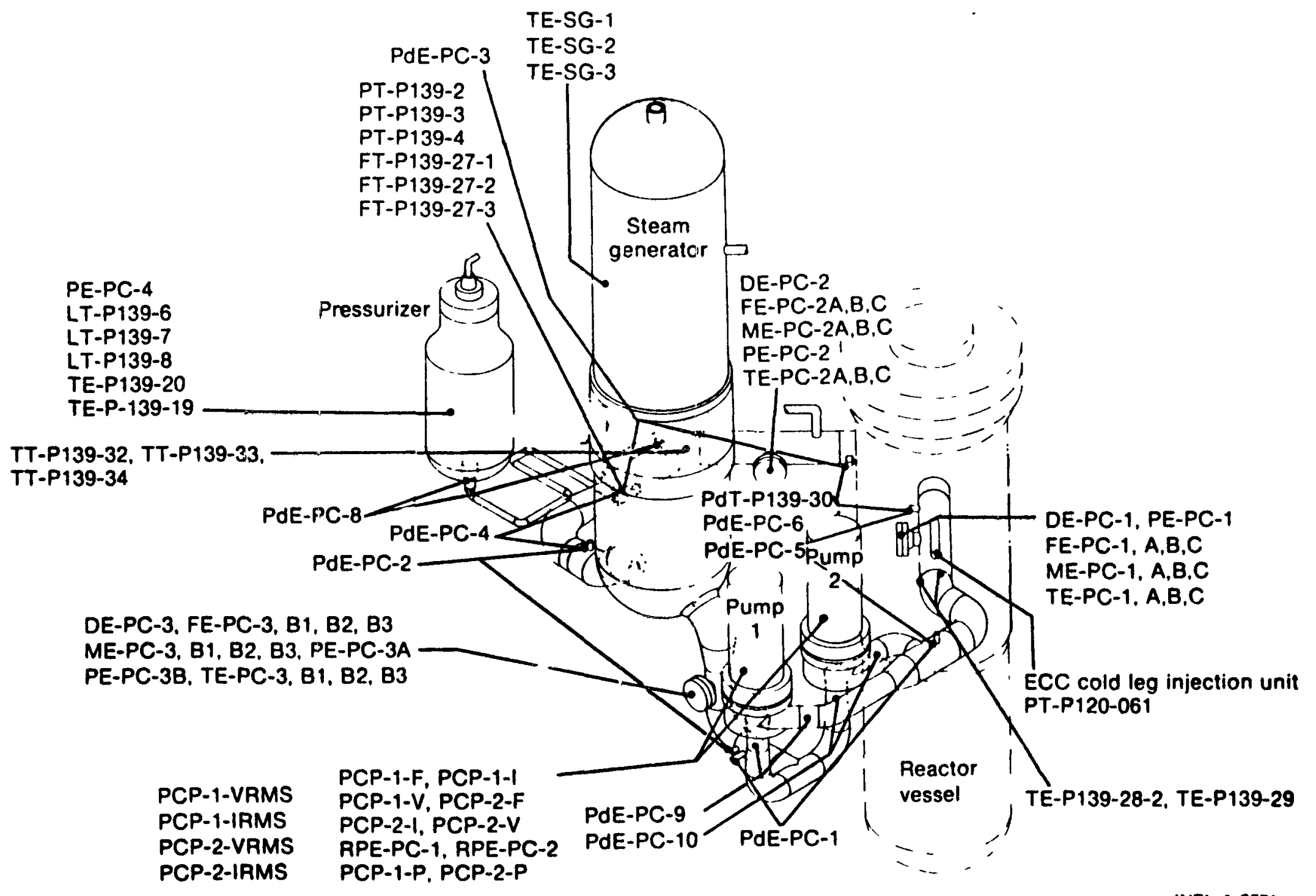

Figure 7.1. Schematic showing the LOFT reactor instrumentation. 
SICNAL NAME- PUMP OELTA P (POE-PC-1)
RECORDED AT POUSR LEVEL 10GX

APGD FUNCTIONS OF MEAGURED SIONALS AND THEIR INERENT NOIGE

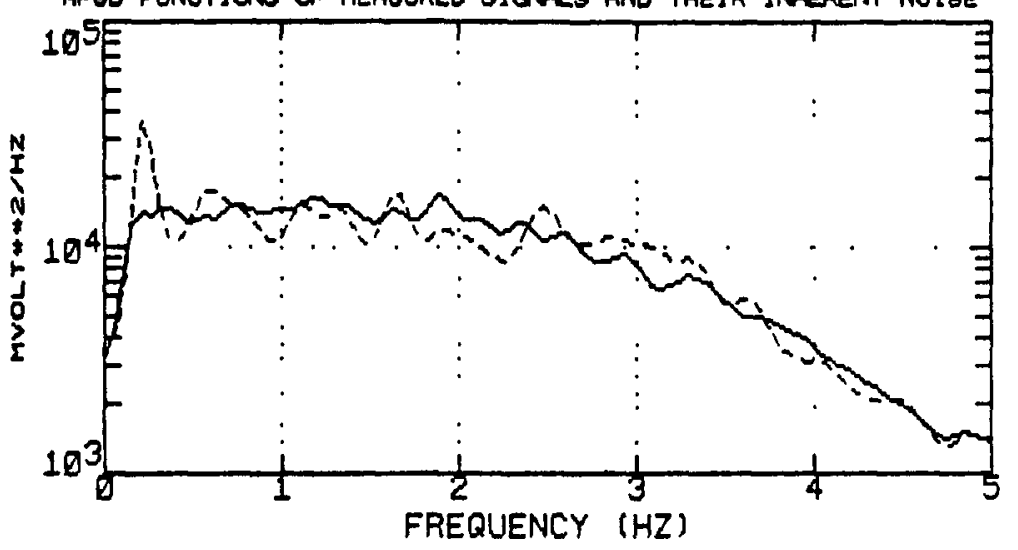

SIGNAL NANE- INCORE NEUTRON (NE-5DQ-61) CHANNEL NO.- 4 RECOROED AT POUER LEVEL 100X DATA FILE- AILE4090. 1940

\section{APSO FUNCTIONS OF MEAGUREO SIGNLS AND THEIR INHERENT NOISE}

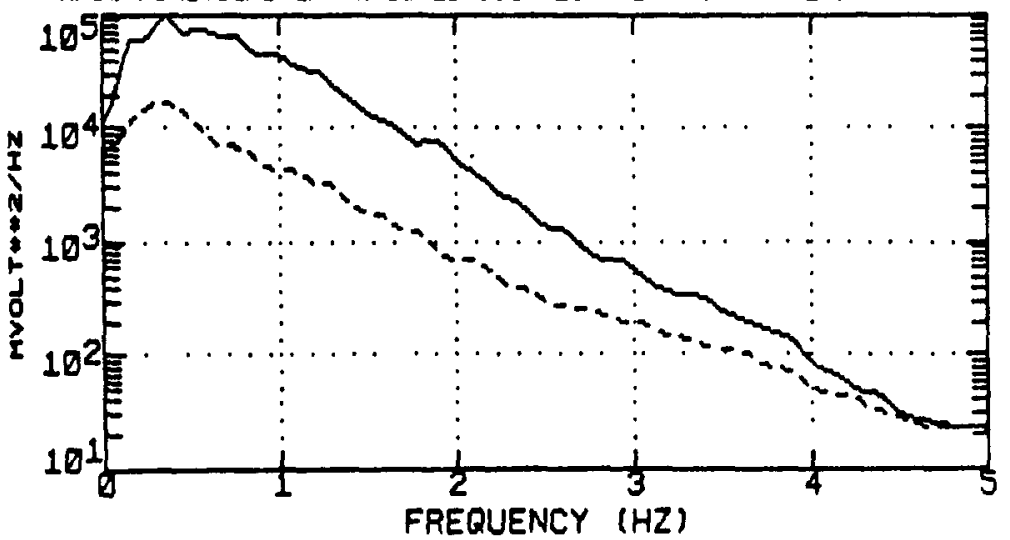

SIGNAL NAME- CORE DELTA P IPOE-PC-E) RECORDEO AT POWER LEVEL 100X

CHANNEL NO.- 3 IMTM FILE- AILEAEQR. 840

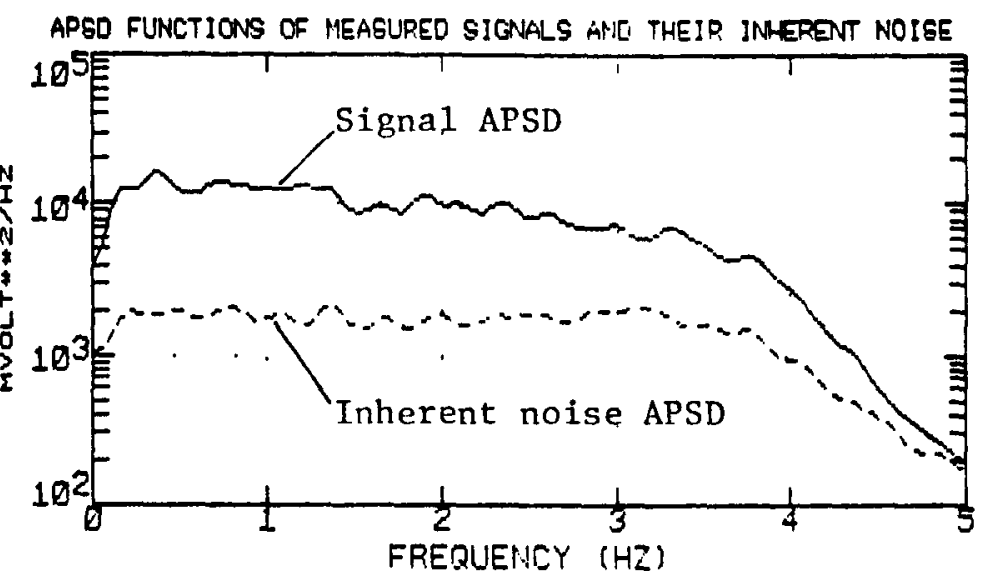

SIGNAL NAME- CORE-EXIT TC (TE-5IIF-3) RECOROED AT POWER LEVEL 100X

CHANNEL NO.- 5 DATA FILE- AILE4BgO. 040

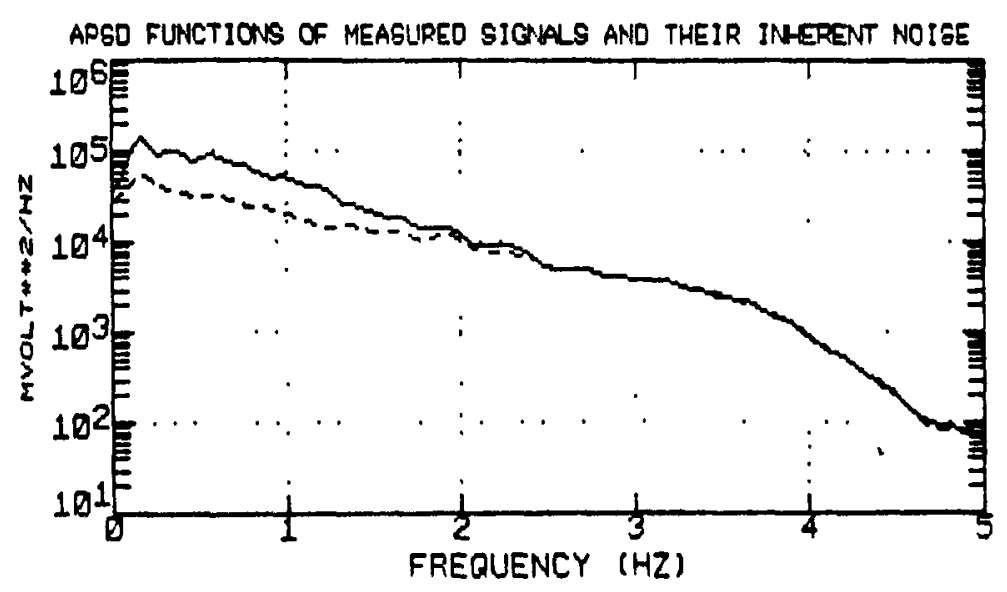

Figure 7.2. Power spectral density functions. (a) Pump DP. (b) Core DP. (c) In-core neutron detector. (d) Core-exit TC. 

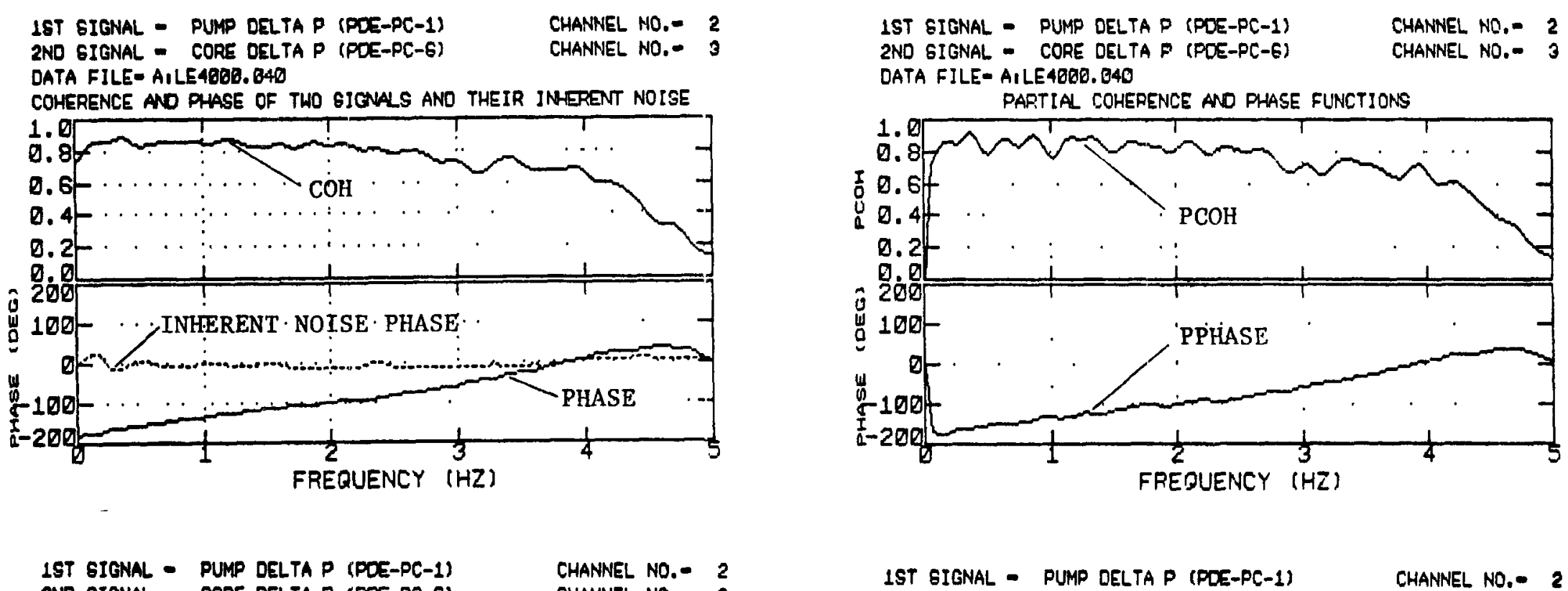

CHANNEL NO.- 3 2NO GIGNAL - CORE DEL

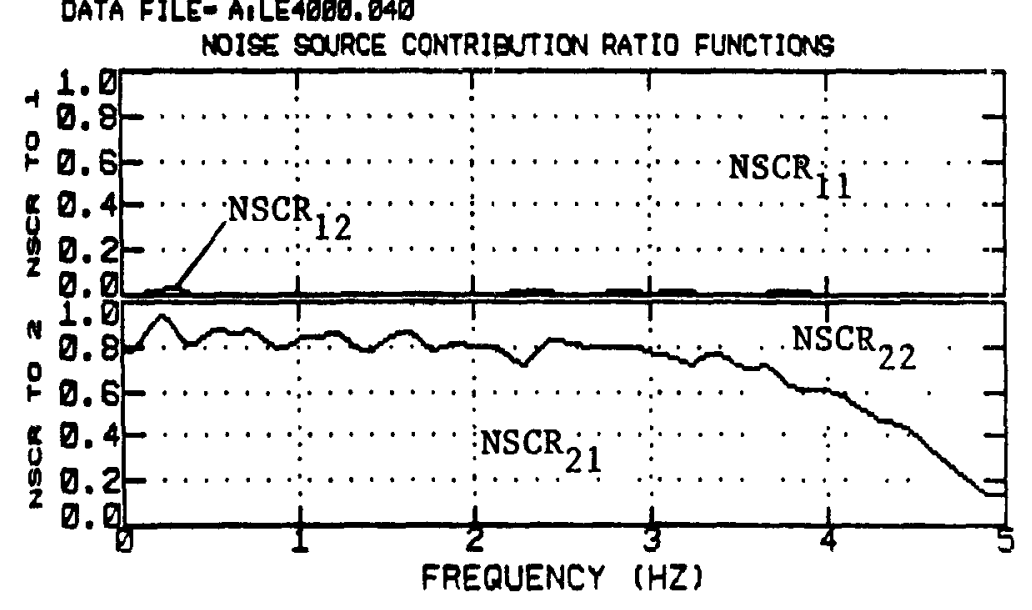

1ST GIGNAL - PUMP DELTA P (PDE-PC-1) 2ND GIGNAL - CORE DELTA P (PDE+PC-6)

CHANNEL NO.- 2 DATA FILE- AILE4600.040

PAATIAL NOISE SOURCE CONTRIEUTION RATIOS TO SIGNAL I AND 2

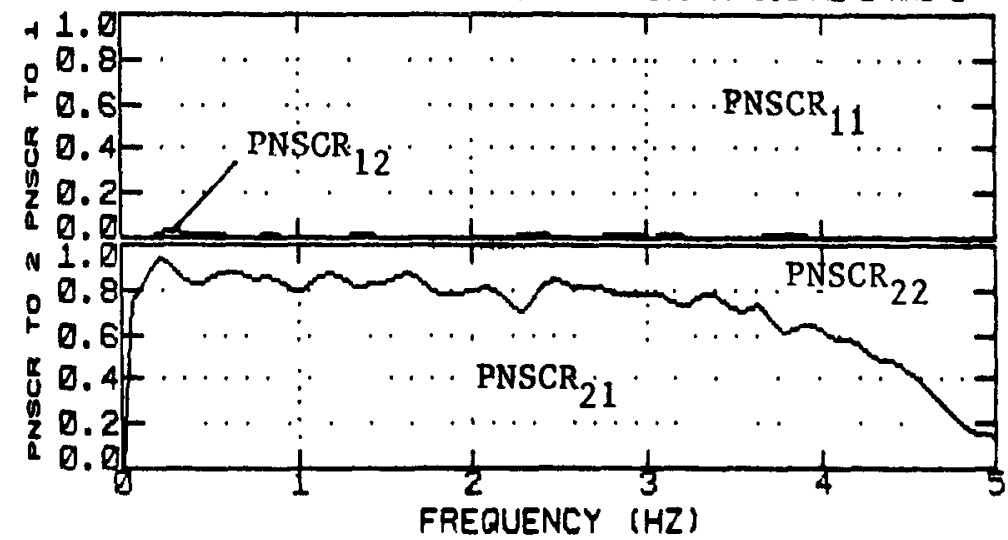

Figure 7.3. Signal pair pump DP-core DP. Coherence, phase and noise source contribution ratios (ordinary and partial). 
1ST GIGNAL - PUMP DELTA P (PDE-PC-1)

2ND SIGNAL - INCORE NEUTRON (NE-5DO-61)

CHANNEL NO.- 2

CHANNEL NO.-

DACA FILE- AILE4989.040

COHERENCE AND PHASE OF TWO SIGNALS AND THEIR IMERENT NOISE

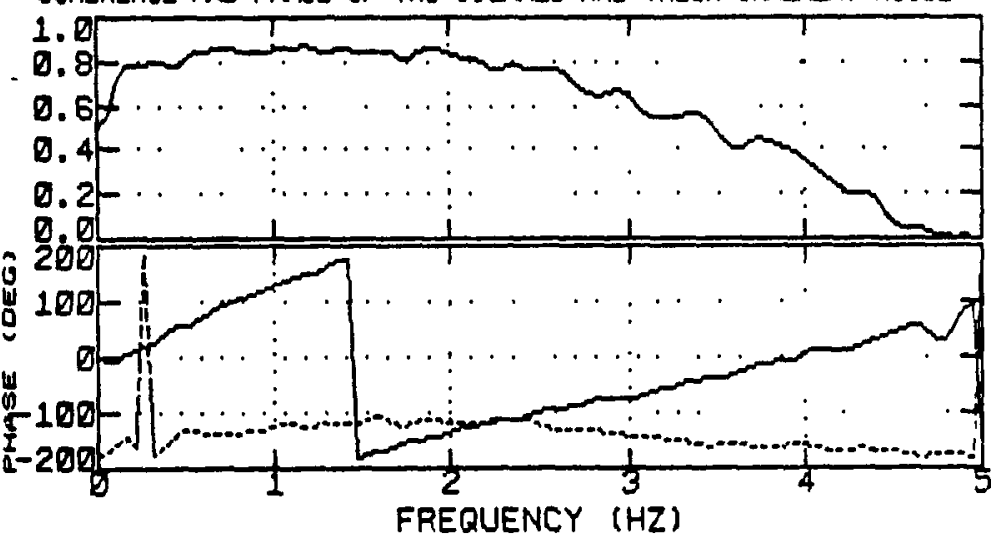

1ST GIGNAL - PUAP OELTA P (PDE-PC-1)

2ND SIGNAL - INCORE NEUTRON (NE-509-61)

CHANNEL NO.- 2

CHANNEL NO.- 4

DATA FILE- AILEA6B日. 640

NOISE SOURCE CONTRIEUTION RATIO FUNCTIONS

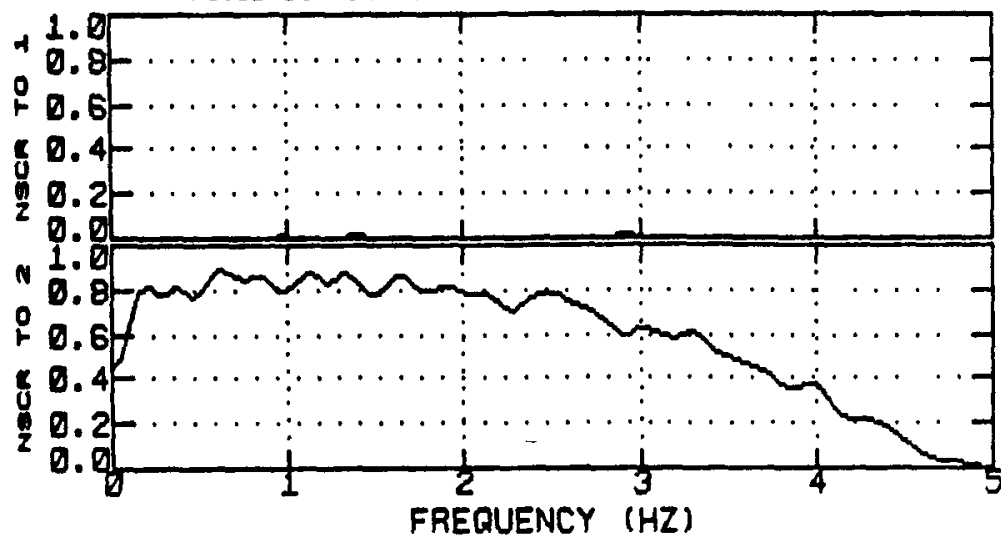

1ST SIGNAL - PUMP DELTAP (PDE-PC-1)

2NID SIGNAL - INCORE NEUTFON (RE-509-E1)

CHANNEL ND.- 2

DATA FILE- A,LE4OOOO. G40

PAFTIAL COHERENCE AND PHASE FUNCTIONS

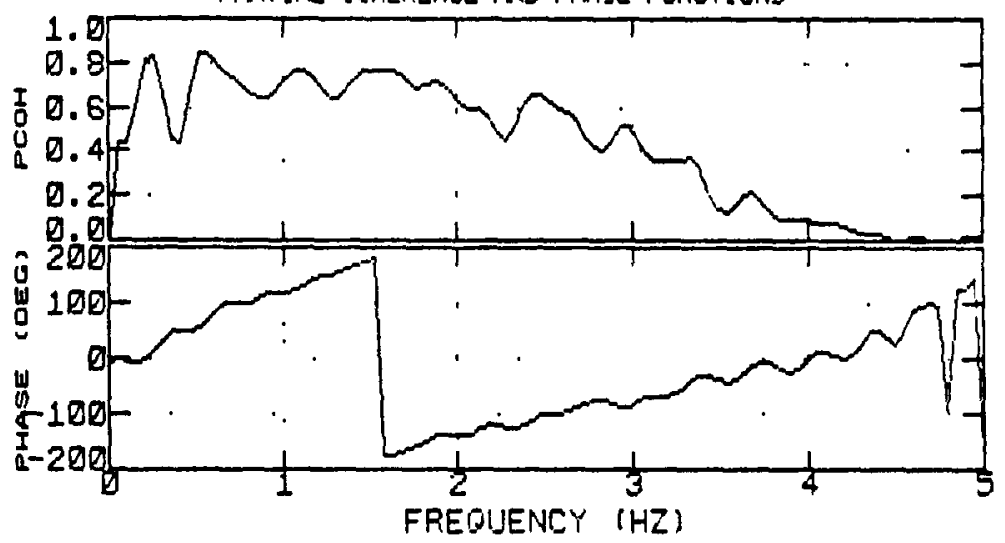

1ST GIGNAL - PUMP DELTA P (FOE-PC-1)

CHANNEL NO.- 2 2ND SIGNAL - INCORE NEIJTRON (NE-5DE-S1)

CHANNEL NO.- a DATA FILE- AILE4000.040

PARTIAL NOISE SOURCE CONTRIOUTION RATIOS TO SIGNAL 1 AND 2

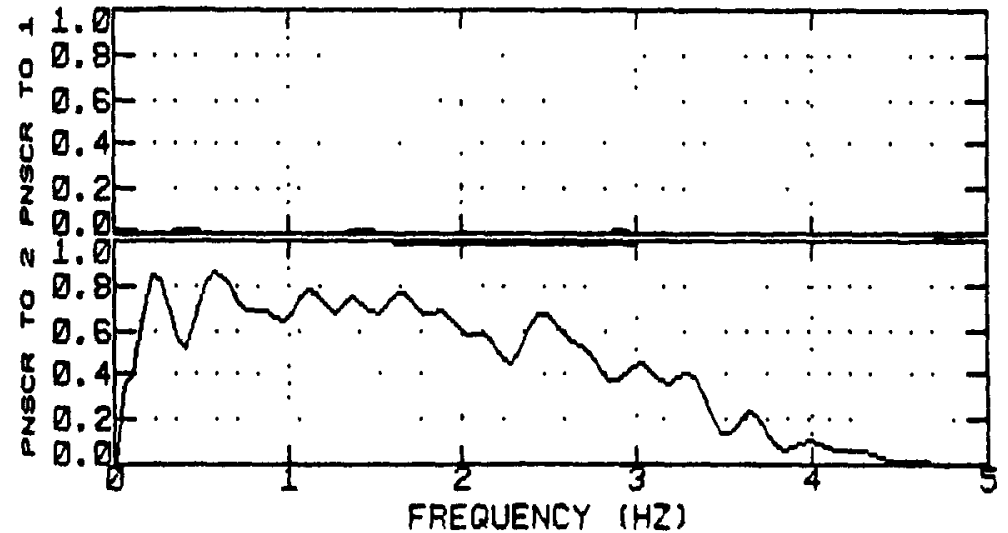

Figure 7.4. Signal pair pump DP-in-core neutron detector. Coherence, phase and noise source contribution ratios (ordinary and partial). 
1ST GIGNAL - PUMP DELTA P (POE-PC-1) 2ND GIGNAL - CORE-EXIT TC (TE-5UP-3) DATA FILE- AILE4600.040

COHERENCE ANO PHASE OF TWO 8IGNALS AND THEIR INERENT NOISE

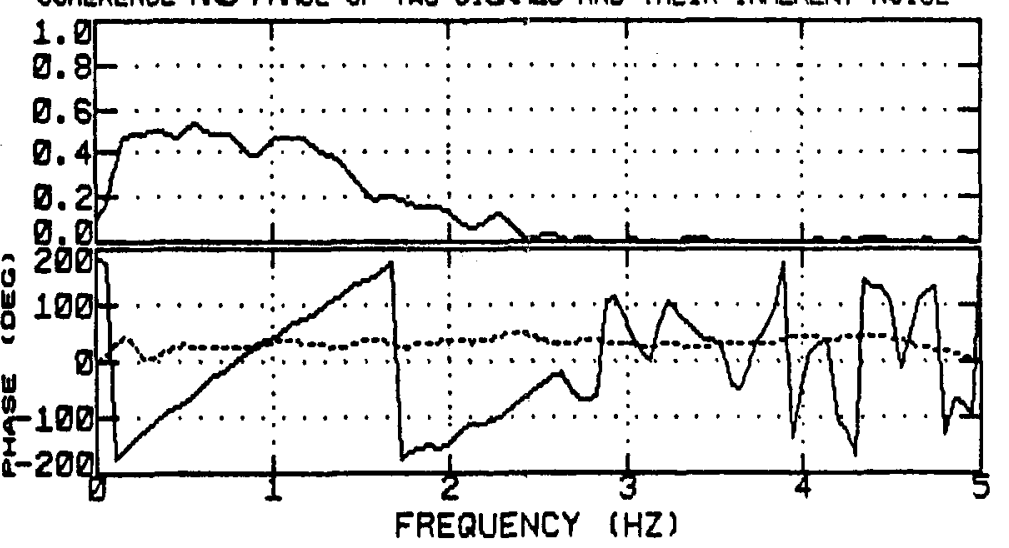

1ST GIGNAL - PUMP DELTA P (POE-PC-1) 2NO GIGNAL - CORE-EXIT TC (TE-5UP-3)

CHANNEL NO.- 2 DATA FILE- AILE4600. 040 NOISE SOURCE CONTRIEUTION RATIO FUNCTIONS

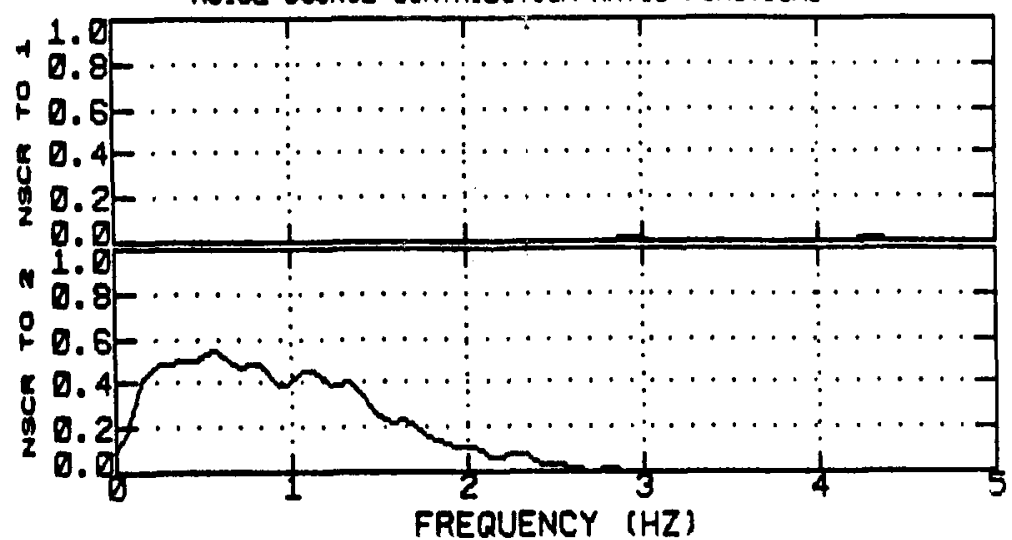

1ST GIGNAL - PUMP DELTA P (FOE-PC-1) 2ND SIGNAL - CORE-EXIT TC (TE-SIJP-3) DATA FILE- AILE4000.040

PARTIAL COHERENCE AND FHASE FUNCTIONS

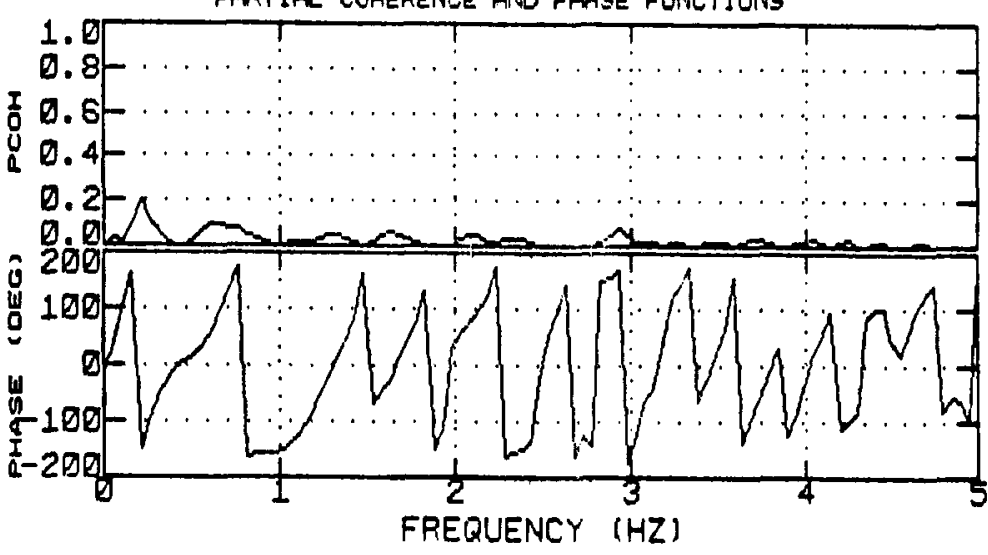

1ST GIGNAL - PUMP DELTA P (POE-PC-1) 2ND GIGNAL - CORE-EXIT TC (TE-SUP-3)

CHANNEL HO. - 2 DATA FILE- AILE4000.040

PARTIAL NOISE SOURCE CONTRIQUTION RATIOS TO GIGHA 1 AND 2

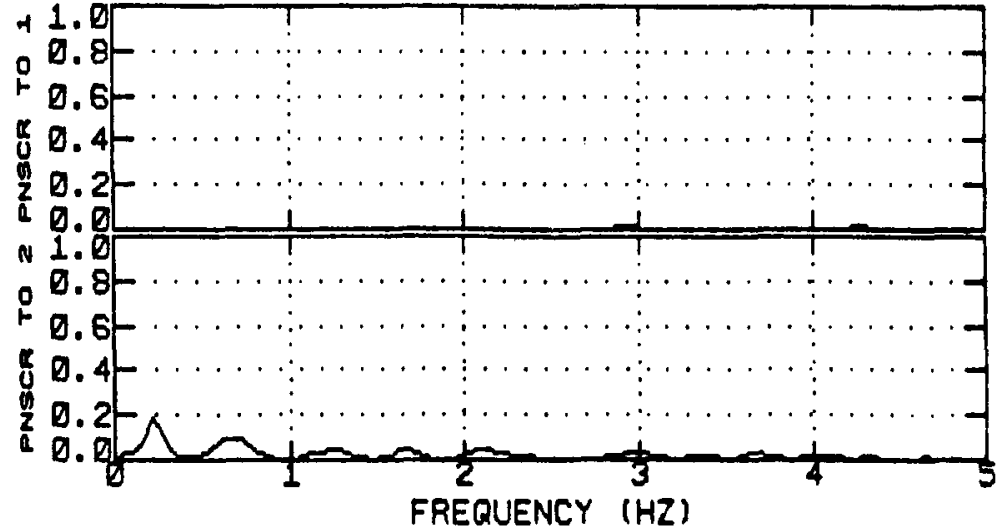

Figure 7.5. Signal pair pump DP-core-exit TC. Coherence, phase and noise source contribution ratios (ordinary and partial). 


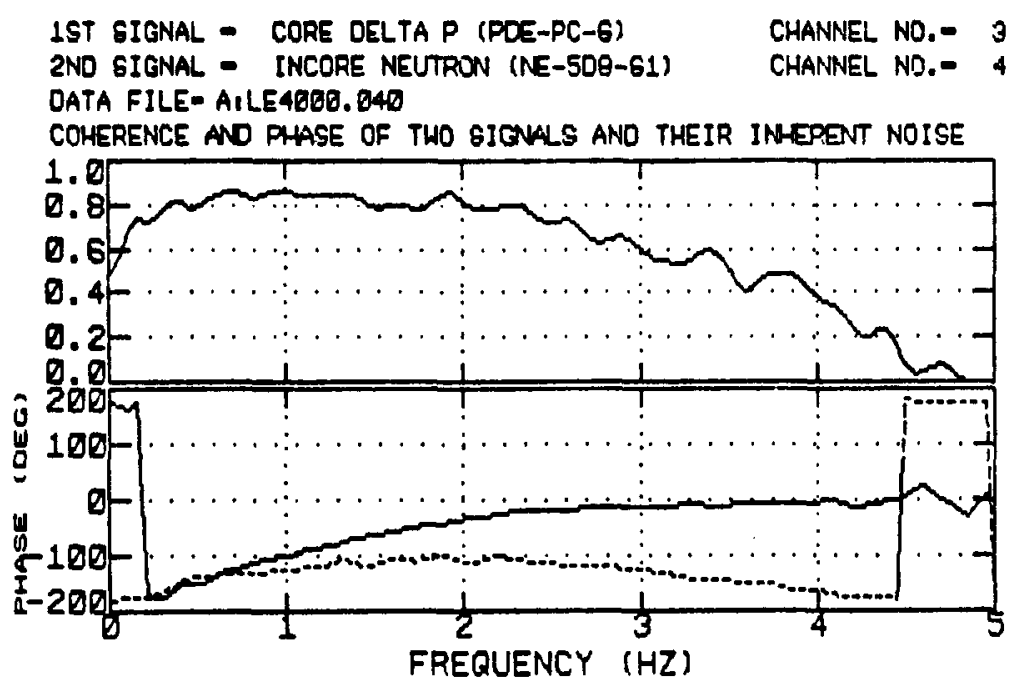

1ST SIGNAL - CORE DELTA P (POE-PC-G)

2NO GIGNAL - INCORE NEUTRON (NE-508-G1)

CHANNEL, NO.- 3 DATA FILE- AILE4000. 040 NOISE SOURCE CONTRIEUTION RATIO FUNCTION

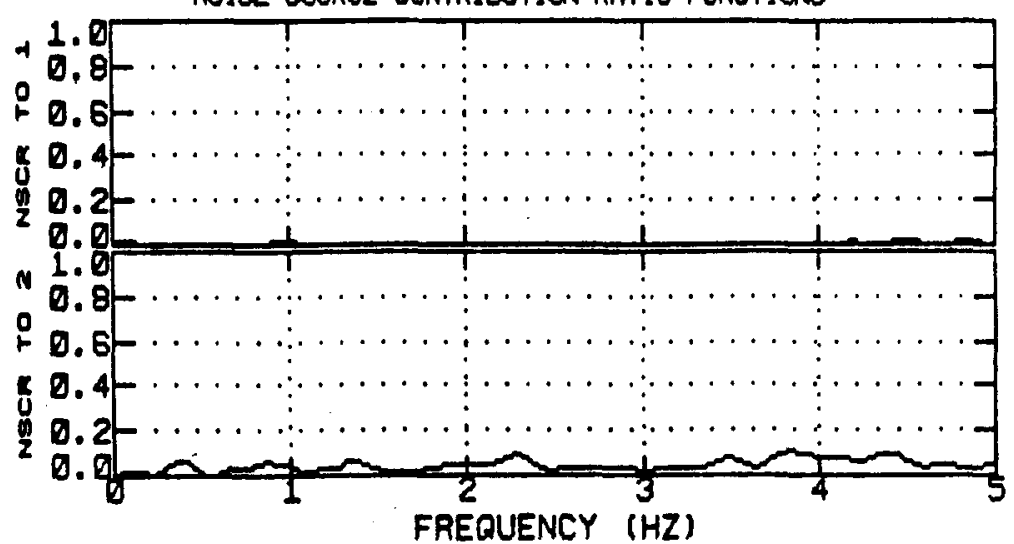

1ST SIGNAL - CORE DELTAP (POE-PC-6) 2ND SIGNAL - INCORE NEIJTRON (RE-SCQ-61)

DATA FILE- A.LE4R00.040

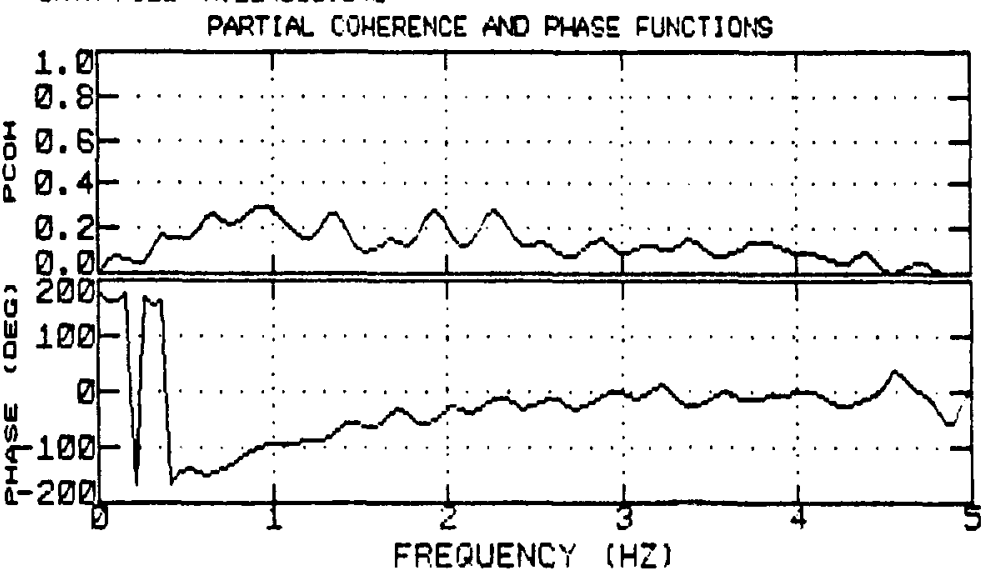

1ST SIGNAL - CORE DELTA P (FDE-PC-G)

CHANNEL ND.- 3 2ND SIGNAL - INCORE NEUTRON (RE-SOQ-61) CHANNEL NO.OATA FILE- AILE4000. 040

PARTIAL NOISE SOURCE CONTRIEITION RATIOS TO SIGNAL 1 AND 2

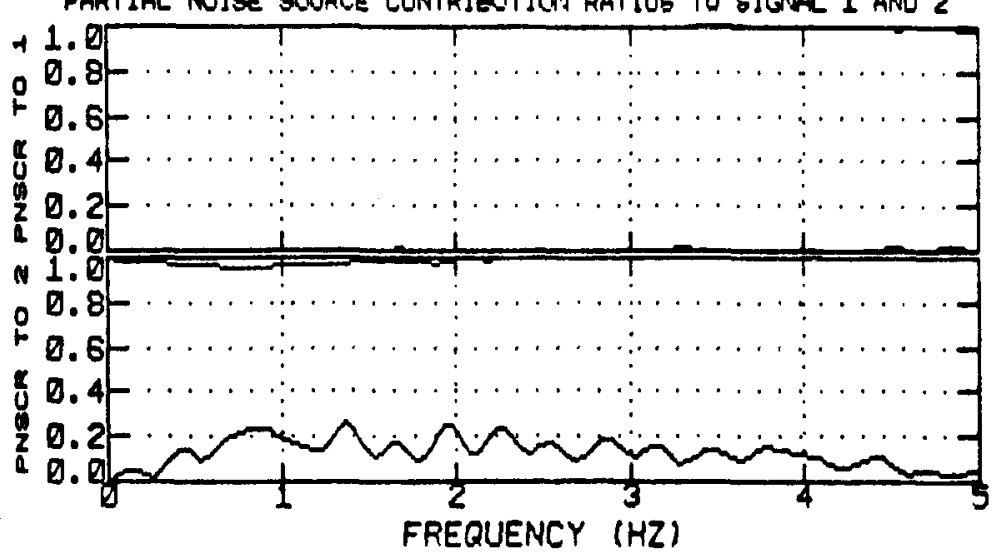

Figure 7.6. Signal pair core DP-in-core ND. Coherence, phase and noise source contribution ratios (ordinary and partial). 


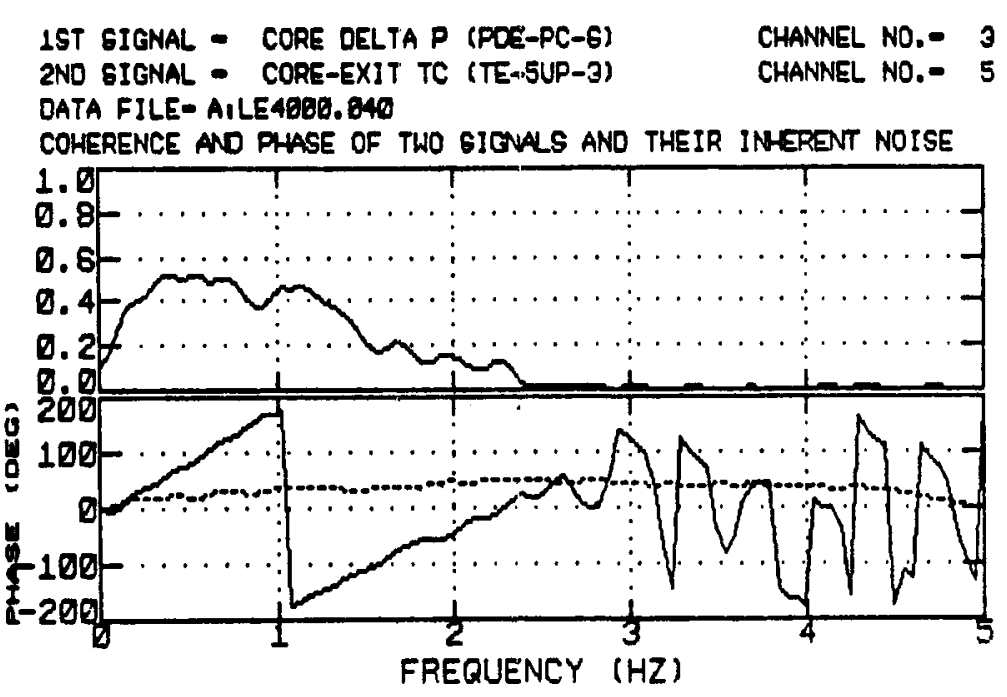

LST GIGNAL - CORE DELTA P (PDE-PC-G) CHANNEL NO.- 3 2ND GIGNAL - CORE-EXIT TC (TE-SUP-3) CHANNEL NO.- 5 DATA FILE- AILE4000.640

NOISE SOURE CONTRIEUTION RATIO FUNCTIONS
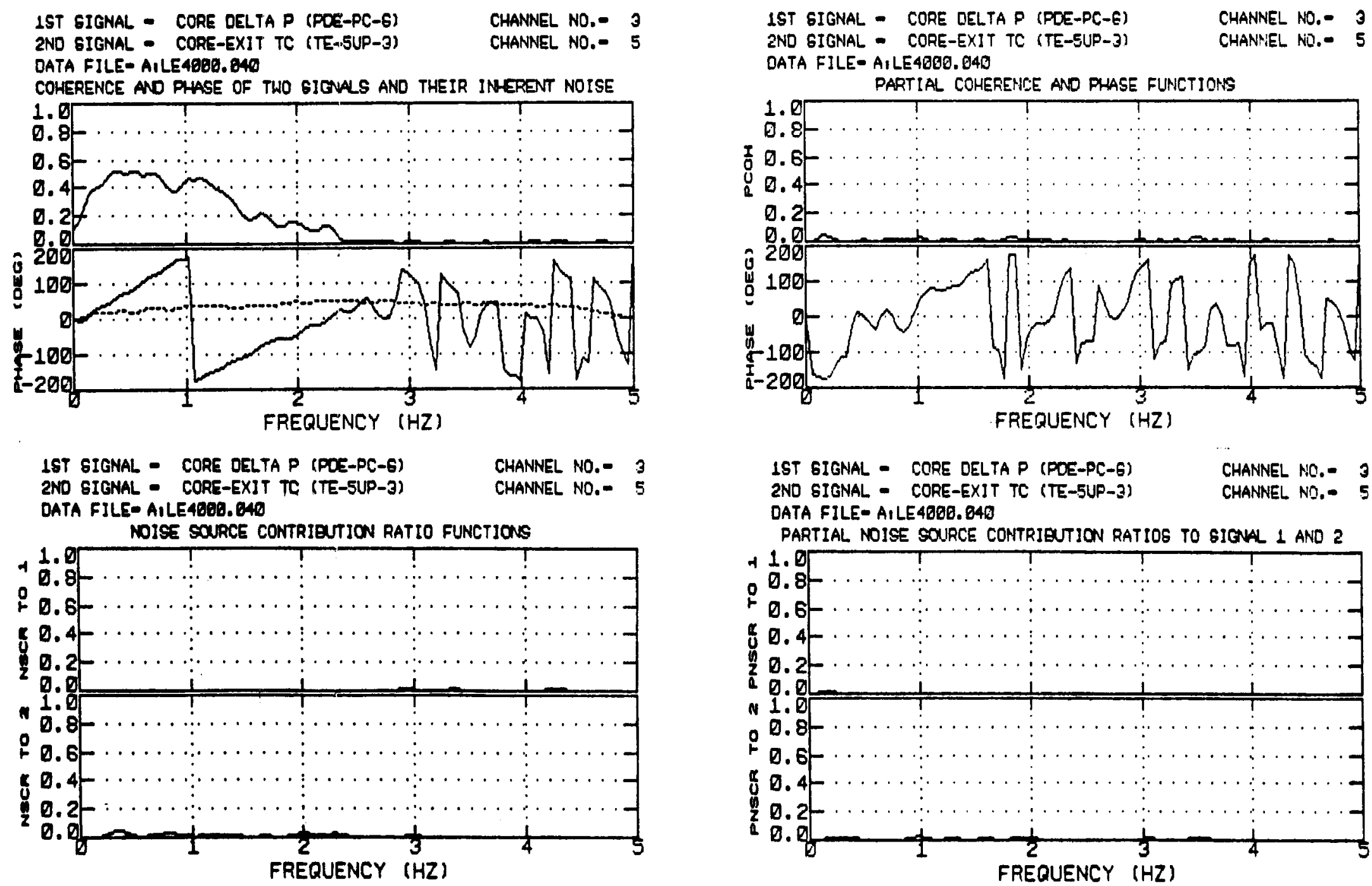

1ST GIGNAL - CORE DELTAP (POE-PC-6)

2ND SIGNAL - CORE-EXIT TC (TE-5UP-3)

DATA FILE- AILE4080.640

CHANNEL NO.- 3

PARTIAL NOIOE COURCE CONTRIEUTION RATIOS TO SIGIAL 1 ANO

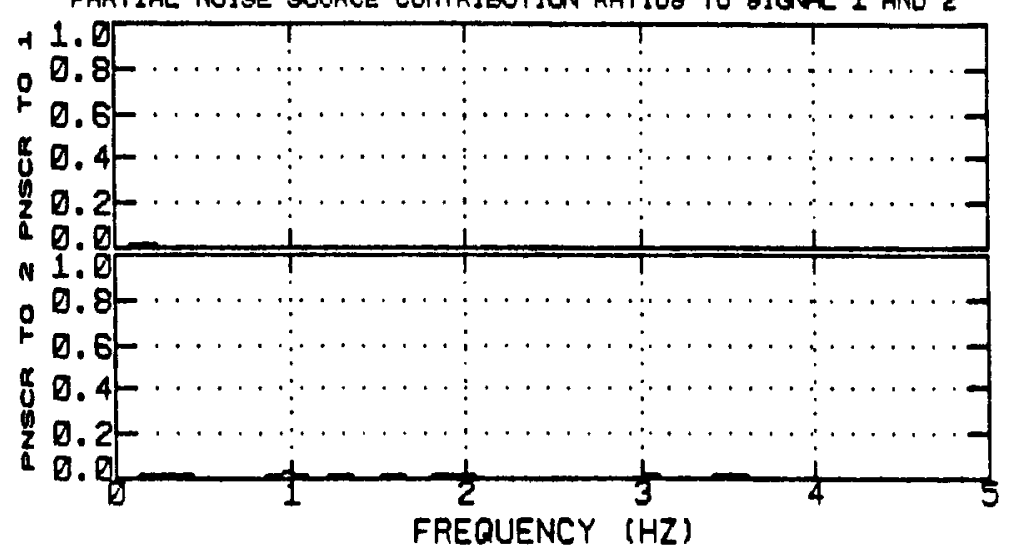

Figure 7.7. Signal pair core DP-core-exit TC. Coherence, phase and noise source contribution ratios (ordinary and partial). 


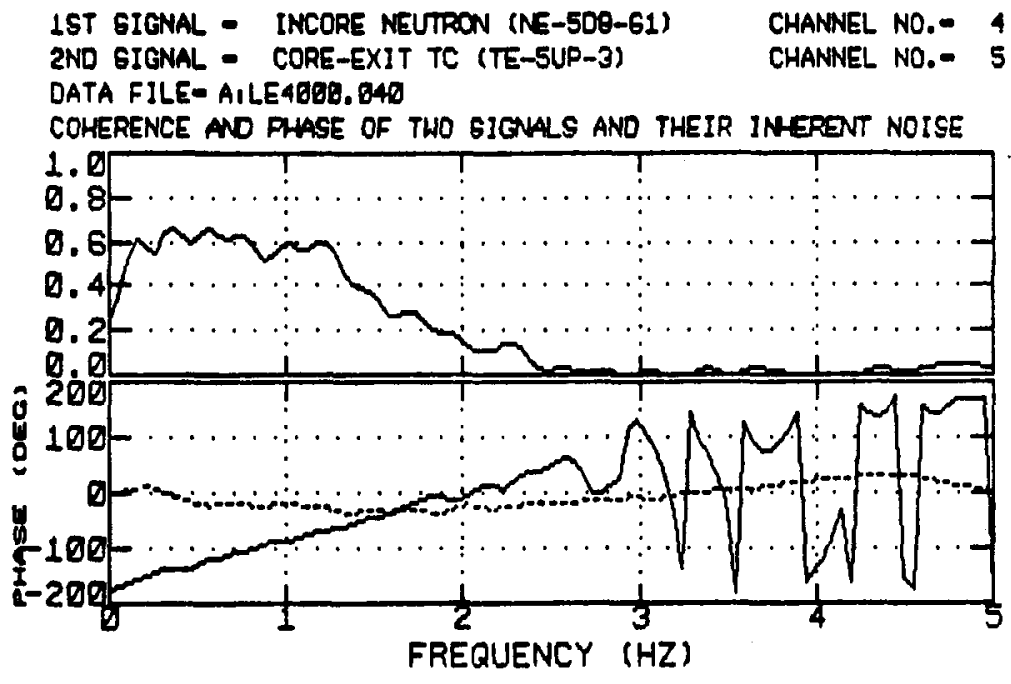

1ST GIGNAL - INCORE NEUTRON (NE-5DO-G1) CHANNEL NO.- 4 2ND GIGNAL - CORE-EXIT TC (TE-5UP-3) CHANNEL NO.- 5 DATA FILE- AILE4690.640

NOISE SOURCE CONTRIEJTION RATIO FUNCTIONS

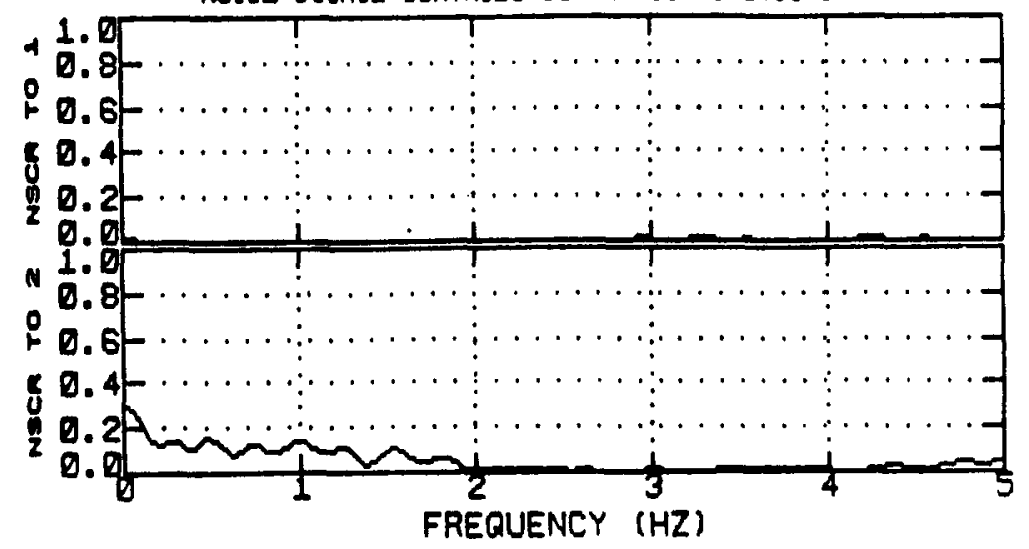

1ST SIGNAL - INCORE NEUTRON (NE-500-61)

2ND GIGNAL - CORE-EXIT TE (TE-5UP-3)

DATA FILE- AILE4000.040

PARTIAL COHERENCE ANO PHASE FUNCTIONS

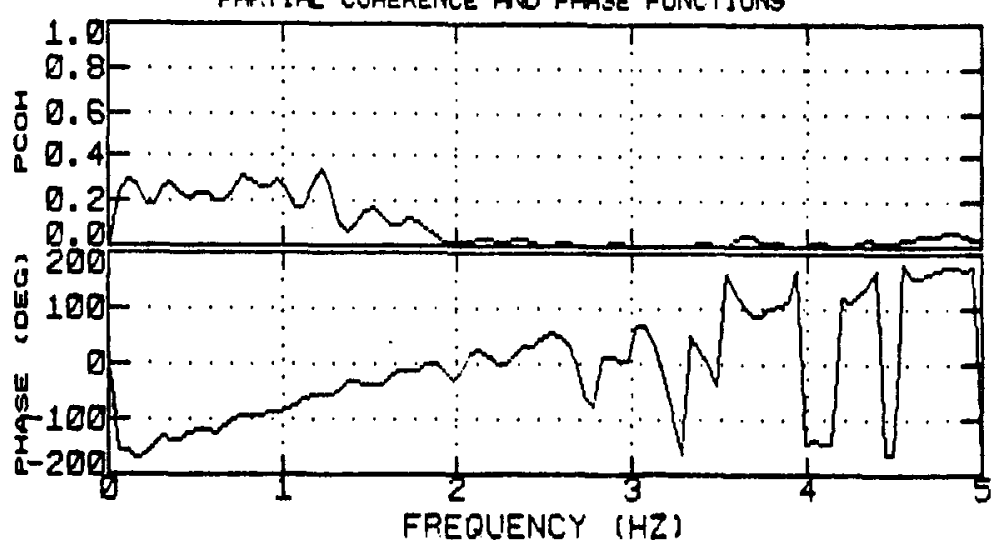

1ST GIGNAL - INCORE NEUTPON (NE-500-61) 2ND GIGNAL - CORE-EXIT TC (TE-5UP-3) DATA FILE- AILE4000. 040

CHANNEL NO.- 4

PARTIAL NDICE COUACE CONTRIETTION RATIOS TO SIONAL $\perp$ ANO 2

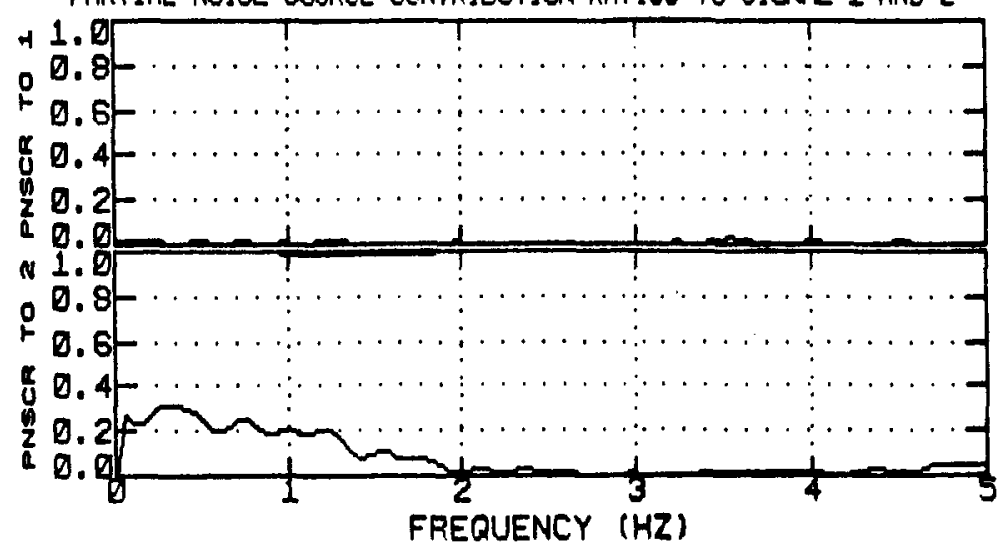

Figure 7.8. Signal pair in-core ND-core-exit TC. Coherence, phase and noise source contribution ratios (ordinary and partial). 
NORMALIZED CONTRIQUTION TO THE APSO OF PUMP OELTA P (PDE-PC-1) FROM THE INOIVIOAAL NOIGE GORCES

DATA FILE- A,LEABO0. B4D

QUM OF NOISE GOURCE CONTRIDUTION RATIO FUNCTIONS

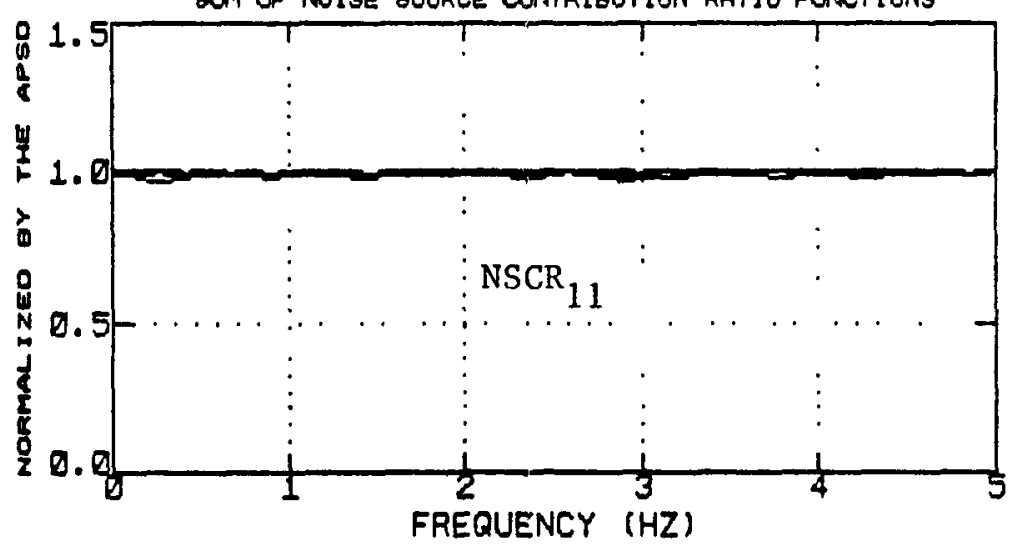

NORMALIZED CONTRIEUTION TO THE APSD OF INCORE NEUTRON (NE-5DQ-(;1) FROM THE INOIVIDUAL NOISE SOREES

DATA FILE- A,LE4B8Q gate

AM OF NOISE SOURCE CONTRLQUTTION RATIO FUNCTIONS

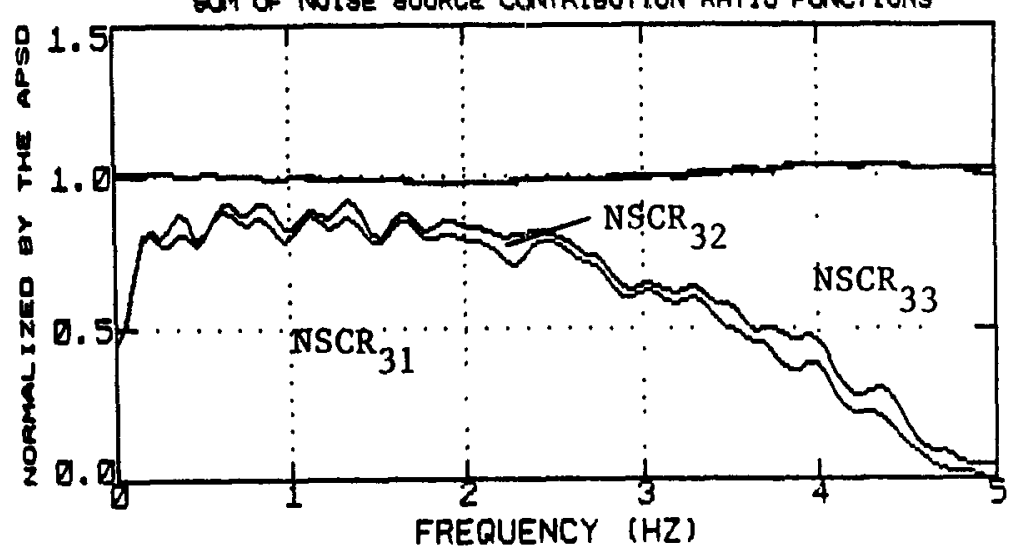

NOAMALIZED CONTRIQUTION TO THE APSD OF CORE OELTA P (DOE-PC-G) FROM THE INOIVIDUAL NOIEE GOLPCES

DATA FILE- AILE4600.040

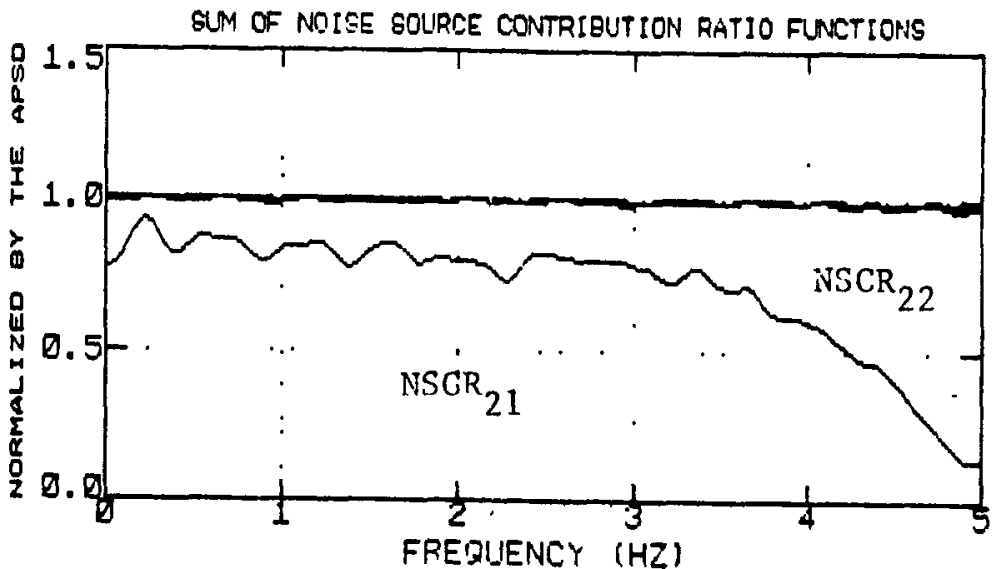

NORMALIZEO CONTRIBUTION TO THE APSO OF CORE-EXIT TC (TE-ELIF-3) FROM THE INDIVIDLAL NOISE SOARCES DATA FILE- AI LE4000.840

SLM OF NOISE GOURCE CONTRIBUTION RATJO FUNCTIONS

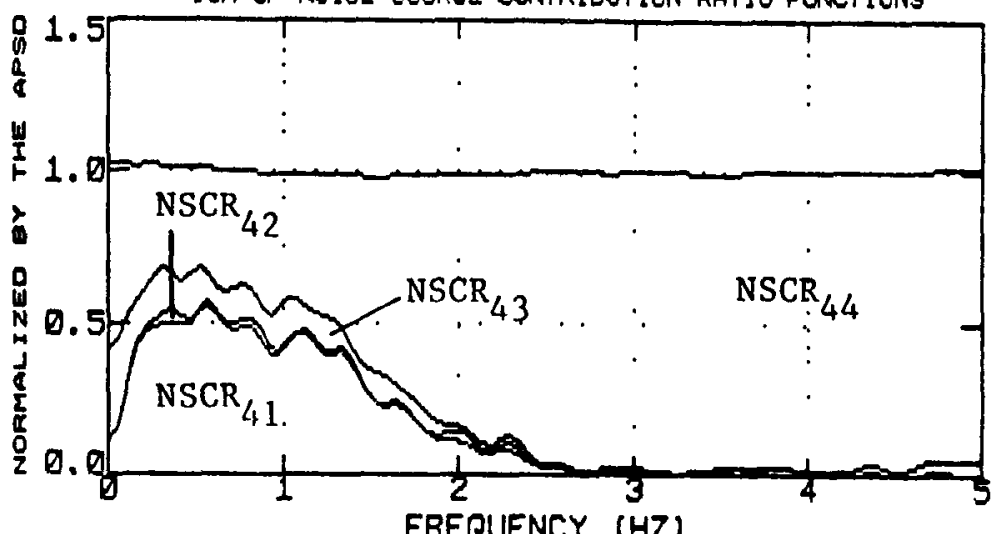

Figure 7.9. Sum of noise source contribution ratios for each of the four signals. 


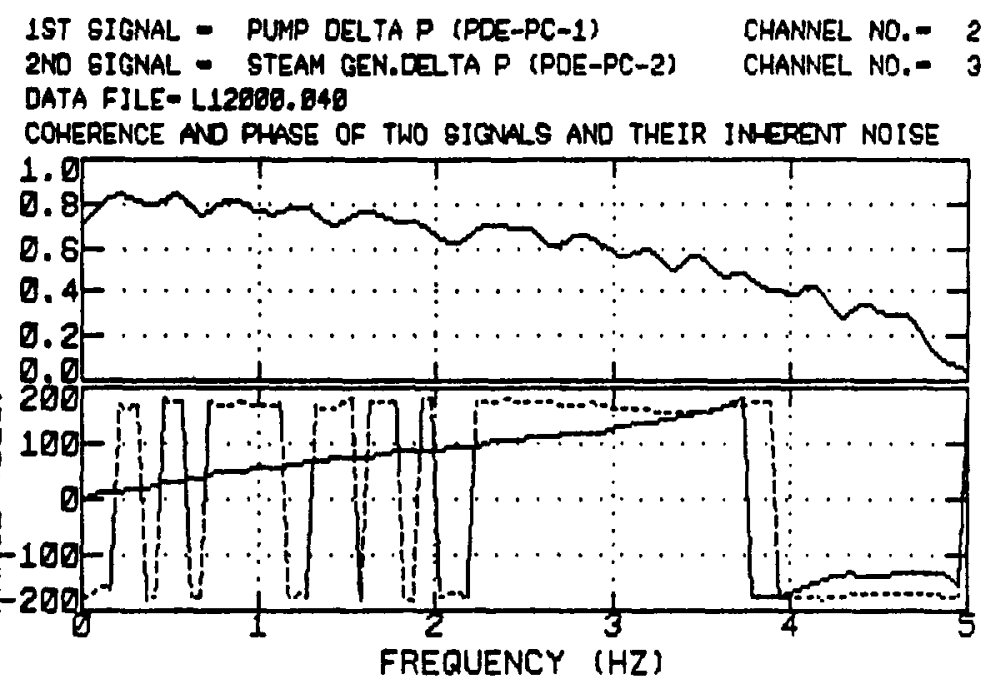

1ST GIGNAL - PUMP DELTA P (POE-PC-1) 2ND GIGNAL - STEAM GEN.DELTA P (POE-PC-2) CHANNEL NO.- 3 DATA FILE- LL2800.640

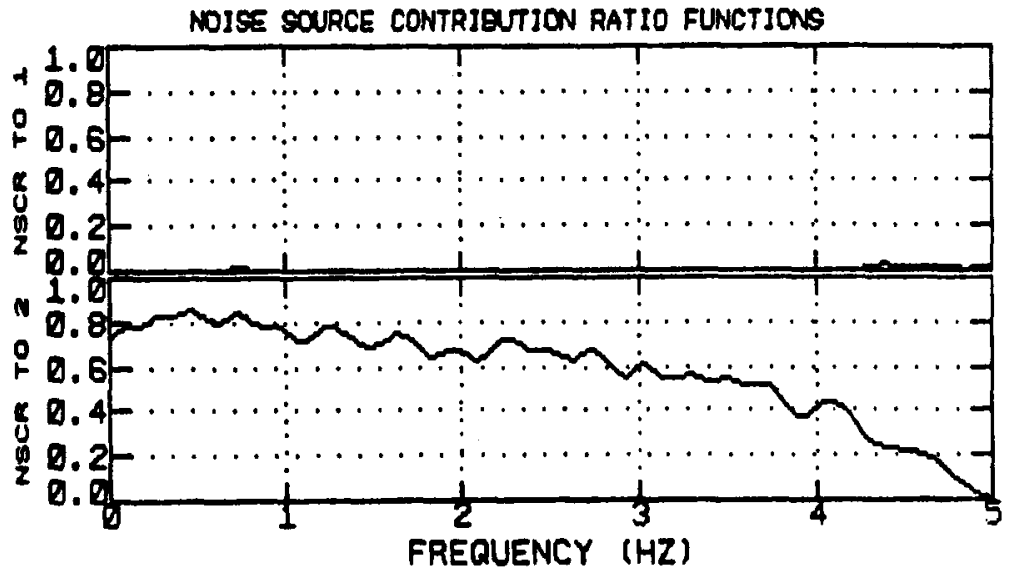

IST SIGNAL - PIMP OELTA F (FDE-FC-1)

CHANNEL NO.- 2 2ND SIGNAL - STEAM GEN.DELTA $P$ (POE-PC-2) CHANNEL NO.DATA FILE- L12000.040 PARTIAL COHERENCE ANO PHASE FUNCTIONS

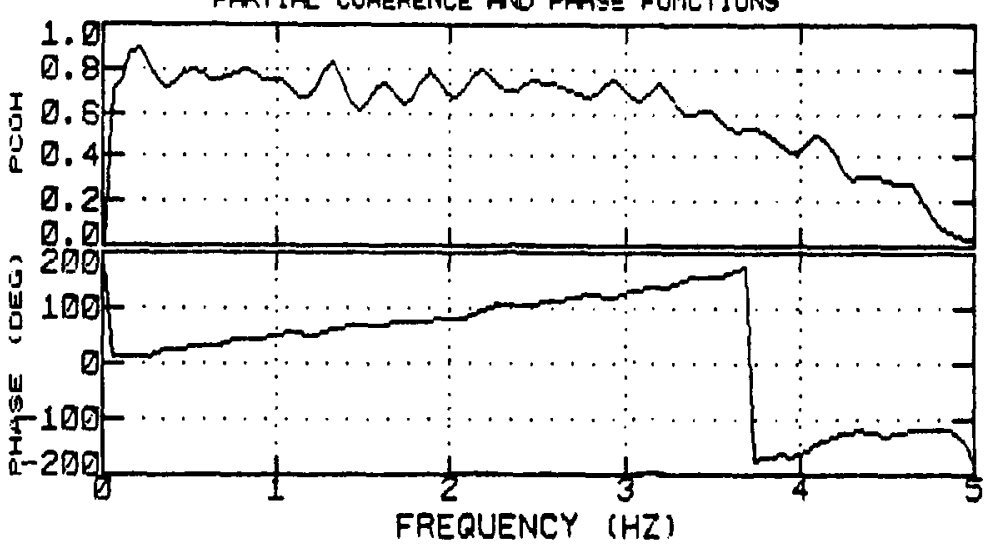

1 ST SIGNAL - PUMP OELTA P (PDE-PC-1)

CHANNEL NO.- 2 2ND SIGNAL - STEAM GEN.DELTA P (POE-PC-2) CHANNEL NO.- 3 DATA FILE- L12808.040

PARTIAL NOISE SOURCE CONTRIEUTION RATIOS TO SIGNAL $\perp$ AND 2

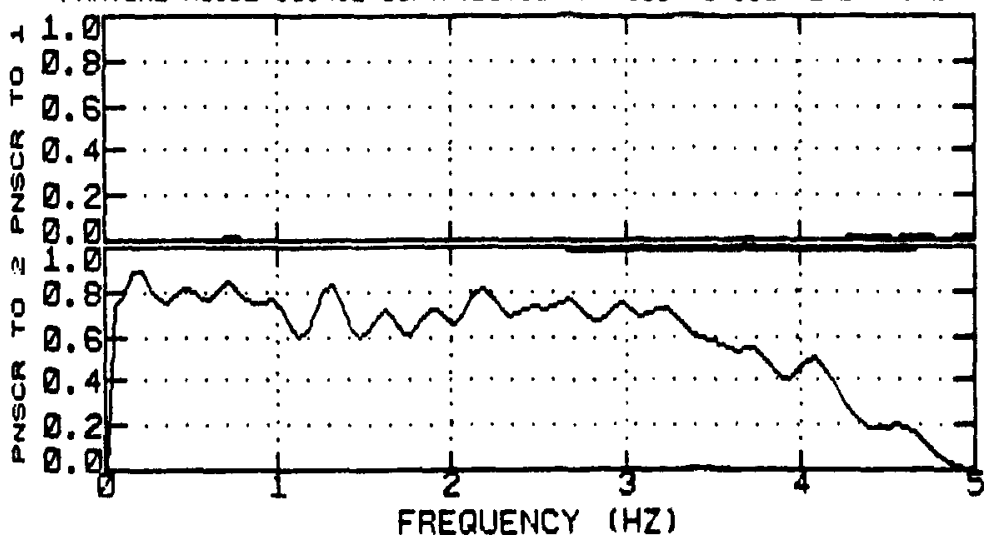

Figure 7.10. Signal pair pump DP-Steam generator DP. Coherence, phase and noise source contribution ratios (ordinary and partial). 


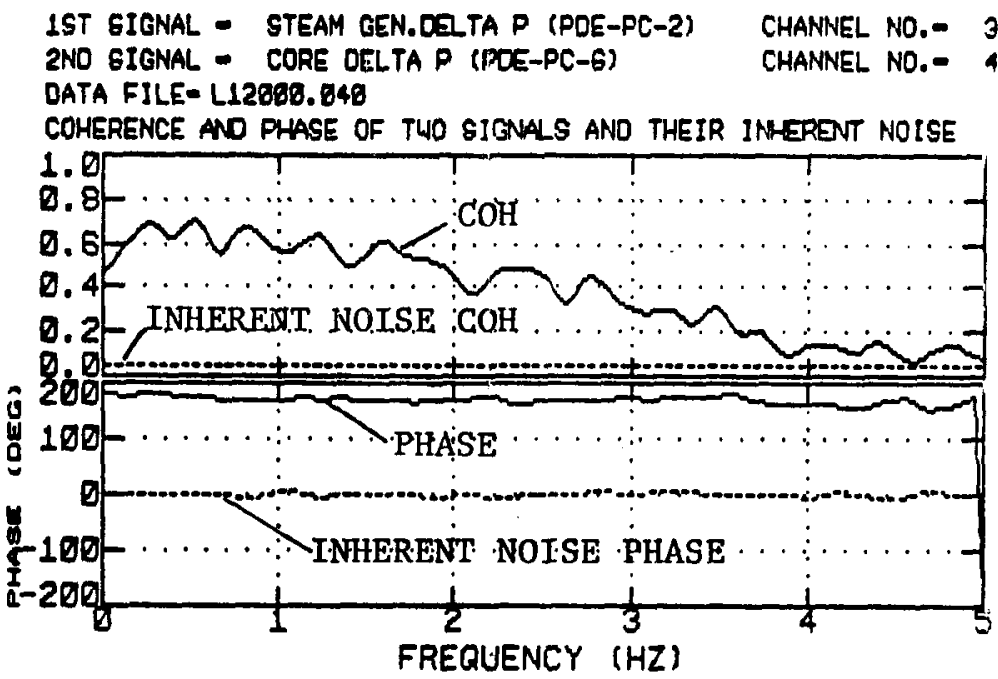

$19 T$ SIONAL - STEAM GEN.DELTA P (POE-PC-2) CHANNEL NO.- 3 2ND SIGNAL - CORE DELTA P (POE-PC-6) CHANNEL NO.- a DATA FILE- L12600.640

NOISE SOURCE CONTRIEUTION RATIO FUNCTIONS

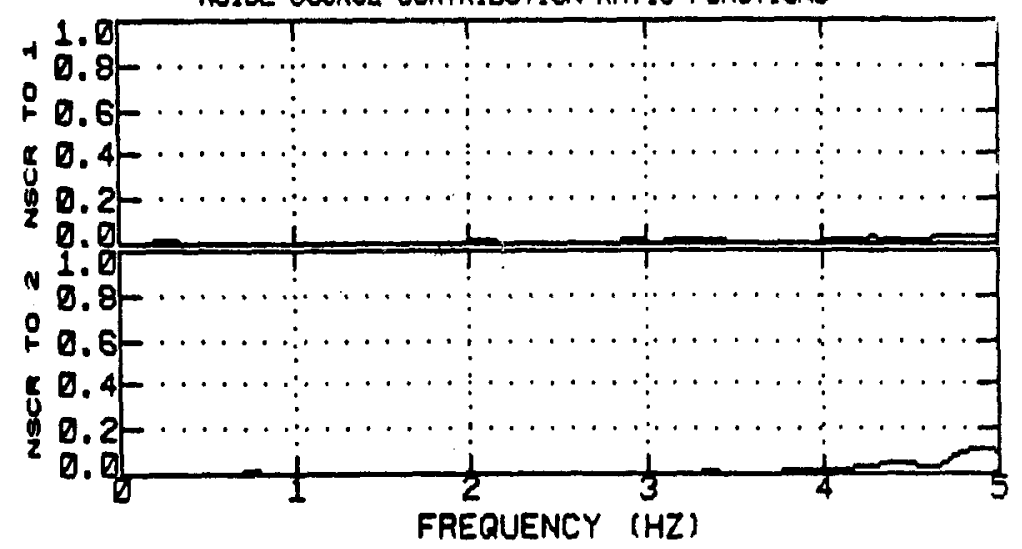

1ST GIGHAL - STEAM GEN.DQLTA P (FOE-FC-2) CHANNEL NO.- 3 2ND GIGNAL - CORE DELTA P (POE-PC-G) CHANNEL NO.- 4 DATA FILE- L12000.040

PARTIAL COHERENCE AND PHASE FUNCTIONS

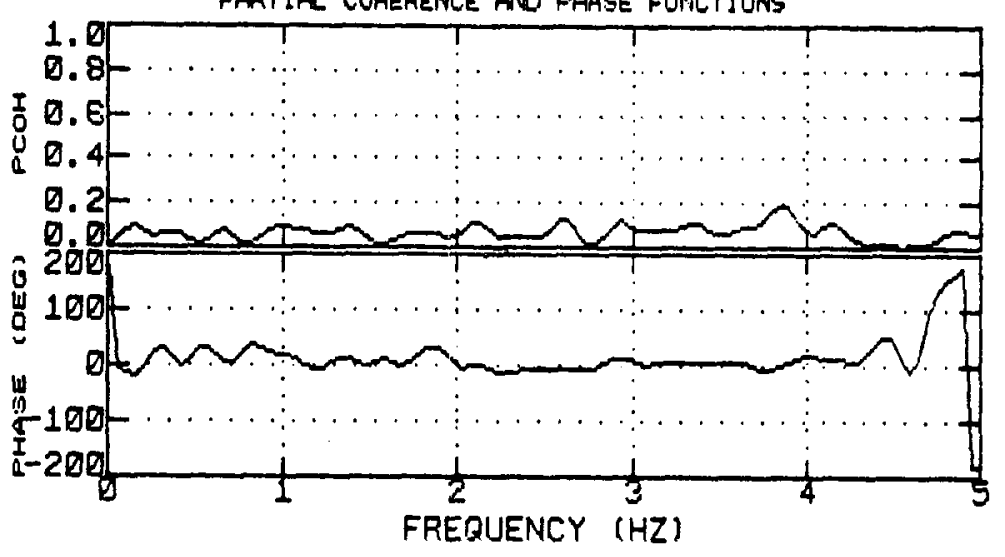

1ST SIGNAL - STEAM GEN.DELTA P (POE-PC-2) CHANNEL NO.- 3 2ND SIGNAL - CORE DELTA P (POE-PC-G) CHANAEL NO.- 4 DATA FILE- LI2000.040

PARTIAL NOISE SOURCE CONTRIEUTION RATIOS TO SIGNAL 1 ANO 2

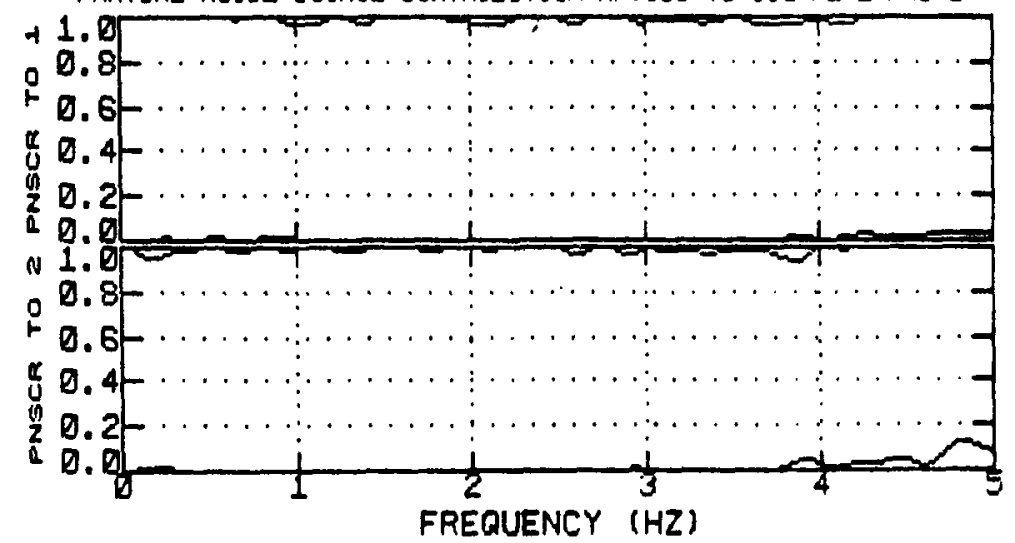

Figure 7.11. Signal pair steam generator DP-core DP. Coherence, phase and noise source contribution ratios (ordinary and partial). 
SIGNAL NAME- CORE-EXIT TC (TE-5UP-3) RECOROEO AT POWBR LEVEL 1BQX

CHANEL NO.- 5 DATA FILE- AILE4802.040

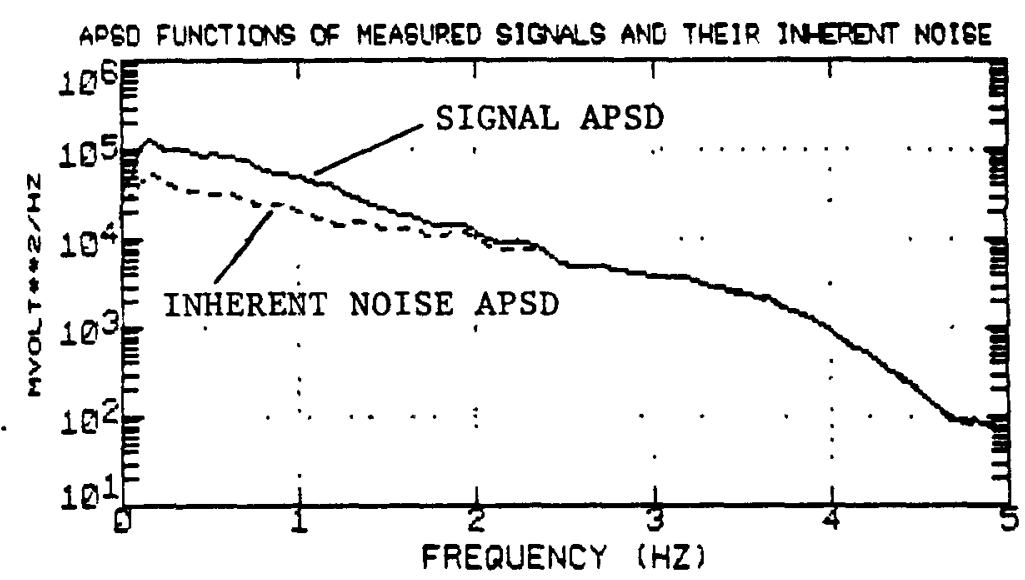

Figure 7.12a. Signal APSD and inherent noise APSD of core-exit TC (TE-5UP-3).
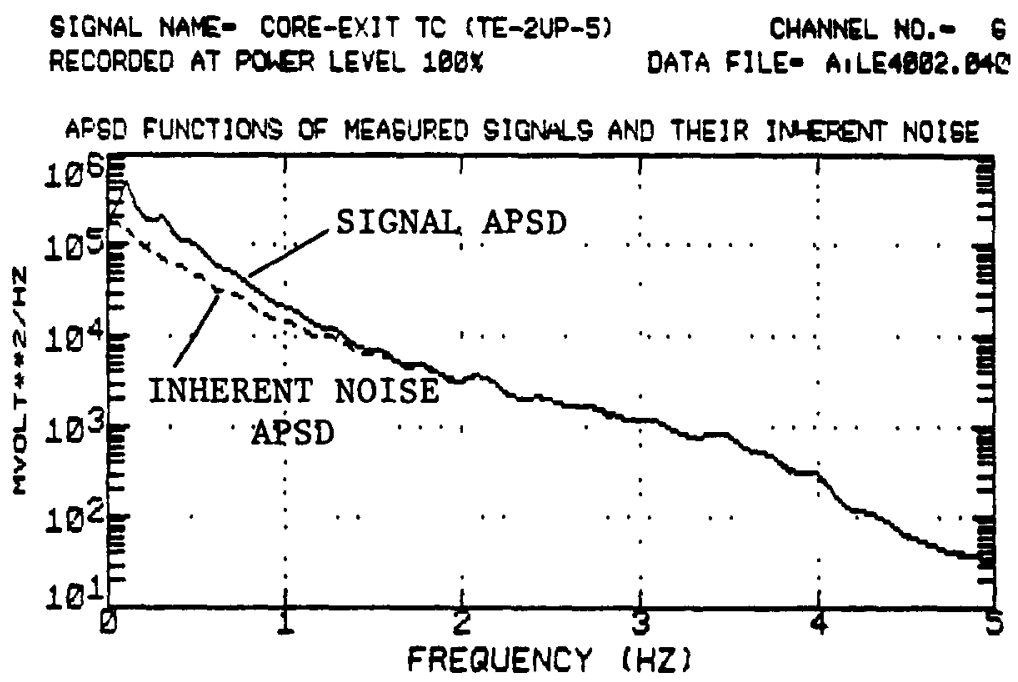

Figure 7.12b. Signal APSD and inherent noise APSD of core-exit TC (TE-2UP-5). 
1ST GIGNAL - PUMP DELTA P (POE-PC-1) 2ND SIGNAL - CORE-EXIT TC (TE-2UP-5) DATA FILE- AILE4EB2, 840

CHANNEL NO.CHANNEL NO.-

COHERENCE ANO PHASE OF TWO SIGNALS AND THEIR IHERENT NOISE

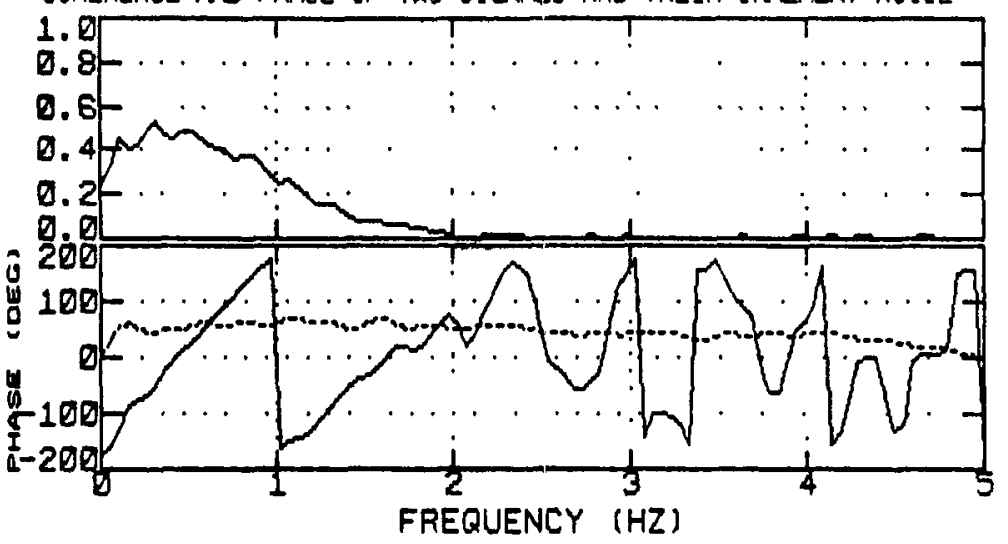

1ST GIGNAL - PUMP DELTA P (PDE-PC-1)

CHANNEL NO.- 2 2ND SIGNAL - CORE-EXIT TC (TE-2UP-5) CHANNEL NO.DATA FILE- AILE4EO2. B40 NOISE SOURCE CONTRIEUTION RATIO FUNCTIONS

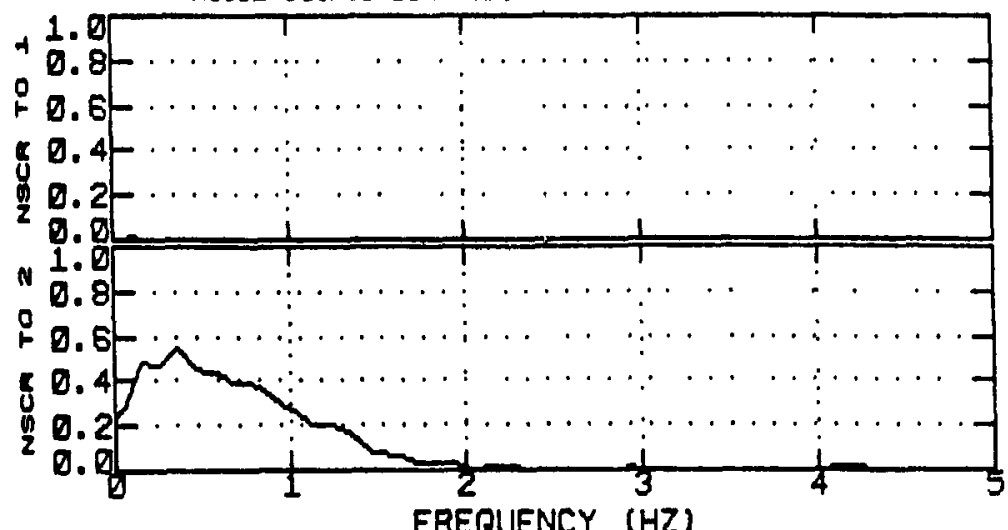

1ST SIGNAL - PUMP DELTA P (FOE-PC-1) 2ND SIGNAL - CORE-EXIT TC (TE-2UP-5) DATA FILE- AILE4062.040

CHANNEL ND.CHAINEL NO.- 6

PARTIAL COHERENCE AND PHAge FLNCTIOHS

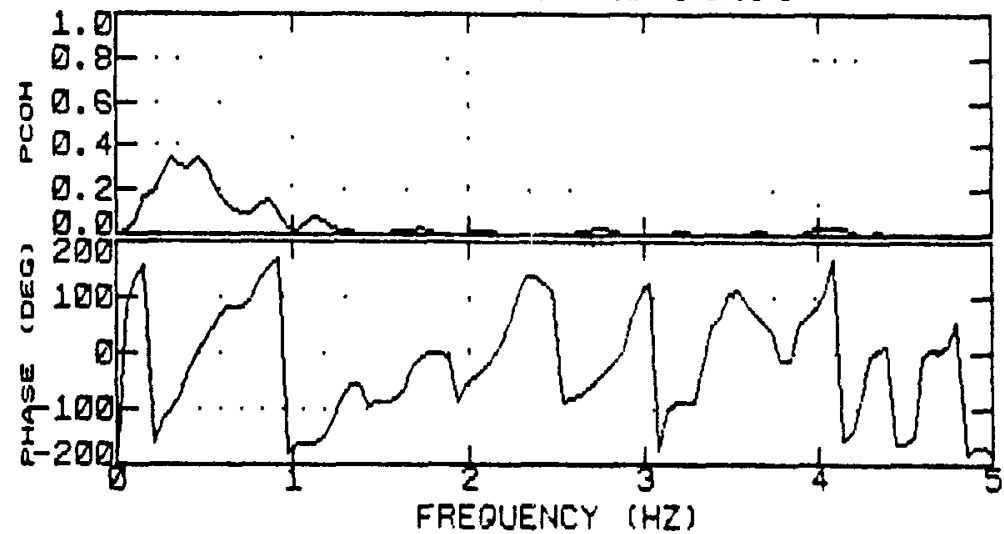

1ST GIGNAL - PUMP DELTA P (POE-PC-1) 2ND GIGNAL - CORE-EXIT TC (TE-2UP-5)

CHANNEL NQ.- 2 DATA FILE- AILE4862.040

PARTIAL NOISE GOURCE CONTRIOUTION RATIOS TO SIGNAL 1 ANO 2

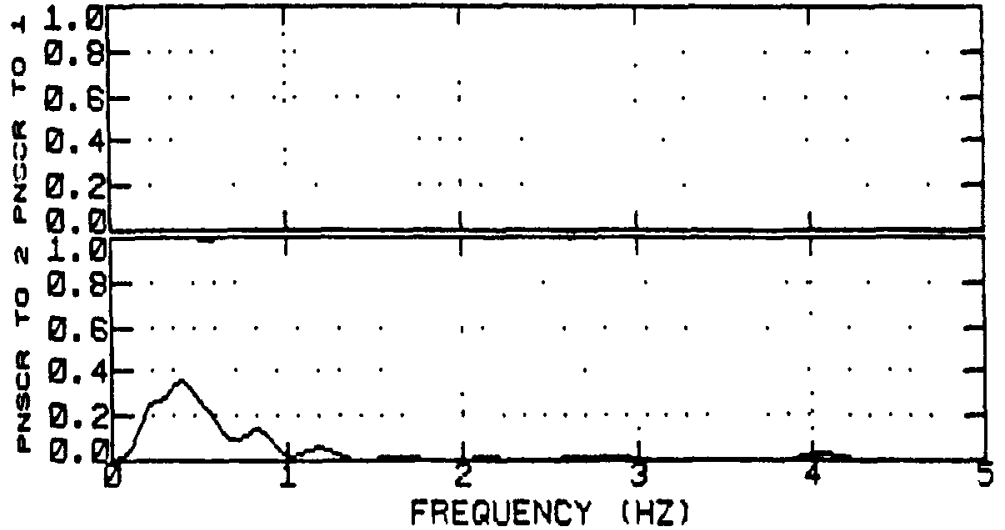

Figure 7.13. Signal pair pump DP-core-exit TC (TE-2UP-5). Coherence, phase and noise source contribution ratios (ordinary and partial). 


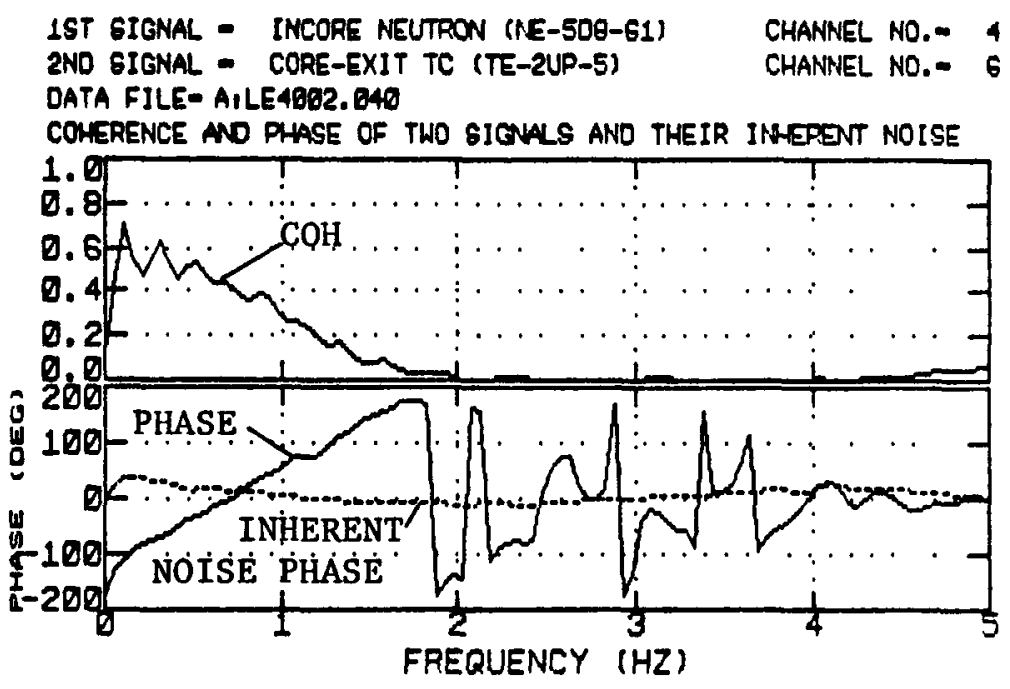

1ST SIGNAL - INCOFE HEUTRCN (TE-SOQ-51)

2ND SIGNAL - CORE-EXIT TC (TE-2UJP-E)

DATA FILE- AILE4BO2.040

CHANNEL NO.- 4

PARTIAL COHERENCE ANO FHASE FUNICTIONS

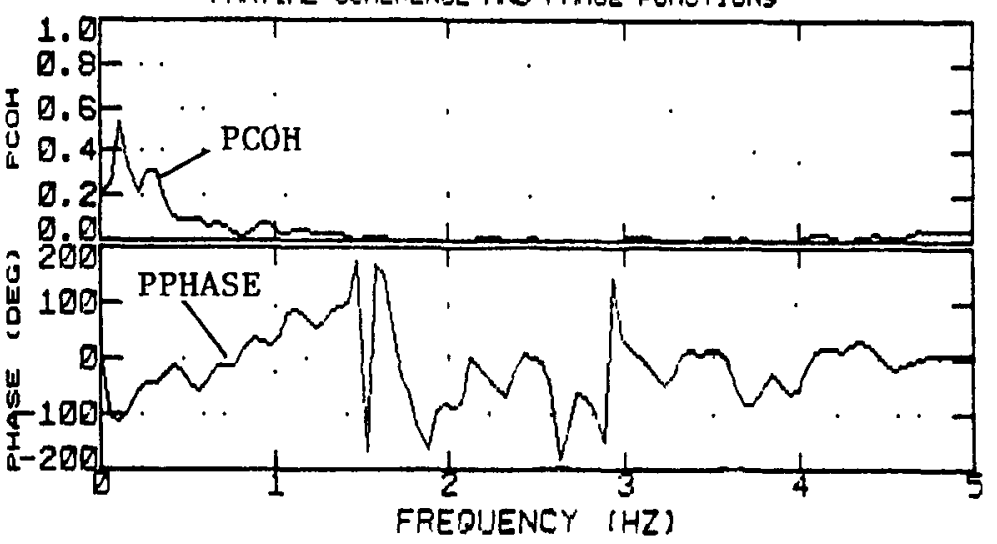

1ST SIGNAL - INCORE NEUTRON (NE-508-61)

CHANNEL NO.- 4 2ND GIGNAL * CORE-EXIT TC (TE-2UP-5)

CHANNEL NO.DATA FILE- AILE4882. B40 NOISE SOURCE CONTRIEUTION RATIO FUNCTIONS

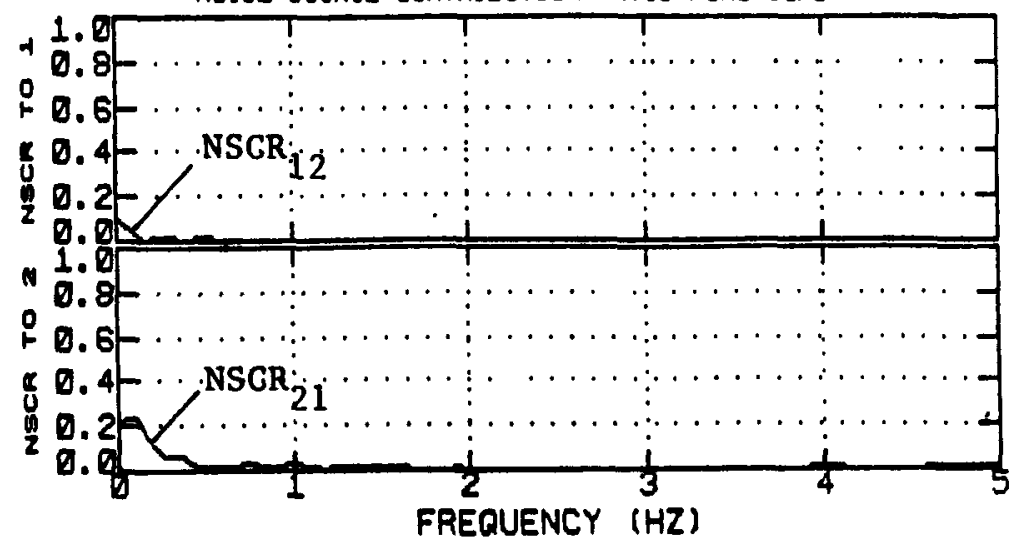

1ST GIGNAL - INCORE NEIJTRON (RE-30Q-S1)

CHANNEL NE.- 4 2ND GIGNAL - CORE-EXIT TC (TE-2UP-S) CHANMEL NO.- 6 DATA FILE- AILE4002. 040

PARTIAL NOISE SOURCE CONTRIESTION RATIOS TO SIGNAL \& ANO 2

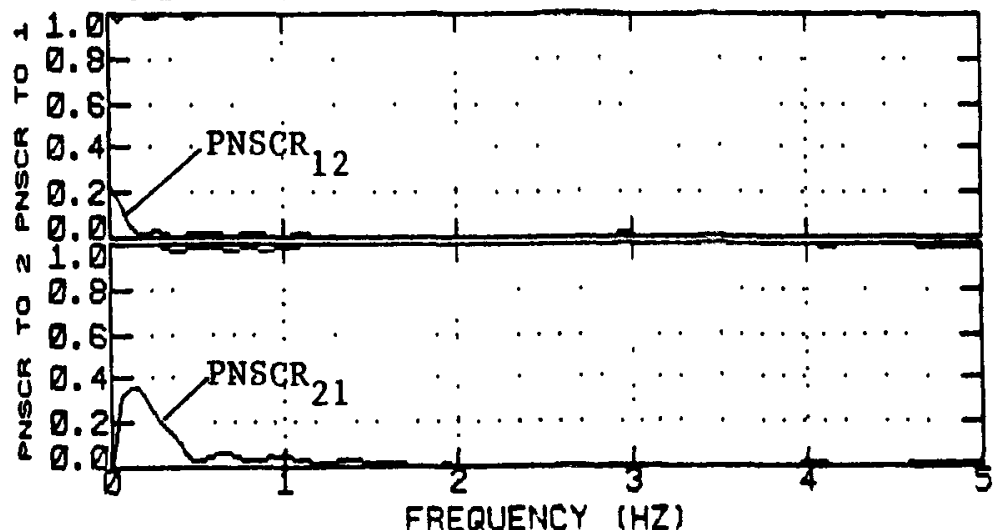

Figure 7.14. Signal pair in-core neutron detector-core-exit TC (TE-2UP-5). Coherence, phase and noise source contribution ratios (ordinary and partial). 


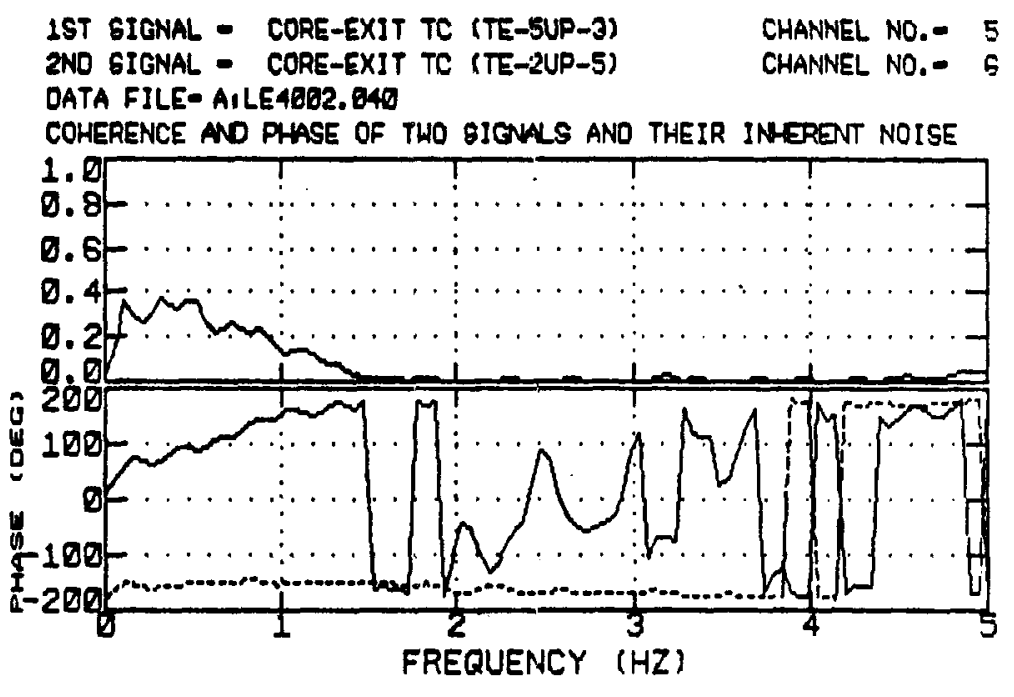

1ST SIGNAL - CORE-EXIT TC (TE-5UP-3) 2ND GIGNAL - CORE-EXIT TC (TE-2UP-5) DATA FILE- AILE40B2. B40

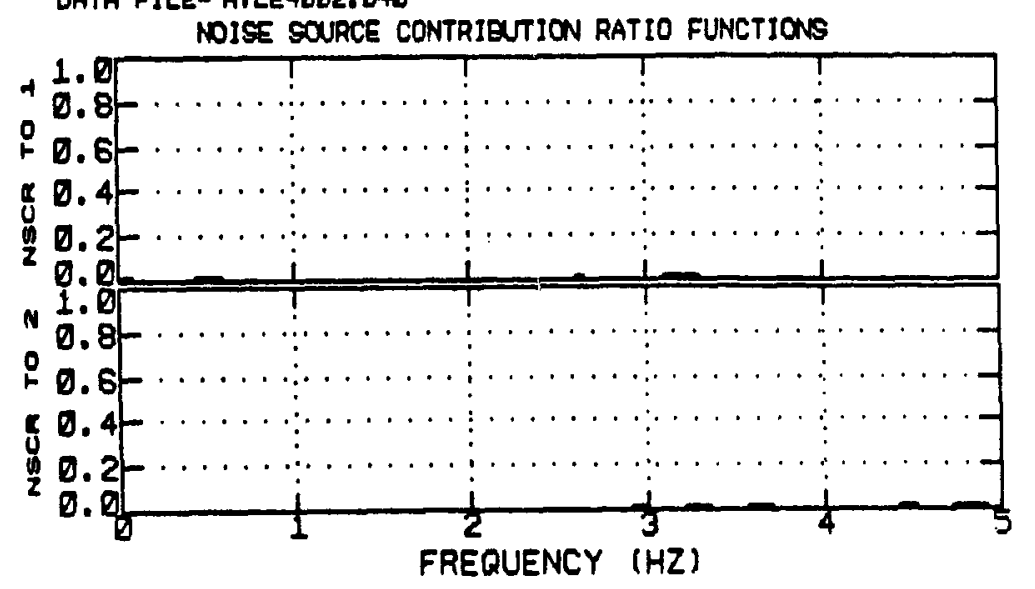

1ST SIGNAL - CORE-EXIT TC (TE-SUP-O) 2ND SIGNAL - CORE-EXIT TC (TE-2UP-5) DATA FILE- A1LE4002.040

PAFTIAL COHERENCE AND PHASE FUNCTIONS

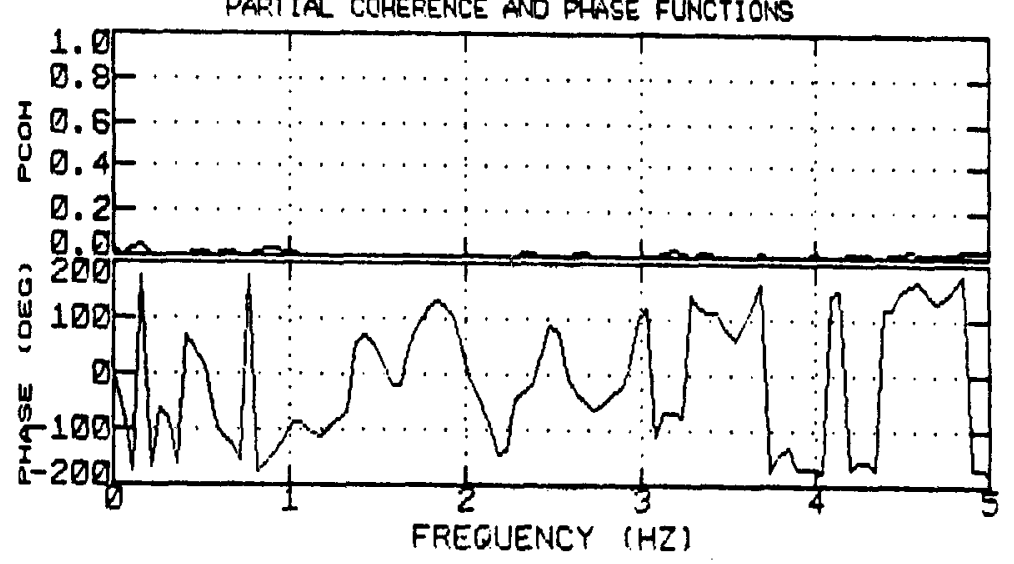

1ST GIGNAL - CORE-EXIT TC (TE-5UP-3) 2ND GIGNAL - CORE-EXIT TC (TE-2UP-5) OATA FILE- AILE4BQ2. D40 CHANNEL NO.- 5
CHANNEL NO.- 6

PARTIAL NOISE SOURCE CONTRIEUTION RATIOS TO SIGNAL 1 AND 2

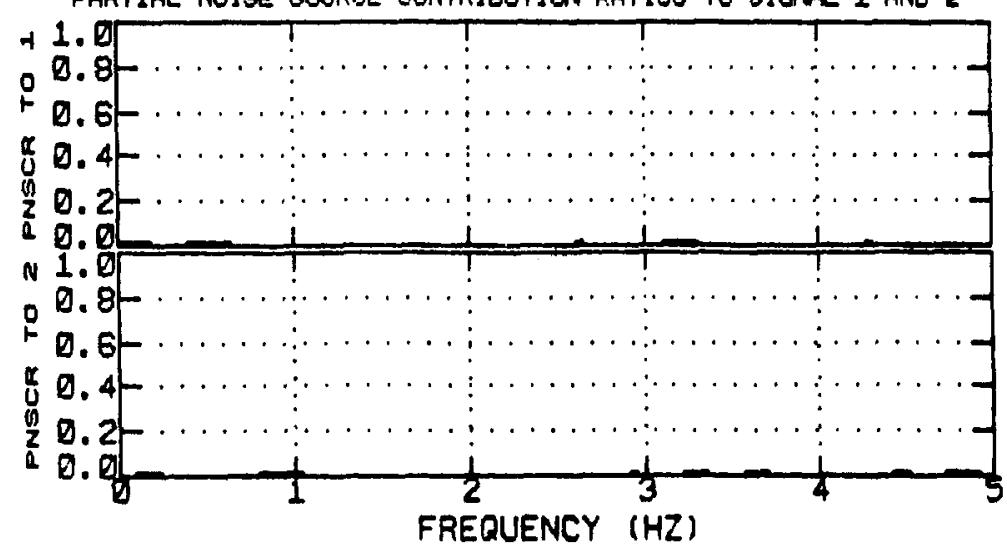

Figure 7.15. Signal pair core-exit TC (TE-5UP-3)-core-exit TC (TE-2UP-5). Coherence, phase and noise source signal contribution ratios (ordinary and partial). 


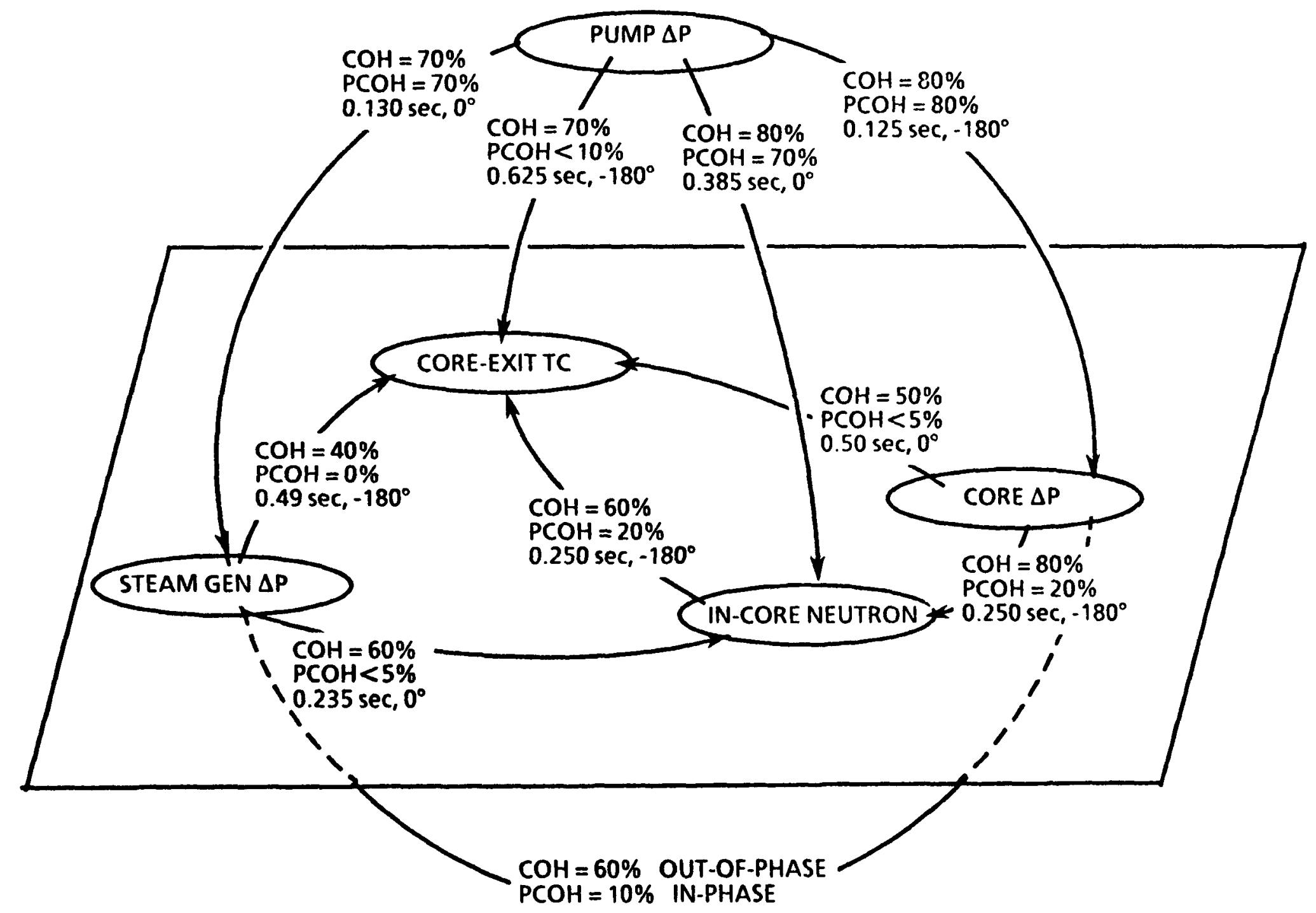

Figure 7.16. Signal transmission path diagram for the LOFT reactor primary system. 


\section{Section 8}

\section{UNIVARIATE AUTOREGRESSION (UAR) TIME SERIES MODELING FOR SENSOR SURVEILLANCE}

\subsection{Introduction}

Low-level fluctuations of variables in a power plant contain information about the process dynamics and sensor characteristics. If the mean values of measured signals are appropriately filtered, the remaining fluctuating components can be preconditioned (amplification and filtering) and recorded for further analysis. A data acquisition and analysis system has been developed at the University of Tennessee for analysis of wide-band signals from nuclear power plants and process control systems.

The univariate time series analysis of a digitized signal may be efficiently processed using autoregression (AR) models. Good prediction models can be generated using the data, and may be applied to monitor process-related characteristics of a signal or the response characteristics of the detector. Univariate autoregression models have been used in various applictions including sensor surveillance [21], BWR stability monitoring [22], geophysical data analysis [23], and others. A complete univariate AR software system has been developed for implementation both in a personal computer and in a VAX workstation. Both timedomain and frequency-domain signatures may be estimated. Unlike classical methods, small data records may be processed to extract information from plant signals.

\subsection{Determination of Univariate Autoregression Models}

A stationary random process $x(t)$ may be represented by a finite order AR model in the form 


$$
x(t)=\sum_{i=1}^{n} a_{i} x(t-i \Delta t)+v(t)
$$

where $\mathbf{x}(t)=$ sampled signal at interval $\Delta t$ sec.

$$
v(t)=\text { driving noise source with variance } \sigma^{2} \text {. }
$$

$\left(\mathrm{a}_{1}, \mathrm{a}_{2}, \ldots, \mathrm{a}_{\mathrm{n}}\right)=\mathrm{AR}$ parameters.

The parameter set $\left(a_{1}, a_{2}, \ldots, a_{n}\right)$ is estimated by solving the set of Yule-Walker equations given by

$$
c(k)=\sum_{i=1}^{n} a_{i} c(k-i), k=1,2, \ldots, n
$$

where $c(k)$ is the autocorrelation function of $x(t)$ at lag $k \Delta t$

$$
c(k)=\frac{1}{T} \int_{0}^{T} x(\tau) x(\tau+k \Delta t) d \tau
$$

The autocorrelation function is estimated from the discrete samples as

$$
a(k)=\frac{1}{N} \sum_{i=1}^{n-k} x(i) x(i+k)
$$

where $\mathrm{N}$ is the number of samples per data block. Several blocks of data are used to obtain a statistically accurate estimate of $\mathrm{c}(\mathbf{k})$.

Instead of solving the set of $n$-equations (8.2) directly, a recursive parameter estimation method [23] is used to update the parameters from one model order to the next. Let $\left\{a_{i}\right\}$ denote the parameters of the AR model of order $n$. Then the parameters of the model of order $(n+1)$ are given by the following recursion equations.

$$
a_{n+1, j}=a_{n j}-a_{n+1, n+1} \cdot a_{n, n-j+1} ; j=1,2, \ldots, n
$$




$$
a_{n+1, n+1}=\frac{(n+1)-\sum_{i=1}^{n} a_{n i} c(n+1-1)}{c(0)-\sum_{i=1}^{n} a_{n i} c(i)}
$$

This procedure minimizes the computational time and numerical errors due to inverting large order matrices.

The error or noise covariance $\sigma^{2}$ is then given by

$$
\sigma^{2}=c(0)-\sum_{i=1}^{n} a_{i} c(i)
$$

The optimal model order is selected such that the mean-squared prediction error is a minimum [21]. The model order is a function of the sampling interval $\Delta t$, and the spectral properties of the signal.

\subsection{Estimation of Frequency- and Time-Domain Signatures}

Once the optimal model of order $\mathrm{n}$ is determined, the power spectral density function of $x(t)$ is estimated as

$$
S_{x x}(f)=\frac{0^{2} \Delta t}{\left|1-\sum_{k=1}^{n} a_{k} \exp (-j 2 \pi f k \Delta t)\right|^{2}}, f \ll \frac{1}{2 \Delta t}
$$

where $\mathrm{f}$ is the frequency in $\mathrm{Hz}$. The changes in the spectral patterns from its normal behavior may be monitored using pattern recognition methods [24]. This signature is useful for tracking harmonic frequencies due to vibration and damped oscillatory behavior of sensors (flow, level, pressure) and systems.

If the frequency bandwidth of the process fluctuations driving the sensor is greater than the sensor bandwidth, then the AR model may be used to monitor sensor response characteristics. This is performed by computing the step response of 
the signal AR model. The impulse response is estimated recursively as follows.

$$
\begin{gathered}
x_{I}(k)=\sum_{i=1}^{n} a_{i} x_{I}(k-i) \\
x_{I}(0)=0, x_{I}(\ell)=0 \text { for } \ell<0 .
\end{gathered}
$$

The step response is then calculated from the impulse response,

$$
x_{s}(t)=\int_{0}^{t} x_{I}(\tau) d \tau
$$

The ramp response is calculated in a similar manner from the step response as

The step response is used to estimate a value of the time constant, $T_{s} . T_{S}$ is defined as the time at which the step response attains $63.2 \%$ of its steady-state value. This definition is borrowed from the results for a first order system. The ramp delay time is determined from the steady-state delay between the ideal input ramp and the ramp response function. These sensor parameters have safety related implications in nuclear power plants.

\subsection{Example of Application to a Pressure Sensor}

Wideband fluctuation signal from a pressure sensor were acquired from the test loop facility at Analysis and Measurement Services (AMS) Corporation. Figures 8.18.6 show the following quantities.
(a) A sample of digitized data.
(b) Autocorrelation function.
(c) Power spectrum estimated from an AR(40) model.
(d) Estimated impulse response function.
(e) Estimated step response function.
(f) Estimated ramp response function. 
Table 8.1 gives important information estimated from the signal.

Table 1. Data Processing Information for RTD

\begin{tabular}{|l|l|}
\hline Model Order & 40 \\
Time Constant (sec) & 0.35 \\
Ramp delay time (sec) & 0.43 \\
Sampling interval (sec) & 0.05 \\
No. of data samples & 51,200 \\
\hline
\end{tabular}

Since the accuracy of the estimation of response time characteristics using this approach depends strongly on the properties of the measured signals, this technique must be used only for monitoring the changes in the signal characteristics. 


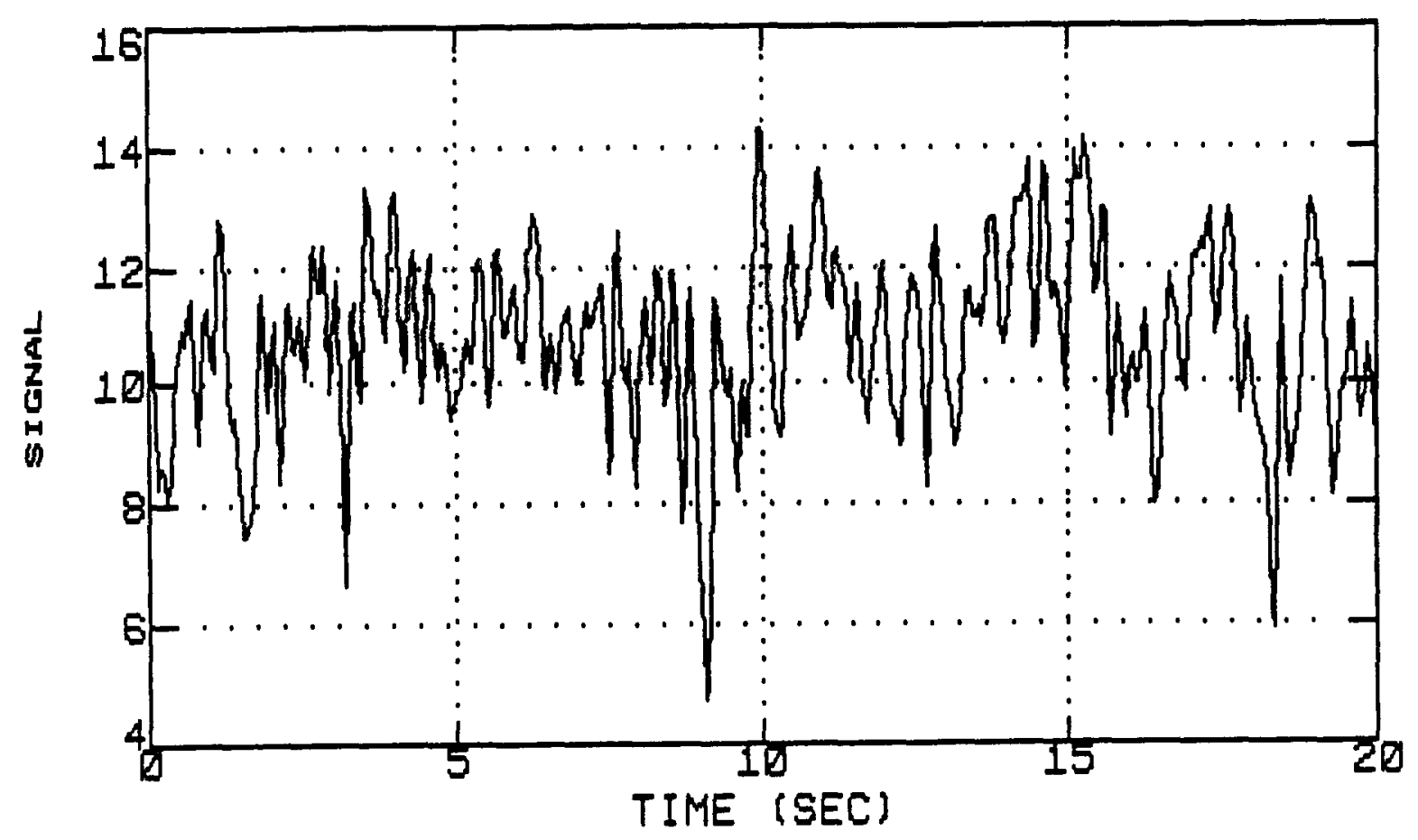

Figure 8.1. A sample plot of the pressure sensor wide-band signal.

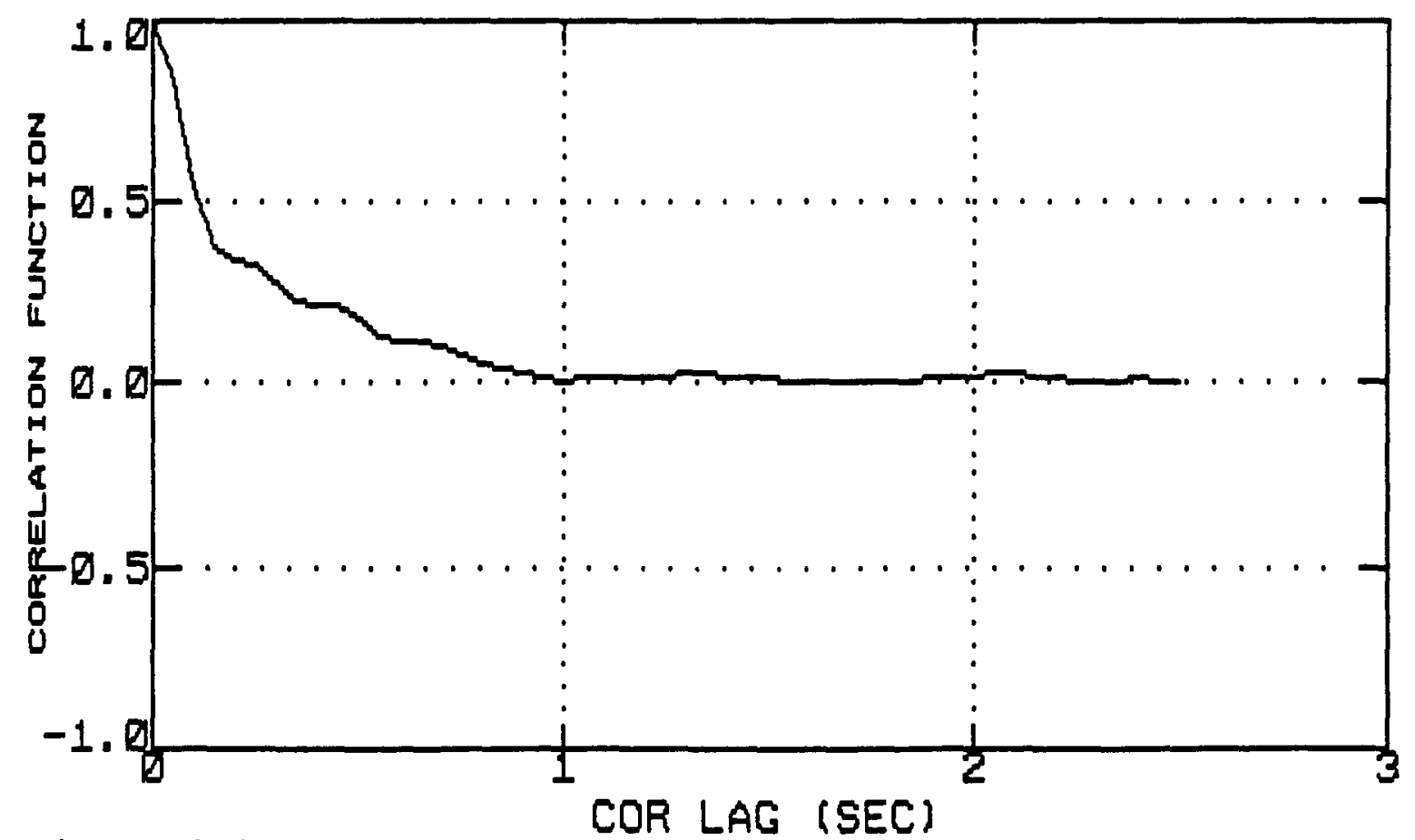

Figure 8.2. Autocorrelatioin function of the pressure sensor data. 


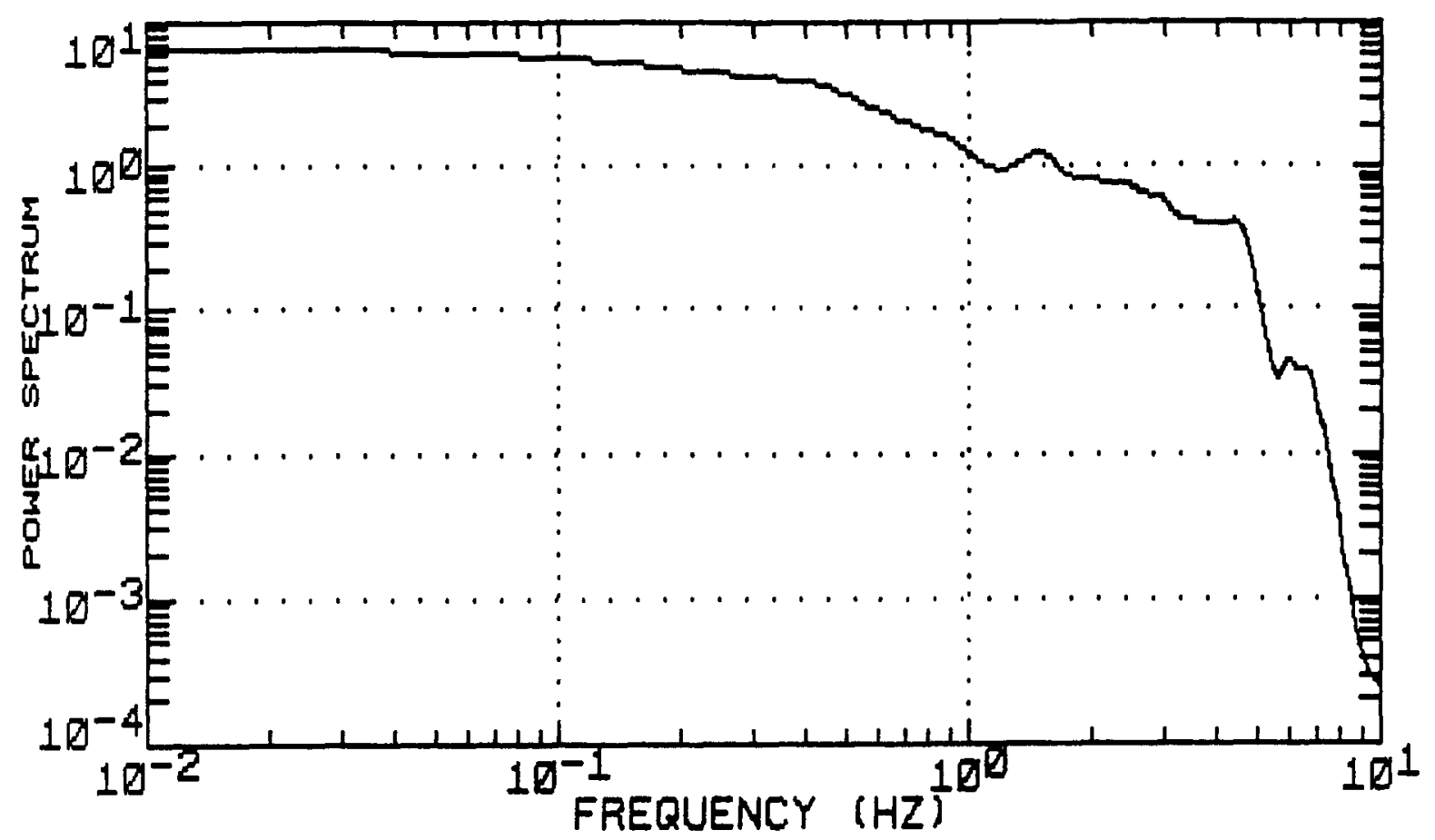

Figure 8.3. Power spectral density function of the pressure sensor signal estimated from a univariate autoregressive model of order $n=40$.

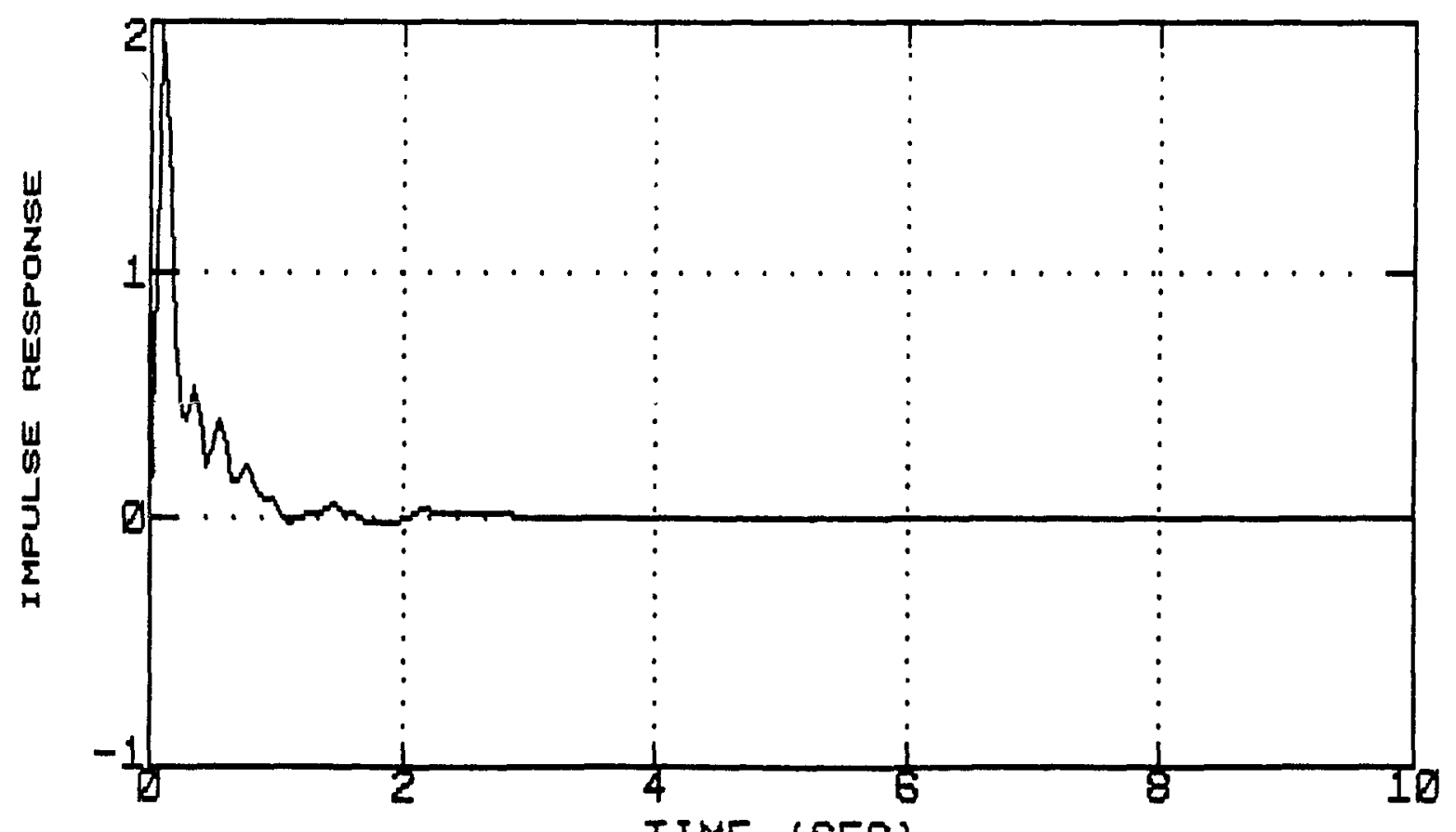

TIME (SEC)

Figure 8.4. Impulse response function of the pressure sensor system as estimated from the univariate autoregressive model of order $n=40$. 


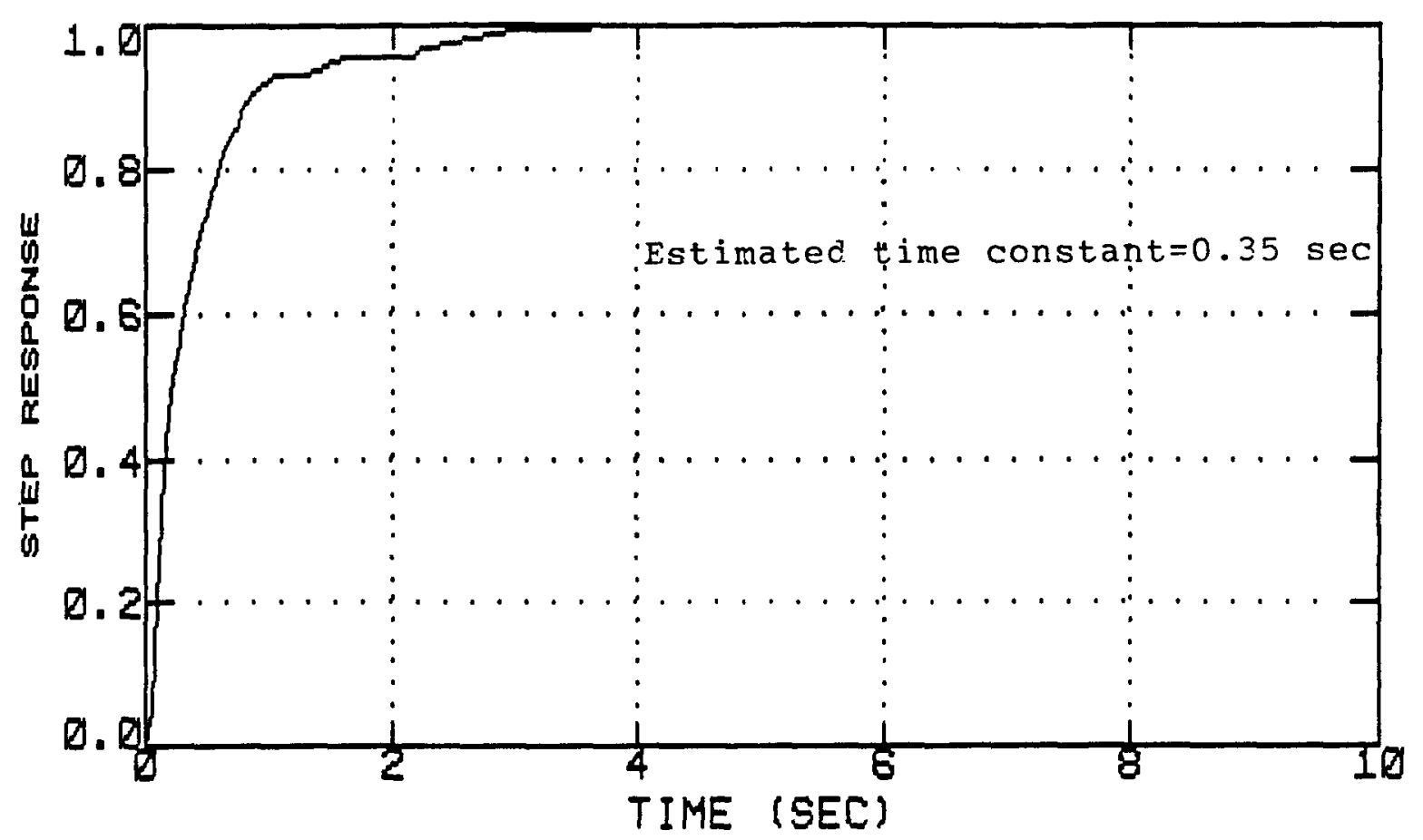

Figure 8.5. Step response function of the pressure sensor system as estimated from the univariate model of oroer $n=40$.

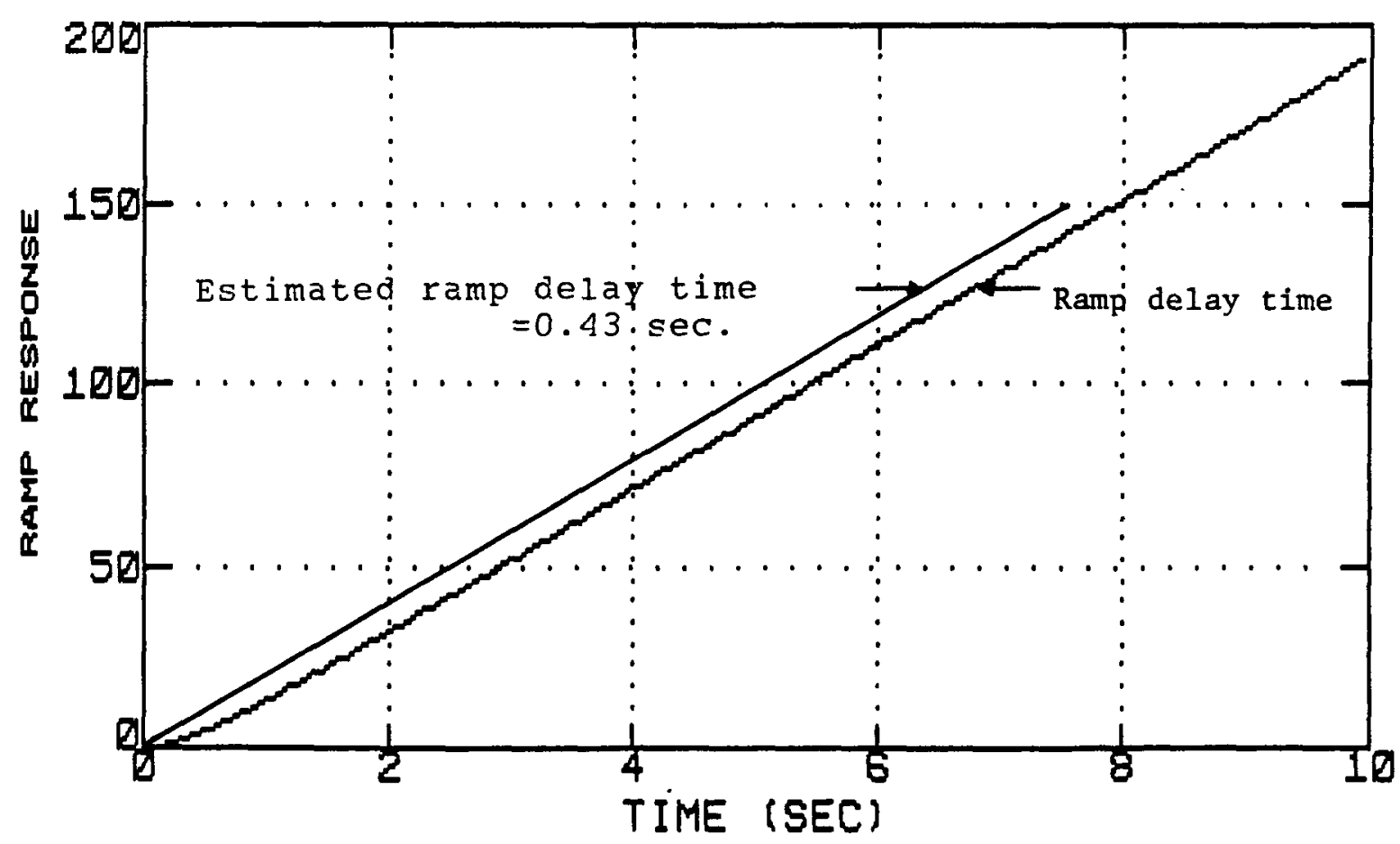

Figure 8.6. Ramp response function of the pressure sensor system as estimated from the univariate model of order $n=40$. 


\section{Section 9}

\section{CONCLUDING REMARKS}

The various signal validation modules implemented in this project are described in detail. Results of applications to data from an operating PWR and the EBR-I are presented. The modular signal validation concept with multiple (and independent) techniques for signal processing are the unique contribution of this research and development program. The signal validation modules may be implemented in various combinations as required for the process under consideration.

Volume 3 of this final report presents a detailed description of the integration of these modules using a system executive. The development and implementation of the system executive, integration of the modules, and the final decision as to the validity of the signals are described in Volume 3 . The architecture of the SVS and the flow of information are also detailed. The practical aspects of implementation are demonstrated using data from a PWR and the EBR-II.

The signal validation technology developed under this program may be applied to other process industry systems. The extensions include instrument calibration verification, predictive maintenance advising, and incorporation of a signalcommand validation unit as part of control systems. Future signal validation approaches will include neural network computing techniques which perform signal prediction based on parallel processing [26]. 


\section{REFERENCES}

1. B. R. Upadhyaya et al., "Development and Testing of an Integrated Signal validation System for Nuclear Power Plants," Annual report prepared for the U. S. Department of Energy, by the University of Tennessee, DOE/NE/3795924, September 1988.

2. B. R. Upadhyaya and T. W. Kerlin, "Development and Testing of an Integrated Signal Validation System for Nuclear Power Plants," Final Report - Volume 1, Executive Summary, Research report prepared for the U.S. Department of Energy, by the University of Tennessee, DOE/NE/37959-34, September 1989.

3. J. C. Deckert et al.,"On-Line Power Plant signal Validation Technique Utilizing Parity Space Representation and Analytic Redundancy," Electric Power Research Institute, Report NP-2110, November 1981.

4. O. L. Deutsch et al., "Development and Testing of a Real-Time Measurement Validation Program," Charles Stark Draper Laboratory, CSDL-R-1592, October 1982.

5. A. S. Willsky, "A Survey of Design Methods for Failure Detection in Dynamic Systems," Automatica, Vol. 12, pp 601-611, 1976.

6. T. T. Chien and M. B. Adams, "A Sequential Failure Detection Technique and Its Applications," IEEE Tran. Aut. Cont., Vol. AC-21, pp 750-757, 1976.

7. F.P. Wolvaardt, B. R. Upadhyaya and O. Glockler, "An Integrated Approach for Sensor Failure Detection in Dynamic Processes," Research report prepared for the University of Tennessee Measurement and Control Engineering Center, Report No. NE-MCEC-BRU-87-01, March 1987.

8. A. Wald, "Sequential Tests of Statistical Hypotheses," Ann. Math. Stat., Vol. 16, pp 117-186, 1945.

9. O. Glockler, B. R. Upadhyaya and T. W. Kerlin, "Signal Validation Algorithms for Consistency Checking and Sequential Probability Ratio Testing of Redundant Measurements," Topical Report prepared for the U.S. Department of Energy, by the University of Tennessee, DOE/NE/37959-6, July 1987.

10. Z. Frei and B. R. Upadhyaya, "Empirical Modeling of Steady-State Behavior of Linear and Nonlinear Systems with Application to Signal Validation," Topical report prepared for the U. S. Department of Energy, by the University of Tennessee, DOE/NE/37959-11, August 1987.

11. B. R. Upadhyaya, K. E. Holbert and E. Eryurek, "Automated Generation of Nonlinear System Characterization for Sensor Failur Detection," Proc. 35th International Instrumentation Symp., Orlando, FL, pp 269-274, May 1989.

12. A. Desrochers and S. Mohseni, "On Determining the Structure of a Nonlinear System," Intl. J. of Control, Vol. 40, No. 5, pp 923-938, 1984.

13. K. E. Holbert and B. R. Upadhyaya, "Multi-Dimensional Process Hypercube for Signal Validation," Topical Report prepared for the U. S. Department of Energy,by the University of Tennessee, DOE/NE/37959-33, July 1989.

14. D. V. Hinkley, "Inference About the Change Point From Cumulative Sum Test," Biometrika, Vol. 58, No. 3, pp 509-523, 1971. 
15. V. M. Morgenstern, B. R. Upadhyaya and O. Glockler, "Signal Anomaly Detection and Characterization," Topical Report prepared for the U.S. Department of Energy, by the University of Tennessee, DOE/NE/37959-18, August 1988.

16. S. Wilks, Mathematical Statistics, John Wiley, New York, 1963.

17. H. M. Hashemian, T. W. Kerlin and B. R. Upadhyaya, "Apparatus for Measuring the Degradation of a Sensor Time Constant," U. S. Patent No. $4,295,128$, October $13,1981$.

18. S. L. Marple, Jr., Digital Spectral Analysis with Applications, Prentice-Hall, Englewood Cliffs, NJ, 1987.

19. O. Glockler and B. R. Upadkyaya, "Results and Interpretation of Multivariate Autoregressive Analysis Applied to Loss-of-Fluid-Test Reactor Process Noise Data," SMORN-V, Progress in Nuclear Energy, Pergamon Press, Vol.21, pp 447-456, 1988.

20. B. R. Upadhyaya, M. Kitamura and T. W. Kerlin, "Multivariate Signal Analysis Algorithms for Process Monitoring and Parameter Estimation in Nuclear Reactors," Annals of Nuclear Energy, Pergamon Press, Vol. 7, pp 1-11, 1980 .

21. B. R. Upadhyaya and T. W. Kerlin, "Estimation of Response Time Characteristics of Platinum Resistance Thermometers by the Noise Analysis Technique," ISA Transactions, Vol. 17, pp 21-38, 1978.

22. B. R. Upadhyaya and M. Kitamura, "Stability Monitoring of Boiling Water Reactors by Time Series Analysis of Neutron Noise," Nuclear Science and Engrg., Vol. 77, No. 4, pp 480-492, 1981.

23. S. M. Kay, Modern Spectral Estimation, Prentice-Hall, Englewood Cliffs, NJ, 1988.

24. J.T. Tou and R. C. Gonzalez, Pattern Recognition Principles, Addison-Wesley, Reading, Massachusetts, 1979.

25. O. Glockler, B. R. Upadhyaya and V. Morgenstern, "Generalized Consistency Checking of Multivariable Redundant Measurements (GCCM) and CommonMode Failure Detection," Research Report prepared for the U.S. Department of Energy, by the University of Tennessee, DOE/NE/37959-26, January 1989.

26. B. R. Upadhyaya, E. Eryurek and G. Mathai, "Application of Neural Computing Paradigms for Signal Validation," Proc. Seventh Power Plant Dynamics, Control \& Testing Symposium, Vol. 1, pp 27.01-27.18, May 1989. 